DOE/EIA-8558-6/1

5 Vol. 6 of 6

Order-No. 522/6

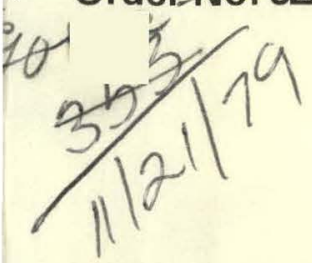

\title{
The Integrating Model of the Project Independence Evaluation System. \\ Volume VI + Data Documentation. Part I
}

February 1979

Prepared for:

U.S. Department of Energy

Energy Information Administration

Assistant Administrator for Applied Analysis

Under Contract No. EC-77C-01-8558 


\section{DISCLAIMER}

This report was prepared as an account of work sponsored by an agency of the United States Government. Neither the United States Government nor any agency Thereof, nor any of their employees, makes any warranty, express or implied, or assumes any legal liability or responsibility for the accuracy, completeness, or usefulness of any information, apparatus, product, or process disclosed, or represents that its use would not infringe privately owned rights. Reference herein to any specific commercial product, process, or service by trade name, trademark, manufacturer, or otherwise does not necessarily constitute or imply its endorsement, recommendation, or favoring by the United States Government or any agency thereof. The views and opinions of authors expressed herein do not necessarily state or reflect those of the United States Government or any agency thereof. 


\section{DISCLAIMER}

Portions of this document may be illegible in electronic image products. Images are produced from the best available original document. 
Available from:

National Technical Information Service (NTIS)

U.S. Department of Commerce

5285 Port Royal Road

Springfield, VA 22161

Price:

Printed Copy: $\$ 11.50$

Microfiche: $\quad \$ 3.00$ 


\section{The Integrating Model of the Project Independence Evaluation System. Volume VI, $\frac{\%}{T}$ Data Documentation. Part I}

February 1979

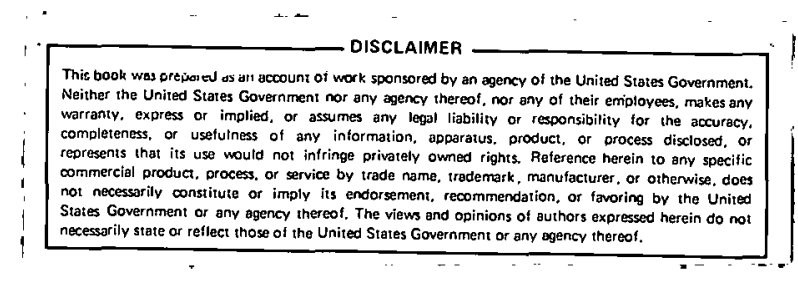

Prepared by:

Brenda J. Allen.

Logistics Management Institute

Washington, D.C. 20016

Under Contract No. EC-77C-01-8558

Prepared for:

U.S. Department of Energy

Energy Information Administration

Assistant Administrator for Applied Analysis

Office of Integrative Analysis

Mid-Terrin Analysis Division

Washington, D.C. 20461

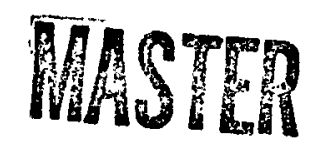


This report was prepared under Department of Energy (DOE) Contract Number EC-77C-01-8558 and does not necessarily state or reflect the views, opinions, or policies of the DOE or the Federal Government. References to trade names or specific commercial products, commodities, or services in this report do not represent or constitute an endorsement, recommendation, or favoring by DOE of the specific commercial product, commodity, or service.

Released for printing October 3, 1979. 
This contractor report was prepared by Logistics Management Institute and provides documentation of the version of the Project Independence Evaluation System (PIES) as it existed on January 1, 1978. Since that date, PIES has evolved into what is now called the Mid Range Energy Market llodel (MEMM), a major component of the liid Range Energy, Forecasting system (MEFS). Major structural changes that have occurred between January 1, 1978 and January 1, 1979, are documented in the supplemental volume entitled, "Revisions to the Midterm Energy Market Model Relating to Natural Gas Regulation, Advanced Technologies, Coal Demand and Dynamics." Together, the six volume set of documentation, plus the supplemental volume on revisions, form the most comprehensive and up-todate version of MEMM documentation currently available, documentation which significantly surpasses in both form and content the single volume published in January, 1977.

Although this documentation has not gone through the appropriate review process and clearance procedures to be published as an EIA endorsed report, it is currently being made available in its present form as an interim measure to satisfy many outstanding requests for MEMM documentation. As this report has not been submitted to comprehensive review, EIA does not endorse any information contained herein. The documentation is presently being subjected to comprehensive review both inside and outside the Department of Energy. A contract is currently in process to update it to the version used for the 1978 Annual Report to Congress (published in July 1979). This new version of the documentation report is intended to bring the MEMM documentation into conformity with EIA's documentation standards and to respond to any issues raised as a result of the review process. The results of the latter effort will result in a set of MEMM documentation fully cleared and endorsed by EIA, available in 1980. 


\section{PREFACE}

This documentation describes the Project Independence Evaluation System (PIES) Integrating Model as it existed on January 1, 1978. The complete documentation consists of six volumes describing the various aspects of the Integrating Model as follows:

- Volume I is an executive summary, providing a simple, nontechnical overview of PIES.

- Volume II is a primer, describing and illustrating the basic inputs to the PIES algorithm.

- Volume III is a user's guide, describing scenario specification and the operational procedures for running the Integrating Model.

- Volume IV is the main model documentation, describing the theoretical basis of the Integrating Model and each of the supply submudels.

- Volume $V$ is code documentation, describing the data processing aspects of PIES: the data flow through the PlES programs, the functions performed by exich program, the data inputs and outputs, and the PIES naming conventions.

- Volume VI is data documentation, containing the standard table data used for the Administrator's Report at the beginning of 1978, along with primary data sources and the office responsible. It also contains a copy of a PIES Integrating Model Report with a description of its contents.

The data and scenarios used in these volumes are those used in the 1977 Annual

Report to Congress, (actually published in 1978), prepared by the Energy Information Administration. In all volumes, we refer to this report as the Administrator's Annual Repurl (AAR). 
PREFACE

LIST OF FIGURES ............................. . . . v

LIST OF TABLES. . . . . . . . . . . . . . . . . . . . v v

LIST OF DATA TABLES IN CHAPTER II . . . . . ........ . vi

\section{PART I}

\section{CHAPTER}

I. OVERVIEW ............................. I- 1

Introduction . . . . . . . . . . . . . . . . I I- 1

Supply Data . . . . . . . . . . . . . . . . . . I- 1

EIA Projection Series and Scenarios . . . . . . . . . I- 2

Demand Data and Assumptions . . . . . . . . . . . . I 6

Supply Assumptions - Oil and Gas Availabilities. . . . . . . . I- 8

II. SUPPLY AND DEMAND DATA TABLES AND SOURCES FOR

THE MID-RANGE SCENARIO FOR TARGET YEARS

1985 AND 1990

II- 1

Introduction . . . . . . . . . . . . . . . . . II- 1

Demand, Price and Elasticity Data . . . . . . . . . . II- 1

Coal Data . . . . . . . . . . . . . . . . . . . II- 4

Imports Data. ... . . . . . . . . . . . . . . . . II-113

Oil and Gas Data . . . . . . . . . . . . . . . . III-126

Refineries Data. . . . . . . . . . . . . . . . . III-168

Synthetics, Shale and Solar/Geothermal Data . . . . . . . . . II-194

Transportation Data . . . . . . . . . . . . . . . III-202

Utilities Data . . . . . . . . . . . . . . . . II-217

\section{PART II}

III. ALTERNATE SCENARIOS .. . . . . . . . . . . . . . III- 1

Introduction . . . . . . . . . . . . . . . . . . III- 1 Low and High Demand Data. . . . . . . . . . . . . . . III- 1 Low and High Oil and Gas Supply Data . . . . . . . . . . III- 52 Refinery and Oil and Gas Data Assuming a 5 Percent Annual Increase in Real World Oil Prices . . . . . . . . . . . III-129 
IV. THE PIES INTEGRATING MODEL REPORT . . . . . . . . . . IV- 1

Introduction . . . . . . . . . . . . . . . . IV- 1

Organization of the PIES Report. . . . . . . . . . . . . IV- 1

Explanation of the Tables . . . . . . . . . . . . . . . . IV- 2

TABLE 1: Executive Data Summary in Standard Units . . . . IV- 1

TABLE 2: Executive Data Summary (TBTU) . . . . . . . IV- 5

TABLE 3: Supply and Consumption Growth Rates from 1975 . IV- 8

TABLE 4: Import Summary ................ IV- 8

TABLE 5: Oil and Gas Production ............. . IV- 8

TABLE 6: Domestic Crude Production by Region . . . . . . IV- 10

TABLE 7: Crude Regional Distribution Summary . . . . . . III- 10

TABLE 8: Gas Regional Distribution Summary . . . . . . . III- 11

TABLE 9: Gas Regional/Sectoral Demand in BCF/YR . . . . III- 11

TABLE 10: Coal Regional Production Summary by Mine Type . IV- 12

TARTE 11: Copl Regional Production Summary by Coal Type. IV- 1.3

TABLE 12: Coal Regional Demand Summary. . . . . . . . IV- 13

TABLE 13: Sectoral/Regional Consumption. . . . . . . . . IV- 14

TABLE 14: Sectoral/Regional BTU Consumption Growth Rates IV- 14

TABLE 15: Regional Consumption Summary . . . . . . . IV- 15

TABLE 16: Refinery Consumption. . . . . . . . . . . IV- 15

TABLE 17: Refinery Production Summary . . . . . . . . . IV- 15

TABLE 18: Refinery Production Summary in TBTU per Year . IV- 16

TABLE 19: Regional Utility Consumption . . . . . . . . IV- 16

TABLE 20: Electricity Generation Summary . . . . . . . . IV- 17

TABLE 21: Price Summary in 1978 \$/Standard Physical Unit . IV- 18

TABLE 22: Regional Price Summary in 1978 \$/MMBTU . . . . IV- 18

TABLE 23: Demand Region Retail BTU Price Growth Rates. - IV- 18

TABLE 24: Demand Region Price Table . . . . . . . . . . IV- 18

TABLE 25: Utility Region Prices... . . . . . . . . . IV- 19

TABLE 26: Refinery Region Prices .............. IV- 19

TABLE 27: Oil Region Wellhead Prices ............ IV- 19

TABLE 28: Regional Gas Wellhead Prices . . . . . . . . IV 20

TABLE 29: Coal Region F.O.B. Mine Prices ........ IV $=20$

TABLE 30: Executive Data Summary - 1975. . . . . . . . IV- 20

TABLE 31: Sectoral/Regional Consumption Summary . . . . . IV- 20

TABLE 32: Demand Regional Retail Prices 1975 ....... . IV- 21 
PIES Preprocessors . . . . . . . . . . . . . .

I-2 EIA Projection Series A Through F . . . . . . . .

I-3 Variations in Projected Levels of U.S. Energy Consumption and Production by EIA Projection Series A Through F . . . . . . I- 9

I-4 Projected U.S. Petroleum Liquid Supply . . . . . . . . . I- 9

II-1 DOE Regions. . . . . . . . . . . . . . . . . II- 3

II-2 PIES Coal Regions .................... . II- 30

II-3 Oil Supply Regions . . . . . . . . . . . . . . . . II-127

II-4 NPC Regions. . . . . . . . . . . . . . . . . II-128

II-5 Natural Gas Supply Regions . . . . . . . . . . . . . . II-129

II-6 PIES Refinery Regions . . . . . . . . . . . . . . II-169

TABLE

\section{LIST OF TABLES}

PART I

I-1 ElA Projectioñ Series Assumptions . . . . . . . . . . . I- 5

I-2 Sectors Affected by Alternate Scenarios . . . . . . . . . I- 6

I-3 Major Assumptions Underlying DRI Macroeconomic Forecasts . . . I- 7

I-4 U.S. Petroleum Liquids Demand, Supply and Imports (Millions of Barrels per Day) . . . . . . . . . . . I I 11

I-5 U.S. Natural Gas Demand, Supply and Imports (Trillions of Cubic Feet per Year) ........... I- 12

I-6 1985 Energy Supply/Demand Balance (Quadrillion BTU per year) . . . . . . . . . . . . I I- 13

II-1 Correspondence Between Oil Import Tables

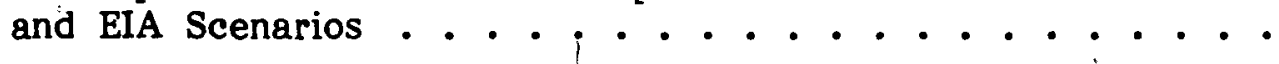

PART II

IV-1 Physical Units in the PIES Report . . . . . . . . . . IV- 3

IV-2 Conversion Factors . . . . . . . . . . . . . . IV- 6

IV-3 Abbreviations for Sectoral Demand. . . . . . . . . . . IV- 22 
DEMAND, PRICE, AND ELASTICITY DATA - LOW-SCENARIO

1 Demand Points in Trillions of Btu's for 1985 . . . . . . . . II- 6

2 Demand Points in Trillions of Btu's for $1990 \ldots . . . . . .$. II- 7

3 Initial Prices in Dollars/Standard Unit for 1985 .......... II- 8

4 Initial Prices in Dollars/Standard Unit for 1990 .......... II- 9

ELASTICITIES: TARGET YEAR 1985

5 Unscaled Elasticities for Demand Region 1 . . . . . . . II- 10

6 Unsealed Elasticities for Demand Region 2 ........... II- 11

7 Unscaled Elasticities for Demand Region 3 . . . . . . . . II- 12

8. Unscaled Elasticities for Demand Region 4 ......... II- 13

9 Unscaled Elasticities for Demand Region 5 . . . . . . . . . II- 14

10 Unscaled Elasticities for Demand Region 6 . . . . . . . . II- 15

11 Unscaled Elasticities for Demand Region $7 \ldots \ldots$....... II- 16

12 Unscaled Elasticities for Demand Region 8 .......... II- 17

13. Unscaled Elasticities for Demand Region 9 . . . . . . . . . II- 18

14 Unscaled Elacticities for Demand Region $10 \ldots \ldots$. . . . . II- 19

ELASTICITIES: TARGET YEAR 1990

15 Unscaled Elasticities for Demand Region 1 . . . . . . . . II- 20

16 Unscaled Elasticities for Demand Region 2 . . . . . . . II- 21

17 Unscaled Elasticities for Demand Region 3 . . . . . . . . II- 22

18 Unscaled Elasticities for Demand Region $4 \ldots . . . . .$. II- 23

19 Unscaled Elasticities for Demand Region 5 ........... II- 24

20 Unscaled Elasticities for Demand Region 6 ........... II- 25

21 Unscaled Elasticities for Demand Region $7 \ldots \ldots$. . . . . II- 26

22 Unscaled Elasticities for Demand Region 8 . . . . . . . . II- 27

23 Unscaled Elasticities for Demand Region 9 . . . . . . . . . II- 28

24 Unscaled Elasticities for Demand Region $10 \ldots . . . . .$. II- 29

COAL DATA - MID-RANGE SCENARIO

TARGET YEAR: 1985

1 Northern Appalachia, High Btu, High Sulfur . . . . . . . . II- 35

2 Northern Appalachia, Medium Btu, High Sulfur. . . . . . . II- 36

3 Northern Appalachia, High Btu, Medium Sulfur ......... II- 37

4 Northern Appalachia, Medium Btu, Medium Sulfur . . . . . . . II- 38

5 Northern Appalachia, Metallurgical Coal, Low Sulfur ....... II- 39

6 Northern Appalarchia, High Btu, Low Sulfur . . . . . . . . . II- 40

7 Central Appalachia, High Btu, High Sulfur ............ II- 41

8 Central Appalachia, High Btu, Medium Sulfur ......... II- 42

9 Central Appalachia, Metallurgical Coal, Low Sulfur....... II- 43

10 Central Appalachia, High Btu, Low Sulfur .......... II- 44 
11 Southern Appalachia, High Btu, Medium Sulfur . . . . . . . . Southern Appalachia, Metallurgical Coal, Low Sulfur . . . . . .

Midwest, High Btu, High Sulfur . . . . . . . . . . . . .

II- 47

Midwest, Medium Btu, High Sulfur ............... II- 48

Midwest, High Btu, Medium Sulfur . . . . . . . . . . . .

II- 49

Midwest, Medium Btu, Medium Sulfur .............. II- 50

Central West, High Btu, High Sulfur . . . . . . . . . . .

II- 51

Central West, Medium Btu, High Sulfur .. . . . . . . . . ${ }_{-}$II- 52

Central. West, High Btu, Medium Sulfur . . . . . . . . . . .

II- 53

Central West, Sub-Bituminous, Medium Sulfur . . . . . . . . . II- 54

Central West, Metallurgical Coal, Low Sulfur . . . . . . . . .

II- 55

Gulf, Lignite, Medium Sulfur . . . . . . . . . . . . . . .. •

II- 56

Eastern Northern Great Plains, Lignite, Medium Sulfur . . . . . . II- 57

Eastern Northern Great Plains, Lignite, Low Sulfur.

II- 58

Western Northern Great Plains, Medium Btu, Medium Sulfur . . . . II- 59

Western Northern Great Plains, Sub-Bituminous

Medium Sulfur

Western Northern Great Plains, Medium Btu

II- 60

Western Northern Great Plains, Sub-Bituminous, Low Sulfur .

II- 61

Rockies, High Btu, Medium Sulfur

II- 62

Rockies, Medium Btu, Medium Sulfur . . . . . . . . . . . .

Rockies, Metallurgical Coal, Low Sulfur . . . . . . . . . . .

Rockies, High Btu, Low Sulfur . . . . . . . . . . . . . . .

Rockies, Medium Btu, Low Sulfur . . . . . . . . . . . . .

Southwest, Medium Btu, Medium Sulfur . . . . . . . . . . .

Southwest, Medium Btu, Low Sulfur . . . . . . . . . . . .

Northwest, Sub-Bituminous, Medium Sulfur . . . . . . . . . .

Alaska, Sub-Bituminous, Low Sulfur . . . . . . . . . . .

II- 63

II- 64

II- 65

II- 66

II- 67

II- 68

II- 69

II- 70

II- 71

38

Total Coal Supply at the Highest Price Step

II- 72

COAL DATA - MID-RANGE SCENARIO

TARGET YEAR: 1990

1 Northern Appalachia, High Btu, High Sulfur . . . . . . . . . .

II- 7.5

Northern Appalachia, Medium Btu, High Sulfur . . . . . . . . . II- 76

3 Northern Appalachia, High Btu, Medium Sulfur . . . . . . . . .

II- 77

Northern Appalachia, Medium Btu, Medium Sulfur . . . . . . .

II- 78

Northern Appalachia, Metallurgical Coal, Low Sulfur . . ... . . :

II- 79

Northern Appalachia, High Btu, Low Sulfur . . . . . . . . .

II- 80

Central Appalachia, High Btu, High Sulfur . . . . . . . . . . II- 81

Central Appalachia, High Btu, Medium Sulfur . . . . . . . . . II- 82

Central Appalachia, Metallurgical Coal, Low Sulfur . . . . . .

II- 83

Central Appalachia, High Btu, Low Sulfur . . . . . . . . . . .

II- 84

Southern Appalachia, High Btu, Medium Sulfur . . . . . . . . . II- 85

Southern Appalachia, Metallurgical Coal, Low Sulfur . . . . . .

II- 86

Midwest, High Btu, High Sulfur . . . . . . . . . . . . II- 87

Midwest, Medium Btu, High Sulfur . . . . . . . . . . . .

II- 88

Midwest, High Btu, Medium Sulfur . . . . . . . . . . II- 89

Midwest, Medium Btu, Medium Sulfur

II- 90 
18 Central West, Medium Btu, High Sulfur . . . . . . . . . . Gulf, Lignite, Medium Sulfur . . . . . . . . . . . . . . Eastern Northern Great Plains, Lignite, Medium Sulfur . . . . . .

II-, 96

II- 97 Eastern Northern Great Plains, Lignite, Low Sulfur . . . . . . . •

II- 98 Western Northern Great Plains, Medium Btu, Medium Sulfur.: . . - II- 99 Western Northern Great Plains, Sub-Bituminous, Medium Sulfur. . - II-100 Western Northern Great Plains, Medium Btu, Low Sulfur . . . . . II-101 Western Northern Great Plains, Sub-Bituminous, Low Sulfur . . . . II-102 Rockies, High Btu, Medium Sulfur. . . . . . . . . . . . II-103 Rockies, Medium Btu, Medium Sulfur . . . . . . . . . . . II-104 -Rockies, Metallurgical Coal, Low Sulfur . . . . . . . . . . . II-105 Rockies, High Btu, Low Sulfur . . . . . . . . . . . . . II-106 Rockies, Medium Btu, Low Sulfur . . . . . . . . . . . . II-107 Suuthwest, Medium Btu, Medium Sulfur . . . . . . . . . . II-108 Southwest, Medium Btu, Low Sulfur .... . . . . . . . . . II-109 Northwest, Sub-Bituminous, Medium Sulfur . . . . . . . . . . II-110 Alaska, Sub-Bituminous, Low Sulfur . . . . . . . . . . . . . . II-111

38 Total Coal Supply at the Highest Price Step . . . . . . . . . . . II-112

\section{IMPORTS DATA - MID-RANGE SCENARIO}

1.1 Crude Oil Import Bundles, 1985, Net Product

Import Level 2.6 MMB/CD . . . . . . . . . . . . . .

1.2 Oil Product Imports, 1985, Net Product Import

2.1 Crude Oil Import Bundles, 1985, Net Product

2.2 Oil Product Imports, 1985, Net Product

3.1 Crude Oil Import Bundles, 1985, Net Product

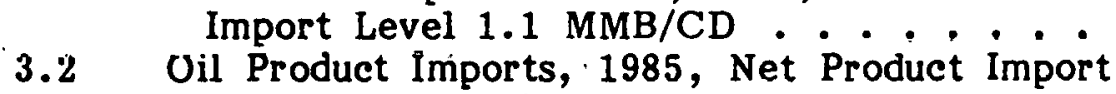

3.3 Oil Product, Exports, 1985 , Net Product Import

4.1 Crude Oil Import Bundles, $198 \dot{5}^{\text {, Net Product }}$

II-118

İmport Level $.8 \mathrm{MMB} / \mathrm{CD}$. . . . . . . . . . . . . . . . . .

II -118

II-119

4.2 Oil Product Imports, 1985, Net Product

4.3 Oil Product Exports, 1985, Net Product Import

5.1 Crude Oil Import Bundles, 1990, Net Product Import Level 3.0 MMB/CD . . . . . .

5.2 Oil Product Imports, 1990, Net Product Import Level 3.0 MMB/CD . 
6.1 Crude Oil Import Bundles, 1990, Net Product

Import Level 2.3 MMB/CD . . . . . . . . . . . . . II-121

6.2 Oil Product Imports, 1990, Net Product Import

Level $2.3 \mathrm{MMB} / \mathrm{CD}$. . . . . . . . . . . . . . . II-121

7.1 Crude Oil Import Bundles, 1990, Net Product

Import Level $1.1 \mathrm{MMB} / \mathrm{CD}$. . . . . . . .

7.2 Oil Product Imports, 1990, Net Product Import

Level $1.1 \mathrm{MMB} / \mathrm{CD}$. . . . . . . . . . . . . . . . . III-122

7.3 Oil Product Exports, 1990, Net Product Level

$1.1 \mathrm{MMB} / \mathrm{CD}$. . . . . . . . . . . . . . . . . . . II-122

8.1 Crude Oil Import Bundles, 1990, Net Product

Import Level, 1.0 MMB/CD ..................

8.2 Oil Product Imports, 1990, Net Product Import

Level, $1.0 \mathrm{MMB} / \mathrm{CD}$..................... III-123

8.3 Oil Product Exports, 1990, Net Product Import

Level $1.0 \mathrm{MMB} / \mathrm{CD}$...................... II-123

9 Natural Gas Imports for 1985 . . . . . . . . . . . . . . . II-124

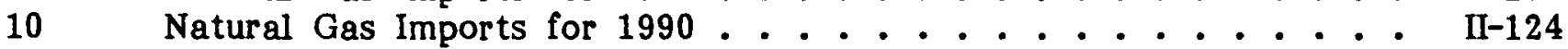

11 Coal Exports for 1985 and 1990 . . . . . . . . . . . . II-125

OIL AND GAS DATA - MID-RANGE SCENARIO

1 Price Trajectories for Oil and Gas Liquids . . . . . . . . . . . .

Price Trajectories for Associated and Dissolved Gas . . . . . . . . .

II-134

Oil Supply Curves for 1985 . . . . . . . . . . . . . . . III-135

Oil Supply Curves for 1990 . . . . . . . . . . . . . . II-138

Price Trajectories for Nonassociated Gas . . . . . . . . . . . II-141

Price Trajectories for Natural Gas Liquids . . . . . . . . . . . II-141

Gas Supply Curves for 1985 . . . . . . . . . . . . . . II-142

Gas Supply Curves for 1990 . . . . . . . . . . . . . . II-146

Distribution of Interstate Gas in 1985 . . . . . . . . . . . . II-150

Interstate Gas Deliveries in 1985 . . . . . . . . . . . . . II-151

Distribution of Interstate Gas in 1990 . . . . . . . . . . II-152

Interstate Gas Deliveries in 1990 . . . . . . . . . . . . . II-153

Oil Well Drilling, Investment and Production Summary for 1985 . . II-154

Oil Well Drilling, Investment and Production Summary for 1990 . . II-155

Nonassociated Interstate Gas Production Summary in 1985 . . . . . II-156

Nonassociated Interstate Gas Production Summary in 1990 . . . . . II-157

Nonassociated Intrastate Gas Production Summary in 1985 . . . . . II-158

Nonassociated Intrastate Gas Production Summary in 1990 . . . . . II-159

Capital Investment Summary for Gas Wells from 1977 thru 1984 . . II-160

Capital Investment Summary for Gas Wells from 1977 thru 1989 . . II-161

Drilling Summary for Gas Wells from 1977 thru 1984 . . . . . . . II-162

Drilling Summary for Gas Wells from 1977 thru 1989 . . . . . . . II-163

Oil and Gas Supply at the Highest Price Steps in 1985 . . . . . . II-164

Oil and Gas Supply at the Highest Price Steps in 1990 . . . . . . II-165

Breakdown of Regional Production by Crude Type. . . . . . . . . II-166

Splitting Factors for Intrastate Associated and Dissolved Gas . . . II-166

Splitting Factors for Intrastate Coproducts . . . . . . . . . II-167 
1 Price Trajectories for Oil and Gas Liquids . . . . . . . . . . . .

2 Barrels of Refinery Products Produced per Barrel

3 Relative Product Pricing Relationships . . . . . . . . . . . . . . II-179

4 Refinery Capacity Data . . . . . . . . . . . . . . II-181

TARGET YEAR: 1990

1 Refinery Costs for Base Yields . . . . . . . . . . . . . .

II-183

2 Barrels of Refinery Products Produced per Barrel of Crude Consumed. . . . . . . . . . . . . . . III-184

3 Relative Product Pricing Relationships .............. II-191

4 Refinery Capacity data . . . . . . . . . . . . . II-193

SYNTHETICS DATA - MID-RANGE SCENARIO

TAKGET YEAR: 1985

1 Production of Synthetic Crude from Coal by Coal Region . . . . . II-196

2 Production of High Btu Gas from Coal by Coal Region . . . . . . II-196

3 Production of Low and Medium Btu Synthetic Gas

from Coal by Utility Region. . . . . . . . . . . . . .II-197

4 Production of Synthetic Gas from Sources Other Than

Coal by Utility Region . . . . . . . . . . . . . . II-197

TARGET YEAR: 1990

1 Production of Synthetic Crude from Coal by Coal Region . . . . . II-198

2 Production of High Btu Gas from Coal by Coal Region . . . . . . II-198

3 Production of Low and Medium Btu Synthetic Gas from

Coal by Utility Region . . . . . . . . . . . . . . . II-199

4 Production of Synthetic Gas from Sources Other Than

Coal by .Demand Region. . . . . . . . . . . . . . . II-199

SHALE DATA - MID-RANGE SCENARIO

1 Shale Input Data . . . . . . . . . . . . . . . . II-200

SOLAR/GEOTHERMAL DATA - MID-RANGE SCENARIO

1 Geothermal . . . . . . . . . . . . . . . . . . . II-201

2 Wind . . . ... . . . . . . . . . . . . . . . II-201

3 Solar Thermal . . . . . . . . . . . . . . . . . . II-201

4 Solar Voltaic. . . . . . . . . . . . . . . . . . II-201 
1 Coal Transportation Costs. . . . . . . . . . . . .

2 Fraction of Coal Demand in Each DOE Region That Is Met from Representative Cities ............. II-206

3 Transportation Costs of Domestic Natural Gas . . . . . . . . II-208

4 Transportation Costs for Imported Natural Gas . . . . . . . II-208

5 Loss Factors for Natural Gas . . . . . . . . . . . . II-209

6 Costs of Transporting Crude Oil by Pipeline . . . . . . . II-211

7 Costs of Transporting Domestic Crude Oil by

Barge and Tanker................ II-211

8 Transportation Costs for Foreign Crude Oil . . . . . . . . II-212

9 Transportation Costs for Alaskan Crude Oil. . . . . . . . . II-212

10 Costs of Transporting Petroleum Products by Pipeline . . . . . . II-214

11 Costs of Transporting Domestic Petroleum Products

(Except Residual) by Barge and Tanker ........... II-214

12 Costs of Transporting Imported Petroleum Products

(Except Residual) by Barge and Tanker .......... II-215

13 Costs of Transporting Domestic Residual Fuel by Barge

and Tanker....................... II-216

14 Costs of Transporting Imported Residual Fuel by Barge

and Tanker................... II-216

UTILITIES DATA - MID-RANGE SCENARIO

TARGET YEAR: 1985

1 Load-Duration Curve Data for 1985 and $1990 \ldots \ldots$. . . . . .

II-220

Capacity Factors for DOE Region 1 (New England) . . . . . . . II-222

Capacity Factors for DOE Region 2 (New York/New Jersey) . . II-223

Capacity Factors for DOE Region 3 (Mid-Atlantic) . . . . . . II-224

Capacity Factors for DOE Region 4 (South Atlantic) ...... II-225

Capacity Factors for DOE Region 5 (Midwest) . . . . . . . II-226

Capacity Factors for DOE Region 6 (Southwest) . . . . . . . II-227

Capacity Factors for DOE Region 7 (Central) . . . . . . . . II-228

Capacity Factors for DOE Region 8 (North Central) . . . . . II-229

Capacity Factors for DOE Region 9 (West) . . . . . . . . II-230

Capacity Factors for DOE Region 10 (Northwest) . . . . . . II-231

2 Existing Capacity. . . . . . . . . . . . II-232

3 Existing Capacities Which May Be Converted to $\ldots$. . . . . II-233

4 Retirement Rates for Fossil-Fuel-Fired Plants ......... II-234

5 Committed Capacity for 1985 and 1990 . . . . . . . . . . II-234

6 Deferrable Capacity for $1985 \ldots . . . . . . . . . \quad$ II-235

7 New Plant Capacity Limits (1985).............. II-235

8 Operation and Maintenance Costs ............. II-236

9 Revenuc Requirements in 1985 for Fxisting Assets ........ II-237

10 Capital Costs of New Plants in 1985............ II-237

11 Capital Charge Rates (Percent). for Revenue Requirements . . . II-238

12 Capital Charge Rates for Investment Decisions . . . . . . II-239 
13 Transmission and Distribution Data............ II-240

14 Heat Rates for Existing Equipent Operated in Base Load .... . II-241

15 Heat Rates for Existing Equipment Operated in Cycling Load . . . II-241

16 Heat Rates for Existing Equipment Operated in Daily Peak Load. . II-242

17 Heat Rates for Existing Equipment Operated in Seasonal

Peak Load ................... . . . . . . . . . 242

18 Heat Rates for New Equipment ............... II-243

19 Heat Values of Fossil Fuels ................ II-243

20 Nuclear Fuel Supply Curve ................ II-244

TARGET YEAR: 1990

1 Load-Duration Curve Data for 1985 and $1990 \ldots . . . . . . . . \quad$ II-247 Capacity Factors for DOE Region 1 (New England) . . . . . . II-249 Capacity Factors for DOE Region 2 (New York/New Jersey) . . II-250 Capacity Factors for DOE Region 3 (Mid-Atlantic) . . . . . II-251 Capacity Factors for DOE Region 4 (South Atlantic) ...... II-252 Capacity Factors for DOE Region 5 (Midwest) . . . . . . . II-253 Capacity Factors for DOE Region 6 (Southwest) . . . . . . . II-254 Capacity Factors for DOE Region 7 (Central) . . . . . . . II-255 Capacity Factors for DOE Region 8 (North Central) . . . . . II-256 Capacity Factors for DOE Region 9 (West) .......... II-257 Capacity Factors for DOE Region 10 (Northwest) . . . . . . II-258

2 Existing Capacity. . . . . . . . . . . . II-259

3 Existing Capacities Which May Be Converted to Burn Alternative Fuel Types ................ II-260 Retirement Rates for Fossil-Fuel-Fired Plants . . . . . . . . II-260 Committed Capacity for 1985 and 1990 ... . . . . . . . . II-261 Deferrable Capacity for 1985 . . . . . . . . . . . . II-262 New Plant Capacity Limits (1985). . . . . . . . . . . . II-262 Operation and Maintenance Costs .............. II-263 Revenue Requirements in 1985 for Existing Assets ......... II-264 Capital Costs of New Plants in 1985 . . . . . . . . . . . II-264 Capital Charge Rates (Percent) for Revenue Requirements .... III-265 Capital Charge Rates for Investment Decisions . . . . . . . . II-266 Transmission and Distribution Data. ........... II-267 Heat Rates for Existing Equipment Operated in Base Load. . . . II-268 Heat Rates for Existing Equipment Operated in Cycling Load . . . II-268 Heat Rates for Existing Equipment Operated in

Daily Peak Load .................. II-269

17 Heat Rates for Existing Equipment Operated in Seasonal

Peak Load .................. II-269

18. Heat Rates for New Equipment ............. II-270

19 Heat Values of Fossil Fuels ................ II-270

20 Nuclear Fuel Supply Curve ................ II-271 


\section{OVERVIEW}

\section{INTRODUCTION}

The purposes of this volume are to document the sources of input data to the PIES Integrating Model and to publish both the data used for the 1978 Annual Administrator's Report $(A A R)^{1}$ and a sample PIES Integrating Model Report, with a description of its contents. We have also included a discussion of the EIA energy supply and demand assumptions underlying the six scenarios used for the AAR and presented in this volume. The demand data documented are the quantities, prices, and elasticities, which are output from the Demand Model, and are input to the PIES equilibrating mechanism. SUPPLY DATA

There are three categories of supply data in this volume: facilities, materials, and transportation. The facilities are refineries and utilities. The materials are oil, coal, natural gas, synthetics, shale, and nuclear fuel. ${ }^{2}$ The third category, transportation, provides linkages between facilities and materials.

Data for these three categories are provided by numerous non-DOE sources, for exàmple, private research firms and Governmental departments (such as the U.S. Geological Survey). The data provided by these sources are referred to as "raw data" tables. These raw data tables are formatted for the convenience of the data collecting office and do not necessarily conform to the data format and unit requirements of PIES.

The PIES preprocessors format the raw data and convert the raw data table entries into a uniform set of "standard tables." ${ }^{3}$ The standard tables contain supply data formatted for use by the PIES matrix generation program, which forms the linear the AAR.

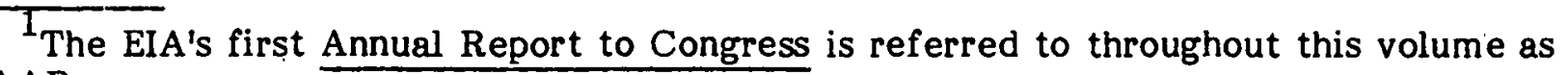

${ }^{2}$ Nuclear and nuclear fuel supply sectors are embedded in the utilities sector. Thére are no preprocessors for imports data.

3 The calculations performed in the PIES preprocessors are discussed in volume IV of this documentation series. 
programming matrix used by the equilibrating mechanism. ${ }^{4}$ Figure $1-1$ illustrates the flow of data from source through the standard table stage.

The supply tables contained in Chapters II and III of this volume are the computer printouts of the automated standard table documentation programs. These computer programs are external to PIES and print the standard tables with clear English headings' rather than mnemonic computer coding.

\section{EIA'S PROJECTION SERIES AND SCENARIOS}

The EIA's first Annual Report to Congress contains energy supply and demand forecasts made under six sets of assumptions. These six sets of assumptions, also referred to as projection series, include five that are combinations of three macroeconomic demand scenarios (low, medium, and high demand) and three oil and gas production supply scenarios (low, medium and high domestic supply). These demand and supply scenarios are discussed below. These five projection series presuppose constant real world oil prices between 1978 and 1990. The sixth, a variant of the medium demand/medium supply projection series discussed below, assumes a 5 percent yearly rise in world oil prices for the same time period. Table I-1 lists the six projection series and their major assumptions.

Figure I-2 shows the ten possible combinations of supply/demand projection series available with our six sets of așsumptions. The 1978 AAR used the six projection series indicated. These six series cover the range of PIES projections. It should be clear to the reader, however, that all of these ten supply/demand combinations as well as other scenarios are available to PIES.

${ }^{4}$ A description of the PIES equilibrating mechanism is contained in Chapter II of Volume IV in this documentation series. 
FIUJRE I-J

PIES PREPROCESSURS
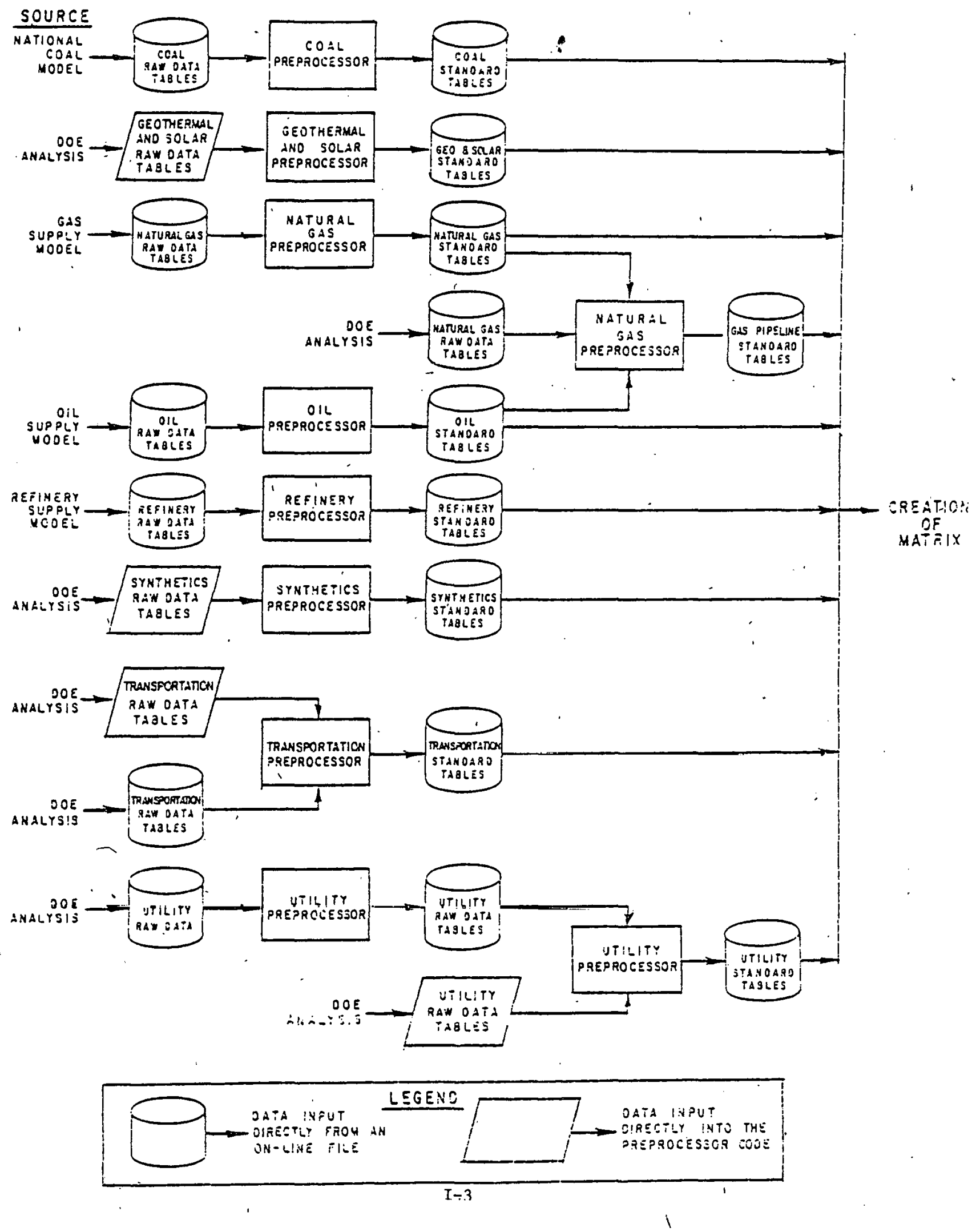
FIGURE I- 2

EIA PROJECTION SERIES A THROUGH $F^{\prime}$

:

ANNUAL ECONOMIC GROWTH RATES (1975-1990)

GROWTH OF

OIL \& GAS

DEMAND

PRODUCTION

(1975-1990)

$H I G H$

MEDIUM

LOW

ANNUAL RATE

$3.9 \%$

$3.8 \%$

$3.4 \%$

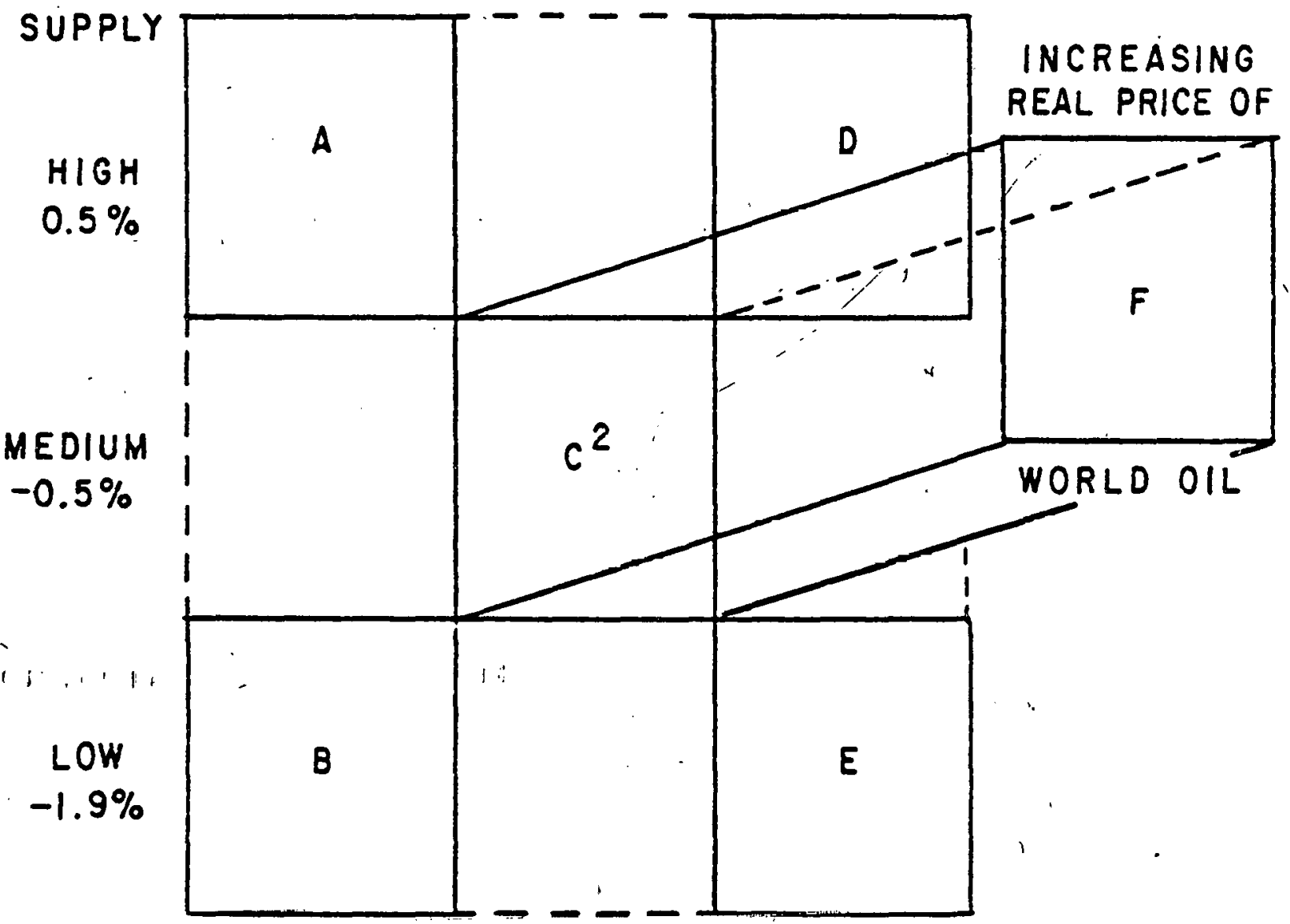

SOURCE: VOLUME II 1977 EXECUTIVE SUMMARY OF THE ANNUAL REPORT TO CONGRESS.

ENERGY INFORMATION AOMINISTRATION, DOE

1 SERIES A THROUGH F ARE THE SIX PROJECTION

SERIES CHOSEN FOR DISCUSSION IN THE AAR.

2) THE COMBINATION ÓF MEDIUM DEMANO/MEDIUM SUPPLY IS SOMETIMES REFERRED TO AS THE MIORANGE OR "BUSINESS AS USUAL" PROJECTION SERIES. 
The mid-range projection series (medium demand/medium supply) input data are displayed in Chapter II. Chapter III contains only the input data directly affected by the five alternative AAR scenarios; namely, high and low demand and supply and a 5 percent increase in world oil prices. The high and low demand tables are given. The high and low supply scenarios are illustrated with the high and low oil and gas data tables. The assumption of increasing world oil price affects the refinery input tables and the oil and gas tables. The tables also appear in Chapter III. Table I-2 summarizes the sectoral data tables affected by alternate scenarios. All other sector tables remain as depicted for the mid-range projection series of Chapter II.

TABLE I-1. EIA PROJECTION SERIES ASSUMPTIONS

\begin{tabular}{|c|c|c|c|c|}
\hline $\begin{array}{c}\text { Projection } \\
\text { Series } \\
\end{array}$ & $\begin{array}{c}\text { Demand } \\
\text { Assumption } \\
\end{array}$ & $\begin{array}{c}\text { Supply } \\
\text { Assumption } \\
\end{array}$ & $\begin{array}{c}\text { World Oil Price } \\
\text { Assumption }\end{array}$ & $\begin{array}{l}\text { Important Series } \\
\text { Characteristic } \\
\text { (relative to constant } \\
\text { price assumption) }\end{array}$ \\
\hline A & High & High & Constant & Maximum consumption \\
\hline B & High & Low & Constant & $\begin{array}{l}\text { Maximum imports; } \\
\text { Maximum relative } \\
\text { price of energy }\end{array}$ \\
\hline $\mathrm{C}$ & Medium & Medium & Constant & Mid-range \\
\hline $\mathrm{D}$ & Low & High & Constant & $\begin{array}{l}\text { Minimum imports; } \\
\text { Minimum relative } \\
\text { price of energy }\end{array}$ \\
\hline$E$ & Low & Low & Constant & Minimum Consumption \\
\hline $\mathrm{F}$ & Medium & Medium & Increasing & Important Contingency \\
\hline
\end{tabular}

Source: AAR, Vol. II 
TABLE 1-2. SECTORS AFFECTED BY ALTERNATE SCENARIOS

\begin{tabular}{ll}
\multicolumn{1}{c}{ Scenario } & \multicolumn{1}{c}{ Sector } \\
Low Supply/Low Demand & \multicolumn{1}{c}{$\begin{array}{l}\text { Imports. } \\
\text { Oil and Gas } \\
\text { Imports } \\
\text { Oil and Gas }\end{array}$} \\
Low Supply/High Demand & $\begin{array}{l}\text { Imports } \\
\text { Synthetics } \\
\text { Shale }\end{array}$ \\
Oigh Supply/Low Demand and Gas \\
High Supply/High Demand & $\begin{array}{l}\text { Imports } \\
\text { Shale }\end{array}$ \\
& $\begin{array}{l}\text { Oil and Gas } \\
\text { Medium Supply/Medium Demand }\end{array}$ \\
$5 \%$ Increase in World Oil Prices & $\begin{array}{l}\text { Imports } \\
\text { Refinery } \\
\text { Oil and Gas }\end{array}$
\end{tabular}

DEMAND DATA AND ASSUMPTIONS

Low demand is represented in PIES by the CYCLELONG macroeconomic forecast. Medium and high demand are called TRENDLONG and CEASPIRIT, respectively. These demand scenarios are taken from the Data Resources, Incorporated (DRI) econometric model. The DRI model provides macroeconomic projections used as input to the Bureau of Labor Statistics Input-Output (BLS/IO) Model. The BLS model then provides, as output, employment levels by economic sector, which are incorporated into PIES. Table I-3 lists the major assumptions underlying the DRI macroeconomic forecasts.

The EIA scenarios used to generate the tables presented in this volume assume a continuation of existing energy policies. This assumption presumes that natural gas regulation will remain in effect between now and 1990. Energy projections could vary widely if different assumptions were used. 
Assumptions

1. Piscul Policy

2. Monetary Policy

3. Consumer Price Index (CPI)

4. Nominal Energy Prices in the United States

5. Nominul Price of linported Oil

6. liorcign (OliC:D) Growth in Industria Production lndex

\section{CEASPIIRIT}

Federal expendilures increase 7.8 percent between 1977 und 1990.

Money supply increuses at a compound annual rate of 6.9 percent from 1977 to 1983 and 5.3 percent between 1983 and 1990.

l'rime lending rate declines ulmost continually from light of 7 percent in 1978 to a low of 5.4 percent in 1990 . Pullern and level of longlerın yields similar to those in TRENDI.ONG but yiclds are slightly lower. at a coinpoind anmual rate of

TIIINDIONG

liediral expenditures increase at a compound nnmual rate of 8.6 percent on a national income basis betwoen 1977 and 1!90.

Norrowly defined money supply (M1) increnses at $n$ compound ainuil rate of 5.7 percent beaimiunt rale of $5 . \%$ parceil be(ween 1977 and 1983 and at a 4.6 percent rate between 1983 and 1990.

Prime lending rate ranges between 7.2 percent and 7.7 percent belween 1978 and 1983 . Subsequently clectines gradually $10 \mathrm{~b} .1$ percent in 1990. A roughly similar phltern (but nol level) of yiclals exists for long-lerin bonds (new $\triangle \wedge \wedge$ ) after soine lime lag.

Cl'l increases at anmual rate of 5 percent - 6 percent through 1983 (except in 1977), declincs continually to 4.1 percent in 1990. 4.9 percent and 5.6 percent during 1978-1983, and then declines continually to 4.0 percent.

Compound annual rate of incrense between 1.978 and 1990 is about 5 percent, declining from 10 percent in 1978 to 3.3 percellt in 1990.

Rntes of increase similar to those in T'Rl:N DI.ONG (hrough 1982. Substantially sinaller rates thereafter which decline brudually from 5.2 percent iil 1983 to 3.6 pereenl in 1990 .

Growll Hiltes: $1977-19805.5$ percent 1980-1985 4.2 percent 1985-19?0 4.0 percent
Compound annunl rate of increase belween 1978 and 1990 is 7.6 percent, deelining from 16 percenl in 1978 to 5.3 percent in 1990.

Rates of incrense range from 7 . i percent to 8.5 percent beIween 1977 and 1980. Rate of incereuse is at constant 7.5 percent after 1981.

\section{Growllh llites:}

$1977-19804.2$ percent

$1980-19855.2$ percent

1985-1990 1.4 percent

\section{CYCLEIONG}

Federal expenditures increase at a compound ammal rate of 12.1 percent between 1977 und 1990 .

Money supply increases at acompoumin anmul rate of 7.3 percent belween 1977 and 1983 and 9.5 percent from 1983 to 1990.

Prime lending rute increnses continunliy to nenrly 10 percent by 1982 with sliarp varialions thereafter betwcen low of 6.7 percent and light of 11.3 perceint. Long-term yields are substantially higher than in TllENDLONG throughoul 1977-1990 reflecting higlser inflation, credit erunches in 1982 and 1986 , higlıer risks of finnneial instability and recurrent expunsionary policies.

Cl'I increnses ul annunl rate of 7 percent during 1977-1983 und at ubout 8 percent thereafter, willt donble digit inflation in, 1986 and 1987 .

Compound antual rate of inerease between 1978 und 1990 is $\mathbf{1 2 . 2}$ percent peaking at 17 perecnt in 1990.

Rutes of incrense similar 10 llose in 'THINALON(; througli 1979. Thereafter, rules range from 8 perceilt to 18 percent and usiully excecd 10 pereent.

Growll Rates:

$19^{\prime} 77-19804.2$ percent

1980-1985 4.9 percent

1985-1990 2.7 percent

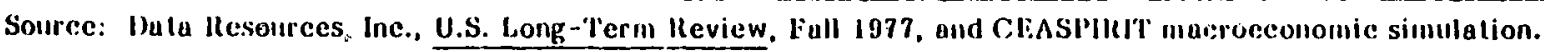


Oil and gas availabilities play important roles in the projection of future energy trends. As can be seen in Figure I-3, oil and gas production, in general, constitutes the bulk of domestic energy levels projected for the six EIA projection series. Tables I-4 and I-5 give oil and gas demand, supply and imports for three projection series. These three scenarios, A, C, and E, represent the range of energy supply and demand over the projection series.

Oil and gas production from mature wells in on-shore areas is expected to decline in the mid-term (now to 1990). Production from Alaska and off-shore areas is expected to offsel these declines, resulting in domestic oil and gas production being maintained at current levels, as a best estimate. Import levels are expected to continue their climb, as seen in Figure $\mathbf{I} 4$.

Imports of crude oil are less sensitive to changes in economic growth assumptions than are refined petroleum product imports. This is because crude oil imports are constrained by the available U.S. domestic refinery capacity and by capacity utilization rates. For the AAR, a 90 percent capacity utilization is assumed.

Petroleum products, on the other hand, are highly sensitive to economic and domestic production assumptions. Petroleum product import projections are highest $(6.9$ quadrillion Btu per year) for the high demand/low supply projection series, series B. They are lowest (3.2 quadrillion Btu per year) for the low demand/high supply series, series D.

This represents a drop of 54 percent from series B to series D. The corresponding decline in crude oil (19.3 to 17.0 quadrillion Btu per year) for the two series, $B$ and $D$, respectively, is only 17 percent. Table $1-6$ gives energy production and consumption summaries in BTU's for target year 1985 for the six EIA projection series. 
FIGURE I-3

VARIATIONS IN PROJECTED LEVELS OF U.S. ENERGY CONSUMPTION AND PRODUCTION BY EIA PROJECTION SERIES A THROUGH F

QUADRILLION

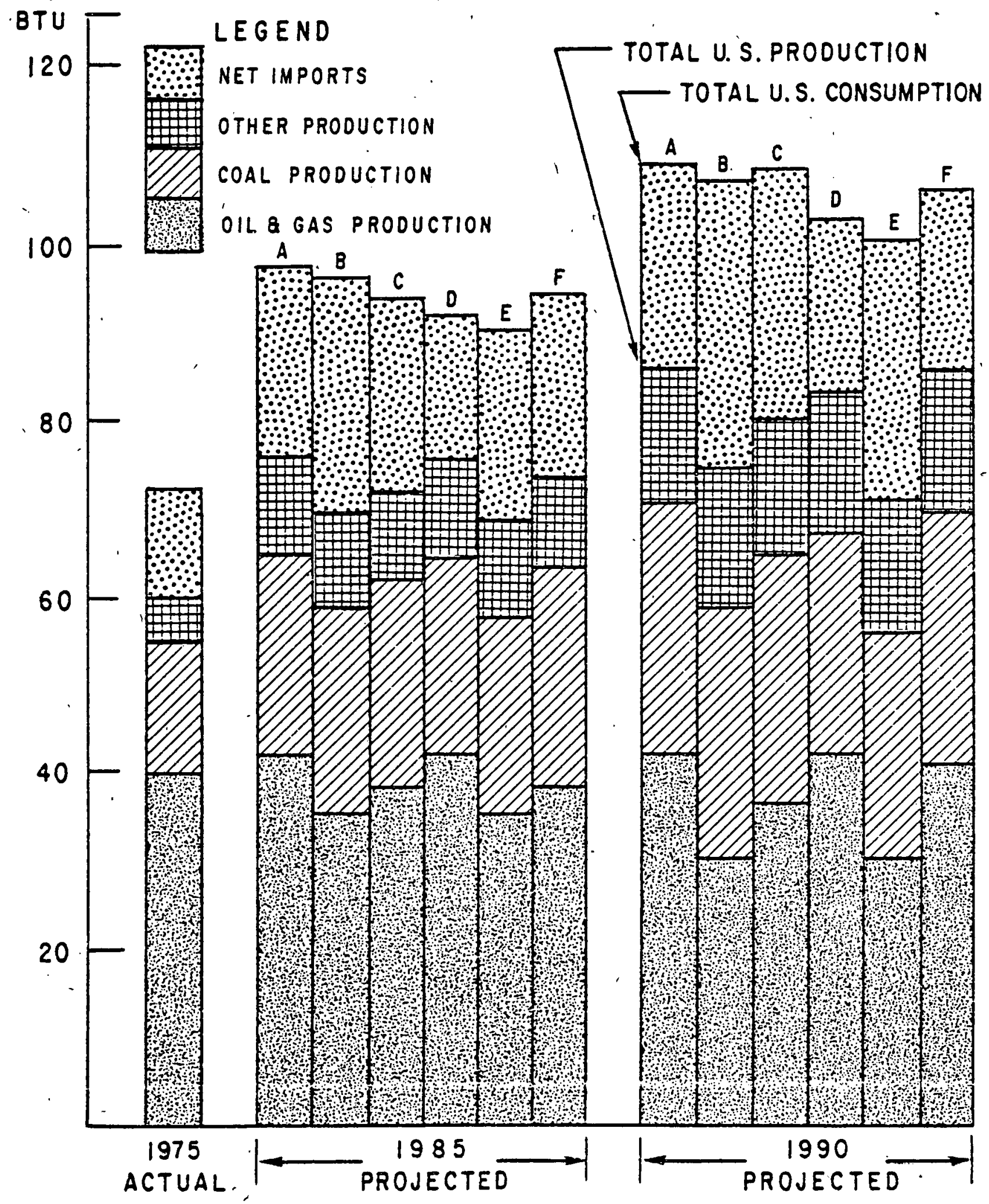

SOURCE: VOLUME II 1977 EXECUTIVE SUMMARY OF THE

ANNUAL PEPORT TO CONGRESS.

ENERGY INFORMATIOIN ALMINISTRATION, DOE 
FIGURE $\quad 1-4$

\section{PROJECTED U.S. PETROLEUM LIQUIDS SUPPLY}

\section{MILLIQN \\ BARRELS / DAY}

25

25

20

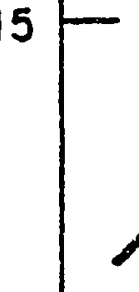

TOTAL SUPPLY

10
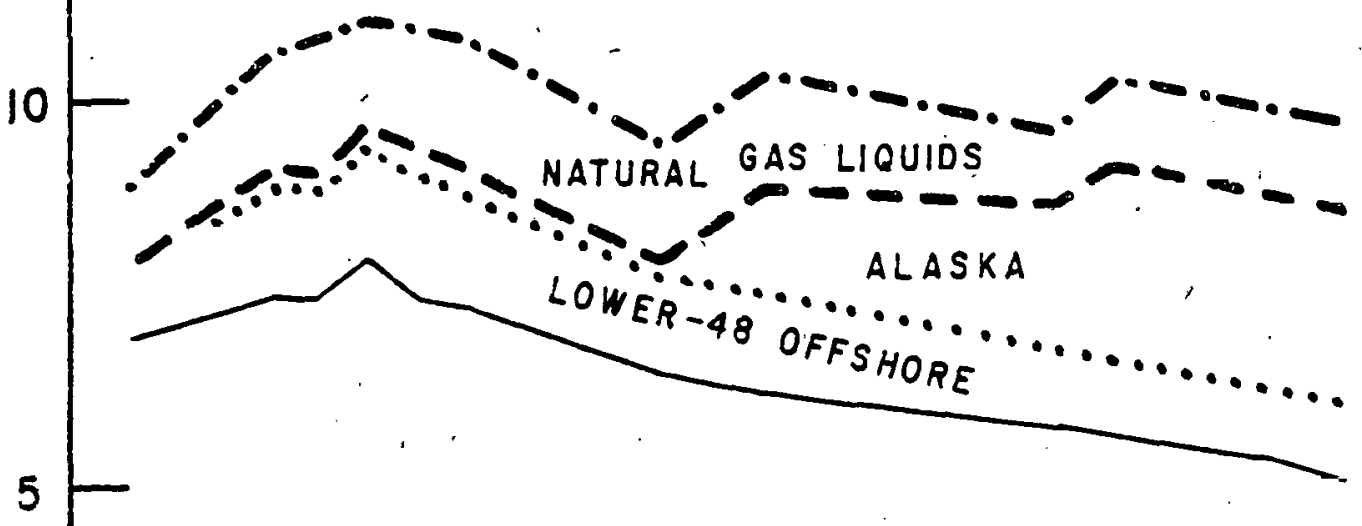

5

LOWER-48 ONSHORE

IMPORTS
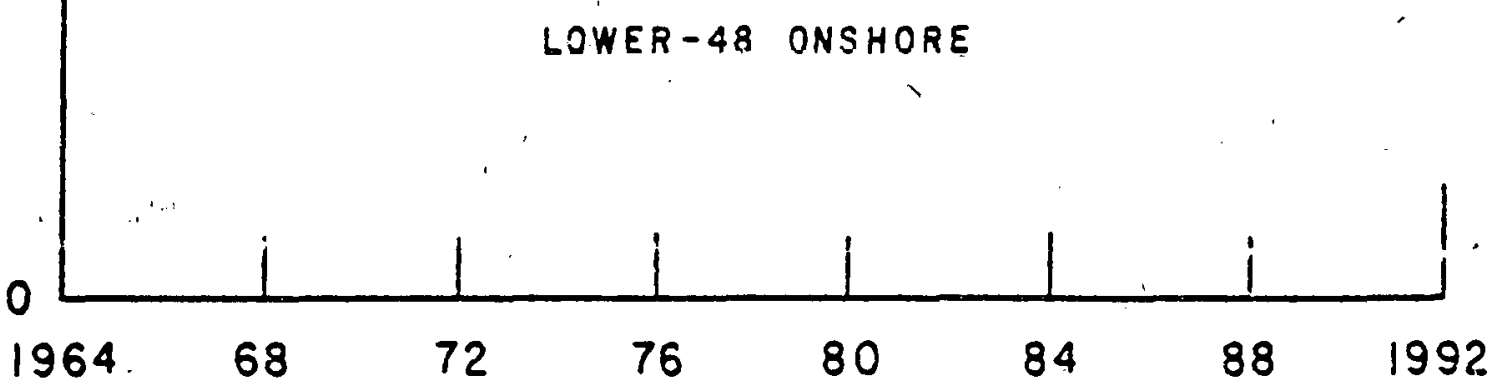

SOURCE: AAR, PROJECTION SERIES C 
TABLE I-4. U. S. PETROLEUM LIQUIDS DEMAND, SUPPLY, AND IMPORTS (Millions of Barrels per Day)

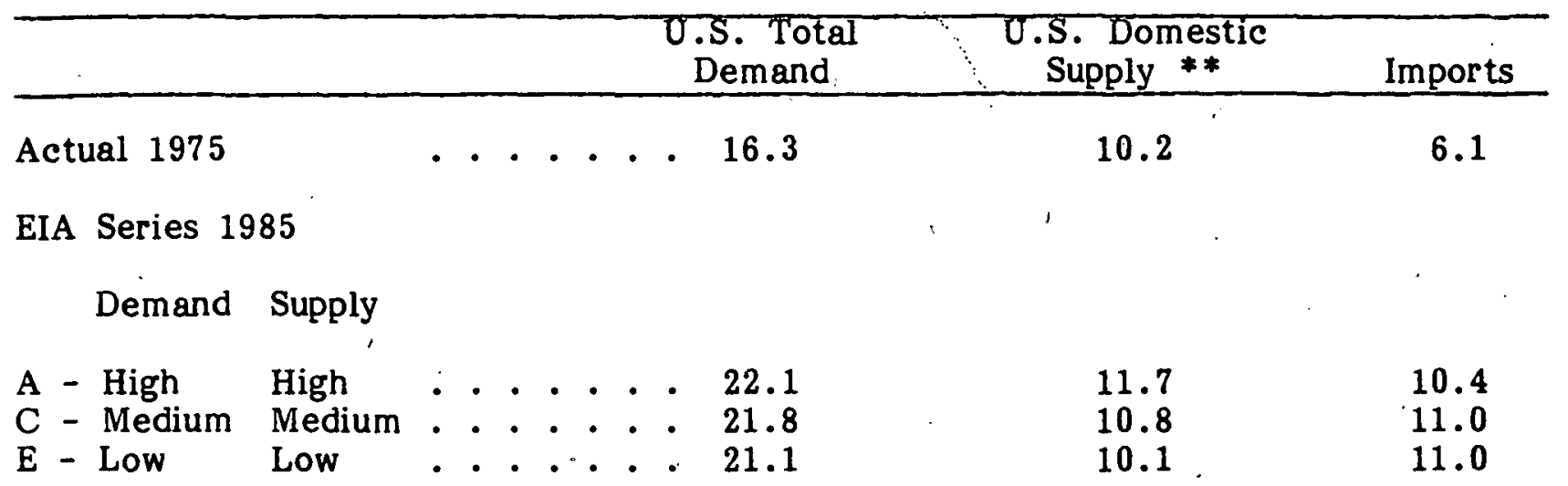

EIA Series 1990

Demand Supply
A - High High ...... 24.0
C - Medium Medium ....... 24.2
12.3
11.7
$\mathrm{E}$ - Low Low ....... 22.7
10.4
8.6
13.8
14.1

Average Annual Growth (percent)
1960-1975 (Actual)
3.4
1.5
8.3
1975-1985 (Projected)

Demand Supply
A - High High . . . . . . 3.1
C - Medium Medium .. . . . 3.0
1.4
5.5
E - Low Low $\quad$ c. . . . . 2.6
0.6
6.1
1985-1990 (Projected)
0.8
6.1

Demand Supply
A - High
High
1.7

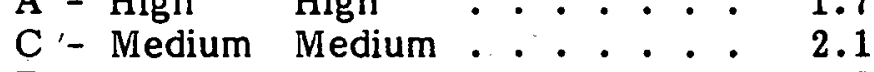
E - Low Low . . . . . . 1.5
1.0
$-0.8$
2.4
$-5.0$
4.6
5.1

* In crude oil equivalents.

* * Domestic supply includes production plus net gains (losses) in conversion, etc.

Source: AAR, Vol. II, Executive Summary. 
TABLE 1-5. U. S. NATURAL GAS DEMAND, SUPPLY, AND IMPORTS (Trillions of Cubic Feet, Annually)

\begin{tabular}{llccc}
\hline & $\begin{array}{c}\text { U.S. Total } \\
\text { Demand }\end{array}$ & $\begin{array}{c}\text { U.S. Domestic } \\
\text { Supply* }\end{array}$ & Imports \\
\hline Actual 1975 & ....... 19.5 & 18.5 & 1.0
\end{tabular}

EIA Series 1985

Demand Supply

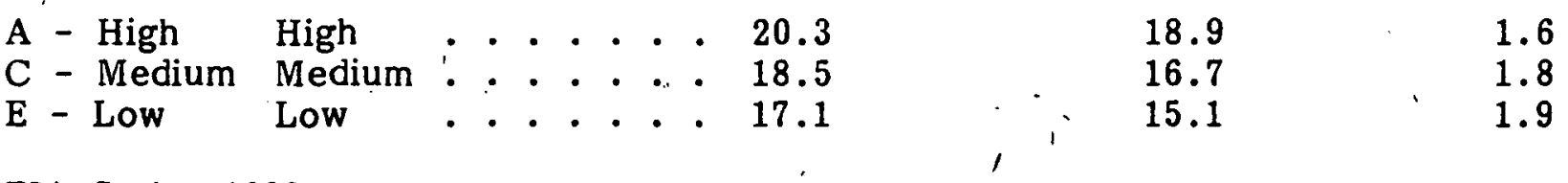

EIA Series 1990

Demand Supply
A - High
High
19.9
17.7
2.2
C - Medium Medium ....... 18.7
16.2
2.5
E - Low
Low
15.4
12.8
2.6

Average Annual Growth (percent)
1960-1975 (Actual)
3.3
3.1
12.8
1975-1985 (Projected)

Demand Supply
A - High
High
0.4
0.2
4.8
C - Medium
Medium ...... -0.5
$-1.0$
E - Luw
Low
$-2.0$
6.1
6.6

1985-1990 (Projected)

Demand Supply
A - High
High
$-0.4$
$-1.3$
6.6
C '- Medium
Medium
0.2
$-0.6$
6.8
E - Low
Low
$-2.1$
$-3.3$
6.5

Note: Data may not add to total demand due lo rounding.

* Does not include production of synthetic gas projected, at 0.9 Tef in 1985 and 1.0 Tef to $1.2 \mathrm{Tef}$ in 1990 . 
TABLE I-6. $\frac{1985 \text { ENERGY SUPPLY/DENAND BALANCE }}{\text { (Quadrillion Btu Per Year) }}$

Projection Series:

$\begin{array}{ccccccc}\text { A } & \text { B } & \text { C } & D & E & F & \\ \text { Actual } & \text { High } & \text { High } & \text { Medium } \\ 1975 & \begin{array}{c}\text { Demand } \\ \text { High } \\ \text { Supply }\end{array} & \begin{array}{c}\text { Demand } \\ \text { Low } \\ \text { Supply }\end{array} & \begin{array}{c}\text { Demand } \\ \text { Medium } \\ \text { Supply }\end{array} & \begin{array}{c}\text { Demand } \\ \text { High } \\ \text { Supply }\end{array} & \begin{array}{c}\text { Demand } \\ \text { Low } \\ \text { Supply }\end{array} & \begin{array}{c}\text { Digh } \\ \text { Price }\end{array} \\ \end{array}$

Domestic Production

Crude Oil Production

Natural Gas

$\begin{array}{lllllll}17.9 & 20.3 & 17.7 & 19.0 & 20.3 & 17.7 & 19.5\end{array}$

Liquids \&

Butane . ...

Shale Oil ...

Natural Gas . .

CORl .....

Nuclear .....

Hydro \& Geothermal

Total Domestic

Production . .

Imports

Crude Oil ....

Petroleum Products

Natural Gas . .

Total Imports. 13.5

$\begin{array}{rr}2.6 & 2.2 \\ 0 . & .2 \\ 119.0 & 19.4\end{array}$

1.8

2.0

2.2

$1.7 \quad 1.9$

.1

$14.6 \quad 23.0$

16.0

.1

23.8

17.2

.2

19.2

$.1 \quad .1$

$1.8 \quad 6.2$

6.2

23.1

22.2

$3.2 \quad 4.2$

4.2

6.2

6.2

$22.8 \quad 23.6$

4

$59.1 \quad 75.5$

69.8

4.2

4.2

6.2

6.2

\begin{tabular}{lllllll}
\hline 59.1 & 75.5 & 69.8 & 71.8 & 74.5 & 68.3 & 73.0
\end{tabular}

Total Supply ..

$\begin{array}{rr}8.7 & 15.5 \\ 3.8 & 6.6 \\ 1.0 & 1.6\end{array}$

19.3

16.5

16.0

17.5

17.0

6.9

6.7

3.2

5.7

4.3

\begin{tabular}{llllll}
\hline 23.7 & 28.3 & 25.1 & 20.4 & 25.2 & 23.2
\end{tabular}

$72.6 \quad 99.2$

98.1

96.9

94.9

93.5

96.2

Domestic Consumption

Oil . . . . . . .

Natural Gas . .

32.8

44.4

45.4

43.9

41.5

42.3

42.4

$20.0 \quad 21.0$

18.1

19.1

20.4

17.6

19.4

12.821 .1

21.9

21.2

20.3

$20.9 \quad 21.7$

Nuclear . . . .

1.8

6.2

6.2

6.2

6.2

6.2

6.2

Hydro \& Geothermal

3.24 .2

4.2

4.2

4.2

4.2

4.2

Total Domestic Consumption

$$
70
$$

70.

$96.9 \quad 95.8$

94.6

92.6

91.2

93.9

Exports

Coal

Refinery Loss.

Total Ccnsumption

\& Exports . .

$\begin{array}{rrrrrrr}1.8 & 1.9 & 1.9 & 1.9 & 1.9 & 1.9 & 1.8 \\ .2 & .4 & .4 & .4 & .4 & .4 & .4\end{array}$

$\begin{array}{lllllll}72.6 & 99.2 & 98.1 & 96.9 & 94.9 & 93.5 & 96.2\end{array}$

Domestic Consumption by Sector

Residential. . ..

Commercial . . .

Industrial . . .

Transportation ..

Total Domestic Consumption

\begin{tabular}{lllllll}
14.7 & 19.3 & 19.0 & 19.0 & 18.9 & 18.6 & 18.6 \\
11.3 & 13.8 & 13.5 & 13.5 & 13.4 & 13.1 & 13.2 \\
26.0 & 42.0 & 41.5 & 40.7 & 39.9 & 39.1 & 40.9 \\
18.6 & 21.8 & 21.8 & 21.4 & 20.4 & 20.4 & 21.2 \\
\hline 70.8 & 96.9 & .95 .8 & 94.6 & 92.6 & 91.2 & 93.9
\end{tabular}

Source: 1978 Annual Administrater's Report, Vol. II. 
II. SUPPLY AND DEMAŃD DATA TABLES AND SOURCES FOR THE MID-RANGE

SCENARIO: TARGET YEARS 1985 AND 1990

\section{INTRODUCTION}

This chapter contains supply and demand data used by the PIES Integrating Model for the mid-range scenario target years 1985 and 1990.

Primary data sources are cited at the bottom of each table. These sources are the sources of the data used to generate the data shown in the following tables, which are used in turn to generate the PIES standard tables. Taking the coal'supply data as an example, the sources cited are, in general, data sources for inputs to the National Coal Model developed by a private consultant firm, ICF, Inc. The output from the National Coal Model is used as input to the PIES pre-processor to generate the PIES coal supply standard tables; thus, the data sources cited are actually sources of the input data to the National Coal Model.

Also given at the bottom of each table is the DOE of fice responsible for collecting and updating the data. This function does not necessarily entail collecting the primary data, but rather, collecting the raw data input to PIES, for example, obtaining the output files from the National Coal Model.

Data for nuclear fuel, imports, and shale are input directly in PIES standard table format. No satellite models are used. Sources cited for these data are the actual sources for what is in the tables.

DEMAND, PRICE AND ELASTICITY DATA

This section contains four tables showing demand point quantities and initial fuel prices for the two target years, 1985 and 1990. The column headings are the 10 demand regions. The row headings are the type of fuel used by five sectors of the economyresidential, commercial, industrial, transportation and raw material. The 12 fuels are: 
electricity, liquid gas, distillate fuel, natural gas, residual fuel, gasoline, oil, naphtha, asphalt, metallurgical coal, steam coal, and jet fuel. Since each fuel is not consumed in every energy sector, the total number of rows is only 30 . These 30 categories, which designate fuel by sector, we refer to as fuel types.

The two tables showing initial prices consider only 18 fuel types - those with "elastic" demands. 1 Products or fuel types with inelastic demands are not considered, since PIES assumes they are completely inelastic, and so their demand is not affected by price.

Also contained in this section are tables showing own-and cross-elasticities for each fuel type. The own-elasticities shown measure the percentage change in the quantity of a specific fuel type with respect to a percentage change in the weighted sectoral average of the prices of that fuel. For example, the own-elasticity for electricity in the residential sector is the percentage change in the quantity of electricity in the residential sector divided by the percentage change in the weighted average price of electricity in all sectors: residential, commercial, industrial, and transportation.

Cross-elasticities measure the percentage change in the quantity of a specific fuel type with respect to the weighted sectoral average price of a different fuel. An example would be the cross-elasticity of residential coal with respect to a weighted average of natural gas prices.

There are two sets of elasticity tables - one for 1985 and the other for 1990. Each set of these tables contains a separate table for each demand region. Demand (and electric utility regions) are the DOE regions shown in Figure II-1. The entries are the own - or cross-elasticities of a fuel type (given by the row name) with respect to the weighted average of a fuel's prices (the fuel being given by the column name). The nine fuels given in the column headings are those with elastic demands.

\footnotetext{
"Elastic" here means not perfectly inelastic!
} 


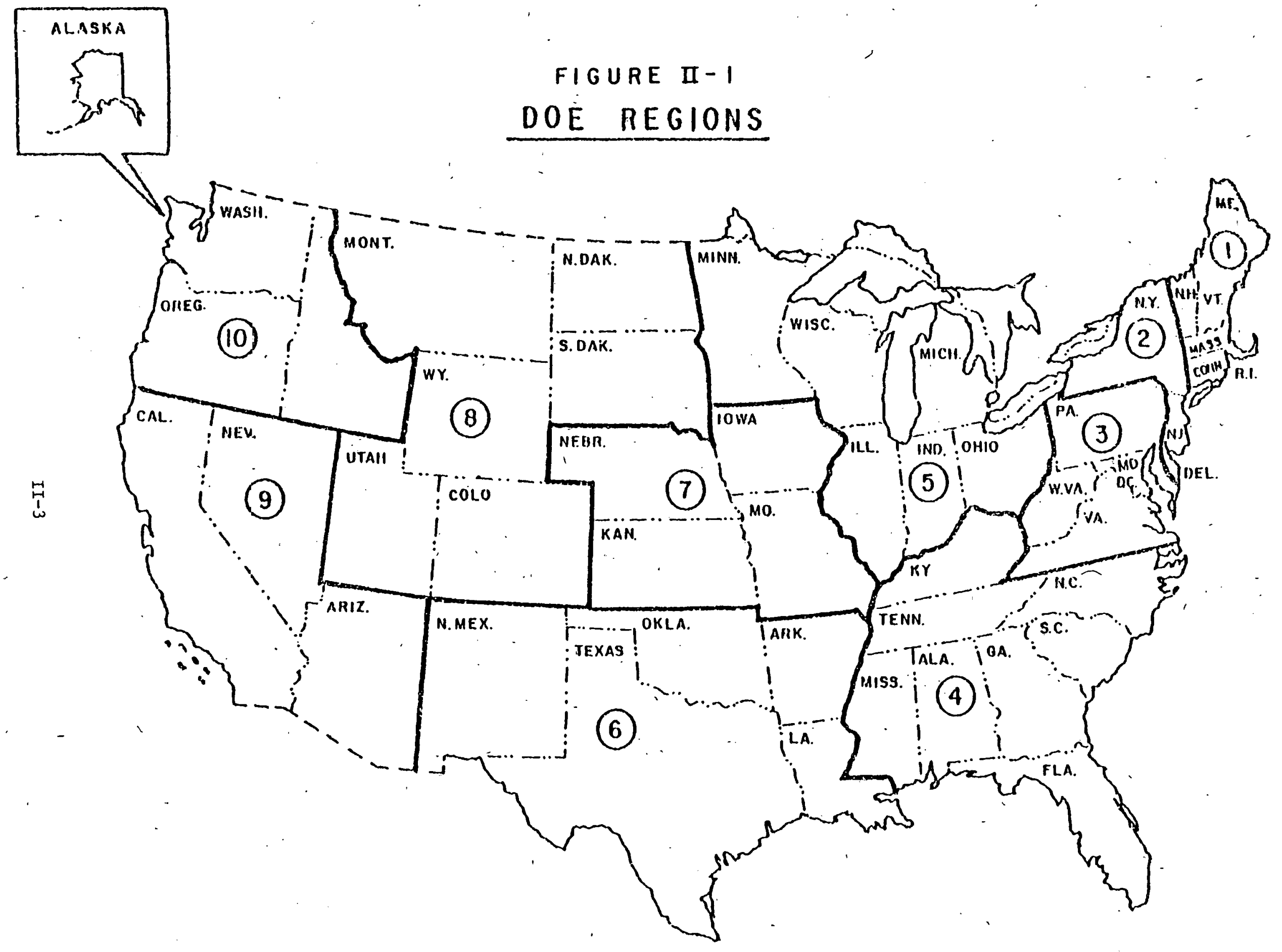


The historical data used to determine the price and demand quantities are derived from three primary sources:

- Electricity data are taken from the Statistical Yearbook, an annual publication of the Edison Electric Institute, Washington, D.C.

- Jet fuel data are taken from newsletters and reports of the Ethyl Corporation of Houston, Texas.

- Petroleum, oil, gasoline, gas and coal data are taken from the various volumes of Mineral Industry Surveys, produced by the Bureau of Mines.

These historical data are combined with macroeconomic data from DRI to produce the price, quantity and elasticity projections for 1985 and 1990 that appear in the following tables. DRI obtains its data from standard sources; for example, population data from the Census Bureau, value added, labor and labor productivity data from the Bureau of Labor Statistics, and so forth.

The following tables are results of the Demand Model; assuming "business as usual" with regard to economic growth expectations. This medium economic growth scenario is referred to as TRENDLONG. By varying the assumptions about economic growth, for instance, growth in GNP, productivity and value added, we can generate a low, or pessimistic, demand forecast or a high, or optimistic, one. The low forecast is referred to as CYCLELONG, the high as CEASPIRIT. 2

\section{COAL DATA}

Two sets of tables' follow, one each for target years 1985 and 1990 . Each set contains information about (1) the minimum acceptable selling price of each coal type, (2) the maximum level of production from each mine type, and (3) the present value of cumulative investment required between 1978 and the target year to continue operating existing mines or to open each new mine. Each table provides data for a specific combination of coal type and region. Coal regions are shown in Figure II-2. The region name and coal type are stated in the table headings.

\footnotetext{
$\therefore$

${ }^{2}$ See Chapter 1 of this volume for a description' of the TRENDLONG, CYCLELONG and CEASPIRIT scenarios.
} 
DEMANO. DATA

THENULUNG

DATA sHuHCES

FETTNICIIY - STATISTICAL YEAHBIIOK, EUISIIN EIECTHIC IHSTITUTE, WASHINGTDN,D.C.

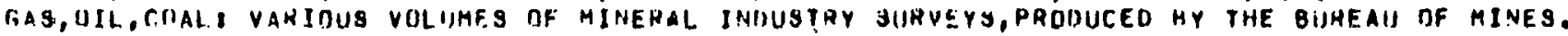
JET FULL L NEWSLETTERS AND RELEASES FHOA THE ETHEL, CUNPIIRATION GF HIUSTUN,TEXAS

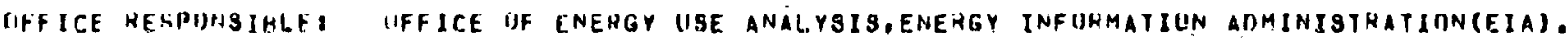


IABLE I DENAN: FCINTS IN TFILLICAS CF HTU S FCR 1985

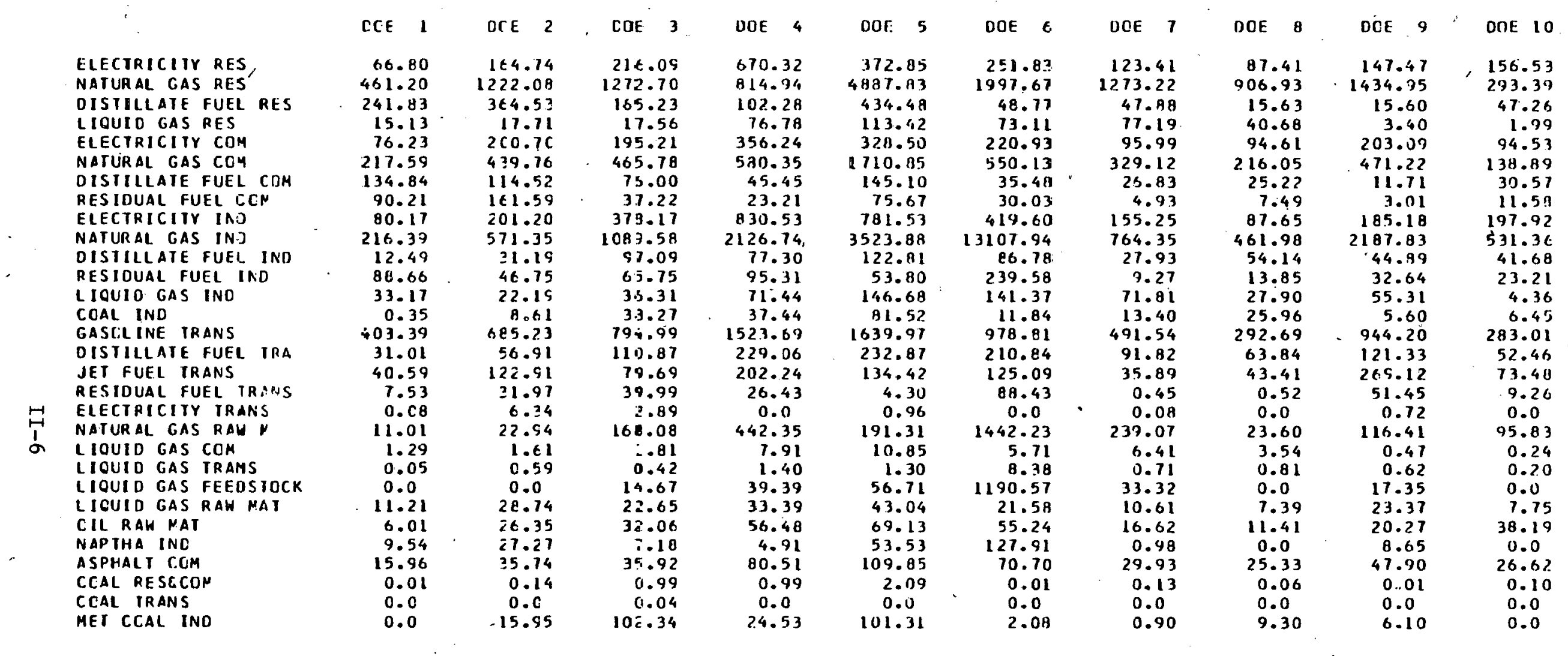




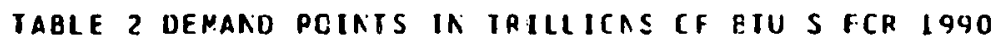

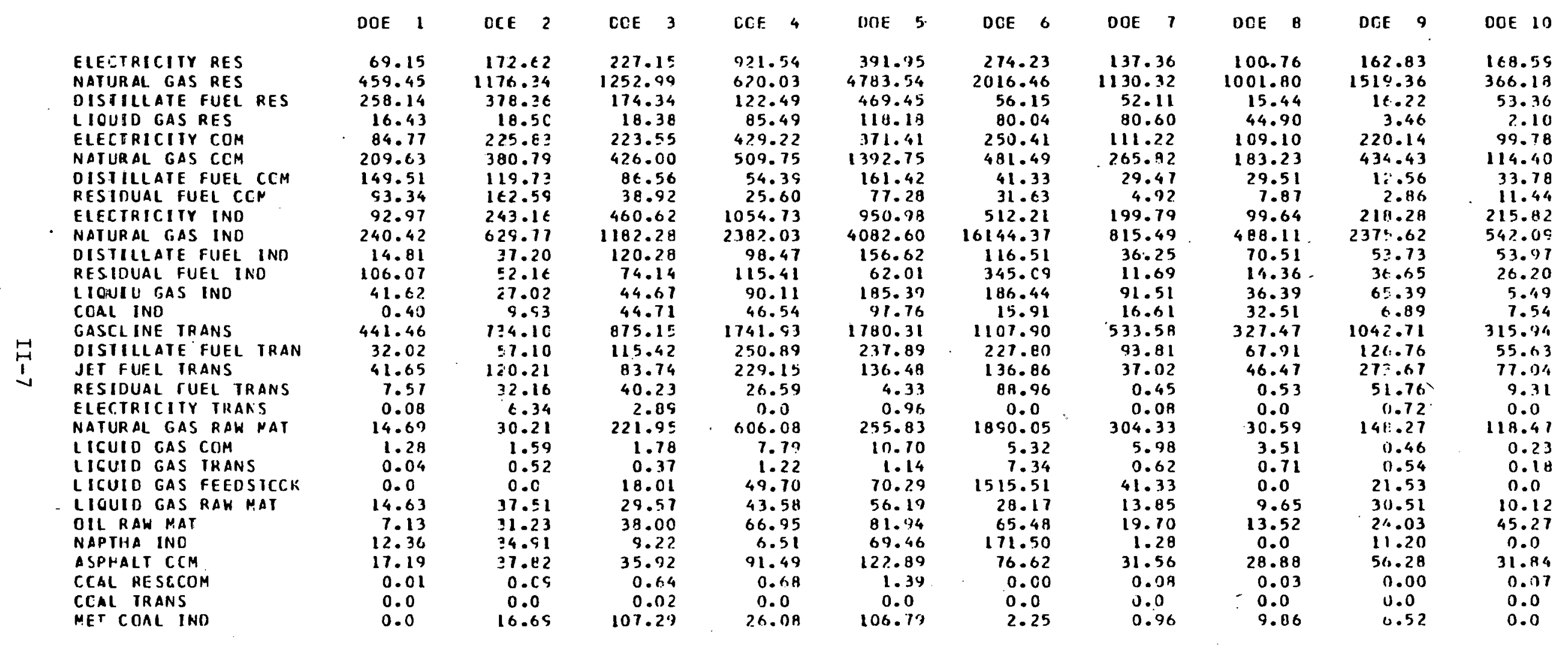


TARLE 3 INITINI, PRICES IN JOLLARS/STANDARD INIT FOR 1985

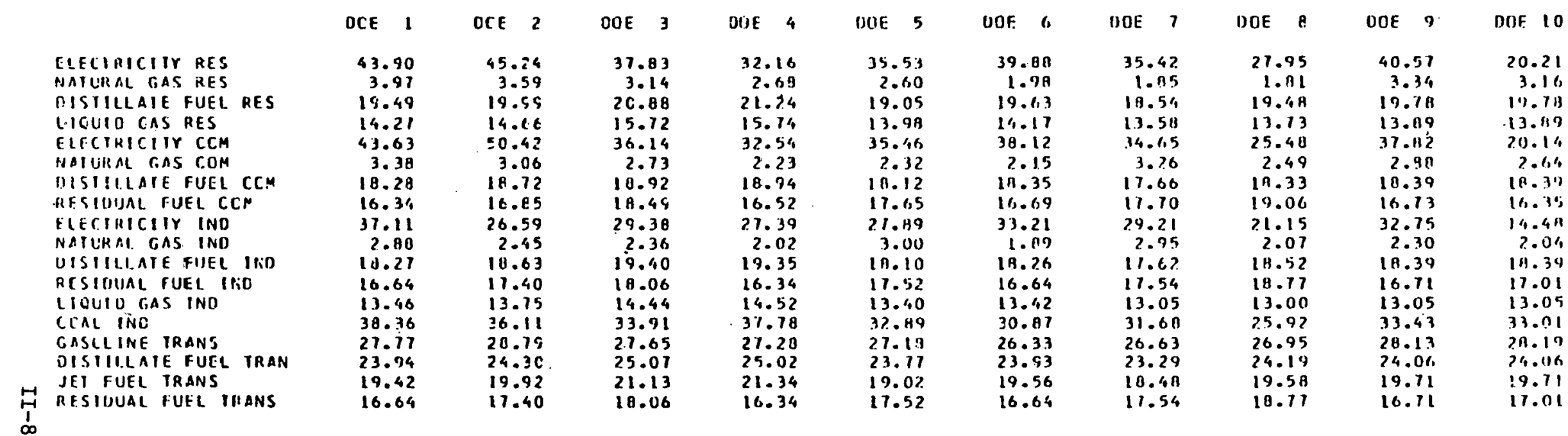


TABLE 4 INITTAL PRICES. IN DOLLARS/STANUARD UNI'T FOR 1990

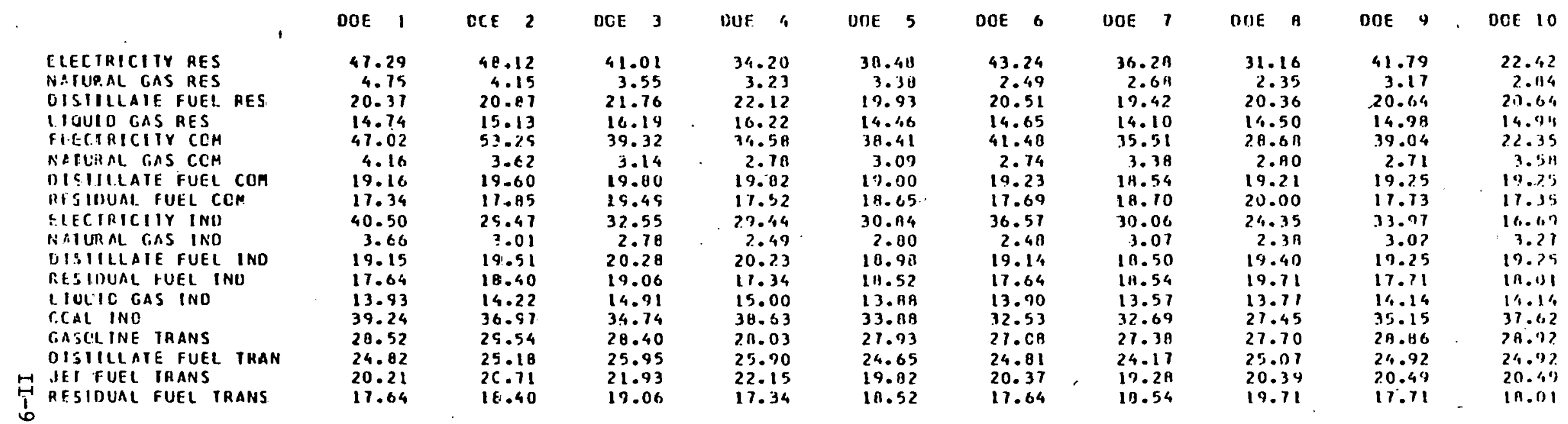


TABLE S UNSCALED ELASTICITIEB FOR DEMANO REGION 1 - 1985

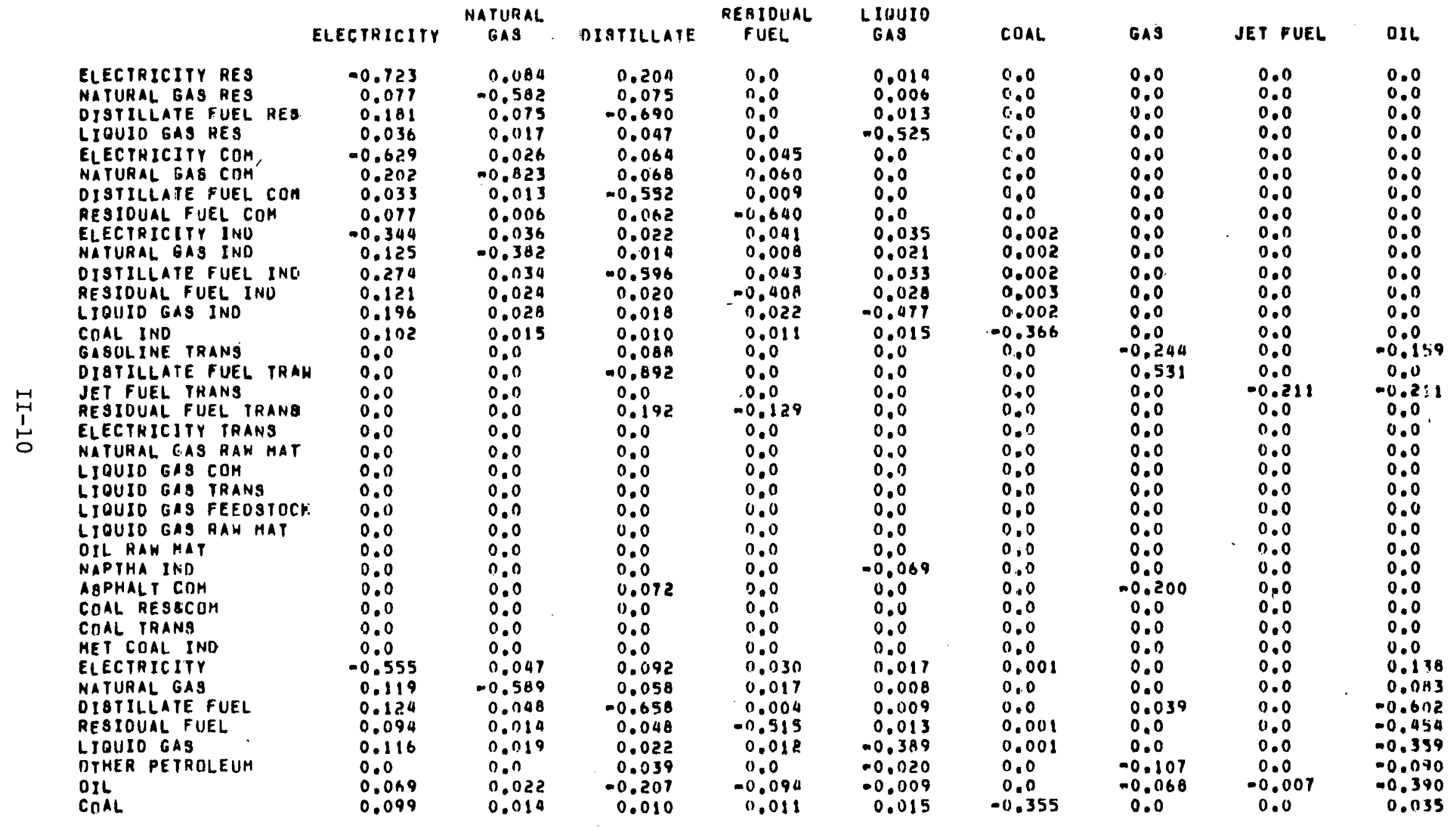


TaOLE 6 unscaled elasticities fór nemano RegiUH 2 - 19AS.

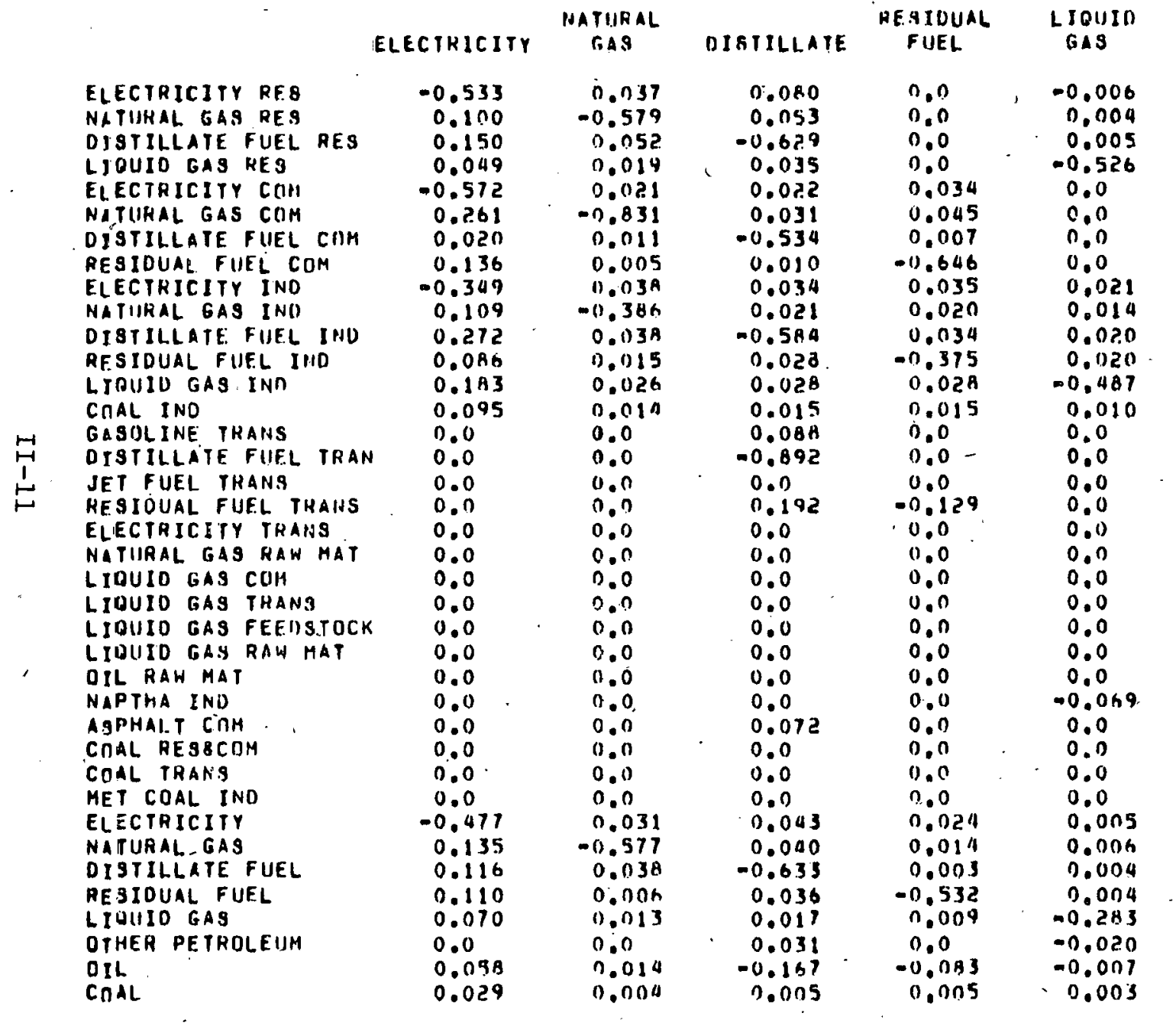

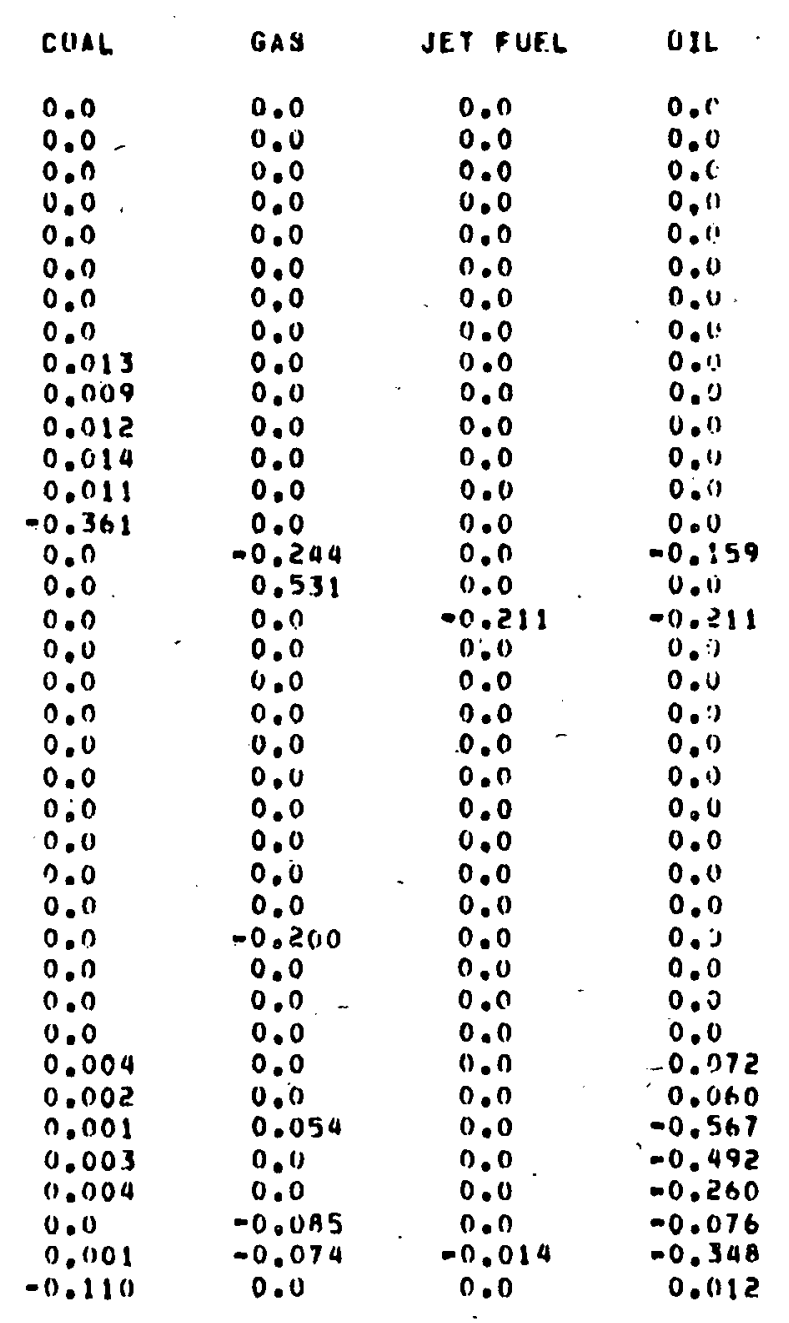


TABLE T UNSCALED ELAGTICITIES FIH DEAAIIJ KEGION 3 - ISUS

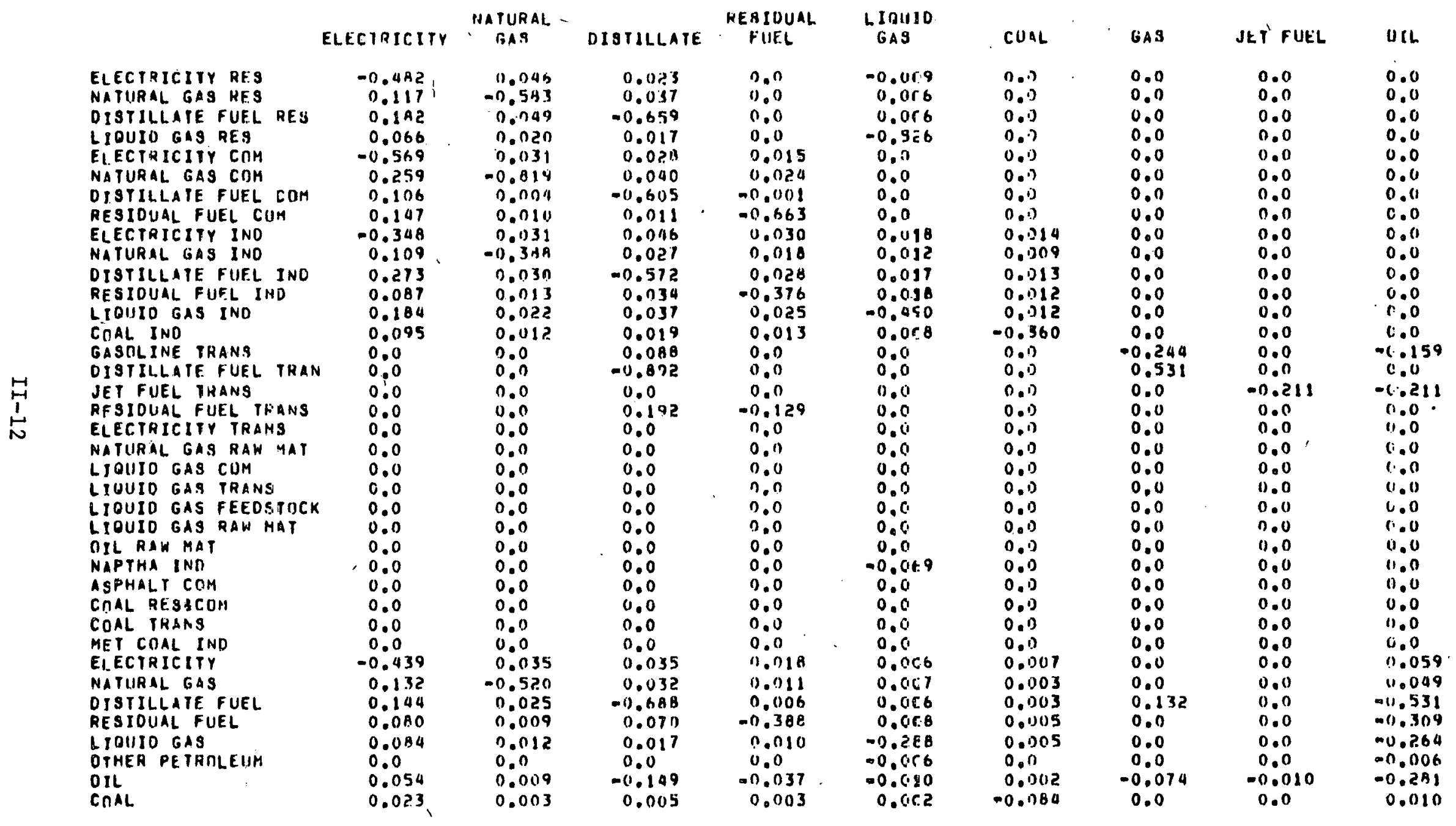


TABLE " UNSCALED ELASTICITIES FUA DEMARD REgIUN .1985

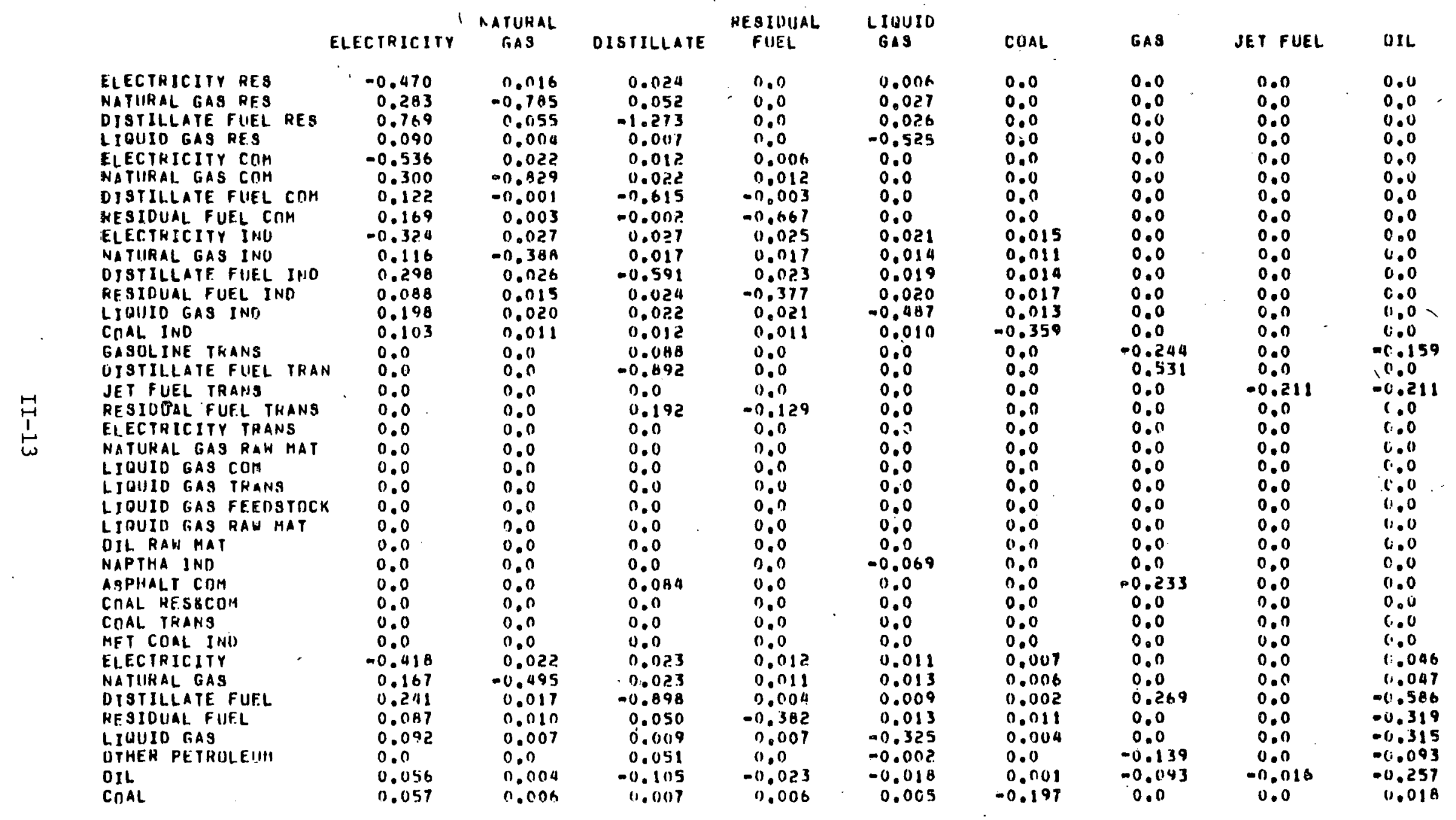


TAULE 9 WHSCALED ELASTICITIFS FÓR DEMANO HEgIUN 5 - 1965

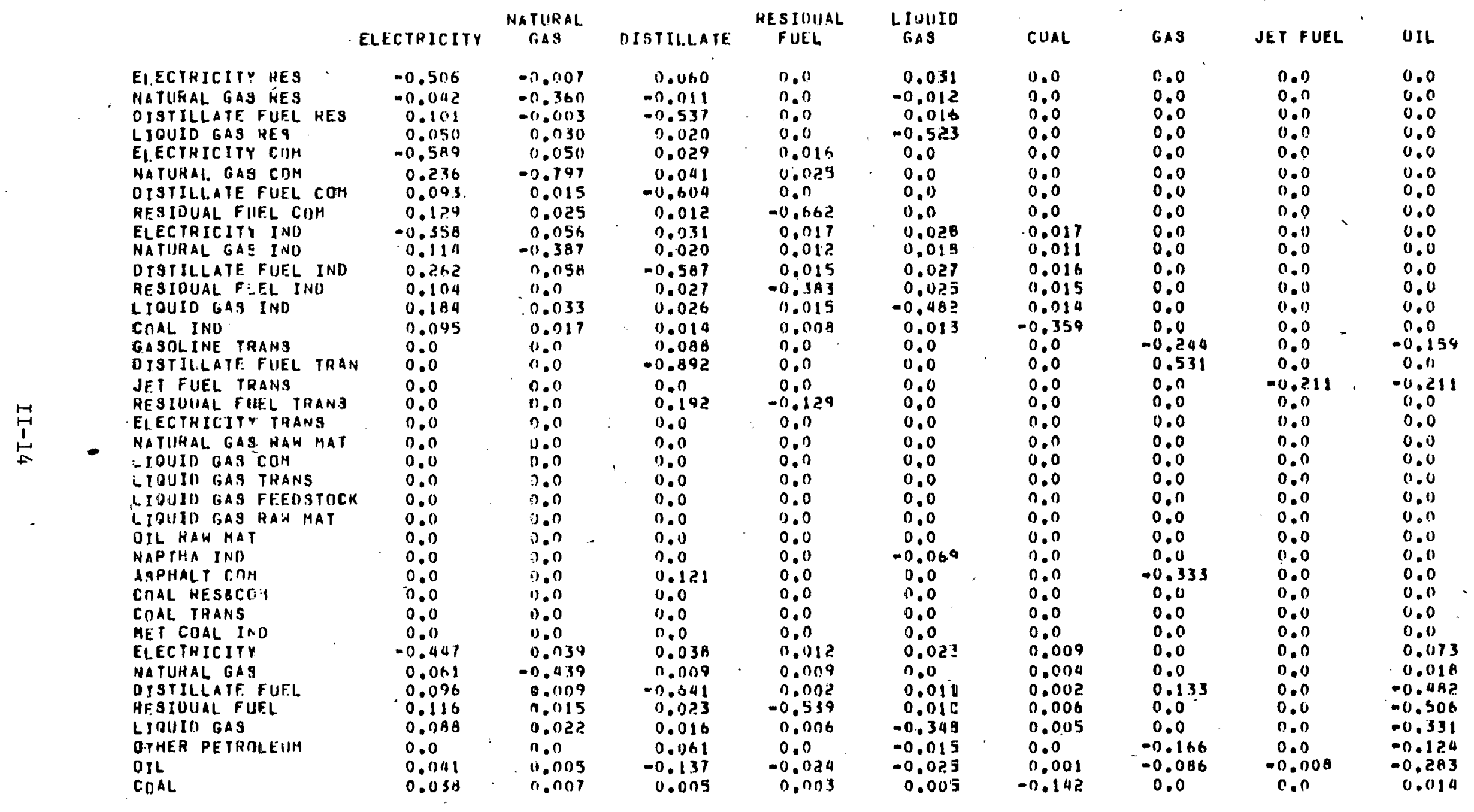




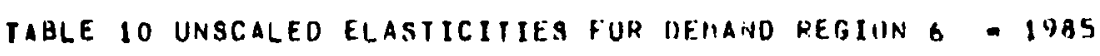

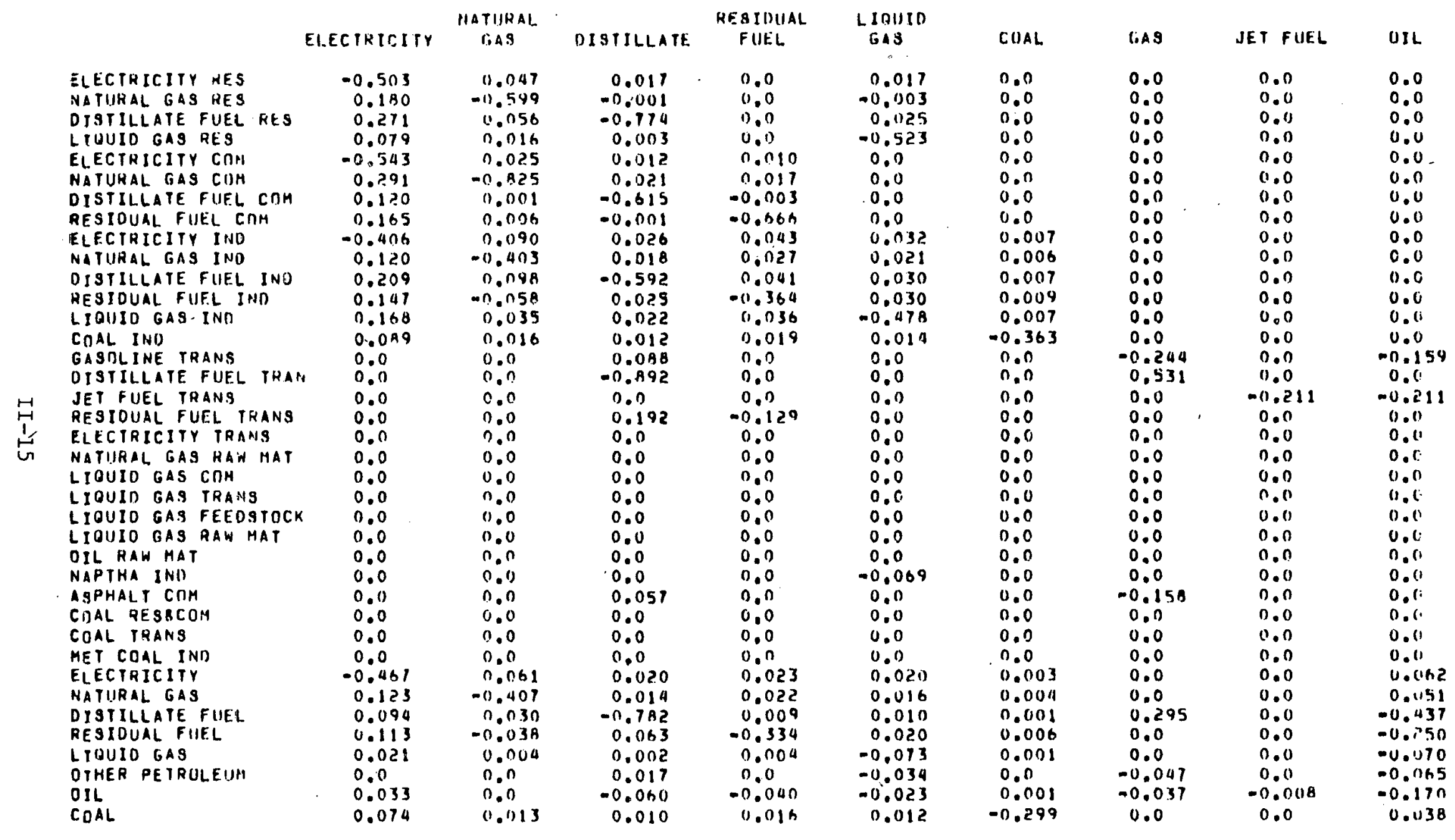


TABLE II UNSCALED ELASTICITIEB FOR DEMAND REgIUN

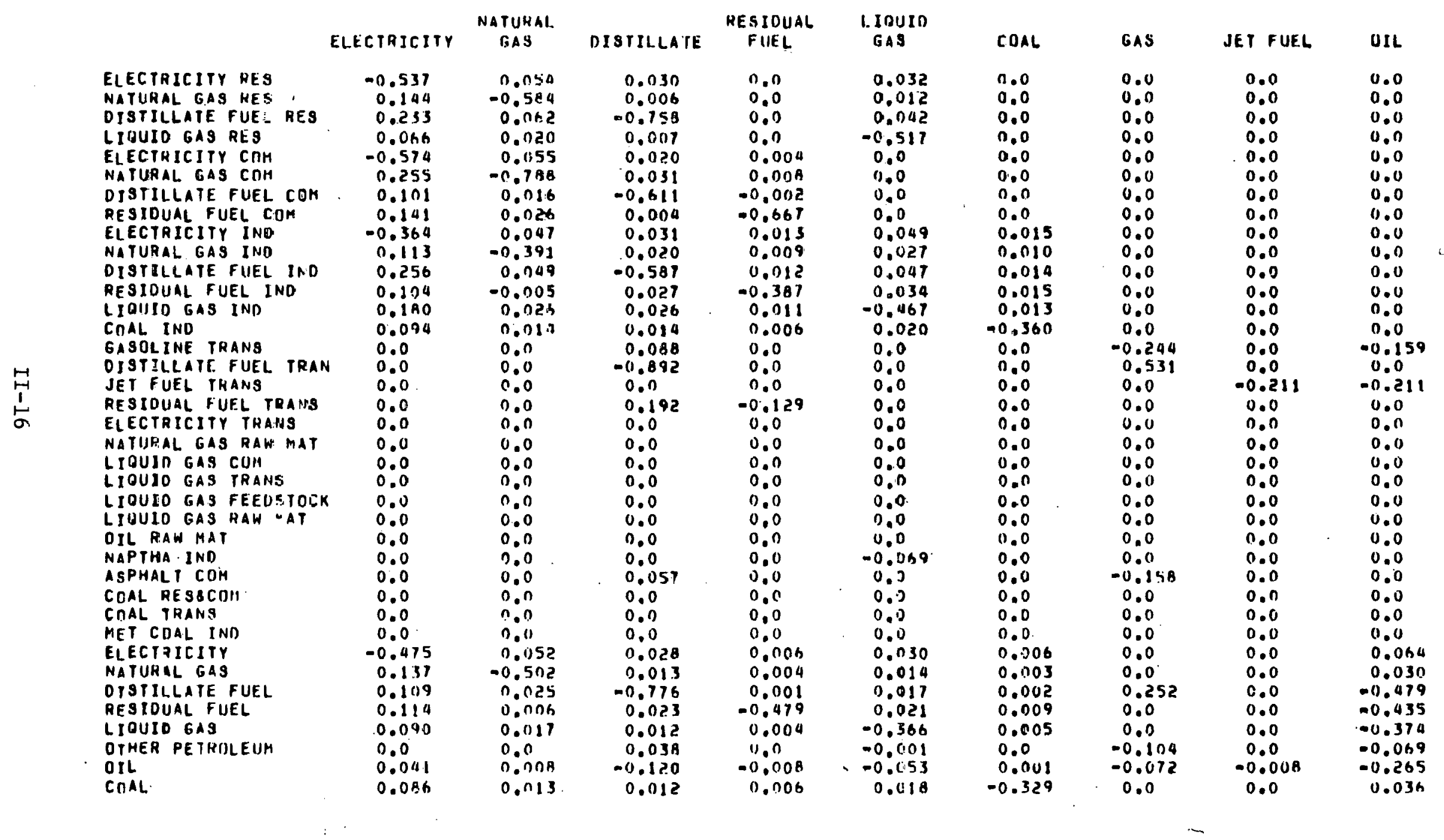


TABLE 12 IJNGCALEO ELASTICITIES FDR DEMANO HFGIUN B D 1995

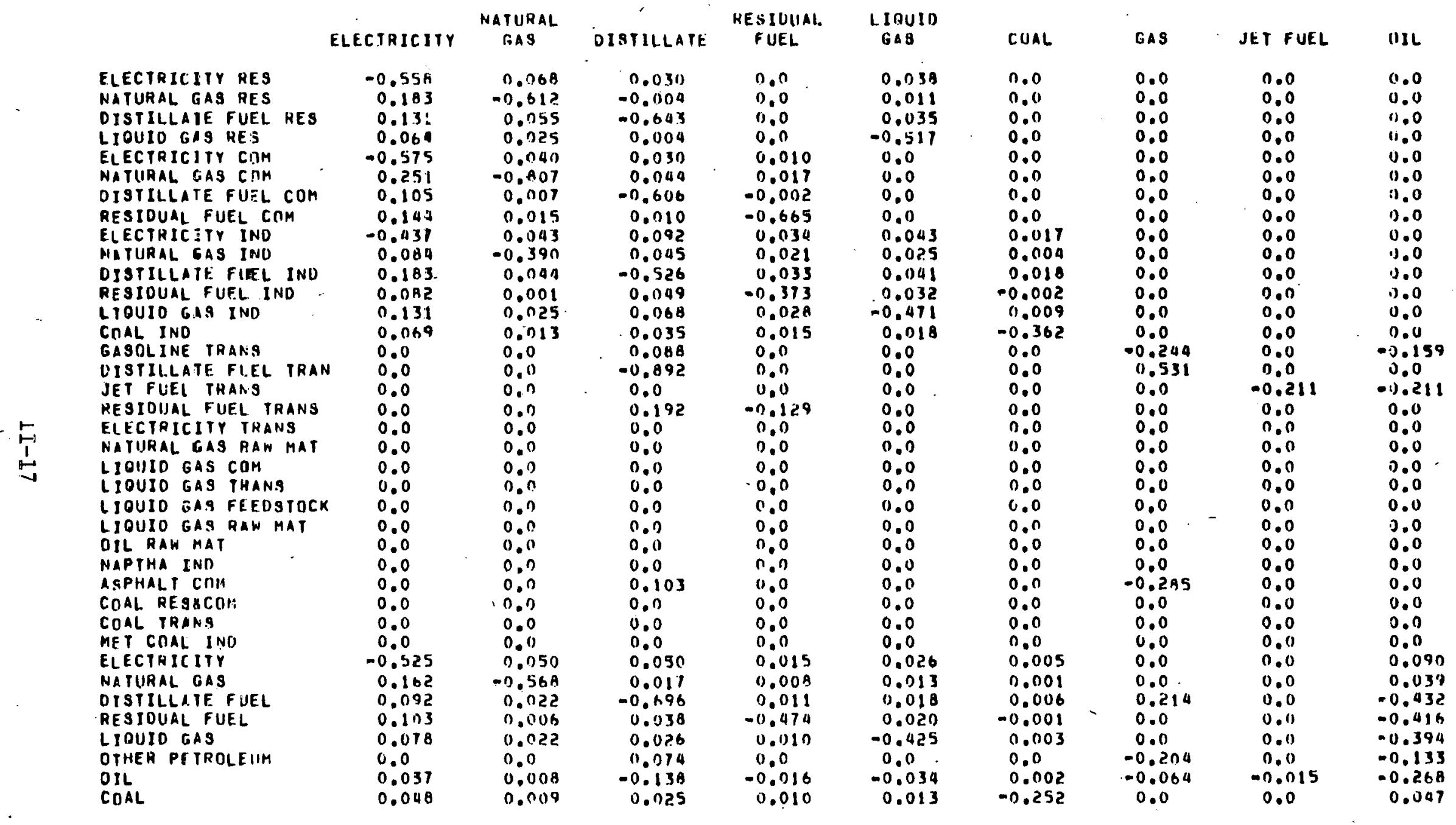


THQLE 13 IINSEALEO ELASIICITIES FOR DEMANO KEGJUH 9 - 1945

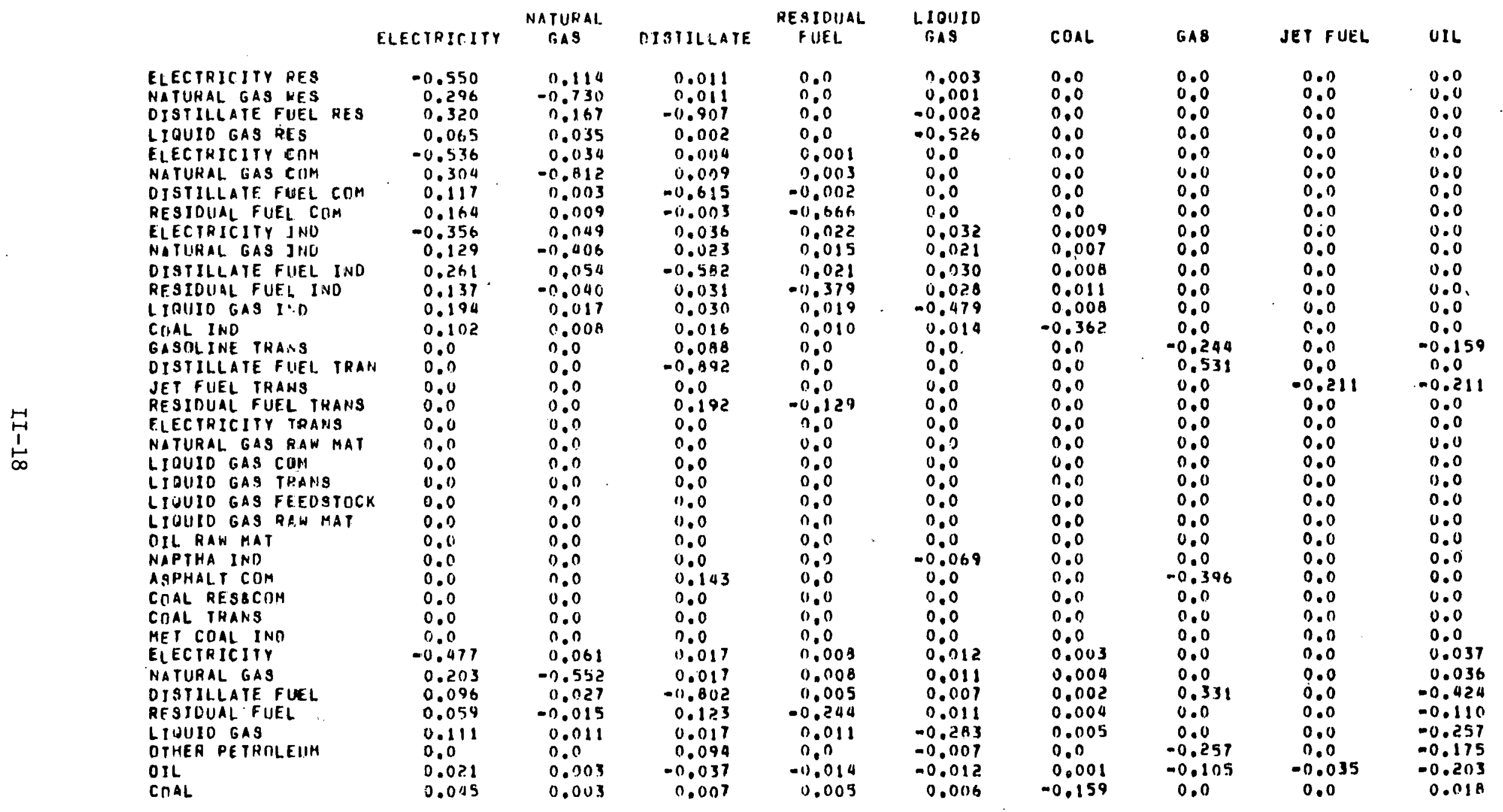


TABLE IQ UNGCALEO ELASTICITIEg FOR DEMANO NEGIIIN 10 - 1905

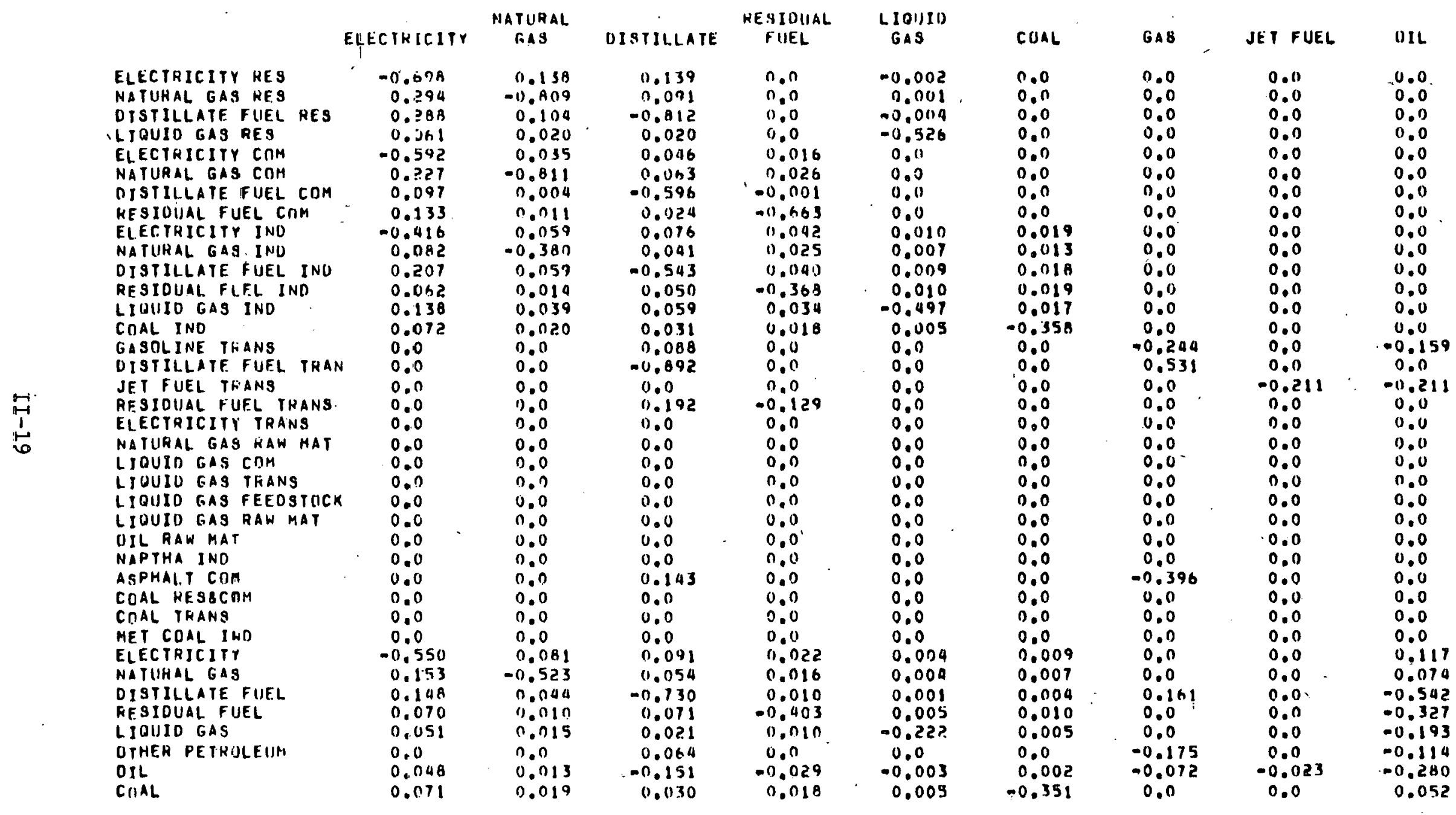


TABLE IS UNSCALEO FLARTICITIES FIIR DEMAHU REGIIN 1 - 1990

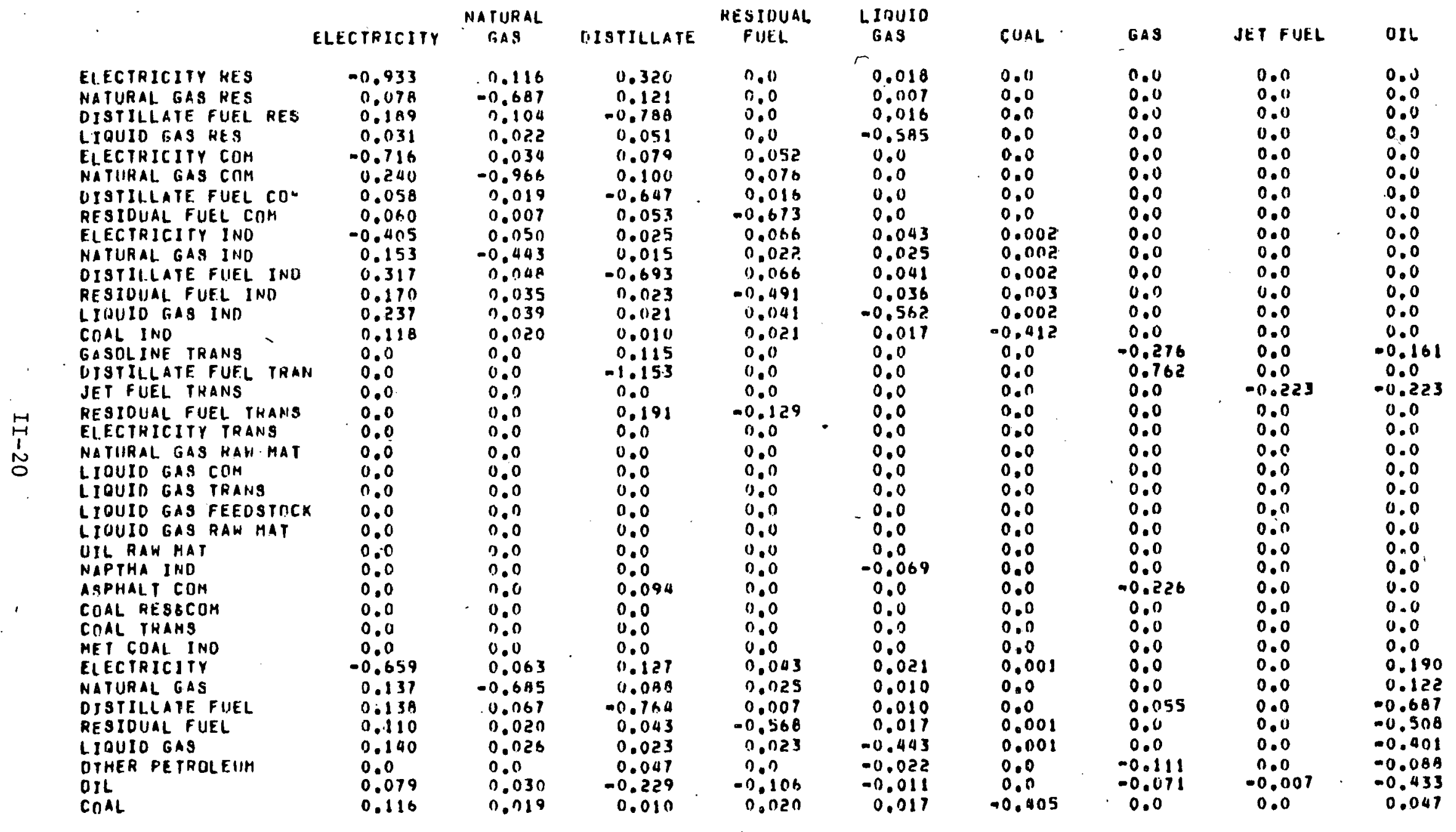


TABLE Io UNSCALEO ELASTICITIES FUR DEMAND HEgION 2 - IYYO

\begin{tabular}{|c|c|c|c|c|c|c|c|c|c|c|c|}
\hline & & & ELECTKICITY & $\begin{array}{l}\text { NATUPALL } \\
\text { GAS }\end{array}$ & DISTILLATE & $\begin{array}{l}\text { RESIDUAL } \\
\text { FUEL }\end{array}$ & $\begin{array}{l}\text { LIDUIO } \\
\text { GAS }\end{array}$ & CUAL & GAS & JET FUFL & UIL \\
\hline $\begin{array}{l}\stackrel{H}{H} \\
\vdots \\
\stackrel{\omega}{\omega}\end{array}$ & 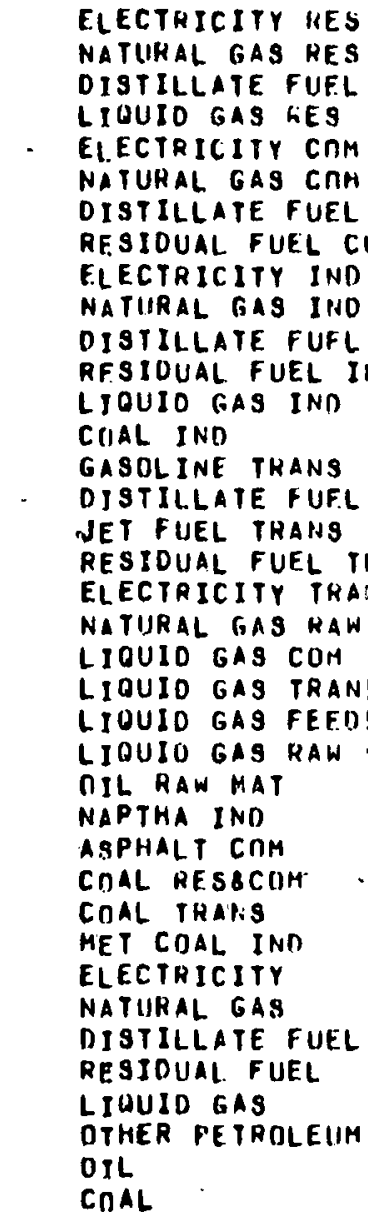 & $\begin{array}{l}\text { RES } \\
\text { COM } \\
\text { IHD } \\
\text { TRANS } \\
\text { TNS } \\
\text { N MAT } \\
\text { NS } \\
\text { ISTOCK } \\
\text { TAT } \\
\text { I }\end{array}$ & $\begin{array}{l}-0.544 \\
0.116 \\
0.114 \\
0.053 \\
-0.648 \\
0.322 \\
0.154 \\
0.111 \\
-0.397 \\
0.143 \\
0.32 .8 \\
0.138 \\
0.233 \\
0.116 \\
0.0 \\
0.0 \\
0.0 \\
0.0 \\
0.0 \\
0.0 \\
0.0 \\
0.0 \\
0.0 \\
0.0 \\
0.0 \\
0.0 \\
0.0 \\
0.0 \\
0.0 \\
0.0 \\
-0.521 \\
0.163 \\
0.143 \\
0.103 \\
0.086 \\
0.0 \\
0.066 \\
0.030\end{array}$ & $\begin{array}{l}0.026 \\
-0.081 \\
0.071 \\
0.014 \\
0.027 \\
-0.977 \\
0.015 \\
0.006 \\
0.055 \\
-0.004 \\
0.054 \\
0.027 \\
0.040 \\
0.020 \\
0.0 \\
0.0 \\
0.0 \\
0.0 \\
0.0 \\
0.0 \\
0.0 \\
0.0 \\
0.0 \\
0.0 \\
0.0 \\
0.0 \\
0.0 \\
0.0 \\
0.0 \\
0.0 \\
0.037 \\
-0.663 \\
0.052 \\
0.009 \\
0.017 \\
0.0 \\
0.019 \\
0.007\end{array}$ & $\begin{array}{l}0.045 \\
0.061 \\
-0.127 \\
0.034 \\
0.028 \\
0.044 \\
-0.037 \\
0.008 \\
0.034 \\
0.023 \\
-0.679 \\
0.034 \\
0.032 \\
0.016 \\
0.115 \\
-1.153 \\
0.00 \\
0.191 \\
0.0 \\
0.0 \\
0.0 \\
0.00 \\
0.00 \\
0.01 \\
0.0 \\
0.00 \\
0.094 \\
0.00 \\
0.00 \\
0.00 \\
0.037 \\
0.057 \\
0.146 \\
0.034 \\
0.017 \\
0.037 \\
00.186 \\
0.005\end{array}$ & $\begin{array}{l}0.0 \\
0.0 \\
0.0 \\
0.0 \\
0.041 \\
0.060 \\
0.013 \\
-0.079 \\
0.042 \\
0.025 \\
0.040 \\
0.066 \\
0.034 \\
0.017 \\
0.0 \\
0.0 \\
0.0 \\
0.129 \\
0.00 \\
0.00 \\
0.0 \\
0.0 \\
0.0 \\
0.0 \\
0.00 \\
0.0 \\
0.0 \\
0.0 \\
10.0 \\
0.00 \\
0.030 \\
0.018 \\
0.005 \\
-0.573 \\
0.011 \\
0.00 \\
-0.089 \\
0.006\end{array}$ & $\begin{array}{l}-0.006 \\
0.005 \\
0.006 \\
-0.587 \\
0.0 \\
0.0 \\
0.0 \\
0.0 \\
0.024 \\
0.016 \\
0.022 \\
0.024 \\
-0.576 \\
0.010 \\
0.0 \\
0.0 \\
0.0 \\
0.0 \\
0.0 \\
0.0 \\
0.0 \\
0.0 \\
0.0 \\
0.0 \\
0.0 \\
-0.049 \\
0.0 \\
0.0 \\
0.0 \\
0.0 \\
0.007 \\
0.007 \\
0.005 \\
0.004 \\
-0.307 \\
-0.023 \\
-0.009 \\
0.003\end{array}$ & $\begin{array}{l}0.0 \\
0.0 \\
0.0 \\
0.0 \\
0.0 \\
0.0 \\
0.0 \\
0.0 \\
0.015 \\
0.011 \\
0.014 \\
0.018 \\
0.014 \\
-0.006 \\
0.0 \\
0.00 \\
0.0 \\
0.0 \\
0.0 \\
0.0 \\
0.0 \\
0.0 \\
0.0 \\
0.0 \\
0.0 \\
0.0 \\
0.0 \\
0.0 \\
0.0 \\
0.0 \\
0.006 \\
0.003 \\
0.001 \\
0.003 \\
0.004 \\
0.0 \\
0.001 \\
-0.132\end{array}$ & $\begin{array}{l}0.0 \\
0.0 \\
0.0 \\
0.0 \\
0.0 \\
0.0 \\
0.0 \\
0.0 \\
0.0 \\
0.0 \\
0.0 \\
0.0 \\
0.0 \\
0.0 \\
00.276 \\
0.762 \\
0.0 \\
0.0 \\
0.0 \\
0.0 \\
0.0 \\
0.0 \\
0.0 \\
0.0 \\
0.0 \\
0.0 \\
00.226 \\
0.0 \\
0.0 \\
0.0 \\
0.0 \\
0.0 \\
0.075 \\
0.0 \\
0.0 \\
0000 \\
00.078 \\
0.0\end{array}$ & $\begin{array}{l}0.0 \\
0.0 \\
0.0 \\
0.0 \\
0.0 \\
0.0 \\
0.0 \\
0.0 \\
0.0 \\
0.0 \\
0.0 \\
0.0 \\
0.0 \\
0.0 \\
0.0 \\
0.0 \\
.0 .223 \\
0.0 \\
0.0 \\
0.0 \\
0.0 \\
0.0 \\
0.0 \\
0.0 \\
0.0 \\
0.0 \\
0.0 \\
0.0 \\
0.0 \\
0.0 \\
0.0 \\
0.0 \\
0.0 \\
0.0 \\
0.0 \\
0.0 \\
.0 .014 \\
0.0\end{array}$ & 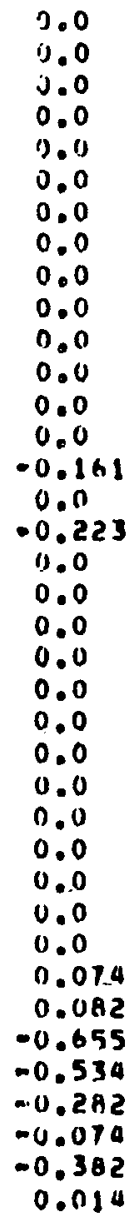 \\
\hline
\end{tabular}


TABLE 17 UNSCALED ELASTICITIES FOR DEMANO REgION 3 - 1440

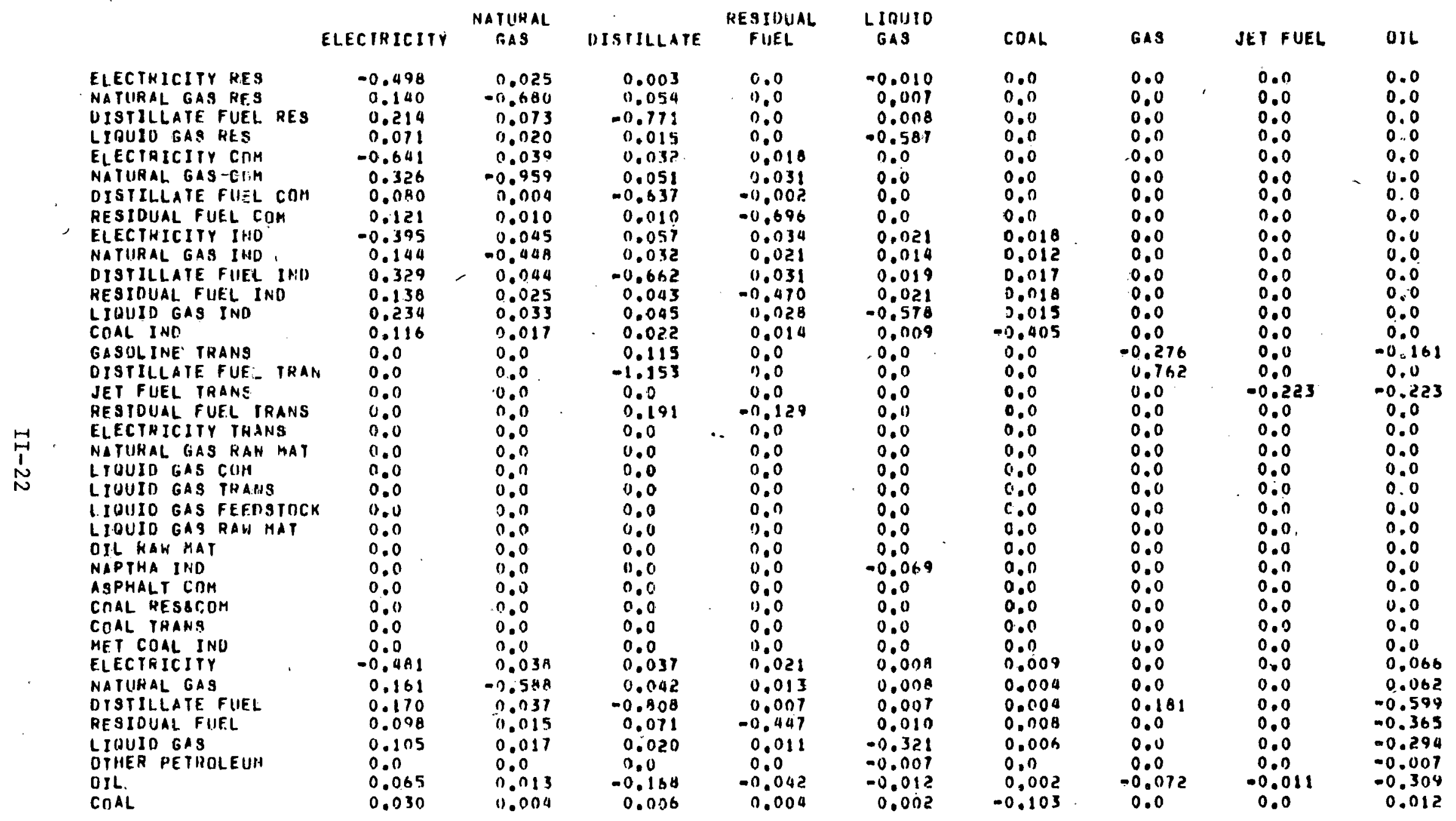


TABLE 18 UNSCALED ELASTICITIES FUH DEMAND HEgIUN 4 - 1990

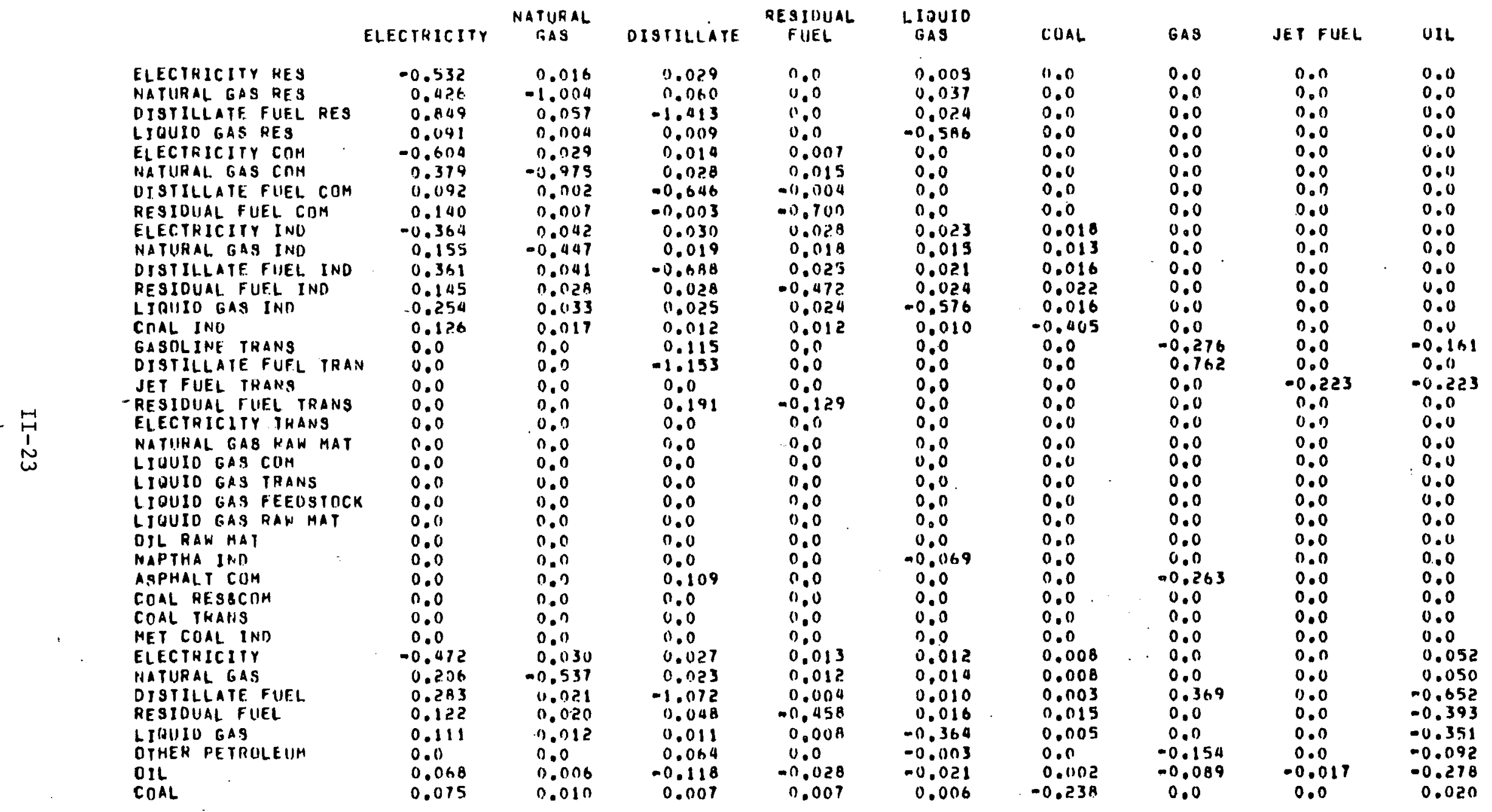


TABLE 19 INSCALED ELAg:ICITIES FIIR DEMAND hEgION 5 - 1990

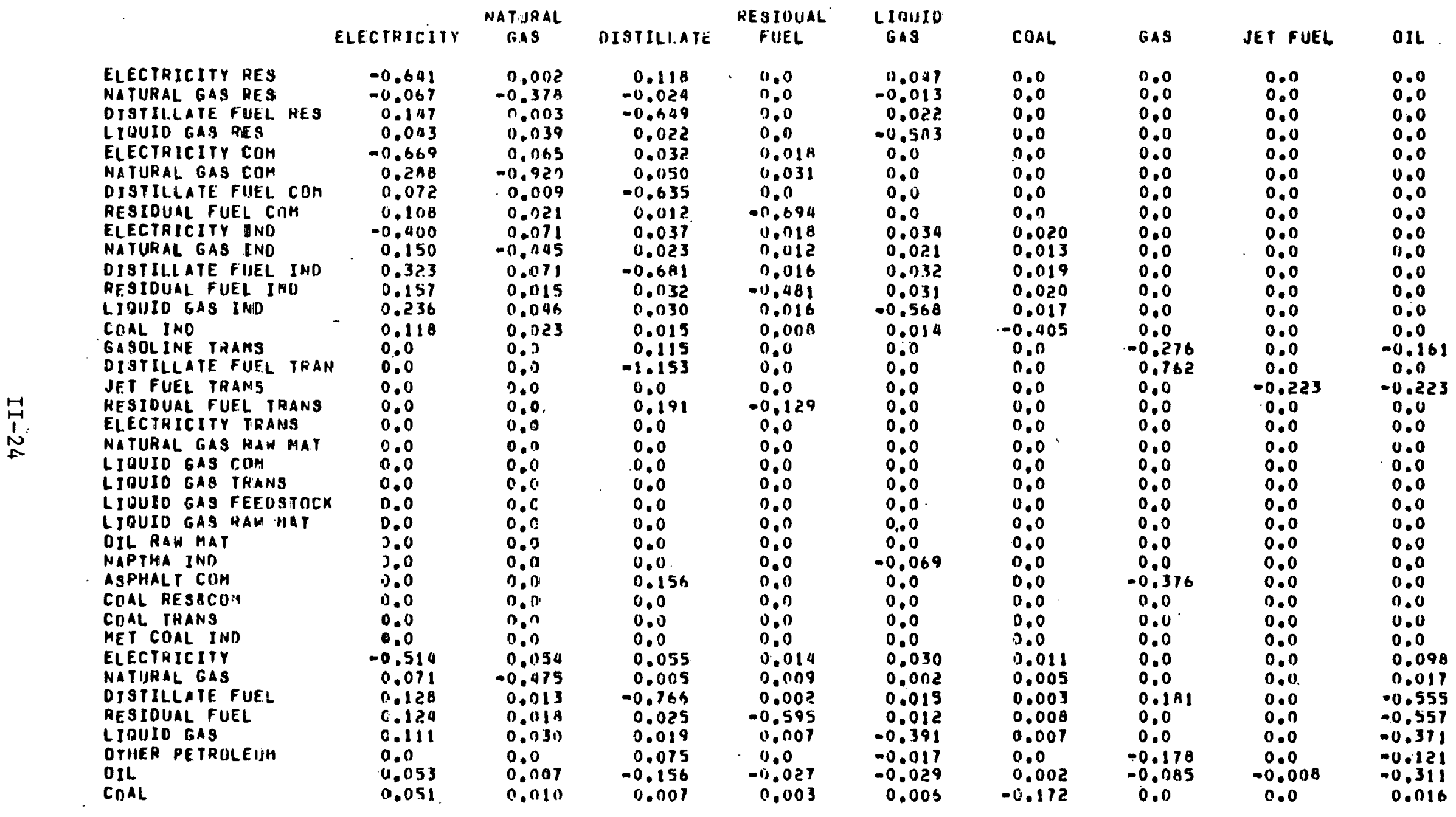


TABLE 20 UNSCALED ELASTICITJES FUR DEMANO HEGION $6=1990$

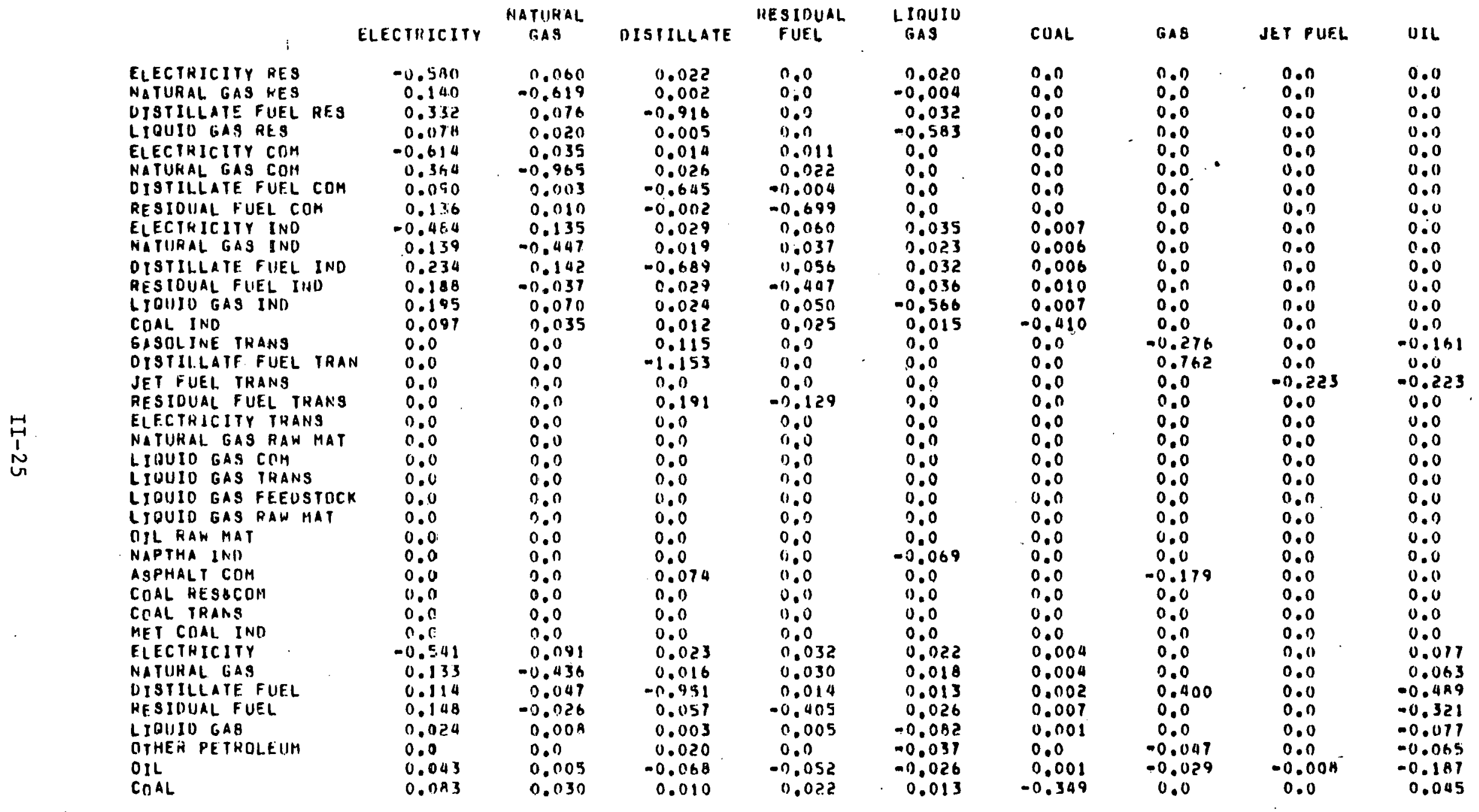


TABLE 2I UNSCALED ELASTICITIES FUR DFHAHO REGIUN $?$ - 1990

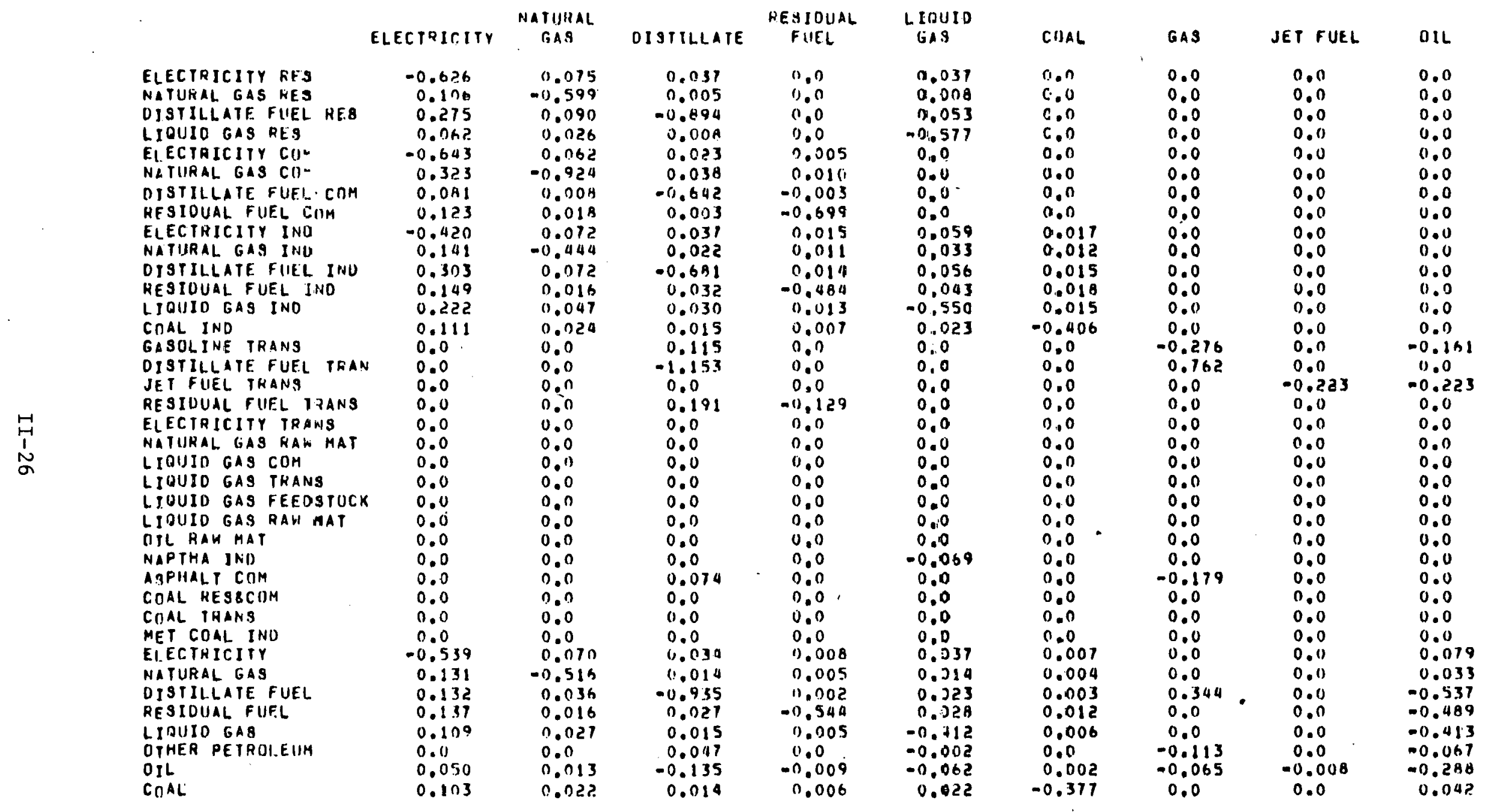


TAGLE 22 UNSCALEO ELÁsTICITIES fUR IEMAMO REgION $A$ - 1990

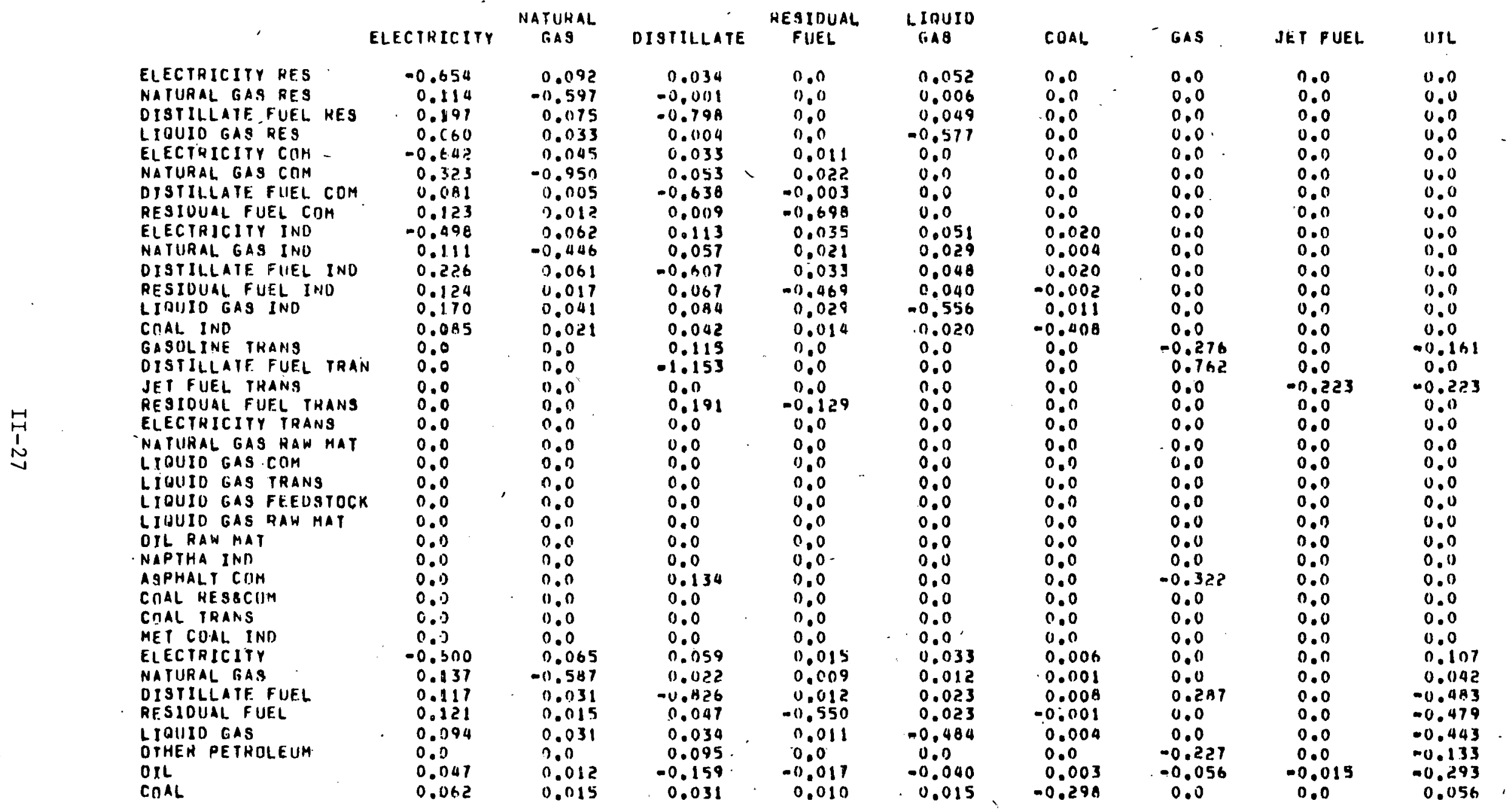


TABLE 23 IINSCALED ELASTICITIES For nEMANO REgIIIN 9 - 1990

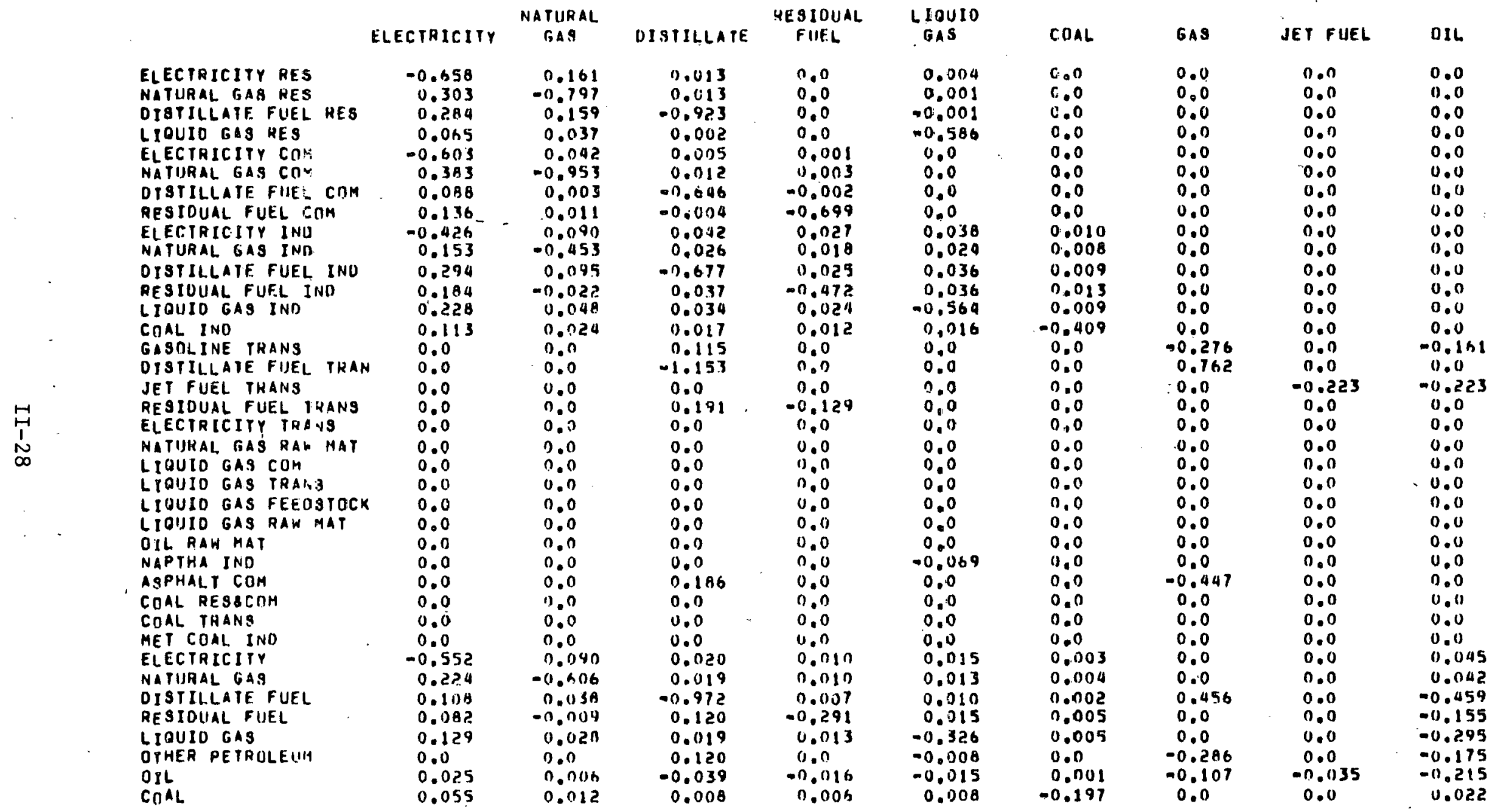


TABLE 24 UNSCALED ELASTICITIES FOR DEMAND KEgIUN 10 - 1990

\begin{tabular}{|c|c|c|c|c|c|c|c|c|c|}
\hline & ELECTRICITY & $\begin{array}{l}\text { NATURAL } \\
\text { GAS }\end{array}$ & DISTILLATE & $\begin{array}{l}\text { RESIDUAL } \\
\text { FUEL }\end{array}$ & $\begin{array}{l}\text { LIQUIO } \\
\text { GAS }\end{array}$ & COAL & GAB & JET FUEL & LIL \\
\hline ELECTHICITY RES & -0.761 & 0.136 & 0.146 & 0.0 & -0.003 & $n .0$ & 0.0 & 0.0 & 0.0 \\
\hline NATUHAL GAS HES & 0.306 & $=0.890$ & 0.101 & 0.0 & 0.0 & 0.0 & 0.0 & 0.0 & 0.0 \\
\hline DISTILLATE FUEL HES & 0.245 & 0.101 & -0.823 & 0.0 & $=0.004$ & 0.0 & 0.0 & 0.0 & 0.0 \\
\hline LIQUIN GAS RES & 0.060 & 0.055 & 0.023 & 0.0 & -0.587 & 0.0 & 0.0 & 0.0 & 6.0 \\
\hline ELECTRICITYY COM & -0.665 & 0.043 & 0.051 & 0.019 & 0.0 & 0.0 & 0.0 & 0.0 & 0.0 \\
\hline NATURAL GAS CUM & 0.293 & $=0.953$ & 0.076 & 0.033 & 0.0 & 0.0 & 0.0 & 0.0 & 0.0 \\
\hline OISTILLATE FUEL COM & 0.071 & 0.004 & .0 .627 & $=n .0 \cap 2$ & 0.0 & 0.0 & 0.0 & 0.0 & 0.0 \\
\hline RESIDUAL FUEL COM & 0.108 & 0.011 & 0.023 & $=10.696$ & 0.0 & 0.0 & 0.0 & 0.0 & 0.0 \\
\hline ELECTRICITY INO & $=0.468$ & D.OAB & 0.085 & 0.044 & 0.011 & 0.022 & 0.0 & 0.0 & 0.0 \\
\hline NATURAL GAS IND & 0.115 & -0.434 & 0.045 & 0.026 & 0.007 & 0.015 & 0.0 & 0.0 & 11.0 \\
\hline DISTILLATE FUEL INU & 0.257 & 0.087 & .0 .635 & 0.042 & 0.010 & 0.020 & 0.0 & 0.0 & 0.0 \\
\hline RESIUUAL FUEL INO & 0.114 & 0.031 & 0.059 & $=0.463$ & 0.012 & 0.023 & 0.0 & 0.0 & 0.0 \\
\hline LIQUID GAS IND & 0.185 & 0.060 & 0.065 & 0.036 & -0.587 & 0.018 & 0.0 & 0.0 & 0.0 \\
\hline COAL IND & 0,092 & $0.03 n$ & 0.032 & 0.018 & 0.005 & -0.404 & 0.0 & 0.0 & 11.0 \\
\hline GASOLINE TRANS & 0.0 & 0.0 & 0.125 & 0.0 & 0.0 & 0.0 & -0.276 & 0.0 & $=0.161$ \\
\hline DISTILLATE FUEL TRAN & 0.0 & 0.0 & -1.153 & 0,0 & 0.0 & 0,0 & 0.762 & 0.0 & 0.0 \\
\hline JET FUEL THANS & 0.0 & 0.0 & 0.0 & 0.0 & 0.0 & 0.0 & 0.0 & $=0.223$ & -0.223 \\
\hline RESIDUAL FUEL THANS & 0.0 & 0.0 & 0.191 & -0.129 & 0.0 & 0.0 & 0.0 & 0.0 & 0.0 \\
\hline ELECTRICITY TRANS & 0.0 & 0.0 & 0.0 & 0.0 & 0.0 & 0.0 & 0.0 & 0.0 & 0.0 \\
\hline NATURAL GAS HAW MAT & 0.0 & 0.0 & 0.0 & 0.0 & 0.0 & 0.0 & 0.0 & 0.0 & 0.0 \\
\hline LIOUID GAS COM & 0.0 & 0.0 & 0.0 & 0.0 & 0.0 & 0.0 & 0.0 & 0.0 & 0.0 \\
\hline LIOUIO GAS THANS & 0.0 & 0.0 & 0.0 & 6.0 & 0.0 & 0.0 & 0.0 & 0.0 & 0.0 \\
\hline LJQUID GAS FEEDSTOCK & 0.0 & 0.0 & 0.0 & 0.0 & 0.0 & 0.0 & 0.0 & 0.0 & 0.0 \\
\hline LIOUIO GAS RAW MAT & 0.0 & 0.0 & 0.0 & 0.0 & 0.0 & 0.0 & 0.0 & 0.0 & 1.0 \\
\hline OIL RAW MAT & 0.0 & 0.0 & 0.0 & 0.0 & 0.0 & 0.0 & 0.0 & 0.0 & 0,0 \\
\hline NAPTHA INA & 0.0 & 0.0 & 0.0 & 0.0 & 0.0 & 0.0 & 0.0 & 0.0 & 0.0 \\
\hline ASPHALP COM & 0.0 & $n .0$ & 0.186 & 0.0 & 0.0 & 0.0 & .0 .447 & 0.0 & 11.0 \\
\hline COAL RES\&COM & 0.0 & 0.0 & 0.0 & 0.0 & 0.0 & 0.0 & 0.0 & 0.0 & $u .0$ \\
\hline CIAL TRANA & 0.0 & 0.0 & 0.0 & 0.0 & 0.0 & 0.0 & 0.0 & 0.0 & 0.0 \\
\hline MEY COAL IND & 0.0 & 0.0 & 0.0 & 0.0 & 0.0 & 0.0 & 0.0 & 0.0 & 0.0 \\
\hline EIECTRICITY & -0.608 & 0.094 & 0.098 & 0.024 & 0.004 & 0.010 & 0.0 & 0.0 & 0.126 \\
\hline NATURAL GAS & 0.185 & $=0.590$ & 0.062 & 0.016 & 0.0134 & 0.007 & 0.0 & 0.0 & 0.082 \\
\hline DISTILLATE FUEL & 0.151 & 0.052 & -0.023 & 0.011 & 0.002 & 0.006 & 0.214 & 0.0 & -0.580 \\
\hline RESIDUAL FUEL & 0.092 & 0.020 & 0.073 & $=0.467$ & 0.006 & 0.012 & 0.0 & 0.0 & $=0.387$ \\
\hline LIOUID GAS & 0.066 & 0.021 & 0.02 .3 & 0.011 & $=0.249$ & 0.006 & 0.0 & 0.0 & $=0.217$ \\
\hline OTHER PETRDLEUM & 0.0 & 0.0 & 0.083 & 0.0 & 0.0 & 0.0 & $=0.197$ & 0.0 & $\infty 0.115$ \\
\hline $0 \mathrm{IL}$ & 0.052 & 0.017 & -0.166 & -0.033 & -0.003 & 0.003 & $=0.069$ & .0 .023 & $=0.294$ \\
\hline COAL & 0.091 & $0.03 n$ & 0.032 & $0.01 A$ & 0.005 & -0.399 & 0.0 & 0.0 & 0.054 \\
\hline
\end{tabular}




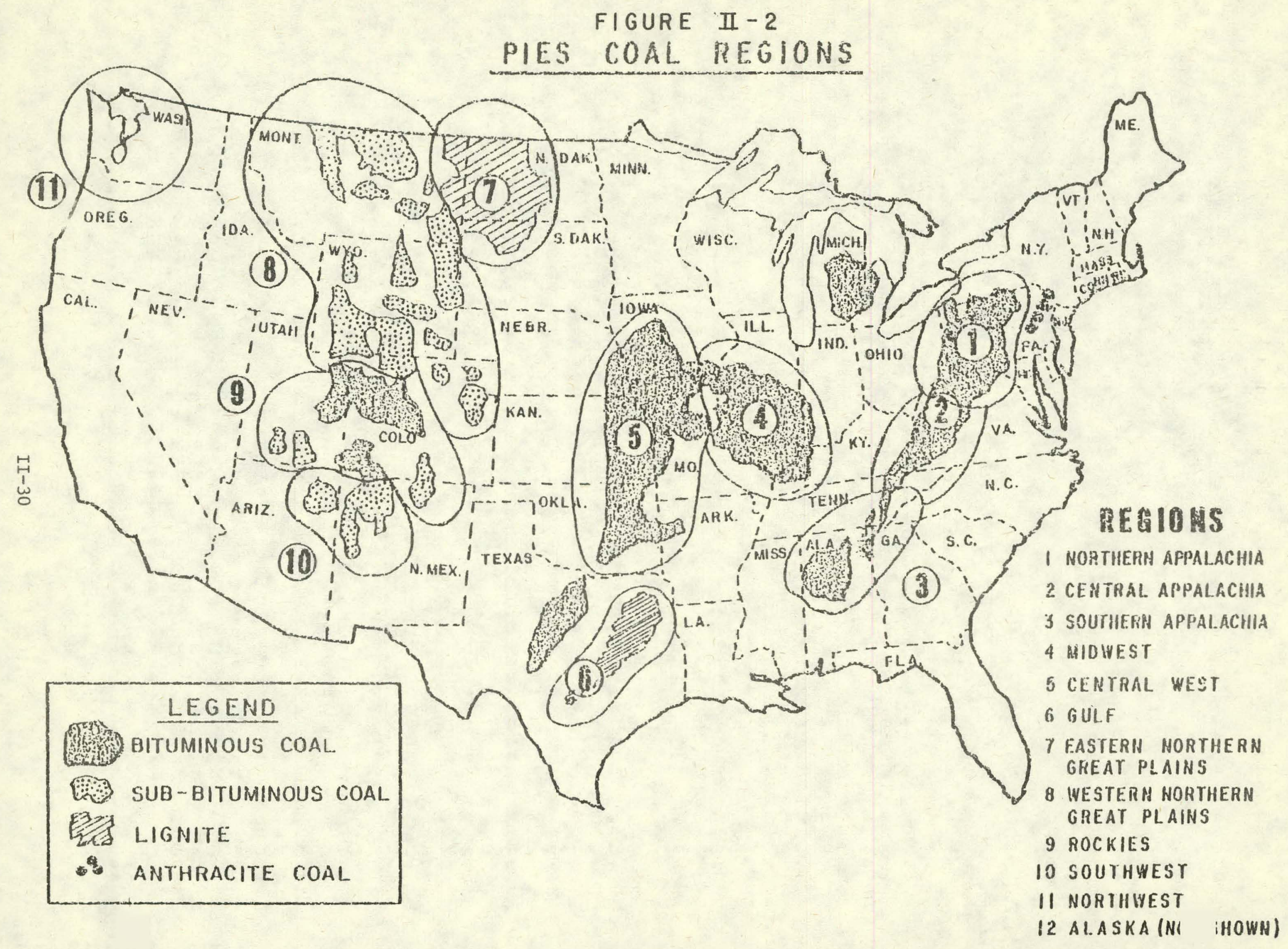


The PRICE STEP column has entries for the minimum acceptable selling price the product will accept, given its rate of return. This price includes all direct costs, severance taxes, reclamation costs and other related costs and takes into consideration the producer's rate of return, miner productivity, black lung insurance, etc. Each price represents the lowest price at which mines will open (NEW MINES) or be brought into operation (EXISTING MINES).

Mine type names are not stated, but are listed in order of ascending price; thus, each price step represents a different mine type.

INCREMENTAL SUPPLY gives the maximum production or supply available at each price step. This supply is the amount of coal available from that mine type and is divided between surface mines and deep mines according to historical patterns. CUMULATIVE SUPPLY lists the total coal supply available to meet demand at e'ach price step.

The CAPITAL column represents the present value of the cumulative investment required between 1978 and the target year to continue operating existing mines or to open each new mine. The units used are millions of 1975 dollars per thousand tons per calendar day.

The last table in each set, TOTAL COAL SUPPLY AT THE HIGHEST PRICE STEP, provides the regional and national production levels, assuming that the highest possible price level is realized by operating both new and existing mines. The regional production levels are also aggregated into EAST and WEST coal region totals. The last column gives the total regional supply of coal in millions of Btus per day. Conversion factors are given below the table under COAL TYPE DESCRIPTORS.

The two main sources for historical production data are the Bureau of Mines and the ICF, Inc. publication, "PIES Supply Curve Methodology," (Final Report for the FEA, under Contract No. CO-05-50198-00, December 1975). One of the main sources for capital data is "Basic Capital, Investment and Costs for Surface and Deep Mines," published in 1975 by the Bureau of Mines. 
Other data sources are:

- Severance taxes: State offices

- Reclamation costs: regional questionnaires, Coal Analysis Division (DOE) estimates.

- Black lung insurance premium: Morgantown Process Evaluation Group

- All other data: Bureau of Mines, ICF, Inc., National Coal Model, Geological Survey, NCA, TRW, and numerous other sources.

A final documentation of the data sources for The National Coal Model is expected in mid-1978 from Arthur Young and Company. 
19A= COA!. SIIPOLY DATA

TNOIT: TI THE PIES INTPERATIN: MODEI.

197A ANNIIAL AMMINISTRATOR'S REPOR'

(OFERATIOH DATE 121977 


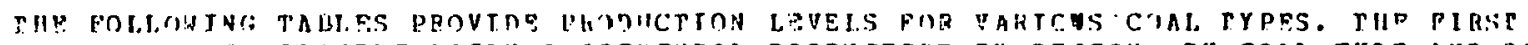

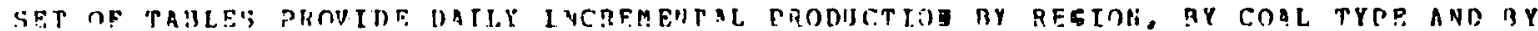
CORRES RUNUTVI: DRTCE I.FUPLS. TIIESF PRTCES ALF TUF RINIMIM ACCFPTABLP DRTCES. ANII

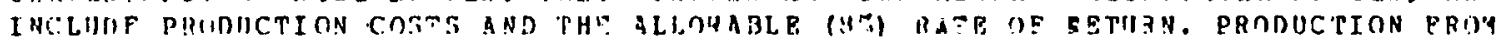

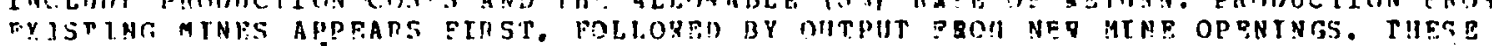

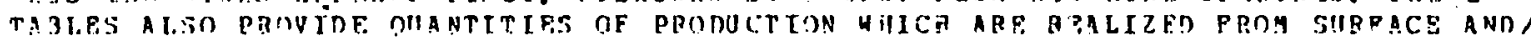

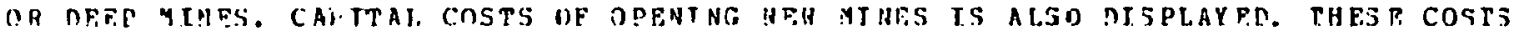
PEPRESENT THE TOTAI. CAPITAI, EXPEVIITIRF ASSOCIATED HITH APH MINBS.

THF IAST TABLP PAOVINES THF. PFIIONAL, AMO NATIONAL PRODIICEION LEVELS ASSUITNG TUP.

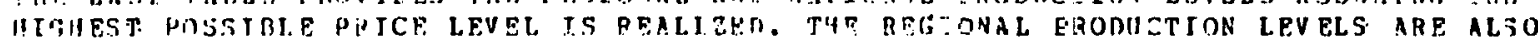

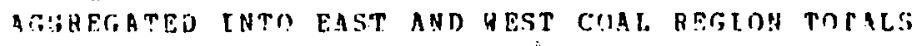




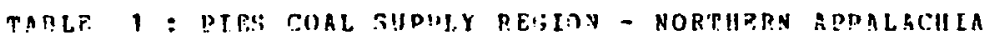

CNAI. TYPP: "ICH BTI (2), M MARTI/TON

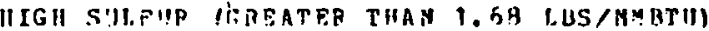

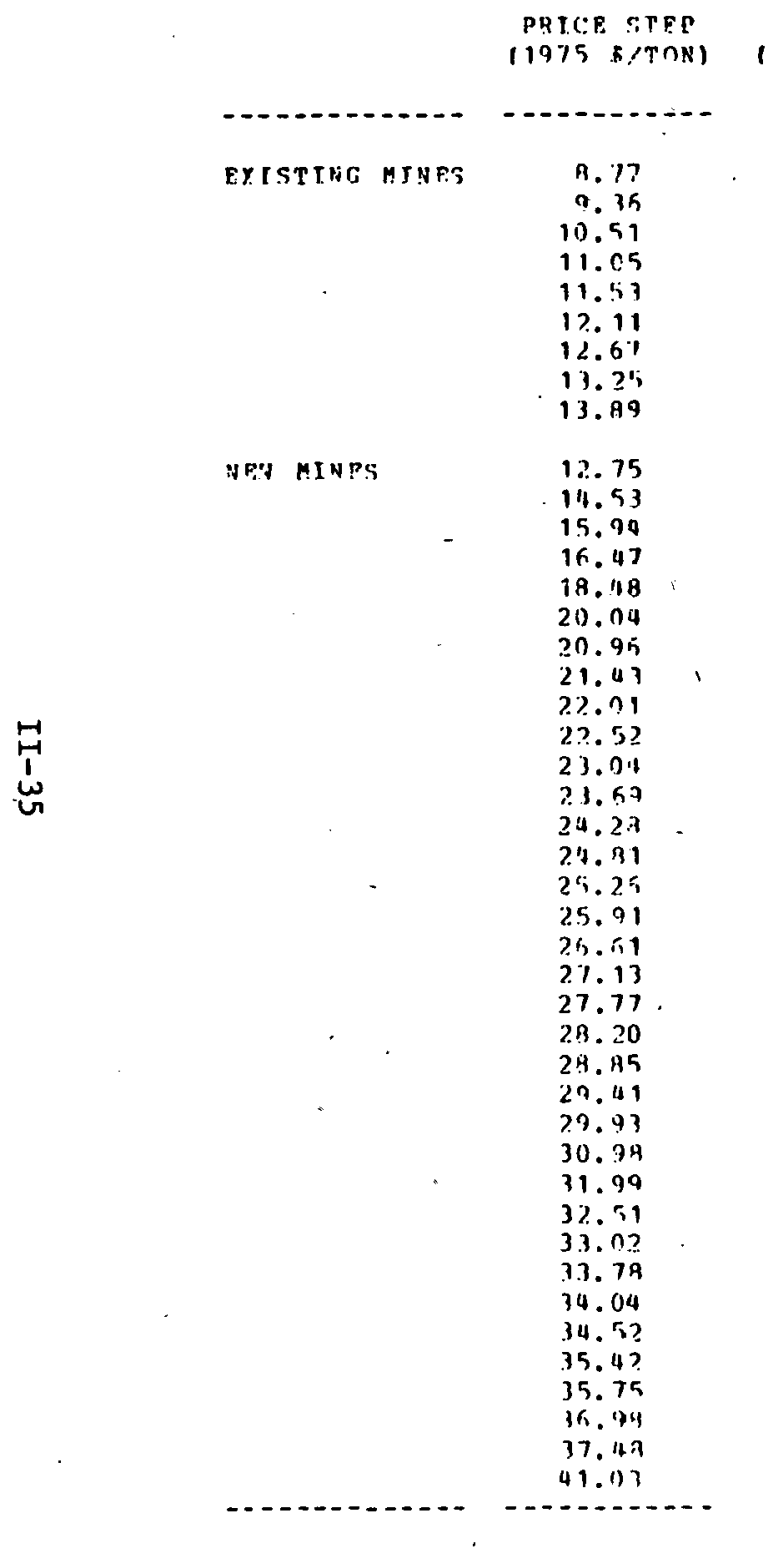

INCREMFNTAL SIIPPLY

TUNISANAS OF TONS PFR DAY SUPFACE DFVD TOTAL:

2.94
11.00
3.91
.36
19.26
3.79
1.411
1.01
1.01

.12
35.97
4.06
44.71
9.05
9.95

1.06

19.37

39. $\$ 5$

4.41

6.2. 95

12.93

17. 5n

1.01
1.01

2.60

2.50
11.71

$2 . \sin$

13.01

13.01

12. .99

14. 91

1.94

2.87

35. 16

5.21

3. 13

3.65

2.

2.45
.52

3.511

3.511

4.16
0.36
3.07
11.52
3.07
5.71
.7319

CII MUIHLATIVE. SUPPLY

TIJUISANGS OP TONS PER DAY) (MTLLIONS OF 1975 \&) SUAPACD DEEP TOTAL

$\begin{array}{rrr}2.94 & .12 & 3.05 \\ 13.94 & 8.49 & 22.42 \\ 17.76 & 114.51 & 52.27 \\ 17.12 & 48.57 & 65.58 \\ 15.37 & 93.25 & 129.54 \\ 10.16 & 192.31 & 142.47 \\ 19.55 & 111.36 & 160.01 \\ 19.55 & 111.36 & 161.07 \\ 512.75 & 111.36 & 162.03\end{array}$

$$
2.60
$$

$$
\begin{array}{r}
2.60 \\
14.31 \\
15.32
\end{array}
$$$$
15.32
$$$$
23.9 .1
$$$$
42.9 .5
$$$$
42.75
$$$$
\begin{aligned}
& 42.75 \\
& 42.95
\end{aligned}
$$$$
\begin{aligned}
& 42.95 \\
& 42.95
\end{aligned}
$$$$
\begin{aligned}
& 42.95 \\
& 5 \% .93
\end{aligned}
$$$$
5 \% .93
$$$$
55.73
$$$$
73.74
$$$$
72.57
$$$$
715.411
$$$$
75.44
$$$$
2.60
$$

14. 31

14.31

16.92

29.9 .3

42.95

53.90

B1. 30

123. 38

151.2?

226.70

275.79

$34 ? .09$

1109.21

45 A. 91

505.60

578.27

$110.60 \quad 559.00 \quad 679.60$

$113.60 \quad 623.57 \quad 734.19$

764.81

7 ค6. 56

786.56
797.72

797.72
914.46

914.46
82.6 .79

962.74

ค7 7.65

999.2?

907.11

924.34

928.50

?314. 96

937.97
949.54

952.61

952.61

950.53

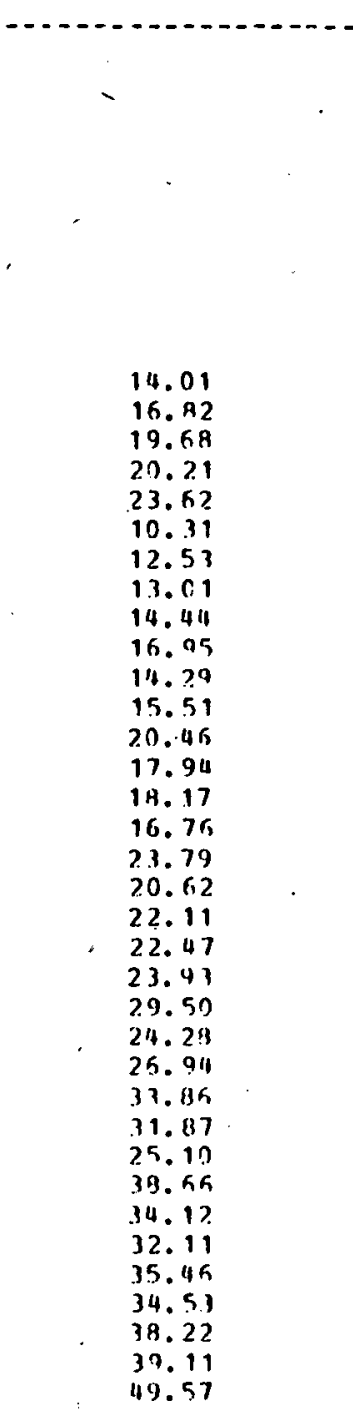

PIfS COD?: Ch

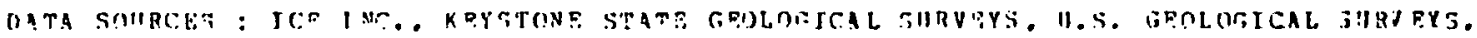

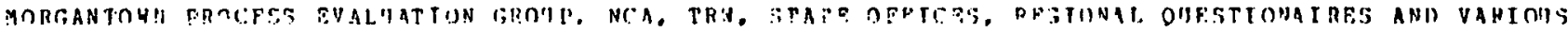

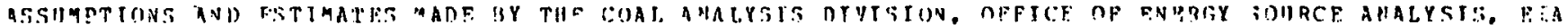

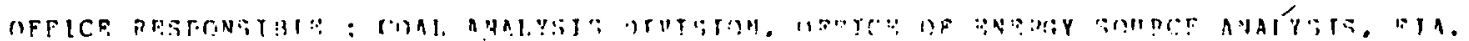




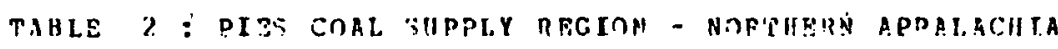

COA. TYPF: YENI'IA BTII (21.4 MMBTH/TON!

HIGII SHI.FIR (TEEATER TIIAN 1. KR LBS/MMBTU)

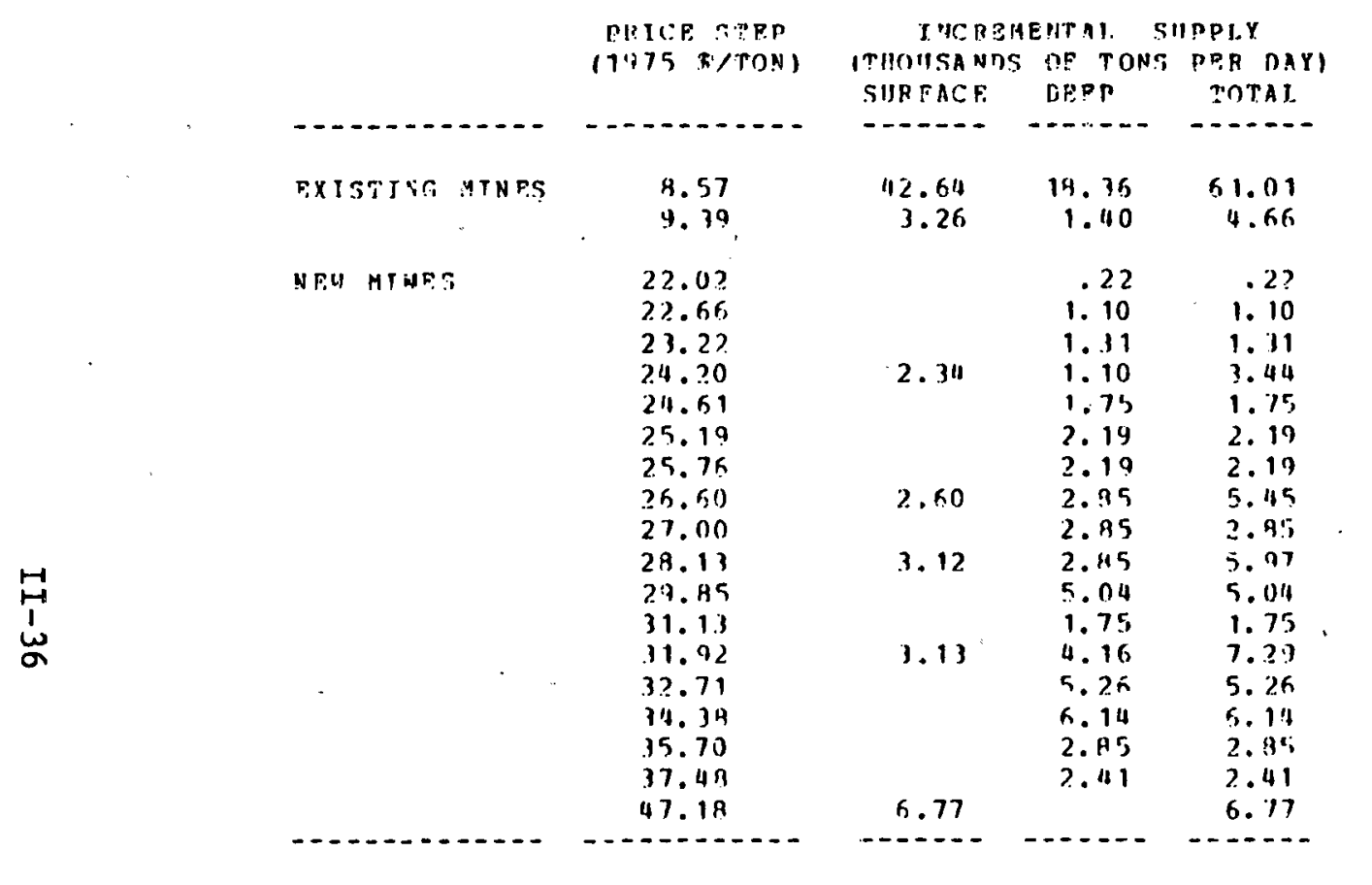

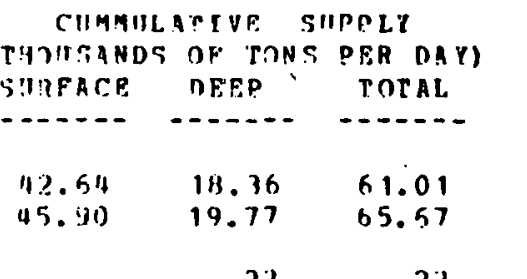

CAPTTAL

IMELLONS OF $1975: 1$

- - - - - - - - - - - - - - - - -

\begin{tabular}{|c|c|c|}
\hline & $\begin{array}{r}.72 \\
1.31 \\
2.53\end{array}$ & $\begin{array}{l}.22 \\
1.31 \\
2.63\end{array}$ \\
\hline 2.34 & 3.71 & 5.07 \\
\hline 2.34 & 5.49 & 7. 22 \\
\hline 2.34 & 7.67 & 10.01 \\
\hline 2.34 & 9.RK & 12.20 \\
\hline 4.94 & 12.71 & 17.66 \\
\hline 4.94 & 15.56 & 20.51 \\
\hline 3.127 & 18.41 & 2.5 .144 \\
\hline B. . ? & 23.45 & 31.52 \\
\hline 8.07 & 25.2 .1 & 33.27 \\
\hline 11.19 & 29.37 & 40.55 \\
\hline 11.19 & 34.57 & 45.82 \\
\hline 11.19 & 40.77 & 51.96 \\
\hline 11.19 & 43.62 & 54.81 \\
\hline 11.19 & 45.03 & 57.22 \\
\hline 17.95 & 46.03 & 63.99 \\
\hline
\end{tabular}

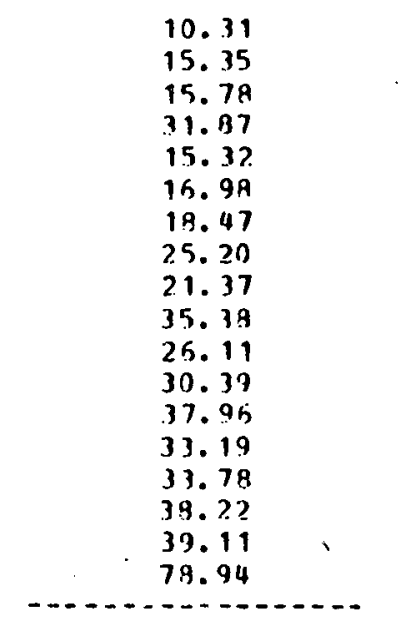

PIES CODE: CH

DATA STIIRCES : ICF I VC̀. KEYSTONF STATE GEOLOGICAL SIBUFYS. H.S. GEOLDGIEAL SURVFYS.

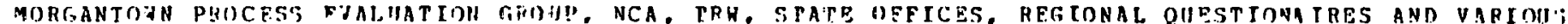

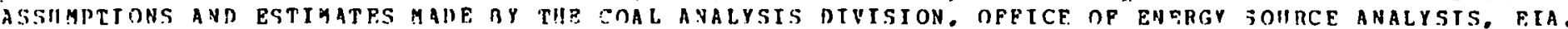

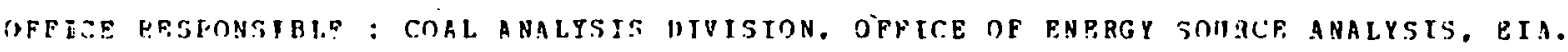


THHLE J : PIES COAT. BIPRLY RHEION - NITTHPRII APPALACHIA

COAL TYPE: HTGH BTH 123. a YMBTIITON

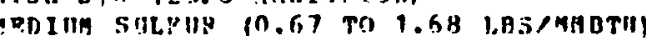

PQICP STPP

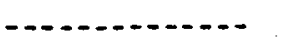

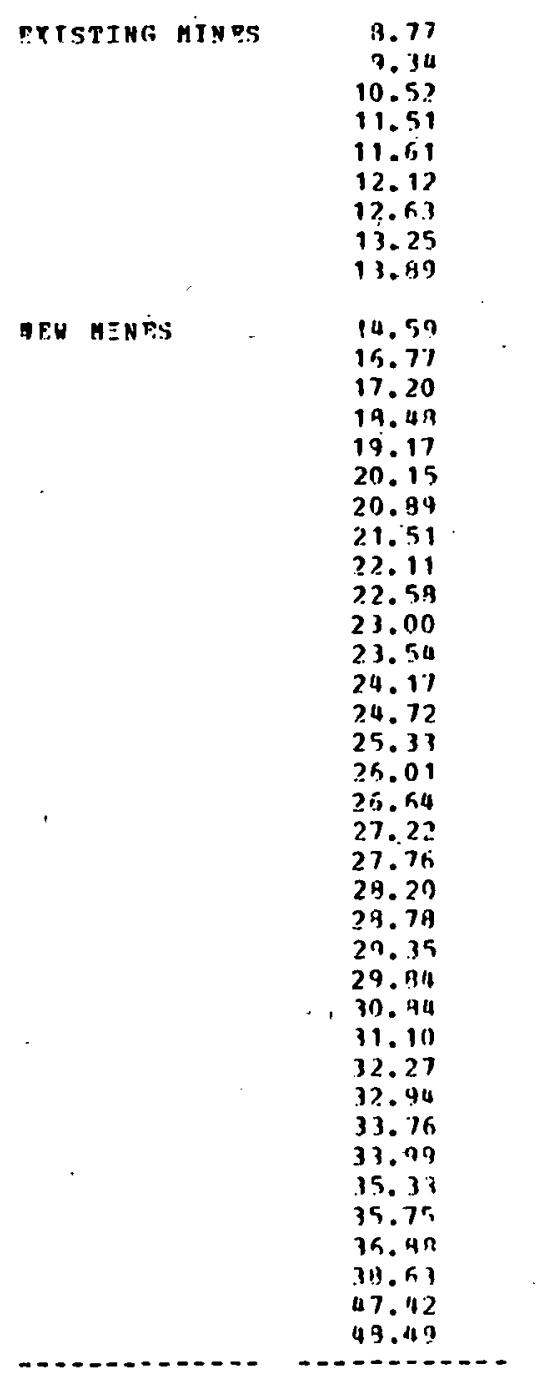

IACRPMENTAL SIIPRLY THOUSANAS OP TONS HER DAYI SURFACP. DEPR TOFAL

.78
.06
2.59
17.48
.42
3.58
23.52
4.38
4.30

.04
2.02
9.17
.23
1.92
11.38
2.02
2.112

.70
.50
4.71
26.65
.65
5.51
34.90
$6.3 \%$
6.32

1.30

2.61
1.30

2.50

1.30
CH MAUI,ATIVE SIIPPLY (TUOYSANDS OF TOYS PER DAY) SITFACE DRP.P

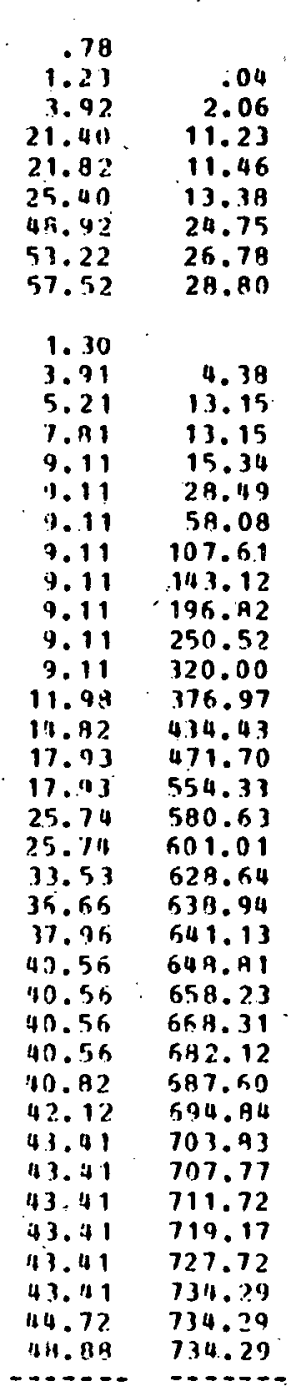
TOTAL .74 .78
1.27
5.98
32.53
33.28
39.78
73.67
90.00
85.32 1.30 1.30
8.29 18. 36 20.96 20.96
24.45 37.60 67.19 116.73 115.73
152.2 .3 152.2.3 205.93 259.63 329.11 388.96 449.25 489.63 572.26 606.37 6?6. 75 6.62.1 1 A 675.60 679.09 6ค9. 37 648.79 708.87 .722 .68 .722 .68
$729.4 ?$ $729.4 ?$
736.95 736.95 747.24 751.19 755.11 762.59
771.13 771.13 779.0 783.17
CAPITAL (GILLYNNS OP 1975 S)

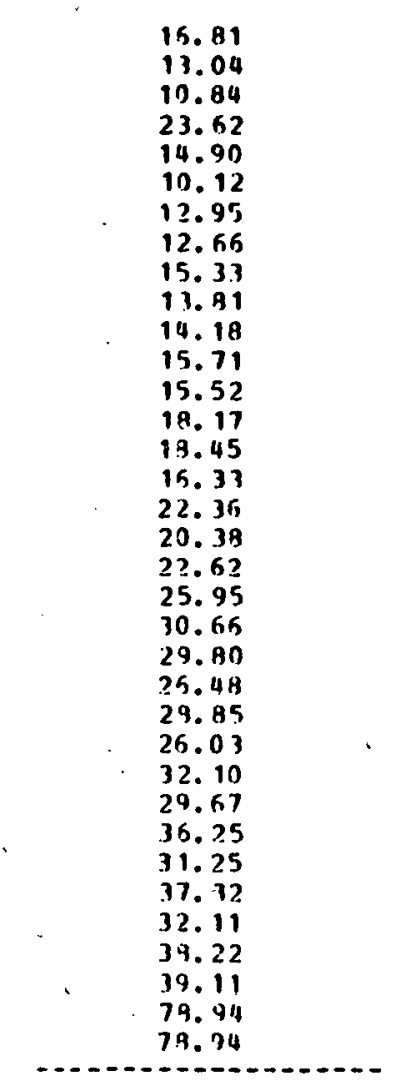

PIES CODP.: CR

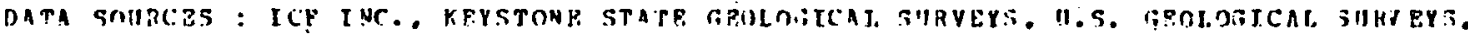

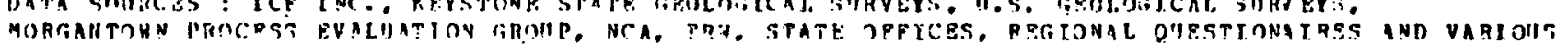

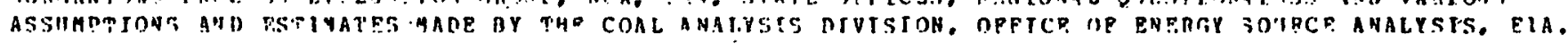

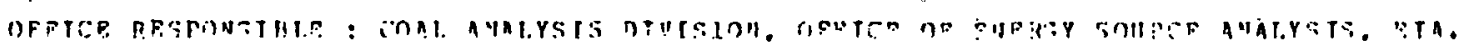




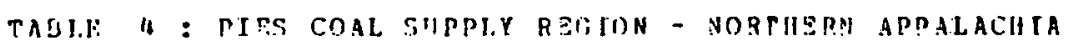

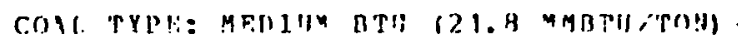

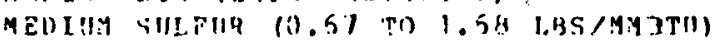

FXISTING MITN

P!ES MINF:
FHTEE $: 2: E R$ $(1972$ s/TON)

- - - - - - . - .

I RCREMENTAL SUPIIIY SIIKFICE DFR? TORAL.

- - - - -

-.......

TOEAL

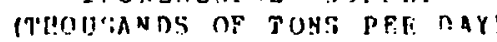

\begin{tabular}{|c|c|c|}
\hline .1 .8 & & $4 \Omega$ \\
\hline .04 & & .04 \\
\hline .36 & & .25 \\
\hline & .32 & .2? \\
\hline & 1.10 & 1.10 \\
\hline .26 & .83 & 1.11 \\
\hline & 2.61 & 2.63 \\
\hline & . В & .89 \\
\hline .26 & 1.53 & 1.79 \\
\hline & 2.111 & 2.111 \\
\hline & 2.19 & 2.10 \\
\hline & 1.10 & 1.10 \\
\hline & 1.10 & 1.10 \\
\hline 1.014 & & 1.011 \\
\hline
\end{tabular}

EUMEULATCVE SUPPLY ('HAII:SANDS O? TONS PER-DAY SIRPAI:P DEEP -...... (...... r......

.46
.51
.25
.26
.26
.52
.52
.52
.78
.78
.79
.74
.78
1.82
$-7 .-5$

.22
1.31
2.19
4.82
5.70
7.23
3.61
111.84
12.93
1.03
1.03

.43
.51
.26
.49
1.58
2.71
5.31
5.22
3.01
10.42
12.52
13.71
14.81
15.85
-2.2
43 51

24.35

26.81

2A. $2 \mathrm{~K}$

23.95

31.91

32.7 ?

34.37

$37.4 \%$

47.17 1.014
CAPITA

IML LIOIOHS OF 1975 . $\cdots$

39.22
11.49
29.53
28.99
26.03
30.39
29.45
39.66
33.67
39.22
39.11
78.94
$\ldots \ldots$

PIES CODE: CS

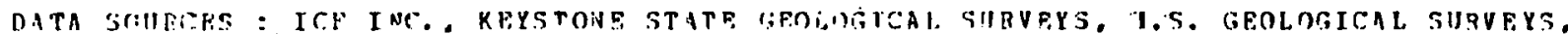

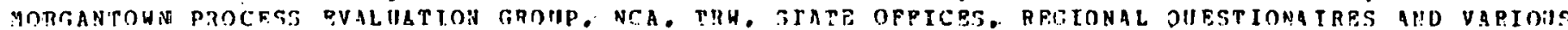

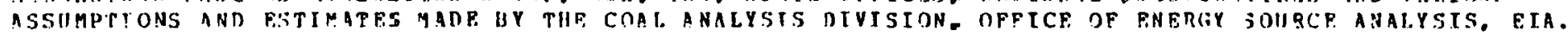

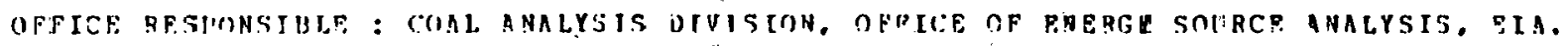


TAMLE 5 : PIES COAL. SIIPEI.Y RESLON - NORTHERN APPALACHIA

CONL TYDE: METALIJR!;ICAT. COAL (27.0 MABT!I/TON)

LOU SULPIR (LESS THAH O.GT LUS/MMBTII)

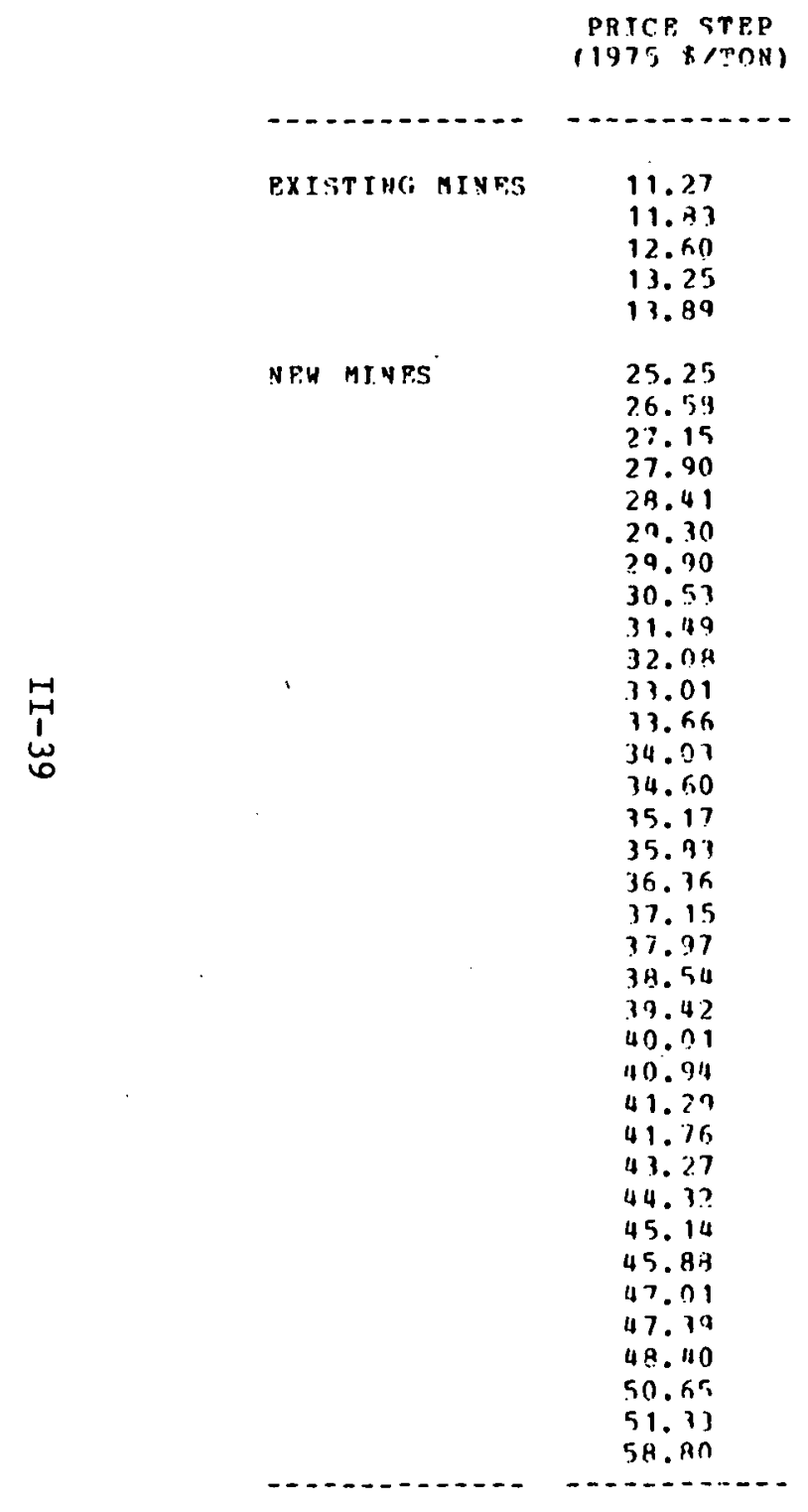

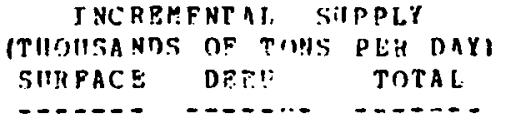

3.84
.33
3.12
.77
.77

$\begin{array}{rr}5.90 & 1.75 \\ .07 & 1.09 \\ 9.70 & 11.82 \\ 2.21 & 2.94 \\ 2.21 & 2.913\end{array}$

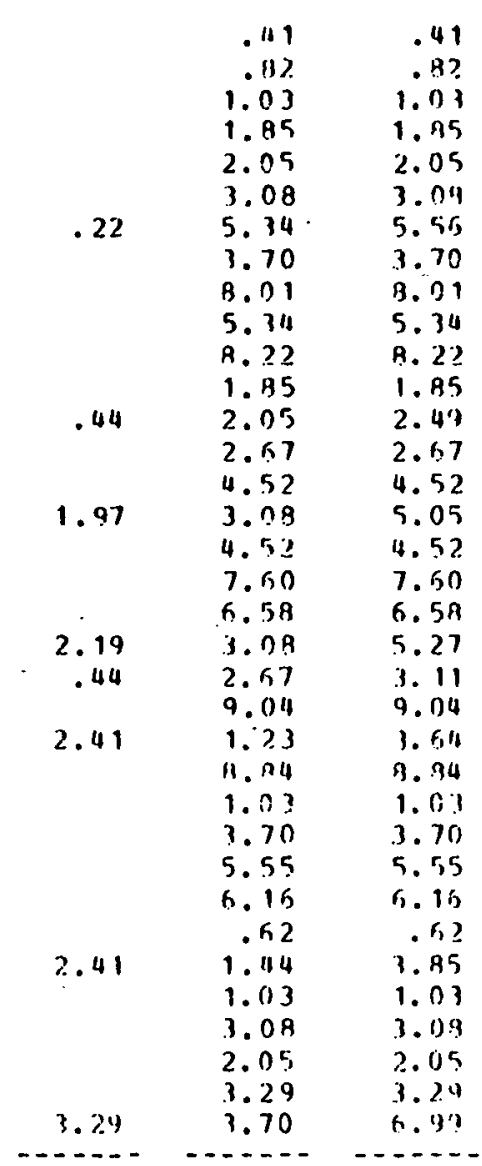

CIJMYILATIVP. SUPELY

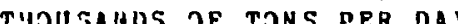
SIIRPACF, DESP TORAT.

$\begin{array}{rrr}2.84 & 5.90 & 8.75 \\ 1.17 & 5.58 & 9.75 \\ 5.211 & 15.28 & 21.57 \\ 7.05 & 17.411 & 24.54 \\ 7.73 & 19.70 & 27.52\end{array}$

\begin{tabular}{|c|c|c|}
\hline & .41 & .41 \\
\hline & 1.2 .3 & 1.2 .3 \\
\hline & 2.25 & 2.26 \\
\hline & 4.11 & 4.11 \\
\hline & 6.16 & 6.16 \\
\hline & 9.25 & 9. 25 \\
\hline .22 & 111.59 & 14.81 \\
\hline .22 & 1R.?!? & 18.51 \\
\hline .22 & 26.30 & 26.52 \\
\hline .22 & 31.65 & 31.96 \\
\hline .22 & 39.85 & 40.08 \\
\hline .22 & 41.71 & 41.93 \\
\hline .65 & 43.77 & $44.11 ?$ \\
\hline .56 & $4 \pi .414$ & 47.10 \\
\hline .56 & 50.96 & 51.62. \\
\hline 2.53 & 54.04 & 56.67 \\
\hline 2.6 .3 & 58.56 & 61.19 \\
\hline 2.6 .1 & 66.17 & 69. 79 \\
\hline 2.53 & 72.74 & 75.37 \\
\hline 4.92 & 75.82 & 30.54 \\
\hline 5.26 & 78.119 & $\begin{array}{l}93.75 \\
?) .79\end{array}$ \\
\hline $7.6,7$ & RA. 77 & 96.44 \\
\hline 7.57 & 97.60 & 105.27 \\
\hline 7.6 .7 & 98.57 & 106.30 \\
\hline $7: 5 ?$ & $102 . .33$ & 110.00 \\
\hline 1.67 & 197.87 & 115.55 \\
\hline 7.57 & 111.04 & 121.71 \\
\hline 7.67 & 114.56 & 122.33 \\
\hline 13.013 & $11 \mathrm{f.10}$ & 126.19 \\
\hline 10.08 & 117.12 & 127.20 \\
\hline 10.014 & 120.20 & $130 . ? 9$ \\
\hline 11).01 & 122.26 & 132.34 \\
\hline 10.0 B & 125.55 & 135.63 \\
\hline 13.17 & 12.9 .24 & 142.61 \\
\hline & & \\
\hline
\end{tabular}

CAPITAL

(AILLIONG OF 1975 \$)

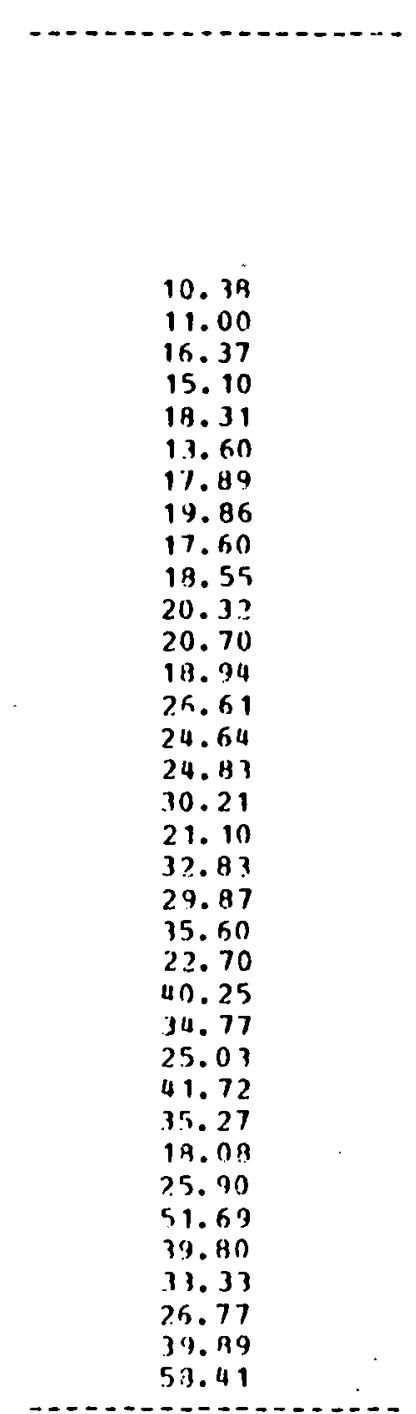

PIES CONF: CM

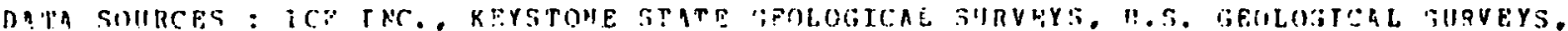

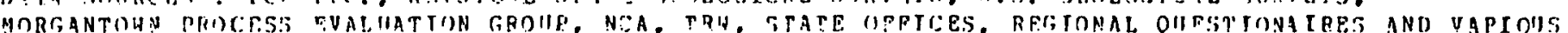

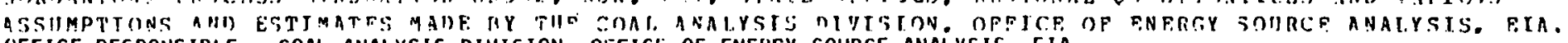
OFFICE RESPONSIBLE: COAL ANALYSIS DIVISION, OFFICE OF ENERTY SOURCE ANALYSIS, EIA. 
TA MLF $r_{1}$ : TIPS COAL SIPHLY RYGION - NOFTHEBH GPPALACHTA

COAL TYPE: HTGA BRIA P?:A YMBTH/TON

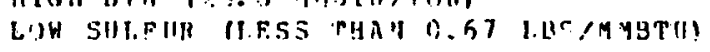

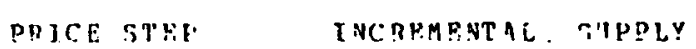
11975 t/TON
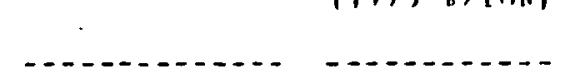

כXTSIML MIVES

vou MINES

11.05
11.57

22.87
COMUILATJYE SIIPLLY (THA)ISHDS OF TONS ?EU DAY) SIPRACE REEP -..... -.... rnTA.T.

.39
.47
.47

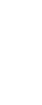

24.21

25.59

27.65

23. 32

$31.0 \mathrm{~A}$

33.116

33.97

35.85

$37.0 \mathrm{~A}$

49.01

DEQP

- 7

04

$.2 ?$

. 27.

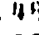

$.2 ?$
.52

.22

-22
.52

.22

1. 5.6.

$\begin{array}{r} \\ .25 \\ .26 \\ .79 \\ .74 \\ 1.30 \\ 1.30 \\ 1.30 \\ 1.30 \\ 2.96 \\ \hline\end{array}$

$\begin{array}{r}.22 \\ .411 \\ .65 \\ .99 \\ .89 \\ 1.10 \\ 1.10 \\ 1.31 \\ 1.53 \\ 1.75 \\ 1.75 \\ \hline\end{array}$

$\begin{array}{r}.31 \\ .43 \\ .47 \\ .2 ? \\ .114 \\ .92 \\ 1.14 \\ 1.65 \\ 1.89 \\ 2.40 \\ 2.62 \\ 2.83 \\ 3.05 \\ 4.62 \\ \hline .92\end{array}$

\begin{tabular}{c}
10.31 \\
10.91 \\
26.51 \\
12.06 \\
47.15 \\
24.29 \\
55.10 \\
31.25 \\
32.11 \\
79.22 \\
78.94 \\
\hline
\end{tabular}

prtes CODE: CI

CAnItAL

MLLLIONS OF 1975 N)

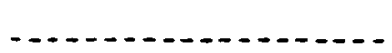

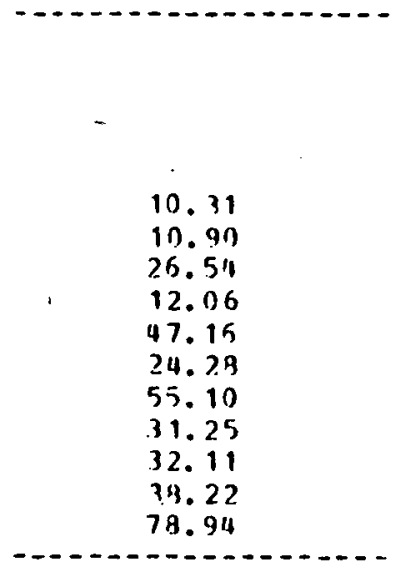

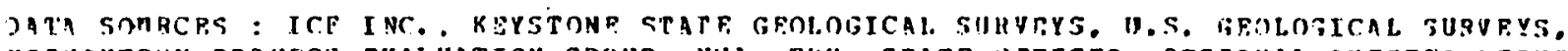

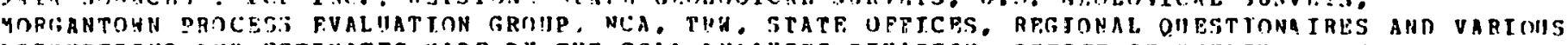

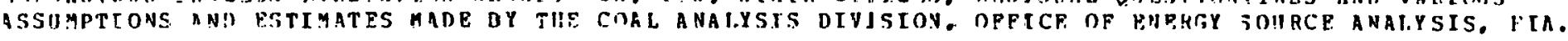

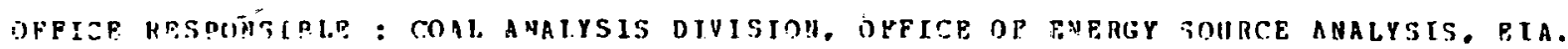


TABLE 7 : PIES COAL SIPPIYY RFIIONA - CEVTRAL APPALACIIA

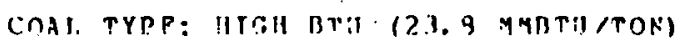

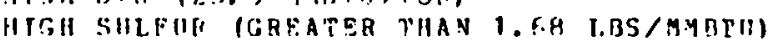

PRICE STFE

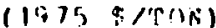

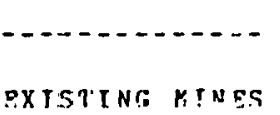

PXISTING KINES
NFIT MINES

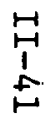

14. $: 1$

?4. 15

24.74

25.

27.111

34.21

33,00

29.51

30.59

12.49
INCRE!HFNTAL SIIPPLLY

I"'liOUSANDS OF TON" DER DAY SURFACF.

DEE:P (T)'? A L

$$
\begin{array}{r}
2.90 \\
2.39 \\
.99
\end{array}
$$

2.51
9.512
5.01
22.82
$? .40$
2.40

5.3
11.0
5.9
22.9
2.4
2.4

1. 30

$33 . \therefore 7$

15. C?

36. 6.1

37.26

19.17

39.19

11.) . ? ?

i1) 11

100.60
CilimIITALIV SIIPJ CUTNOS OF TONS TEA DAYI SURFACE DEE! TIRAL

-...-.-

-

$-0 .--$

MILLONS OF 1975 E)

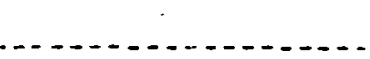

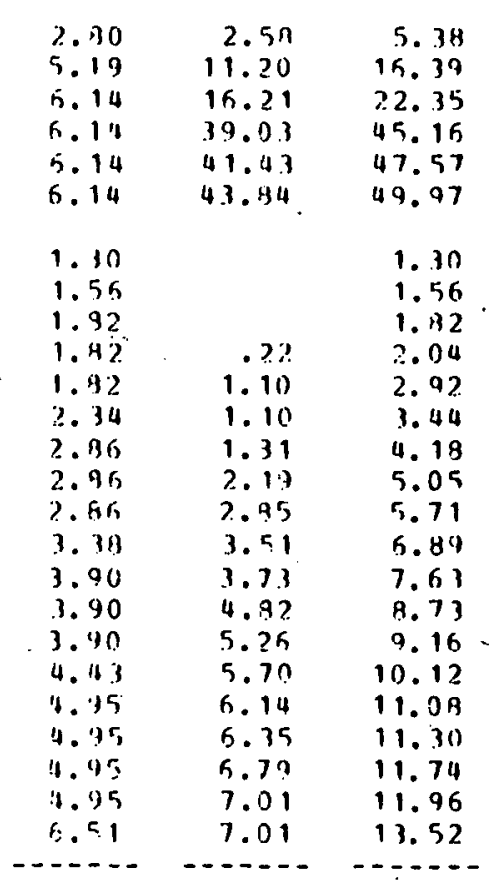

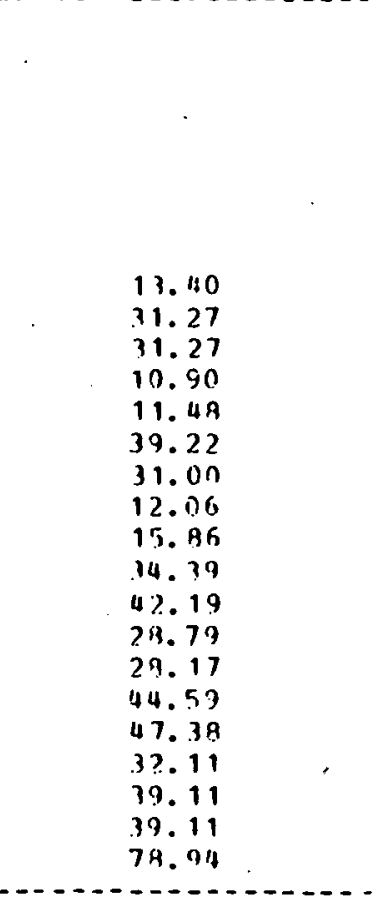

PIFS CONE: CH

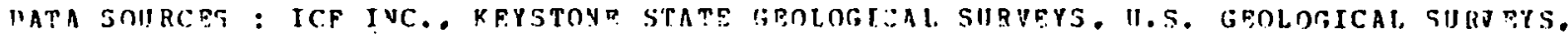

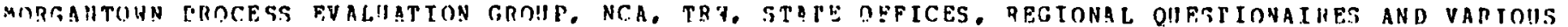

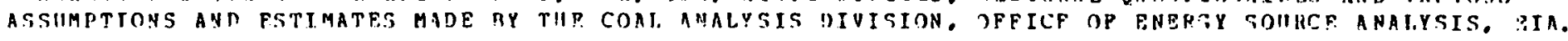

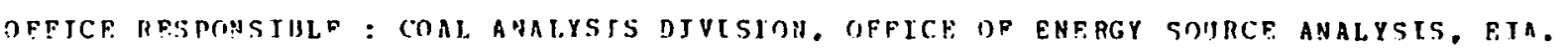


TABIE A : TIEG COAL. FHPQI.Y PEGIOA - ERNTRAL APPALACIIA

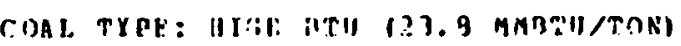

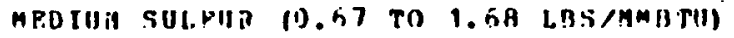

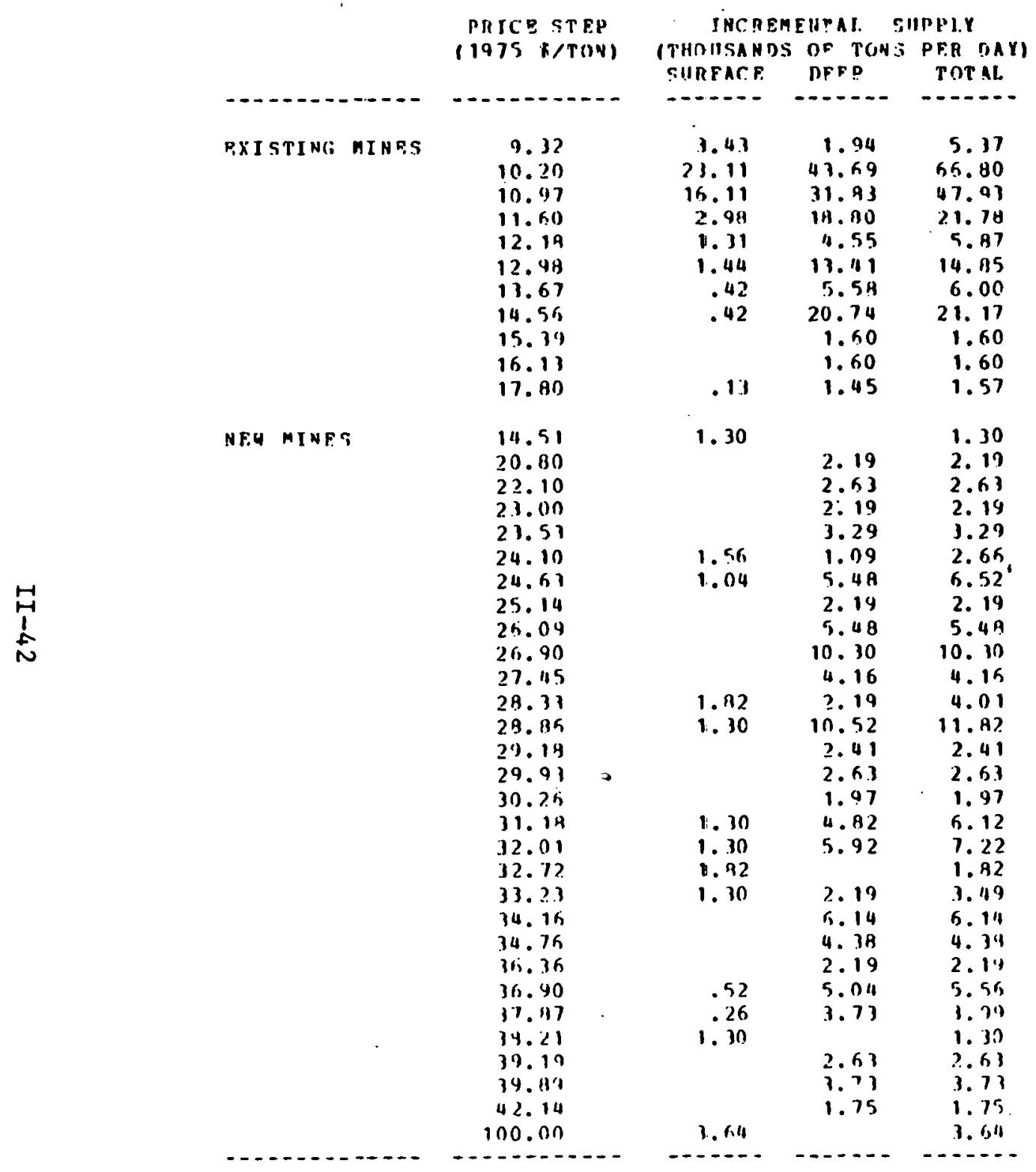

CUMEULATIVD SHEPLY (AILLLONS OP 1975 R) SURFACP. DEEP TOTAL

$\begin{array}{rrr}3.43 & 1.94 & 5.37 \\ 26.55 & 45.61 & 72.19 \\ 42.65 & 77.46 & 120.11 \\ 45.54 & 96.26 & 141.89 \\ 46.45 & 100.81 & 147.76 \\ 48.19 & 114.22 & 162.51 \\ 49.91 & 119.90 & 169.61 \\ 49.24 & 140.54 & 189.71 \\ 19.24 & 142.14 & 191.37 \\ 49.24 & 143.74 & 192.97 \\ 49.16 & 145.19 & 194.55\end{array}$

1.30

1. 30

1.30

1. 30

1.30

2. 166

3.91

1.91

3.91

3.91

3.91

5.71

7.03
7.01

7.01
7.03

1.01

9. 13

$9.6 ?$

11.46

12.76

12.76

12.76

12.76

13. 24

11.54

14.114

14.334
14.816

14.114

11. 119

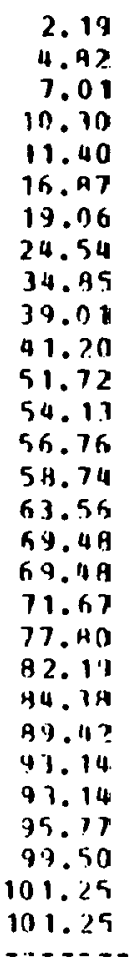

1.49
6.12

6. 12

B. 11

11. 60

14.26
20.79

22.97

24.45

3. 75

42.92

46.91

5月. 75

61.15

63.73

65.77

71.99

79.11

50.91

84. 112

90.56

94.94

47.14

102.70

106.69

107.98
110.61

114.34

116.09

119.74

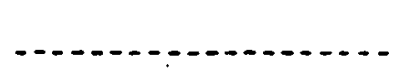

\begin{tabular}{|c|}
\hline $\begin{array}{r}13.40 \\
9.73 \\
3.97 \\
12.71 \\
12.14 \\
24.53 \\
15.17 \\
10.90 \\
12.94 \\
12.79 \\
16.55 \\
27.31 \\
16.54 \\
14.67 \\
25.42 \\
23.116 \\
20.44 \\
25.68 \\
155.03 \\
37.37 \\
24.72 \\
31.36 \\
27.54 \\
32.97 \\
38.69 \\
53.15 \\
32.11 \\
38.43 \\
39.11 \\
73.60\end{array}$ \\
\hline
\end{tabular}

PIES CONE: C.H

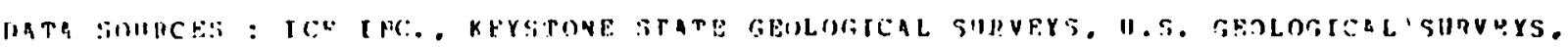

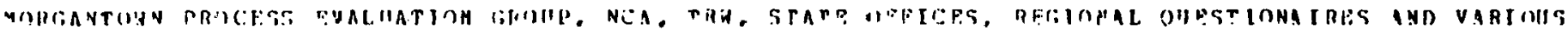

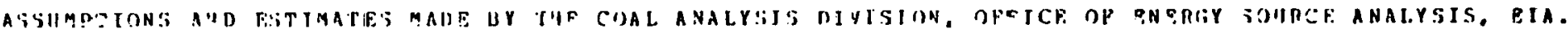

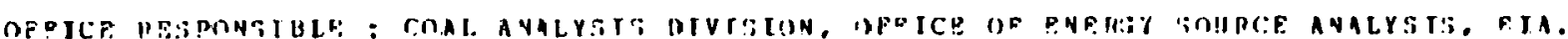




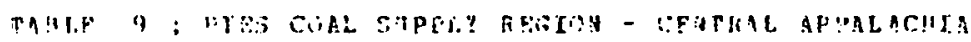

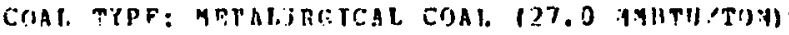

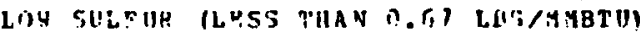

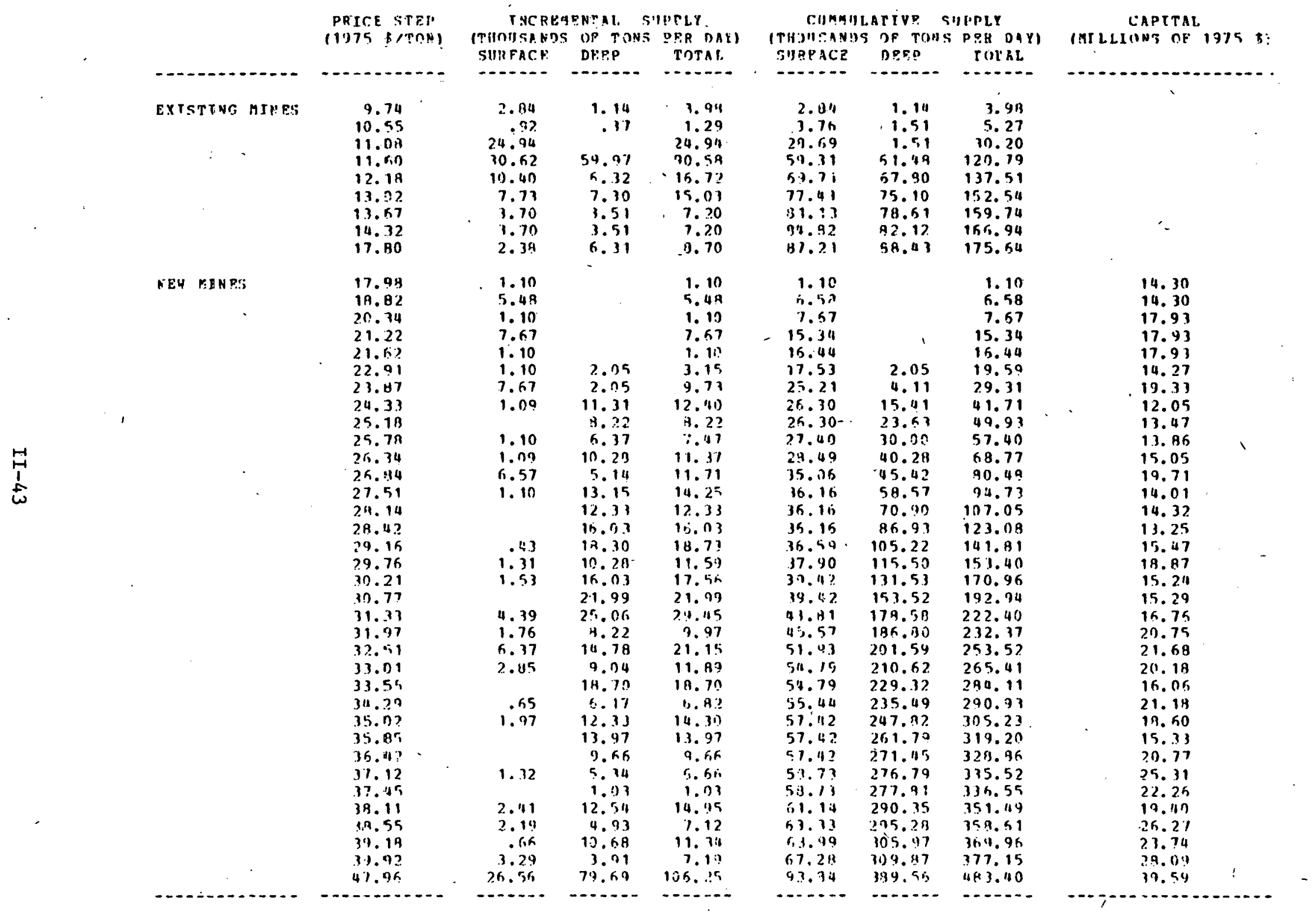

Fros cone: cy

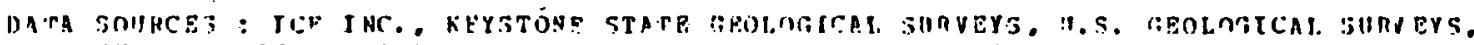

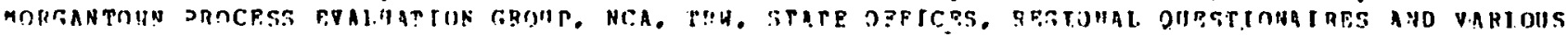

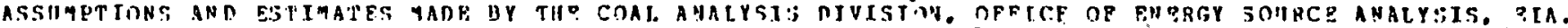

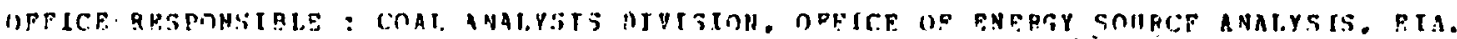


TABIP. 10 : PIPS COAL SUIPELY REGIUN - CENTEAL APPALACHIA

COAL TYEP: HISH BSU (23.8 MMBTU/TOH)

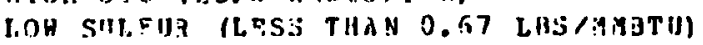

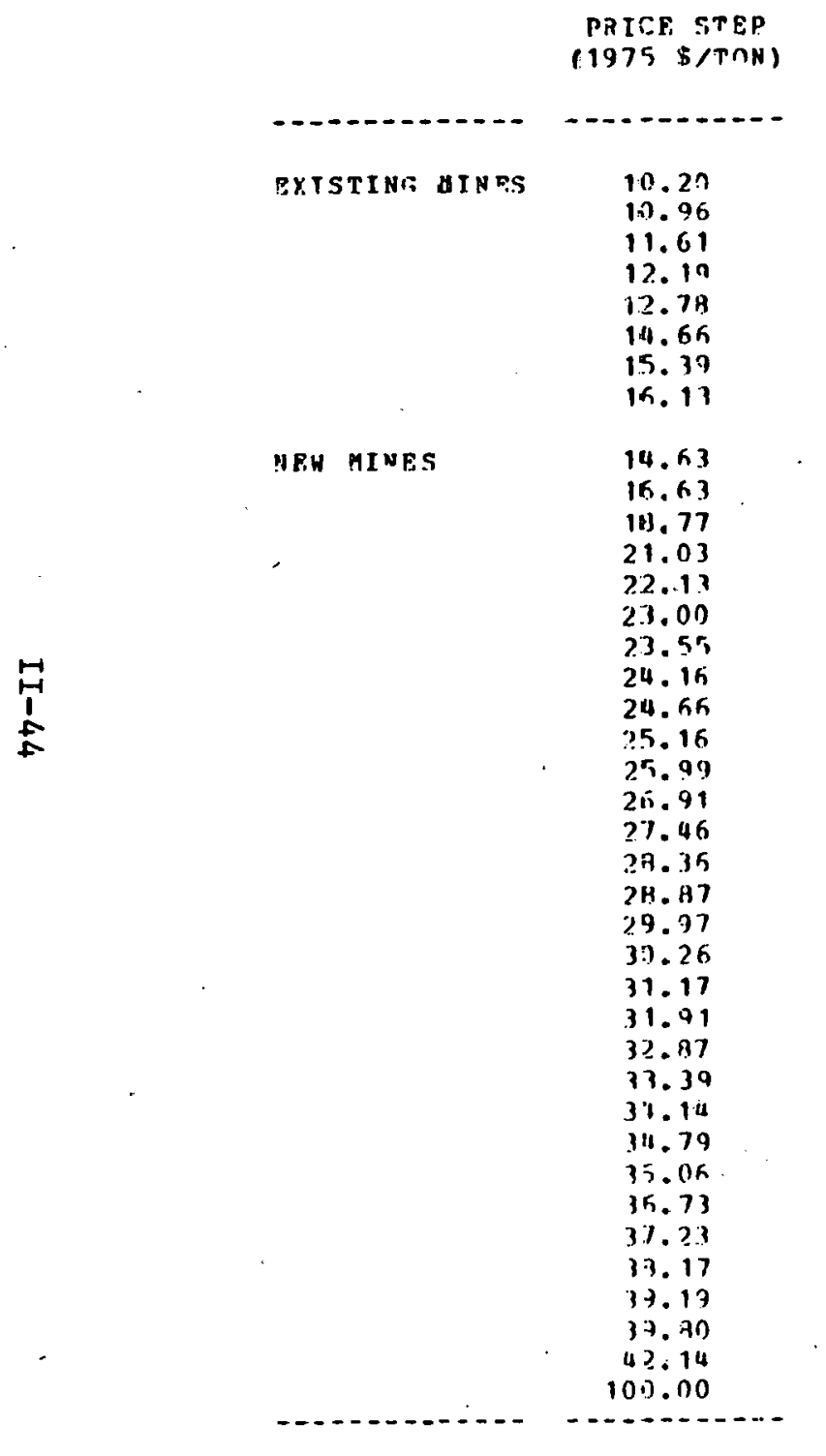

IHCRPMBNT AL SUPUI.Y IT!UOISA NDS DE TONS PER DAYI

CIIMIMIILATIVP. SIIPPLY

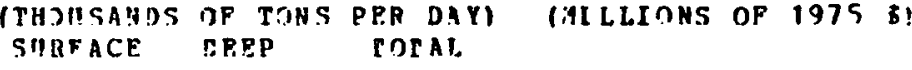
SUHFACE DFPC TOTAI. SIIRFACE

IREP

10.22
6.57
2.25
1.08
9.09
3.02
.32
.32

.
.89
.43
.143

10.22

\subsection{0}

2.60

2.60
2.60

2. 50

2.60

2.60

1.03
3.95

3.95

2.19

1.55
2.08

3.73
1.09

1.09
.1 .29

1.29
2.63
1.10

6.58

1.10

2.09

1.97
6.36

6. 36

2.19
1.11
2.111

5.20

3.90

2.08

2.11

3. 15

15.73
19.05

20.14

21.32

$? 1.22$

$\begin{array}{rlll}1.07 & 24.24 & 1.74 & 25.9 .7 \\ .12 & 24.55 & 1.74 & 25.29\end{array}$

24. 9 ?

.89
1.3
1.74
1.74
1.7

10.2

19.94

1.7421 .45

$\begin{array}{rlll}1.07 & 24.24 & 1.74 & 25.9 .7 \\ .32 & 24.55 & 1.74 & 25.29\end{array}$

3.12

1.77

2.50
5.21

5.21

2.69

3.70
3.95

2. 19

3.73

10.41

10.41

10.41

10.41

11.97

14.05

5.37

2. 63

1.111
6.54

1. 19

14.05

14.06

14.05

15.14

4.49

9.49

2. 19

1. 31
7.52

19.26

19.26

13.26

211.46

2). 36

$\because .05$

$3.29 \quad 3.12$

3.29
4.16

4.16

1.97
2.135

4.16
1.97

13.

3.3 .5

33.57

33.57

33.57

33.57

36.64

$35.1,9$

35.59

35.59
45.58

1.74

26. 61

1.09

$$
\begin{aligned}
& 2.60 \\
& 5.21
\end{aligned}
$$

$$
5.21
$$

$\begin{array}{ll}7.2 ? & 15.45 \\ 17.64\end{array}$

1D.96 21.37

1.2.09 24.03

$15.34 \quad 29.40$

$17.97 \quad 32.03$

$19.05 \quad 33.12$

$\begin{array}{ll}.5 .64 & 39.70 \\ 25.74 & 110.79\end{array}$

28.7144 .85

$15.07 \quad 54.13$

37.2656 .5 ?

$18.57 \quad 57.83$

$40.99 \quad 65.45$

43.6271 .99

$05.54 \quad 76.04$

$45.59 \quad 70.16$

QB.คA 92.145

$53.04 \quad 85.51$

$55.0 ? \quad H 8.59$

$57.97 \quad 91.14$

$\$ 9.40 \quad 92.97$

$59.94 \quad 95.53$

$67.7 \pi \quad 97.41$

6.21799 .16

$\begin{array}{rr}63.13 & 99.82 \\ f .3 .1 .1 & 109.71\end{array}$

A.).

1).....

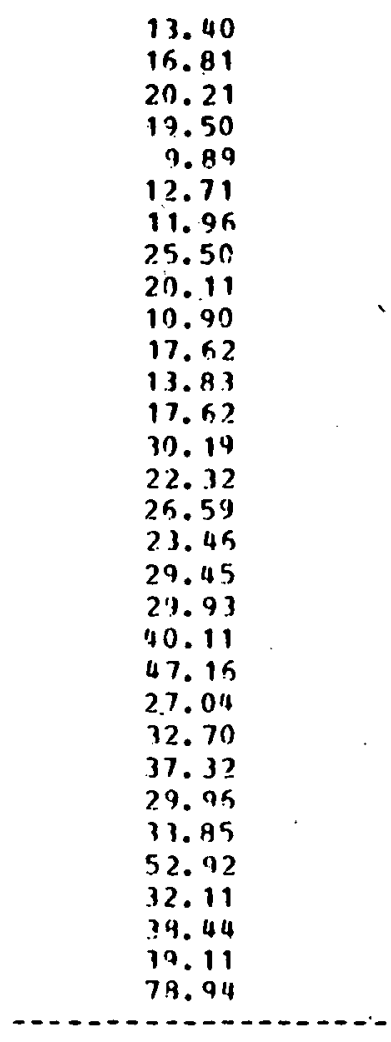

PIES CODE: CI

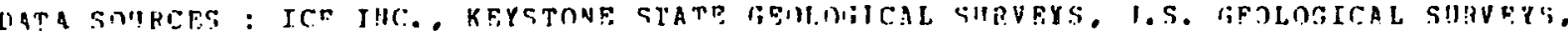

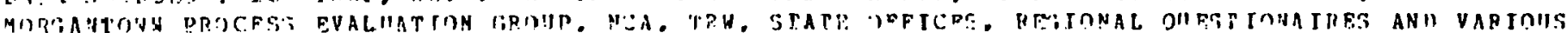

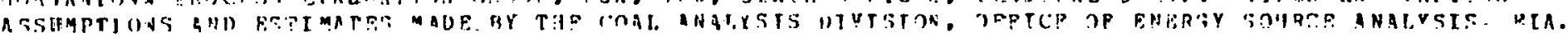

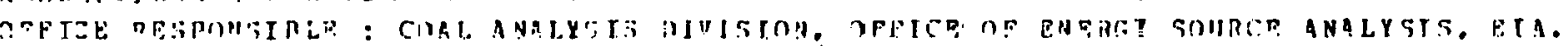


TABLF 11 : PIES COAL SIITLY FE:AION - SNIITIFRY APPALACHIA

COAL TYPF: HISH MUN i2?.8 MMATH/RON

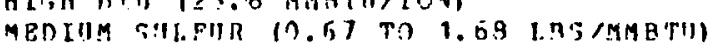

PRICE TTE! $(1975$ औ/2ON)

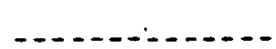
-..............

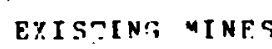

$$
\begin{aligned}
& 12.74 \\
& 13.74 \\
& 14.02 \\
& 11.54 \\
& 15.23
\end{aligned}
$$

บEบ ตโกลร
TNC REMBNTHI, SIIPELYY (TH)USANDS OF TOIS PEH DAY) SIIRFACE DFFP TOTAL sin

21.01
5.91
1.26
.29
.29

DFFP

$\begin{array}{r}12.66 \\ 3.15 \\ .15 \\ .11 \\ .14 \\ 4.34 \\ 1.97 \\ 1.97 \\ 1.31 \\ \hline\end{array}$

9.05

4?
QUAYYIT. ATIVE SIIPPLY (PILLIONE OP 1975 B) SIPFACF DEEP TOTAL

(19.0. DEP -

$\begin{array}{lll}21.01 & 12.56 & 31.67 \\ 26.92 & 15.81 & 42.73 \\ 24.17 & 15.57 & 44.75 \\ 2.466 & 15.72 & 45.17 \\ 24.74 & 16.86 & 45.59\end{array}$

$29.74 \quad 16$. AF $\quad 15.59$

29.740

$\begin{array}{ll}4.39 & 4.39 \\ 5.36 & 6.36 \\ 9.33 & 9.33 \\ 9.64 & 9.54\end{array}$

12.06
25.10
32.11
19.11

PIES CODP: CH

DATA SOIRCES : ICF INC. KFYSTONF STATE GPOLOFICAI. SIRVEYS, "S. GROLOTICAL SURVEYS.

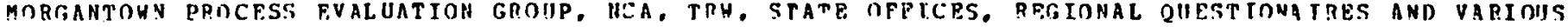

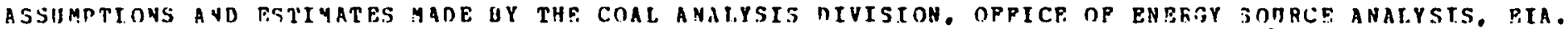

OFFITE RESPONIIBIF, : COAL ANALYSTS DTVISION, OFPICE OE ENERGY SOIRCE ANALYSIS, PIA. 
TAHIE 12 : PIES COAL SIIPPLY HEISON - SMUTHERH APPELACHIA

COAL TYPE: METTALIREITSAI. COAL 127. O MMBTUITONI

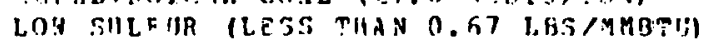

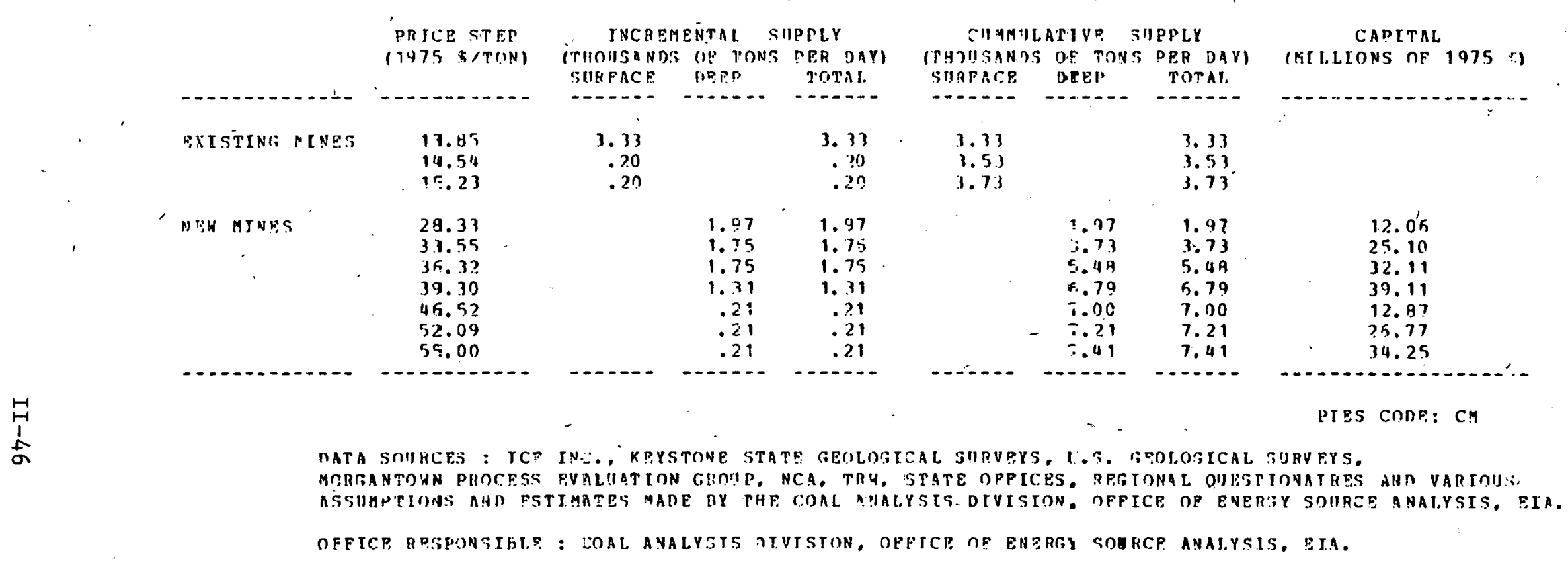




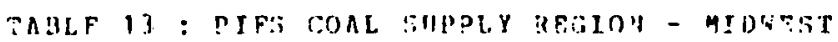

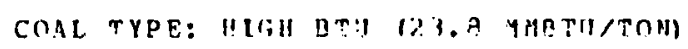

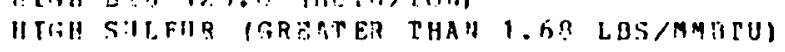

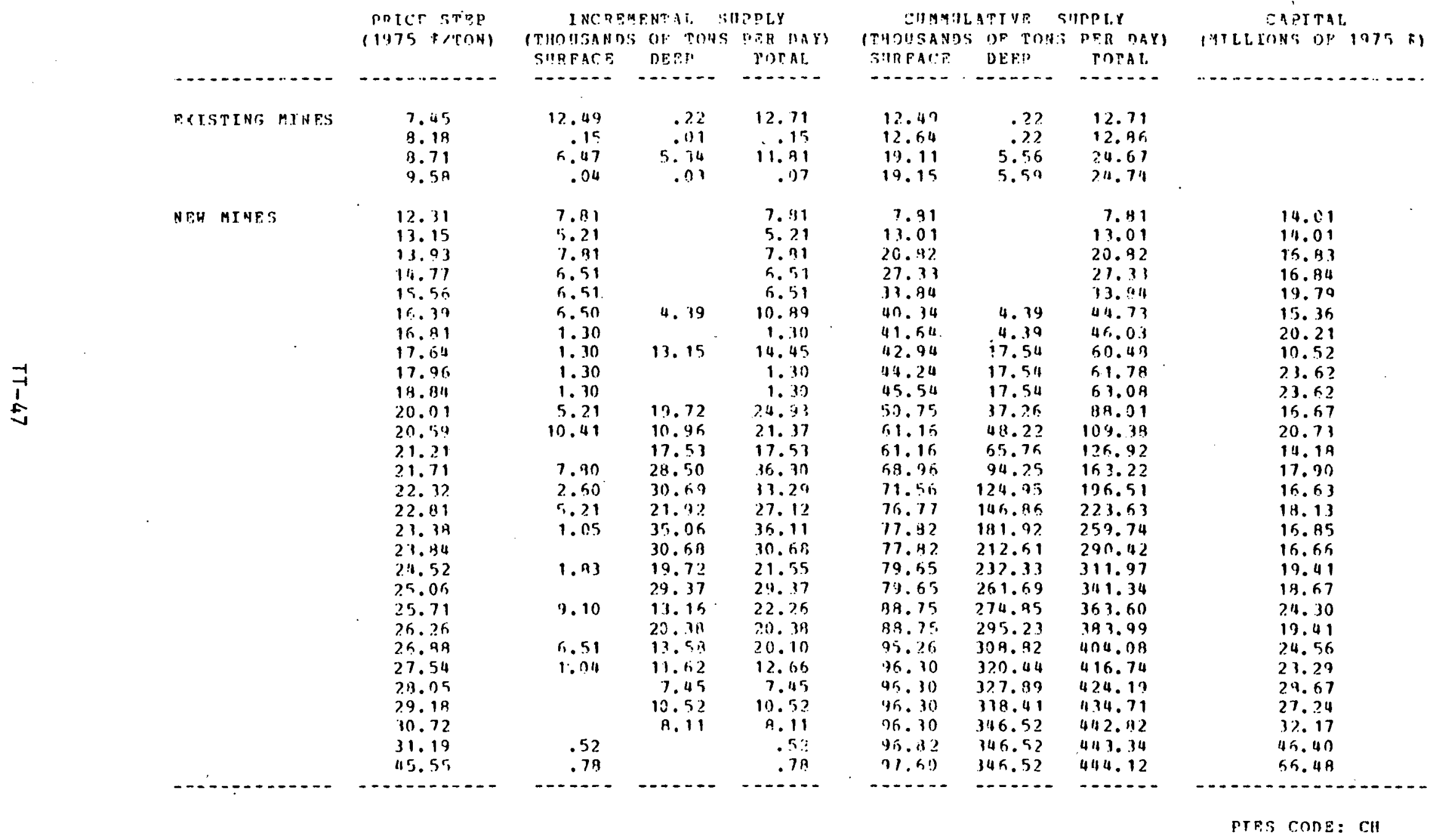

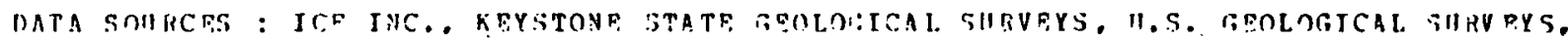

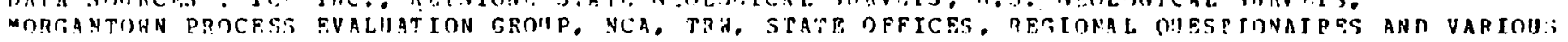

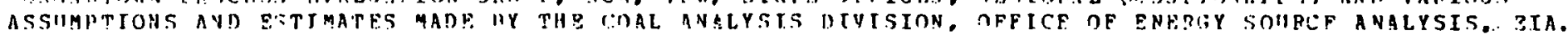

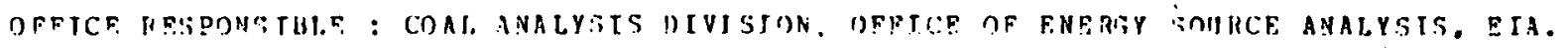


TAHLE 14 : PIES COAL SHPPI.Y RETION - MIWHFST

GOAL. TPE: MEQDIUM NYU (21.A MMBTI/TRM)

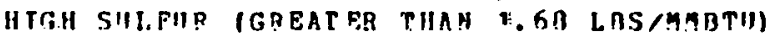

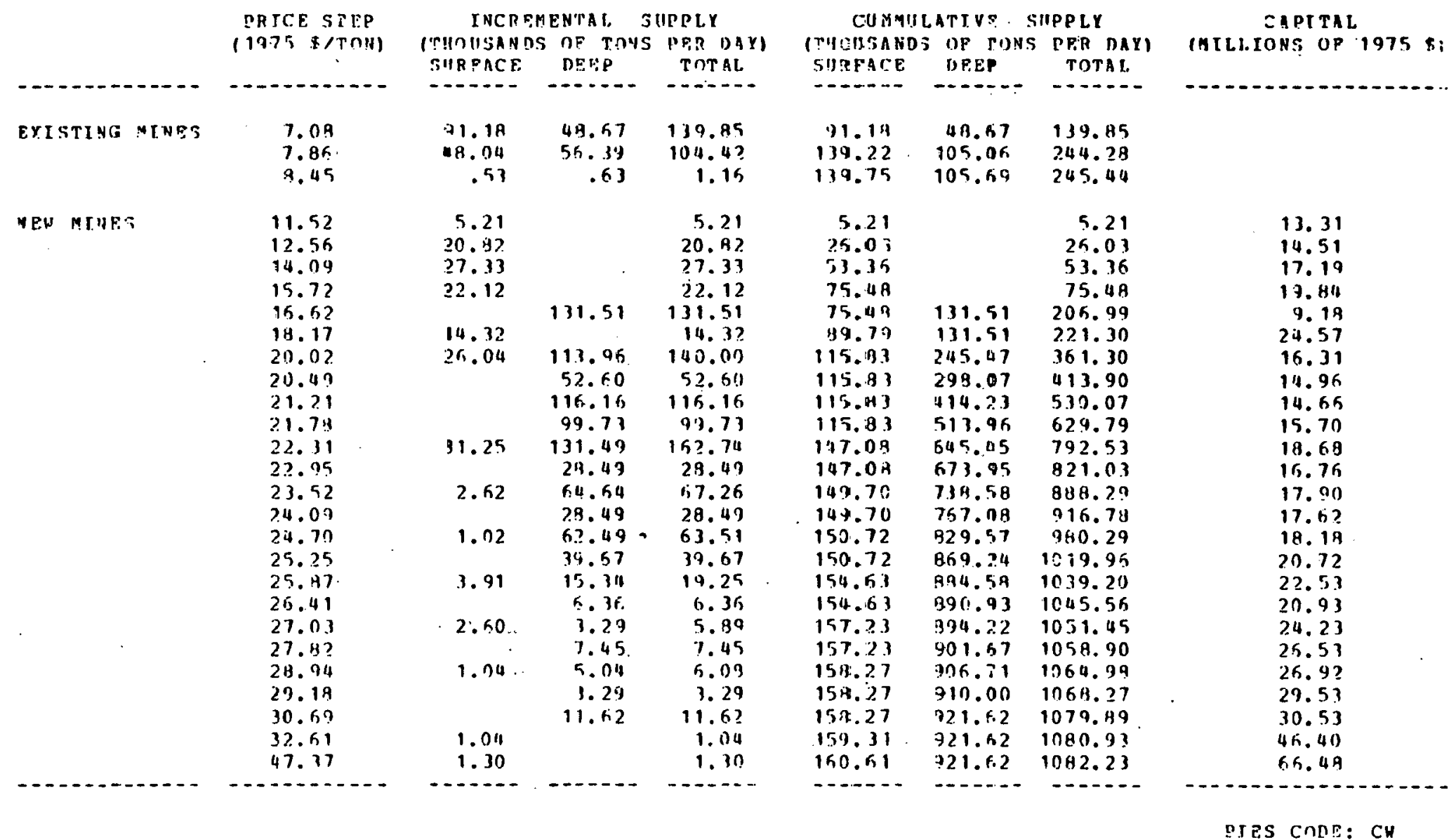

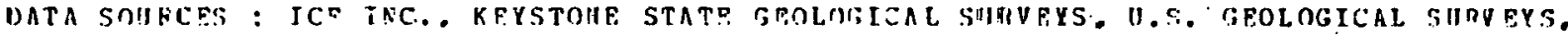

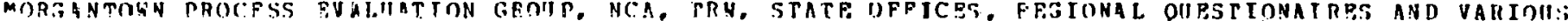

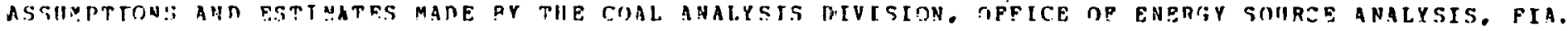

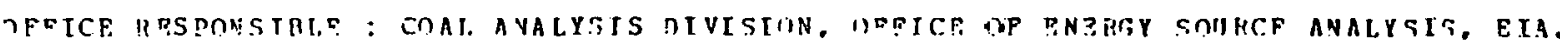


TABLF. 15 : PIFS COAL SIURLY REGTOH - MITORST

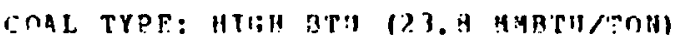

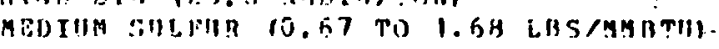

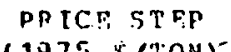

TICFFINENTAT. SISPLI.Y

(197, (ITON) THOUSANDS OF TONS: PEY TAY

CIMYMILATI VP SUPPLY

CAPITAL.

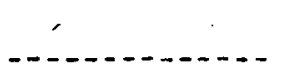
SIIRFAC. THOUSAHDS OF TOHS PPR DAY SIIRFACE DEEP TOTAI.

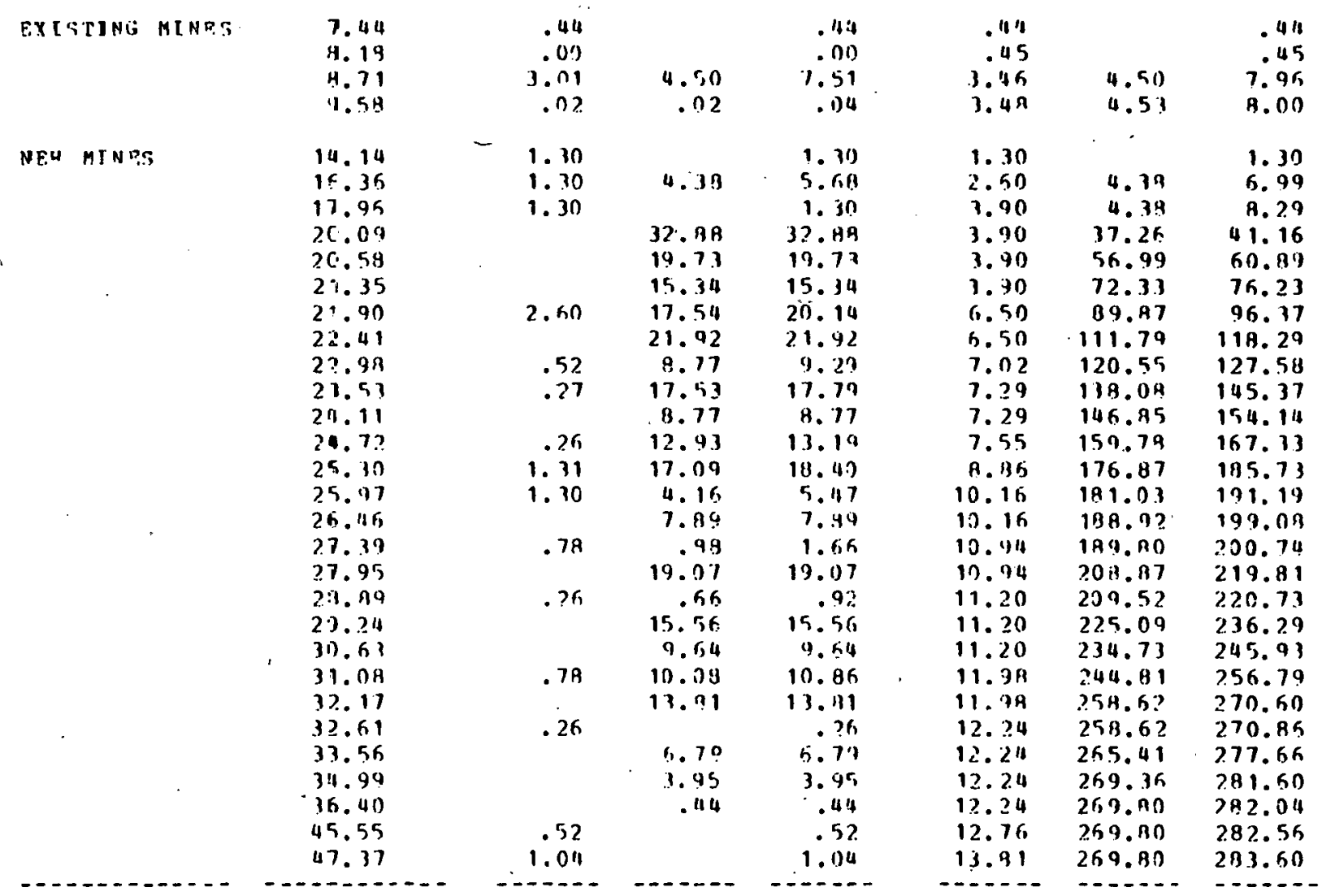

\begin{tabular}{l}
16.81 \\
11.40 \\
23.62 \\
13.94 \\
14.86 \\
14.53 \\
17.35 \\
16.50 \\
19.02 \\
17.49 \\
17.62 \\
19.01 \\
22.09 \\
27.70 \\
22.65 \\
34.63 \\
25.52 \\
28.65 \\
27.09 \\
30.85 \\
36.93 \\
14.17 \\
46.49 \\
36.94 \\
39.22 \\
19.11 \\
79.91 \\
65.48 \\
\hline 9.99
\end{tabular}

Pres conNe: CP

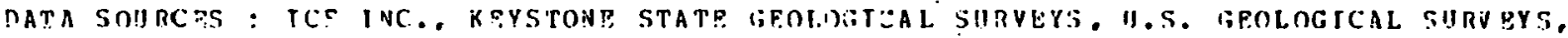

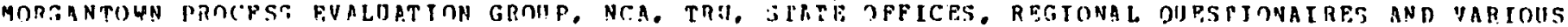

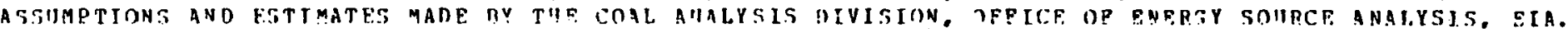

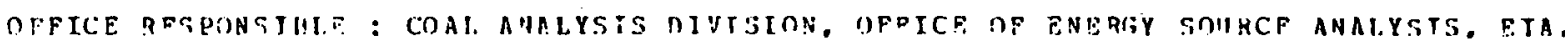




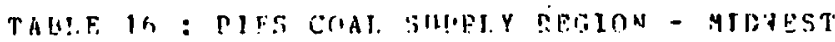

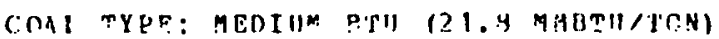

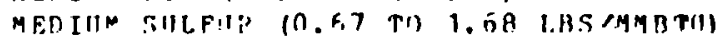

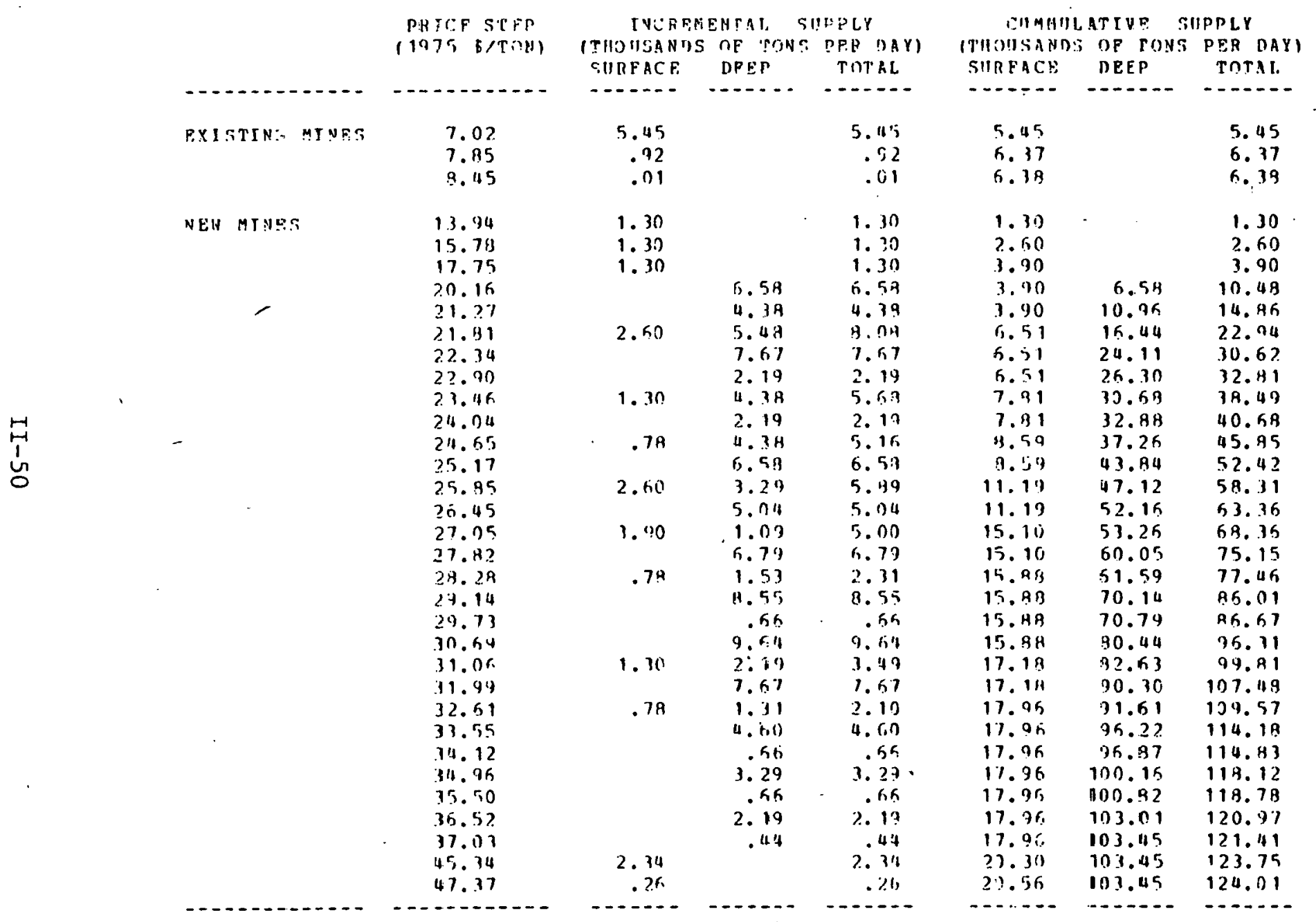

CA BTTAL

(111LLTONS OF $1975 \$ 1$

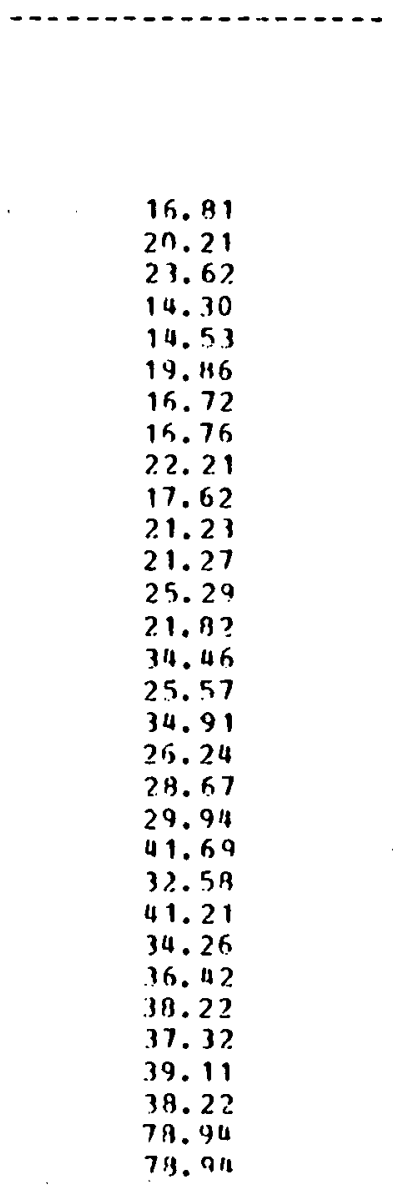

PIBS CODDF: CS

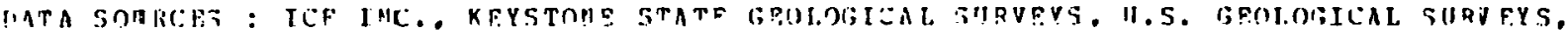

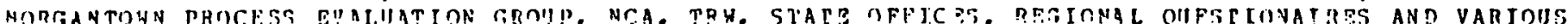

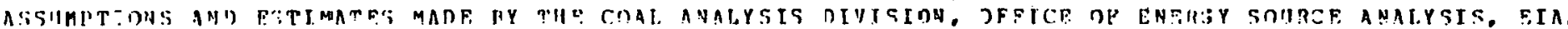

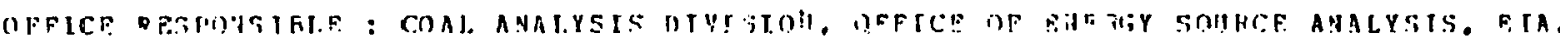




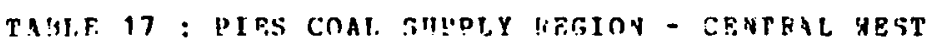

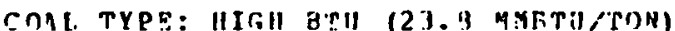

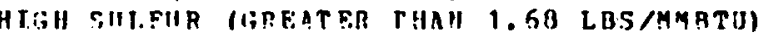

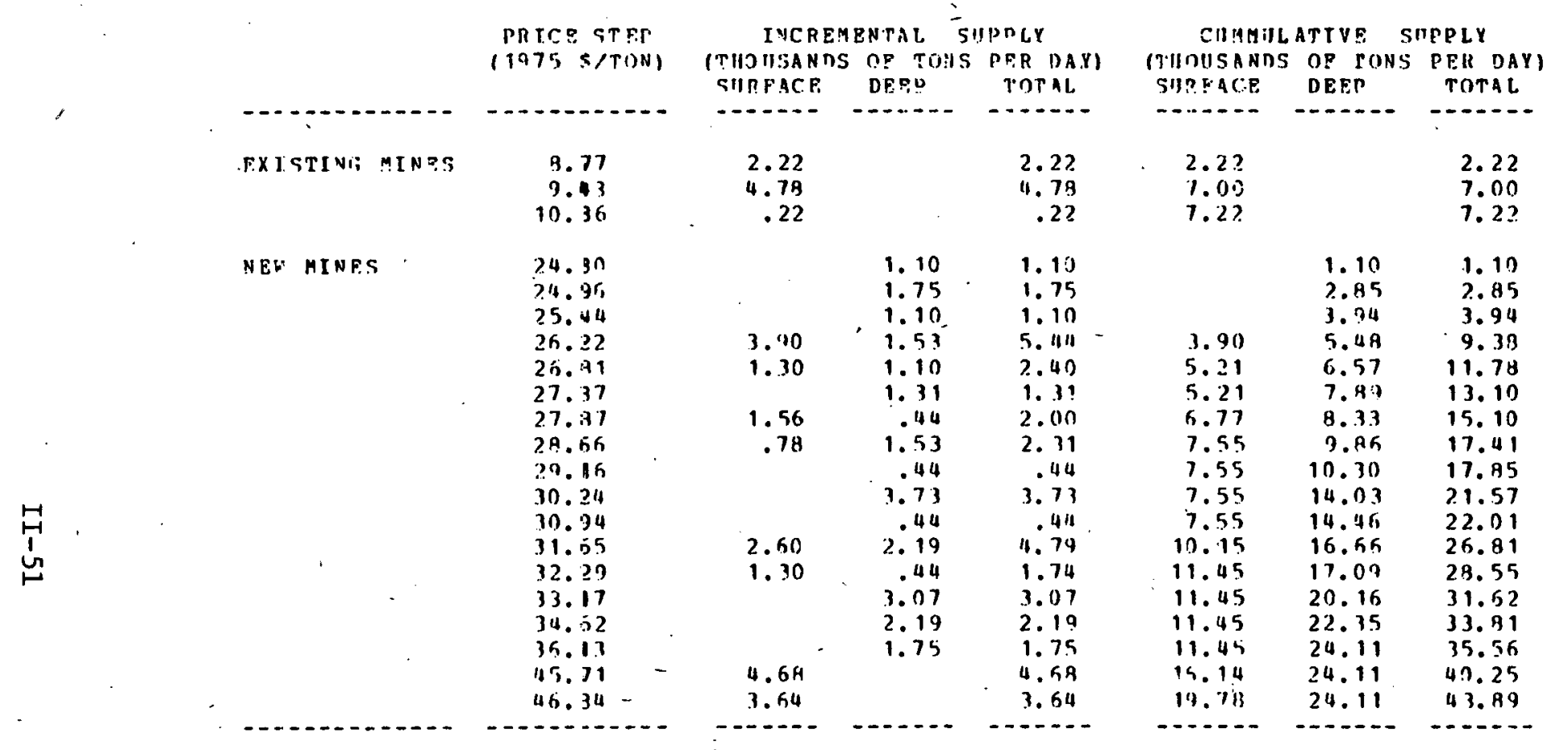

CAPITAL

MTLLTONS OF 1975 NI

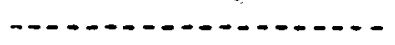

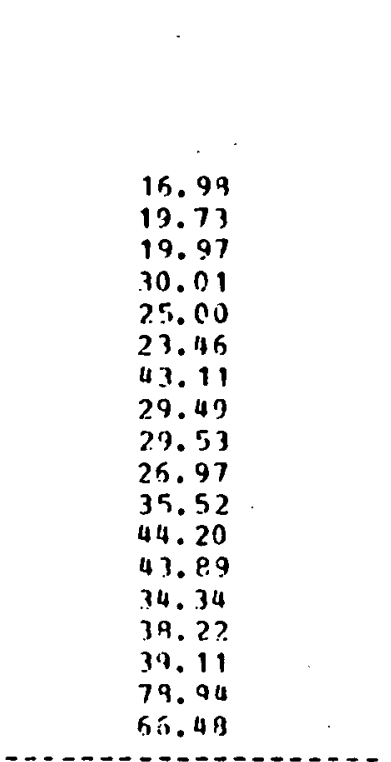

PIPS CODE: CH

DATA SOIIREE : ICF I YC. KEYSTONE STATE GFOLOCICAL SURVEYS. D.S. TFOLOGICAL SURVEYS.

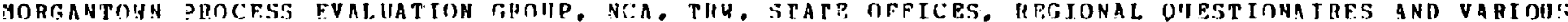

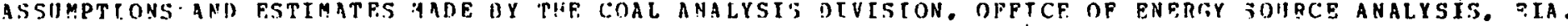

OFFIEF RESPTNGTILF : COAL AVALYSIS DIVTSION, OPFICE OP RNFRGY SOURCF ANALYSTS, PIA. 


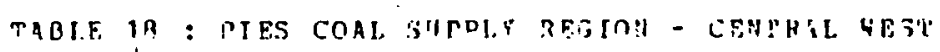

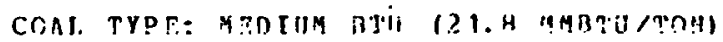

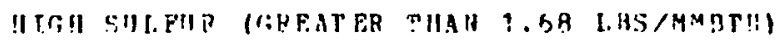

PRTCE STEF
1975 TOOH

- -

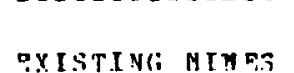

ifid MIMES

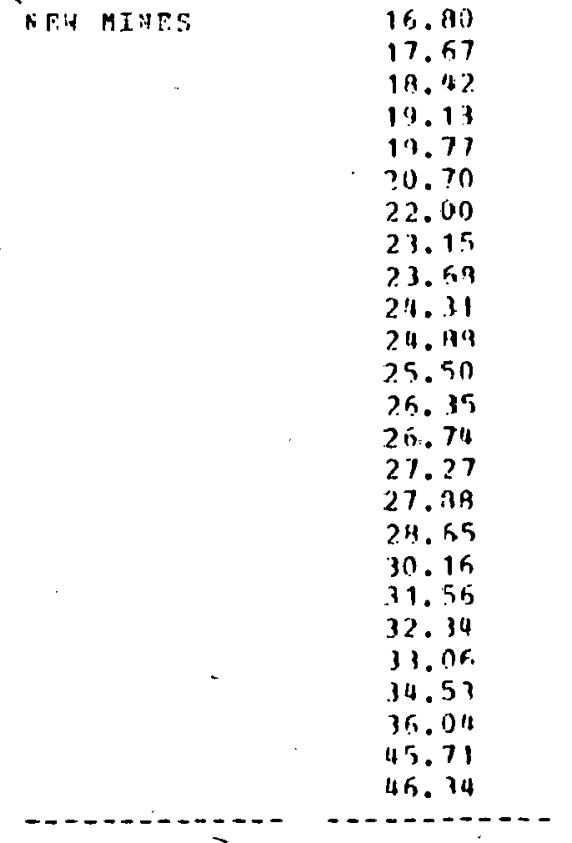

INC.RFMENTML STIPLY (THU) IJSANDS OF TONS DFR BAY) SIIRFACE bPF: TOIA AL.

12.15
1.50

1.30

2. 74

$$
\begin{aligned}
& 2.51 \\
& 5.95
\end{aligned}
$$$$
\begin{array}{r}
6.95 \\
5.45
\end{array}
$$$$
4.56
$$$$
4.56
$$$$
\begin{array}{r}
4.11 \\
10.41
\end{array}
$$$$
\begin{array}{r}
10.41 \\
3.59
\end{array}
$$$$
\begin{array}{r}
3.59 \\
2.19
\end{array}
$$$$
\begin{array}{r}
2.79 \\
14.79
\end{array}
$$$$
7.01
$$

14.79
7.01
18.08

5. ?0

18.08
9.05

$$
\text { 4. } 38
$$$$
\text { 2. } 63
$$$$
\text { 2. } \cos
$$$$
3.29
$$$$
7.90
$$

2.60

$$
\begin{aligned}
& 8.11 \\
& 9.21
\end{aligned}
$$$$
.26
$$
9. $9 F$ 9.? 1$$
7.45
$$

.79 CUYAII.ATIVF SIIPRLY ITIID!SAUDS OF TOHS PER DAYI (MILLTONS OF 1975 \%) SUAFACE: DFEF

12.15
1.57
2.74
9.59
9.15
5.14
4.55
4.11
10.41
4.54
2.19
14.79
7.01
19.09
11.25
4.39
2.63
5.37
7.89
8.11
11.91
.26
9.96
3.21
7.45
6.71
.79
2.27
9
TOPAL

-

\begin{tabular}{|c|c|}
\hline $\begin{array}{r}2.14 \\
12.31 \\
19.18\end{array}$ & $\begin{array}{r}2.74 \\
12.33 \\
20.49\end{array}$ \\
\hline 24.65 & 25.96 \\
\hline 29.11 & 30.52 \\
\hline 33.42 & 34.73 \\
\hline 43.93 & 45.14 \\
\hline 53.42 & 54.73 \\
\hline 55.51 & 56.92 \\
\hline 70.111 & 71.71 \\
\hline 77.42 & 78.73 \\
\hline 95.50 & 96.81 \\
\hline 94.55 & 111.05 \\
\hline 08.9 .3 & 115.44 \\
\hline 11.56 & 118.07 \\
\hline 14.85 & 123.44 \\
\hline 22.74 & 131.33 \\
\hline 30.85 & 139.44 \\
\hline 40.06 & 151.25 \\
\hline $40.3 \pi$ & 151.51 \\
\hline 49.92 & 161.37 \\
\hline 59.13 & 170.57 \\
\hline 66.59 & 179.03 \\
\hline $66.5 n$ & 184.79 \\
\hline F6. 58 & 195.57 \\
\hline
\end{tabular}

12.15
11.65

12.15
$17: 65$

\begin{tabular}{l}
12.84 \\
13.65 \\
16.03 \\
15.37 \\
16.20 \\
19.04 \\
19.15 \\
20.43 \\
17.62 \\
21.81 \\
19.68 \\
23.58 \\
23.31 \\
29.85 \\
23.46 \\
37.44 \\
24.28 \\
25.67 \\
35.51 \\
45.110 \\
31.39 \\
39.22 \\
37.11 \\
79.911 \\
65.49 \\
\hline 9
\end{tabular}

PIES CODE: CH

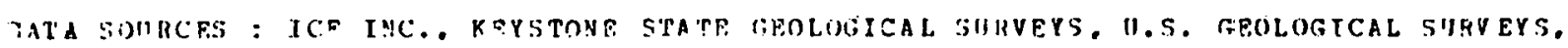

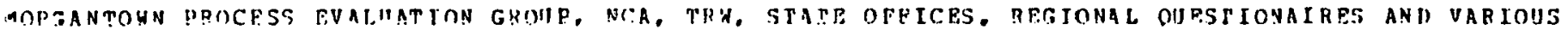
ISTMMUMIUNS AND ESTIMATES YADE FY THE COAT AUALYSTS DIVISION. OFFICE OF ENERGY SOUREE ANALYGIS, ?IA.

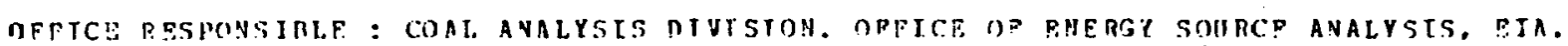




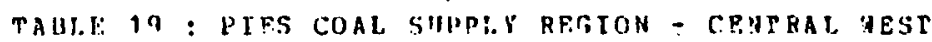

COAL TYPF: HISH ITTI 123.9 MATTH/TON)

MFDIUM SULEIIR (0.67 TO 1.59 LAS/MYRTII)

PRTCE. STEP

(1)75 $\$$ TION)

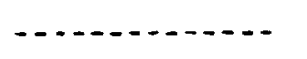

-

PXTSTING MTNES

10.15
10.47
11.39
15.34
16.11
15.97

NFA MINES:

占

2.5. 11

26.29

2.8 .47

29. 32

29.91

$30.5 \mathrm{~A}$

32.92 .

33.53
INCREMENTEL SISPMLY THOUSANDS OF TONS POP DAY

314.311

36.39

36.79

46.34

49.177 SIIRPACP. -....-

.22
.01
.01
1.08
.36
.36

$$
\text { DF F.F }
$$

TOR

TnTIL

.22
.01
.01
1.08
.35
.36

1.56
.78
.52
1.82
.22
1.56
4.42

.22

.114

1. 10

1. 53

1.31

1.53

1.10

1.71

4. 42
$.2 ?$
CUMMULATIVE. SJPPLY ITIOISANDS OF [ONG PRE DAYI TIREAC:E DERP -......

$$
\text { DEEP }
$$

TOTAL.

.22
.023
.24
1.33
1.63
2.05

.22
.24
1.56
1.10
.79
1.93
1.24
1.35
1.10
1.38
1.22
1.56
1.112
$. .2 . .5$

\section{$(2.05$}

1.56

1.56
1.56

1.55
2.34

2.34
2.14

2.74

4.69

4.69

11.69

4.91 .

5.47

10.94.

-.......

$\begin{array}{r}.22 \\ .23 \\ .24 \\ 1.33 \\ 1.67 \\ 2.05 \\ \\ .22 \\ .114 \\ .98 \\ 2.44 \\ 3.53 \\ 4.31 \\ 5.85 \\ 7.69 \\ 11.04 \\ 12.14 \\ 13.45 \\ 13.67 \\ 15.23 \\ 19.66 \\ \hline .0 .0\end{array}$

$\approx$ APITAL

(MTLLIONS OP 1975 B)

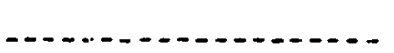

,

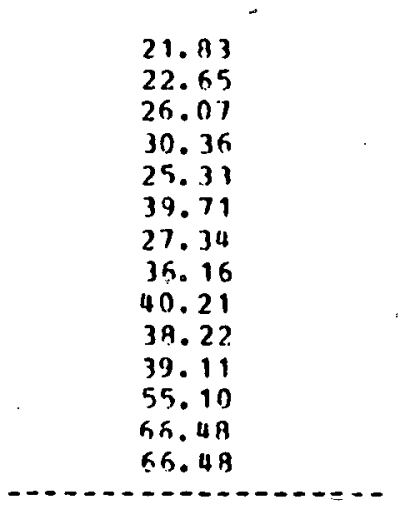

PIFS CODR: CR

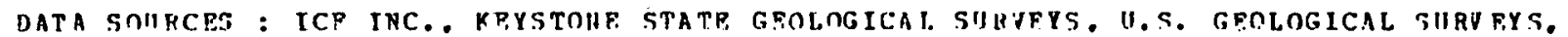

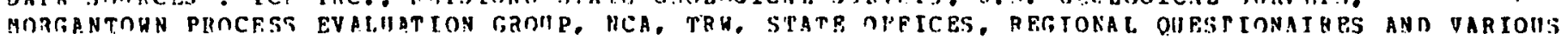
ASSIMPTIONS AND ESTT MATES MADE MY THE COAT. AIALYST: DTYISION. OFPICF DP PNERSY SOIRCF ANALYSIS. BIA.

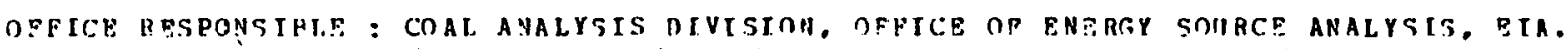




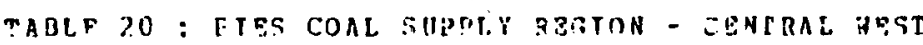

COAI. TYEF: SIR-AITIIAIMO!IS (19. 3 MMBTIJ/TON)

MFOIUM SUFIIA (GRFATPR THAN 0.6$)$ LBS/MMBTH)

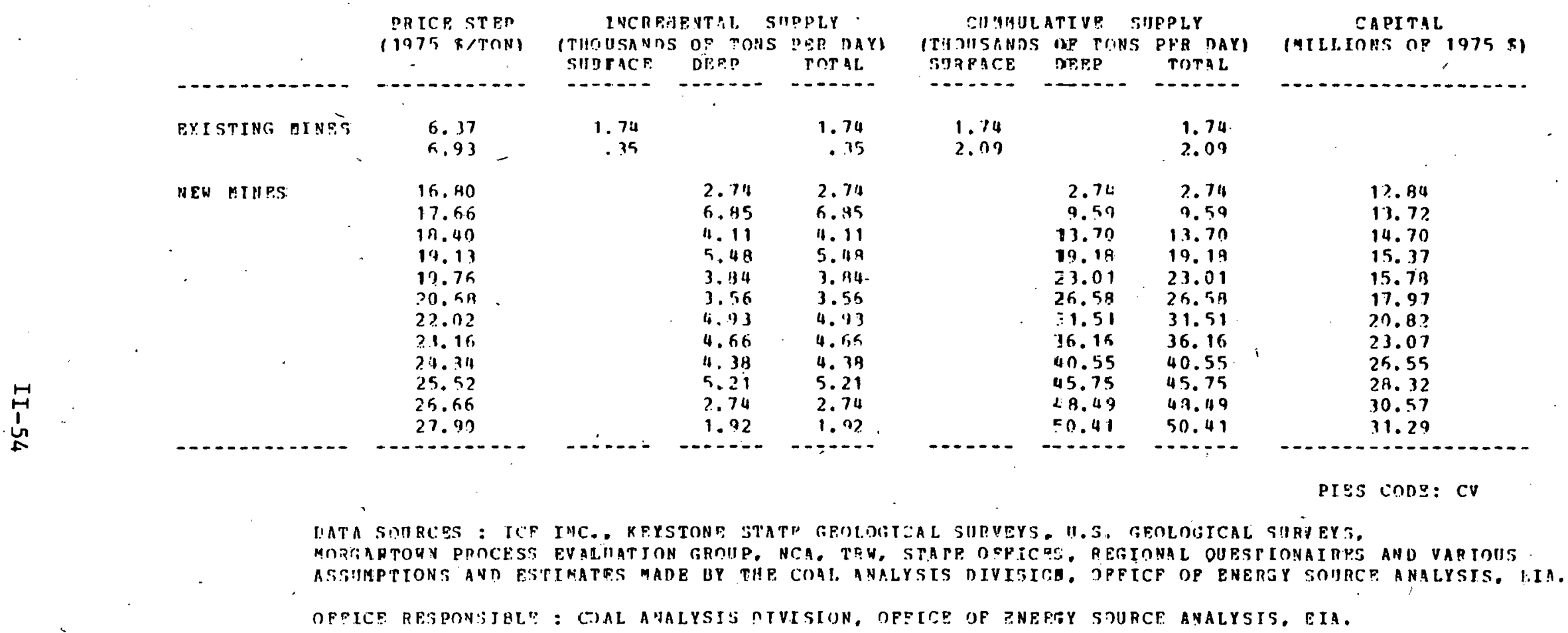




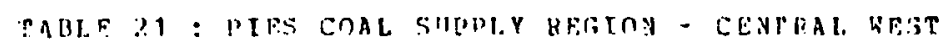

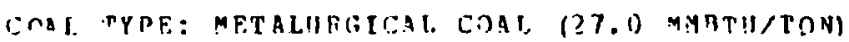

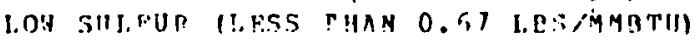

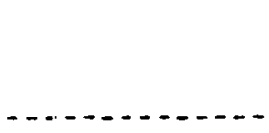

FFICF, STFF

$(1475 \quad 5 / T \cap N)$

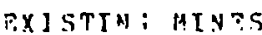

- - - - - -

NEF MIMTS

占

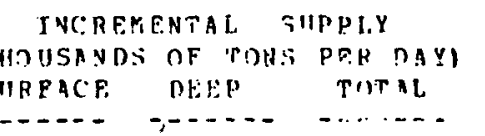

\begin{tabular}{|c|c|c|}
\hline .78 & & $.7 \mathrm{~B}$ \\
\hline $\begin{array}{l}011 \\
.04\end{array}$ & & $\begin{array}{l}0.14 \\
0.04\end{array}$ \\
\hline .26 & & .26 \\
\hline & $\begin{array}{l}111 \\
.62\end{array}$ & $\begin{array}{l}01 \\
06 ?\end{array}$ \\
\hline .22 & $\begin{array}{r}.52 \\
.82 \\
.52\end{array}$ & $\begin{array}{r}54 \\
092 \\
.62\end{array}$ \\
\hline $\begin{array}{l}.41 \\
1.53 \\
1.31\end{array}$ & .92 & $\begin{array}{l}1.25 \\
1.5 \\
1.3:\end{array}$ \\
\hline
\end{tabular}

\section{3 in \\ 10.87}

11. 39

18

- . 2?

.26

.26
.26
.26

32.99

34.56

36.00

77.59

39.211

40.77

$5 \% .1$

59.74
:UMAII.ATTVE SUPPLY TILISANDS OP TONS PER DAYI SIIIFACE DFEP TिTAL

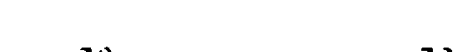

1.41
1.03
1.54
7.47
3.08
3.90
3.90
3.90
.- .90

OAPITAL

(4TLLIONJ OF 1979 ?)

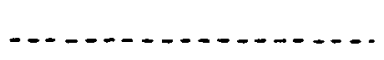

\begin{tabular}{rr}
.76 & 1.03 \\
.48 & 1.54 \\
.48 & 2.47 \\
.92 & 3.08 \\
2.45 & 3.90 \\
3.77 & 3.90 \\
\hline $.0 .-90$ & $-.0 .-$.
\end{tabular}

.78

.97

.25

.67

1. 2 ?

2. 12

2.94

3.56
4. 92

4.92
6.36

7.57

\begin{tabular}{c}
46.40 \\
25.90 \\
26.77 \\
16.95 \\
71.25 \\
40.75 \\
46.39 \\
11.95 \\
78.94 \\
\hline
\end{tabular}

PIES CODP: CM

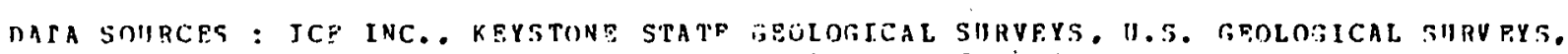

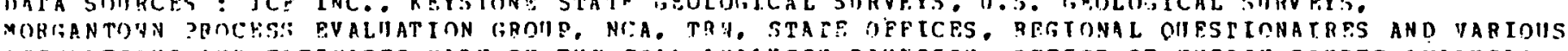

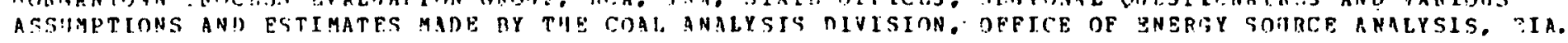

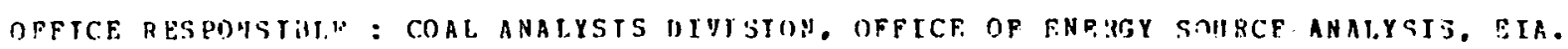


TABIF 2? : PIFS COAL SUPPLY FPGION - GILLF

CON. TYPE: I.IGNTTE (13.0 MMHTI/TON)

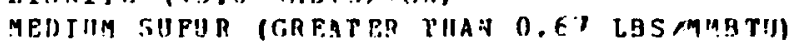

\begin{tabular}{|c|c|c|}
\hline & $\begin{array}{l}\text { PRTCE STEH } \\
11975 \text { Q TTONI }\end{array}$ & $\begin{array}{l}\text { INEPEM } \\
\text { ITHOUSANDS } \\
\text { SIP.FACE. }\end{array}$ \\
\hline FXISTINA MITES & 2.47 & 27.42 \\
\hline NEY MINFS & $\begin{array}{r}6.09 \\
6.93 \\
7.41 \\
8.17 \\
9.63 \\
7.49 \\
9.92 \\
10.91 \\
11.71 \\
12.54 \\
15.05 \\
18.46 \\
21.93 \\
23.41 \\
25.61 \\
27.96\end{array}$ & $\begin{array}{r}24.56 \\
43.94 \\
35.67 \\
60.27 \\
32.84 \\
32.89 \\
10.96 \\
10.96 \\
1.64 \\
2.74 \\
1.37 \\
1.37 \\
.92\end{array}$ \\
\hline
\end{tabular}

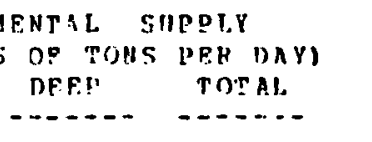

CIBMHI.ATIZF. SUPPJ.Y

CAPITAL SIITISANDS OF TONS PEA DAY) (MILLIONS DF 1975 k) ACE DP, EP TOMnt.

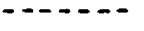
D.-.-...-.

\begin{tabular}{l} 
\\
4.10 \\
5.60 \\
5.25 \\
7.42 \\
9.49 \\
8.78 \\
10.64 \\
10.96 \\
12.31 \\
13.45 \\
13.66 \\
25.02 \\
31.37 \\
19.42 \\
25.00 \\
30.57 \\
\hline$\ldots$
\end{tabular}

PIES CODR: Cu

MATA SOIRCES : ICF INC. KRYSTONP STATZ GEOLOGTCAI. SIRUP.YS. D.S. GEJLOGTCAL SURVEYS. MOQGANTOHN PROCFSS FVALUATION GROIIE. NCA, TRA. STATP IYF!CES. REGIONAL OIIESTIONAIBES AND VARIO!S

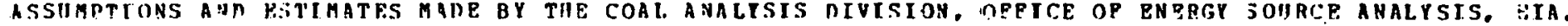

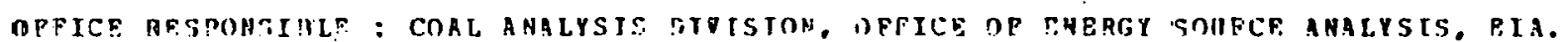




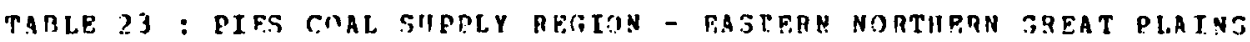

COAL TYPE: LIGNITE (13.) MYGTIJTONI

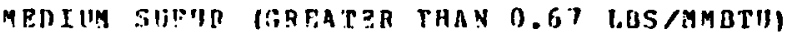

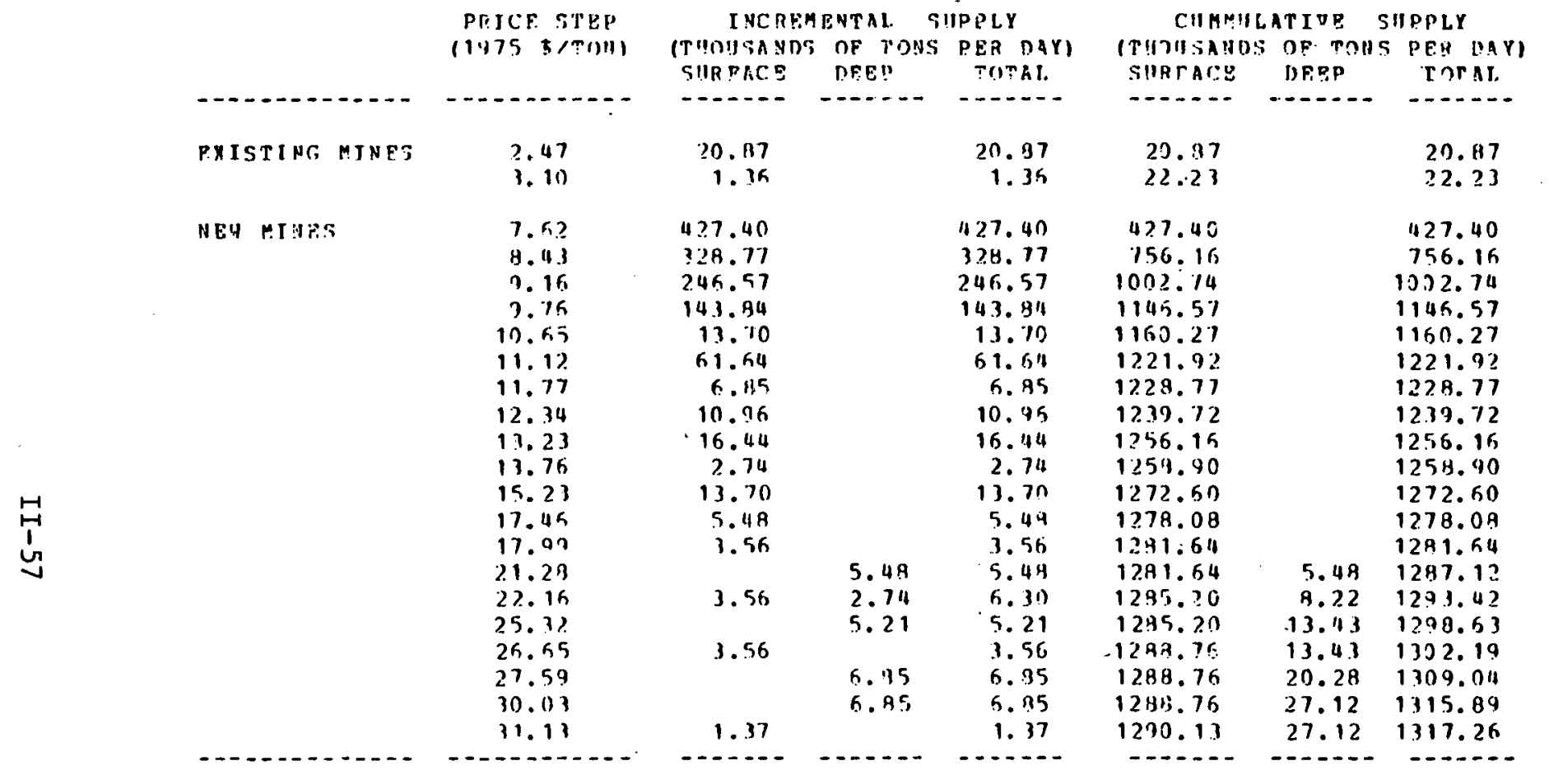

CATTAL

IMLLLONS OF $-1975 \$ 1$

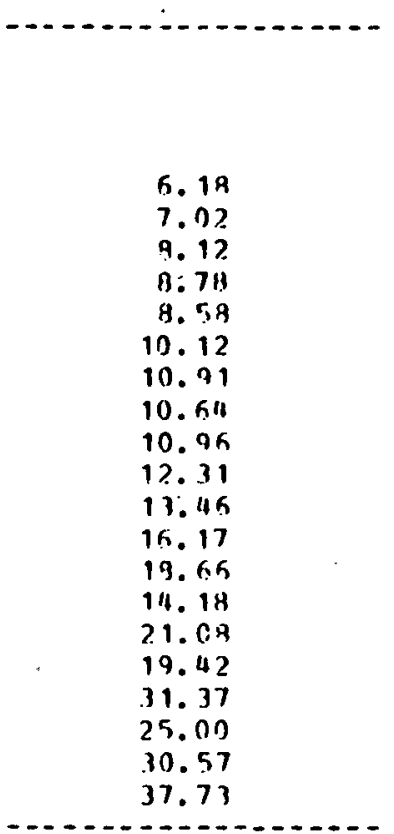

PIES CODE: CII

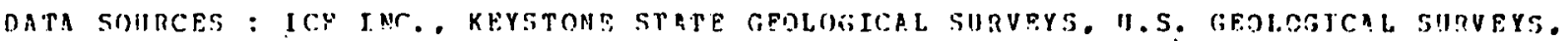

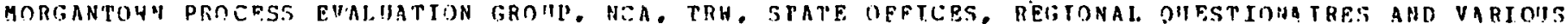

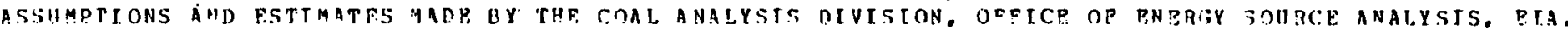

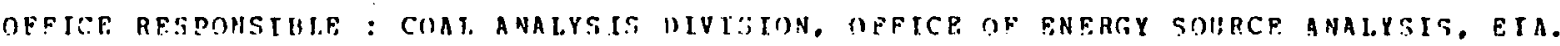




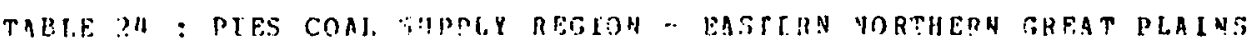

COAL TYPE: LTRETYE (13.) MMBTU/T(IN)

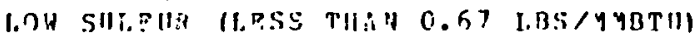

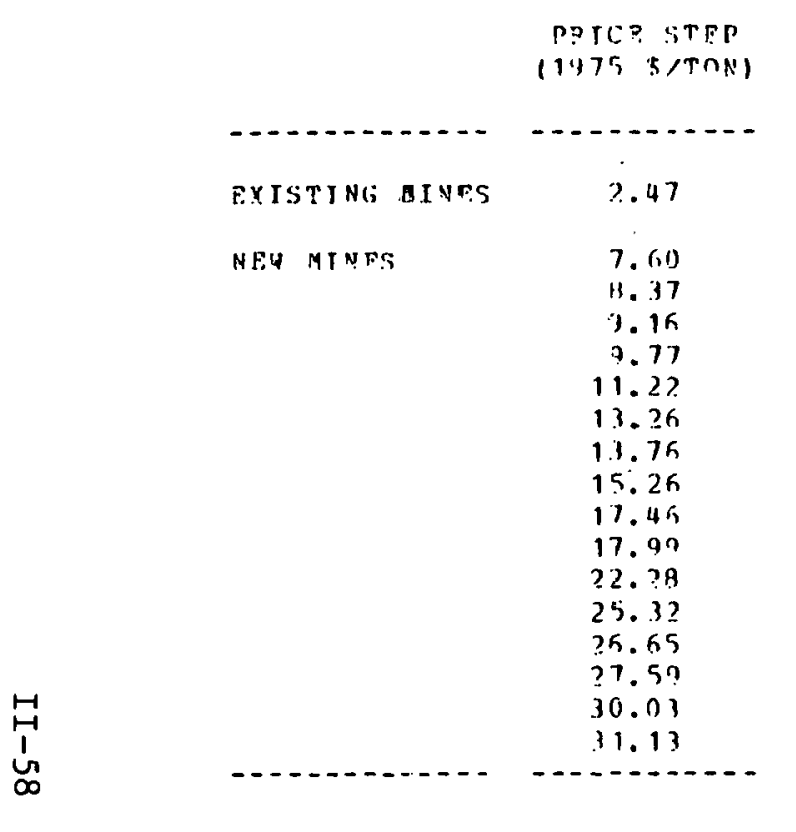

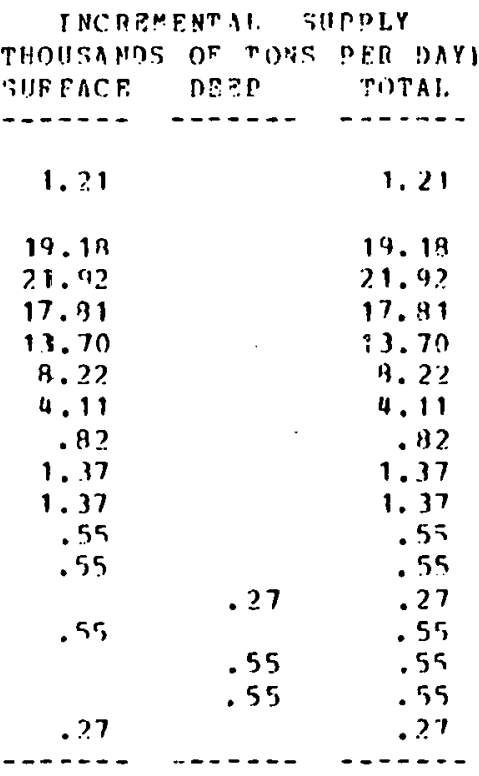

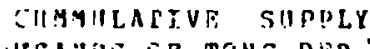

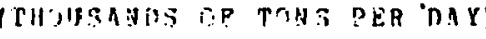
SIITEACE JEEP TOTA.T.

\begin{tabular}{|c|c|c|}
\hline 1.21 & & 1.21 \\
\hline 19.13 & & 19.18 \\
\hline 41.10 & & 41.10 \\
\hline 53.90 & & 58.90 \\
\hline 72.50 & & 72. (1) \\
\hline 80.32 & & BO.B? \\
\hline 811.73 & & RI. 93 \\
\hline 85.75 & & 95.75 \\
\hline 27. & & R7. 12 \\
\hline $39 .+9$ & & 89.49 \\
\hline ค3.8) 4 & & ค9.04 \\
\hline 49.57 & & 89.54 \\
\hline ค7. 59 & .27 & 89.96 \\
\hline 90.4 & .27 & 90.41 \\
\hline 90.14 & . 12 & 90.96 \\
\hline 90.14 & 0.17 & 91.51 \\
\hline 9.9 .4 & 1.37 & 91.78 \\
\hline
\end{tabular}

CAPITAL

(KILIIINNS OF 1975 \$)

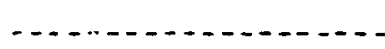

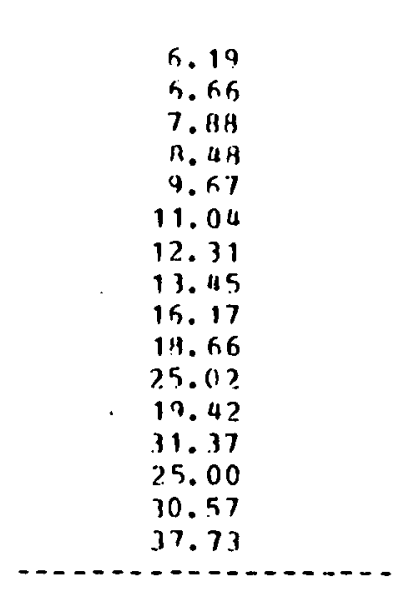

DIPS CODF: CY

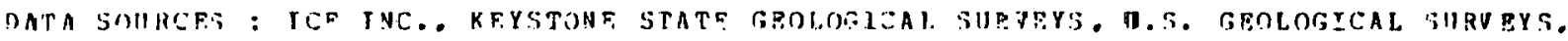

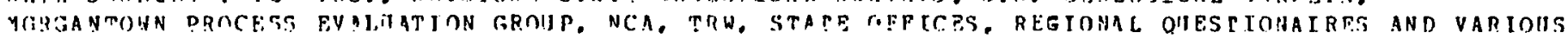

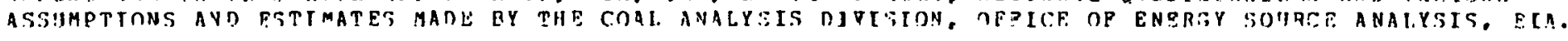

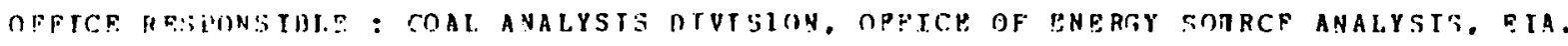




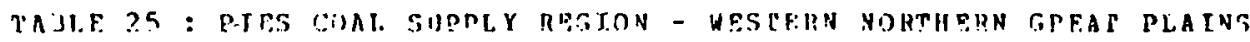

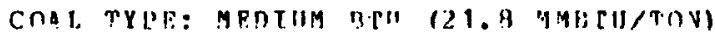

EDDIIIM SIIIFII? 0.67 TO 1.58 T.IIS/A4MBTII)

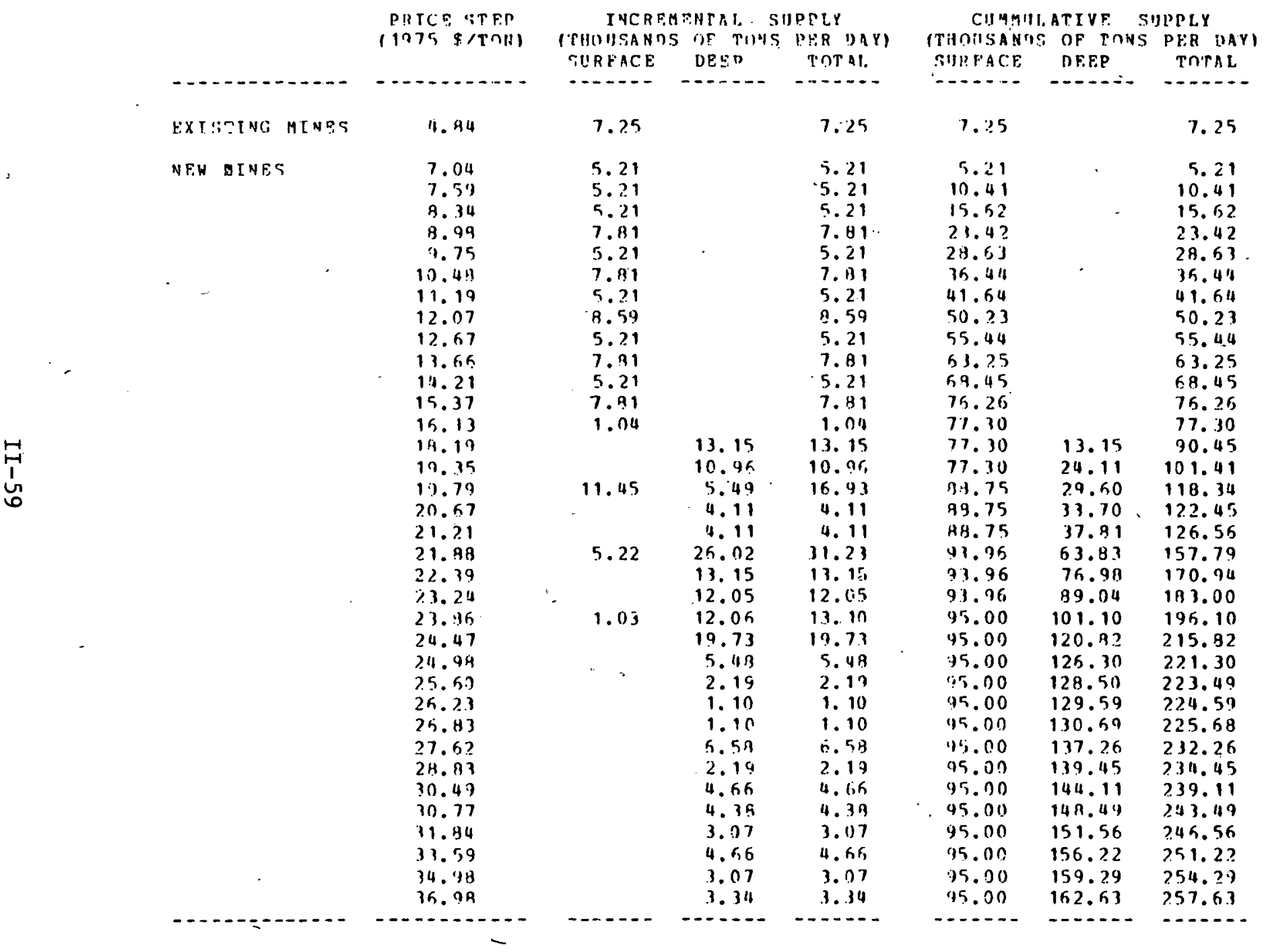

EAPTTAL.

MILLTOHS OP 1975 \$1

-

点

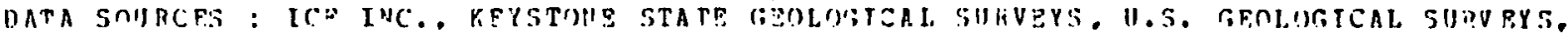

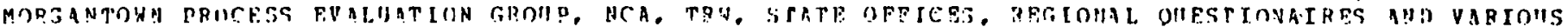

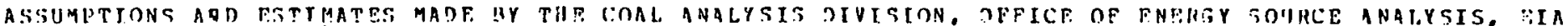

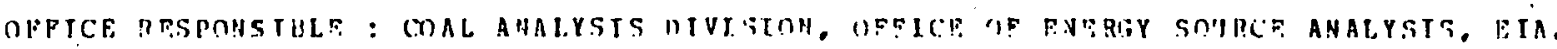




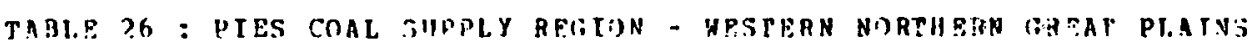

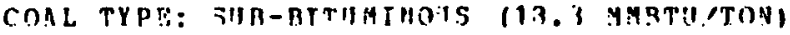

MEUIUM SIIFIT UBRFATFA THAH 0.67 LUS/MEBTI!

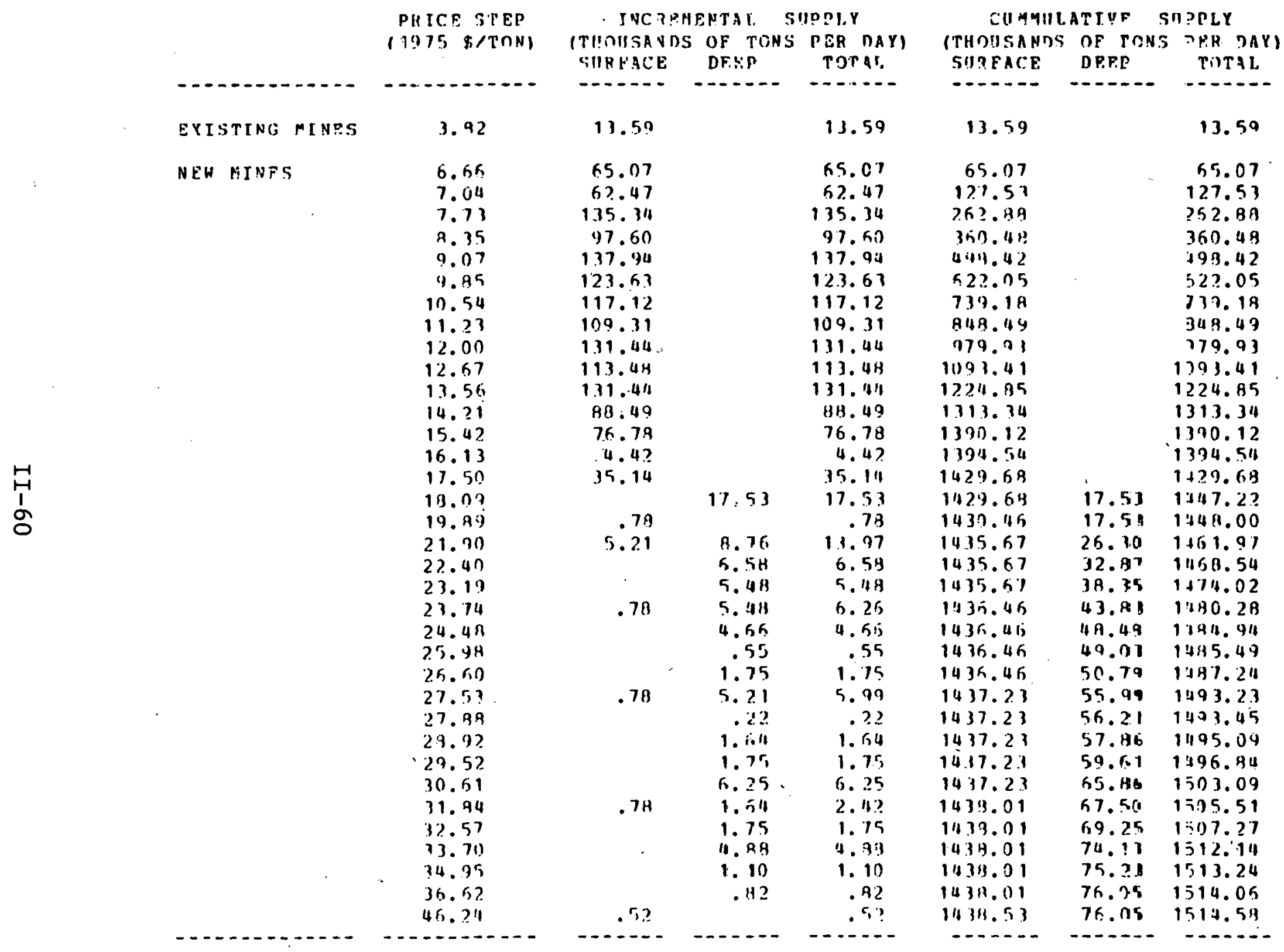

CAPTTAL

IMLLTONS OP 1975 P)

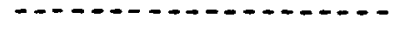

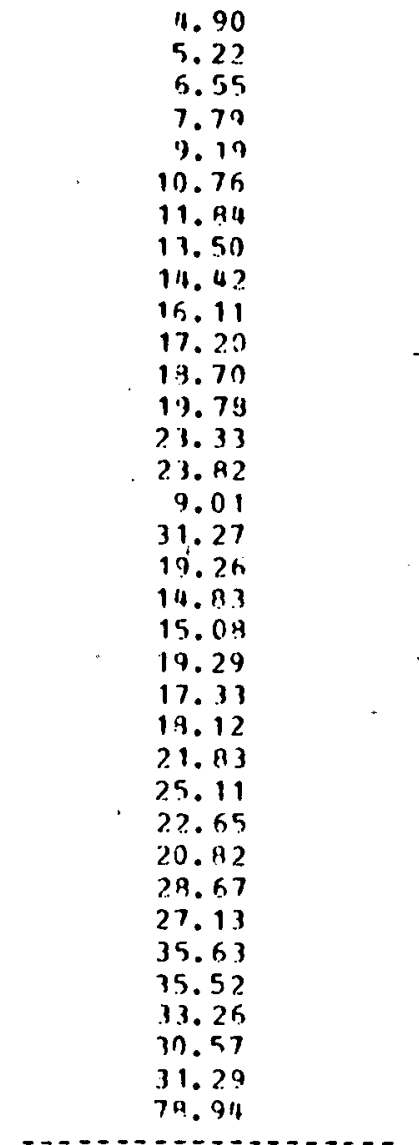

PIES CODP: CV

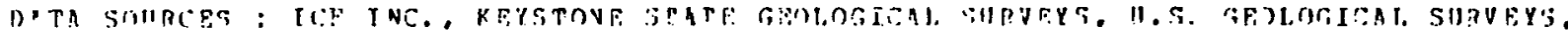

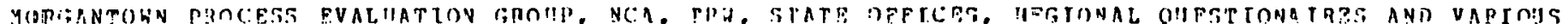

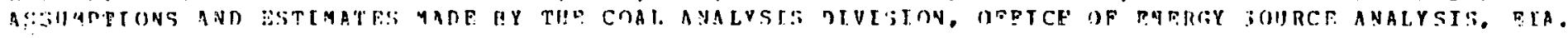

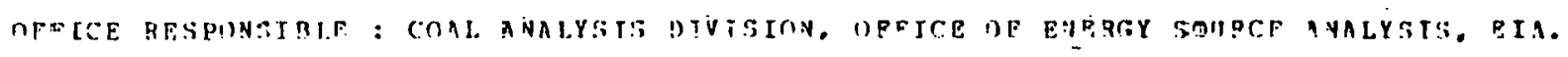




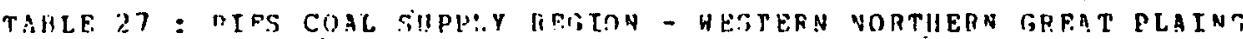

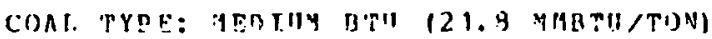

I.OW SULPUR (LESS THAN 0.57 LHG/AMBT!)

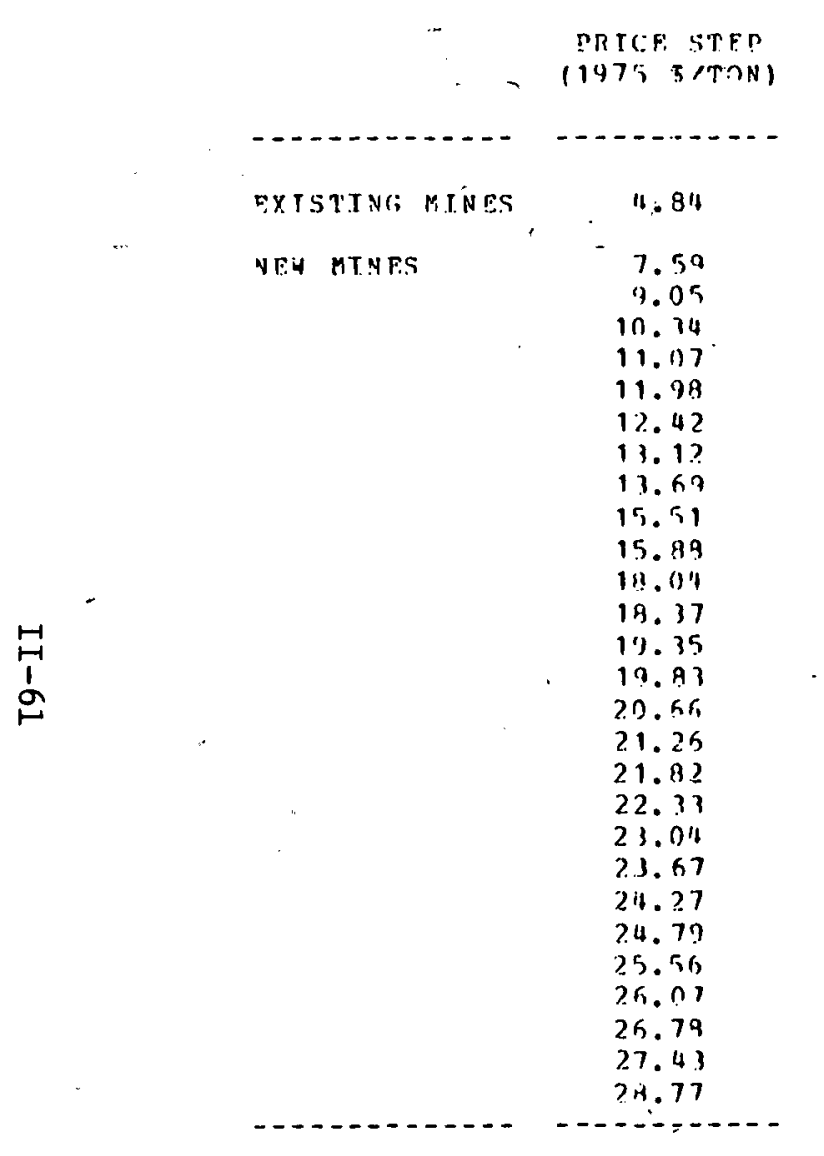

L NCREMEUT AL SI!P?!.Y ITHOISANDS OF TON:S PRII DAY SIIEFAC F. DFE:P DF r:P POTAl.

\begin{tabular}{|c|c|c|}
\hline 20.35 & & 20.35 \\
\hline 2.6 .0 & \multirow{5}{*}{ - } & 2.50 \\
\hline 3.97 & & 3.37 \\
\hline 3.40 & & $3.90^{\prime}$ \\
\hline 1.37 & & 1.37 \\
\hline 3.90 & & 3.90 \\
\hline $.7 H$ & & .79 \\
\hline 1.37 & & 1.37 \\
\hline 4.45 & & 4.45 \\
\hline 5.27 & 5.148 & 10.75 \\
\hline .25 & 10.96 & $11.2 ?$ \\
\hline 2.40 & R.76 & 11.16 \\
\hline & 4.38 & 11.33 \\
\hline & 21.92 & 21.92 \\
\hline .27 & 16.47 & 16.79 \\
\hline & 16.44 & 16.44 \\
\hline & 16.44 & 16.44 \\
\hline 2.60 & 27.95 & .70 .55 \\
\hline 1.10 & $\begin{array}{l}11.21 \\
20.27\end{array}$ & $\begin{array}{l}12.33 \\
20.27\end{array}$ \\
\hline .26 & 13.42 & 13.69 \\
\hline & 19.45 & 19.45 \\
\hline & 16.71 & $1 \mathrm{~K} .71$ \\
\hline 1.31 & 9.31 & $10.6 ?$ \\
\hline & A. 77 & 8.77 \\
\hline 1.10 & 3.23 & 14. $3 n$ \\
\hline$\cdot 26$ & 11.18 & 11.144 \\
\hline & 2.63 & 2.63 \\
\hline
\end{tabular}

CIJMYIILATIVE ZIIDPLY

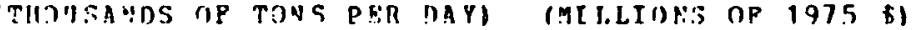
ror Al

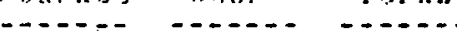

\begin{tabular}{|c|c|c|}
\hline $20=15$ & & 20.35 \\
\hline $\begin{array}{l}2.60 \\
5.54\end{array}$ & & $\begin{array}{l}2.60 \\
6.58\end{array}$ \\
\hline 10.48 & & 10.18 \\
\hline 11.95 & & 11.9 .5 \\
\hline 15.75 & & 15.75 \\
\hline 15.51 & & 16.51 \\
\hline 17.90 & & 17.90 \\
\hline 2.2 .35 & & 22.36 \\
\hline 27.51 & .5 .48 & 33.11 \\
\hline 27.99 & 16.45 & 44.31 \\
\hline 30.28 & 25.21 & 55.49 \\
\hline 30.21 & 29.59 & $59.8 A$ \\
\hline 30.28 & 51.51 & 81.79 \\
\hline 30.57 & 67.94 & 93.49 \\
\hline 30.55 & 64.38 & 114.93 \\
\hline 30.55 & 100.92 & 1.11 .37 \\
\hline 13.15 & 128.77 & 161.92 \\
\hline 14.21 & 140.00 & 174.25 \\
\hline 14.24 & 160.28 & 194.52 \\
\hline 34.50 & 173.79 & 20.4 .21 \\
\hline 34.50 & 193.15 & 227.65 \\
\hline 34.50 & 209.96 & 2414.37 \\
\hline 35.41 & 2.19 .18 & 254.99 \\
\hline 35.91 & 227.94 & 253.75 \\
\hline 16.91 & 231.23 & 269.111 \\
\hline 17.11 & 2112.41 & 279.57 \\
\hline 37.17 & 245.01 & 242.20 \\
\hline & & -1 \\
\hline
\end{tabular}

CA ITAL (2-10.

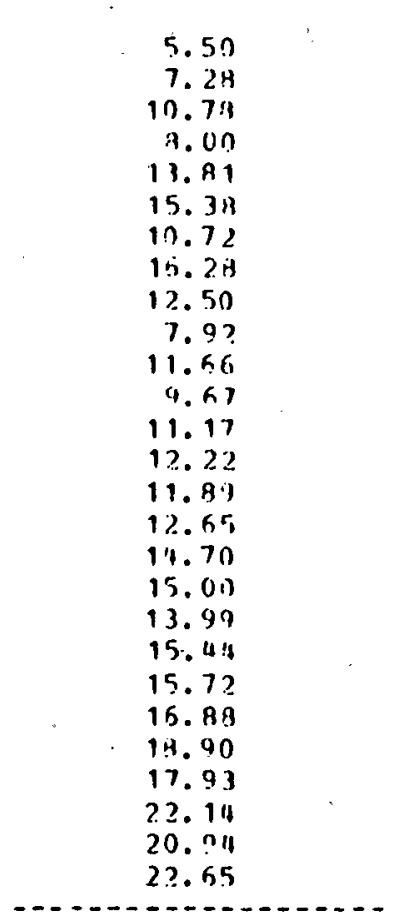

PIES CODÉ: CZ

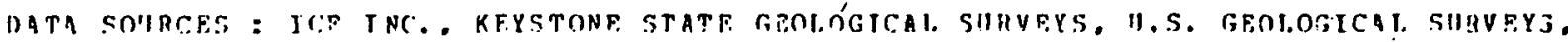

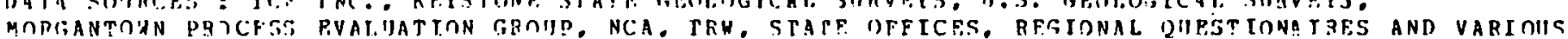

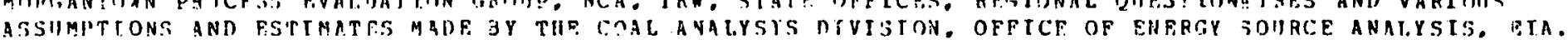

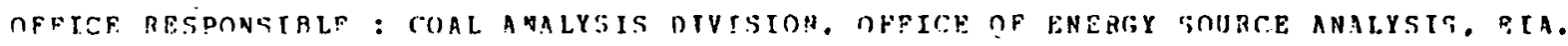




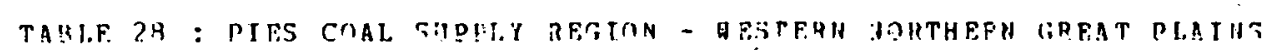

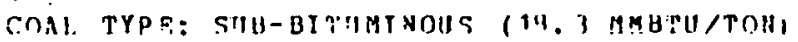

L.OH SIILFIN (LESS TIIAN 0.57 LBSGMYTI!)

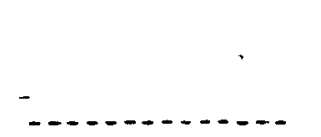

SEISE STEP

$(1975 \$ / 2121)$

TNCREMENTAL SIIPPLY

(T!OUSANISS OF TONS PQR DRY) TURPA:S: DE TEP

CIJMA JLATIVP SIIPPLY

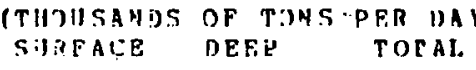

F.XISTTH, MINFS

B1. 43

1.16

82.61

131.413

1.15

Q2.63

NF.H MLMES

7. 14

$\therefore .61$

9.41

2.37 .911

251.23

$463.94)$

361.72

499.52

327.67

3.92

19.67

11.38

328.112

11.69237 .17

.25.

\section{$23 \% .81$}

$23 \% .81$
251.21

251.27
453.90

153.90
151.92

151.92
449.52

327.67
329.42
237.12 .26

\subsection{7 .81}

489.04

952.74

1311.96

1314.18

2142.05

2470.48

2707.60

27117. $\mathrm{HK}$

-...-.

$\begin{array}{r}237.31 \\ 489.04 \\ 952.94 \\ 1314.86 \\ 1814.34 \\ 2142.05 \\ 2470.48 \\ 2707.60 \\ 2707.86 \\ \hline\end{array}$

CAPITAL

(MILLIONS OP 1975 \$)

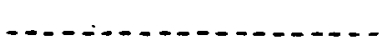

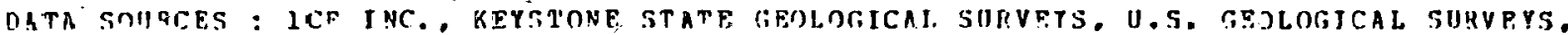

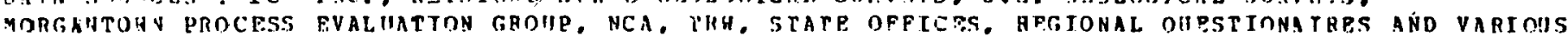

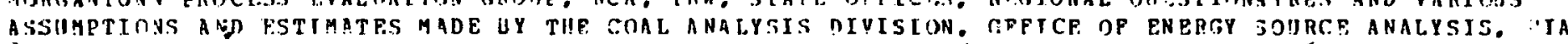

OFFIEF. PFSPONSTRLE: : COAL ANALYSIS DIVISUON, OFFICF OF EYEPGY SOUPCF ANAIYSIS. ELA. 


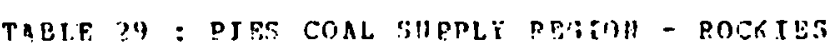

COMJ. TYPP: HIRI BTH (23. A MABTH, TON)

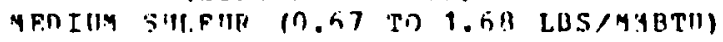

PRTCE STEP THCREAENTAL SIILPLY 11975 क/Trinl

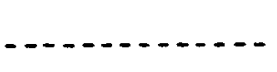

FXISTIMF, MINFS NEM MIVPS

6.51
CIMMILATIVE SIIPLIY TTH SIISANDS SURFACE DEEP rOT AI. SIIPFACE DETS TOTAL.

10.111

7. 91

3.12

16. 9

20.7

22.55

23.09

23.54

24.33

24.33

25.45

25.01

26.60

27.99

29.45

30.94

32.50

33.79

15. 36

35.90

18.51
.01

.01

6.51

10.4

7.91
3.12

6.062

3.27

3.29

3.29
4.39

5. 5 ?

2.17

1. 11

1.10

3.51

2. 94

4. R?

3.71

6. 14

3.71

2.6

3.4

1. 11

-.....

0.51

15.92

24.73
27.65

3?. 28

32.213

32. $2 \mathrm{H}$

32.28

36.70

35.70

35.70

15.70

36.70

39.26

34.25

34.26

33.26

39.25

3日. ? 5

19.26

38.26
.01

.01

5.51

16. 9 ?

24.73

27.95

$\begin{array}{ll}2.19 & 34.47\end{array}$

13.15 45.11?

14.25

16.114

17.53

18.63

22.14

23.45

2 ค. ??

12.00
38.14

41.45

44.49

46.90

48.22

50.94

53.14
54.23

55.33

58.93

51.71

66.53

70.26

76.40

40.12

82. 75

45. 16

Bर. 18
3.48
8.77
CAPITAL

(MILIINNS OF 1975 b)

\begin{tabular}{l}
10.47 \\
13.55 \\
15.81 \\
22.03 \\
25.42 \\
111.53 \\
15.90 \\
15.79 \\
34.75 \\
17.17 \\
17.62 \\
11.07 \\
21.87 \\
35.46 \\
27.25 \\
26.14 \\
$3 ? .19$ \\
31.07 \\
34.71 \\
39.22 \\
39.11 \\
\hline
\end{tabular}

PIES CONE: CR

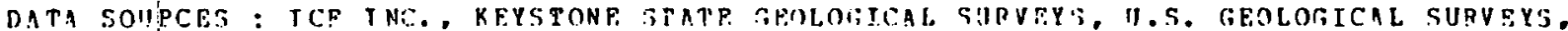

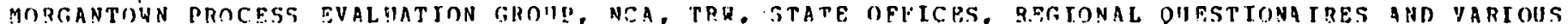

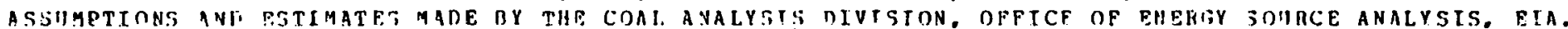

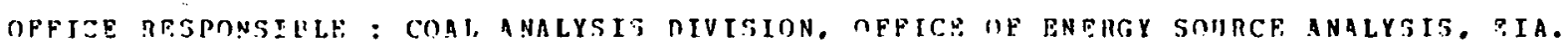




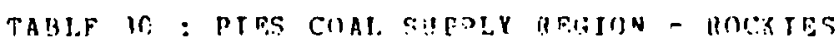

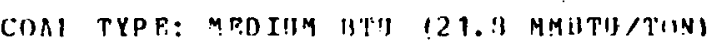

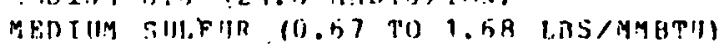

\begin{tabular}{|c|c|c|c|c|c|c|c|}
\hline & PRICE TTP.P & TNCREM & ERTAI. $S$ & IIPLI.Y I I & ruymlit & ATIVE 5 & I P?I.Y \\
\hline & $(1) 75$ (1) & $\begin{array}{l}\text { ITHDIISA NOS } \\
\text { SURFACE }\end{array}$ & $\begin{array}{l}\text { OE TOAS: } \\
\text { DEEP }\end{array}$ & $\begin{array}{l}\text { PER 1Y4Y } \\
\text { POPAI. }\end{array}$ & $\begin{array}{c}\text { (THOJTANIS } \\
\text { SURPACE }\end{array}$ & $\begin{array}{l}\text { OE TUNS } \\
\text { DEEP }\end{array}$ & $\begin{array}{l}\text { DEP DAY) } \\
\text { TOPAL }\end{array}$ \\
\hline 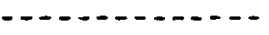 & $-\cdots+--\infty-\infty$ & $-\cdots--\cdot$ & $\cdots \cdots$ & 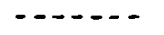 & $\cdots+\cdots$ & $\cdots----$ & $\ldots \ldots$ \\
\hline EXISTING; MTAFS & 9.21 & & 2.07 & 2.07 & & 2.07 & 2.07 \\
\hline NEU MTNFS & 21.07 & & 2.19 & 2.11 & & 2.19 & 2.19 \\
\hline
\end{tabular}

LAPITAL

MILLLONS OF 1975 \$)
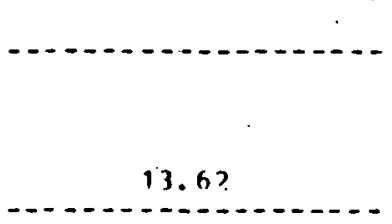

PIES CODE : CS

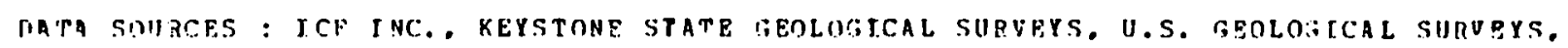

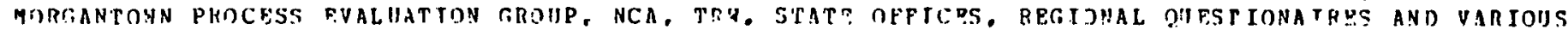

ASSIMDTIONS AND ESTI MATES MADE BY THF COAL ANALYSIS DIVISION, OPFIZE OF FNERGY SOIIRES ANALYSIS. EIA.

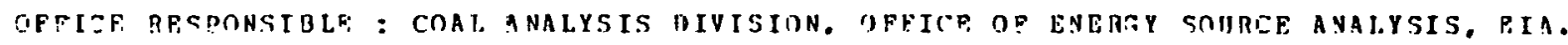




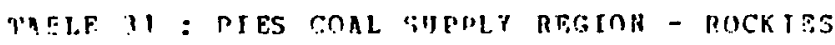

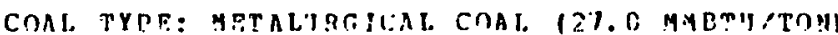

LOW SILLEUR (LESS TIAN D.

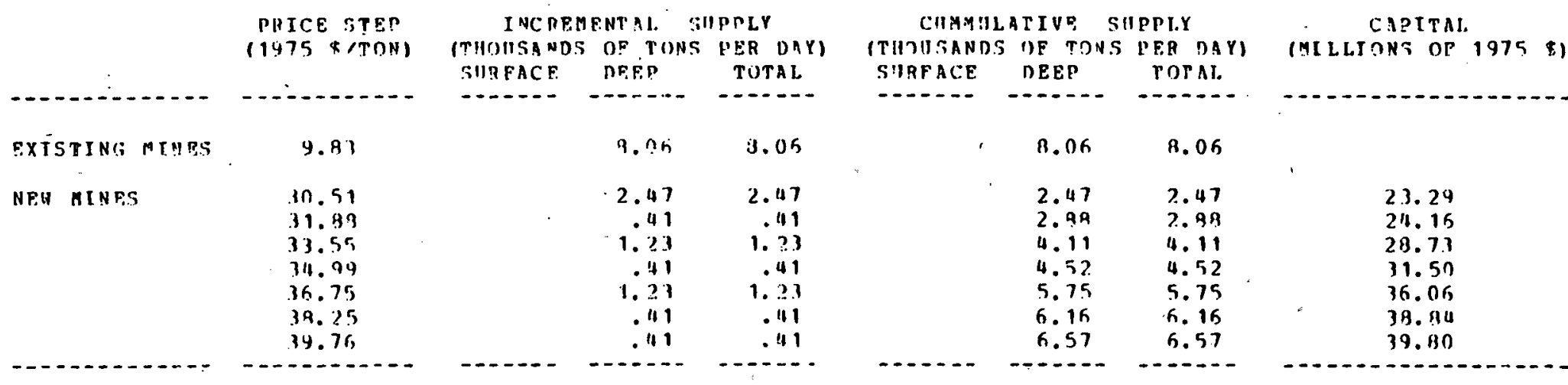

PIF.3 CODP: CA

DATA SOURCES : ICP INC. . KEYSTONE GTATE GEULOSICAL SURVEYS. II.S. GFOLOGICAL SIJRVEYS.

MORTANTOHN PSOCPSS FVALIIATTIM GROIP. NCA. TRK STATE OFFICPS. REGIONAL OUEST LONA TRPS ANI VARIOIIS ASSIIMPTIONS AND ESTIMATES MADE BY TIIE COAL ANALYSTS DIVISIOH, OFFICE OP PNPRGP BOHHCP ANALYSIS. PEA.

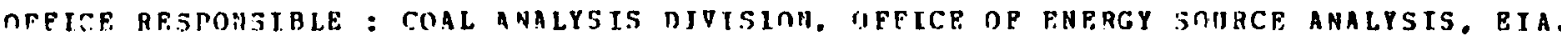


TARI.F, 12. : PIES COAL, SUPPI.Y PEGIINN - hOCKIES

COAI. TYPE: HIBH HT:I (3.) g MASTU/TONI

LOW SUITFOR II.ESS THAN 0.57 L.HS/RABTII

\begin{tabular}{|c|c|c|c|c|c|c|c|c|}
\hline & $\begin{array}{l}\text { PRTCF STFE } \\
(1475 \text { क } / \text { TON })\end{array}$ & $\begin{array}{l}\text { IYCREM } \\
\text { (THOISANDS } \\
\text { SURFACR }\end{array}$ & $\begin{array}{l}\text { ENTA i. SI } \\
\text { OF I!UNS } \\
\text { DEEP }\end{array}$ & $\begin{array}{l}\text { DPEY } \\
\text { PEP DAYI } \\
\text { TOTAL }\end{array}$ & $\begin{array}{l}\text { EIIMHUL } \\
\text { ITHOUSANOS } \\
\text { SURPACR }\end{array}$ & $\begin{array}{l}\text { ATTVE SI } \\
\text { OF TONS } \\
\text { DFE? }\end{array}$ & $\begin{array}{l}\text { JPELY } \\
\text { PEH DAYI } \\
\text { TOTAL. }\end{array}$ & $\begin{array}{c}\text { CAPITAL } \\
\text { (YILIIONS OP } 1975 \$)\end{array}$ \\
\hline - & $----\infty-\infty-\infty$ & 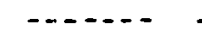 & $\cdots+-\infty$ & 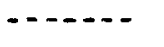 & $\cdots-\cdots$ & $\cdots-\cdots$ & $\cdots \cdots$ & $-\cdots,-\infty,-\infty$ \\
\hline PXISTING MINPS & 13.35 & & 17.91 & 17.81 & & 17.91 & 17.81 & \\
\hline NEH MINES & $\begin{array}{l}13.04 \\
11.75 \\
17.11 \\
20.98 \\
21.44 \\
22.43 \\
22.95 \\
23.54 \\
214.18 \\
24.75 \\
25.36 \\
25.92 \\
26.44 \\
27.80 \\
23.99 \\
29.13 \\
20.52 \\
30.59 \\
39.86 \\
32.05 \\
12.53 \\
33.64 \\
31.95 \\
34.67 \\
35.35 \\
35.80 \\
314.51\end{array}$ & $\begin{array}{r}1.30 \\
1.30 \\
.78 \\
.79\end{array}$ & $\begin{array}{r}10.95 \\
10.116 \\
5.48 \\
5.48 \\
9.36 \\
4.38 \\
8.77 \\
4.38 \\
4.38 \\
5.79 \\
3.07 \\
5.92 \\
3.95 \\
3.07 \\
2.41 \\
5.92 \\
5.92 \\
3.07 \\
2.41 \\
.16 \\
4.35 \\
2.91 \\
2.19\end{array}$ & 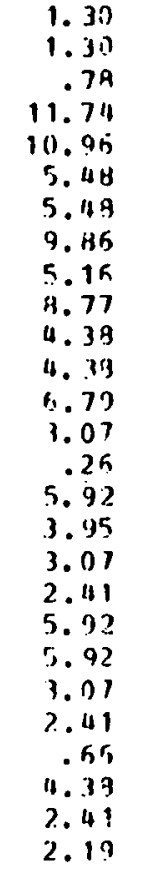 & $\begin{array}{l}1.30 \\
2.50 \\
3.34 \\
4.17 \\
4.17 \\
4.17 \\
4.17 \\
4.17 \\
4.95 \\
4.95 \\
1.95 \\
4.95 \\
4.95 \\
4.95 \\
5.21 \\
5.21 \\
5.21 \\
5.21 \\
5.21 \\
5.21 \\
5.21 \\
5.21 \\
5.21 \\
5.21 \\
5.21 \\
5.21 \\
5.21\end{array}$ & $\begin{array}{l}10.95 \\
21.91 \\
27.79 \\
12.47 \\
42.7 .7 \\
47.12 \\
55.89 \\
60.77 \\
54.65 \\
71.45 \\
74.52 \\
74.52 \\
80.43 \\
84.39 \\
87.45 \\
99.96 \\
95.78 \\
101.69 \\
104.76 \\
107.17 \\
107.83 \\
112.21 \\
114.62 \\
116.82\end{array}$ & $\begin{array}{r}1.30 \\
2.50 \\
3.39 \\
15.12 \\
26.09 \\
31.56 \\
37.04 \\
46.90 \\
52.07 \\
60.811 \\
65.22 \\
69.60 \\
76.40 \\
79.47 \\
79.73 \\
95.611 \\
189.59 \\
92.65 \\
95.07 \\
100.99 \\
105.90 \\
109.97 \\
112.31 \\
113.04 \\
117.42 \\
119.83 \\
122.03\end{array}$ & $\begin{array}{l}13.40 \\
15.81 \\
.11 .33 \\
14.92 \\
14.71 \\
14.53 \\
15.39 \\
16.42 \\
20.15 \\
17.17 \\
17.67 \\
19.07 \\
21.93 \\
22.65 \\
47.15 \\
25.97 \\
29.57 \\
29.53 \\
24.20 \\
32.85 \\
32.04 \\
32.35 \\
35.4 ? \\
37.32 \\
34.97 \\
33.29 \\
39.11\end{array}$ \\
\hline & 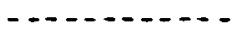 & & 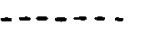 & $---\cdot-$ & $\ldots . .$. & $\cdots-\cdots$ & 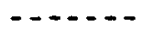 & $6-----\rightarrow--$ \\
\hline
\end{tabular}

PIRS COINE: CI

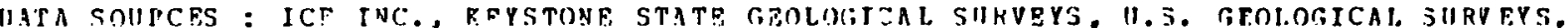

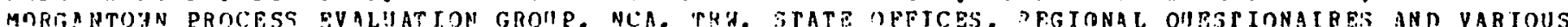

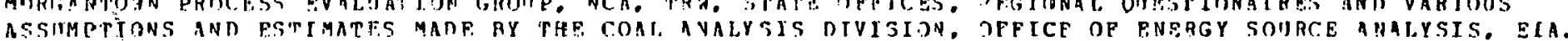

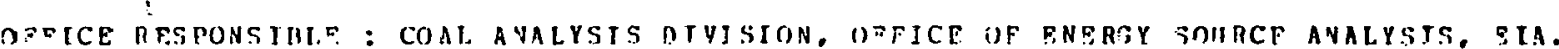


TASTE 13 : PIES COAL. SUPEI.Y HPGIOH - ROCKTES

CONI. TYPF: MEDIU: BTH 121.B YMBTIITON

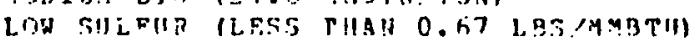

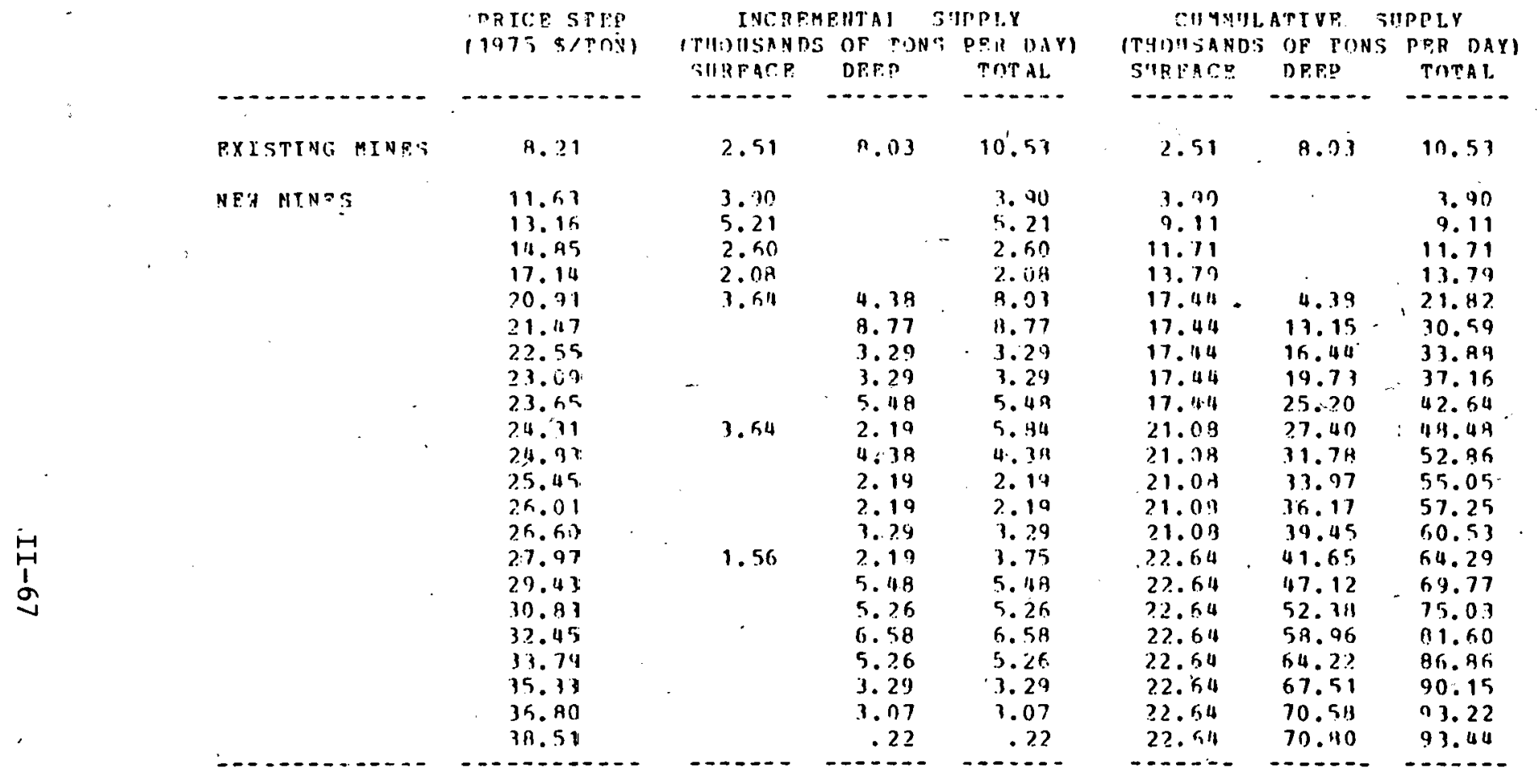

ZAPITAL

AYILLION:S OF $1975 * 1$

-

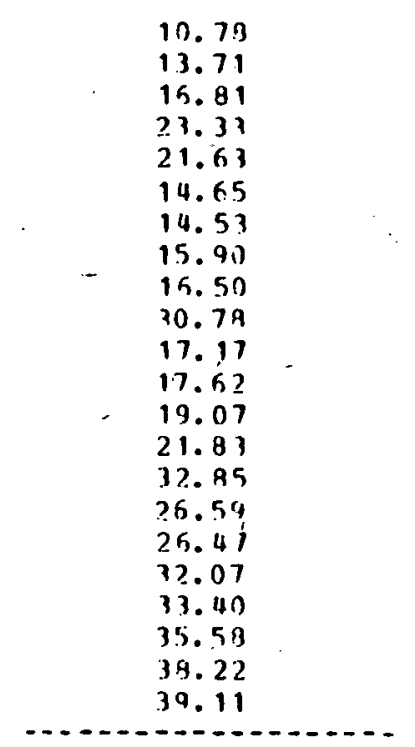

PIES CODE: CZ

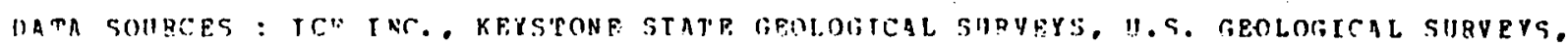

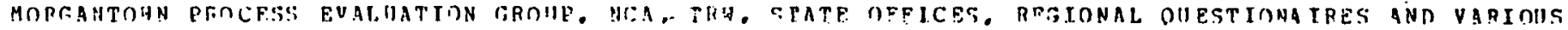

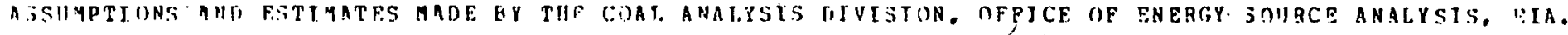

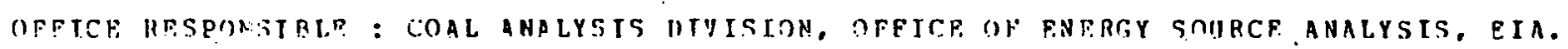




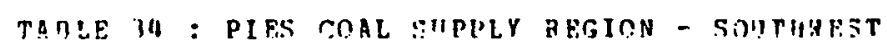

COAL TYPE: MEDIJM NT! 121.8 MMTTIJUNI

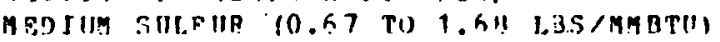

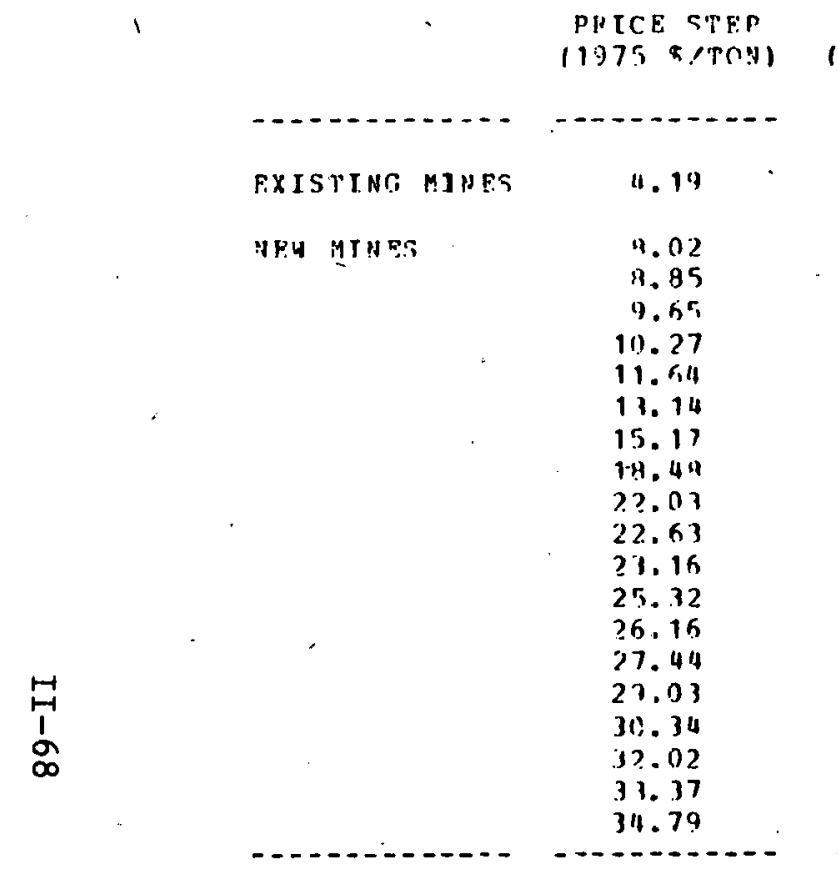

INCPFMEHTAT. SUUPLY (CUOISANDS OF TOKS IVER DAY) SHRPACE DFFT TOTAL - - - -

73.73
26.03
26.39
26.03
20.96
13.22
6.71
.55
.92
.92
.27

$$
2.13) 41.5 \%
$$

26.03 25.30 25.07 20. 96 i3. 2?

6.71

.55

3.01

2.17

2.13

2.19

2. 17

2. 27

2. 19

2.114

2.53

2.63

2.63
.44

.44
. .44$$
2.63
$$$$
.44
$$$$
2.63
$$$$
.44
$$

.44
SUPPL (TIIOIISAYD' OF TONS PE'R DAY) SIIRPAI: DREP TIITAI.
2.83

41.55

52.33

$78.3 \mathrm{~h}$
91. 1 ?

112.53

119.23

119.79

$121.4 \%$

121.44

121.41

121.71

121.71

121.71

$121.7 \mathrm{i}$

121.11

121.71

121.71

121.71

-.......

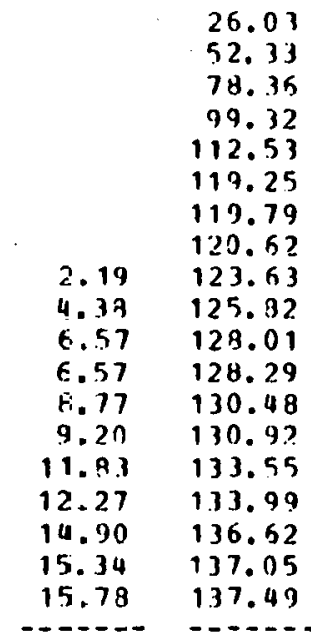

25.03
CAPITAL

IMILLONS OF 1975 \$1

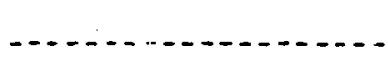

7.55
7.97
10.49
17.89
17.91
11.76
14.56
25.02
19.12
15.90
17.27
37.73
21.83
27.65
27.81
29.51
34.65
36.42
37.32

PIES CONDE: CS

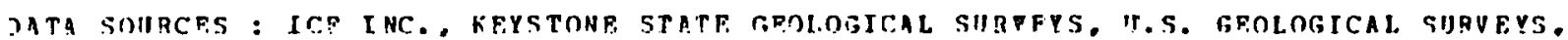

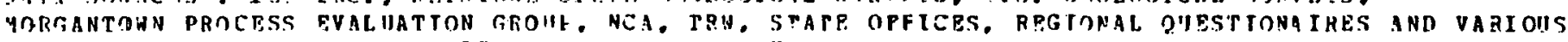
ASSIIMPTINNS AND ESTIMATES MADE BY THE COAL ANAIYSIS DIVISIOH. OFFTCE OP PNERTY SOIRCE ANALYSIS. FIA.

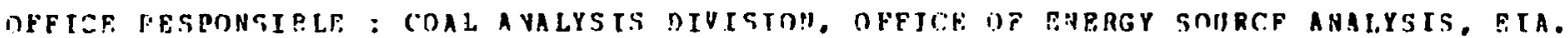




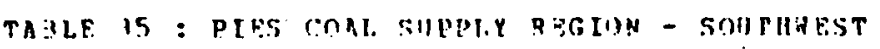

COAJ. TYPF: MEIJIUM BTU (21. A MUTIJTON)

LOH SULFUR IITSSG THAN 0.57 LBS/AYBTUI

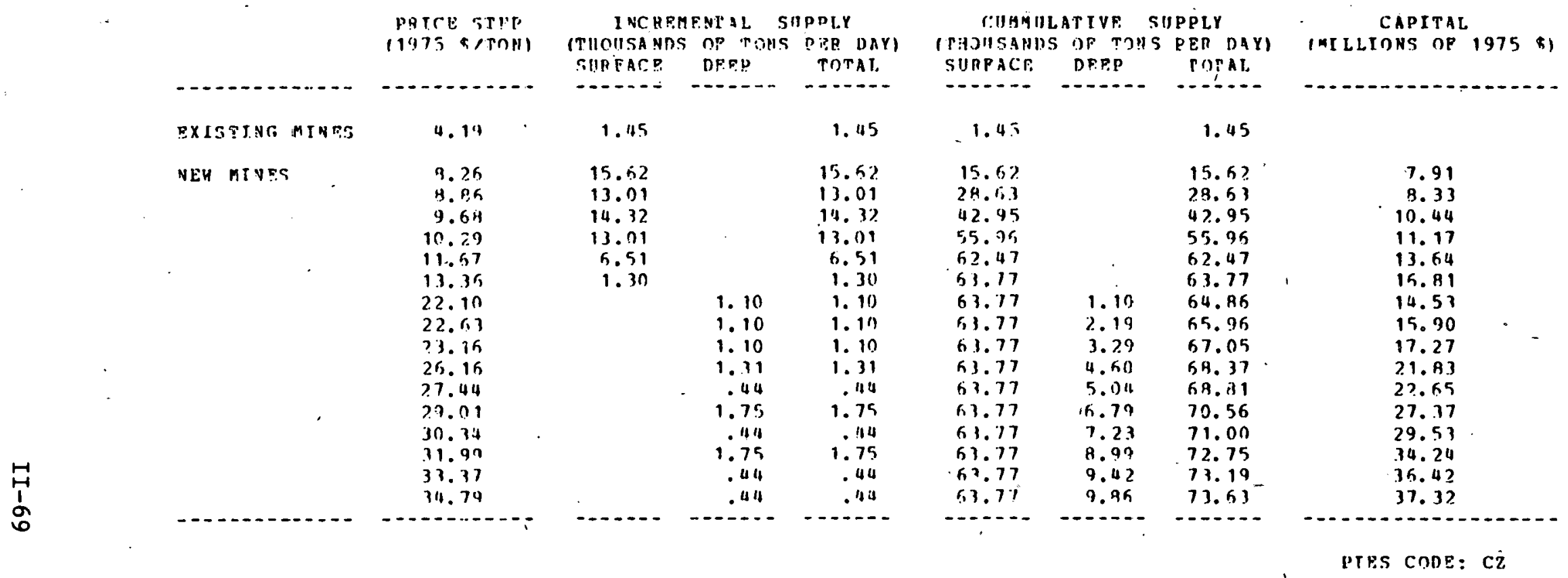

DATA SOIIRCE: : ICP INC.. KFYSTONE STARE GPOLOGIZAL SIIRVPYS. U.S. GEOLOFICAL SURVFYS.

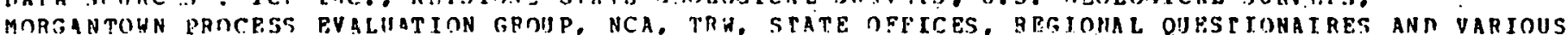
ASSIMPTIONS AND ESTIMATFS MADE RY TUE COAL ANALYSTS DIVISION. DPFICP OF ENFRGY SOURE ANAIYSIS, PIA.

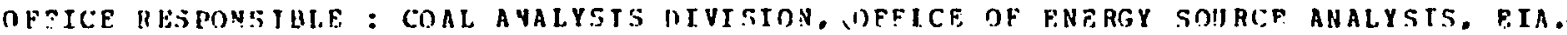




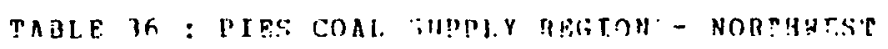

COAL TYPF: SIIB-RITUMTINUIS (14. 1 MMRTU/TON)

MEOIUM SIIFUH IOREATF.R THAN $0.6,7$ LAS/MHOTIII

\begin{tabular}{|c|c|c|c|c|c|c|c|c|}
\hline \multirow{2}{*}{. } & \multirow{2}{*}{$\begin{array}{l}\text { PHTCE } \$ T E P \\
(1775 \$ / Y O H)\end{array}$} & \multicolumn{3}{|c|}{.. INCFEMFNTAL SUPPLY } & \multicolumn{3}{|c|}{ C!MMULATIVE SHFPLY } & CAPITAI. \\
\hline & & $\begin{array}{l}\text { I PUOUISAMDS } \\
\text { SURFACE }\end{array}$ & $\begin{array}{l}5 \text { OF TIINS } \\
\text { DFFP }\end{array}$ & 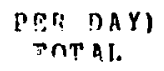 & $\begin{array}{l}\text { (THOUSANDS } \\
\text { SUPVACF. }\end{array}$ & $\begin{array}{l}\text { OE TONS } \\
\text { DEER }\end{array}$ & $\begin{array}{l}\text { EFR DAY } \\
\text { TOTAI. }\end{array}$ & (MILLIONS OP 1975 \$) \\
\hline 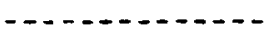 & $--\infty \cdots+\cdots-\infty$ & $\cdots-\cdots$ & $\therefore \ldots$ & $\cdots+\cdots$ & $\cdots-\cdots$ & $\cdots+\cdots$ & $\cdots---$ & $n-n-n-n-n$ \\
\hline FXIITING MINET & 7.97 & 10.05 & ' & 10.03 & 10.05 & - & 10.05 & . \\
\hline NES' MINES & $\begin{array}{r}9.39 \\
10.71 \\
12.30 \\
13.95 \\
15.91 \\
17.94 \\
19.71 \\
22.97\end{array}$ & $\begin{array}{l}2.60 \\
5.21 \\
5.21 \\
5.21 \\
2.60 \\
5.21 \\
7.91 \\
1.90\end{array}$ & & $\begin{array}{l}2.60 \\
5.21 \\
5.21 \\
5.21 \\
2.60 \\
5.21 \\
7.131 \\
3.90\end{array}$ & $\begin{array}{r}9.60 \\
7.91 \\
13.01 \\
18.2 ? \\
20.32 \\
26.017 \\
33.44 \\
37.74\end{array}$ & & $\begin{array}{r}2.60 \\
7.91 \\
13.01 \\
19.22 \\
20.82 \\
26.03 \\
33.84 \\
37.74\end{array}$ & $\begin{array}{l}3.41 \\
11.5 ! 1 \\
14.11 \\
16.89 \\
17.99 \\
27.55 \\
23.74 \\
28.49\end{array}$ \\
\hline$-m-n-m-1-n$ & 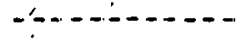 & $----\cdot$ & ----- & $\cdots-\cdots$ & ------ & ----- & $\cdots--\cdots$ & $n---n---n-n$ \\
\hline
\end{tabular}

DATA SOIJPCES : ILF INC. KEYSTONE STATE GEOLOGICAI. SIIRVFYS, IJ.S, GECLOGICAL 'SIJPVEYS,

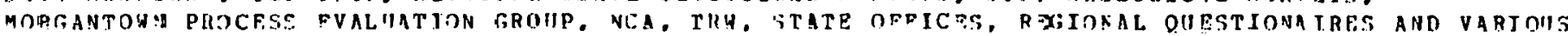

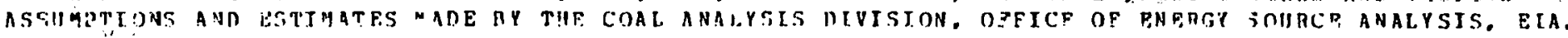

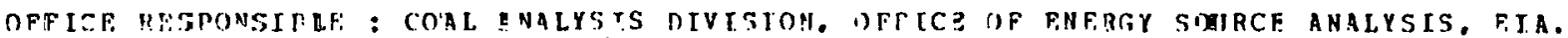




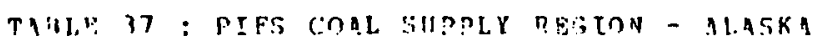

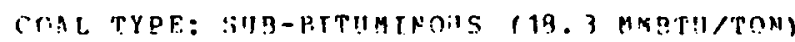

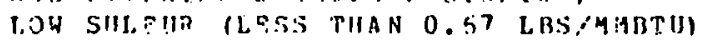

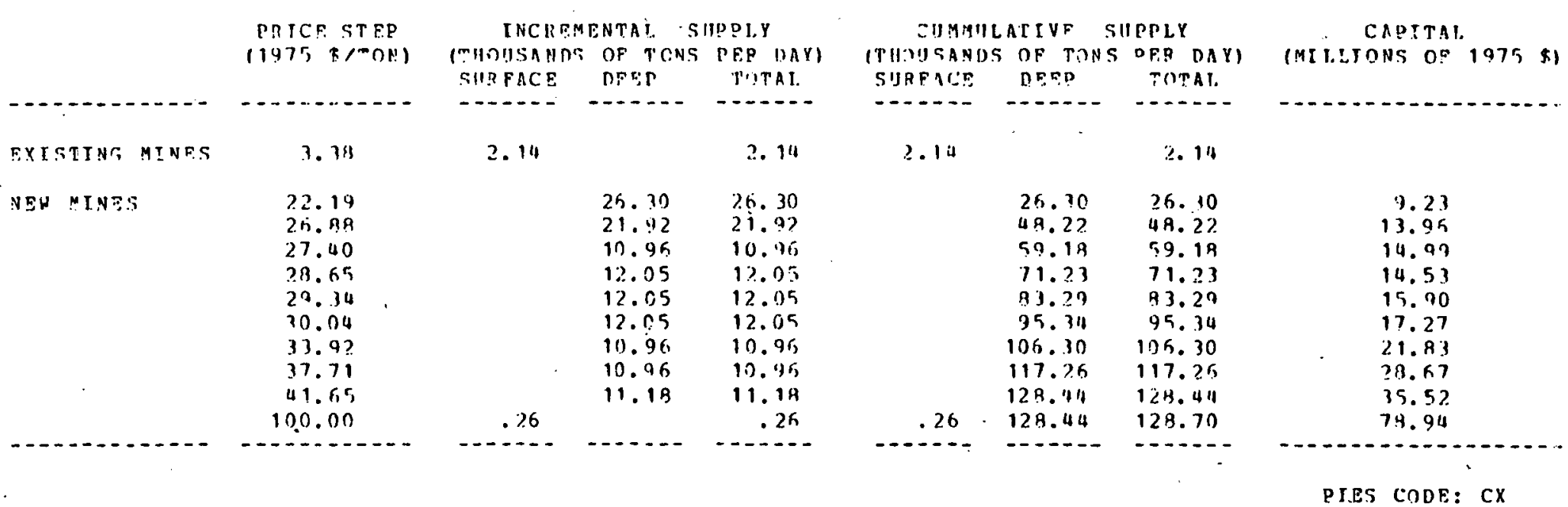

DATA SOIIRLFS : ICF TNC. . KFYSTONE STATF GEOLOGICAL SURVPYS, U.S. GEOLOSICAL SURVEYS.

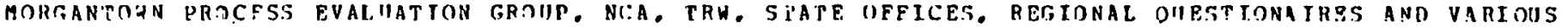

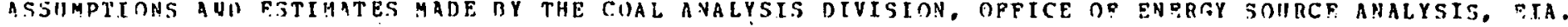
OPFICF. RESPONGIULE: COAL ANALYSTS UIVISION, OFFICP OF FNERGY SOURCE ANALYSIS, SIA. 


\begin{tabular}{|c|c|c|c|c|c|c|c|c|c|c|c|c|}
\hline PIES COAL PEGIONS & $\begin{array}{l}H I-U \Gamma I I \\
H I-S\end{array}$ & $\begin{array}{l}\text { YDSE-BT!! } \\
\text { HT-S }\end{array}$ & $\begin{array}{l}\text { HI-LT" } \\
\text { MPD-S }\end{array}$ & $\begin{array}{l}\text { YRD-BrT } \\
\text { TED-5 }\end{array}$ & $\begin{array}{l}\text { LO-BTII } \\
\text { MPD-S } \\
\text { THOISAADS }\end{array}$ & $\begin{array}{l}\text { VID-kro } \\
\text { neo-s } \\
\text { ne rons }\end{array}$ & $\begin{array}{l}\text { VHII-BT'II } \\
\text { I.O-S } \\
\text { S PER DIY }\end{array}$ & $\begin{array}{l}\text { HI - BTU } \\
10-5 \\
n\end{array}$ & $\begin{array}{l}\operatorname{mro}-82 ! I \\
\log 5\end{array}$ & $\begin{array}{l}\text { LO-BrU } \\
\text { LO-S }\end{array}$ & $\begin{array}{l}\text { VLO-ATU } \\
\text { LO-S } \\
\end{array}$ & $\begin{array}{l}\text { TOTAL ETIS } \\
\text { PMTLIOAS NF } \\
\text { BTU PRR DAYI }\end{array}$ \\
\hline $\begin{array}{l}\text { MOPTIIERN ARPALACIITA } \\
\text { CENTPAL MPPALACIIIA } \\
\text { SOIJHERN APPALACHIA } \\
\text { MIDHPST }\end{array}$ & $\begin{array}{r}1122.7 \\
63.5 \\
468.9\end{array}$ & 1327.7 & $\begin{array}{r}869.5 \\
314.3 \\
55.2 \\
291.6\end{array}$ & 130.4 & & & $\begin{array}{r}170.1 \\
659.0 \\
11.1\end{array}$ & $\begin{array}{r}5.1 \\
136.3\end{array}$ & & & & $\begin{array}{r}55311.0 \\
30029.4 \\
1615.5 \\
4986.7 .7\end{array}$ \\
\hline TOTAL EAST & 1655.0 & 1457.3 & $\begin{array}{r}1530.6 \\
-130.5\end{array}$ & 146.8 & & & 840.1 & $\begin{array}{r}141.4 \\
-. . .5\end{array}$ & & & & 1.36940 .6 \\
\hline $\begin{array}{l}\text { CENTHAL HEST } \\
\text { G"LF } \\
\text { Y.F. GREAT PLAINS } \\
\text { N.H. GRFAT PLAINS } \\
\text { ROCKIES } \\
\text { SOIIUHEST } \\
\text { NORTIINPST } \\
\text { ALASKA }\end{array}$ & 51.1 & $\cdot 199.2$ & 21.7 & $\begin{array}{r}264.9 \\
4.9 \\
179.0\end{array}$ & $\begin{array}{r}1528.2 . \\
47.8\end{array}$ & $\begin{array}{r}269.2 \\
1339.5\end{array}$ & 14.6 & 139.8 & $\begin{array}{r}302.5 \\
104.0 \\
75.1\end{array}$ & 13).9 & 93.0 & $\begin{array}{r}7269.0 \\
3747.1 \\
18622.2 \\
915.21 .0 \\
8141.1 \\
5579.8 \\
975.0 \\
2.398 .2\end{array}$ \\
\hline rOTAL WEST & $\begin{array}{r}51.1 \\
-.0 .\end{array}$ & 199.2 & 108.2 & 448.2 & 16.24 .5 & 1627.7 & 2.1 .2 & 139.8 & 481.6 & 2321.3 & 9.3 .0 & $1381: 4.3$ \\
\hline NATIOHAL TOTALS & $\begin{array}{r}1705.1 \\
-\end{array}$ & 1656.5 & $\begin{array}{r}1638.8 \\
-\end{array}$ & $\begin{array}{r}594 . ? \\
-5.2-\end{array}$ & $\begin{array}{r}1528.5 \\
-\end{array}$ & $\begin{array}{r}1627.7 \\
-\end{array}$ & $\begin{array}{r}863.5 \\
-\end{array}$ & $\begin{array}{r}2 A 1.2 \\
-\end{array}$ & $\begin{array}{r}481.6 \\
-\end{array}$ & $\begin{array}{r}2921.3 \\
-\end{array}$ & $\begin{array}{r}93.0 \\
- \\
-\end{array}$ & 274964.9 \\
\hline
\end{tabular}

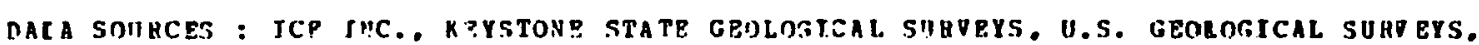

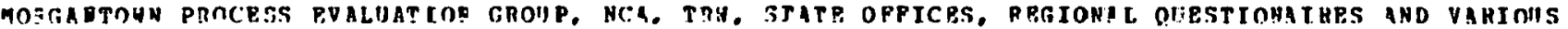

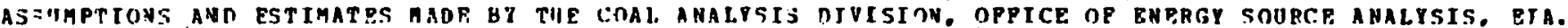

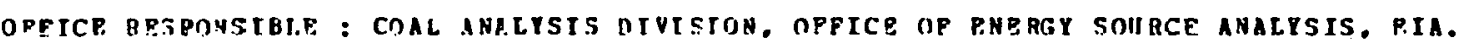

COAI. TYPE DESCIPTOES

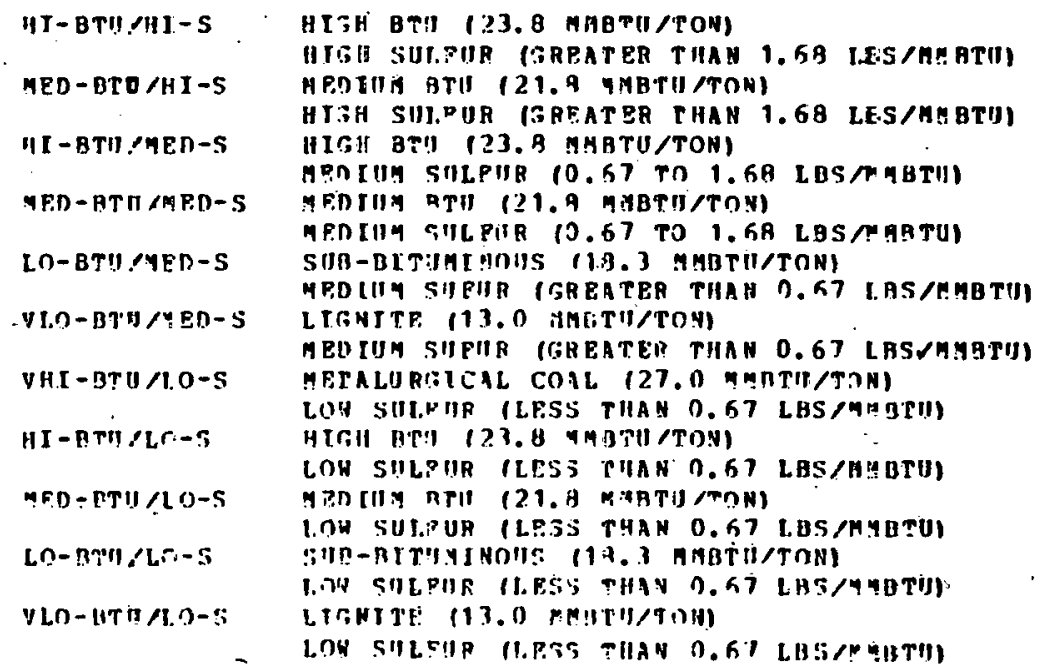


1990 LOAL SIPPLY DATA

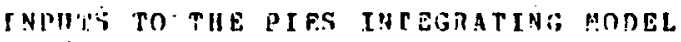
1978 ANNIAL ADMINTSTRATIOR'S FEPORT

GFHERALION DATE 12.1977 
DOAL GIIDUIY DATA

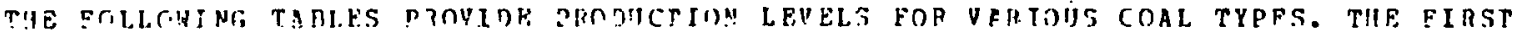

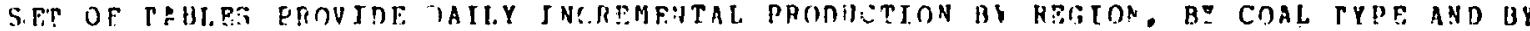

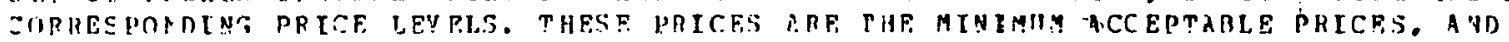

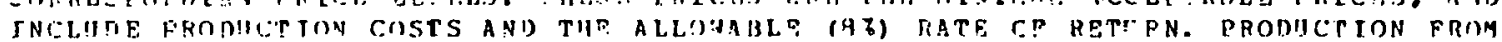

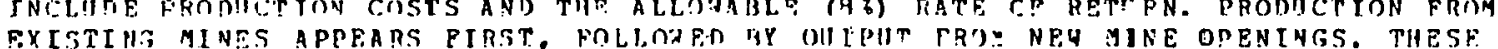

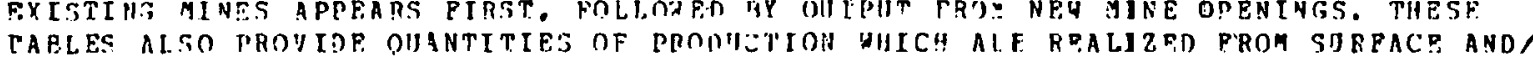

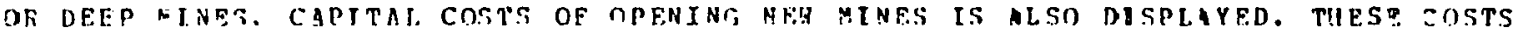
PEPRFSEN THF TOTAL CAPTTAT. EREENIITIRF. ASSOLIATED HITH VEU MTNES.

THF LAST TABLF PFOVIDES THE BFGIONM. AND MATIONAL PRCE!ICTIOR LFTEIS ASSUMTNG TIIE

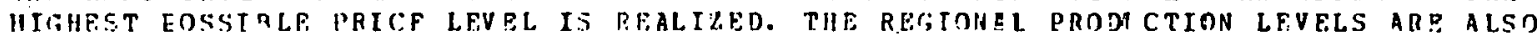
ArGReraten INTO FAST AUI WEST COAL RFGION TOTAL.S. 


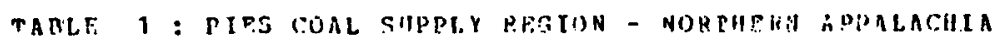

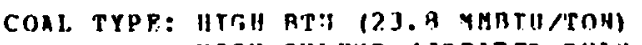

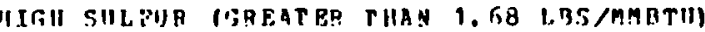

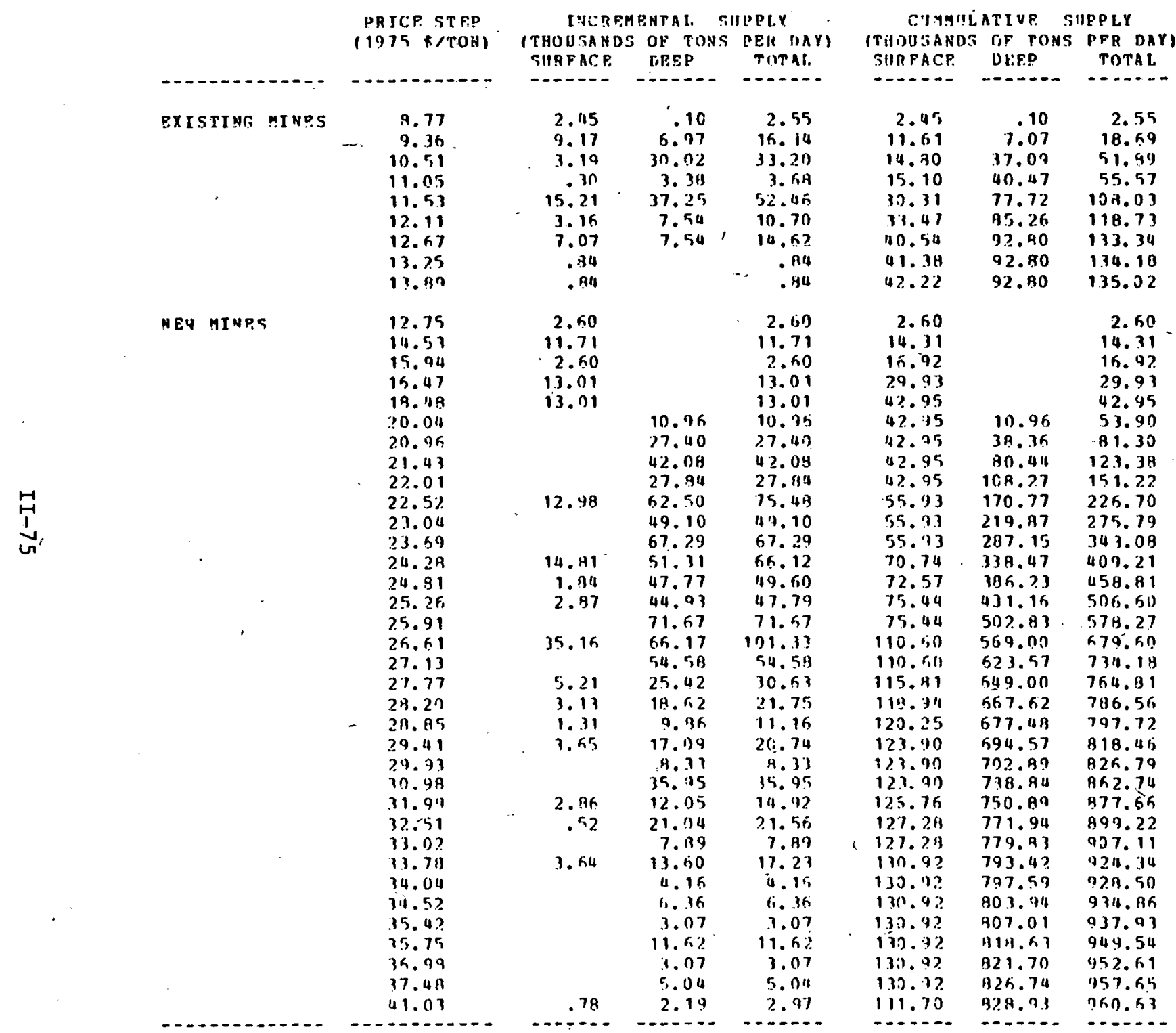

SATTAL.

IMILLINAS OP 1975 \$1

-

14.33
17.21
20.15
21.69
24.19
10.84
13.15
13.53
15.11
17.63
14.95
15.21
21.19
19.67
19.94
17.43
24.59
21.39
22.89
23.27
24.79
30.29
25.07
27.69
34.73
32.64
25.93
39.57
31.89
17.91
316.23
35.34
31.00
39.94
50.69
959

,

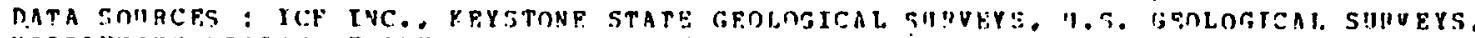

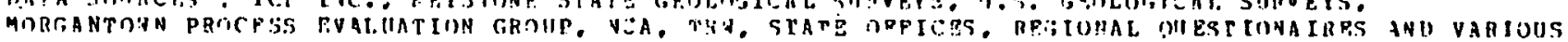

A SSIIMPTIONS AMD FSTIATES M IDP. BY TUP COAL ANMLYSTS DIVISION, OFPTCE OF ENERGY SOIIIER ANALYSTS. ELA.

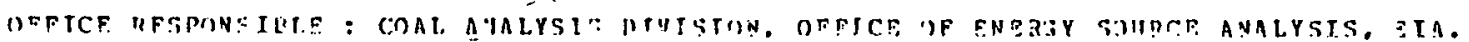




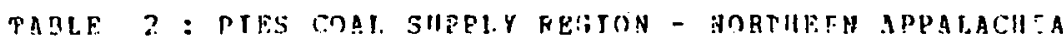

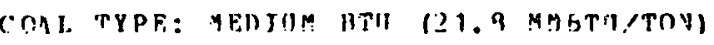

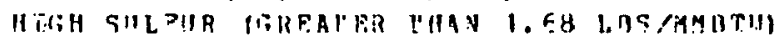

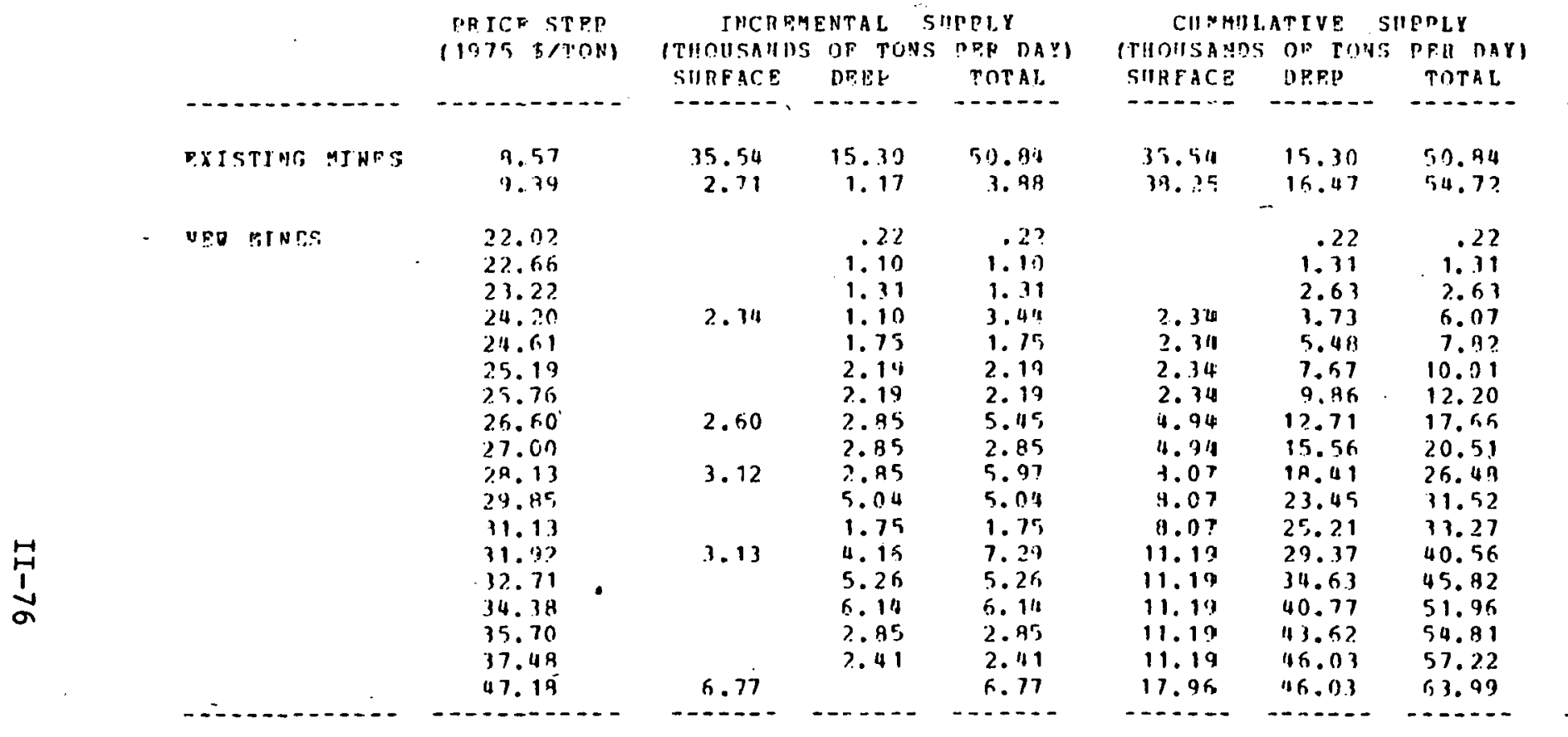

工APITAL

IMTLLRE: DE 1975 \$)

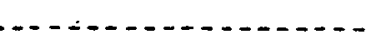

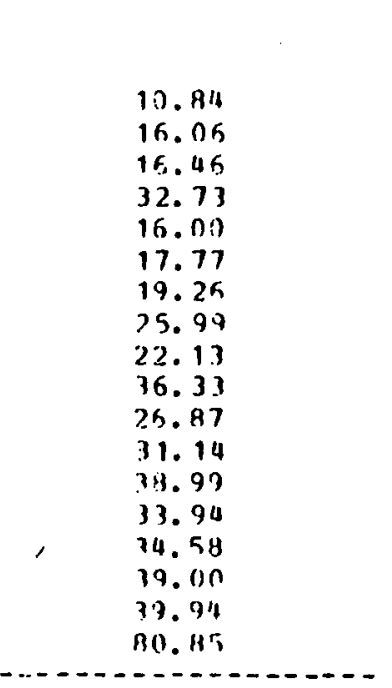

PIES CODE: CH

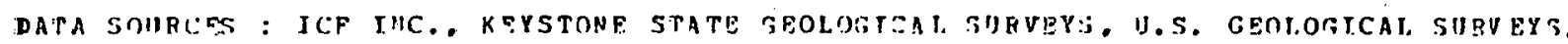

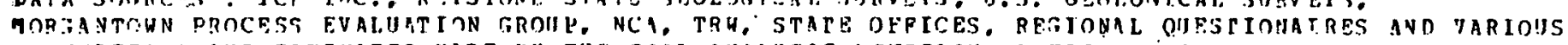

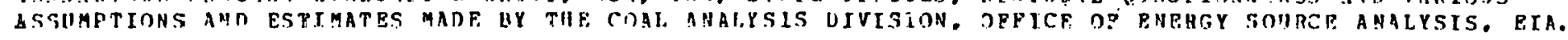

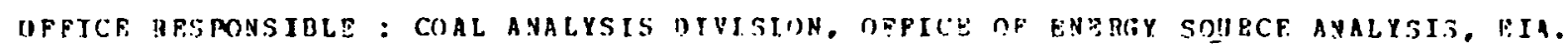




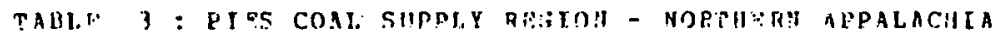

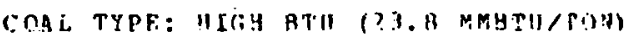

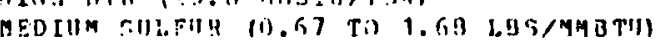

PRICE ST R.P $11975^{\circ} \$$ TON

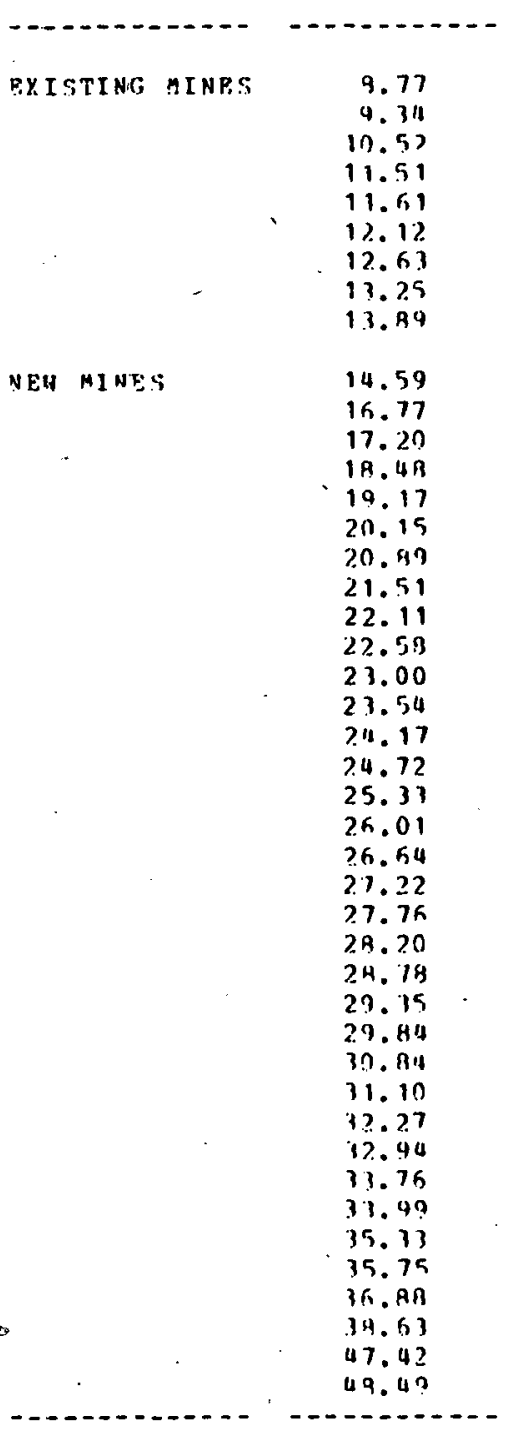

TNCPEMENTAT. SUIPI.Y THOUSANDS DF TONS PFF DAY SIIPFACE DFFP TOTAL

.55
.38
2.24
14.57
.35
2.98
19.60
3.58
3.59

.03
1.58
7.64
.14
1.60
9.42
1.69
1.69

1.30

1.51
2.31
1.30

1.30
2.50

1.30

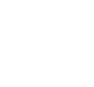

봄

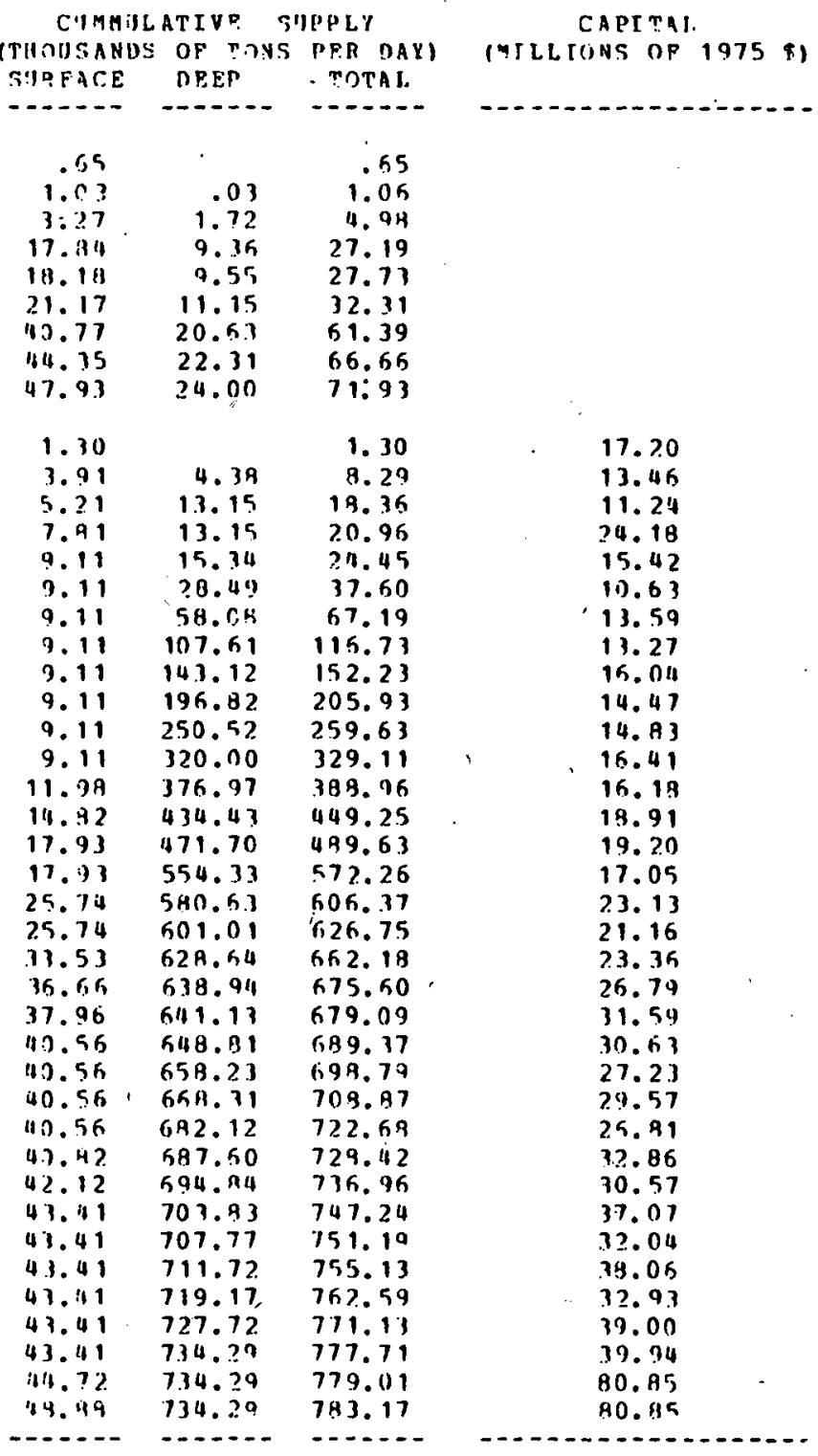

PIR:S COnz: CB

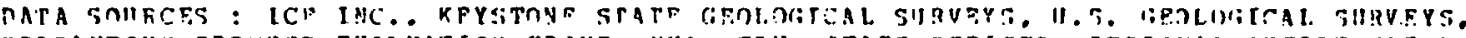

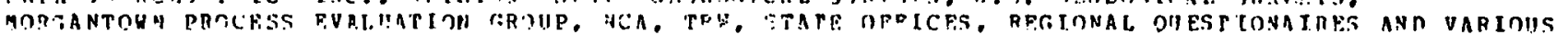

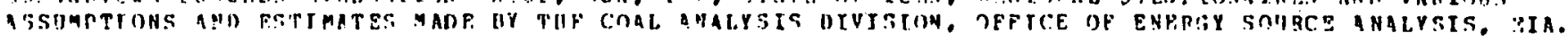

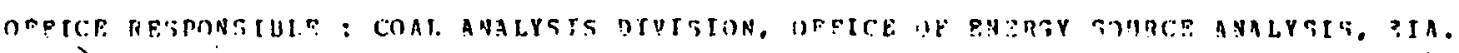




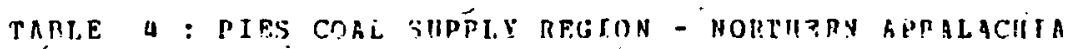

COAI. TYPE: MEDIUM BTII (?.1. B MARPU/TONI

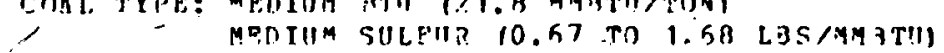

PRICF, STF.P $(1975 *$ TRN

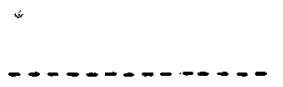

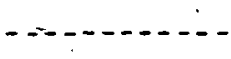

FYISTING MINTS

NEN MTNGS

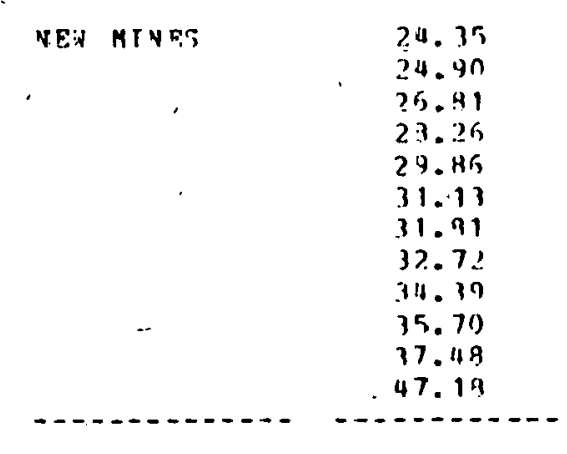

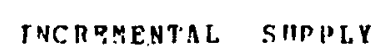
(THOIISANAS OE TONS PER DAY) GIIPA'ACF.
EMMILLATtVF SUDPLY (THOLSAHIS OP TONS PF, DAY) SUPFACP DREP (2.)-..c-....

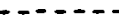
TOOAT.

\begin{tabular}{|c|c|c|}
\hline $\begin{array}{l}.40 \\
.07\end{array}$ & & $\begin{array}{l}.40 \\
.03\end{array}$ \\
\hline .26 & & .25 \\
\hline & .22 & .22 \\
\hline & 1. 10 & 1.10 \\
\hline .26 & .49 & 1.14 \\
\hline & $\begin{array}{r}2.63 \\
88\end{array}$ & 2.67 \\
\hline .26 & 1.53 & 1.71 \\
\hline & 2.41 & 2.41 \\
\hline & 2.19 & 2. 19 \\
\hline & 1.10 & $1.10)$ \\
\hline & 1.10 & 1. 10 \\
\hline & & \\
\hline
\end{tabular}

.10
.117
.25
.26
.25
.57
.52
.52
.73
.73
.73
.73
.73
1.32
.97

.70
.117 - . . . n TOTAI.

(MILIOSN OF 1975 क)

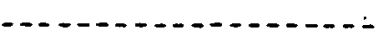

DATA SOURCES : ICF TNC. KFYSTONE STATE GEOLOGICAL SUIUEZS. I.S. GEOLOICAL SURVFYS

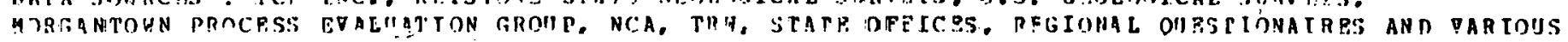

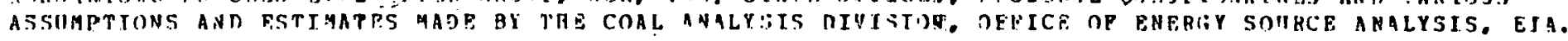

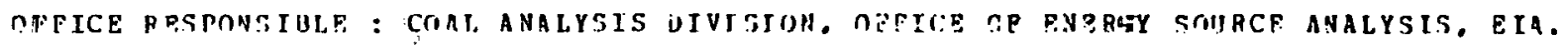




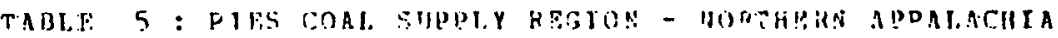

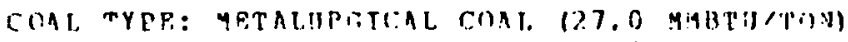

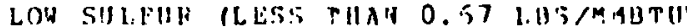

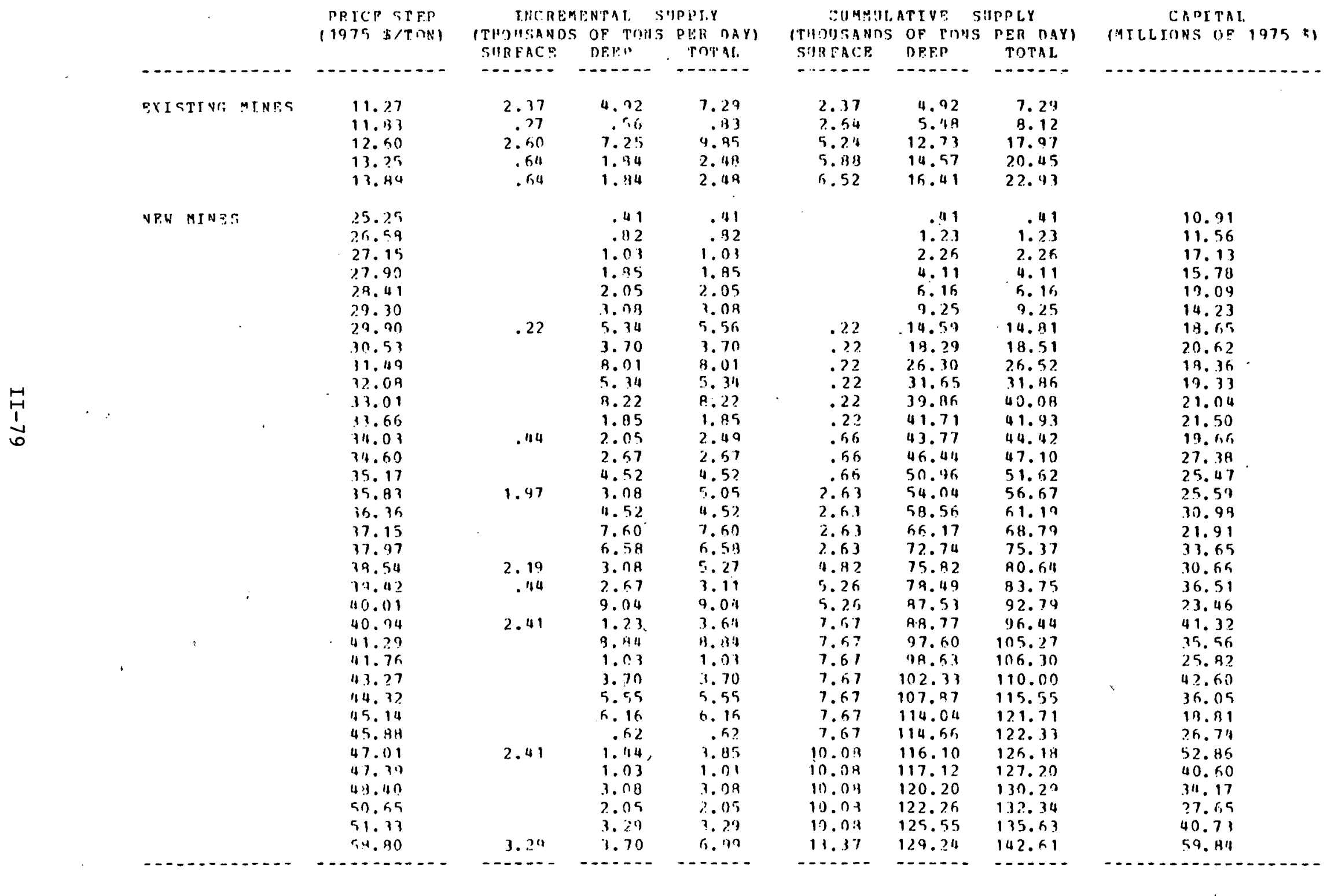

PRF; CODF: CM

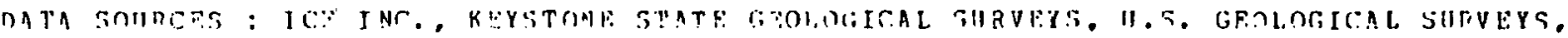

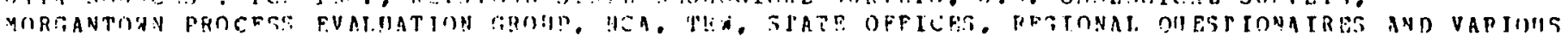

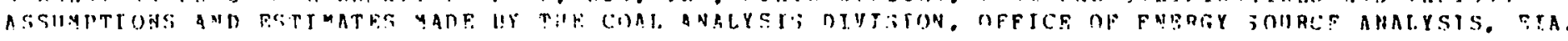

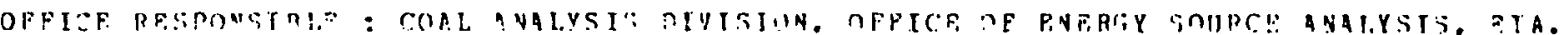




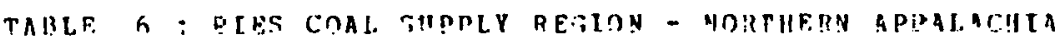

COMI. TUEE: HIGH BTU (23.? MIBSU,TON

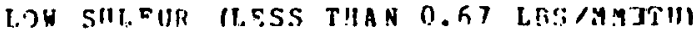

PRICE STFP

$11.375 \quad 3 / T \cap N$

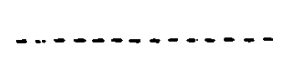

FrTSTINS HTNES $\quad 10.52$

11.05

11.57

MEY MINES:
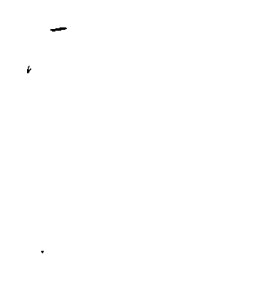

$2 ? .47$

24.27

25.59

27.65

$29.3 ?$

$31.0 \mathrm{~A}$

13.46

3.99

30.85

3.7 .03

49.09
INCERMENTAL. SIIPOLY

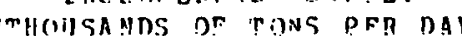
SHEFACE DFTP TNIALI. -.....

- - - - -

32
.014
.14

\begin{tabular}{rrr} 
& .22 & .22 \\
.26 & .22 & .22 \\
& .22 & .49 \\
.52 & .22 & .22 \\
.52 & .22 & $.5 ?$ \\
& .22 \\
& .22 & .52 \\
1.56 & .22 & .22 \\
\hline .0. &. .22 & .22 \\
\hline
\end{tabular}

CIIMHIILATIVE SIIPOLY (THOI)SANOS DP TR.NS OER DAYI SIIPPACE JEE? IOTAL

-.....-

- n...

-.-.-

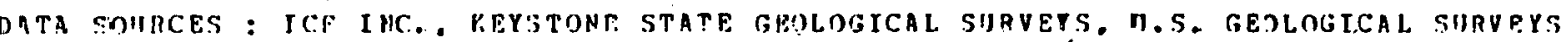

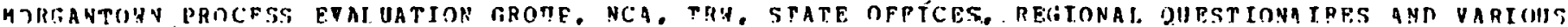

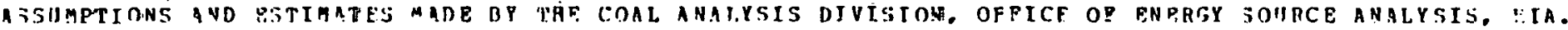

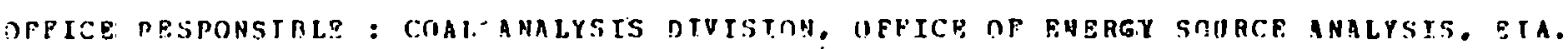




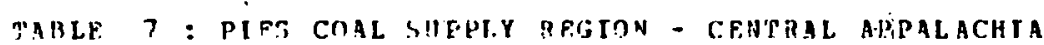

COAL. TYPF: HIGH ETH (23.9 M.UTH/TOH)

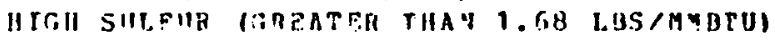

PPICE TTFP

11075 E $/$ RON

- - - - - - - - - -

ERISTING MINRT

9.32
10.19
10.96
11.65
15.17
16.11

NEY hINFC

14.51

\begin{tabular}{|c|c|c|}
\hline I NCRF,M & ENTAI. ! & IP ?.Y \\
\hline T THOUSANDS & OF TONS & PEO DAYI \\
\hline SIIRFACF. & DFF.P & TกTAI. \\
\hline$-\cdots,-\infty$ & $--\cdots-$ & - \\
\hline 2.13 & 2.15 & 4.4 .3 \\
\hline 1.99 & 7.111 & 9.11 \\
\hline .79 & .4 .17 & 4.77 \\
\hline & 19.02 & 19.132 \\
\hline & 2.00 & 2.00 \\
\hline & 2.00 & $2.8)$ \\
\hline 1.30 & & 1.30 \\
\hline .26 & & .25 \\
\hline .76 & & $.2 h$ \\
\hline & .22 & $.2 ?$ \\
\hline & 98 & $8^{3}$ \\
\hline $.5 ?$ & & .52 \\
\hline .52 & .22 & .74 \\
\hline & 89 & $\therefore A$ \\
\hline .52 & .65 & 1.14 \\
\hline .52 & .22 & .74 \\
\hline & 1.10 & 1. 10 \\
\hline & .144 & .144 \\
\hline .52. & .44 & .96 \\
\hline .52 & .44 &. . \\
\hline & .22 & .22 \\
\hline & .114 & .111 \\
\hline 1.56 & .22 & $\begin{array}{r}2 ? \\
156\end{array}$ \\
\hline & & \\
\hline
\end{tabular}

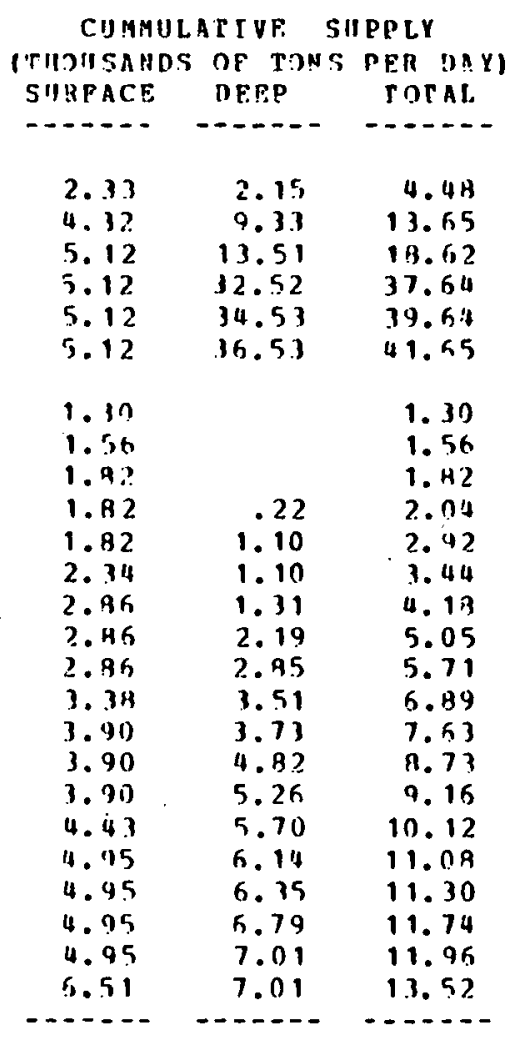

CAPITAL

NITLLLONG OF 1975 \&)

11.71
31.99
31.99
11.45
12.07
40.13
31.81
12.69
16.52
15.32
43.19
29.59
.38 .99
45.53
19.45
32.93
.39 .94
39.94
80.85
91.99

24. 16

2.1. 14

27.41

211. 31

2 i. 911

27.413

29.57
30.39

3?. 49

.13 .31

35.02

16.61

37. 26.

11.17

39.19

$40.3 ?$

$4 ? .14$
100.00

1.56

1.56
.--1.

PIES CODE: $\mathrm{CH}$

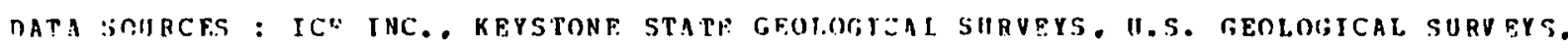

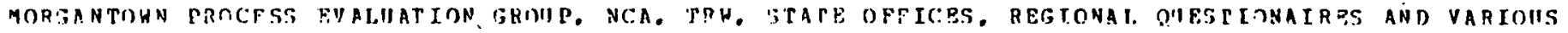

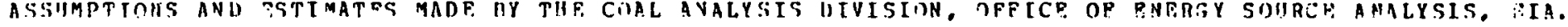

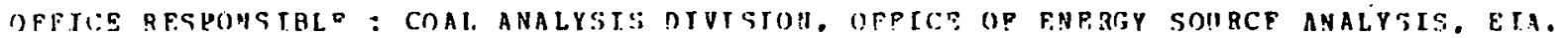




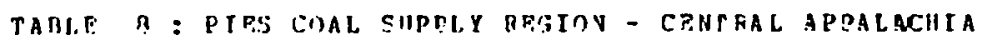

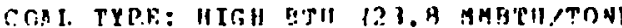

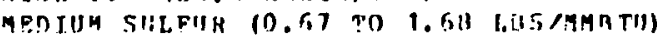

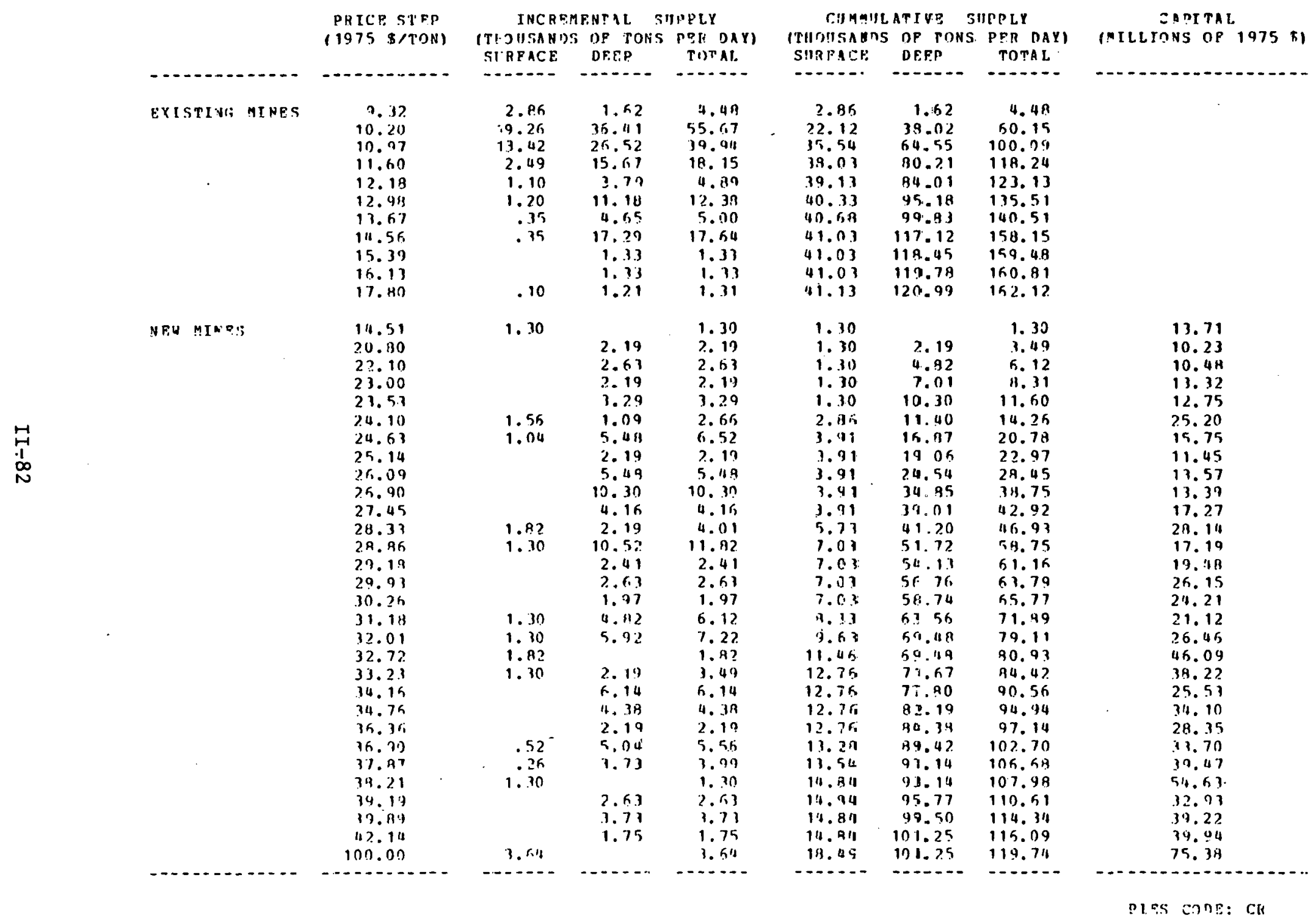

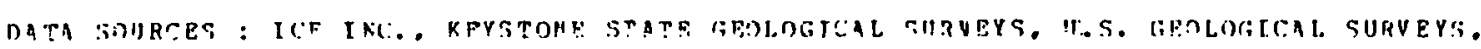

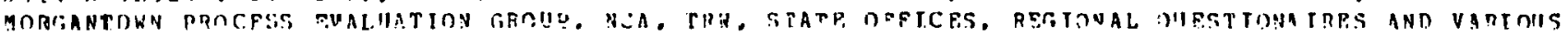

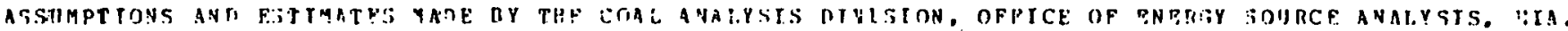

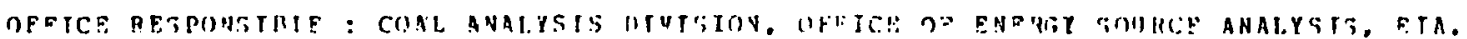




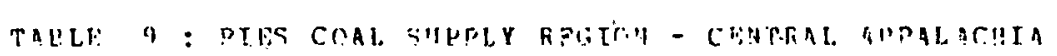

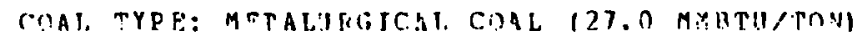

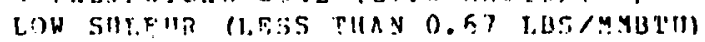

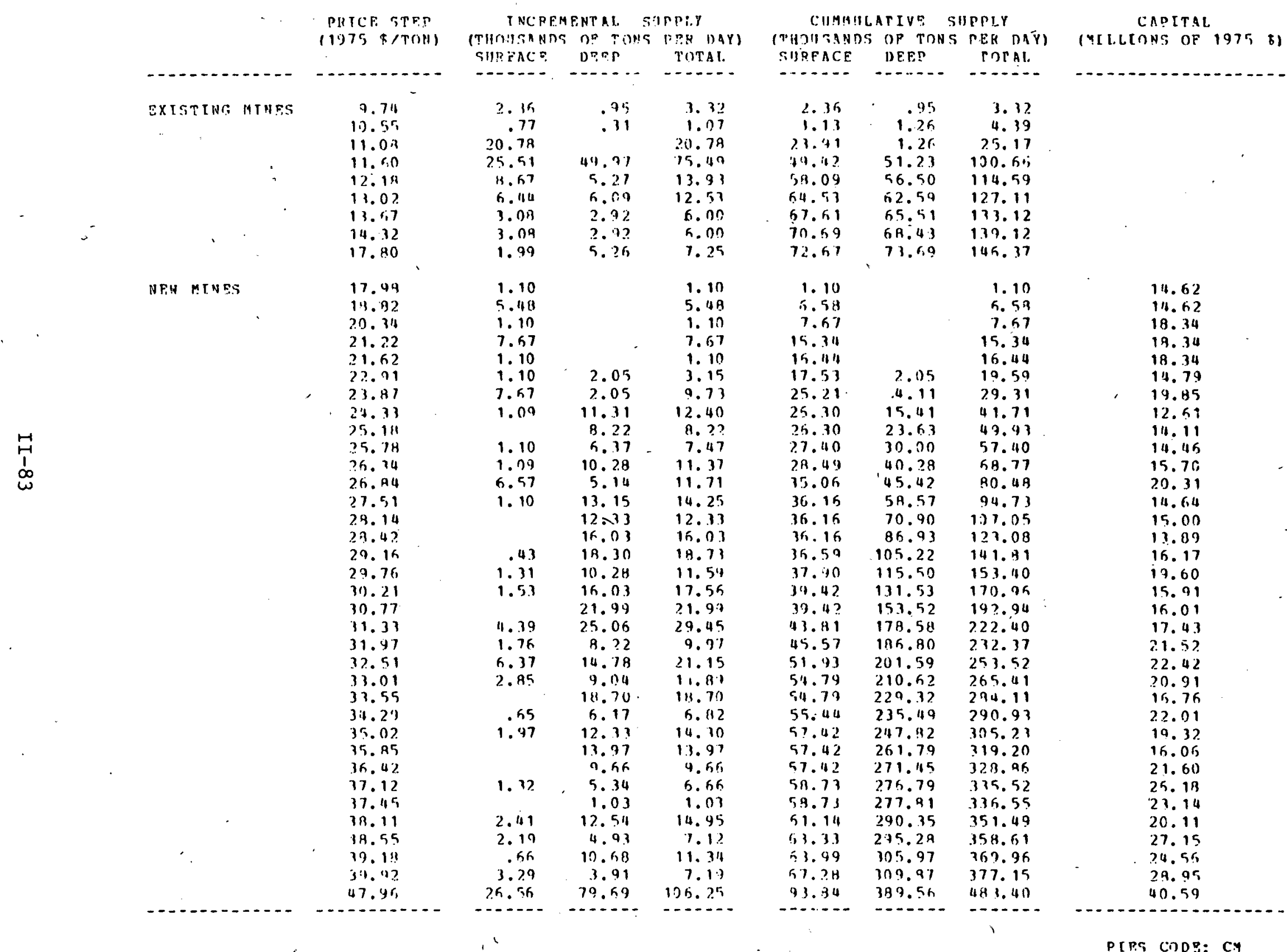

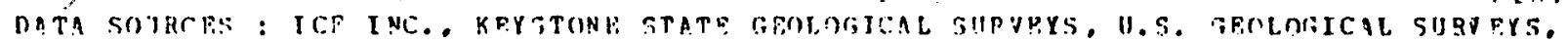

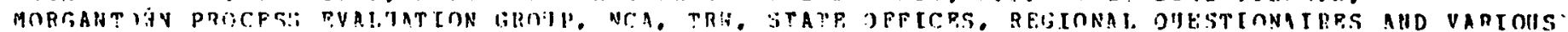

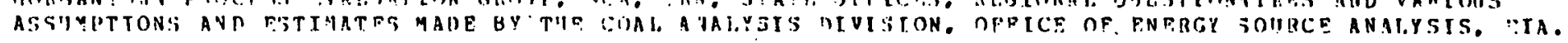

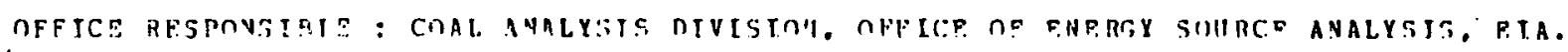




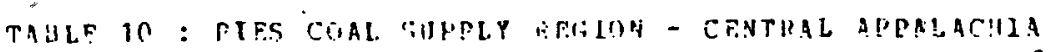

COAL TYPF: HISH BTH (2.]. A MHBTIITUN)

I.ON SUIFIIR ILESS THAN O.rT L.BSEMYFTIII

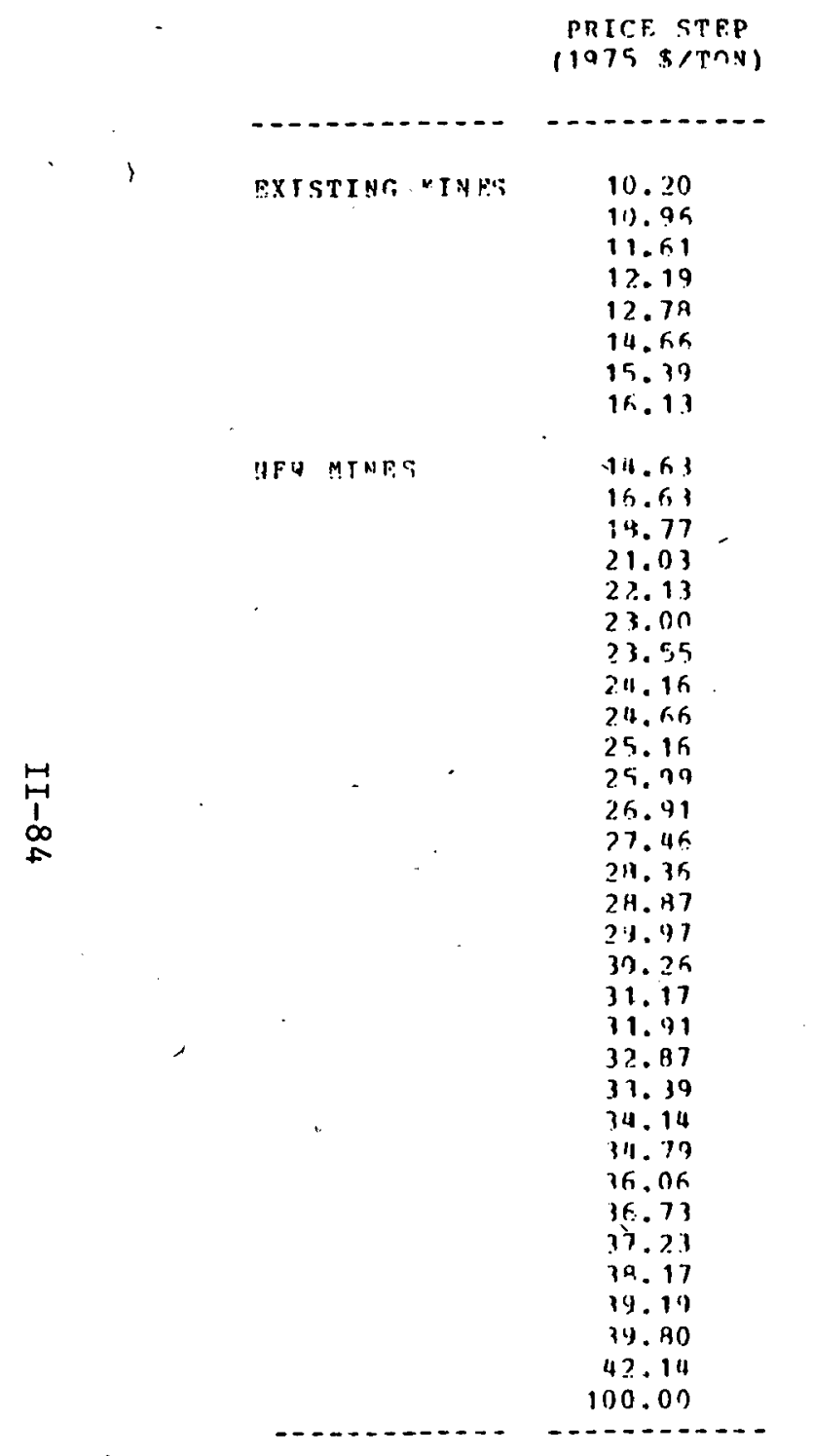

\begin{tabular}{|c|c|c|}
\hline I HC REM & FNTAL $\quad$ G & IPPLY \\
\hline הת אח THOU & OF & $D E R \quad D \cap Y)$ \\
\hline SURPACF. & DE.F.? & TOTA! \\
\hline
\end{tabular}

COMMILLATIVE SITPEY (THJUSANBS OE TONS PER DAY) SIS IIFACE $0 \geq$ P.P TOPAL

8.92
5.48
1.89
.90
.90
2.51
.26
.26

$\begin{array}{rr} & 0.52 \\ .74-\quad 5.48 \\ .15 & 2.52 \\ .35 & 1.26 \\ 1.26 \\ 2.51 \\ .26 \\ \end{array}$

2. 60

2.60

¿. 50

2.50

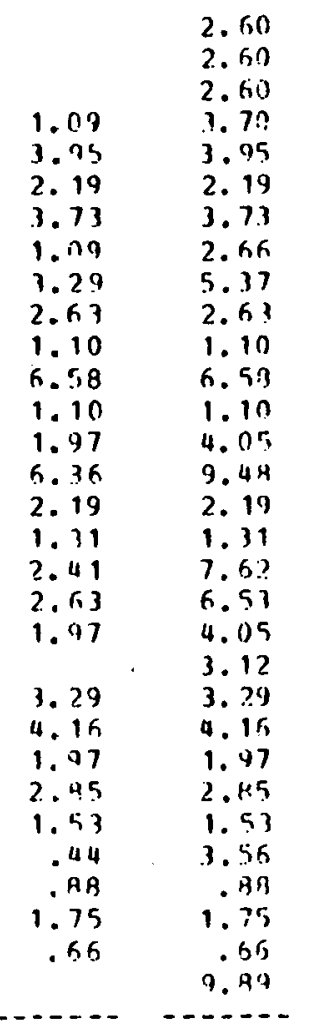

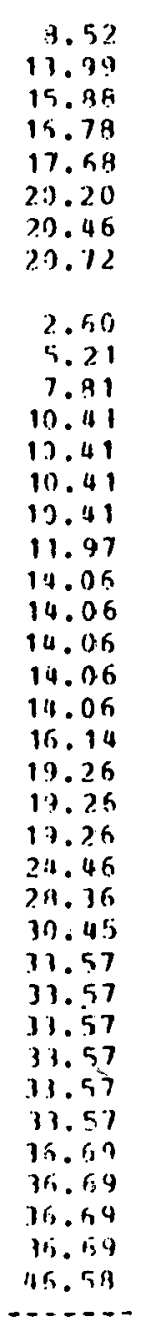
- n...-

-......

$$
\text { 8. } 52
$$$$
13.99
$$$$
.740 .16 .62
$$$$
1.09 \quad 17.87
$$$$
1.45 .14 .13
$$$$
1.45 \quad 21.54
$$$$
1.45 \quad 21.91
$$$$
1.45 \quad 22.17
$$

20

2. 50

$$
\begin{array}{r}
5.21 \\
7.81
\end{array}
$$

17.64

$\begin{array}{ll}10.95 & 21.37 \\ 12.05 & 2.4 .03\end{array}$

$15.34 \quad 29.40$

$17.97 \quad 32.03$

$19.06 \quad 31.12$

$2.5 .64 \quad 39.70$

$26.74 \quad 40.79$

$\begin{array}{ll}26.74 & 40.79 \\ 28.71 & 44.85\end{array}$

$\begin{array}{ll}28.71 & 44.85 \\ 35.07 & 54.37\end{array}$

$37.26 \quad 56.52$

38.5757 .03

$40.96 \quad 65.45$

$4.52 \quad 71.99$
$4.59 \quad 76.04$

$45.59-76.04$

4.5976 .04

45.59 .79 .16
$+8.8 E .42 .45$

$53.04 \quad 46.61$

$55.02 \quad 83.59$

$57.37 \quad 91.44$

$79.40 \quad 92.97$

$59.84 \quad 76.53$

ร0.7 97.41

52.14794 .16

53.1199 .82

เ. 1.1109 .71

10.....$$
1.09 \quad 11.51
$$

$5.04 \quad 15.45$

CAPITAL

IMTLLIOHS OP 1075 \$)

-

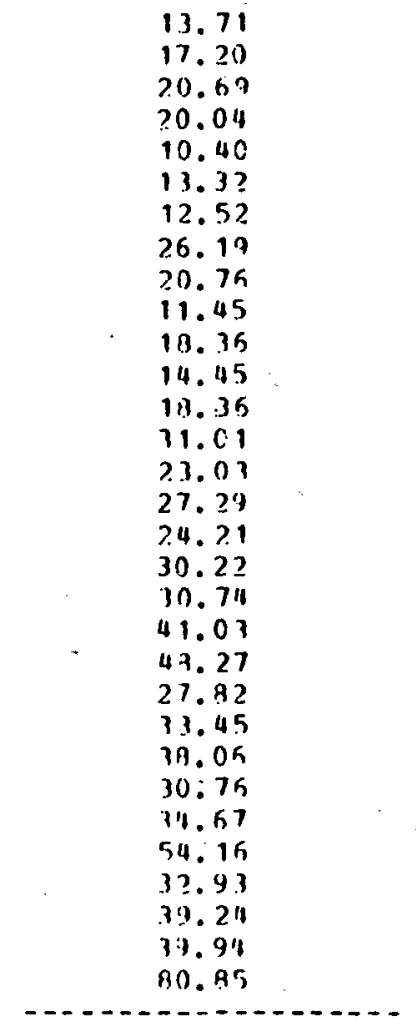

PIES CODP: CI

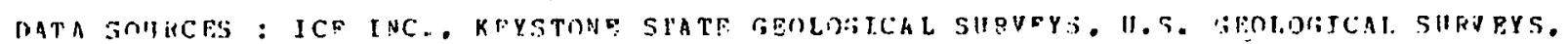

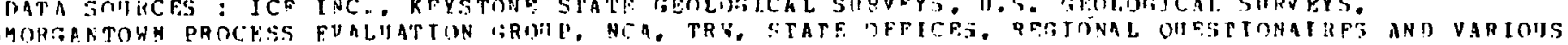

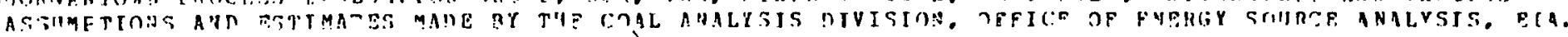

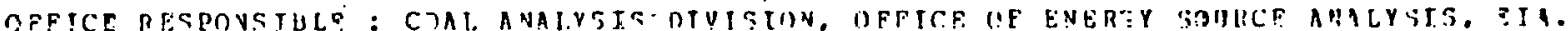




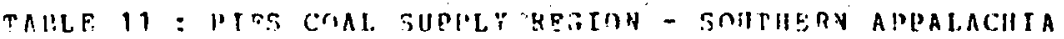

(OAL TYPF: HLAU UTH (2.3. H HMBTH/TON)

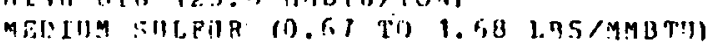

PRICE ST FD $(1375$ h/TON)

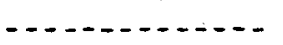

F.Y. ISTING: MTNFS

NE! MERES

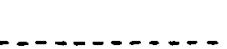

$$
\begin{aligned}
& 12.74 \\
& 13.74 \\
& 10.02 \\
& 14.54 \\
& 15.21
\end{aligned}
$$

28,31

33.55

3 कि. 32

39.30
INCREMFHTAL SIIPPI.Y (? HOUISANTS CF TOMS PFII DAY SIREAC AC SIRTACE

$$
\text { DFin }
$$

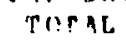

$$
\begin{array}{r}
17.51 \\
4.92 \\
1.05 \\
.23
\end{array}
$$$$
.2 .1
$$

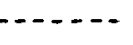

$$
10.55
$$$$
\text { 2. } 6
$$$$
.5 .3
$$$$
.12
$$

24.06

$$
\begin{array}{r}
28.05 \\
7.55 \\
1.68 \\
.35
\end{array}
$$$$
.15
$$

1.. 19

1.97
4. 33
1.37
1.91

1. 3.1
C!I MHILATIVR. SIIPCLY

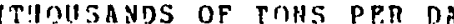
SIIRFACE DEEP TOTAI. SIIRFAC:

17.51
23.43
23.49
23.71
23.95

2.8. 05

35.61

37.29

$14.05 \quad 34.00$

\begin{tabular}{rr}
4.31 & 4.39 \\
6.35 & 6.36 \\
9.31 & 8.31 \\
9.64 & 9.64 \\
\hline
\end{tabular}

EAPITAL

(MTILLIONS OF 1075 क)

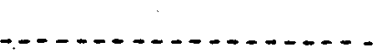

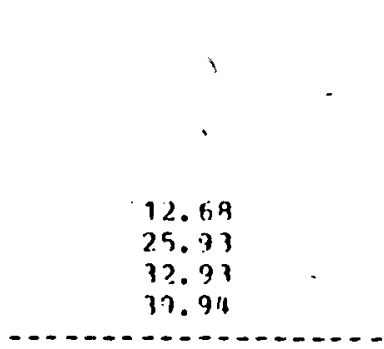

DEES CONE: CR

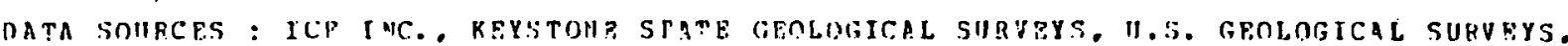

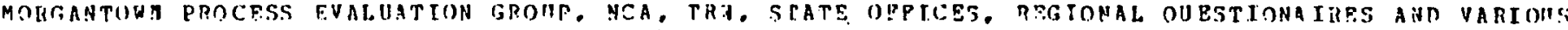

ASSIIMPTIODS AMD FSTJMATES MIDE BY TIIE COAL ANALYSTS NIVTSION, DEFICF OP ENERTY ;OURCE ANALYSIS. PIA.

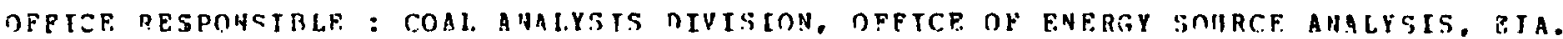


TAGIF 12 : PIES COAL SHPPLT REGJOH - SOITIIERN APQALACHEA

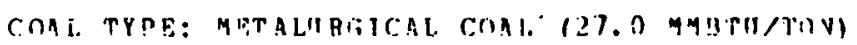

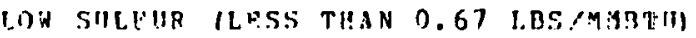

PQTCE STPR

$(1775 \pi / T O N)$

\begin{tabular}{|c|c|}
\hline PXISTINAT MINF: & $\begin{array}{l}13.95 \\
11.94 \\
15.23\end{array}$ \\
\hline NPH MINFG, & $\begin{array}{l}28.33 \\
33.55 \\
36.12 \\
33.30 \\
46.5 ? \\
52.09 \\
55.00\end{array}$ \\
\hline
\end{tabular}

IVCRF.MEHTAT. SIIPPLI (TIOUISANS OF TAMS DYG DAY SIIEFAC DEEP TOTAT. - . - . .

- - - -

$$
\begin{array}{r}
2.79 \\
.17 \\
.17
\end{array}
$$$$
.17
$$

$1.9 \%$

1.75

1.75

1. 31

. 21

221

01
1. 97

1. 75

1.75

1. 31

. 31

.21
.21

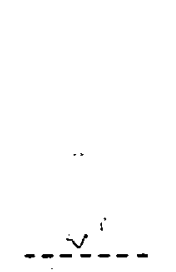

CIIMMILLATIVE SIPPT.Y (THIISAND' OF TONS PFB DAYI SURFICE DFF.P

2.78 .

$$
\therefore .94
$$$$
1.11
$$

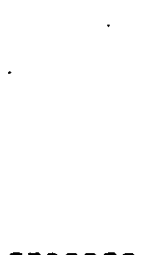

9.97
3.73
5.41
6.74
7.00
7.21
7.111

CADITAL

(MILLIONS OF 1975 \$1 TOTAL

2.78
2.911
3.11
1.97
3.73
5.49
6.79
1.00
7.21
7.41

$\begin{array}{r}12.63 \\ 25.93 \\ 12.93 \\ 39.94 \\ 13.52 \\ 27.65 \\ 35.13 \\ \ldots . .13 \\ \hline\end{array}$

PIES CODE: CM

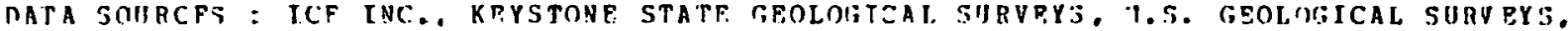

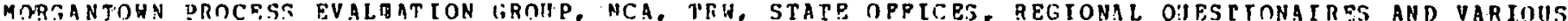

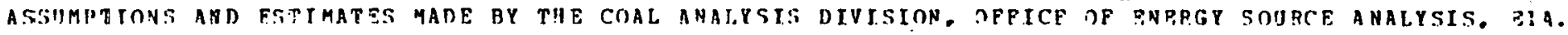

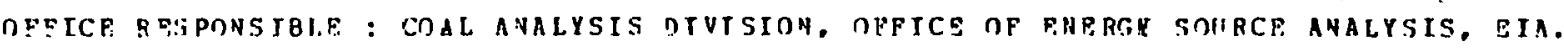


TABT.F 13 : PIES COAL SHPPIYY RPIOIOH - MIOIEST

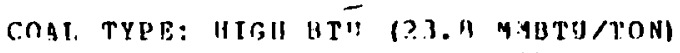

IIGII SIITFIR (EREATER THAH 1. GR LBS/MMATU)

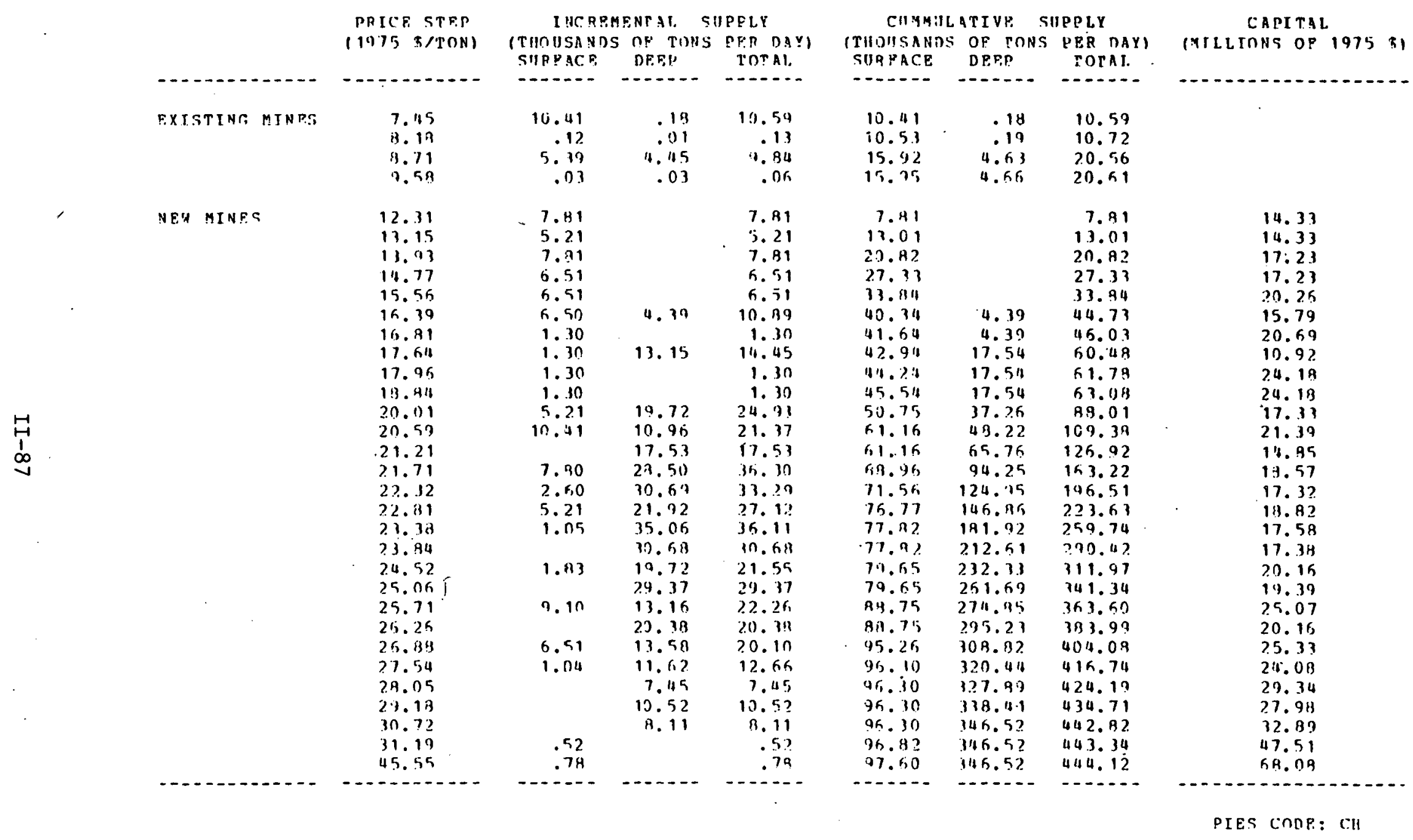

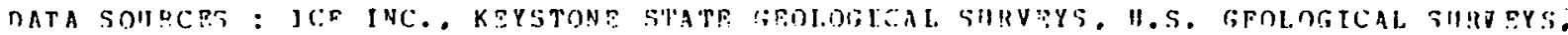

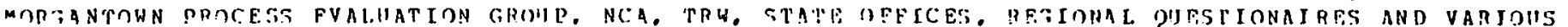

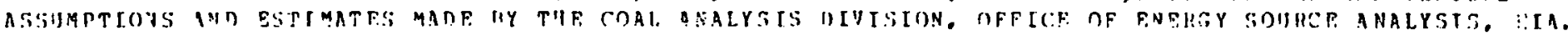

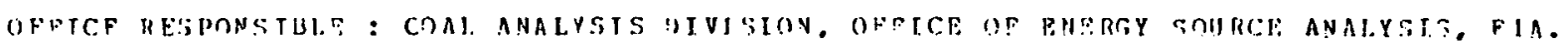


TARI.E 1I : PIPS COAL THPHIY PBRION - MLUWEST

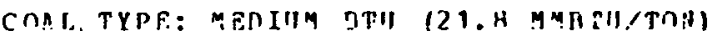

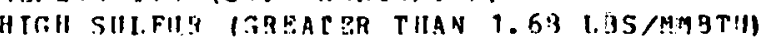

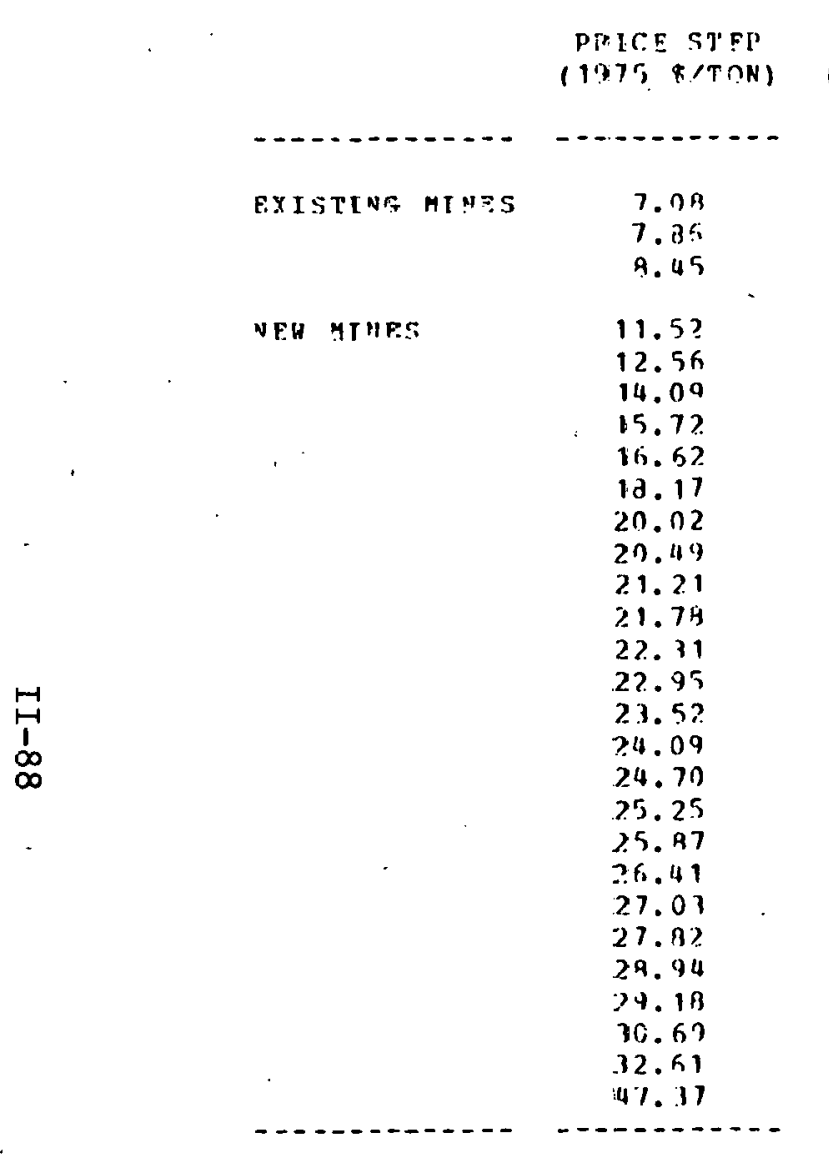

\begin{abstract}
INCREMEHTAI. SHPDLY (TUOUSANDS (IF TUYS PEF DAY) IIIPFACF DFPO Drep ():AL
\end{abstract}

7.09 40.03 $40.56:$
46.117 116.51

5.21

5.21
20.92 20.92 22.12

11.12

26.04

131.51$$
.53
$$$$
37.02
$$$$
.97
$$

5. 21

20. 42

37.31

2.2. 12

26.04

113.

52.60
115.16

131.51
14.3 ?

$14.3 \%$
40.00

$-$

52.60

99.73

116.16

31.25

131.49

99.71

2.62

28.49
64.64

162.74

2月. 4 ?

67. 26

2i. 49

$1.0 ?$

2.49

62.49

63.51

39.67

3.91

15.34

37.67
11.25

2.60

6. $3 \hat{n}$

3.29
-7.45

6. 35

5. 89

7.45

5.04

5. 0 2

3. 29

11.62

11.62

1.04

1.011

1.10
115.02

115.46

:E! YMIII.ATTVD TIIPPLY FIIOIJAAN): OF TONS PF.R DAY SJYFACP. DPE? TOTKL

5.31

25.03

5.1 .35

75.48

75.14

17.79

115.93

115.83

115.13

115.83

147.05

147.09

141.70

153.72

150. 7 ?

154.63

154.63

157.23

157.2 .3

1513.27

158.27

15.3 .27

159.31

160.51

\begin{tabular}{|c|c|}
\hline $\begin{array}{l}40.56 \\
87.55 \\
98.09\end{array}$ & $\begin{array}{l}116.54 \\
207.57 \\
204.53\end{array}$ \\
\hline & $\begin{array}{r}5.21 \\
26.03 \\
53.36 \\
75.48\end{array}$ \\
\hline 131.51 & 205.99 \\
\hline $\begin{array}{r}131.51 \\
245.47\end{array}$ & $\begin{array}{l}2.21 .30 \\
361.30\end{array}$ \\
\hline 298.07 & 41.3 .90 \\
\hline $\begin{array}{l}14.23 \\
513.36\end{array}$ & $\begin{array}{l}510.07 \\
679.79\end{array}$ \\
\hline $\begin{array}{r}35.45 \\
34.45\end{array}$ & 792.53 \\
\hline 573.95 & 821.03 \\
\hline 838.59 & คคค. 29 \\
\hline $\begin{array}{l}767.08 \\
729.57\end{array}$ & $\begin{array}{l}916.78 \\
990.29\end{array}$ \\
\hline 369.24 & 1019.96 \\
\hline 984.58 & 1037.20 \\
\hline 790.93 & 1045.55 \\
\hline 1994.22 & 1251.45 \\
\hline 901.57 & $105 \mathrm{H} .90$ \\
\hline 705.71 & 1954.99 \\
\hline 710.00 & 1068.27 \\
\hline 921.52 & 1079.89 \\
\hline 921.52 & $10: 0.93$ \\
\hline 921.52 & $13 \times 2.23$ \\
\hline
\end{tabular}

CAPITAI.

(MILLIONS OF 1975 ז)

-... . . . . . . . . . .

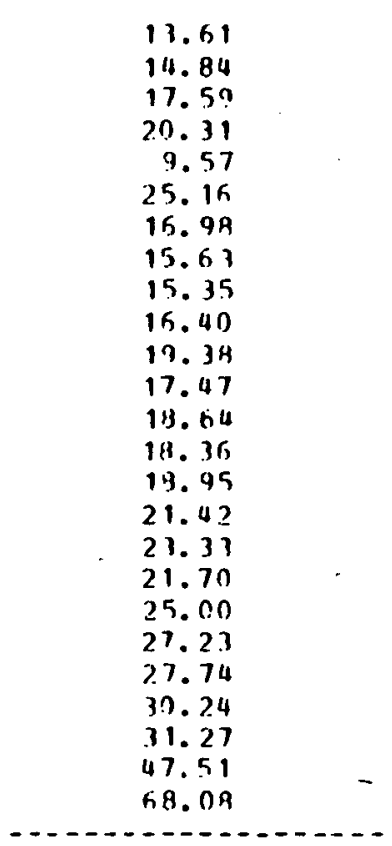

PISS CODE: CH

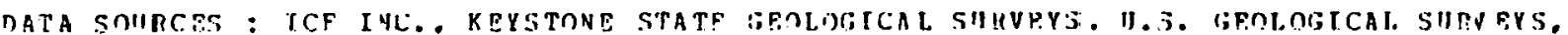

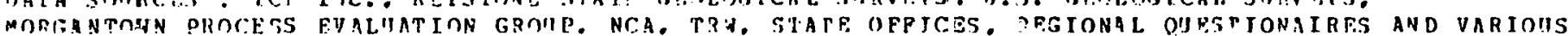

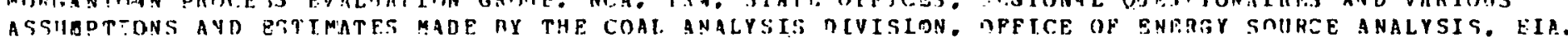

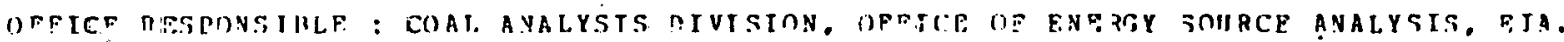




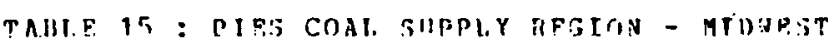

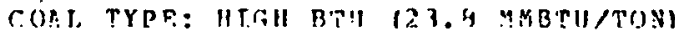

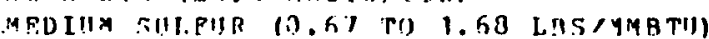

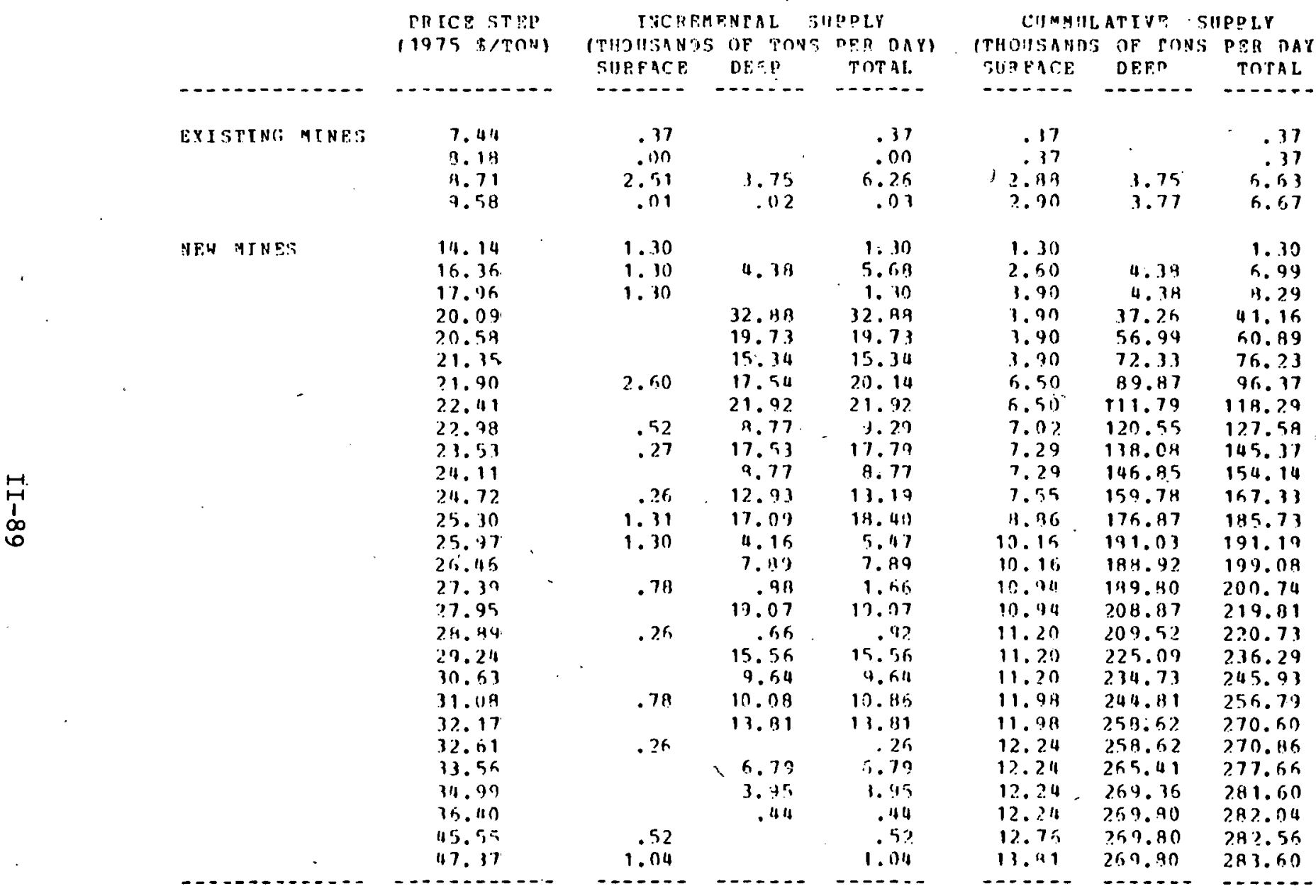

- CADTTAL

MILLIONS OF 1975 RI

- - - - - - - - - - - - - - -

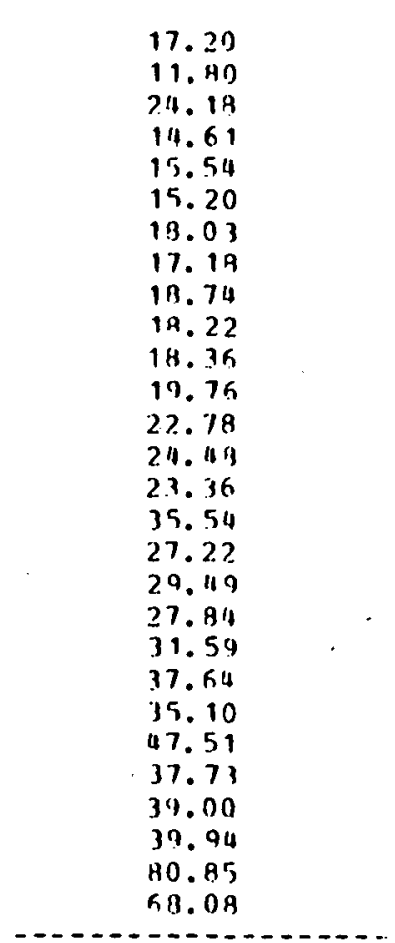

PIFS CODE: CR

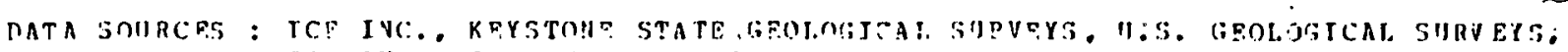

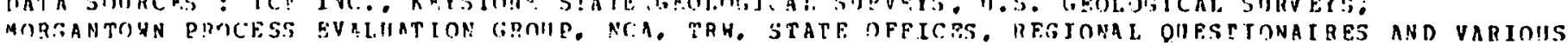

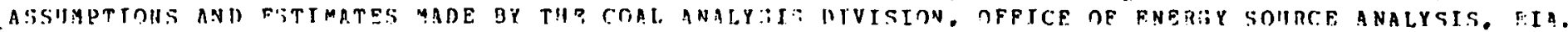

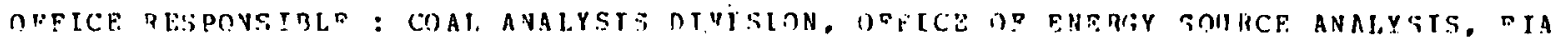




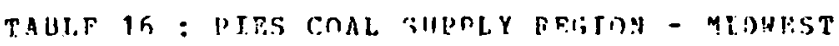

COA. TYPF: YEDIUM ? III (21.9 MABTI/TOY)

4EDTUM SUTEUR (O.G) TO 1.5U LBS/MMTTII)

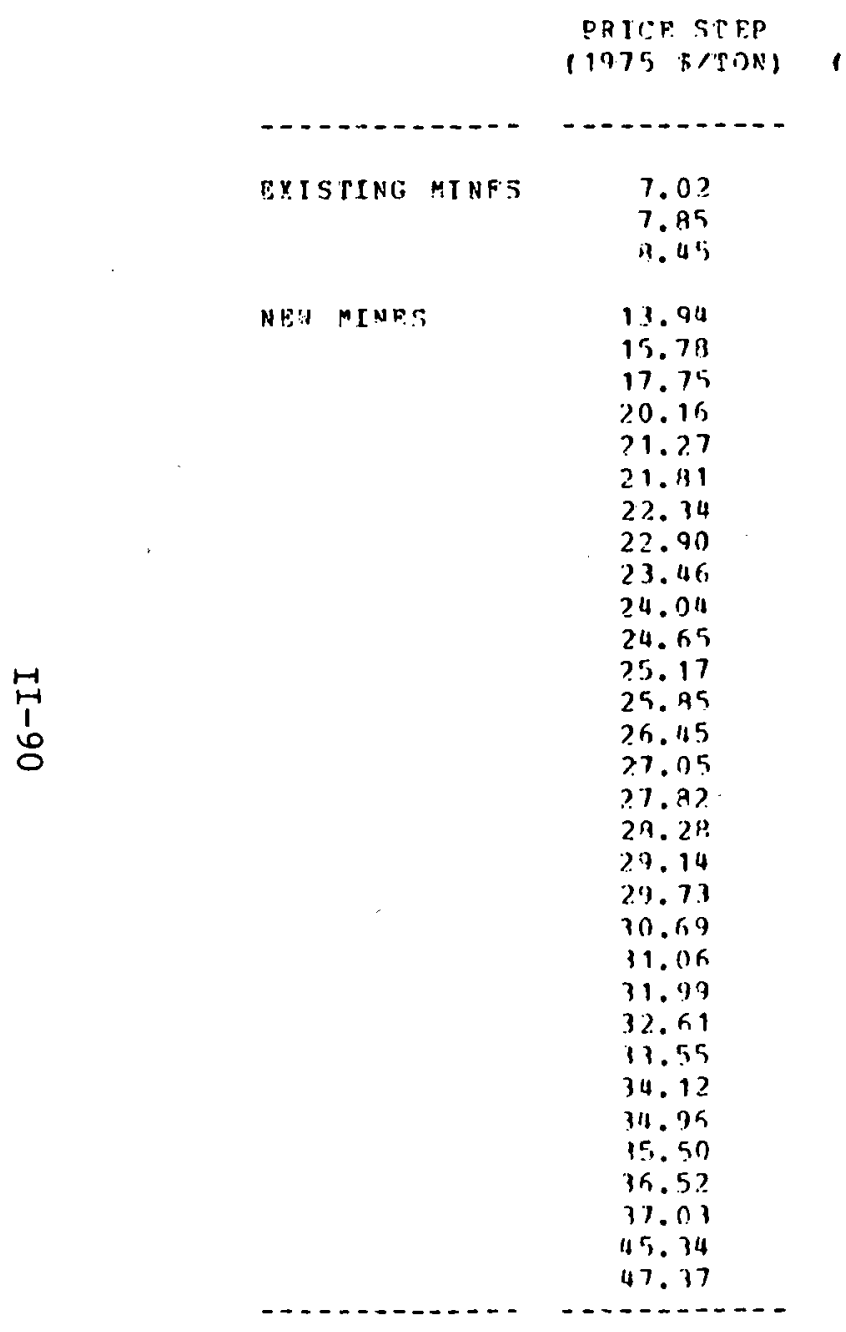

INC TFHFNTAL FIPSLY

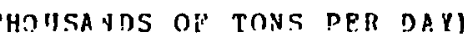
BURFACE DER? TOTAL

\subsection{4
.77 \\ .77}

1.30

1.30

1. 30

2.50

1. 30

.7 A

2.60

3. 90

.78

1.30

1. 30

.78

$$
.78
$$$$
\begin{array}{r}
2.34 \\
.25
\end{array}
$$

CIIMNIL ATIVF. SIIPPI.Y S PER DAY SIIPEACE DERP TOTAL

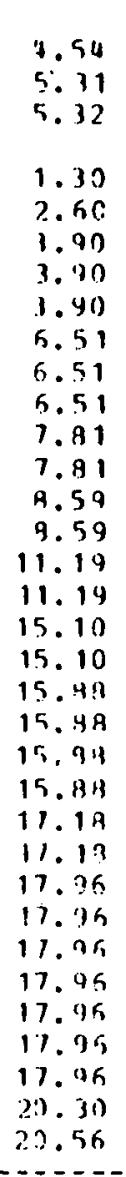

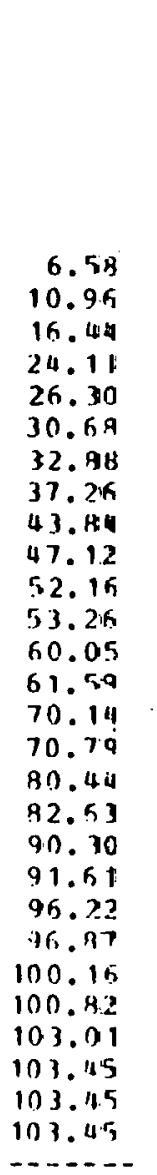

4. 54

5.31

\section{30}

2., 60

1. 9 ?

$10.4 \mathrm{~A}$

11.86

2.94

30.62

12.01
79.49

40.58

45.95

52.42

58.31

63.36

68.35

75.15

77.46

Bh. 01

BG. 57

96.11

99.91

107.118

1.) 1.57

114.19

114.8 .3

118,12

118.12

113.78

12.9 .97

121.41

123.75

124.01

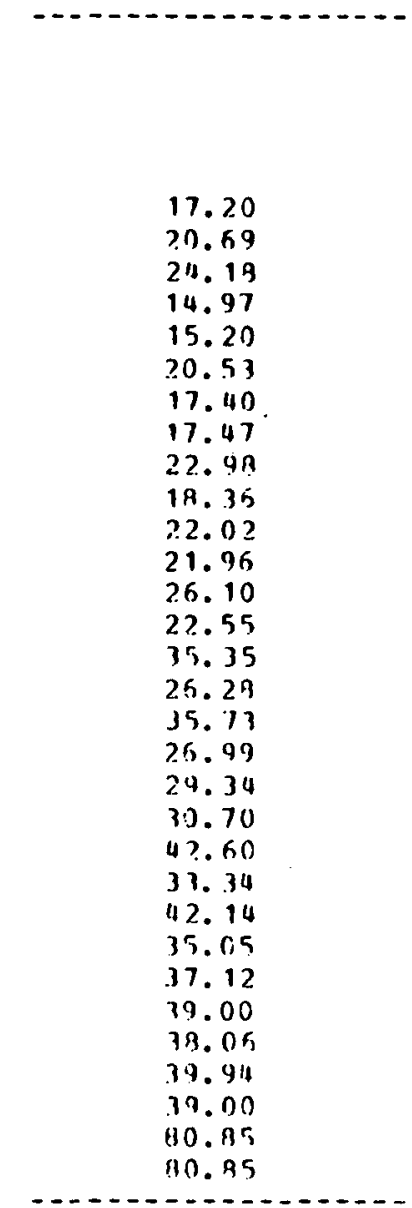

CAPT TAL

(MILI.IONS-OF 1975 \$)

PIFS CODP: CS

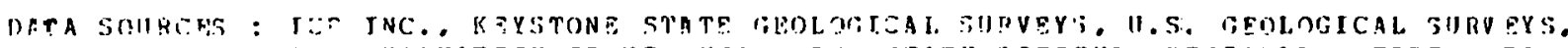

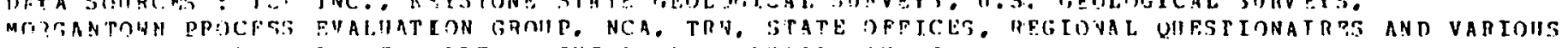

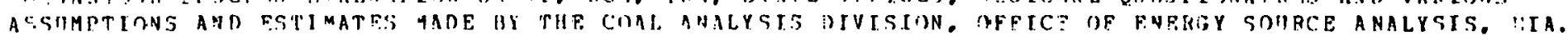

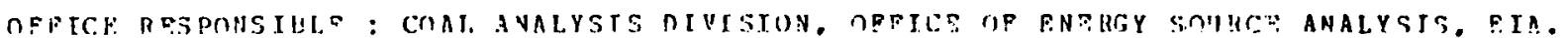




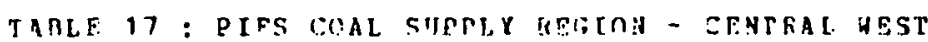

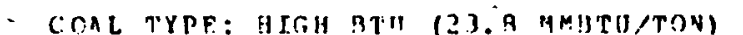

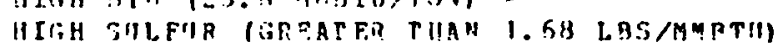

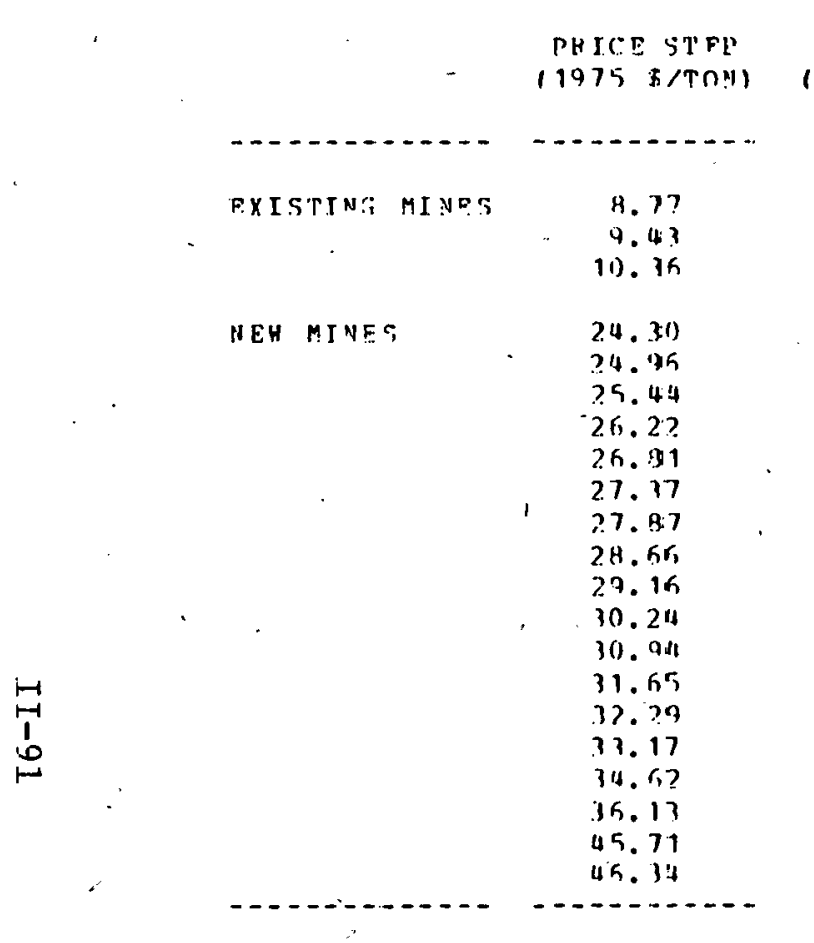

$$
\begin{aligned}
& \text { INC: REMFNTAL. TIOPI.Y } \\
& \text { DFEP }
\end{aligned}
$$

TOTAI.

zUMMUI,ATTVE SUPPLY ITHOIISANIS OP TONS PER DAY SITHACE -...(n) TOTA

$$
\begin{array}{r}
2.23 \\
4.73 \\
.22
\end{array}
$$$$
2.2 ?
$$$$
\text { 4. } 79
$$$$
\text { 2. } 22
$$$$
\begin{aligned}
& 2.00 \\
& 7.27
\end{aligned}
$$$$
\text { - - - - - }
$$

-.....-

$\begin{array}{rr}: & 1.10 \\ & 1.75 \\ 3.30 & 1.10 \\ 1.30 & 1.53 \\ 1.56 & 1.10 \\ .78 & 1.31 \\ & 1.54 \\ & .911 \\ 2.69 & .73 \\ 1.30 & .114 \\ & 2.19 \\ & .44 \\ & 3.07 \\ & 2.19 \\ & 1.75\end{array}$

4.68

3.64

$$
.22
$$

1.10
1.75

1.75

1.11
5.44

2.40

i. 31

2.00

2. 11

.144

3.73

.44

4.79
1.711

1.71

2.19

2.79

1.75

1.53
3.54
3.90

5.21

5.21

5.17

7.55
7.55

7.55

7.55

7.55

1.). 15

11.45

11.115

11.45

11.115

16.14

19.79

\begin{tabular}{l} 
\\
1.19 \\
1.19 \\
2.85 \\
3.94 \\
5.118 \\
6.57 \\
7.89 \\
7.33 \\
9.86 \\
10.30 \\
14.03 \\
14.46 \\
16.65 \\
17.09 \\
20.15 \\
22.15 \\
24.11 \\
24.11 \\
24.11 \\
\hline
\end{tabular}

\subsection{2}

7.09
7.22

1. 10

2.85

3.94

9.39

11.78

1.3. 10

15. 10

17.41

17.85

21.57

22.010

26. 81

29.55

31. 62

33. ค 1

35.56

40.25

43.87
CAPITAL

(M] I.LIOHS OP 1975 क)

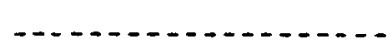

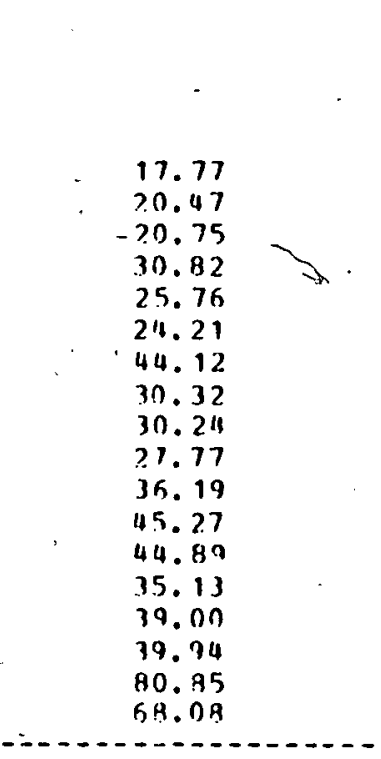

PIF.S Cont: CH

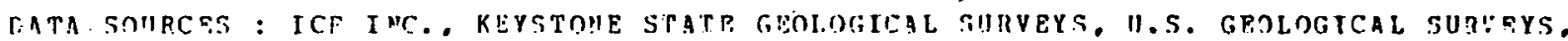

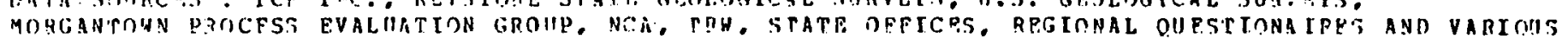
ASSIMPTIONS AND FITIMATES MADE BY T!IE COAL ANALTGIS MIVISION. OFPICE OF ENERGY BOIIRCF ANALYSIS. ETA.

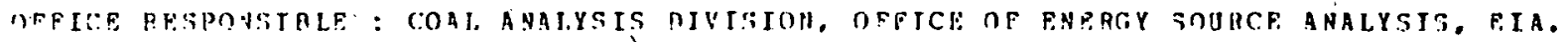




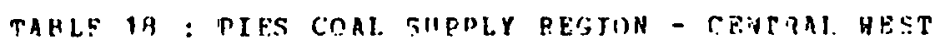

COAL TYPE: MEDTIM BTII 121.8 MMBTI/TONI

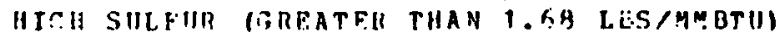

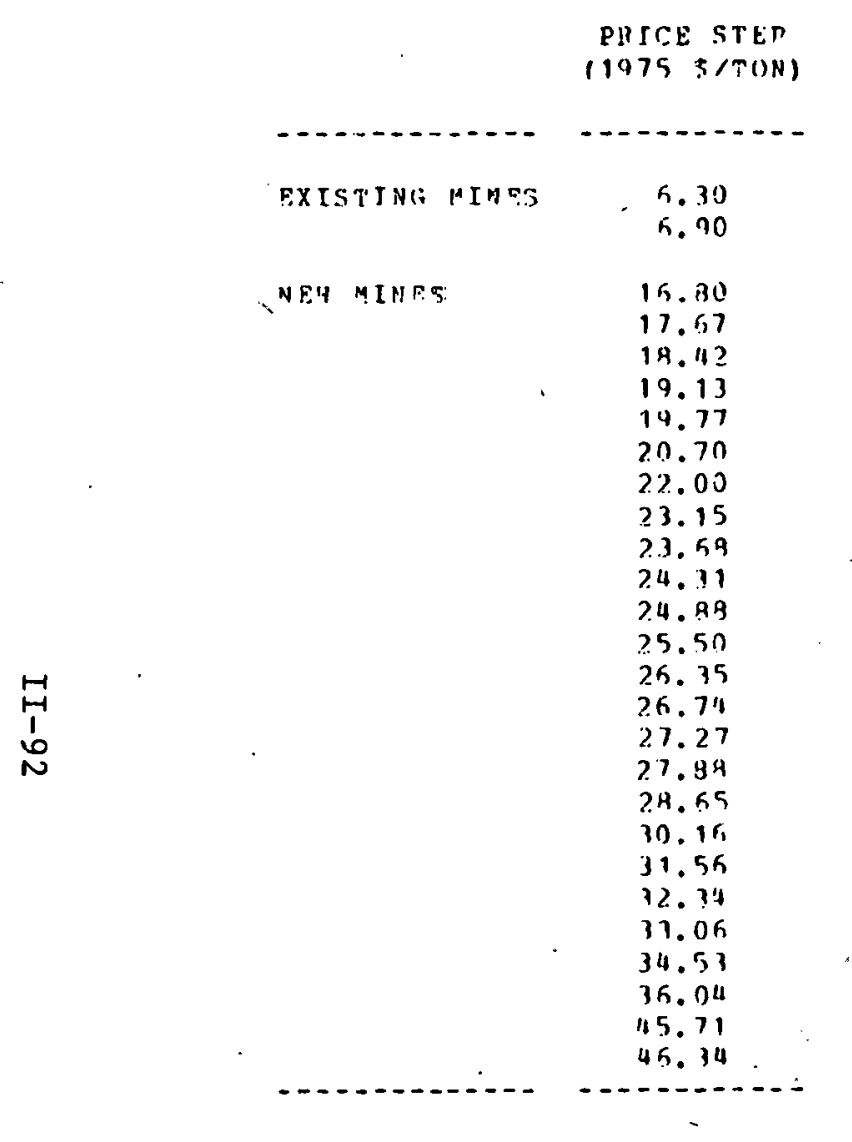

INICRFAEETAL SIIPPI,Y ITHOISANDS OF TOMS E.E. IOYY SURFACE DEFP TOTA T.

12.15
1.50
.

1. 30 $-\cdots \cdot-\cdot$

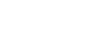

$\cdot$

.

5.20

\section{2. $\cap B$}

2.50
.26

5.77
.79

$$
\begin{array}{r} 
\\
7.71 \\
2.74 \\
9.59 \\
6.85 \\
9.118 \\
4.56 \\
11.11 \\
11.41 \\
9.59 \\
2.19 \\
11.79 \\
7.01 \\
16.04 \\
9.05 \\
4.38 \\
2.63 \\
3.29 \\
7.89 \\
17.11 \\
9.21 \\
9.89 \\
9.21 \\
9.45 \\
7.45
\end{array}
$$

$$
\begin{aligned}
& \begin{array}{r}
12.15 \\
1.50
\end{array} \\
& \text { 2. } 74 \\
& \text {.). 53 } \\
& \text { ก. } 15 \\
& \text { 5. } 44 \\
& 4.65 \\
& \text { 4. } 11 \\
& \begin{array}{r}
10.41 \\
9.59
\end{array} \\
& 9.59 \\
& \text { 2. } 19 \\
& \text { 111. } 79 \\
& 7.01 \\
& 11.03 \\
& \text { 14. } 25 \\
& \begin{array}{l}
4.38 \\
2.53
\end{array} \\
& \begin{array}{l}
2.53 \\
5.37
\end{array} \\
& \begin{array}{l}
5.37 \\
7.89
\end{array} \\
& \begin{array}{r}
\text { 9. } 11 \\
11.41
\end{array} \\
& \begin{array}{r}
11.41 \\
.36
\end{array} \\
& \text { ว. } 36 \\
& \text { 4. } 21 \\
& \begin{array}{r}
7.45 \\
6.77
\end{array} \\
& \begin{array}{r}
6.77 \\
.79
\end{array}
\end{aligned}
$$

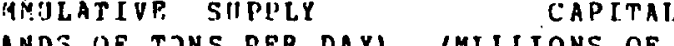

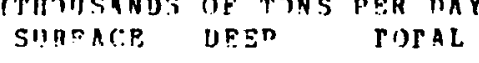
טיב TORAL

$$
12.15
$$$$
13.65
$$$$
12.15
$$

(n)

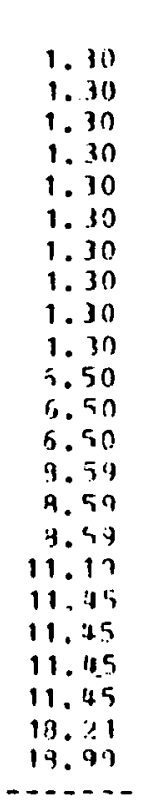

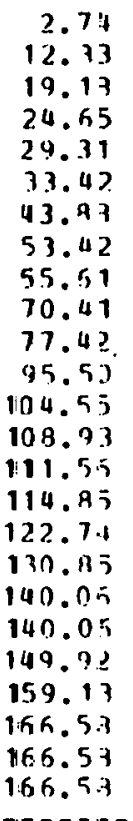

2.74

12.33

20.48

25.96

25.96

30.62

34.73
15.14

54.73

56.92

71.71

$7 \mathrm{~B} .73$

96.81

111.05

115.44

114.07

$123.44 \%$

139.44

151.25

151.25

151.51
161.37

161.37
170.57

17 月. 03

184.79

195.57

$0 . . .$.
CAPITAI

IMILLIONS OF 1975 \$!

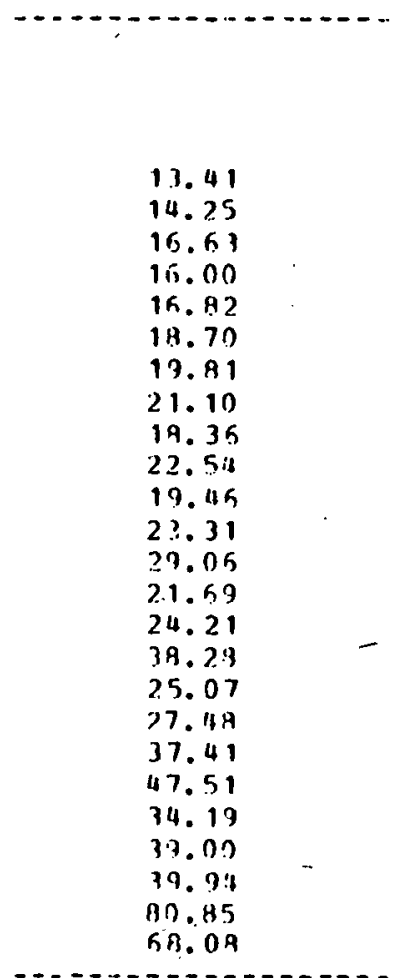

PIES CODE: $\mathrm{CH}$

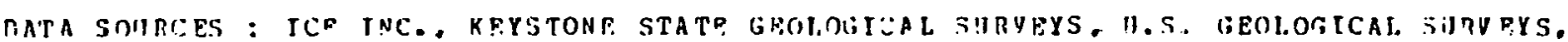

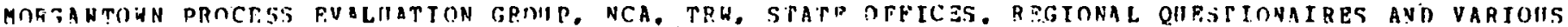

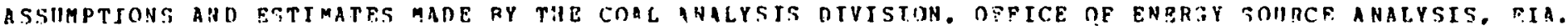

OFFTCF RESPONSTUI.F : COAL AIALYSTS OTVTSTOH, OFFTCE OE ENDIGYY SOIIRCE INALYSIS. DISA. 
TAHIF 19 : DIDS COAL SUPRIY BHGION - EENTGL WIST

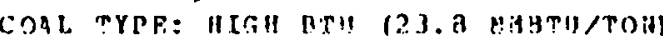

MBIIIM SULPIR 10.

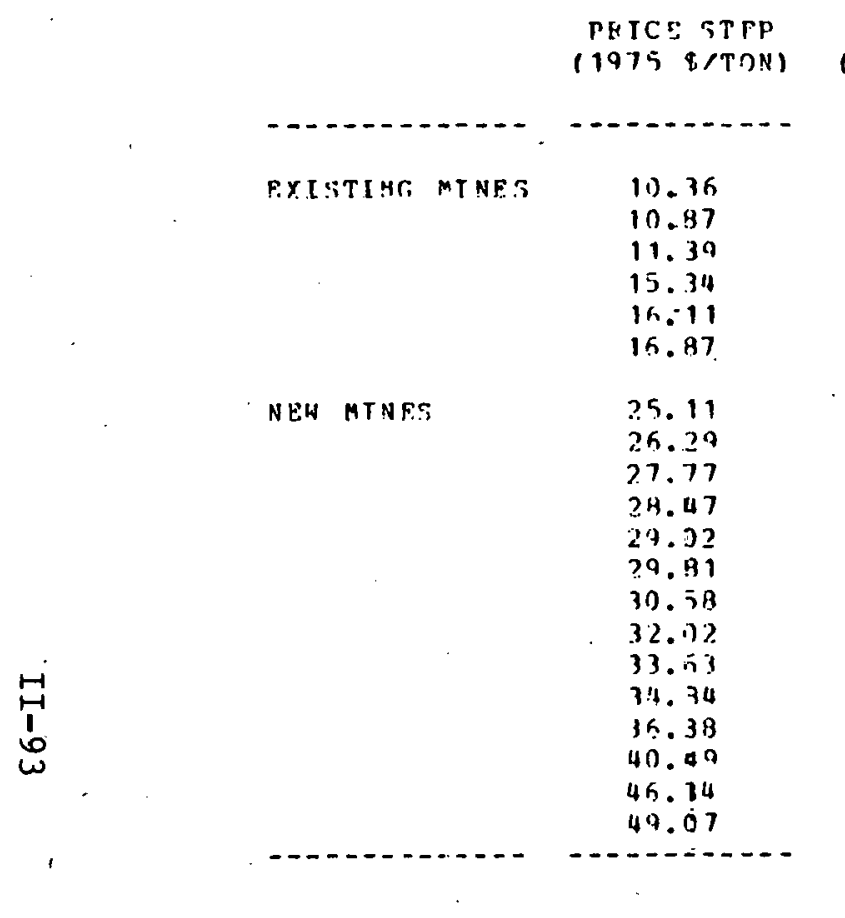

INCIRMENPAI, SUDPLY

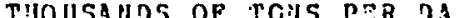
SURFACE DEE? TORAI.

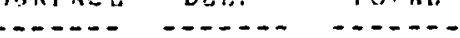

.22
.01
.01
1.08
.16
.36

.

$\begin{array}{r}1.56 \\ .78 \\ .52 \\ 1.42 \\ .22 \\ 1.56 \\ 4.42 \\ \hline . .25\end{array}$

.22
.22
.144
1.10
1.53
1.31
1.53
1.10
1.31

.27
.01
.01
1.08
.36
.36
.27
.22
.44
1.56
1.10
.78
1.53
1.34
3.36
1.10
1.31
.22
1.56
$4.14 ?$
.- .5

CADTTAL

IAILLTONS OF 1975 t)
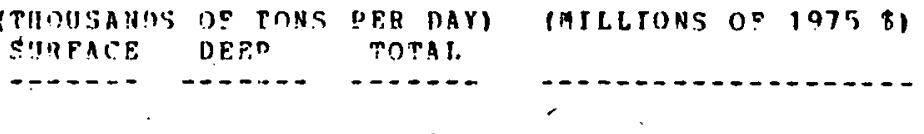

.22
.23
.211
1.31
1.59
2.05

.22

.23

1.37

1.59

2.0 .5

\section{.22}

.22

.44
.98

2.44

1. 53

4. 31

5.85
7.68

7.68
11.04

12. 14

13.45

13.57

15.23

19.66

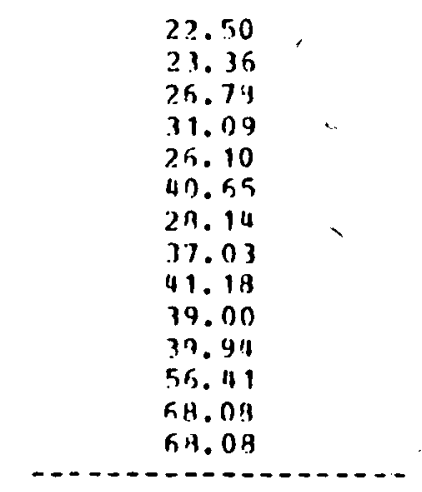

PIE; CODE: CR

DATA SOIRCEE : ICP INC., KFYSTOVP, S?ATE GPOLOGIEAL SIURYPYS . I.S. GFOLOGICAL TURVFYG.

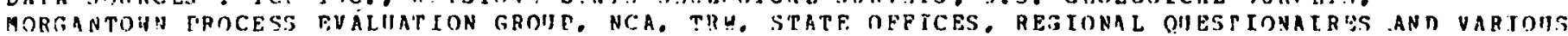

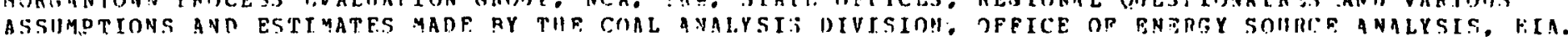

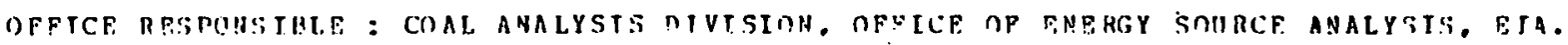




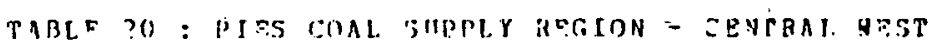

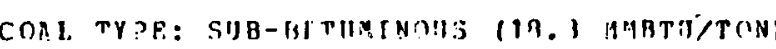

METIEM SIIFIR (GREATFR THAN 0.67 LBSTMMETUI)

phice set

11975 D/TOH

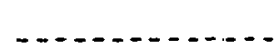

TRY STINI MINF

NEN MTNES

ก. .93
TCHPMPNIAT. SHPILL THIISUN:S OF TONS PER RAYI "HIRFA:F REEP TOTAI.

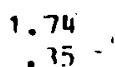

.35

$? .74$

6. 95

4. 11

5.49
1.84
3.56

4.93

4. 56

11.38

5.21

2.74

1. 92

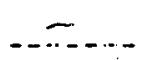

-....

27.900
0

2. 711

6. 95

11.11

5.119
3.814

1.55

11.73

4. 56

4. 313

5.21

2.74

1.92
CIIMAULATIVE SUPPLY TIIOHSANDS OF TONS PER OYY S!IPAC? STIRACL DFEP TOTA

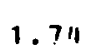

$\begin{array}{ll}1.711 & 1.711 \\ .15 . & 2.07\end{array}$

.7 .74
5.59
1.70
19.19
2.7 .01
26.59
11.51
36.16
40.55
45.75
48.49
50.41
.0 .7$.

1.71
2.09

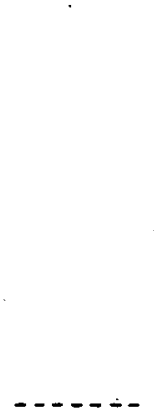

2.74

9.59

13.70

19.19

23.01

26.59

31.51

36.16

40.55

45.75

48.49

50.41

......
IMILLIIINS OF 1975 *

CATITAL
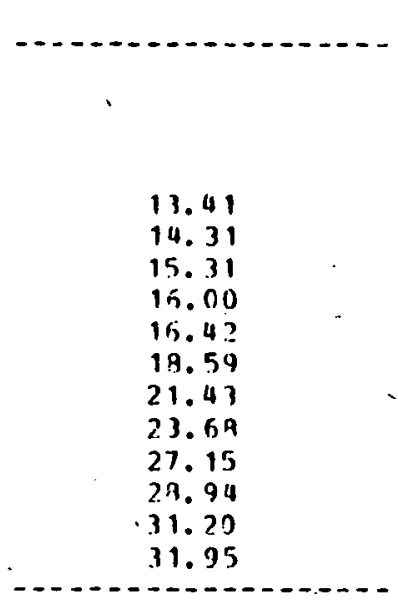

PIES CODE: CV

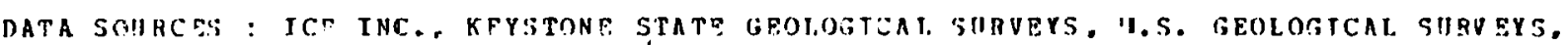

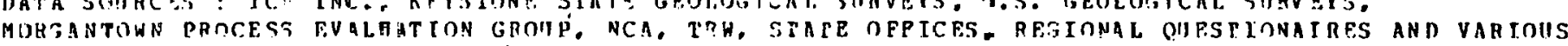

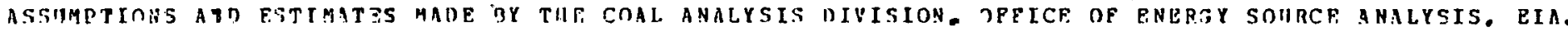

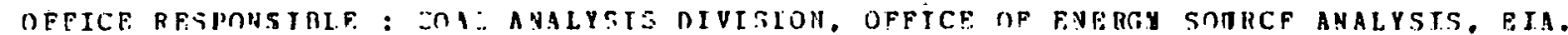


TAELF ?I : PIES COAL SIIPLY REGIOU - CENTEAI. WEST

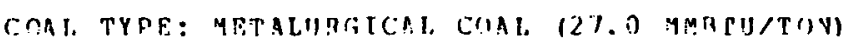

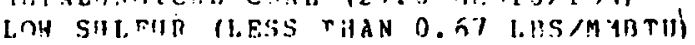

PRIC STE

$(1975 \% /[\cap N)$

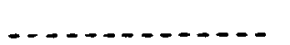

-.........

E.Y. ISTINR: MIN NQS

10. 36

10.87
11.87

NFW MINFS
INCREMENTAT. SHPQLY (?HOUSAHIS OP TONS PTR DAY SIIRFAC:

DE.PP POTAI. -.....

$-\cdot-\cdot-$

CII:AMULLATIVE SIIRFACE DEPD -...-. oEe?

$S$ PER DAY

CAPITAL

MILLIONS OF 1975 \$) TOTAL.

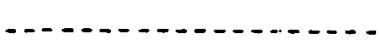

.78
.04

$\begin{array}{r}3 \% .34 \\ 3 ? .99 \\ 31.65 \\ 36.00 \\ 77.69 \\ 39.24 \\ 40.77 \\ 57.11 \\ 58.74 \\ \hline\end{array}$

\begin{tabular}{rrr}
.25 & & .26 \\
& .41 & .41 \\
.23 & .62 & .53 \\
& .52 & .814 \\
.122 & .92 \\
.44 & .52 & .52 \\
1.53 & .92 & 1.25 \\
1.31 & & 1.53 \\
\hline
\end{tabular}

.74
.87
.87
.25
.25
.25
.164
.49
.48
.92
2.115
3.77

$\begin{array}{r} \\ \\ .41 \\ 1.03 \\ 1.54 \\ 2.47 \\ 3.04 \\ 3.90 \\ 3.90 \\ 3.90 \\ \hline\end{array}$

.79
.49
.97
.26
.57
1.29
2.12
2.94
3.56
11.42
6.36
7.57
. .27

\begin{tabular}{l}
47.51 \\
25.74 \\
27.65 \\
37.87 \\
35.13 \\
41.60 \\
47.41 \\
83.93 \\
90.85 \\
\hline
\end{tabular}

PIRS COIIE: CM

DATA SOIRCES: ICF INC., KEYSTONF STATE GEOLOGLZAT. SURYEYJ. U.S. GEOLOGICAL SURVEYS.

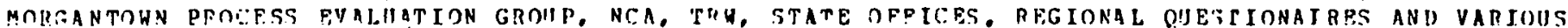

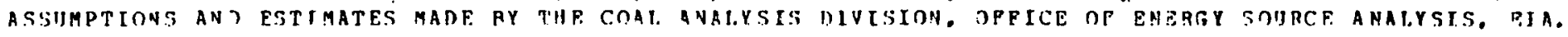

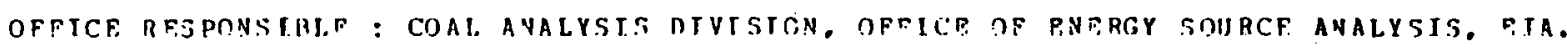




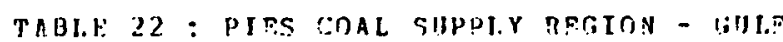

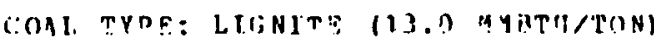

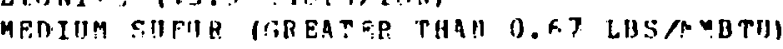

PPICF, ITEP

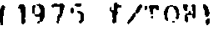

IMCROMFNTAL SIIPPLY ITUOUSANOS OF TUNS PBB DAYI

CUMMIII. ATIVF, SHPPLY

CAPITAL

-..............

SIIPFACE DPE!

TกT 4 I

(TIUISAYTS DF POIS FER DAY

SIPPACF: DEER

TOTAL

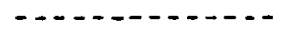

2.47

27.42

27.42

27.47

-.....

-......

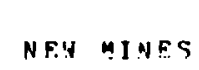

6. 04

6.93

7.41

B. 12

ค. 63

9.43

$9.9 ?$

10.91

11. 71

12. 54

15.05

113.46

?.1.9?

27.4

25.61

27.95

\begin{tabular}{|c|c|}
\hline \\
\hline \multicolumn{2}{|l|}{$\begin{array}{l}24.55 \\
43.84\end{array}$} \\
\hline \\
\hline \multicolumn{2}{|l|}{60.21} \\
\hline \multicolumn{2}{|l|}{ 12. \&A } \\
\hline \multicolumn{2}{|l|}{32.89} \\
\hline \multicolumn{2}{|l|}{10.96} \\
\hline \multicolumn{2}{|l|}{10.95} \\
\hline \multirow{2}{*}{\multicolumn{2}{|c|}{1.54}} \\
\hline & \\
\hline \multicolumn{2}{|l|}{1.37} \\
\hline \multicolumn{2}{|l|}{1.37} \\
\hline \multicolumn{2}{|l|}{.92} \\
\hline & $\begin{array}{l}.27 \\
.27\end{array}$ \\
\hline & .27 \\
\hline
\end{tabular}

$\begin{array}{r}21.66 \\ 41.914 \\ 35.62 \\ 60.27 \\ 12.94 \\ 32.89 \\ 10.95 \\ 10.96 \\ 1.611 \\ 2.74 \\ 1.37 \\ 1.17 \\ .92 \\ .27 \\ .27 \\ .27 \\ \hline .2 .0\end{array}$

\subsection{5}

68.47
1018.11

19.11

16.4. 33

197.26
2.30 .14

241.10

2.5?.05

253.70

256.44

257.41

$259.1 \mathrm{~B}$
250.00

250.00
260.00

250.00

26.0 .00
27.42

24.65
68.49
104.11
164.38
197.25
230.11
241.10
252.05
253.70
256.44
257.91
259.18
260.00
260.27
260.55
250.82

MILI.IONS OF 1975 \$1

- - - - - - - - - - - - - -

$\begin{array}{r}4.19 \\ 5.72 \\ 6.39 \\ 7.54 \\ 11.59 \\ 8.99 \\ 10.89 \\ 11.21 \\ 12.56 \\ 13.76 \\ 19.07 \\ 25.59 \\ 32.10 \\ 20.05 \\ 25.61 \\ 31.20 \\ \hline\end{array}$

PLFS CODE: CU

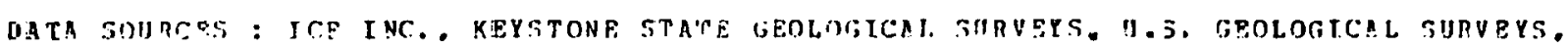

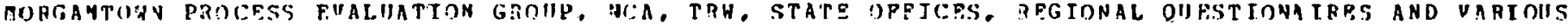

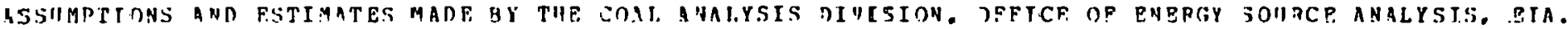

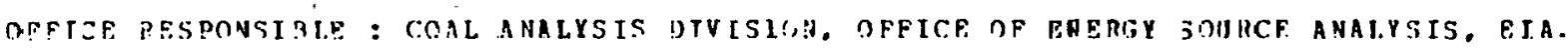


TAMLE J3 : PIES COAL. SIPPLIY RERIOH - LASTSRN NORTHERP GREAT PLAIH:

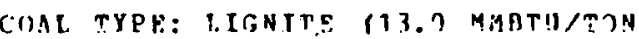

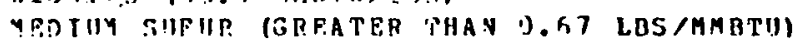

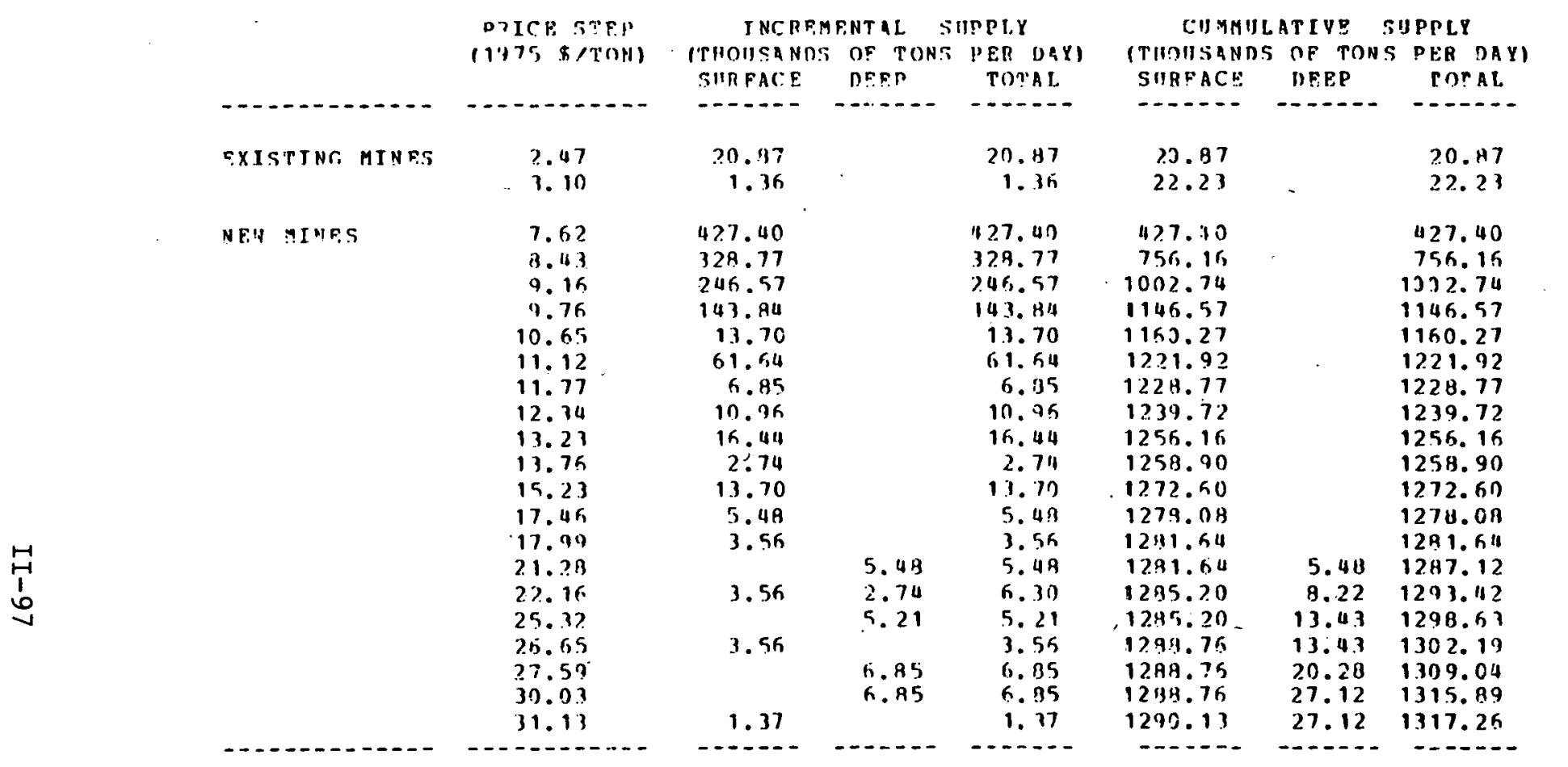

CAPITAL

IHLLLIOHS OP 1979 \&I

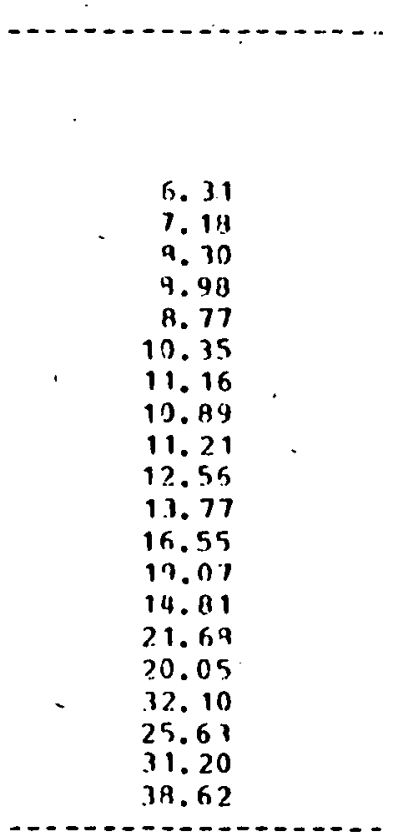

PIES CNDF: CII

DATA SOHRCES : ICF INC. KEYSTONP STATE GEOLOETCAL SIIRVEYS. 11. S. GEOLOGICAL SURVFYS.

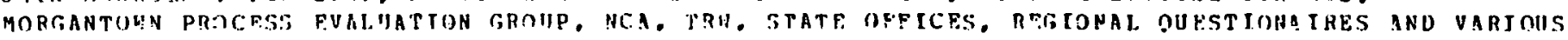

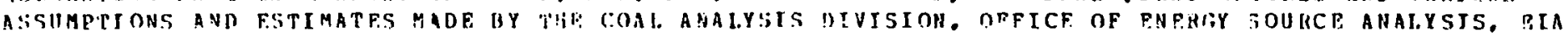

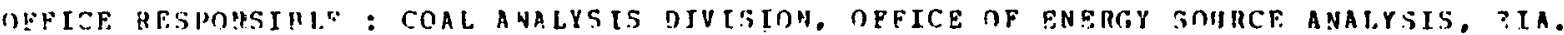




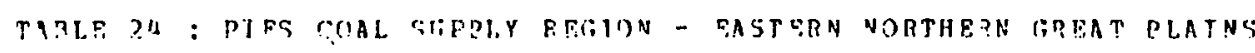

COAT. TYPF: I.IBNITR: 113.5 MMETH/TONI T.O' SIII.FU? II.ESS TIIAN 0.57 LUS/YMBTII.

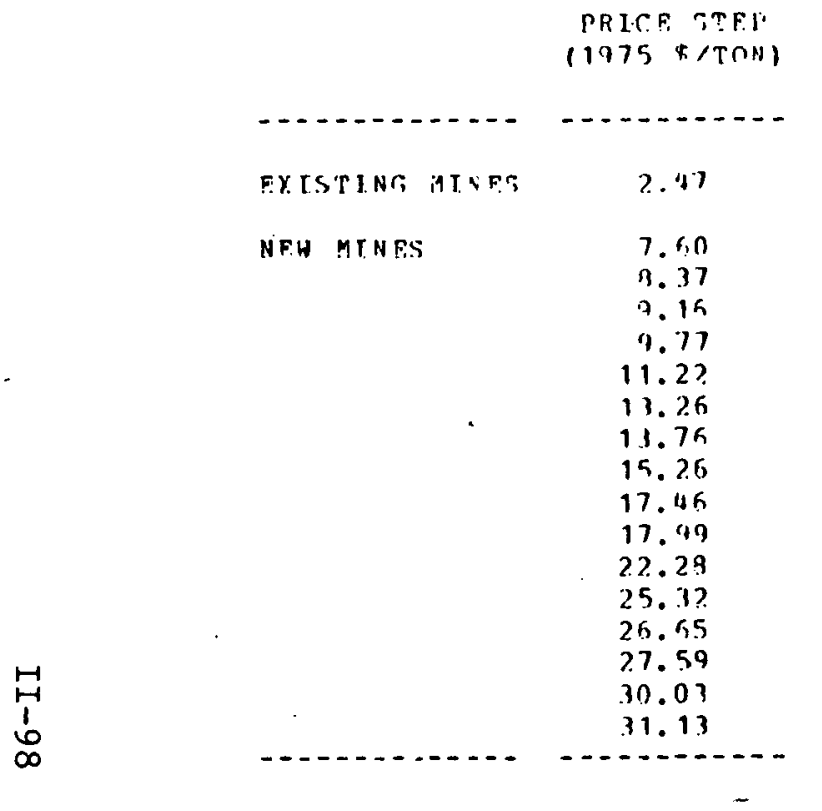

\begin{tabular}{|c|c|c|c|c|c|}
\hline & $\Lambda \mathrm{L}$ & & (:1) & A ITV & \\
\hline & NF The & QER DIYY & IT!III IANDS & of rans & PFE DAYI \\
\hline & DFF? & TOTAI. & SURFAC: & DEE? & TOPAL \\
\hline
\end{tabular}

CAPITAL

IMLLLIONS OF 1975 \$1 (1).

$1 . ? 1$

\begin{tabular}{|c|c|c|}
\hline 1.21 & & 1.21 \\
\hline 19.18 & & 19.17 \\
\hline 21.92 & & $21.9 ?$ \\
\hline 97.91 & & 17.11 \\
\hline 13.70 & & $1.3 .7 n$ \\
\hline A.?? & & 9. 2? \\
\hline 4.11 & & 4.11 \\
\hline .82 & & .82 \\
\hline 1.37 & & 1.37 \\
\hline 1.37 & & 1.37 \\
\hline $\begin{array}{l}.55 \\
.55\end{array}$ & & $\begin{array}{l}.55 \\
.55\end{array}$ \\
\hline & .27 & .27 \\
\hline .55 & & $\begin{array}{l}.55 \\
.55\end{array}$ \\
\hline .27 & & $\begin{array}{r}.27 \\
- \\
\end{array}$ \\
\hline
\end{tabular}

1. 21

19.18

19.13

41.10
53.90

7. 90
$7 \% .60$

คi). B?

9.1. 93

45.75

ค7. 12

58. 199

89.04
R9. 54

84. 50

90.14

99.14

90.14

90.11

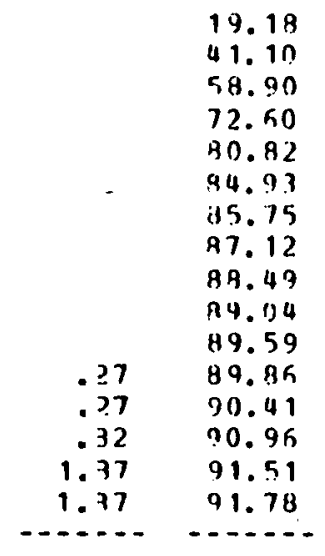

\begin{tabular}{|c|}
\hline $\begin{array}{r}6.33 \\
5.81 \\
8.05 \\
9.57 \\
9.89 \\
11.30 \\
12.56 \\
13.76 \\
16.55 \\
19.07 \\
25.59 \\
20.05 \\
37.10 \\
25.63 \\
31.27 \\
39.62\end{array}$ \\
\hline
\end{tabular}

PI.FS CODE: CY

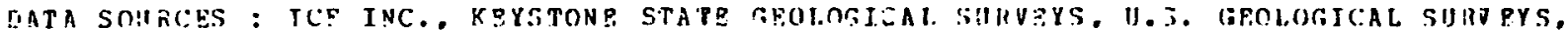

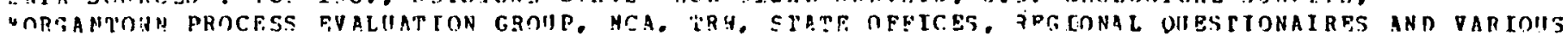

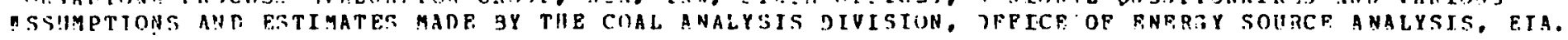

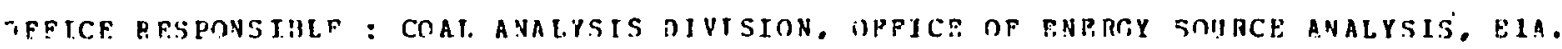




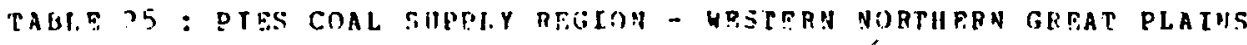

(GAT. TYPF: MEDIUM RTII 121.9 MMBTH/TONI

MPDIUM SULE!IP, 10.67 TO $1.5 B$ LASMMATII)

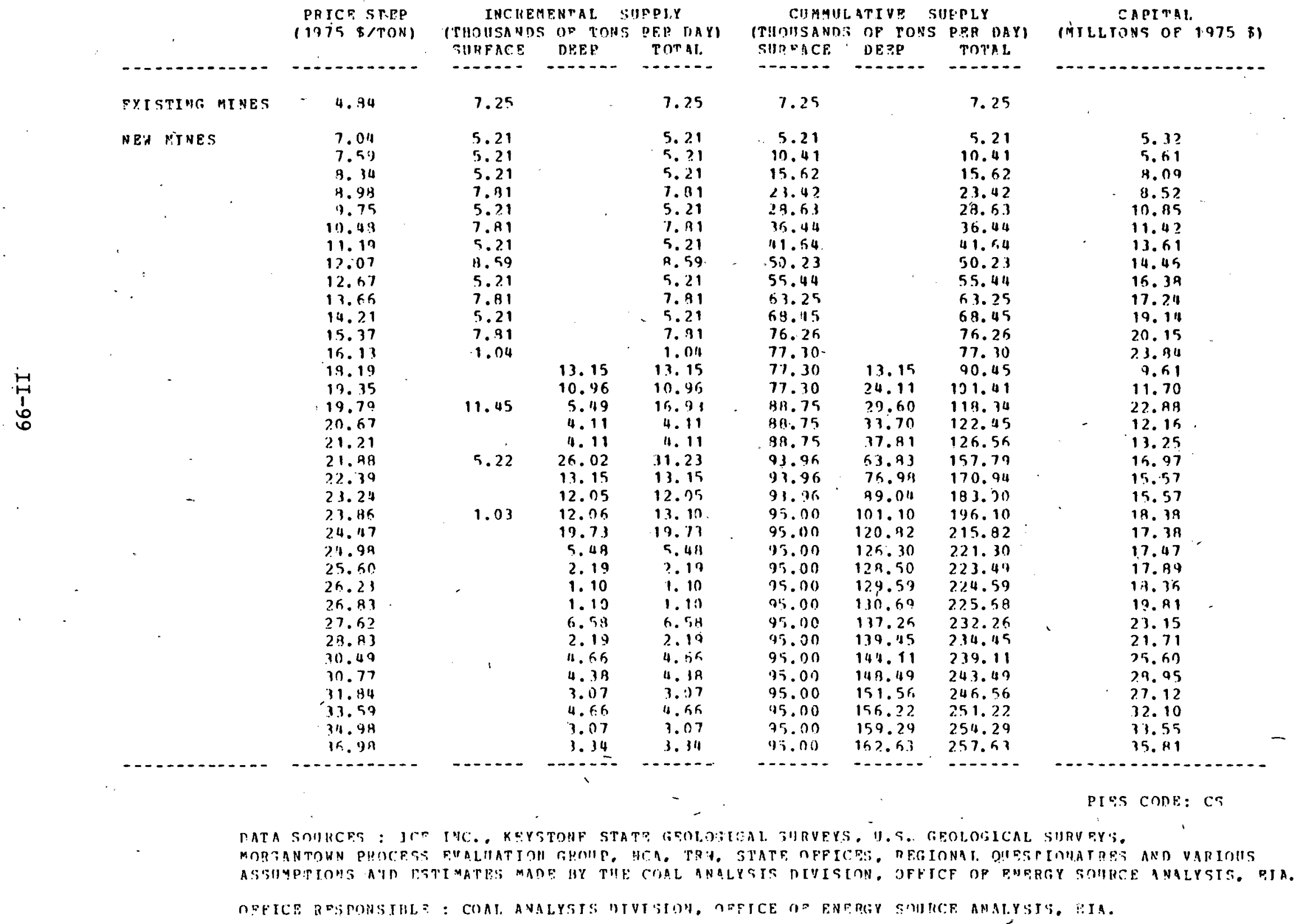




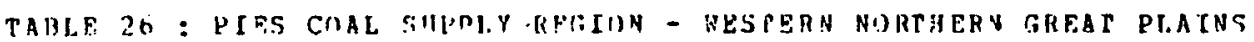

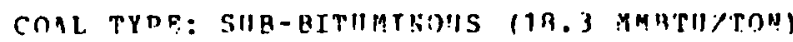

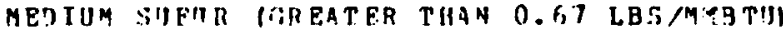

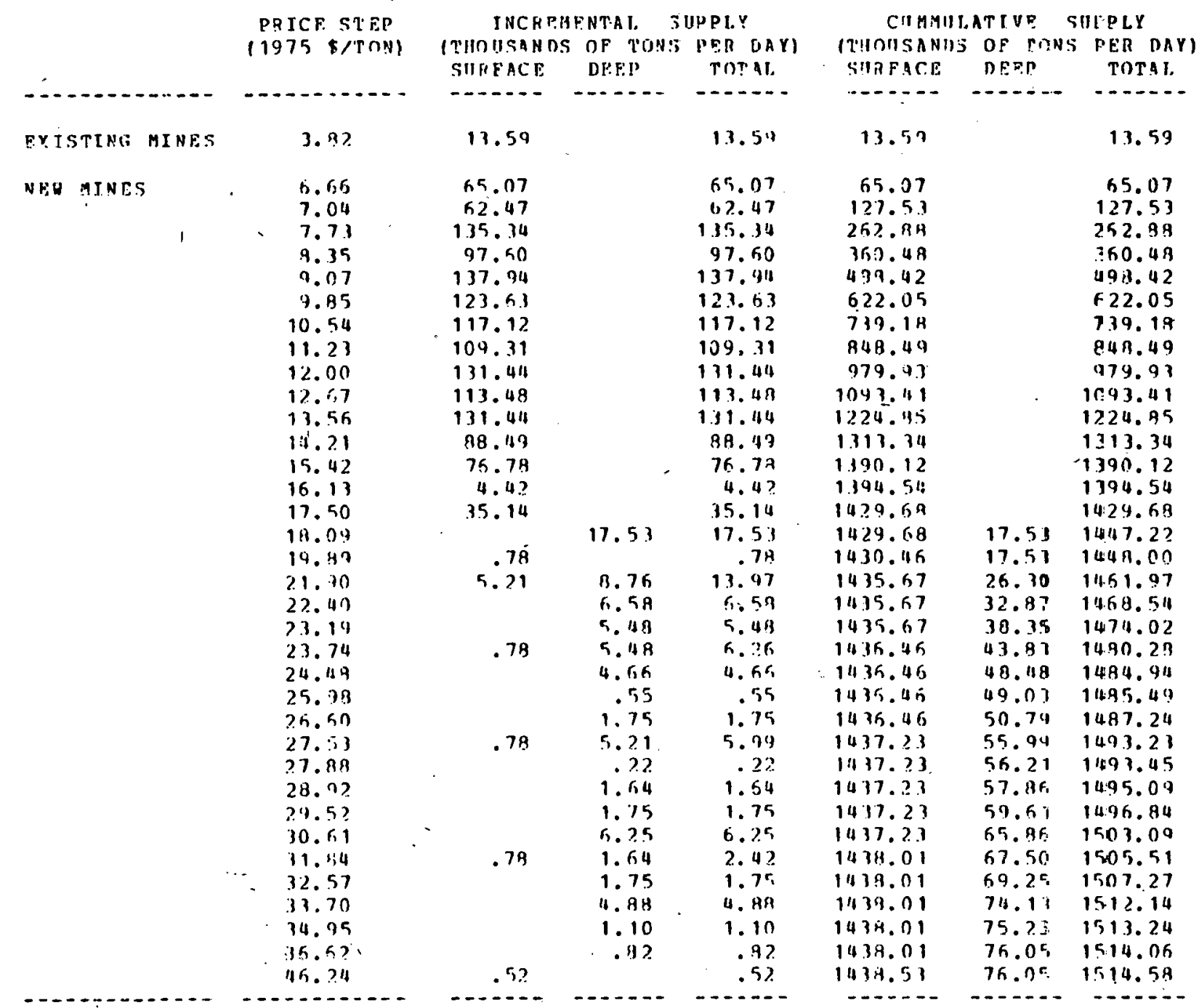

CAPTTAL

(YILLIONS O? 1975 5)

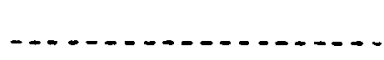

$\stackrel{1}{-1}$
$\stackrel{5}{8}$

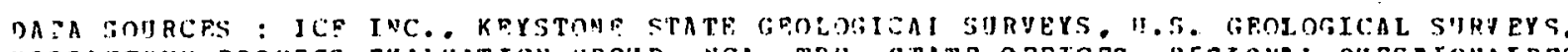

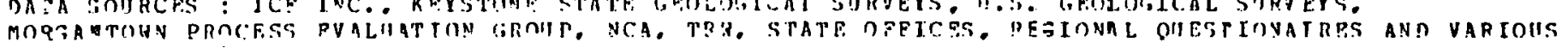

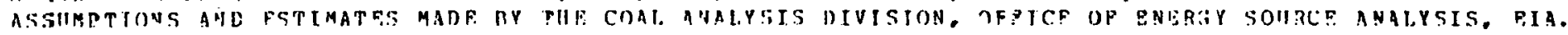

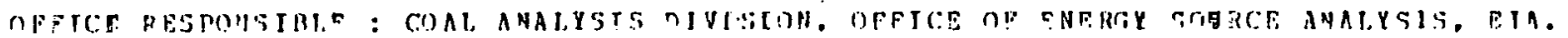




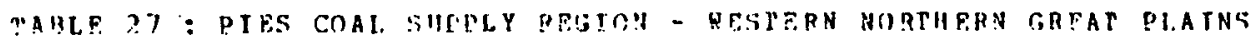

ZOR TYPF: MFEI!IM BT!I 121. M MMTII/TONI

LOS SULPIIP. II.ESS THAH 0.67 LISS/MIITTUI

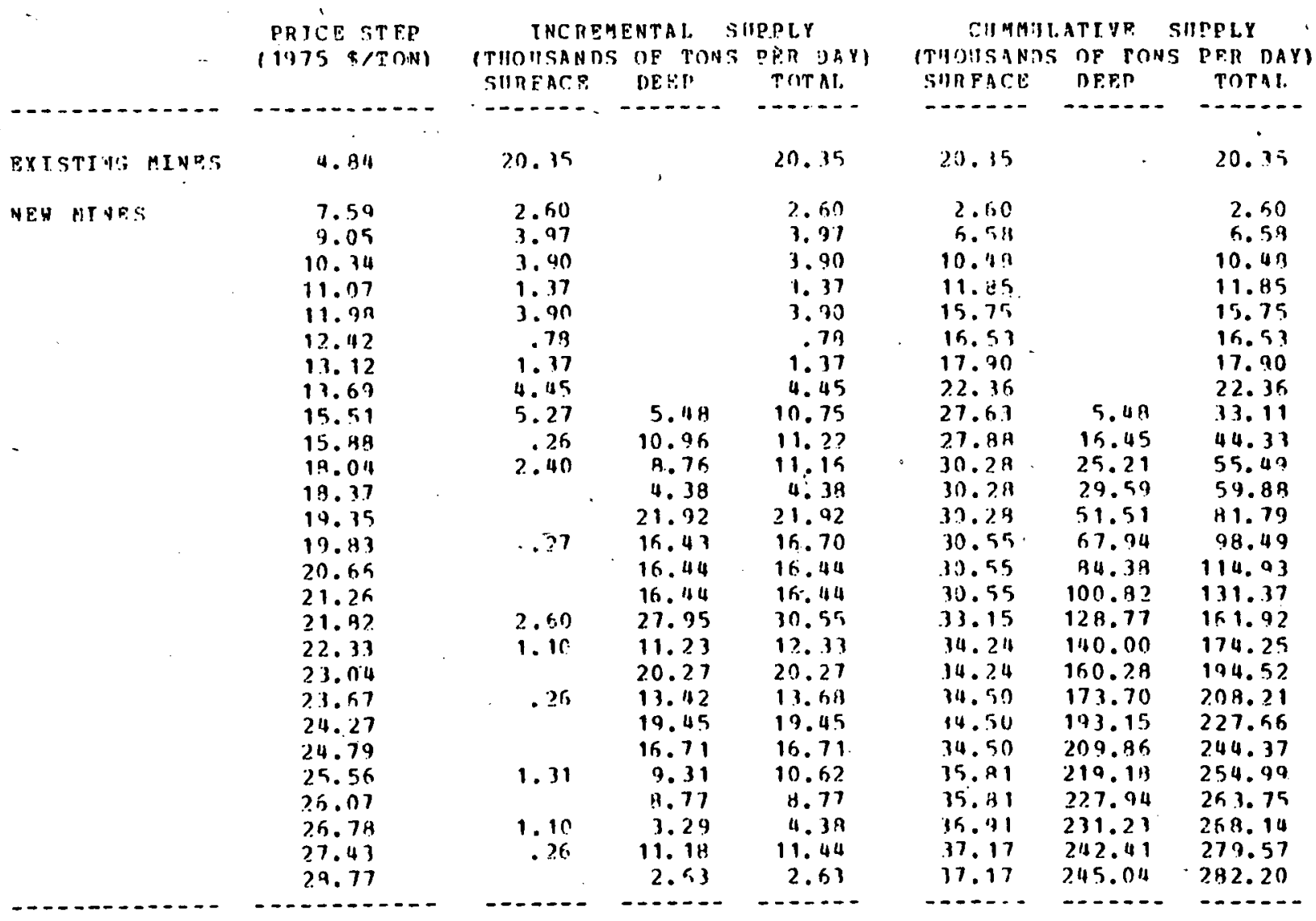

iptrat

(4ILLIONS OP 1975 B)

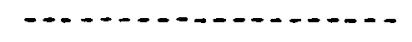

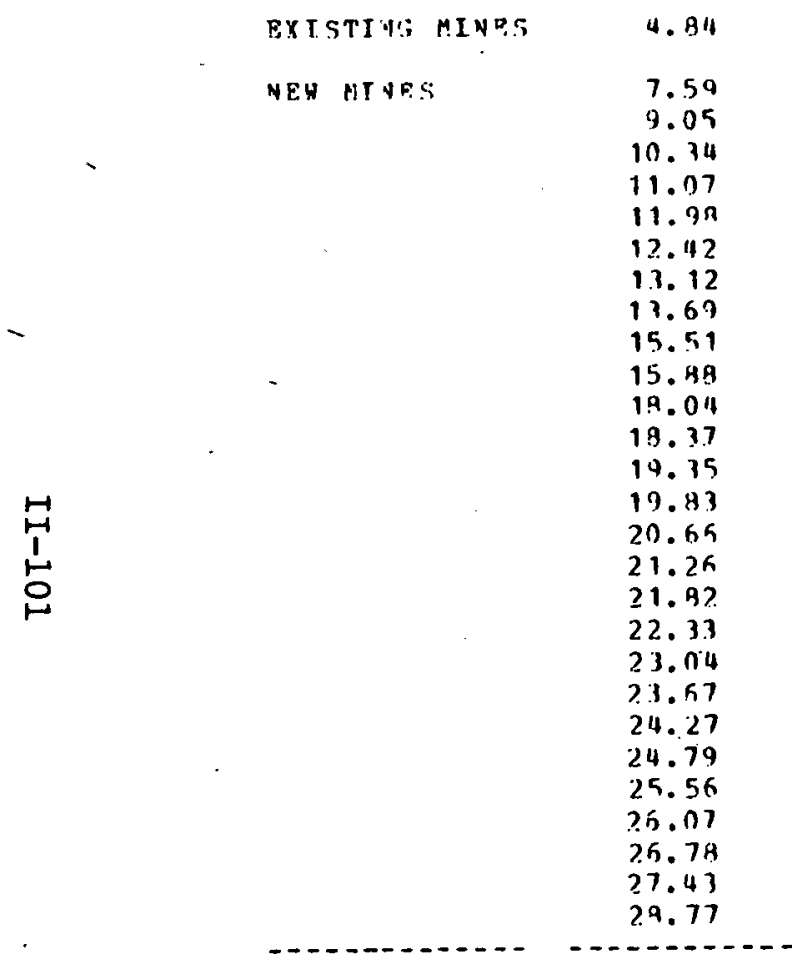

$0.7 i$

5.61
7.47
11.02
4.17
14.12
15.69
10.97
15.65
12.87
9.24
12.06
10.05
11.70
12.76
12.47
13.20
15.30
15.69
14.60
16.08
16.36
17.47
19.61
19.56
22.99
21.59
23.36
19.96

PIES CONE: C.Z

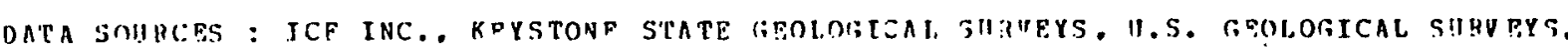

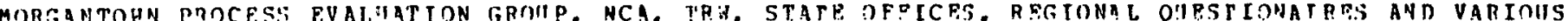

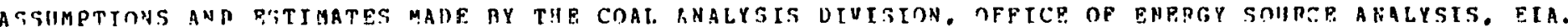

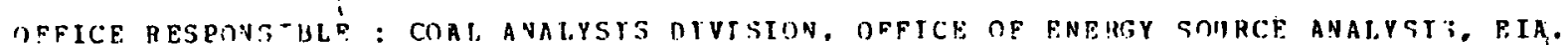




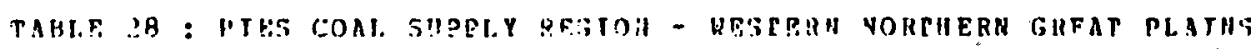

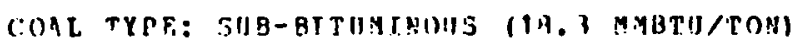

LOH SI!LFIJ ILEST PHAN 0.57 LAS/MHATIII

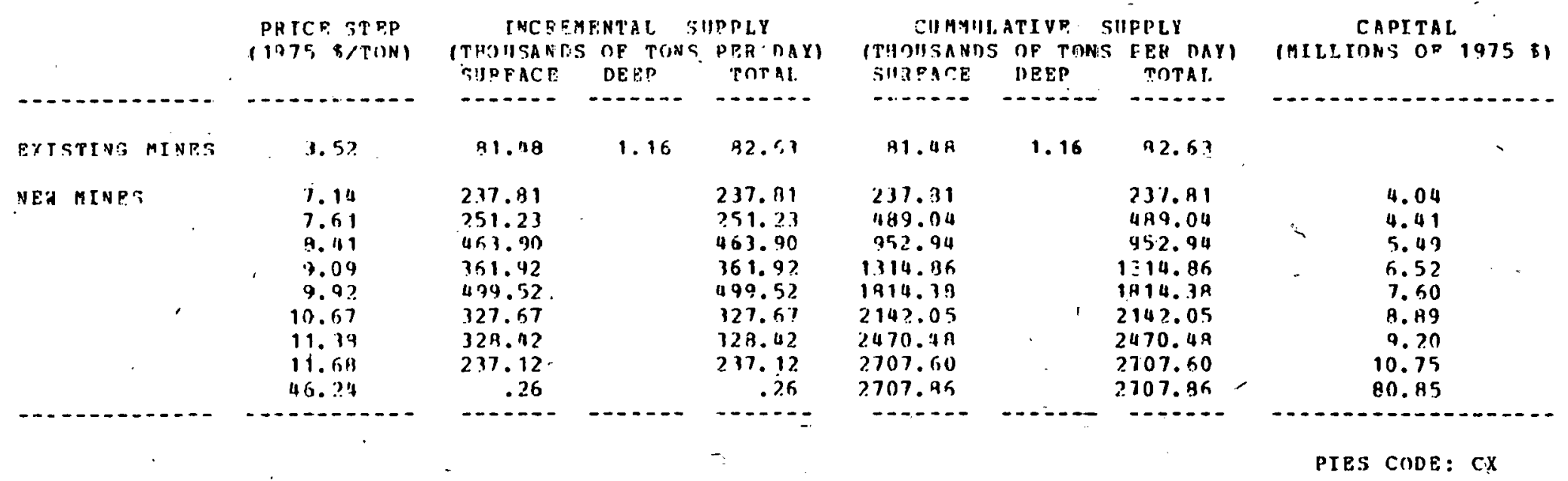

DATA SOHICFS : TCV IHC. KPYSTONI STATF GEOTOCICAL SÜRVSYS. I.S. GROLOGICAL SUROEYS.

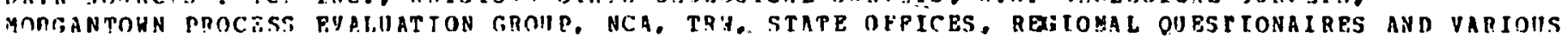
ASSUMPTIONS AND FSTIMATES YHDE BY THF COAL ANALYSIS UIVISION. DFPICP DF PNERGY SOIRCE ANALYSIS. FIA.

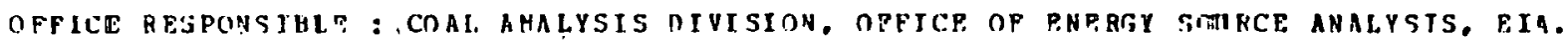




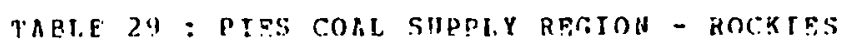

COAL TYPE: HISH RTU ISB. M MXBTU/ROHI

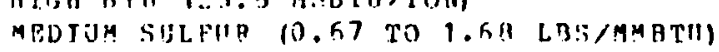

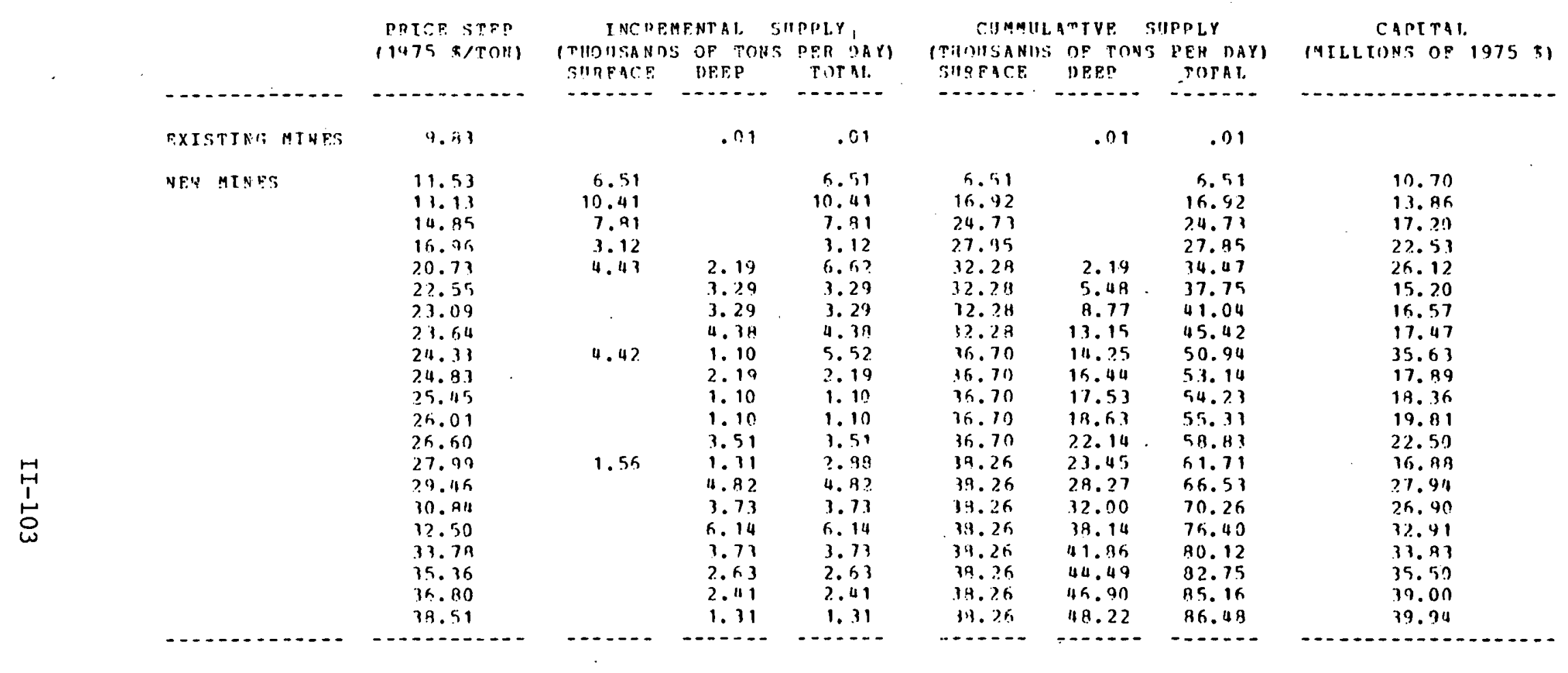

PIES CODE: CR

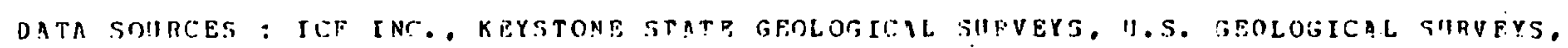

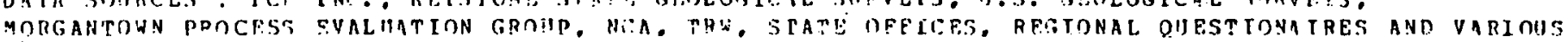

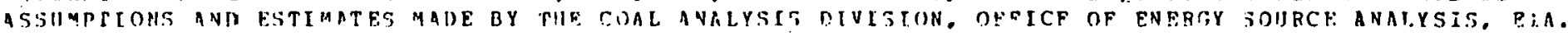

OFFICE RESPONGIPIE : COAL ANALYSIS DIVJSIOA, OFFJCE OF FNERGY SOIRCF ANALYSIS, FIA. 
TAIIT.F BU : PIES COAL SIPPLY REIOTON - RIOCK TES

COAL TYPE: MENIIIM BT! (21. A MBT!/TON)

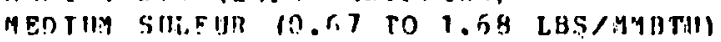

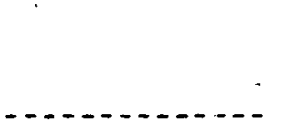

PQICF. STEP 11975 S/TONI

FXISTLNG MINFS -..-.-.-.

IACRFYFNTAL SIPDLE (TIOHSANDS OF TOHS PTR DAY) SURFACF DFFE DFF 'COTA I.

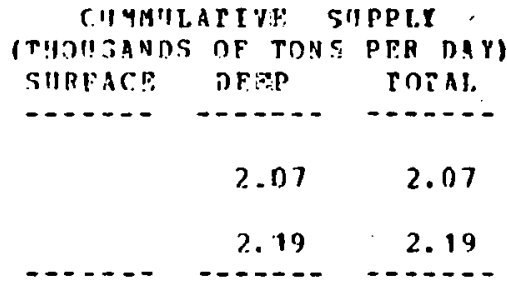
2.07 - - - 2. 19 2.19

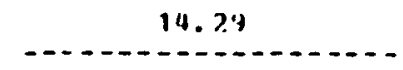

PIES CODER: CS

CAPITAL.

MI LLTONS OF 1975 \$)

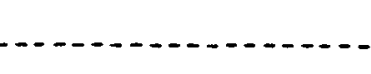

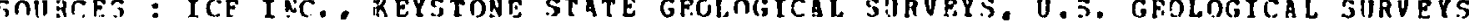

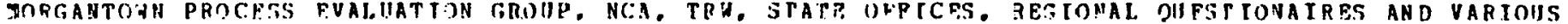

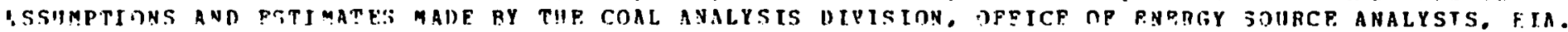

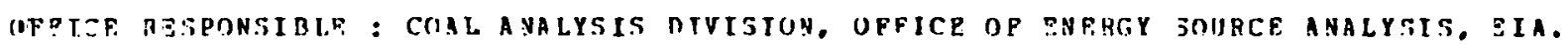


TABIF M : PIES COAL SUPELY BE!SION - ROCYIZS

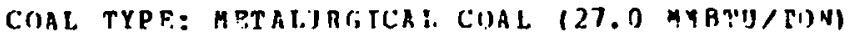

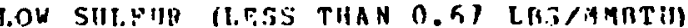

\begin{tabular}{|c|c|c|c|c|c|c|c|}
\hline & PAICF TTEP. & INC RPM & ENTAL. & I!nt.y & CIIMMUL & LATIVF. & IPPLY \\
\hline & $(1975: T(I N)$ & $\begin{array}{l}\text { ITHOUSANANS } \\
\text { SHRFACP. }\end{array}$ & $\begin{array}{l}\text { OF TONS } \\
\text { DE:PP }\end{array}$ & $\begin{array}{l}\text { PER DAYI } \\
\text { TOTAL. }\end{array}$ & $\begin{array}{l}\text { TTIOISANDS } \\
\text { SURPACB }\end{array}$ & $\begin{array}{l}\text { OP TONS } \\
\text { DPRP }\end{array}$ & $\begin{array}{l}\text { PEA I:AY } \\
\text {-FOTAL }\end{array}$ \\
\hline EXISTING MTNDS & 1.97 & , & 9.06 & 3.05 & & 8.06 & 8.05 \\
\hline FH MINES & $\begin{array}{l}30.51 \\
11.39 \\
31.55 \\
34.79 \\
16.75 \\
39.25 \\
39.76\end{array}$ & & $\begin{array}{r}2.17 \\
.41 \\
1.23 \\
.411 \\
1.23 \\
.111 \\
.111\end{array}$ & $\begin{array}{r}2.117 \\
.41 \\
1.23 \\
.111 \\
1.21 \\
.41 \\
.41\end{array}$ & & $\begin{array}{l}2.47 \\
2.84 \\
4.11 \\
4.52 \\
5.75 \\
6.16 \\
6.57\end{array}$ & $\begin{array}{l}2.147 \\
2.89 \\
4.11 \\
4.52 \\
5.75 \\
6.16 \\
6.57\end{array}$ \\
\hline
\end{tabular}

CAPITAL

(MILLIONS OF 1975 \$)

-

$0.0-0.041$

-..-.

6.57

\begin{tabular}{l}
24.00 \\
24.91 \\
29.47 \\
32.26 \\
36.00 \\
39.60 \\
40.60 \\
\hline
\end{tabular}

PIPS CODF: CM

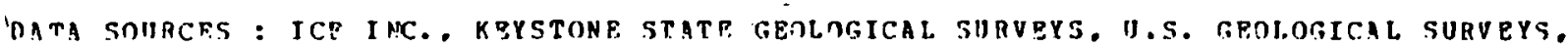

MORGANTOYN PPICESS EVALIMTION GROJP. NCA, TRH. STATE OFPLCES, RPGIONAL OUESTIONATRES AND VARIOIS

ASSUMPTIONG AND ESTIMATF.S MADE BY THE COAL ANALYSIS DIVISION, OFPICE OP BNERGY BOUHCD AUALYSIS. ELA.

OFFIEE RPSPOHSIBLE : COAL ANALYS DIVISION, OFEICE OP PNFRGY SOIICE ANALYSIS. EIA. 
TABIE 3? : PIES COAL SIPUI,Y RFOION - POCYIPS

COAL TYPE: HIGH BT! (23. А M.MBTH/POH

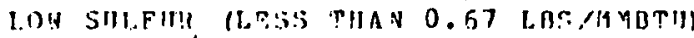

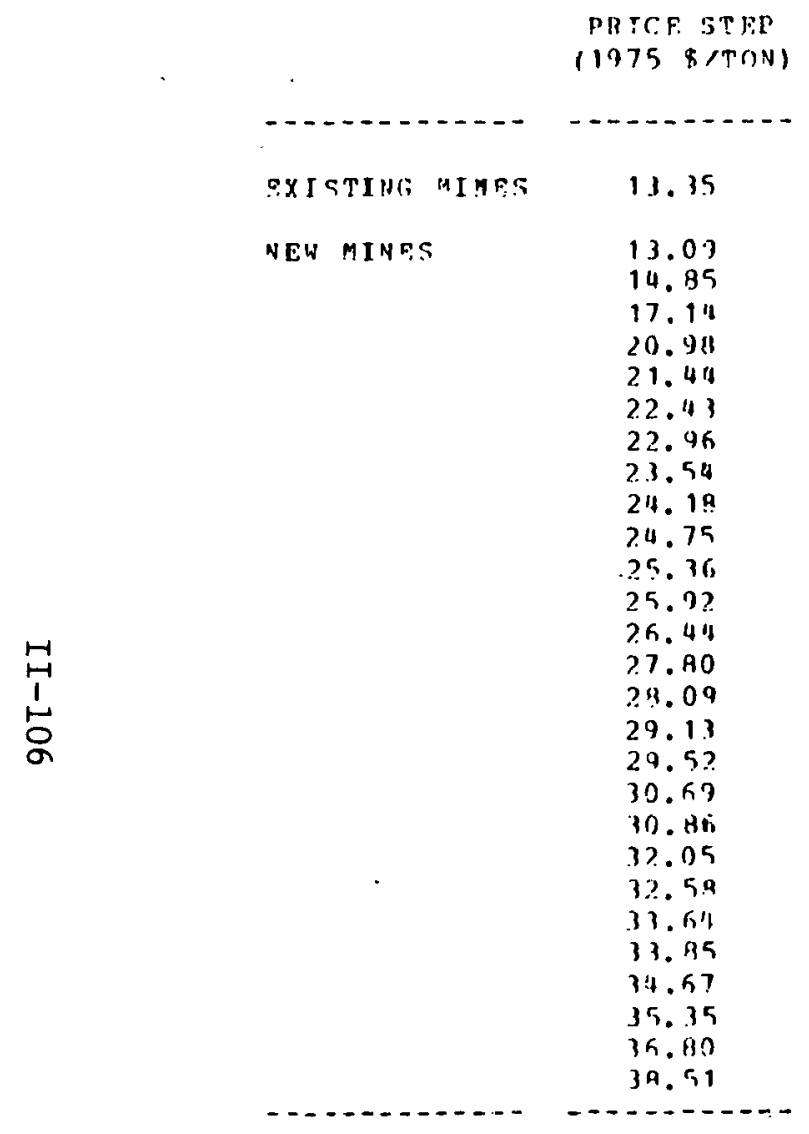

LVZREMENTAL TIJPLLY

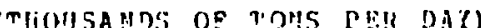
SURFAEE DEEP TOTAL

17.111

17.81

$$
\begin{array}{r}
1.70 \\
1.32 \\
.73 \\
.73
\end{array}
$$

7

39

.25

$$
\begin{aligned}
& \\
& 10.95 \\
& 10.96 \\
& 5.48 \\
& 5.48 \\
& 9.85 \\
& 4.38 \\
& 8.77 \\
& 4.18 \\
& 1.38 \\
& 6.79 \\
& 3.07 \\
& 5.92 \\
& 3.95 \\
& 3.07 \\
& 2.41 \\
& 5.92 \\
& 5.92 \\
& 3.07 \\
& 2.41 \\
& .65 \\
& 4.311 \\
& 2.41 \\
& 2.19
\end{aligned}
$$$$
\text { 1. } 10
$$$$
\text { 1. } 30
$$$$
\begin{array}{r}
.79 \\
11.74
\end{array}
$$$$
\begin{aligned}
& 11.74 \\
& 10.46
\end{aligned}
$$$$
\begin{array}{r}
10.45 \\
5.47
\end{array}
$$$$
5.48
$$$$
\text { 9. } 8 \% \text {. }
$$$$
\text { 9.8. } 16
$$$$
\text { 5. } 16
$$$$
\text { 4. } 77
$$$$
\text { 4. } 39
$$$$
\text { 4. } 33
$$$$
6.79
$$

\begin{tabular}{|c|c|c|}
\hline & \% & PER DAYI \\
\hline S'JHFAC:E & DFEP & TOTAI. \\
\hline
\end{tabular}$$
3.01
$$$$
35
$$$$
\text { 5. } 92
$$$$
3.19
$$

\begin{tabular}{|c|c|c|}
\hline & 17.41 & 17.91 \\
\hline $\begin{array}{l}1.30 \\
2.60\end{array}$ & & $\begin{array}{l}1.30 \\
2.60\end{array}$ \\
\hline 3.19 & & 3.39 \\
\hline 11. 17 & 10.35 & 15.12 \\
\hline 4.17 & 21.91 & $2 \mathrm{~K} . \mathrm{CH}$ \\
\hline 14.17 & 27.79 & 31.56 \\
\hline 4.17 & 32.87 & 37.04 \\
\hline 4.17 & 42.73 & 46.90 \\
\hline 4.95 & 47.12 & 52.07 \\
\hline 4.95 & 55.89 & 60.84 \\
\hline 4.75 & 60.27 & 65.22 \\
\hline 4.95 & 64.65 & 69.60 \\
\hline 4.95 & 71.45 & 16.40 \\
\hline 4.75 & $74.5 ?$ & 79.47 \\
\hline 5.21 & 74.52 & 79.73 \\
\hline 5.21 & 80.43 & 85.54 \\
\hline 5.21 & ค. .79 & 89.59 \\
\hline 5.21 & 97.45 & 92.65 \\
\hline 5.21 & 89.96 & 95.07 \\
\hline 5.21 & 95.78 & 100.99 \\
\hline 5.21 & 101.59 & 106.30 \\
\hline 5.21 & 104.75 & 109.97 \\
\hline 3.21 & 107.17 & $112.3 A$ \\
\hline 5. 2.1 & 107.93 & 113.04 \\
\hline 5.21 & 112.21 & $117.4 ?$ \\
\hline 5.21 & 114.62 & 119.93 \\
\hline 5.21 & 116.87 & 122.03 \\
\hline
\end{tabular}$$
3.07
$$$$
2.41
$$$$
5.92
$$$$
\text { 5. } 192
$$$$
3.07
$$$$
\begin{aligned}
& 3.07 \\
& 2.41
\end{aligned}
$$$$
.65
$$$$
\text { 4. 3A }
$$$$
2.41
$$$$
\therefore 19
$$

CAPITAL

IMILIIONS OF 1975 का S'JHFACE DFEP TOTAI.

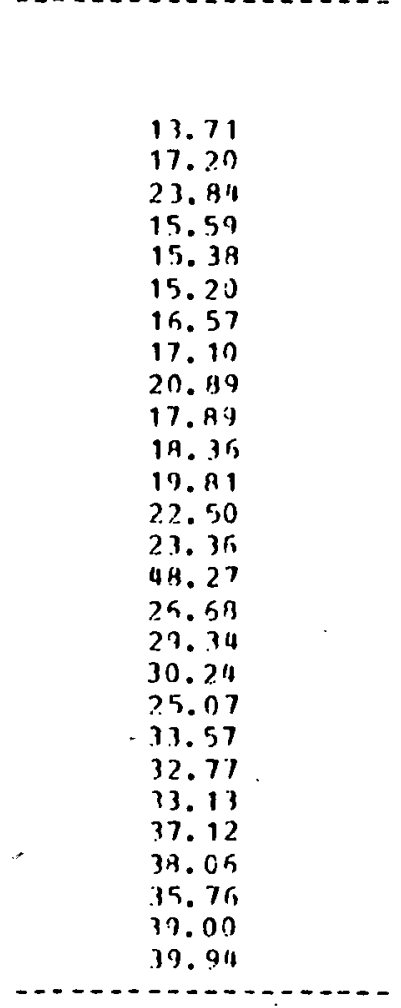

PIST CODF: 'C1

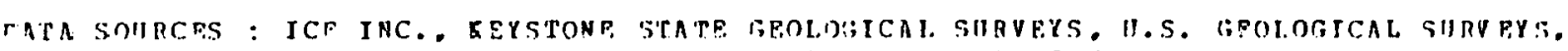

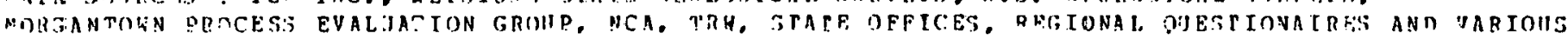

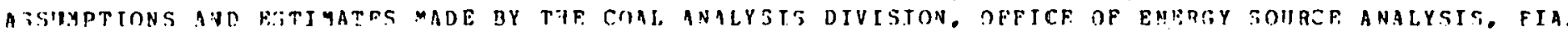

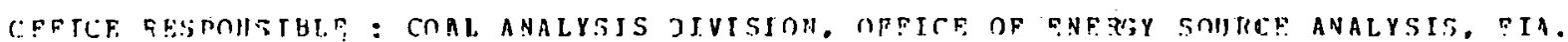




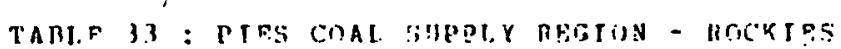

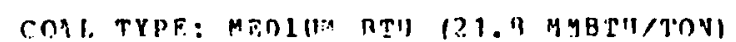

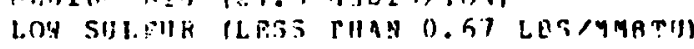

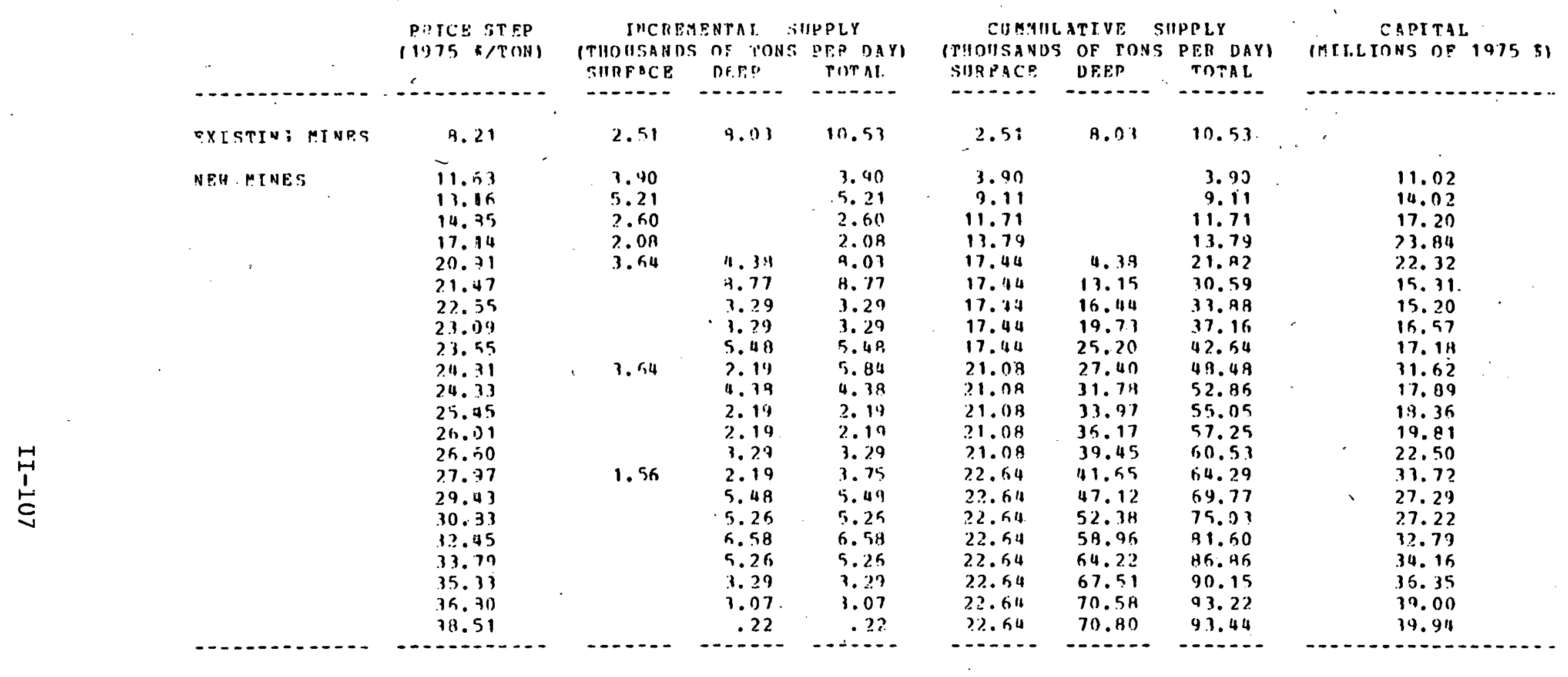

PIES CONE: $\mathrm{CZ}$

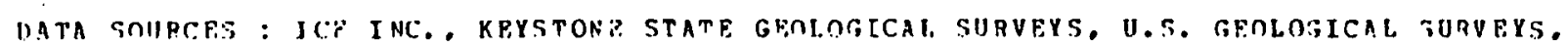

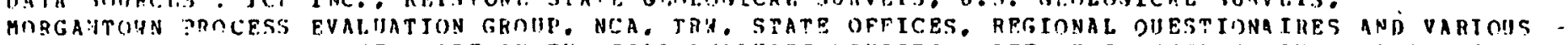

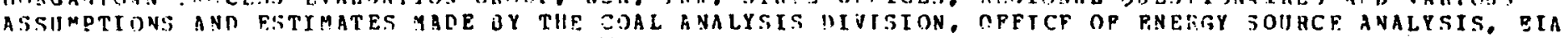

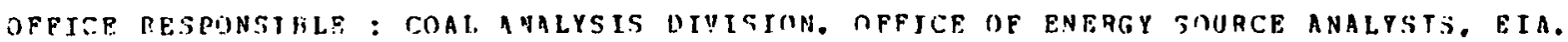


TALILF iL : PIES COAT BIIPLT RFGION - SOUTHUPST

COIL TYPE: MEDIUM BT!I $T 1$ R MMUTI/TON!

MODIII SULEUR 10.67 TO 1.58 LAS/MMUTU)

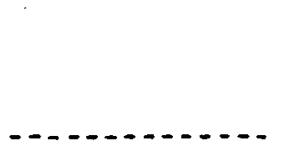

PFICE STPP (1475 $\$ / T$ (T) $)$

existigr hin

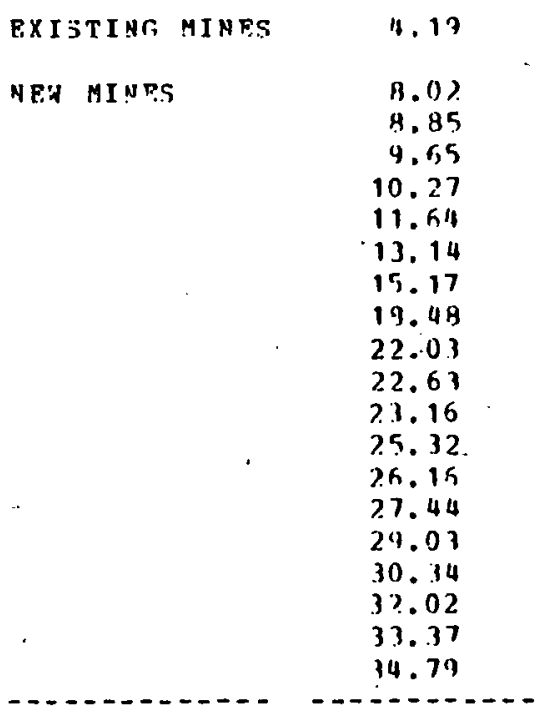

CII MH!ILATIVE SUPPLY (TIOUISANDS OF TCN' PER DAY) SURFACF DEED

$$
\text { DEEP }
$$

$$
38.73
$$$$
2.33
$$

\subsection{3}

26.03

26.07

20.96
13.22

6.71

.55

.02

.92

.27

TOTAL

ITIIISANDS

39.73
25.01
52.13
78.36
99.12
112.51
117.25
119.79
120.62
121.44
121.44
121.44
121.71
121.71
121.71
121.71
121.71
121.71
121.71
121.71
-2.0 .9

2. H 3 TOTAL

$\begin{array}{r}41.55 \\ 26.03 \\ 26.10 \\ 26.03 \\ 20.96 \\ 13.22 \\ 6.71 \\ .59 \\ .97 \\ 3.01 \\ 2.19 \\ 2.19 \\ .27 \\ 2.19 \\ .44 \\ 2.63 \\ .44 \\ 2.57 \\ .44 \\ .44 \\ \hline .0 .9\end{array}$

26.03 52.33

78. 35

79.32

112.53

119.25

119.79

$1 ? 0.62$

2.19 123.53

4. 35 125.9?

$6.57 \quad 128.01$

$\begin{array}{ll}6.57 & 128.01 \\ 6.57 & 128.29\end{array}$

ค.77 1.30.4A

9.25130 .32

$11.83 \quad 133.55$

$12.27 \quad 133.99$

$14.90 \quad 136.62$

$15.3 \mathrm{~L} \quad 137.05$

$15.78 \quad 177.49$
CAPITAL

(MILLIONS OF 1975 s)

- - - - - - - - - - - - - - - -

$\begin{array}{r}7.83 \\ 8.16 \\ 10.72 \\ 11.13 \\ 13.20 \\ 15.10 \\ 17.07 \\ 29.57 \\ 19.81 \\ 16.57 \\ 17.94 \\ 39.62 \\ 27.53 \\ 23.36 \\ 28.49 \\ 30.24 \\ 35.34 \\ 37.12 \\ 19.06 \\ \hline\end{array}$

PTES CODE: CS

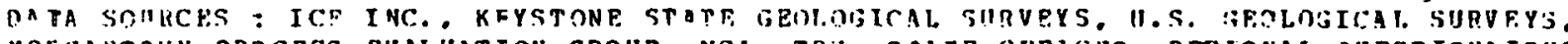

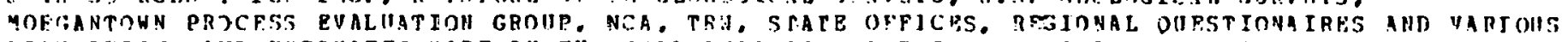

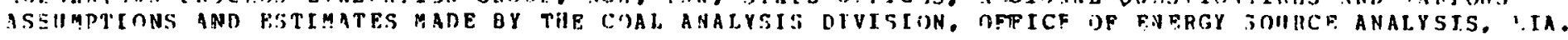

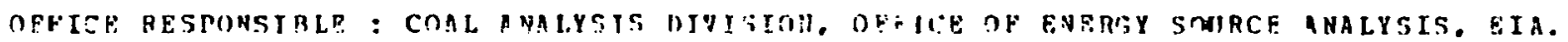


TAB:Y 15 : PIFS COAL S!PPI,Y HEGION - SOIPHHEST

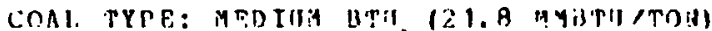

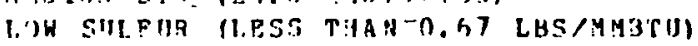

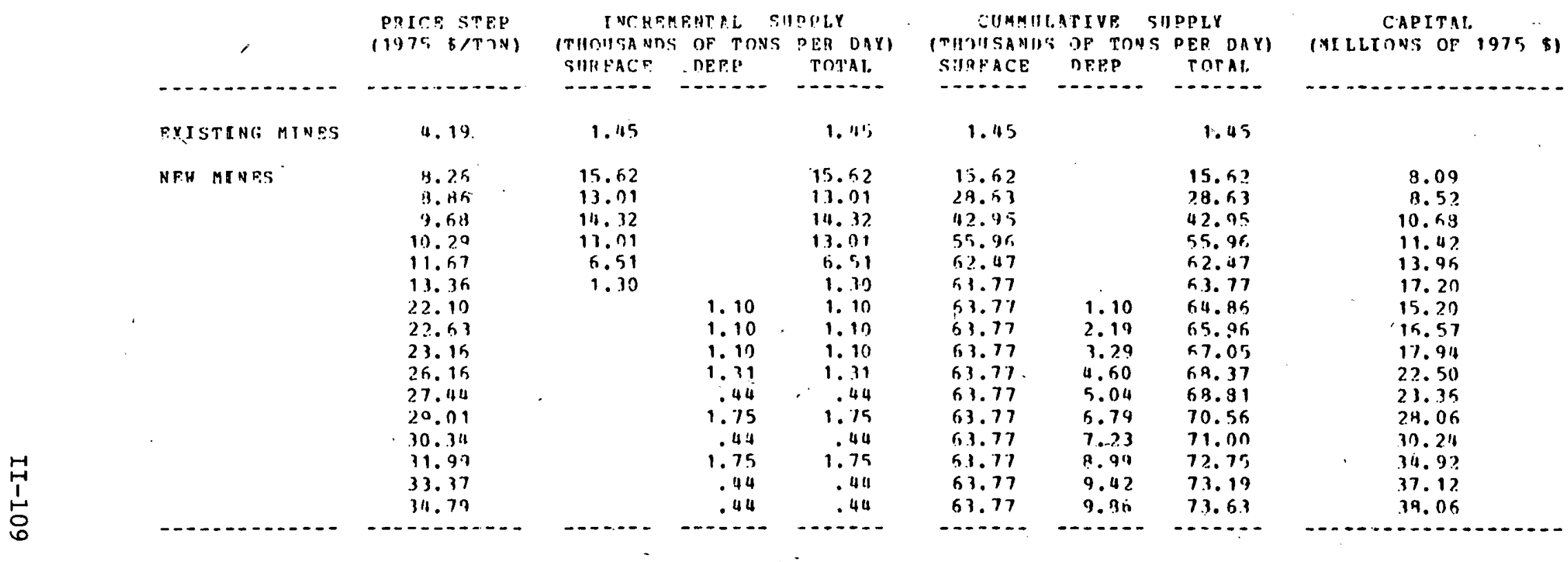

PIES CODE: CZ

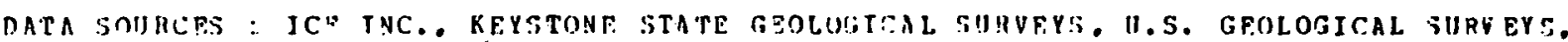

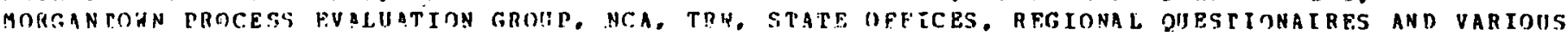

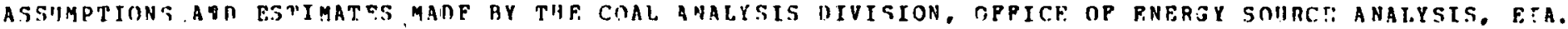

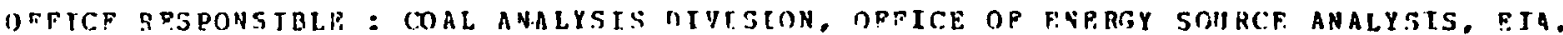


TALLE If. : PTES COAL SIIPPI.Y RPGTON - NOFTHUEST

COAI TYPE: SIB-DITHMLNOIIS (19.3 MMRTH/TON)

MPDIIM SUFUR IGREATFR THAN 0.57 LASMMJTUI

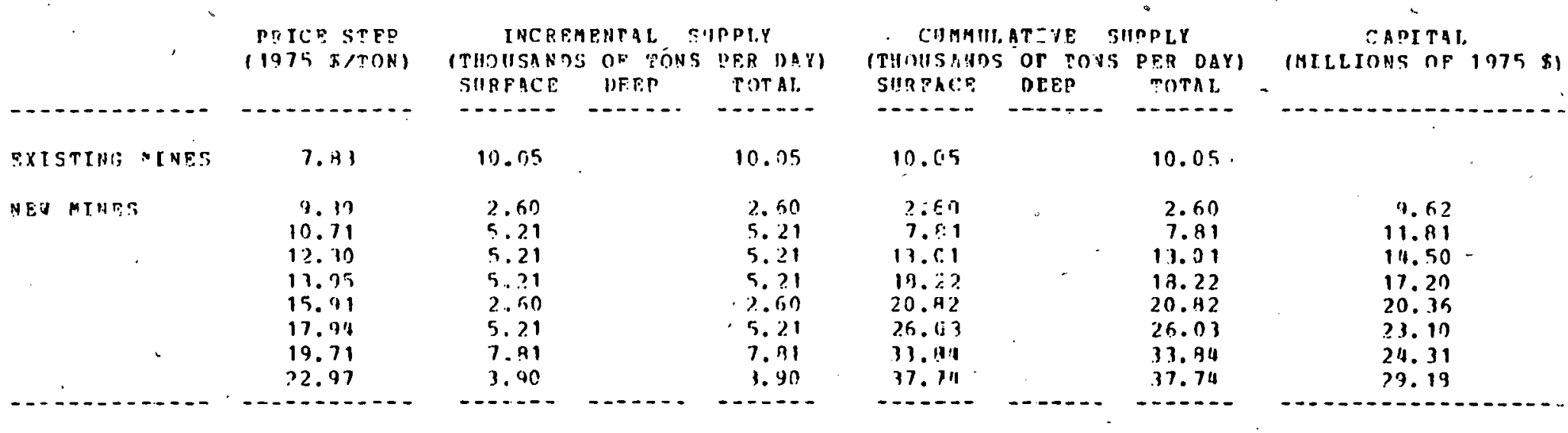

PIPS CODE: CY

DATA SOIRCES : ICF INC. KEYSTONE STATE COFOLOGICAL SURVEYS, D. S. IFFOLOICAL SURVEYS.

MOIGANTOHN PRTCESS EVALIIATION GROIP. NCA, TRE, STATE OFPICES, REGIONAL OUESTIONAJRES AND VARIOIS

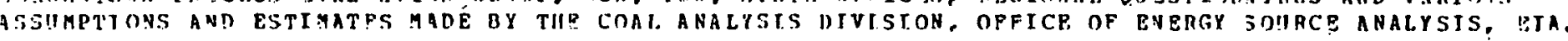

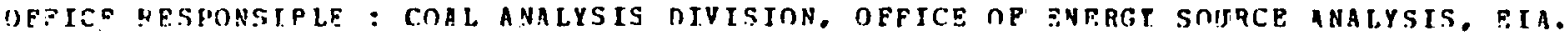


TARIF 17 : PIES COAL SUPPLT HERTOUN - AJASSA

COML TYPE: SIR-RITINIMOTS (14. 1 MMBTH/TON)

- LOW SIII.PUR ILESS TIAN D.67 LRS/MYBTH

PRTCE STFP

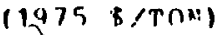

-...-........

FXISTIH: MIHFS

NFU MINES

\begin{abstract}
3. 34
\end{abstract}
22.19
IACREMENTAT. SIMPTI.Y ITHOUSANDS OF TONG PER DAY) SHRFACE DFPL

-....-

2. 111

27.40

2.65

2.3 .65
2.9 .34

$30.0 \%$

33.92

$37.7^{\circ}$

11.65

100.00

-.....

- rothl.

$$
\text { S }
$$

CUMMULATTVF SUPPLY SIIUI:ACF OF TONS PFO DA InTAi.

2.111

\begin{tabular}{rr}
26.10 & 26.30 \\
$21.9 ?$ & $31.9 ?$ \\
19.96 & 10.95 \\
12.05 & 12.05 \\
12.05 & 12.05 \\
12.05 & 12.05 \\
10.96 & 10.96 \\
10.96 & 10.96 \\
11.18 & 11.14 \\
& .26 \\
\hline
\end{tabular}

.

$\rightarrow-\cdots+$

\begin{tabular}{rr} 
& 2.14 \\
26.30 & 26.30 \\
48.27 & 49.22 \\
59.14 & 59.14 \\
71.23 & 71.23 \\
93.27 & 83.29 \\
95.34 & 95.34 \\
106.30 & 106.30 \\
117.26 & 117.25 \\
128.44 & 127.14 \\
128.44 & 128.70 \\
\hline
\end{tabular}

CAPTTAL

(AILLEONS OF 1975 \&)

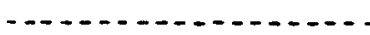

$\begin{array}{r}9.61 \\ 14.67 \\ 15.65 \\ 15.29 \\ 16.57 \\ 17.41 \\ 22.50 \\ 29.311 \\ 36.19 \\ 80.85 \\ \hline\end{array}$

PIES CODP: CX

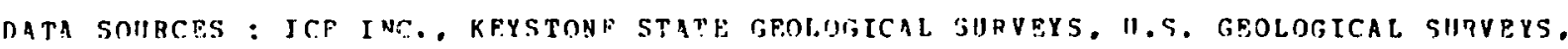

MORIIANTOHN PIOCFSS EVALIIATION GROIP. NCA, TRA, STATE OFFICES, RFGTONAT OUESTIONATRES AND VARTOUS

ASSUMPTIONS END FSTIMATES MADE BY THF COA. ANALYSIS DTUISION, OFYICE OF ENERGY SOIIREE ANALYSIS. FIA.

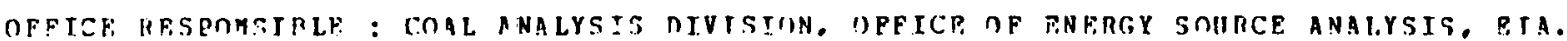


THDLE 3H: ROPGL COA: SIIRLY AT TIE HIGHEST PRICD STFP

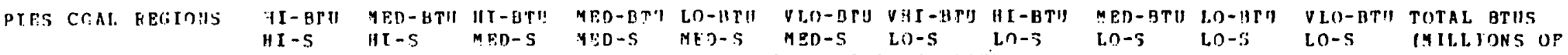
(THU)SANIS OF TONS PQR DAY) BTU PFR DAY)

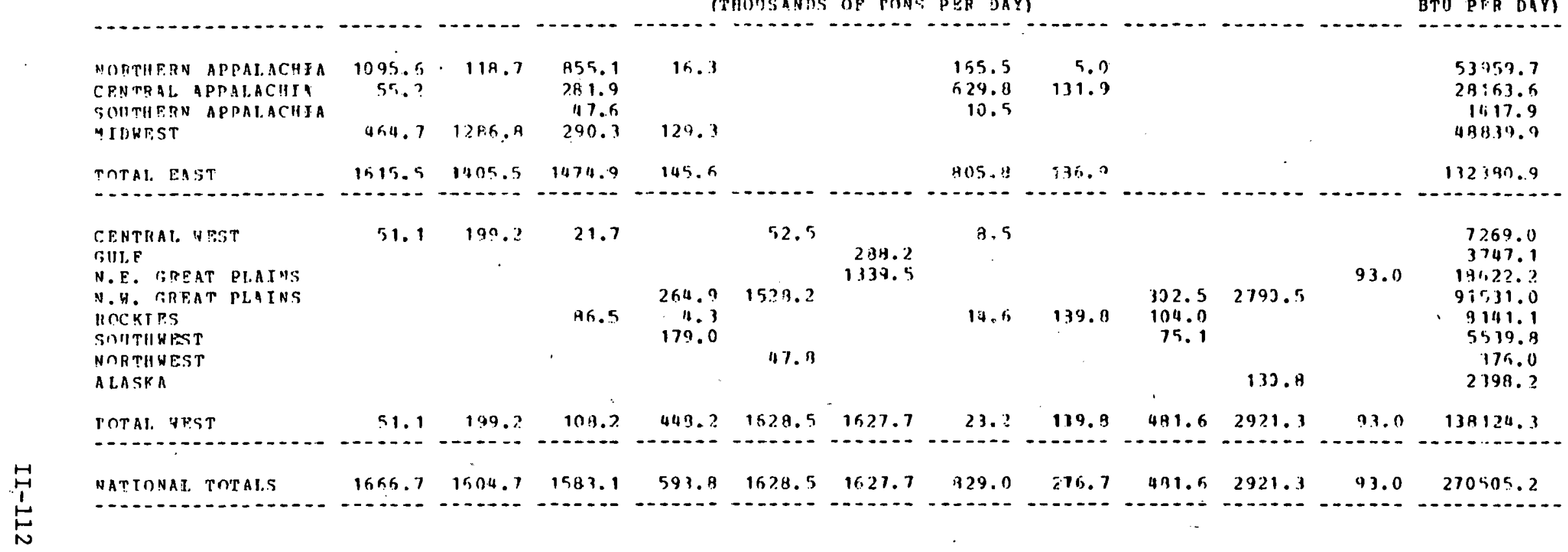

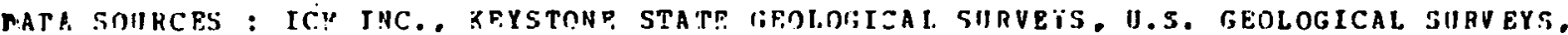

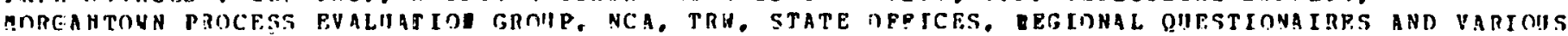

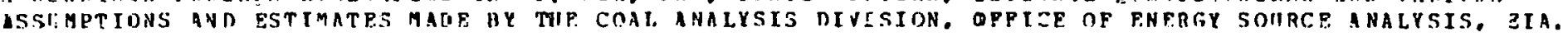

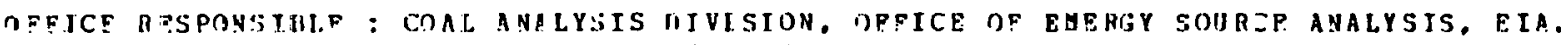
COAL TYPF. DESCIPIORS

\begin{tabular}{|c|c|}
\hline \! I-BTI/HI-S & $\begin{array}{l}\text { HIZH BPI (23. B YYBTU,TON) } \\
\text { HIGH SULFUR I'BHSATEQ THAN } \\
\end{array}$ \\
\hline$m E n-\pi r \| \prime I-S$ & 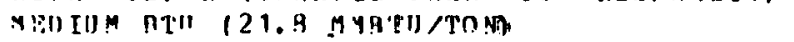 \\
\hline HJ - RT:I/MER-G & 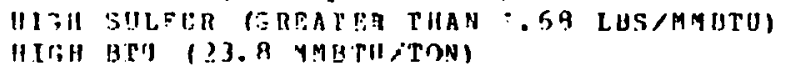 \\
\hline & 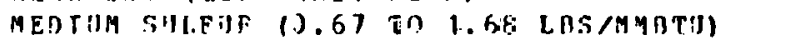 \\
\hline$M F R-n C U / M E D-S$ & 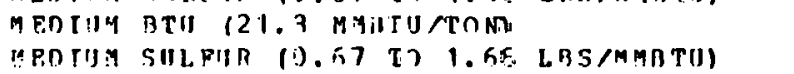 \\
\hline I.0-BTH/Y!D-S & 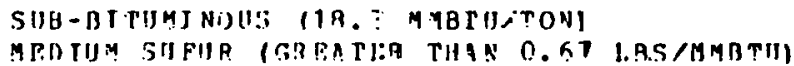 \\
\hline VI.O-BTH/ & LTANITE 113.0 MUBTHITO!I \\
\hline$V\|! I-B ?\| / L O-S$ & 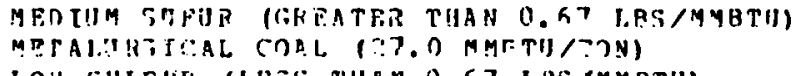 \\
\hline "II-BTE/LC -S & 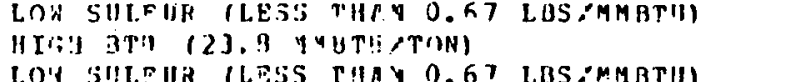 \\
\hline$\because F n-F+1 / / L O-F$ & 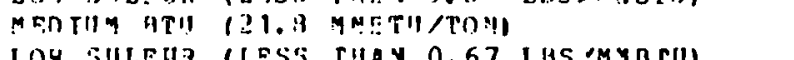 \\
\hline$I, n-j \cdot n ! / L(i-5$ & 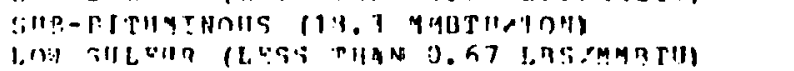 \\
\hline$" 1 . n-3-21 / 1 .: 10-! ;$ & 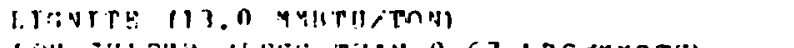 \\
\hline
\end{tabular}


IMPORTS DATA

This section contains data documentation for the PIES imports sector. TABLES 1 through 4 and 5 through 8 give alternative levels of crude and oil product imports for the target years, 1985 and 1990, respectively. From the four tables given, for each target year, one must be chosen to maximize the level of product imports while maintaining at least 90 percent current capacity utilization in domestic refineries. Each of the four tables corresponds to a specific scenario as indicated in Table II-1. Petroleum product import costs and availabilities are given in sub-tables. Petroleum product export levels and revenues are also shown where they apply.

TABLES 9 and 10 give natural gas imports by demand region for 1985 and 1990, respectively. TABLE 11 breaks out steam and metallurgical coal exports and amount of fixed export by target year. TABLES 9 through 11 correspond to the mid-range scenario.

TABLE II-1. CORRESPONDENCE BETWEEN OIL IMPORT TABLES AND EIA SCENARIOS

\begin{tabular}{rrll}
$\frac{1985}{1}$ & $\frac{1990}{2}$ & & \multicolumn{1}{c}{ Scenario } \\
2 & 6 & Medium Supply \\
3 & 7 & Low Supply \\
4 & 8 & $5 \%$ real increase in world oil prices
\end{tabular}



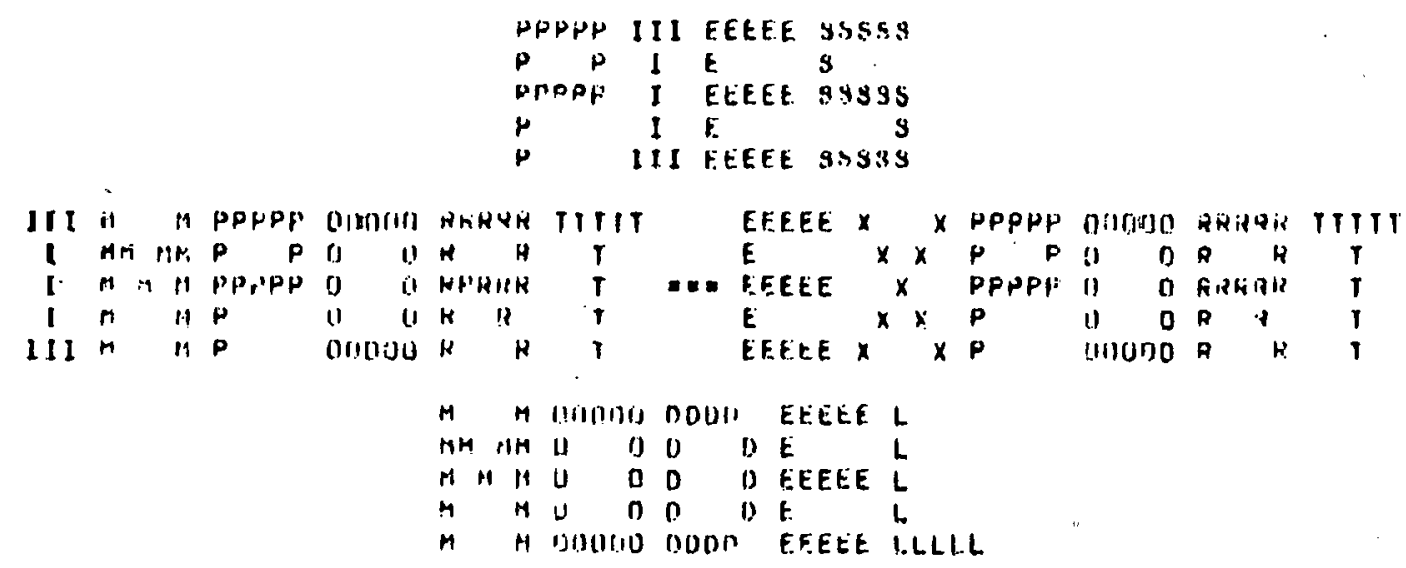

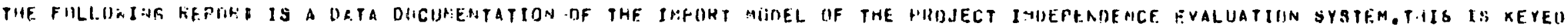

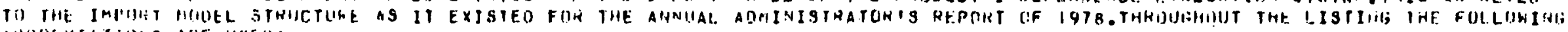
AHBREVIATEBSS AFE USGE

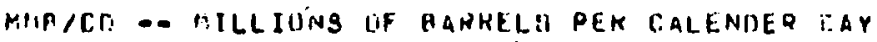

HH/CD - THIIUGANDS UF HAIIHELS PER CALENDEN JAY

S/WUL - 1978 DULLAWS PER HARHEL

MACF/CD - MILLIUNS TIF CUIAIC FFET MEH CALENISE DAY

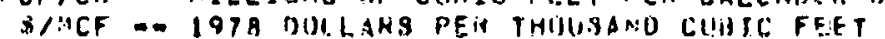

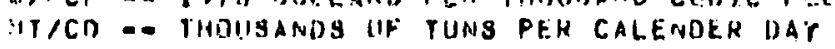




\begin{tabular}{|c|c|c|c|c|c|c|c|c|c|c|c|}
\hline 116111611$]$ & III & l. & 111 & 11 & 14 & PIPPPP & filiditi & RHK & KN & TIT & S\$53S \\
\hline 1) & I & L & I & $H M$ & 1111 & $p \quad p$ & (1) 0 & $\mathbf{k}$ & $R$ & $T$ & \\
\hline () & 1 & l & I & 111 & $M A$ & PPPPP & (1) & KRK & रH & $T$ & 9SSS9 \\
\hline 11$)$ & I & & 1 & $M$ & .1 & $P$ & u & R & a & $T$ & 5 \\
\hline
\end{tabular}

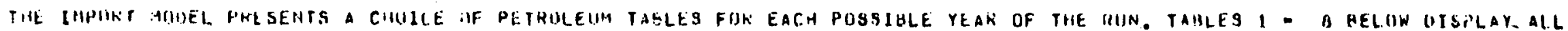
AVAILAGLE TAHLE. IIIHE SPECIFICALLYI

\section{TABLES 1 - W.FUH YEAY 1985
TABLES. S- A FUK YEAK 1990}

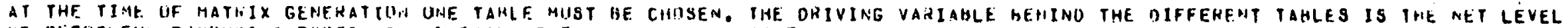

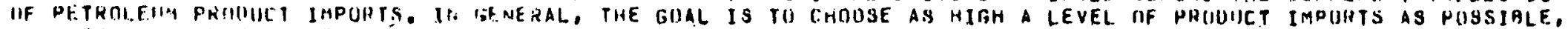

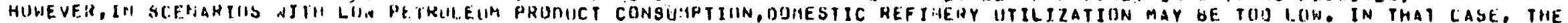

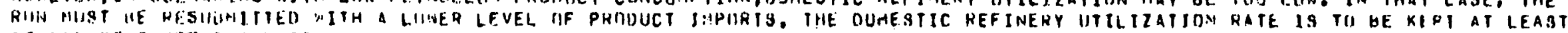

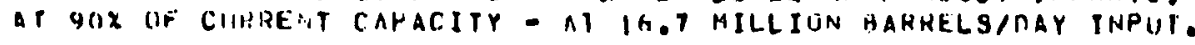

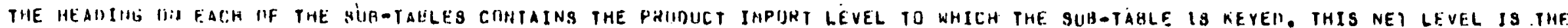

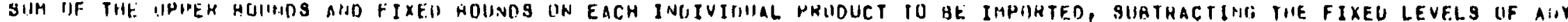

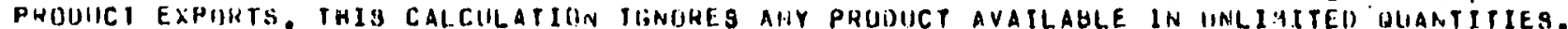

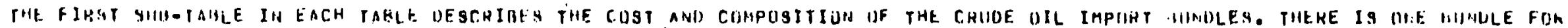

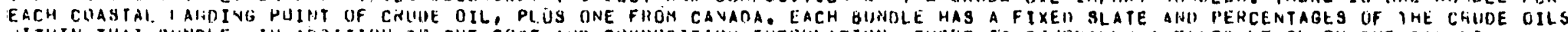

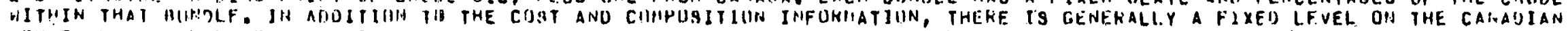

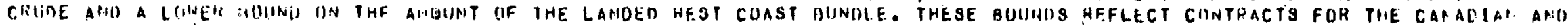

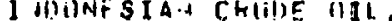

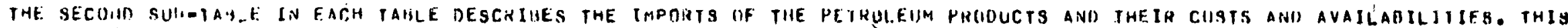
SIIU-TAHLE WAY INCI.UIIE AIIY IH ALI. UF THE FILLULING CATAGNHIES:

$$
\begin{aligned}
& \text { 1. THOSE PRODIICTS IAPIJA IFI) AT A FIXE() LEVEL, }
\end{aligned}
$$

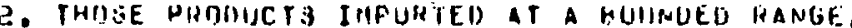

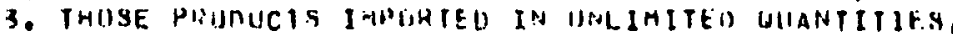

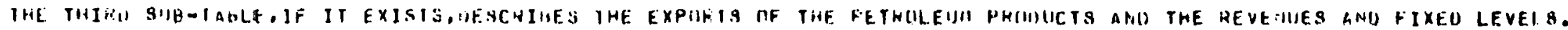

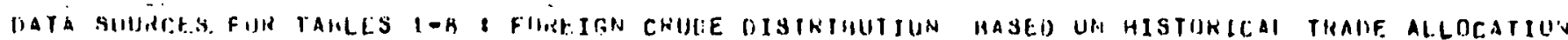

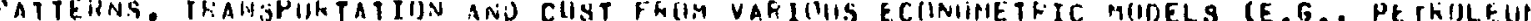

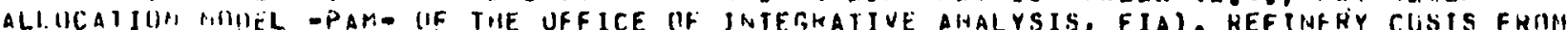

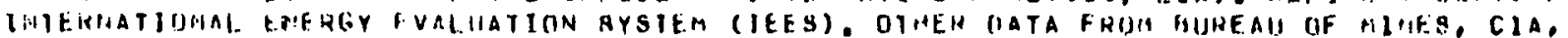
H'FC, IIECN, EIC.

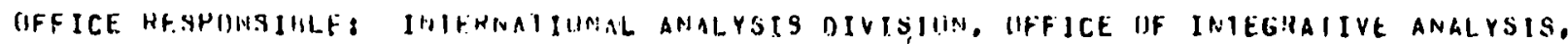
APMI. IFI) APALYSISEEIA, BIIL 
YABL.F I

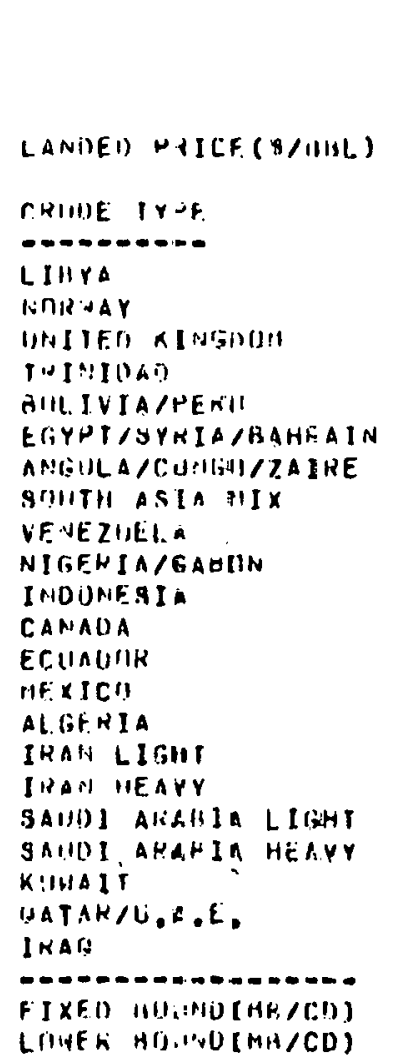

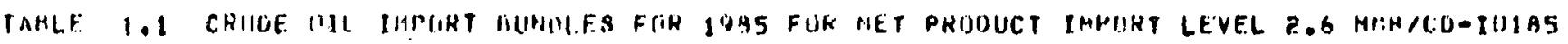

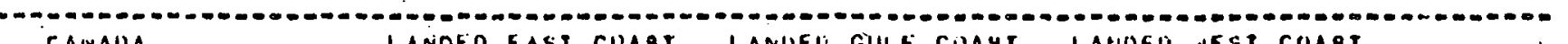

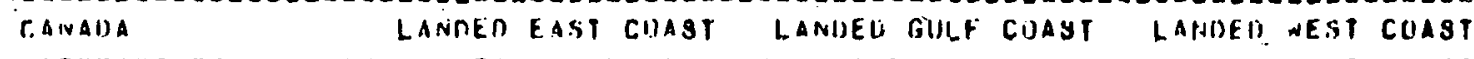

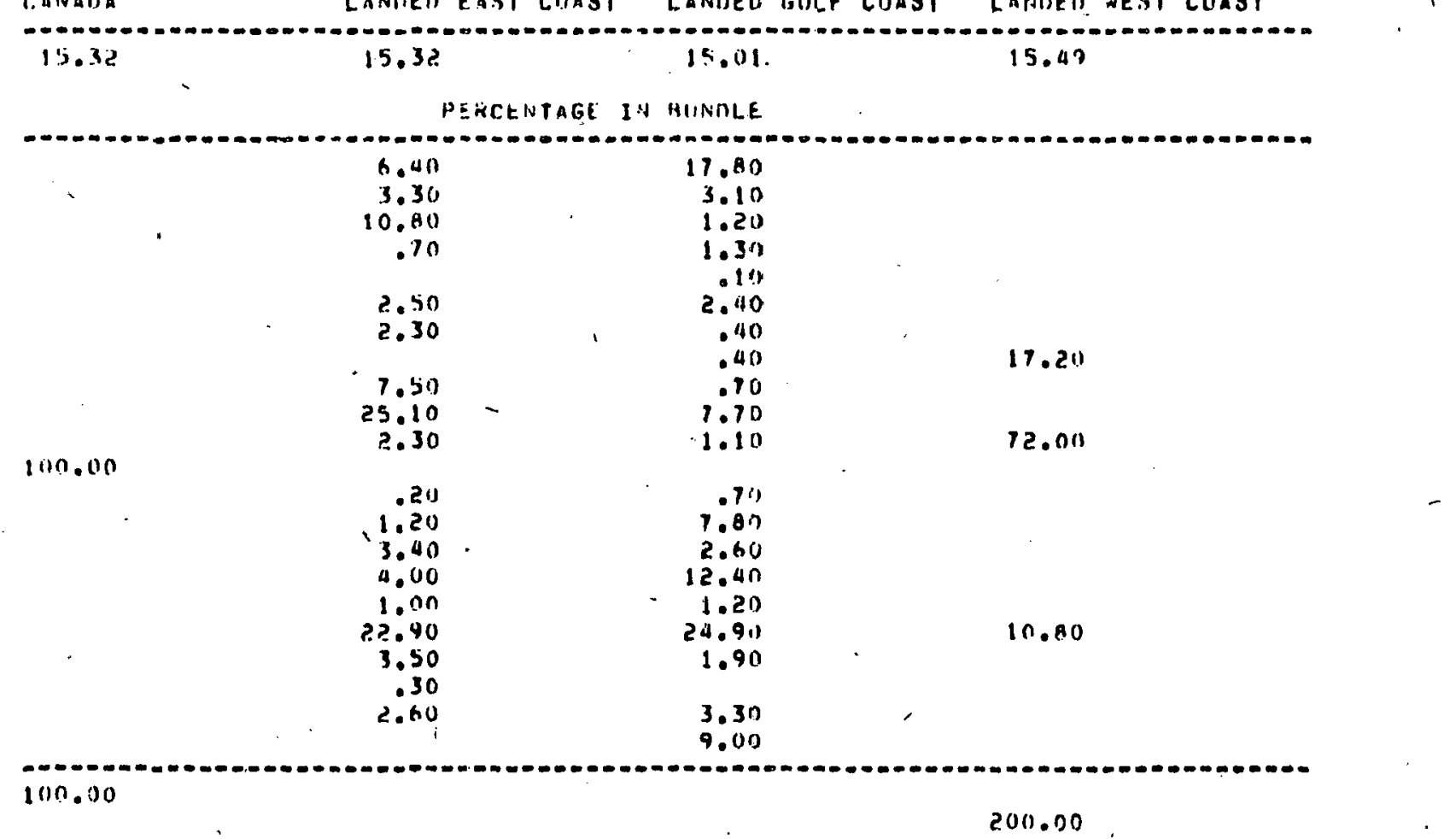

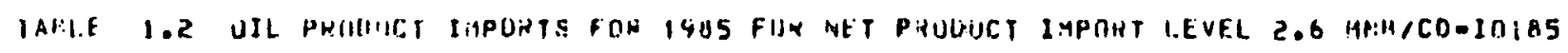
FIXEN CUUANTITY (AH/CU) PHICE (S/GBL)

\begin{tabular}{|c|}
\hline $\begin{array}{l}\text { LASIILIME, ALL. GHADES } \\
\text { OISTILLAIE, ALL GHAUE. } \\
\text { JET FUEL JET A } \\
\text { NAPIITHA }\end{array}$ \\
\hline UTHER KFFINED PETHOL. \\
\hline WESIMIIAL ALL GHAEES \\
\hline
\end{tabular}

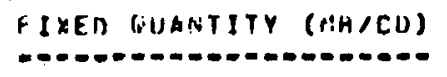

21.90

$\begin{array}{ll}112 & 21.90 \\ 376 & 18.26 \\ 216 & 15.63 \\ 54 & 16.81 \\ 104 & 16.81\end{array}$

BOUNAEU HICAHTITY (MH/CO)

LLEEH MIIINO (HIICO)

PNICE (S/GAL)

h94

15.65

IS AVAILABLE IIS AN UNLIMIIED JUANTITY AI 13.10 SIHBL 
IABLL 5

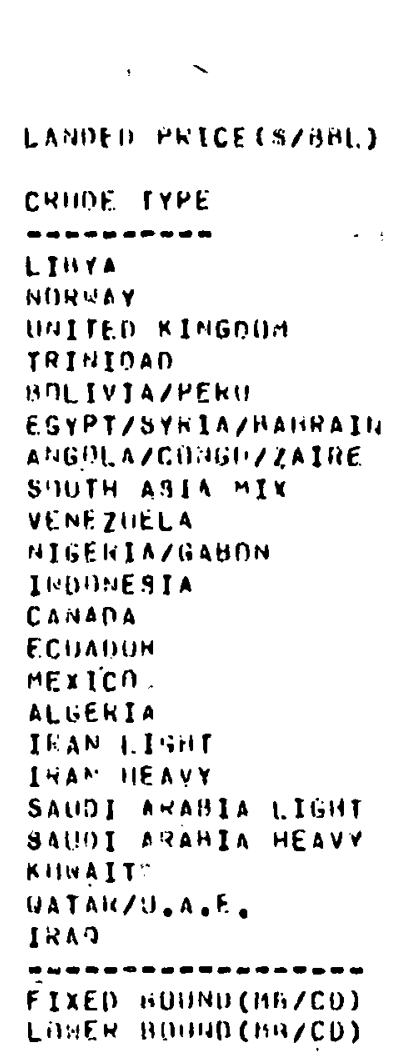

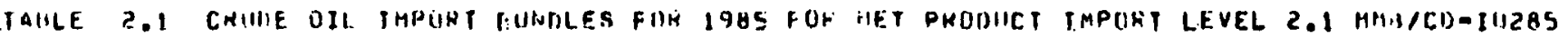

LANAIIA
15.32
NERCENTAGE IN BUNDLE

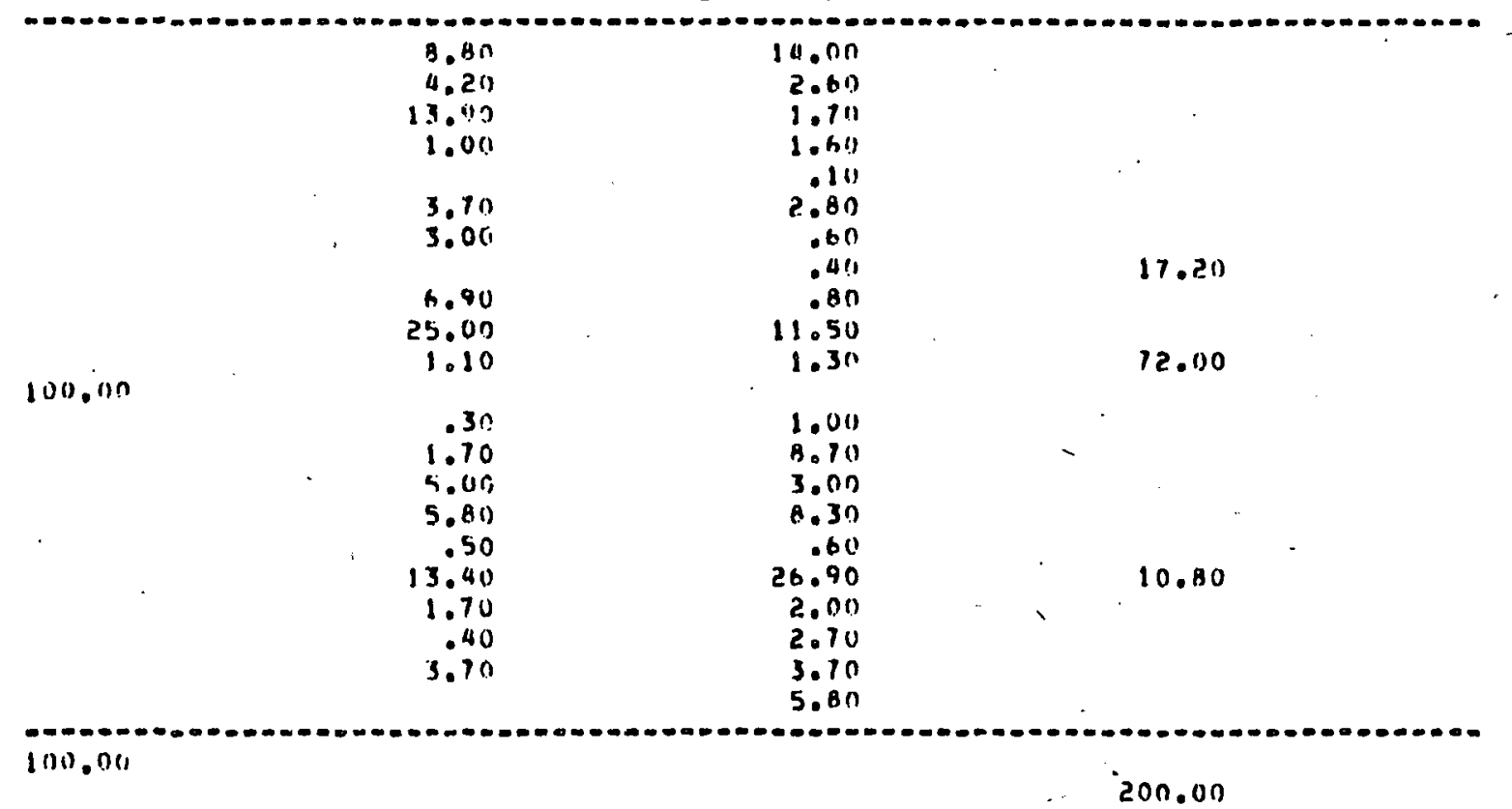

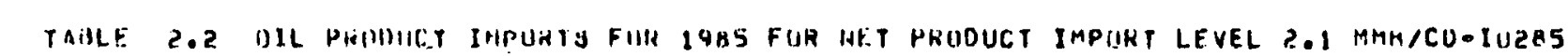

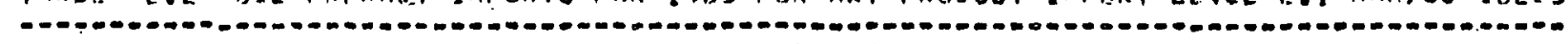

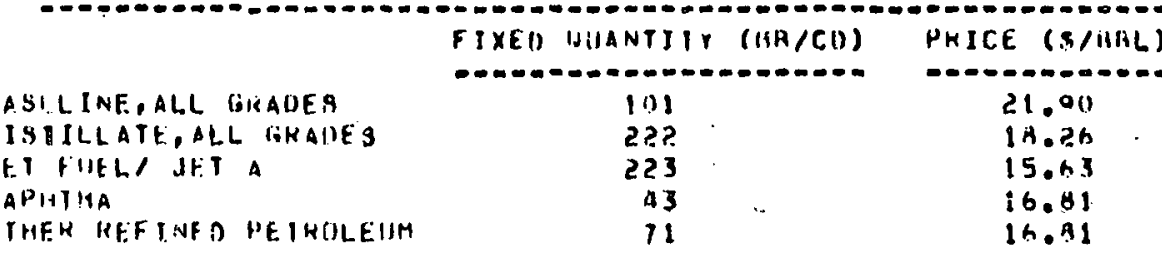

DTHEK HEFINF PEIHOLEIIM

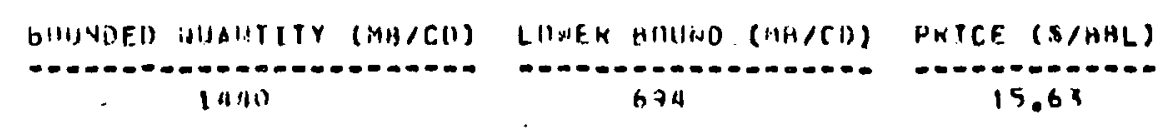

WHEJIHIAL, ALL GHAIIT:S

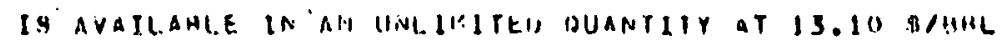




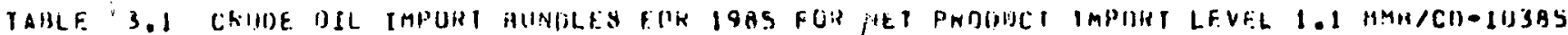

LAINDEO PNICE ( $/ H H L)$.

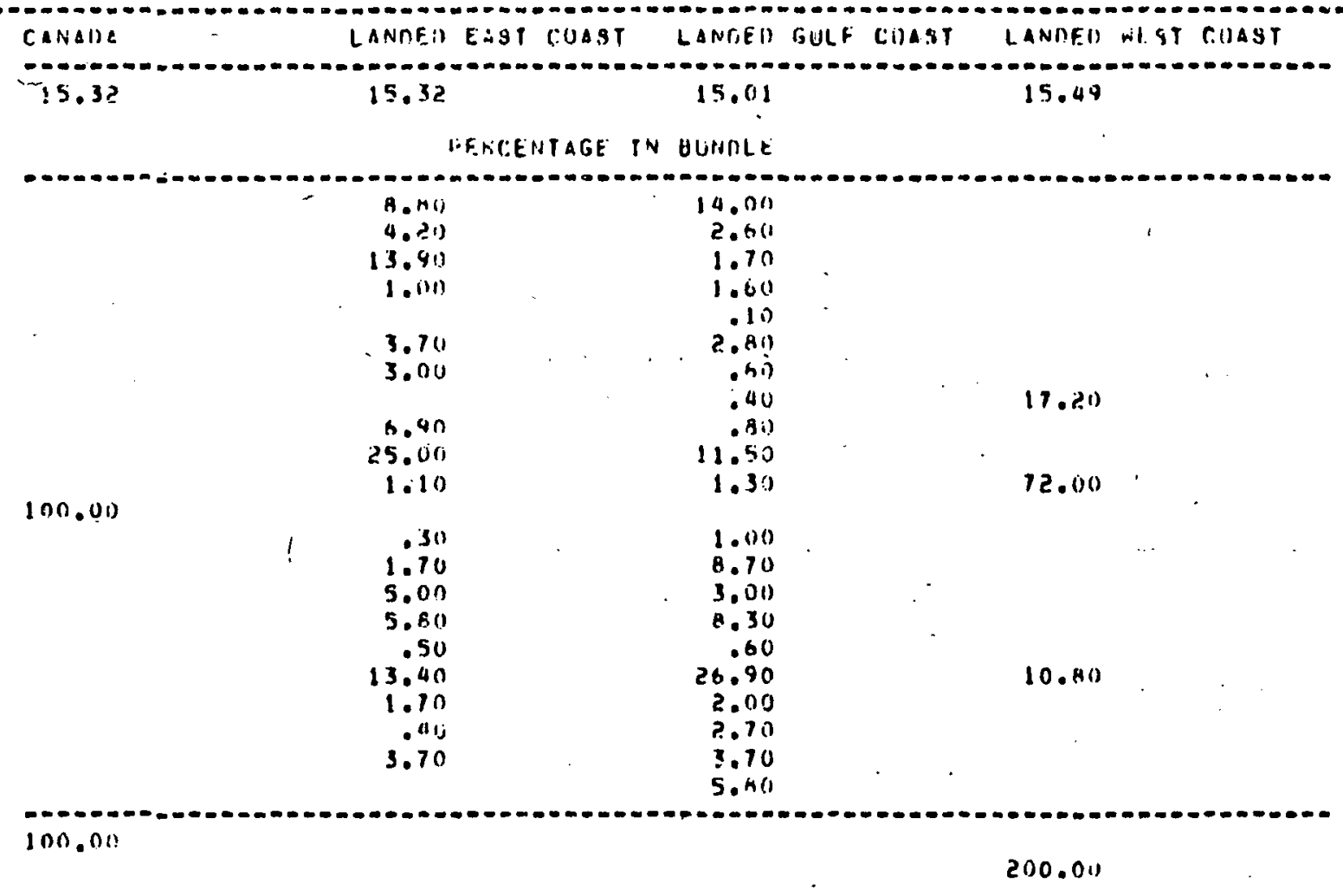

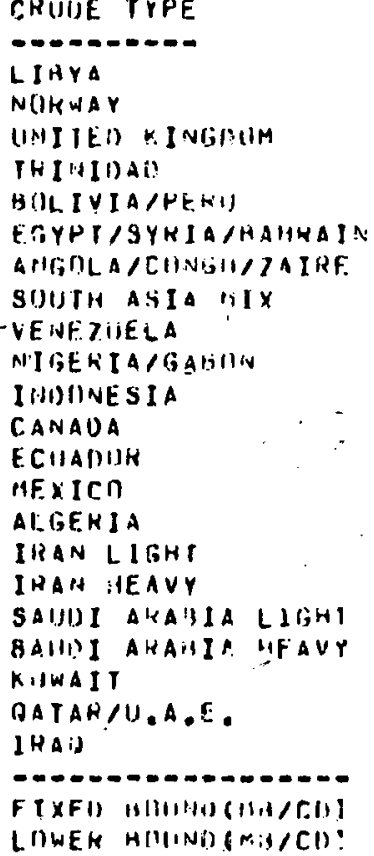

LIWEH H(IINT) (miS/Cl):

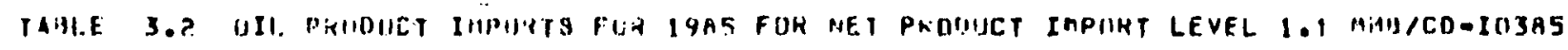

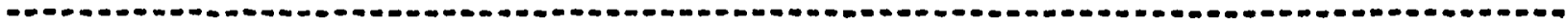

\begin{tabular}{|c|c|c|}
\hline & FIXEN HIIAIATITY $(A B / C O)$ & PIICE ISH.6HL \\
\hline 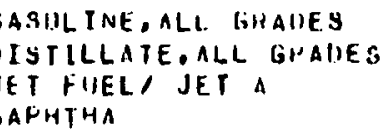 & $\begin{array}{r}10 \\
27 \\
177 \\
14\end{array}$ & $\begin{array}{l}21.90 \\
18.26 \\
15.63 \\
18.81\end{array}$ \\
\hline
\end{tabular}

DET FUEL, JET A

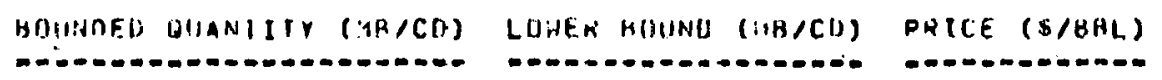

HFS IMUIAL, ALL. GHAUES

641

15.63

LIHUIU PET GASSFS

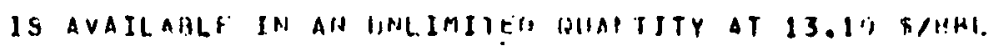

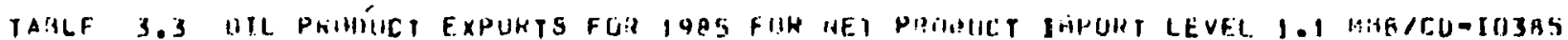
1AILF J.

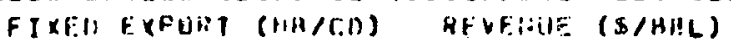




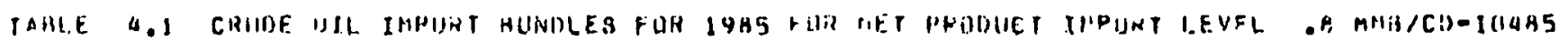

LAWHF.I) PHICE (E/HIHI.)

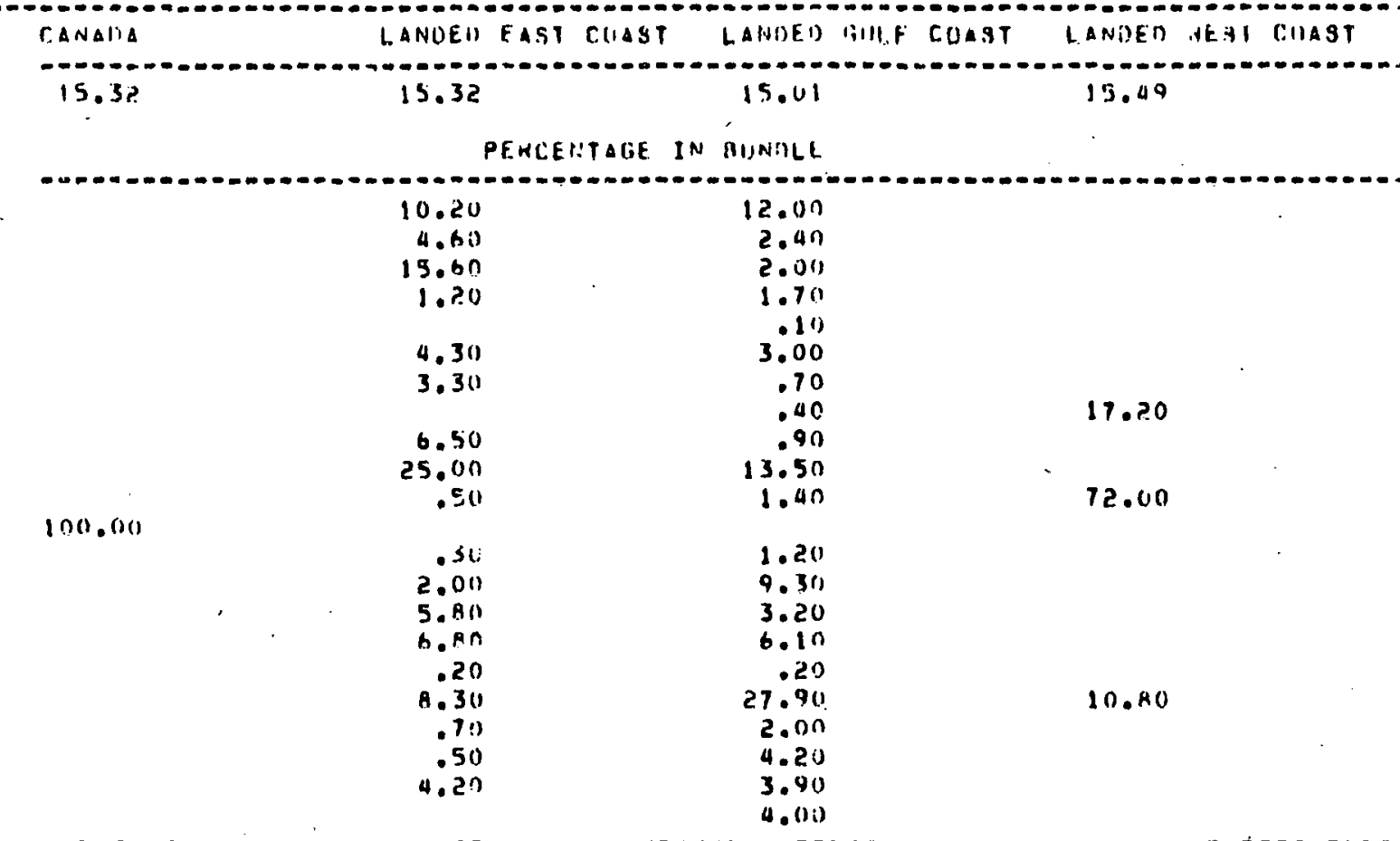

\section{CaUIR. Irite}

Lillya

NHINAAT

UNITEO KINGOUII

TIINIIIA!)

HIILIVIAIPEHII

EGYPT/SYNIA/BAHEAII

A INGOLL / CUPIGO/ZAINE

SHUTH ASIA HIX

VENEZIIEI.A

NIGEKIA /IFABIOIV

I WDIINE.SIA

CANATIA

Ecunulia

ME. XI I . U

Al IiFWJA

Jhan listill

lirais lif A Q W

SALIUI atiabia l.trilt

SAUIDI AHALIJA HEAVY

KII:ifal

TATAN/U.A.E.

JWAH

tixeris

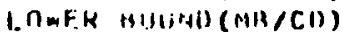

(1)(1.00)

$200.0 n$

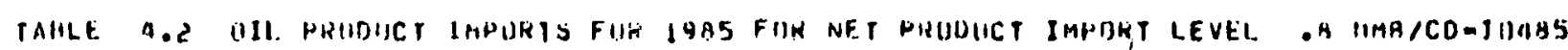

Fixen DUANTIYY (11M/CR) PKICE (s/HuL)

Fixen

HESIIIIAL, AI,L GNADES

JE. P UEI.I JFT A

NAP It TIIA

624

19.63

$\begin{array}{ll}118 & 15.03 \\ 5 & 16.81\end{array}$

L IIIJI!I PET, GASGHS

IY ANAILAII.E JN AN UNLIMITEII MUANTITY AT 13.10 S/II+1.

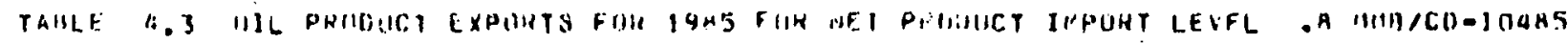

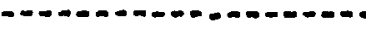

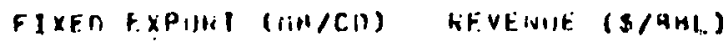

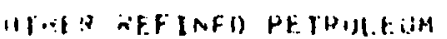

47

16.61 
IAIILE: 5

-

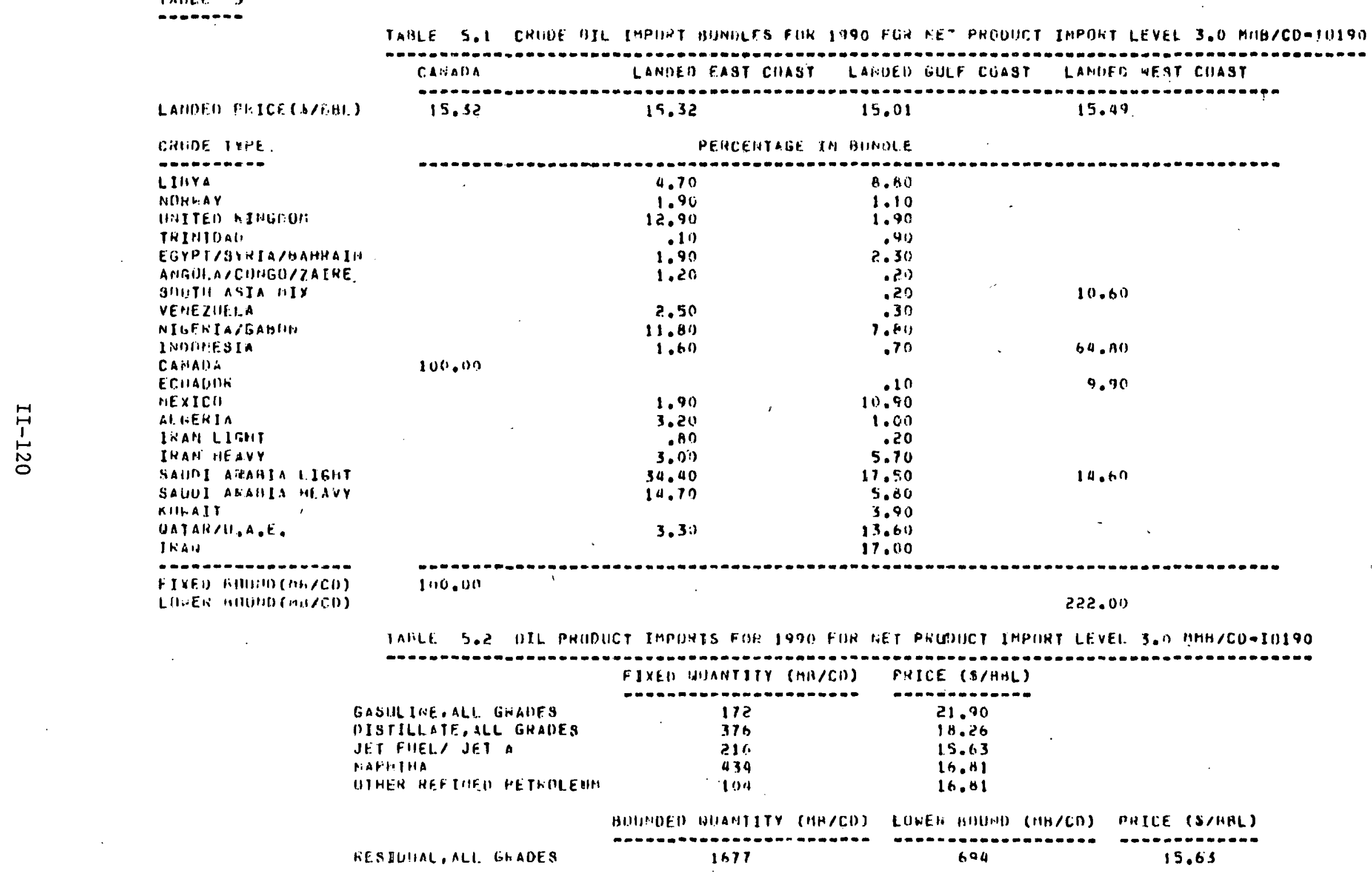

LIUI II) PE]. ISA 39E

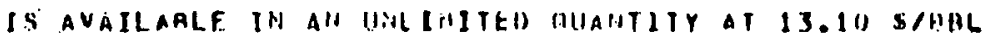


TABLE 6

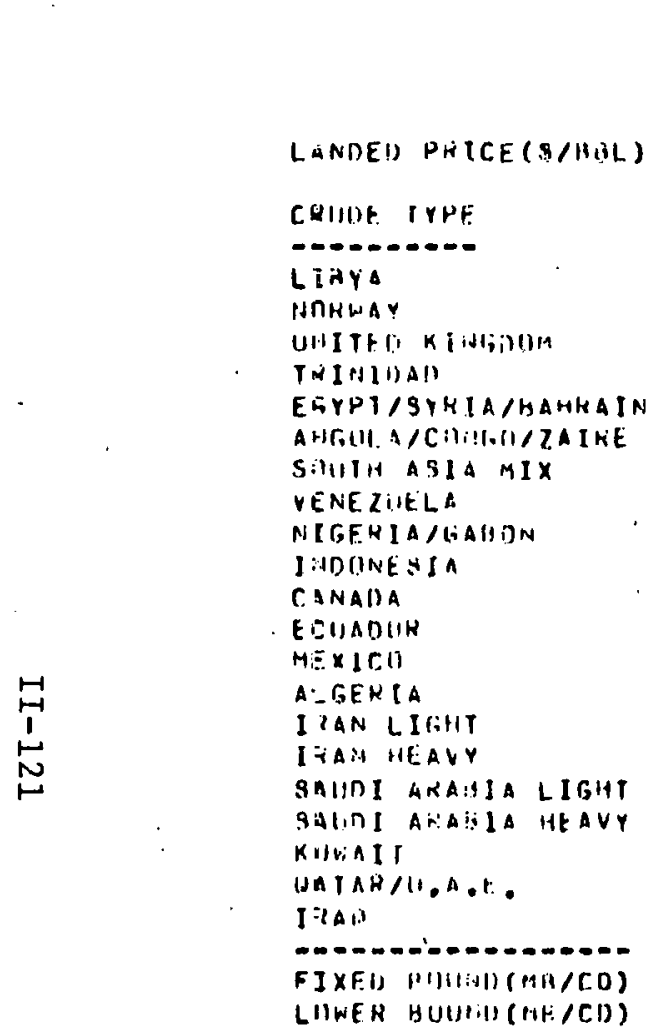

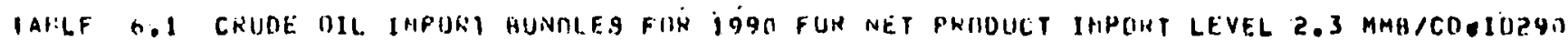

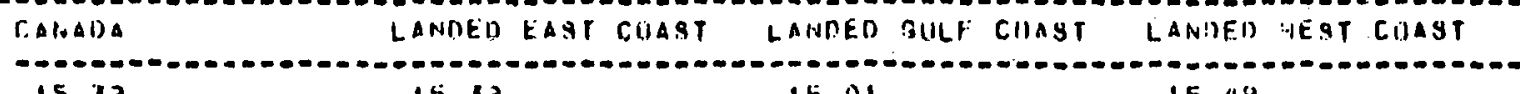

$15.3 ?$

15,32

15.111

15.49

PERCENTAGE IN BUINDLE

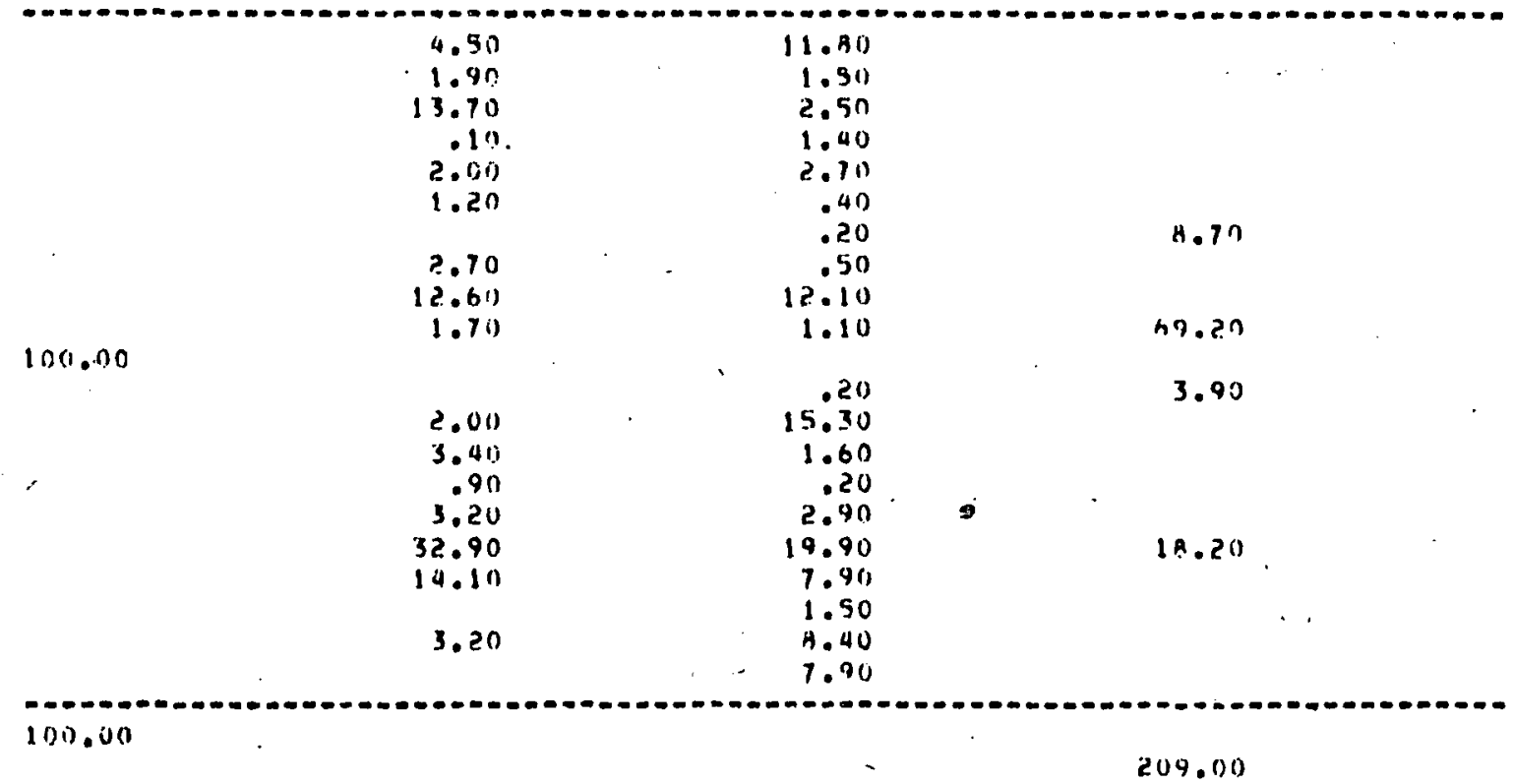

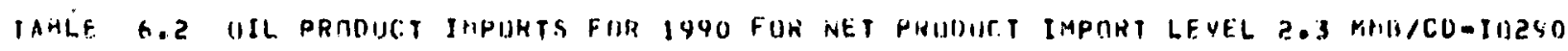
EIXET WUANTITY (IMH/Cn)

GASIIL INE, AI.L GKKALES IIISTILLATE, ALL BHAUES JET FIIELL JFT A HAPHTHA

MTHEA REF INEO HETRIIIEUM

17
171
221
4011
sh

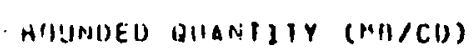

1337 PRICE (S/BRiL)

PH.

21.90
19.26
15.63
16.81
16.81

LIVER MUIJN) (MH/CW) 691

PHICE (S/BHL)

METJUIIAL, ALL GKAIESS

LIIIII!) PET, FABSES 
TABLL. I

LAIRED PRICF(S/HILL)

CRIJDE IYPE

LIHYA

NIJR'NAY

UIIITEO KIPIR,On!?

ININIDAO

EGYPT/SYHIAIIIAHHEAIV

A IIGIILA / CIINGGIPZATKE.

3OUITH ASIA MIY

VENEZIIR.I.A

NIGE I I / GAMIOIH

INDIINESIA

CANADA

ECUADIIT

HEXICO

AI.GER IA

IRAN LISGH

IQAII liEAYY

SAIIIII AKABIS LIGHT

SAIIIII AHAIIA HEAVY

AIHA 11

IATAHAU.A.t:

IHA(1)

F(1)

LOAEY HIIIII) (IIE/CD)

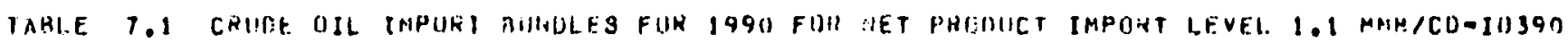

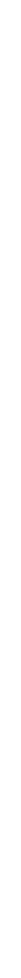

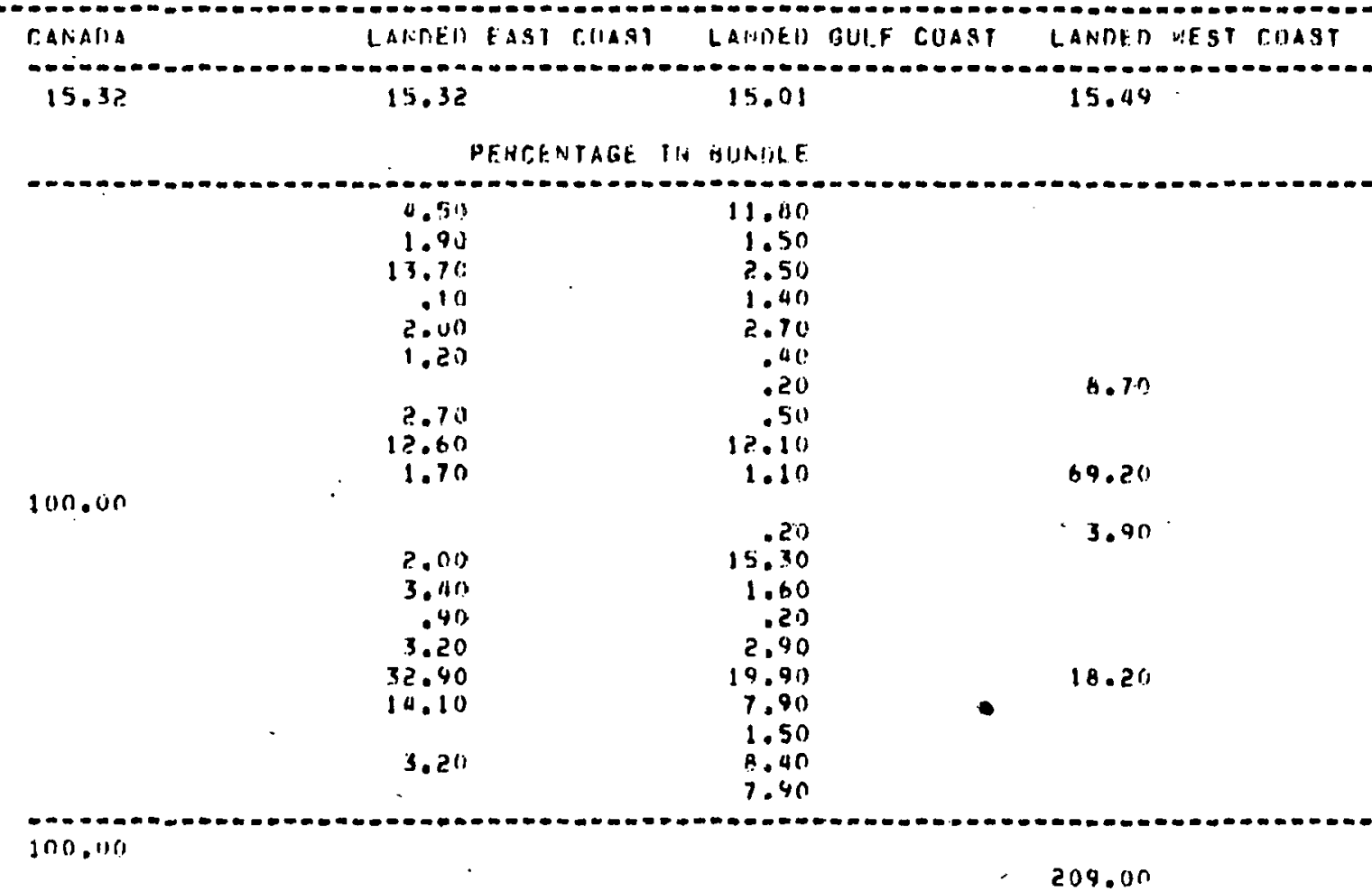

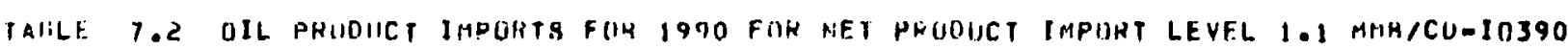
FIXEO BIJAITIIY (HA/CO) PKICE (S/HHL)

$\begin{array}{cc}159 & 18.26 \\ 210 & 15.63 \\ 210 & 16.81\end{array}$

DIEIILLAYE, ALL GKAUE: JET FIIEL, JET a WAFHTHA

BOINOEO UUAHITY (HH/CO)

LIWEK RIIUND (HH/CW) HUICE ( $\$$ /HHL)

694

15.6 .3

KEE IIIUAL, ALI GIIAUES

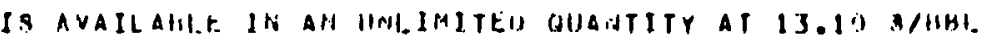

LII.IIIU PET. BASSES

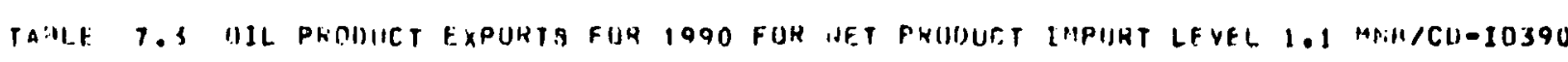
FIXEN EXP(IRT (HIR/CH) KEVENIIE (S/HHL) 


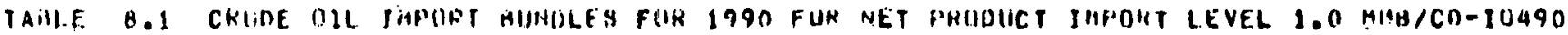
LaNOE! PHICE (x/HISL)

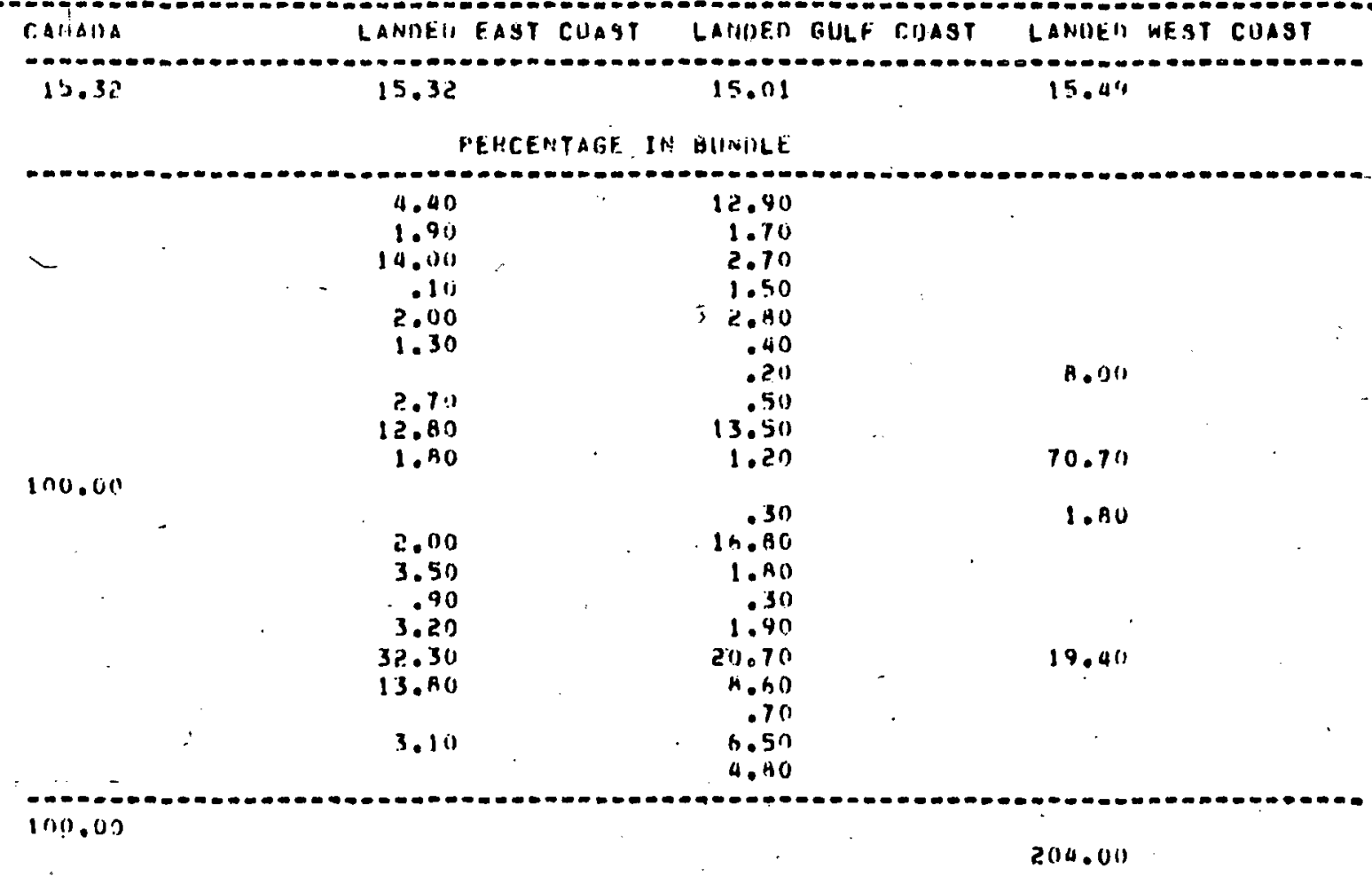

CNIIDE IYHE

LIAYA

NuHivaY

UHITEG) Rlil(jp:U1!

IRINIGAC

ERYPI/SYYIA/BAHAIIN

ANF,ULA/CIIHGO/ZAIHE

SIIIIII ASIA $M I X$

VENETIIELL

NIGEHATGAHOH

INOINES 3 IL

CAHADA

ECliñnllK:

1:EXIC:1

ALISEKTA

IRAH I. I ISH I

JWAW. HEAYY

SAUII ANALIA LIFHI

SALOI RWALIA HEAVY

KIIWA I T

HATAR/II.A.t.

INATI

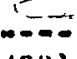

61XE(1) H(1)

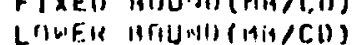

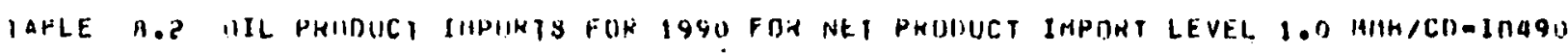

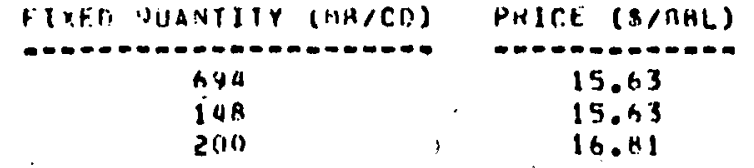

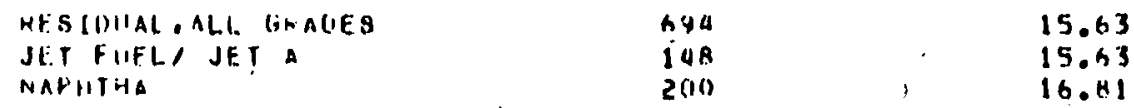

LIJUIR PET, GASSES

13 AVAILAHLE IH AN UIVLIMITEl HIIANTIIY AT 13.10 S/HHL

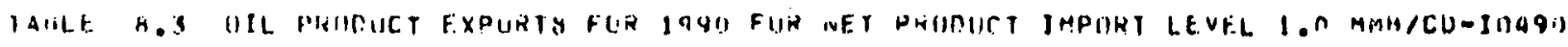

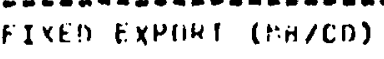
WEVEHIIE (s/tiBL)

UTIE: WEF IWFL PETEOLF UA

47

16.61 


\begin{tabular}{|c|c|c|c|c|c|c|c|c|c|}
\hline$n$ & $A \cap \cap A C$ & & 11 & 1 HAIISH & $A A A A A$ & $\mathrm{~L}$ & BriblicifG & $\triangle A A A$ & 9935s \\
\hline 114 & 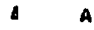 & I & 11 & H H & - A & 1 & G & & \\
\hline 114 & $A \cap A \cap A$ & $T$ & $"$ & I NAHIRY & MAAAA & I. & fil; & $A A A A$. & A 39583 \\
\hline $1: \mathrm{V}$ & 14 & 1 & $" 1$ & $J H \quad H$ & A & L & F & A & $A$ \\
\hline is & 1. & $\boldsymbol{T}$ & (IIII)III) & $1 \mathrm{H}$ & a & LLLLL & & $A$ & s9s53 \\
\hline
\end{tabular}

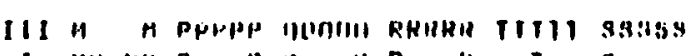

I III III $P$ P II

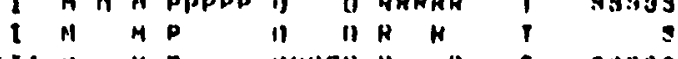

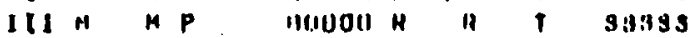

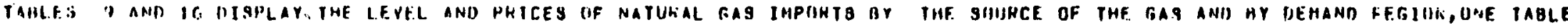

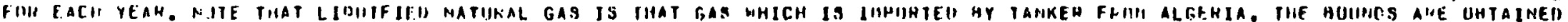

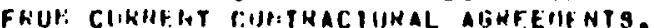

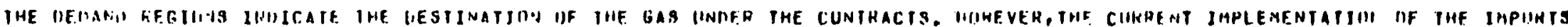

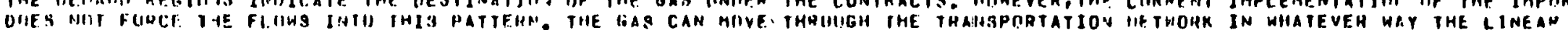

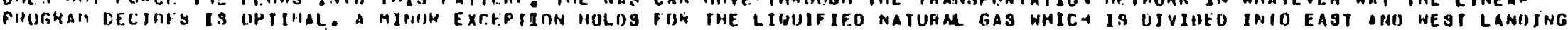

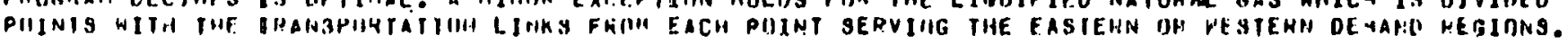

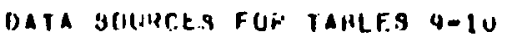

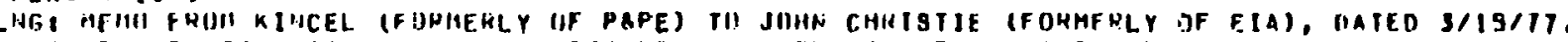
SUISJFCTI SPFCIFICATIUHY EIIH COMPUTEN MODEL TU PHUVIOE FIIH LNG IHPOKTS.

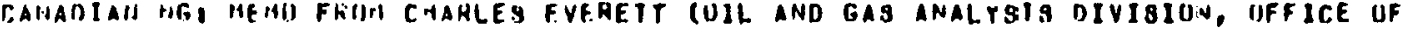

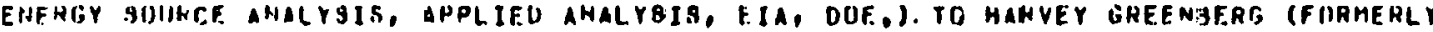

IIF MFFICE IIF AMALYTIC HETHOOS, APPLIEO ANALYYIS, EIA, DOES HNOATED.

SHBJECT I DIL L LAS SUPP:-Y AŚgIMPPTIONS (TABLE 3).

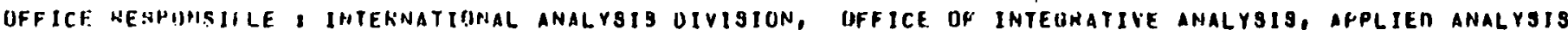
EIA, UREF:

TAIII.F. $\quad 4$

沜

NATUKAL GAS IMPUNTS FIJH 19A5-JMIBS

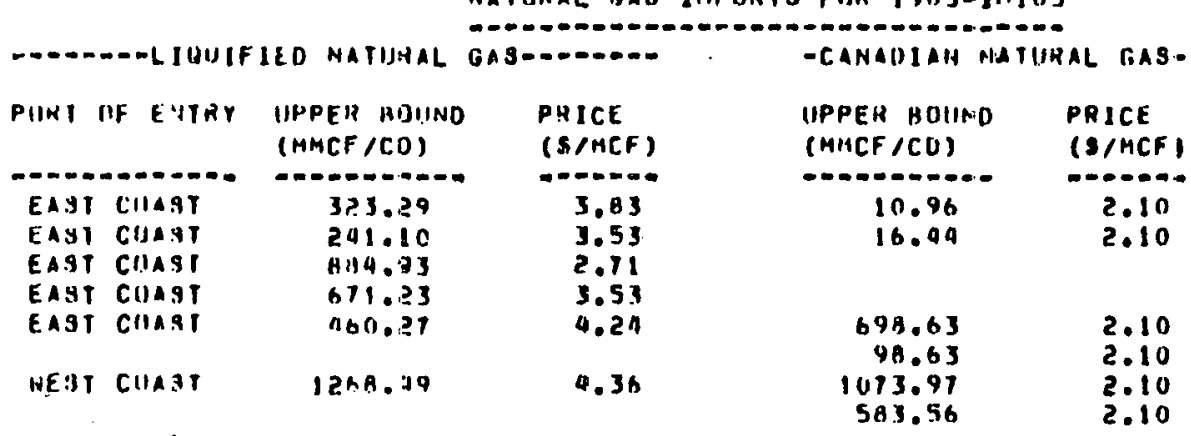

I AIII. III

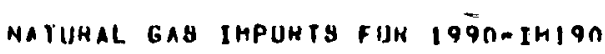

DEMAHU FEGIIII"

iar/:IJ

III)-AIL

s. -All

Hillot at

CENIHAL

$M=\operatorname{Con} 2$ iा.

WF: 31

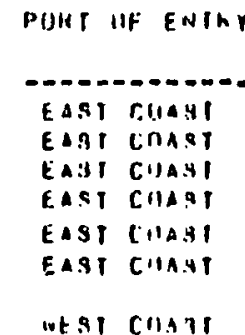

IUPPES IIIIIMO

(190........

-canauiali natiral gago

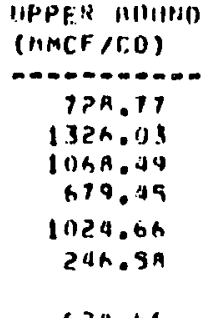

\begin{tabular}{|c|}
\hline $\begin{array}{l}\text { PHICE } \\
\text { (SIP:CF) }\end{array}$ \\
\hline $\begin{array}{l}5.07 \\
5.31 \\
5.33 \\
1.111\end{array}$ \\
\hline $\begin{array}{l}4.119 \\
11.11\end{array}$ \\
\hline
\end{tabular}

(IinCF $/$ CII)

(11)

PHICt

(M) IACF

5.07

3.33

0.111

11.11

648,63

2.10

4.36

99.63
1073.97

2.10 


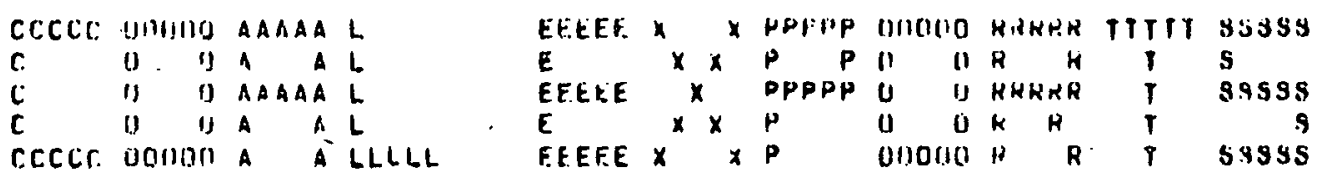

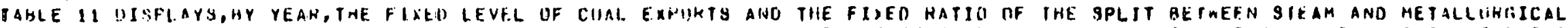

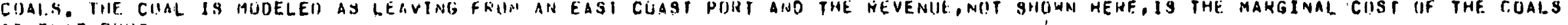
AI THAT PII:I I.

DAPA SULIHCA FIIR TABLE 11 ,

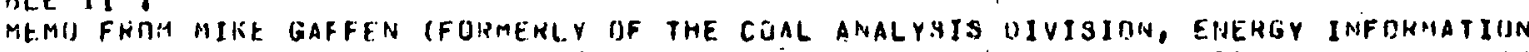

AWALYSIS) THKU PHIL CIILOHFSS (COAL ANALYSIS IIVISIOIV, ENFHGY INFIMMATIJN ANALYSIS,EIA)

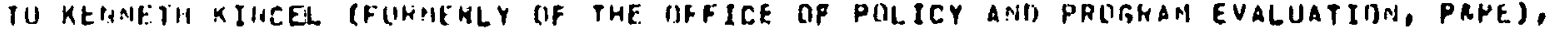

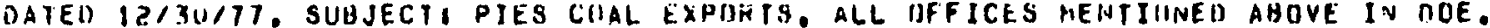

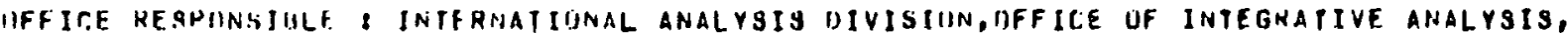

IABI.E 11

$$
\text { APPLIED GHALYSIS, FNERGY IHFORMATIUN ADHINISTKATIIII, UOE. }
$$

CDAL EXPIHTTSOIMIAS IMI9O

CDAL EXPIHTSOIMIAS

FIXEI) F.XPURT

$(\mathrm{H} / \mathrm{C} / \mathrm{U})$

CUAL HIX

YEAK

.202 .74

HE I ALLUKGICAL

.76

.73
HIGH SILFUR STEAM

$.24 !$

.27 
This section contains oil and gas standard tables. The printout includes detailed descriptions of each table and lists of the abbreviations used preceding the tables themselves.

TABLES $1,2,5$ and 6 give the price trajectories for each year from 1977 to 1991 . Tables 3, 4, 7 and 8 contain the price and production data that define the steps of the supply curves. Only one price step, step 5 , is given for oil.

TABLES 9 through 24-are obtained by taking various combinations of the supply curve tables, TABLES $3,4,7$ and 8 . The last three tables, TABLES 25,26 , and 27 contain factors showing the proportions of crude, and intrastate gas and co-products among supply, NPC and DOE regions. NPC, DOE Oil Supply and DOE Gas Supply regions are shown in Figures II-3, 4 and 5 respectively: 


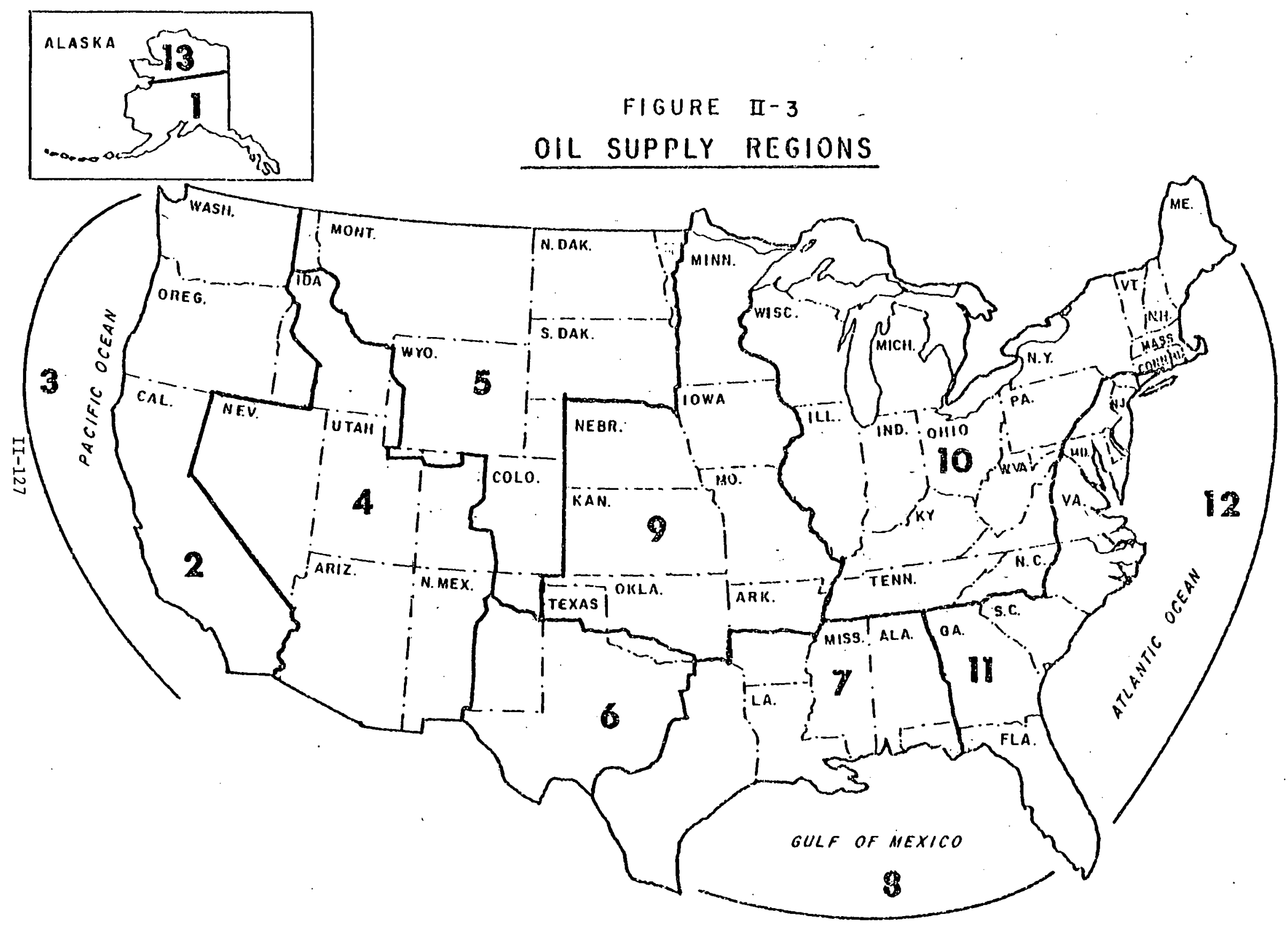




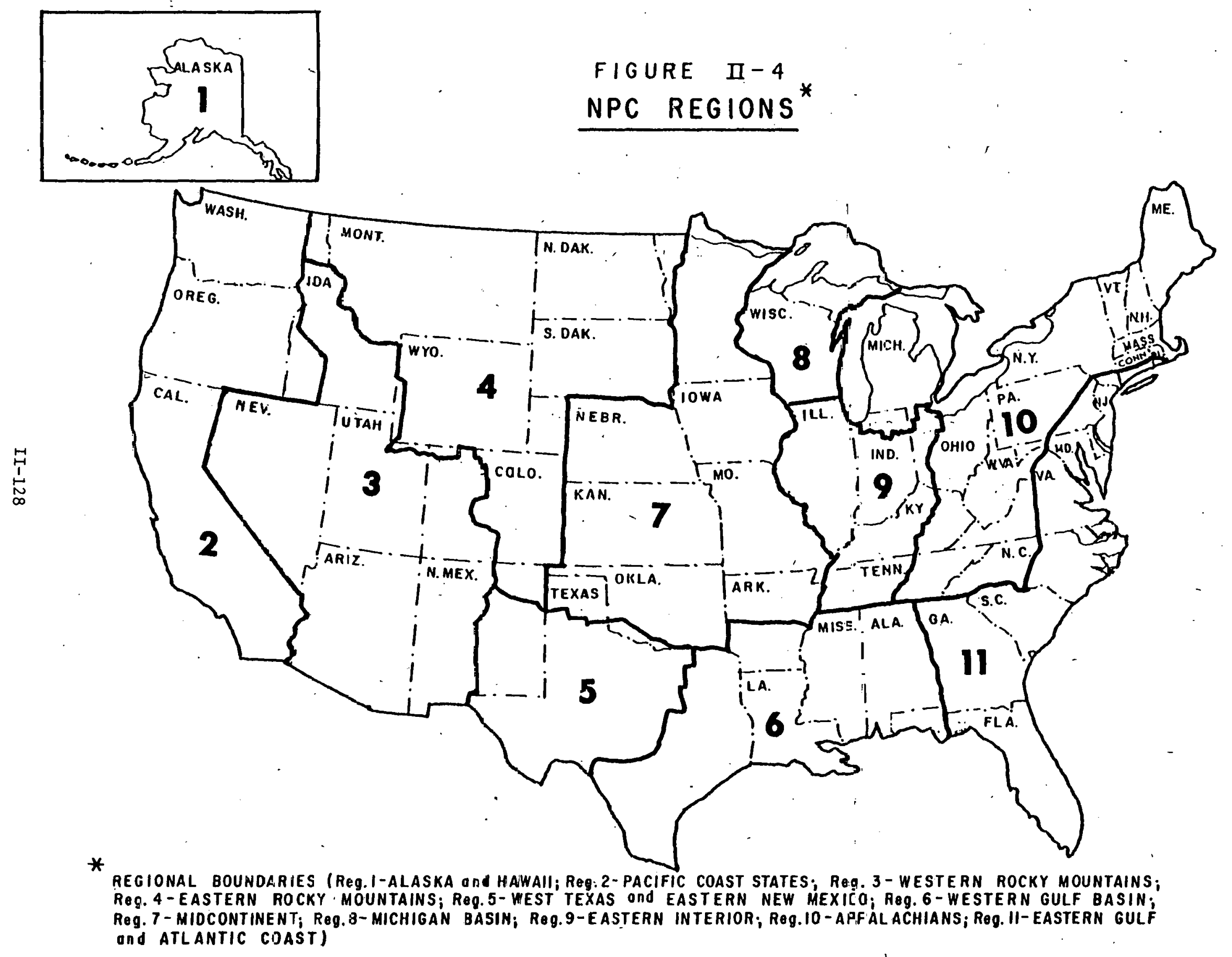


FIGURE II -5

NATURAL GAS SUPPLY REGIONS

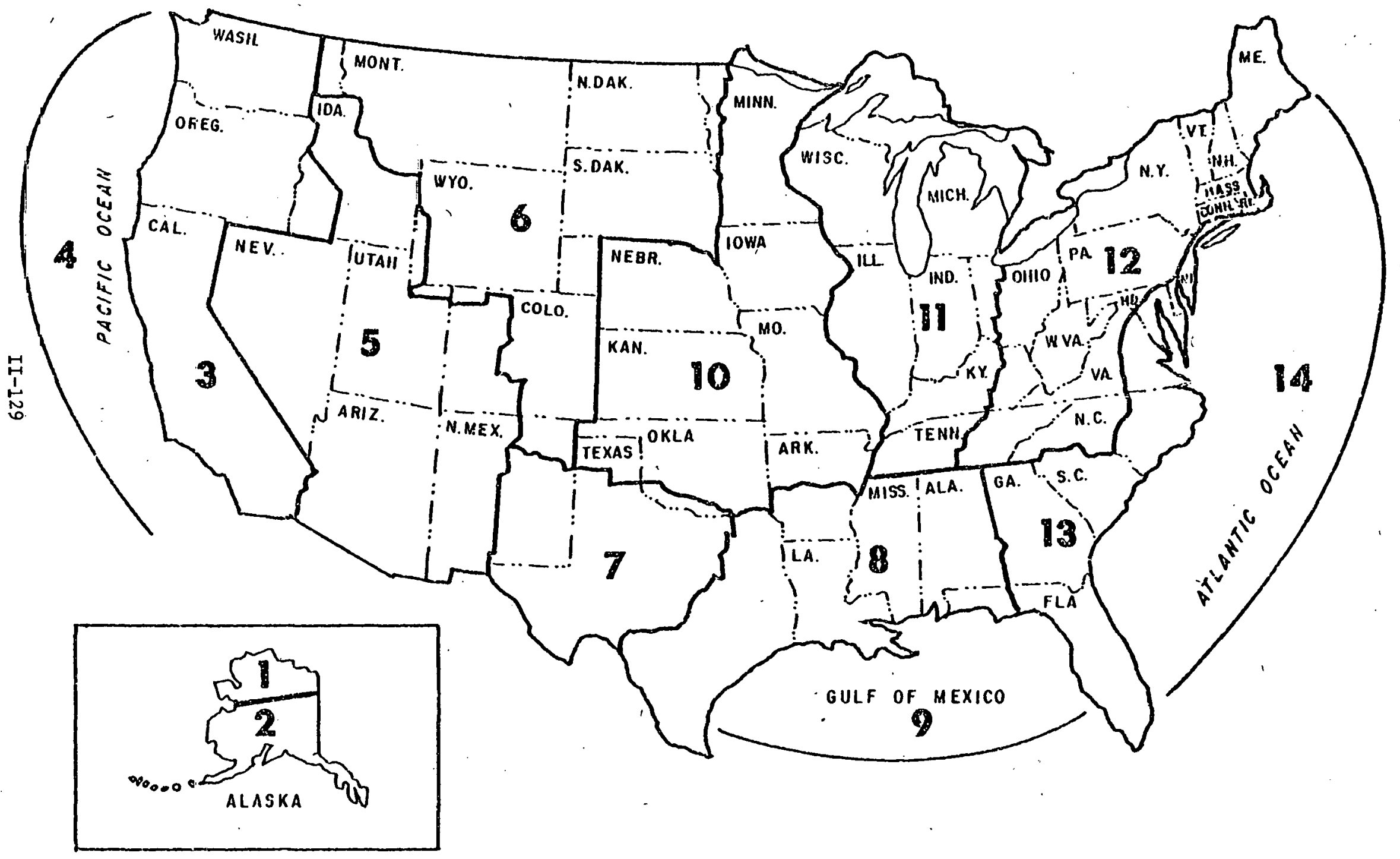


OEL AND GAS SIIPPLI DATA

INPIJTS TO THE P IES IHTBGRATIHG MODEL

1978 ANNUAL ADHENISTRATOR'S RRPOAT

MID-RANGE FOBECAST 
THE PRODUCTIOH OP CMIIIE OIL. NATURAC GAS AHD NATURAL GAS LIOIIIDS IS GEPRESENTED TN THE PIPS I RTEGRATTNG MONEL BY A COLLECTION OP SIIPPI,Y CIIRVES. DHE FOL EACII COABINATION DF PEGION (NPC REGION), TYPR. OF WELL (OTL OR GASI. VINTAGE INEW UR OLDI. AND, IH THE CASP DP OIL MELI.S. TYPE OY PRODICTION (PRIAARY, SECONDARY OR TERTIARY), PACH SUPPIY CIIRVE IS A STRE FUNCTION CONSISTING OF NINE PRICE STEPS. AS SHUH IN THE FIGURE AELOH. EACH STEP GIVES OUANTITY OF PRODUCTION ASSOCIATED UTH GIVEN YELL-HEAD PHICB.

A H.INE STEP SUPPLY CUNVE

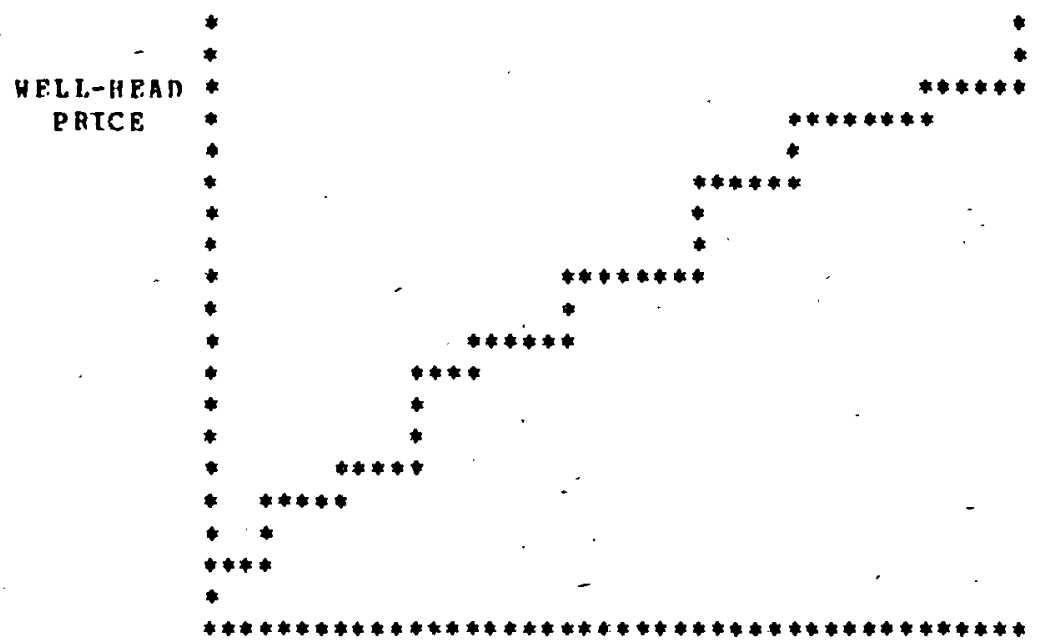

OIIANTITY PRODICED

HOHFVER, THP ABOVE PIGURP UNDERSTATBS THE INFORAATION CONTENT OP PACH STFP. TN PACT. AT EACH STEP PRICES ARE ASSUKRD AND OUARTITIES ARP. GTVEN POA FACII OP FOUR PRODICTS (CRHDE OIL. ASSNCIATED AND DISSOLVEN FAS. BUTANE. AND OTHER GAS LIQJIDSI. IN THF CASE OP OIL AFLL.S. AND THREE PRODUCTS (NON-ASSOCIATED GAS,-B!TANE AND OTHER FAS LIOUIIDS) TN THE CASE OP GAS MELLS. JN ADITTION. BACH STEP ALSO CARRIRS HITH IT THP AMOUNT OF DRTLLING; AND CAPITAL INVESTMENT REUII IRED-TO REACH TIIE INDTCATED LEVF.L OF PRODICTTON.

THP SUPPI.Y CIIRVES ARE GPNERATEN BY THE PIES OIL AND GAS SIPPLY MODEL. THIS MODEL IS DYNAMIC IN THAT SUPPLY CIURVES POB ALL YRARS PROA 1977 TO. 1991 ARE GERPRATED SIMULTRNROUSLY USING THE PRICE TRAJECTORIPS SHONA IN TABI.ES 1 AND 2 FOA OIL WELLS AND T.N TABLPS 5 AND 6 FOR GAS HELLS. THE SUPPLY MODEL IS RUN ONCP POR FACH STPE AUD GRNERATPS THE IISTORY OF

CAPTTAL INVESTAENT, DRILLING, AND PRODUCTION FOR BACH PRODUCT 
THAT HOTLD RESIILT IP MEILL-HEAD PRICPS VERP TO FOLLON THE PRICE TRAJPCTORIES FOR TH'P RIVPN STRP. TIIE PIES SIIPPLY CURVES ARE TIIEN OBTAINED BY SOI.EETING THE DRICES AND CORKPSPONDING DUANTITIES FOR THR YFARS 1985 AND 1990.

THP GAS PRODICTYON PROIECTED BY TUE OTL AND GAS STPPLY MOOEL IS DIVIDED BETYEEN THE INTERSTATE ANA INTAASTATE MARKETS ACCORDING TO TPR POLLOHJNG RILES: (I) ALL. ALASKAN AND DCS GAS IS INTERSTATE, 121 ALL NEN LOHER 118 ONSHOAE GAS IS TNTRASTATE AH! (3) ALL OI.D ONSIIOHE GAS IS DIVIUED ALCOHUING TO RPGION SPECIFIC HISTORICAL (1974) PROPORTIOHS. IT SHOILD BE HEHTIOURD THAT THIS A PRIORI DIVISION TS SUB.JECT TO REVISIOH BY THE. INTEGRATING MODEL WIICH IIAS THE CAPABII. ITY DF SHTPTING SOME GAS HHICH HAS BFEH RESIGNATED AS INTRASTAT. BY THR ABOVE RULPS INTO THR INTERSTATE MARKET AS REQUTRRD TO KEEP THE INTAASTATE PBICE FROM FALLING BELOW THP INTERSTATP, PRICP.

TABLES 3,4, 7 AND 8 GIVE THP SUPPI.T CURVES BY REGIOH. VINTAGP. AND TYPE. THE .PRICE STRP IS GIVPN BY TIL STEP NISHBRA. AIIONI NG RPFRRENCE TO THE PRICE TRAJECTORY TABLES TO PIND ASSIIMPD PRICES FOR EACII PRODUCT. THESE PRICES ARP NOT USPD IN THE.INTEGRATING MODEL. HOHEV BR. SINCE IT SRBS THE IMCREAENT IN PRODIJCTION FRMY ONE STEP TO TIIE NEXT AS A BINDLE OP COMMODITIES WHICH IT MUST PITHEN PRODICE OR NOT PRODUCE TOGETIIER. THIS A SINGLE PRICE, CALLED THF BUNDLE PRICE, IS ASSIGNED TO EACH STEP. AY DRPINITION, THE BIINDL PRICE IS THE RATIO OF THE TOTAL. VALIIE OP THE INCRPMPAT IN PRONICTION FROA THE PREVIOUS STEP IIHCLUNING ALL FOUR OR THHER PRODUCTS. USING PRTCES PROA TABLES 1.2, 5 AND 61 TO THE QUANTITY OF CRIIDE OIL DR NON-ASSOCIATED GAS IN THAT IHCREARNT. THIS SOHENIIAT AUKHARD CONVENTION IS MECESSITATED BT THE FACF THAT THE BIJ NDLE CONTAINS COHMODTTIES MEASURED IV DTPPEBENT UNITS. NOTE TIIAT THESE TADIES SHON NATORAL GAS BROKBN OOT ACCORDIHG TO WHICH MARKET (INTERSATE DR INTRASTATE) IT BELONGS.

IT SHOHLD BE ERPHASIZED THAT VI IIAGE. AS USPD IN $\mathrm{PHIS}$ DOCUNENTATION, MERPI.T DESCRIBES YIIETHER A HRLL WAS DRILbED BPPORE OR AFTER 1/1/77. AND. AS SIICH, IS DIPPERENP FROA THE LEFAL CONCEPTS OF OIL AND GAS VINTAGE.

THP ALASKAN NORTH SI.OPE AND BLK'S HILL ARE NOT INCLUDED In THF OIL AND GAS SUPPI.Y MDDEL. ACCORDINGLY EHGIHEERING ESTIMATES OF PRODUCTION AND COST ARE USBO FOR THESE RESIONS. THESE REgIOHS ARE INDCCATEO IN THE TABLES BY AN * IN IHE PRICE STRP COLIMN.

THP SCENARIOS RHICH HODFL OIL RESIILATIOA ARP DISTINCTIVR. SINCE UELL-HEAD PRICES ARE CONTROLI.PD. ONLY ONP PTICE STEP IS USPD AHD THE OUANTITY OP DOAESTIC CRUDE AVAILABLE IS PIYED. ALSO, THR BUNOLE PAICE IS HOT CALCUIATED AND IS LISTPD PIXED. ALSO, THR BU NOLR
IN THP TABLPS AS $* * 1$ ?

IN GAS REGULATION SCENARIOS THE DISTRIBUTION OP INTRR-

STATE RAS IS ESSENTIALLY FIXED. THIS DISTRIBUTION ANO RESTLT ING DPLIVERIES ARE GIVEN IN TABLES 9-12, THP INT RGRAT ING 
MODPL HAY ALTPR THESE DISTRIBUTIONS IA TIL POLLONING HAYS: (i) A REGION MAY TAKR LESS I TTERSTATE GAS THAN IS ASS IGHED TO IT. (2) EXCYSS INTPRSTATE GAS TH ONE RBGTON MAY BE REASSIGHED TO AROTHER REgTON AND (3) GAS ORIGIHALLT DES IGNATED AS INTRASTATE BOT SHIFTED TO TIIE INTERSTATB MARKET HIL ATGABNT THE STATED DISTRIBOTION.

TABLES 13-24 SUMHARIZE THE INPORAATION IN THE OIL AHD gas SUPPLY CUHVES. TABLES 13 ANB 14 GGREGATE OIL AND (iAS PHODUCTION. INPFSTKENT AND DR ILLING STATISTICS OVER VINTAGE AND TYPR OF PRODICTION. TABLPS 15-22 DO TIIP SAIE POR GAS VELI.S AT AIL PAICE STRPS. THEN TARLES 23 AND 24 COABTHE THE PRODHCTJON PROM OIL AND GAS HELIS AND SHOH THE MAXIMIS. OIIANTITY OP EACH PIEL AVAII,ABLE TO THP. IVTEGRATING HODEL.

TABLE 25 SHOGS THE IIX OP CRODE TYPES ASSUHED POR RACH NPC REFION. THESE PROPORTIONS ARE APPLIEO IRRPSECTIVF DP THE VINTAGS. OR TTPE OP PRODUCTION.

TARLPS 26 AND 27 RROVIDE SOMP INPORMATION RFOUTRED BECAIISE OP THE WAY INTRASTATP GAS PRODICTION AND DISTRIBUTIOA IS MODELED. ASSOCTATED AND DTSSOLVED INTRASTATE GAS IS PRODIC ED IN NPC REGIONS BUT IS DISTRIBUTED PROM DOE REGIOHS. THIS REOUIRES A LOGICAL SHIET OF ASSOCIATED INTRASTATE SAS FROM NPC REGIONS TO DOE REGIONS. TABLE 26 GIVES THE PRACPION OF INTRASTATE GAS PRODICED IN EACH NPC RESION THAT IS SHIPTRO TO FACH DOR REGION POR DISTRIBITIOA. SIMILARLY, GAS LIODIDS PROM I NTRASTATE GAS MELLS aRe PRODICED IN DOE REgIONS BUT DJSTRIBIITEN FEOA HPC REGIONS. TABI.E 27 GIVPS THP. PRACTIOH OP I NTRASTATE GAS LIOUIDS PRODHCED IN EACI DOP. REGION THAT IS SHTPTED TO FACH HPC REGTON.

ALL DOLLARS ARE CONSTANT 1975 DOLLARS. VOLUMES OP NaTURAL GAS ASSUME A HRAT VALIP. OP 1032 BTU/CP. THE EHTRTRS INDRR THE

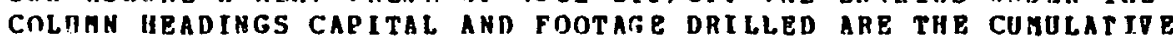
CAPITAL INVESTAENT AND CURILATTVE POOTAGE URILLED PROA 1977 TO ONE YPAR PRIOR (19R4 OR 1983) TO TIE TARCET YEAR (1985 OB 19901. THR ABBREVIATIDNS ISED IN THIS REPORT POR THE VARIOUS UNITS OP MEASURENENT ARE AS POLLONS:

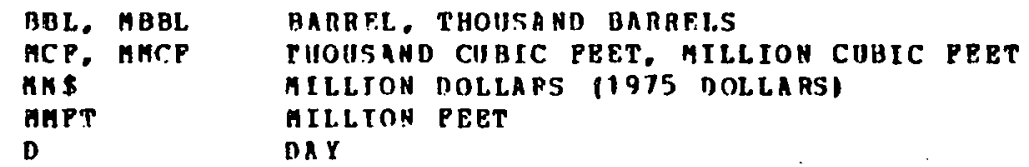


TABLP: 1

PRICE TRA.TPCTOIIES FOR OIL AHD GAS LIOUILC

$(\$ / B ! \mathrm{BL})$

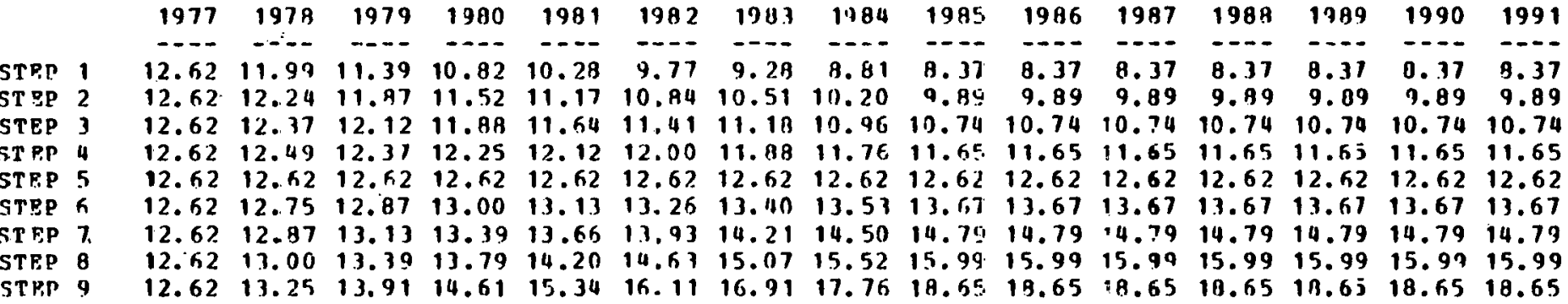

DATA SOIJCES : OUTPUT FROA THE PIES OIL 6 GAS SOPPLY MOCEL DERTGPD PROM AV ERAGE RRPFRPNCF ACOIISITION COSTS. TRANSDORTATION COSTS.

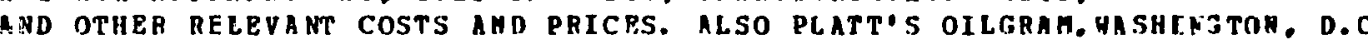
A HERKLY PIIELICATION.

JPPICE RRSPCNSIBLE : OTL AMD GAS INALYSIS DIVISION, OPPICE OP ERERGY SOURCE AMALYSIS. APPLIED AMALYSIS, ?IA.

TABLP 2

\begin{tabular}{|c|c|c|c|c|c|c|c|c|c|c|c|c|c|c|c|c|}
\hline & & 1977 & 1978 & 1979 & $19 A 0$ & 1981 & 1982 & 198.3 & 1984 & 1985 & 1986 & 1987 & 19AA & 1989 & 1990 & \\
\hline$:$ & 1 & 2.17 & $\overline{2.06}$ & 96 & $-\overline{86}$ & $--\cdot$ & 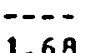 & -- & --- & --- & --- &.-- & $1 .-\overline{40}$ & (44 & $\overline{4}$ & \\
\hline E? & 2 & 2.17 & $\begin{array}{l}2.14 \\
2.11\end{array}$ & 2.04 & 1.98 & 1.92 & 1.86 & 1.81 & & 1.44 & 1.70 & 1.10 & 1.90 & 1.73 & 1.70 & \\
\hline$P P$ & 3 & 2. 17 & & 2.08 & & 2.00 & 1.96 & 1.92 & 1. & 1.85 & 1.85 & & & & & \\
\hline EP & 4 & 2.17 & & 2. & & & & & & & & & & & & \\
\hline rep & 5 & 2.17 & 2.17 & 2.17 & .17 & 2.17 & 2.17 & 2. 17 & 2.17 & 2.17 & 2.17 & & & 2.1 & 7 & \\
\hline BP & 6 & 2.17 & 2.19 & 2.21 & .24 & 2.26 & $2.2 A$ & 2.30 & 2.33 & 35 & & & & 3 & & \\
\hline P.P & 7 & 2.17 & 2.21 & 2.26 & & & 2. & & & & & & & & & \\
\hline & 8 & & & 2. & & & 2. & & & & & & & & & \\
\hline P.P & 9 & 2. 17 & 2.29 & 2.39 & & 2.64 & 2.77 & 2.91 & 05 & & 3.21 & & & 3.21 & & \\
\hline
\end{tabular}

MBTA SOUFCES : NUTPUT PRON THE PIES OIL F. GAS SIJPLY MODEL DERIYED PROH APERAGE BEPERENCE ACOUISITION CUSTS, TRAKSPORTATION COSTS.

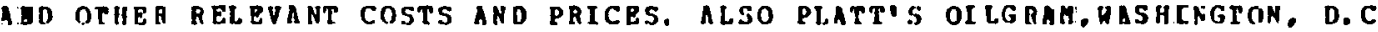
AEEKLY publication.

OFFICE RESPOASIRLP : OIL AND GAS ANALYSIS DIVISION. OPPICP OP BNERGY SODRCE ANALYSIS. APPLTED ANALYSIS, EIA. 
TABLE 3

OLL SIIPPLY CURYES FOR 1985

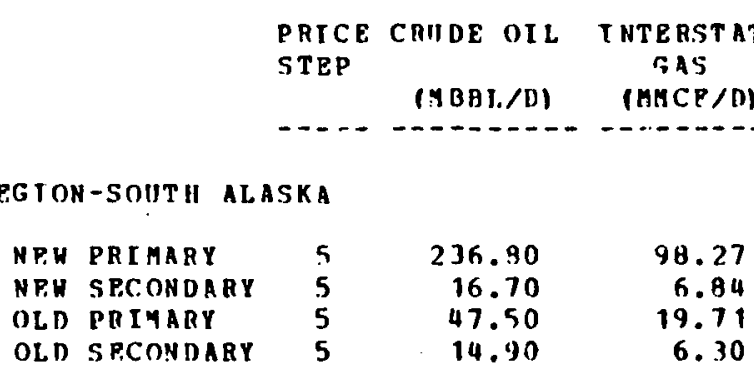

GAS L I OUI I DS (MHCP/D) (MBBL/D) IMBOL/D)

BกT DLE PRICR

(\$/DAL OIL)

CAPITAL

(Ant)

POOTAGE

19/DBL

NPC REGTON-SOUTH ALASKA

1284.00
$4 \quad 47.00$
$* \quad 73.00$

NHC RRGION-PACTFIC COAST

\begin{tabular}{|c|c|c|c|}
\hline \multicolumn{4}{|c|}{ EXCLIIDING } \\
\hline N P.W & PRTHARY & 3 & \\
\hline NEH & SECONDARY & 5 & \\
\hline oLn & PRI MARY & 5 & 3 \\
\hline OLD & SECONDARY & 5 & \\
\hline OLD & TERTIARY & 5 & \\
\hline
\end{tabular}

$$
\begin{array}{r}
69.52 \\
18.61 \\
131.96 \\
10.40
\end{array}
$$

$$
\begin{array}{r}
1.69 \\
.40 \\
3.08 \\
.20
\end{array}
$$$$
\begin{array}{r}
.79 \\
.19 \\
1.57 \\
.10
\end{array}
$$

$\begin{array}{rr}* & 2926.00 \\ * & 75.00 \\ * & 71.00 \\ * & 350.00\end{array}$

LK' S HILL

NPU PRIMARY

175.00

$\bullet$

728.44

NPC REGION-PACIPIC OCEAN

$\begin{array}{llr}\text { NEW PRIMARY } & 5 \\ \text { NEH SECOMDARY } & 5 \\ \text { OLD PRIMARY } & 5 \\ \text { OLD SECONDARY } & 5\end{array}$

$\begin{array}{rr}43.80 & 59.96 \\ .50 & .82 \\ 105.90 & 153.86 \\ 58.50 & 80.76\end{array}$

1.60

1.20

$*$

154.00
2.00

2.20

1.70

247.00

MPC RFGION-YEST ROCRIES

$\begin{array}{llr}\text { NEW PRIMAAY } & 5 & 105.40 \\ \text { NEH SECONDARY } & 5 & 18.80 \\ \text { OLD PRIMAPY } & 5 & 41.80 \\ \text { OLD SECONDARY } & 5 & 105.80\end{array}$

1.05 .40
18.80
41.80
105.80

29.29

2.79
.50
1.40
2.79

2.10
.30
1.00
2.09

$\begin{array}{rr}* \quad & 1301.00 \\ * \quad & 16.00 \\ * \quad & 180.00\end{array}$

124.00

6. 00

VINTAFP. (OLD, NEH) IN DICATES WHETHER OIL WRLLS DRILLED AEPORE OR APTPR 1/1/77 (NOTF DIPPERENCE PROA LBGAL DEFINITION OP OIL VINTAGRI

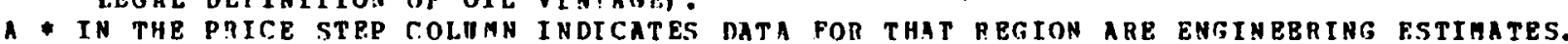

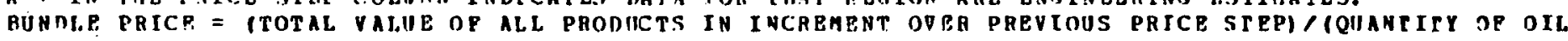
IN THAT INCRERERTI.

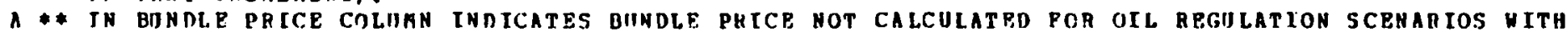
POINT FSTIMATES DP SHPPLY.

CAPITAL INVFSTHENT AND DRILI.ING STATISTICS ARE COHILTIVE FAOM 1977 TO 1984. 
TABLE 3

CONTIUUEU

OLL SUPPLY CURVES POR 1985

\begin{tabular}{|c|c|c|c|c|c|c|c|}
\hline $\begin{array}{l}\text { PICE } \\
\text { TEPP }\end{array}$ & $\begin{array}{l}\text { CRIJEE OIL } \\
\text { (ABBL/D) }\end{array}$ & $\begin{array}{c}\text { INTERSTATE } \\
\text { GAS } \\
\text { (nKCP/D) }\end{array}$ & $\begin{array}{c}\text { IRTRASTATE } \\
\text { GAS } \\
\text { (AMCP/D) }\end{array}$ & $\begin{array}{l}\text { BUTANR } \\
\text { (ABBL/D) }\end{array}$ & $\begin{array}{c}\text { GAS } \\
\text { LC QUI InS } \\
\text { (ABBL, } \mathrm{A} \text { ) }\end{array}$ & $\begin{array}{l}\text { BUNDLE } \\
\text { PRICE } \\
\text { ( } 8 / \text { BBL OIL) }\end{array}$ & $\begin{array}{l}\text { CA PITAL } \\
\text { (nns) }\end{array}$ \\
\hline
\end{tabular}

POOTAGE

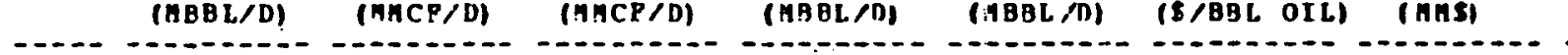

NPC REGION-EAST ROCKIES

$\begin{array}{llr}\text { HPH PRIMARY } & 5 & 133.50 \\ \text { NEH SECNHDARY } & 5 & 19.50 \\ \text { OLD PBIMARY } & 5 & 155.80\end{array}$

155.80

$\begin{array}{ll}82.13 \\ 47.71 & 12.59 \\ 61.63 & 37.70 \\ 49.69\end{array}$

10
5.80
.09

1.70

OLD PBI AARY

1 A3. 10

61.63

49.69

$6.5 \mathrm{P}$

1.30

2.20

$*$

19.00

85.00

MPC REGIOH-D. TEX. /P. N. HEX.

$\begin{array}{llr}\text { NPH PEIMARY } & 5 & 110.50 \\ \text { NEH SECOHDART } & 5 & 27.10 \\ \text { OLD PRIMARY } & 5 & 748.80 \\ \text { OLD SECOHDARY } & 5 & 307.80\end{array}$

27.10
748.80

$\begin{array}{lll}\text { OLD SECONRARY } & 5 & 307.80 \\ \text { OLD TERTIARY } & 5 & 3.73 .10\end{array}$

421.50

98.01

24.09

119.95

536.66

6.100

1. 70

43.73

15.48

2.30

.60

16.17

5.69

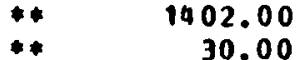

103.00

BPC REGIOR-GULP COAST

NEW PRIMARY
NEH SECONAAHY
OLD PRIMARY
OLD SECOKDARY
OLD TERTIARY

276.80

4.60

716.90

910.60

40.20

423.04

.312 .37

5.20

418.17

509.48

19.49

.30

56.29

6. 17

-10
17.78

17.78

20.22

$$
\begin{array}{rr}
* & 2593.97 \\
* & 5.00 \\
* & 1740.98 \\
* & 242.00
\end{array}
$$

APC REGION-GULP DF AEXICO

$\begin{array}{llrr}\text { NPW PIIMARY } & 5 & 268.10 & 304.16 \\ \text { NPW SPCONDARY } & 5 & 9.60 & 11.22 \\ \text { OLD PRI IARY } & 5 & 236.70 & 263.64 \\ \text { OLD SECANDARY } & 5 & 50.110 & 56.40\end{array}$

7.48
.20
0.50
1.70

$4.5 n$

.10

5. 18

$\begin{array}{rr}* * & 1604.98 \\ * & 9.00 \\ * & 113.00\end{array}$

168.99

$1.70 \quad 1.10$ 
OII. SIIPPLY CIRYRS POR 1905

\begin{tabular}{|c|c|c|c|c|c|c|c|c|}
\hline $\begin{array}{l}C B \\
P\end{array}$ & CPIJDE OIL & $\begin{array}{l}\text { I NTERSTATE } \\
\text { GAS } \\
\text { (MACP/D) }\end{array}$ & $\begin{array}{c}\text { INTRASTATE } \\
\text { GAS } \\
\text { (MHCE/D) }\end{array}$ & $\begin{array}{l}\text { BUTANR } \\
\text { (ABBL/D) }\end{array}$ & $\begin{array}{c}\text { GAS } \\
\text { LIOUI IDS } \\
\text { (IBBC/D) }\end{array}$ & $\begin{array}{l}\text { BUHDLB } \\
\text { PRICE } \\
\text { (\$/BBL OIL) }\end{array}$ & $\begin{array}{l}\text { CA PITAL } \\
\text { (nก) }\end{array}$ & $\begin{array}{l}\text { Fno } \\
\text { DR }\end{array}$ \\
\hline
\end{tabular}

NPC REGION-MIDCONT IN PNT

$\begin{array}{llr}\text { NEH PRIMARY } & 5 & 125.50 \\ \text { NEW SECONARR } & 5 & 42.6 \\ \text { OLD PRTMARY } & 5 & 213.10 \\ \text { OLD SECONDARY } & 5 & 135.11 \\ \text { OLD TERTIARY } & 5 & 27.00\end{array}$

$\begin{array}{rr}202.32 \\ 205.39 & 188.72 \\ 111.12 & 104.62\end{array}$

2.70

.90

3. 39

OLD TERT IARY

27.00

111.12

104.62

2.90

2.09

1.79

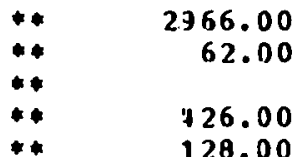

NPC REGION-MI. B./ INT./ APP.

$\begin{array}{llllr}\text { HEN PRIMARY } & 5 & 89.90 & & .39 .05 \\ \text { NEW SPCONDARY } & 5 & 14.30 & & 6.30 \\ \text { OLD PRIYARY } & 5 & 70.30 & 12.52 & 18.41 \\ \text { OLD SECONDARY } & 5 & 65.50 & 11.19 & 16.46\end{array}$

$\begin{array}{rr}* * & 1014.00 \\ * & 14.00 \\ * & 121.00 \\ * & 24.00\end{array}$

NPC REGIOH-ATLANTIC COAST

$\begin{array}{llr}\text { NEH PRIMAPY } & 5 & 101.80 \\ \text { NEM SECONGART } & 5 & 6.00 \\ \text { OLD PRIMARY } & 5 & 4.50\end{array}$

$$
\begin{aligned}
& 6.00 \\
& 4.50
\end{aligned}
$$

$$
\begin{array}{r}
42.71 \\
2.74 \\
1.00
\end{array}
$$

$* * \quad 32 \mathrm{R} .00$
$* \quad 5.00$

22.06

NPC REG ION-ATLANT IC OCEAN

$\begin{array}{lrrr}\text { NEN PRIMARY } & 5 & 263.20 & 199.85 \\ \text { NEH SECONDARY } & 5 & 6.70 & 5.20\end{array}$

5.20

9.58

.29

$* 4$
$* \quad 56.98$
$\quad 560$

5.97

NPC REGION-NOHTH SLOPE

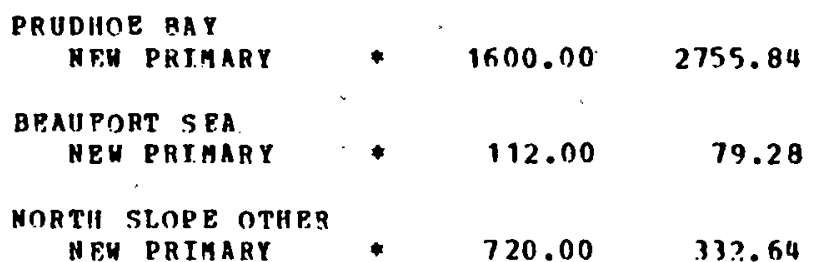

$\begin{array}{rrr}* * & 6776.00 & 6256.00 \\ * * & 1072.96 & 779.52 \\ * * \quad 5745.60 & 5018.40\end{array}$


TAB LP. 4

OIL SIIPPLY CURVES POR 1990

\begin{tabular}{|c|c|c|c|c|c|c|c|c|}
\hline $\begin{array}{l}\text { PRICE } \\
\text { STEP }\end{array}$ & $\begin{array}{l}\text { CRIIDE OIL } \\
\text { (ARBL/D) }\end{array}$ & $\begin{array}{l}\text { I NTPRSTATE } \\
\text { GAS } \\
\text { (AHCF/D) }\end{array}$ & $\begin{array}{l}\text { THTAASTATE } \\
\text { GAS } \\
\text { (MACF/D) }\end{array}$ & $\begin{array}{l}\text { BUTANP } \\
\text { (ABBL/DI }\end{array}$ & $\begin{array}{c}\text { GAS } \\
\text { LIQUEDS } \\
\text { (ABBL /D) }\end{array}$ & $\begin{array}{l}\text { BIINDI.E } \\
\text { PRICE } \\
\text { (\$ S BBL OIL) }\end{array}$ & $\begin{array}{l}\text { CAPITAL } \\
\text { (An\$) }\end{array}$ & $\begin{array}{c}\text { POATAGE } \\
\text { DRILLED } \\
\text { (RAPT) }\end{array}$ \\
\hline
\end{tabular}

NPC REIIION-SOITH ALASKA

$\begin{array}{llrr}\text { HEN PRIMARY } & 5 & 678.90 & 281.40 \\ \text { REA SPCONDARY } & 5 & 41.10 & 16.97 \\ \text { OLD PRIMARY } & 5 & 22.00 & 9.03 \\ \text { OLD SECONDAHY } & 5 & 9.80 & 4.11\end{array}$

1969.96
$* \quad 272.00$
$* \quad 91.00$

NPC REGION-DACIPIC CONST

BXCLUDLAG ELK'S HILL

$\begin{array}{lrr} & \\ \text { HEY PRIMARY } & 5 & 262.50 \\ \text { NFA SECANDARY } & 5 & 112.90 \\ \text { OLD PRIMARY } & 5 & 204.30 \\ \text { OLD SECONDARY } & 5 & 36.50\end{array}$

2.62 .50
112.90

OLD CFC

OL TFRTIARY 5

4.11

91.00

RLK'S HELL

NEH ERIMARY $\bullet \quad 150.00$

$\begin{array}{rrr}92.24 & 2.18 & 1.09 \\ 39.70 & .89 & .50 \\ 89.24 & 2.08 & .98 \\ 12.87 & .30 & .10\end{array}$

$\begin{array}{rr}* * & 5118.98 \\ * * & 340.00 \\ * & 147.00 \\ * * & 685.00\end{array}$

207.98 .

$+630.00$

NPC REFION-PICIPIC OCEAN

$\begin{array}{lrrr}\text { NEW ERIMARY } & 5 & 73.80 & 96.09 \\ \text { NEH TECONDARY } & 5 & 1.60 & 1.92 \\ \text { OLD PRIMARY } & 5 & 64.60 & 93.63 \\ \text { OLD SECONDART } & 5 & 50.30 & 67.35\end{array}$

2.60

.10

3.00

1.90

2.20

$\begin{array}{rr}* & 907.00 \\ * & 11.00 \\ * & 330.00\end{array}$

MPC REGION-HEST ROCKIES

$\begin{array}{llrrr}\text { NP.H PRIMARY } & 5 & 126.90 & & 116.35 \\ \text { NEH SPCONDARY } & 5 & 34.70 & & 11.49 \\ \text { OLD PRIMARY } & 5 & 17.20 & 12.02 & 5.49 \\ \text { OLD SECONDARY } & 5 & 79.10 & 51.07 & 2.31 .31\end{array}$

3.09
.80
.60
1.99

2.30
60
1.40
1.99

$\begin{array}{rr}* * & 2224.00 \\ * * & 91.00 \\ * * & 273.00\end{array}$

99.99

VINTAFP (OLD, NEW) INDICATES HHETHER OIL NELLS DRILIED BEFORE OR AFTER 1/1/77 (NOTE DIPFERENCE PROA LPGAL DEFTHITION OP OIL VINTAGP)

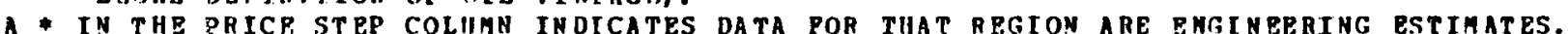

BONDLE PRICE= ITOTAL YALIIE OP ALA PRODIICTS IN INCREMPNT OV RR PREVIOTS PRICE STEPI IOUANTITY OP OTL IN THAT THCREM ENTI

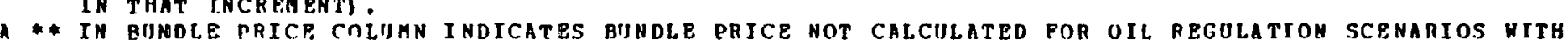
POT NT. ESTIMATPS OP SUPPLY.

CAPITAL INVESTHENT AND DRILLING STATISTICS ARE CHMILATIVE FROM 1977 TO 1989. 
TABI.E 4

(CONT INI) ED)

OIL STPPLY CURVES POR 1990

\begin{tabular}{|c|c|c|c|c|c|c|c|c|}
\hline $\begin{array}{l}\text { PAICB } \\
\text { STEP }\end{array}$ & $\begin{array}{l}\text { CRUDE OIL } \\
\text { (MBEI. } / O)\end{array}$ & $\begin{array}{c}\text { I NTERST ATE } \\
\text { GAS } \\
\text { (KACP/D) }\end{array}$ & $\begin{array}{c}\text { INTRASTATE } \\
\text { rAS } \\
\text { (HACP/D) }\end{array}$ & $\begin{array}{l}\text { BUTANR } \\
\text { (ABBL/D) }\end{array}$ & $\begin{array}{c}\text { GAS } \\
\text { LIQUIDS } \\
\text { (ABAL/D) }\end{array}$ & $\begin{array}{c}\text { BUNDCE } \\
\text { PRICE } \\
\text { (s/BBC OII.) }\end{array}$ & $\begin{array}{l}\text { CAPITAL } \\
\text { (An\$) }\end{array}$ & $\begin{array}{l}\text { FOHTAGF } \\
\text { DRTLLED } \\
\text { (YUFT) }\end{array}$ \\
\hline
\end{tabular}

HPC RERTION-BAST ROCKIES

$\begin{array}{llr}\text { NEH PRIMARY } & 5 & 167.60 \\ \text { NEW SPCONDARY } & 5 & 38.10 \\ \text { OI.D PRIAARY } & 5 & 73.60\end{array}$

OLD SECONDARY 5 146.5

22.48

112. 79

26.28
17.76

52.61

41.56

6.99
1.60
2.10
5.70

2. 30

.50

NPC REGIOn-H. TRX. /E. h. hEX.

$\begin{array}{llr}\text { NEH PRIMANY } & 5 & 141.80 \\ \text { HPD SECONDARY } & 5 & 55.00 \\ \text { OLD PRIMARY } & 5 & 390.90 \\ \text { OLD SECANDARY } & 5 & 181.50\end{array}$

55.00
390.90

$\begin{array}{lll}\text { OLD SECNNDARY } & 5 & 181.50 \\ \text { OLD TERTIARY } & 5 & 429.70\end{array}$

$\begin{array}{rr} & 125.66 \\ 319.76 & 48.73 \\ 70.69 & 407.12 \\ & 90.01\end{array}$

$$
\begin{array}{r}
7.90 \\
3.10 \\
37.37
\end{array}
$$

$70: 69$

90.01

NPC REGIUN-GUI.F COAST

$\begin{array}{llr}\text { NPU PRIMARY } & 5 & 343.30 \\ \text { NER SECONDARY } & 5 & 6.30 \\ \text { OLD PAIMARY } & 5 & 359.50 \\ \text { OLD SFCONDARY } & 5 & 765.40 \\ \text { OI.D TBRTIARY } & 5 & 60.20\end{array}$

275.43

392.87

10.40

232.70

1132.60

24.58
.60
31.07
54.57

7.79

.20

9.89

17.30

$\begin{array}{rr}* * & 4382.02 \\ * & 17.00 \\ * & 2702.93 \\ * & 745.00\end{array}$

NPC RFGTON-GILF OP HEXICO

$\begin{array}{lrrr}\text { NEH PRIMARY } & 5 & 354.60 & 411.76 \\ \text { NEW SPCONDARY } & 5 & 15.60 & 18.62 \\ \text { OLD PRIMARY } & 5 & 102.70 & 114.44 \\ \text { OLD SFCONDARY } & 5 & 21.90 & 21.37\end{array}$

11.76

111.44
211.37

$\begin{array}{rr}8.59 & 5.28 \\ .40 & .20 \\ 3.70 & 2.29 \\ .70 & .50\end{array}$

$\begin{array}{rr}* & 2526.98 \\ * & 45.00 \\ * \quad 113.00\end{array}$

52.98 


\begin{tabular}{|c|c|c|c|c|c|c|c|c|}
\hline $\begin{array}{l}\text { PAICR } \\
\text { STEP }\end{array}$ & $\begin{array}{l}\text { CAUDE OLL } \\
\text { (NBOL/DI }\end{array}$ & $\begin{array}{c}\text { I HTE. RST ATP } \\
\text { GAS } \\
\text { (NNCP/D) }\end{array}$ & $\begin{array}{c}\text { IWTAASTATE } \\
\text { WAAS } \\
\text { (nNCP/(1)) }\end{array}$ & $\begin{array}{l}\text { GUTAME } \\
\text { (MARL/D) }\end{array}$ & 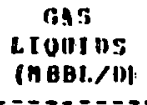 & $\begin{array}{l}\text { DUAOL. } \\
\text { PHICR } \\
\text { (S)DUL DII.) }\end{array}$ & $\begin{array}{l}\text { CapItat } \\
\text { (nns) }\end{array}$ & $\begin{array}{l}\text { Pootafis: } \\
\text { DRILLBo } \\
\text { InAPT: }\end{array}$ \\
\hline
\end{tabular}

HPC DEGIOM-AIDCONT IMENT

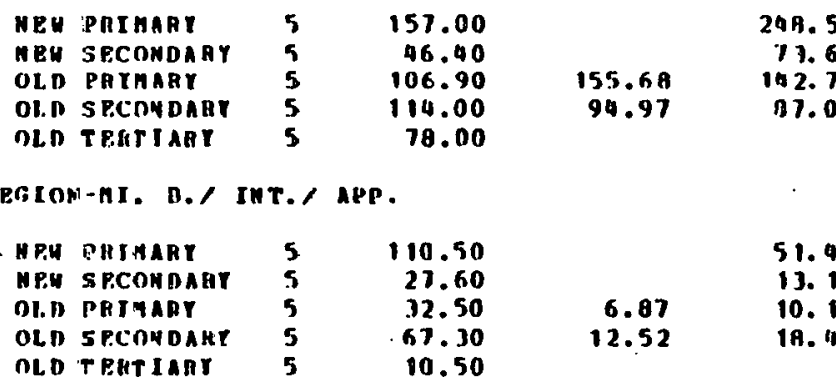

$\begin{array}{rrrr}3.30 & 1.29 & \because & 5357.00 \\ 1.00 & 1.60 & \because & 91.00 \\ 2.99 & 1.89 & \because & 542.99 \\ 2.19 & 1.49 & \because & 383.00\end{array}$

383.197

2.99

.

(2)

$\begin{array}{rr}\because * \quad 1730.00 \\ \because & 65.00 \\ \because & 229.00 \\ \because & 32.00\end{array}$

151.00

14

ค. 11

WPC AETLIOA-ATLANTIC COAST

$\begin{array}{llr}\text { HRW PRIMART } & 5 & 109.60 \\ \text { MPU SPCOMDAR } & 5 & 10.20\end{array}$

$\begin{array}{llr}\text { MRU SPCOHMART } & 5 & 10.60 \\ \text { OI.D PRTMARY } & 5 & 1.20\end{array}$

.05

$\begin{array}{rr}60.50 & 2.89 \\ 6.02 & .10 \\ .50 & \end{array}$

$\because \quad \begin{array}{r}1320.00 \\ \because \quad\end{array}$

15.99

GPC RETION-ATI.ANT IC OCPAN

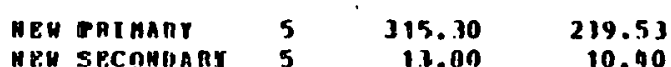

MUC AETION-NOITII SLOPE

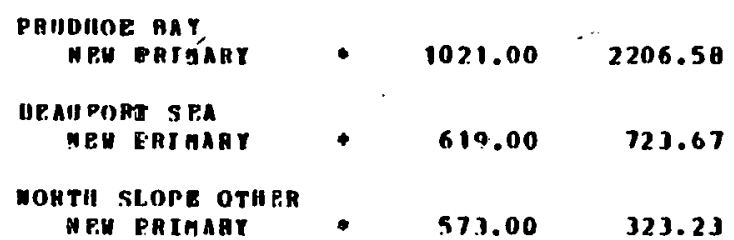

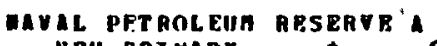

$$
\text { HRU PAIMARY GOS.00 }
$$

DATA SOURC.S : PRODUCTION PIGURPS ARE TAKBW PROM THP PIBS OIL AMt. Gas MODRL

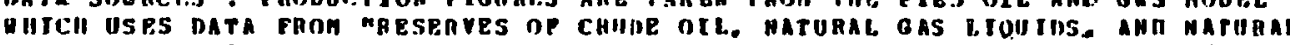
GAS IN THE UNITED STATES AHD CANBOA AND CAPACITT AS OF DECEnBP: 31, 19\%", D.C.

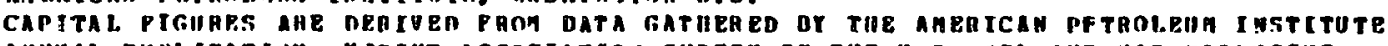

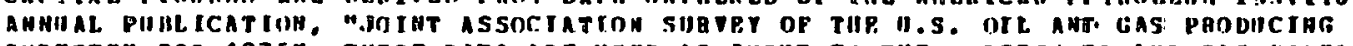

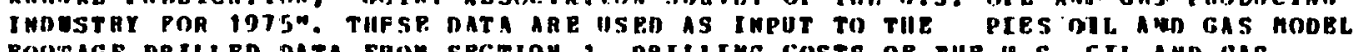

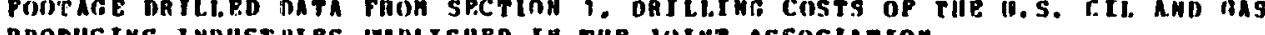
P AMD YAFTUIS OTHBR PAERG

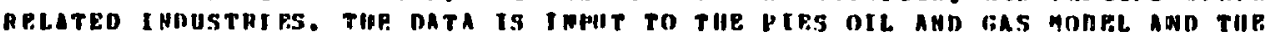

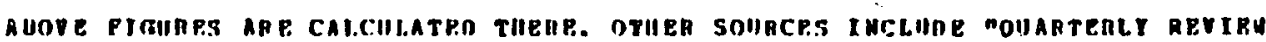

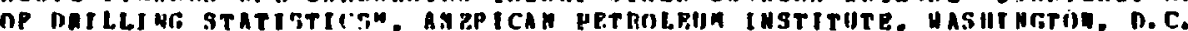

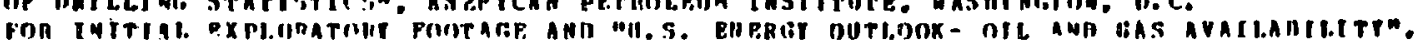

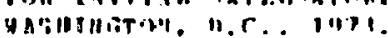


TAILE 5 .

PRTCE THA.TECTORIES FOR HONATSOCTATED GAS

$(\$ / M C P)$

\begin{tabular}{|c|c|c|c|c|c|c|c|c|c|c|c|c|c|c|c|c|}
\hline & & 1977 & 1978 & 1979 & 1980 & 1981 & 19ค2 & 1981 & 1984 & 1995 & 1986 & 1987 & 1988 & 1989 & 1990 & 1991 \\
\hline STE.P & 1 & 1.63 & 1.61 & 1.58 & 1.55 & 1.53 & 1.51 & 1.49 & 1.47 & 1.44 & 1.44 & 1.44 & 1.411 & 1.44 & 1.44 & 1.44 \\
\hline$T E P$ & 2 & 1.6 .1 & 1.51 & 1.60 & 1.58 & 1.57 & 1.55 & 1.53 & 1.52 & .50 & 1.50 & 1.30 & $=0$ & 1.50 & 1.50 & 1.50 \\
\hline STEP & 3 & 1.63 & 1.63 & 1.63 & 1.63 & 1.6 .3 & 1.63 & 1.63 & 1.63 & 1.63 & 1.53 & 1.53 & 1.63 & 1.63 & & 63 \\
\hline iTP.P & 5 & 1.63 & 1.6 & 1.70 & 1.73 & 1.76 & 1. 00 & 1.811 & 1.87 & 1.91 & 1.91 & 1.91 & 1.91 & 1.91 & 1.91 & 1.91 \\
\hline TEP & 6 & 1.63 & $1.6 \mathrm{~A}$ & 1.73 & 1.78 & 1.8 .3 & 1.89 & 1.95 & 2.00 & 06 & 2.06 & .06 & 2.06 & 2.05 & 2.06 & 2.06 \\
\hline IT EP & 7 & 1.53 & 1.69 & 1.76 & 1. $\mathrm{A3}$ & 1.91 & 1.98 & 2.06 & 2. 114 & 2.23 & 2.23 & 23 & 2.23 & 2.23 & 2.23 & 2.23 \\
\hline$T P P$ & 8 & 1.63 & 1.71 & 1.80 & 1.89 & 1.98 & 2.08 & 2.10 & 2.29 & & 2.41 & 41 & & 2.41 & 2.41 & .41 \\
\hline
\end{tabular}

DATA SOURCES : OUTPIT FROM THE PIPS OIL E CAS SIIPLY MODEL DERTVED PROA

AVERACE REPFRPNCE ACOUISITION COSTS, TRANSPOATATION COSTS.

AND OTHEY RPLEVANT COSTS AND PRICES. AlSO PLATT'S OLgGRA, WASHINGTON, D.C

A gFELY PIIBLICATION.

OPPICE RESPONSIBLE : OIL AND GAS ANALTSIS DIVISION, OPFICE OP EHERGY

SOIRCP. ANALYSIS, APPLIED ANALYSIS, RIA.

TABLE 6

PRICE TRAJFCTORIES FOR NATURAI. GAS LIOIIIDS

$(\$ / B \cap L)$

STEP

ST EP 2

$\operatorname{sTBP} 3$

ST E.P

STPP 5

STEP 6

ST EP 7

$S T R, P B$

STEP

DATA SOURCES : OUTPUT FROM THE PTPS OIL E GAS SUPPLY MOUPL DERTVED PROM

AVERAGE REPEABNCP ACOUISITION COSTS. TRANSPOTTATION COSTS

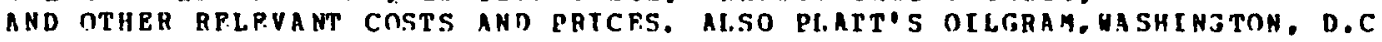
A YPEKLY PIJBI.TCATION.

OFFICE RESPONSIALE : OIL ANR GAS ANALYSIS DIVISIOH, OFPICE OP ENTRGT

SO!IRCE ANAIYTIS, APPLTPR ANAI.YSIS, ETA. 
PABLE 7

GAS SIJPPLY CURPES POR 1985

PRICE STEP WATURAL GAS
(HACP /DI

RITANE
(MBBL/D)

BIN DLP PRIC (S/HCP GAS)

CAPITAL

DAILI.ING

(nHCP/D)

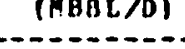

IMIsBL/D

(n) (HAFT)

HPC RRGIOH-SOITH AI,ASKA

INTERSTATE GAS

$\begin{array}{ccc}\text { NEX GAS } & 1 & 122.10 \\ & 2 & 160.98 \\ & 3 & 229.42 \\ & 4 & 370.14 \\ & 5 & 433.94 \\ & 6 & 626.68 \\ & 9 & 629.41 \\ \text { OED TAS } & 1 & 262.93\end{array}$

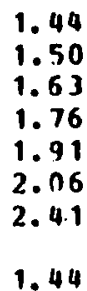

NPC REGION-PACIPIC OCEAN INT P.RST AT P GAS

NEW GAS

55.30

56.67
57.22

58.32

53.41

15.61

OLD GAS

6

NPC REGION-UEST ROCKIES

INTERSTATE GAS
OLD GAS

663.31

1.06

142.98

192.38

239.97

48 A. 96

587.96

881.75

883.95

1.44

NPC REGION-EAST ROCKIES

INTE RSTAT $E$ GAS

OLD GAS

1

243.60

3.73

2.53

1.66

NPC REGJION-N, TFK, /E. N. MEX.

TNTERSTATE GAS

OLD GAS

332.61

1.44

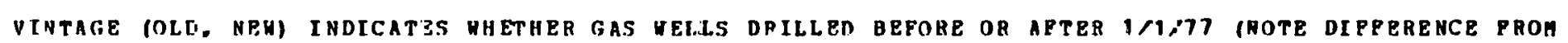
LETAL TIEEINITIOA OP GIS VINTAGE).

ALL OUUATITIES (PRODNCTION. INVESTHBNT AND DRILI.THG) ARE CUNULATIVE OVER PBICE STEPS.

PRICE STEPS AITH NO ADIITIONAL ERODICT ION OV RR PREVIOISS STEP ARE OMITTED.

BUNDLE PRICE = (TOTAL VALIE OP ALL PRODNCTS IN INCREMENT OVRR PREYTOOS PRJCE STEP)/(OHANTITY OP NATURAI. GAS IN THAT I MCRPMENTI.

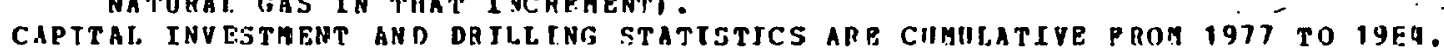


TAB T.E 7

(CONTTHUED)

GAS SUPRLY CURYES POR 1985

PRICP STEP

- - - - - - - -

NPC RFigION-GHJ.P COAST

INTRRSTATE GAS

$$
\text { oln Gas }
$$

NPC REGION-GIILF OE HEXICO I NTEISTATE GAS

NEH GAS
OID GAS

NPC REGION-MTDCONTINENT

I NT ERST ATE GAS

$$
\text { OLN GAS } 1
$$

NPC REGTON-HI. BASIN AND INT INTERSTATP GAS

$$
\text { OLn Gas , }
$$

317. 11

$$
\text { OLD GAS }
$$

NPC REGJON-ATLANTIC OCPAN

INTERST ATE GAS

NP, G GS

1
3
8

395.34

402.46
419.71

7.59
7.69

7.69
7.99

3.08
3.19

J. 1 A
3.28

2.13

.04

A LIOII D

MOE REGTON-n. Y./N.J.

INTRASTATP GAS.
B!JN DI.E PRICE

(\$ / MCF GASI

CAPITAL

(AM \$)

DRILLING

(BBBL $/ D$ )

(HBBL/D)

-...........

1.

(MAF'CI

-

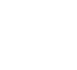


TADLE 7

(CONTINIIED

IAS STPPLY CINRYES FOR 1985

HATURAL GAS

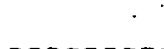

DOE REGION-AID-ATLANTIC

THTRASTATE GAS

OLD GAS 1

DOE REFION-SOITU ITLANTIC INTRASTATE GAS

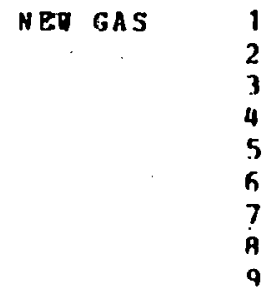

OLE GAS

ATURAL GAS (AMCP /!))

AHT ANE
(MBDL $/ M$ )
(ABBL/D)

3I NDLR PRICE GS /HCP GASI

CAPITAL (AMS)

DR I LLIH: (BกPT)

123.98

2. 37

1.30

1.69

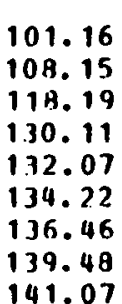

1.23

1. 37.

1.44

1.59

1.61

1.64

1.67

1.70

1.72

1.43

73.46

1.58

1.69

1.84
2.03

2.05

2. 0.9

2.13

2.17

2.20

.99

1. 68

1.75

1.89
2.05

2.05
2.22

2.22
2.39

2. 59

2.81

3.26

1.72

71.06
76.10
85.29
95.99
97.95
100.36
102.38
105.55
107.78

3. 14

3. 37

3.73

4.19
4.24

4. 33

4.42

4.53

4.60

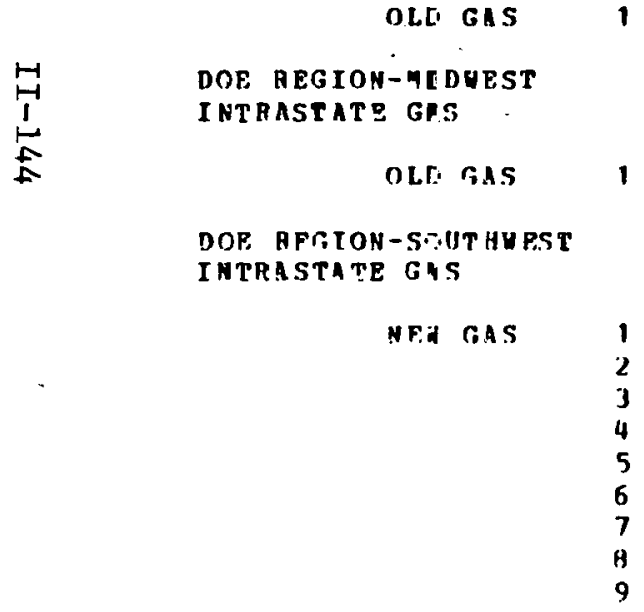

92.84

.84

.41

1.56

15022.00

15820.28

17024.78

18340.18

18605.20

18769.77

18998.17

19391.28

19845.21

198.29

20月. 35

223.65

240.22

243.27

245. 34

248.04

251.85

256.03

71.60
204.10
215.110
231.65
250.20
$253.3 \mathrm{~A}$
255.93
258.83
26.3 .43
267.56

5303.40

54.63

1.57
1.7
1.8
2.34
2.1
2.40
2.55
2.7
3.

10905.97

11570.05

12629.41

13845.23

14091.13

14257.70

14489.70

14907.26

15440.50

1.64
491.22

520.211

$565.4 \%$

619.21

628.87

634.77

643.91

$660.8 ?$

685.00 
TABLE 7

[CONT INIJ ED]

GRS SUPRLY CURVES FOR 1985

PRICE STEP NATIIRAL GAS ( IACF /D)

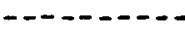

DOE REGIUN-CRNTPAL

I NT RASTATE GAS

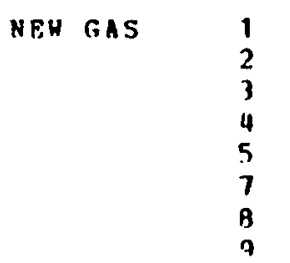

OLD GAS

1

DOE RFGIOH-RORTH CENTRAL

INTRASTATE GAS

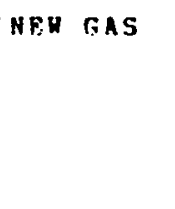

OLD GAS

4
5
5
7
8
9

DOE RECION-WEST

INT RASTATE GAS

NF.H GIS

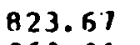

863.01

921.21

975.95

1007.11

1024.51

1038.32

1076.58

335.85

290.21

365.50

453.97

548.94

697.5

A92. 32 .

308.25

BUTANE

( $\mathrm{BBB} / \mathrm{n}$ )

GAS LTQUIDS
(ABBL/D)

BUNDLE PRICE (S/MCP GAS)

Capital (n)\&)

DAILLING (HAPT)

-

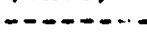

9.47
9.89
10.48
11.03
11.34
11.51
11.61
11.94

8.32
8.59
9.20
9.59
9.97
10.12
10.21
10.51

$1.6 \mathrm{~B}$

1.81

1.96

2.12

2.47

2.60

.07

609.73

644.16

696.38

749.49

777.11

795.57

10.25

ค50.69

1.59

2.25

2.99

3.79
4.68

4.68
5.68

5.68

8.81

6.09

9.53

3. 95

2.60

ค6. 24

197.67

299.24

326.07

353.45

392.33

441.88

515.53

.70
1.69
2.58
2.78
2.94
3.28
3.78
1.37

1.10

2.49

3.69

4.09

4.49

4.88

5.48

208.62
6.31

4.37

.
1.98

2.16
2.32

2.32
2.50

2.68

3.11

330.38

421.98

529.97

649.97

944.51

1113.55

1.63
116.00
212.9 月
$330.9 \mathrm{~B}$
363.98
396.98
444.97
500.97
606.97

15.99

19.99 .

24.98

30.98

40.31

53.40

2. 16

2.46

2.72

1.44

4.99
8.98
13.99
14.98
15.98
17.98
20.97
24.97

. 
TA BLE H

GAS SIIEPI.Y CIIRVES ROR 990

\begin{tabular}{|c|c|c|c|c|}
\hline PRICE STE? & $\begin{array}{l}\text { HATURAL GAS } \\
\text { (HACP /D) }\end{array}$ & $\begin{array}{l}\text { BUTAUE } \\
\text { (ABBL/D) }\end{array}$ & $\begin{array}{c}\text { GAS LIOUIDS } \\
\text { (ABOC/L) }\end{array}$ & $\begin{array}{l}\text { BISNDL? PRIEE } \\
\text { (S/AC? GAS) }\end{array}$ \\
\hline
\end{tabular}

CAPITAL

DRI LLINA: (14) 8 ) (KNPT)

NPC REGION-SCIITH ALASKA

INTERSTATE GAS

NEW TIAS

196.57

473.91

777.81

922.91

1315.24

1317.43

OLD GAS

233.53

$\begin{array}{lr}1.50 & 59.00 \\ 1.63 & 254.98 \\ 1.76 & 555.96 \\ 1.91 & 724.95 \\ 2.06 & 1188.91 \\ 2.41 & 1191.91 \\ 1.44 & \end{array}$

1.00

1.97

2.96

4.92

4.92

NPC REFTON-PACIFIC OCEAN

INTER STATP. GAS

NEW GAS

1
4
5
8
5

点
134.37

136. 34

139.62

140.99

9.85

459.25

160.47
1.62

1. 76

2. 25

2.41

1.91

1.79

1.79

1. 19

.7 .3

.46

1. 47

NPC REGTON-FAST ITOCKIET

INTERSTATE, GAS

$$
\text { OLD GAS } 1
$$

NPC REGION-W, TEX./B., N. HEX, INTERSTATB GAS

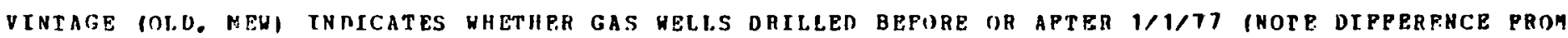
LE(FAL. DEFIUITION OF GAS VI NTAGFI.

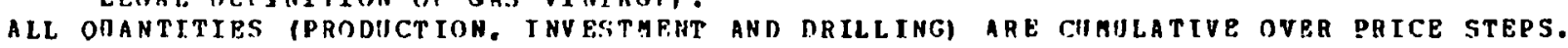

PRTCE STEPS WII $\|$ NO ADDITIONAL PRODUCTION OVER PREVINIIS STEP ARE OATTIED.

BINDLE PRICE = ITOTAL VALUE OF ALL PRODICTS IN INCREMPNT OVER PREVIJIS PRICE STEPI/IOUANTITY OP NATURAL GHS IN THAT INCREMEN'T).

CAPITAL INVESTAFYT AND DRILLING S'PATISTICS ARE CUMILATIVE FRON $197 \%$ TJ 1999. 


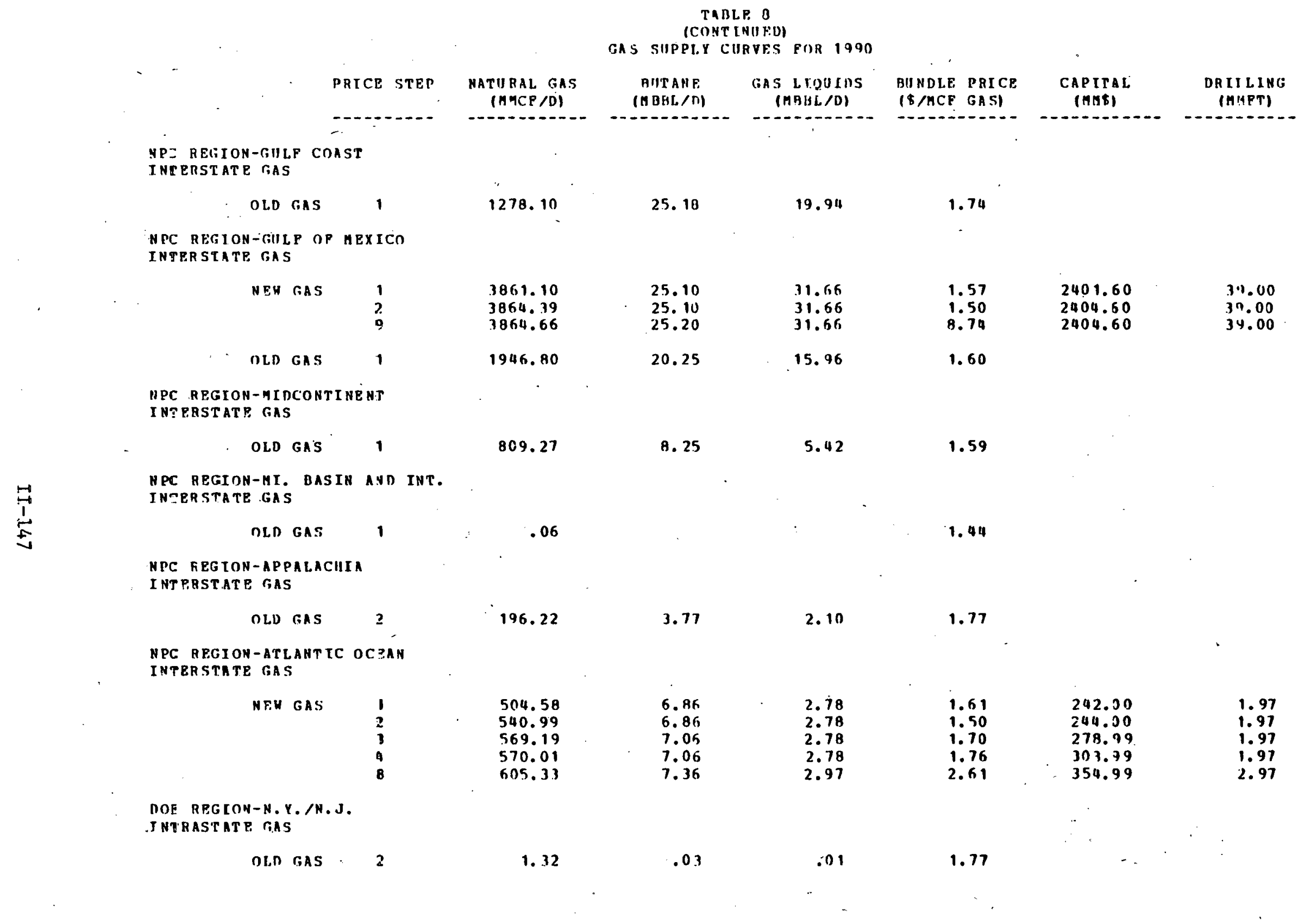


TABLE 8

(EOHTYNOED)

GAS SIJPLY CURVES FOE 1990

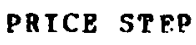

PRTCE STEP

DOP REGION-MID-ATLANTIC

INTAASTATE GA

$$
\text { OLD GAS } 2
$$

DOE REgION-SOUTH ATLANTIC INTRASTATE TIAS

NEH GAS

NATIJAL GAS

(A) $C F / D)$

BUTARE

(MGBL/D)

Gas LIgOIns (ABBL/O)

BIIN DL: PRICE (S/ACP GAS)

CAPITA L (AB)

DAI LI.ING (HA) T)

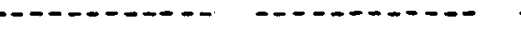

76.72

1.47

.82

1.77

83. 31

96.78

117.72

134.78

141.48

147.53

155.32

167.19

179.80

OLD GAS

21.98

40.85

1.02
1.19
1.44
1.65
1.73
1.00
1.90
2.05
2.20
.43
.80

1.30

1.51

1.8 .3

2.10
2.20

2.20
2.70

2.42

2.60

2.40

.34

.54

1.68

1.75

1.90

2.05

2.22

2.40

2.59

2.80

3.26

1.74

1.77

DOE REGION-MIDHEST

INT RASTATE GAS

CLD GAS

46.28

.46

.26

1.44
1.77

DOE REGION-SOITH URST

I NTRASTAT P, GAS

MEW GIS

1604.00

13778.00

16263.50

1 18232.30

19108.07

19760.11

20693.61

22245.71

24130.91

2687 . 80

OLn gas
1

149.69
176.43
208.00
232.21
242.54
250.59
261.70
279.39
300.32
37.09

156. E. 5

184.15

2.18. 10

244.68

255. 99

264.82

276.95

296.20

318. 26

28. 2

37.09
88.96
98.18
116.09
136.68
144.36
151.90
161.49
177.06
194.32

3.90

4.30

5.04

5.8 B

6. 19

6.49

6.86

7.43

8.07
1.67
1.72
1.88
2.03
2.18
2.38
2.56
2.74
3.17

13464.11

605.73

14747.42

$17095.96 \quad 765.28$

$19582.55 \quad 871.79$

$20598.52 \quad 915.66$

$21379.25 \quad 947.42$

$22550.79 \quad 995.77$

$24535.26 \quad 1082.38$

$27376.53 \quad 1200.02$

1.65 
TARLP: 1

GAS SIPPIY CURVES POA 1990

\begin{tabular}{|c|c|c|c|c|c|c|}
\hline PATCP. STEP & $\begin{array}{l}\text { SATUPAL GAS } \\
\text { (AYCP/OI) }\end{array}$ & $\begin{array}{c}\text { PHTARE } \\
\text { (nOBL/ח) }\end{array}$ & $\begin{array}{c}\text { GAS LIOUIIIS } \\
\text { (YBDL/D) }\end{array}$ & $\begin{array}{l}\text { MIINDLE PRTCP } \\
\text { (S/MCP (BASI }\end{array}$ & $\begin{array}{l}\text { CAPITAL } \\
\text { (AAB) }\end{array}$ & $\begin{array}{l}\text { DRILLIRG } \\
\text { (HAPT) }\end{array}$ \\
\hline
\end{tabular}

NOF. RPTION-CFNTRAT.

ENTRATATE GAS

NP.W GIAS

OLD GAS

1
2
3
4
5
6
7
8
9

1

DOE REGION-NORTH CENTEAL

INTRASTATE, GAS

NEW GAS

4
5
6
7
9
9

OLD GAS

635.03
765.11
859.97
950.09
1004.16
1030.59
1082.86
1155.86
126.61

179.63

6.71
7.77
9.60
9.36
9.95
9.98
10.39
10.75
11.79
1.81

5.91
6.82
7.55
8.22
8.65
8.77
9.14
9.62
10.36
1.20

1.20

\subsection{49 \\ 5.78 \\ 7.18 \\ 9.32
12.31}

925.23
1240.20

204.91
10.10
13.21

.06
.02

1.51
1.64
1.79
1.9 .3
2.09
2.20
2.43
2.61
3.04

1.59

$\begin{array}{rr}760.93 & 38.36 \\ 821.20 & 41.36 \\ 942.52 & 47.38 \\ 1060.26 & 53.02 \\ 1139.45 & 56.77 \\ 1159.20 & 57.71 \\ 1231.05 & 60.91 \\ 1331.68 & 65.42 \\ 1501.71 & 73.12\end{array}$

$\begin{array}{rrr}1.99 & 466.00 & \\ 2.14 & 613.98 & 21.99 \\ 2.33 & 792.97 & 37.98 \\ 2.51 & 1007.95 & 47.98 \\ 2.68 & 1385.31 & 66.26 \\ 3.12 & 1953.15 & 94.07\end{array}$

1.4 .5

DOE REGION-WRST

NEU GAS

2
3
4
5
6
7
7
9

7.12
209.17
330.73
373.71
416.97
477.75
567.55
698.11

.10
1.90
2.79
1.19
3.59
4.09
1.78
5.97

129.22

OLO IFAS

DATA SOIJRCES : PRONIICTION PIGURES ARE TAKEN PROA TIIT. PIUE OIL AHD GAS MODEL

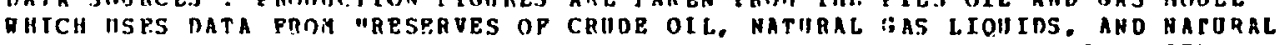

GAS IN THE INITED STATPS AND CANADA AMD CAPACITY AS OP URCEMBER 31 : 974 "

AHERTCLH PETROLEIN INSTTTUTE. MASHINGTON D.C.

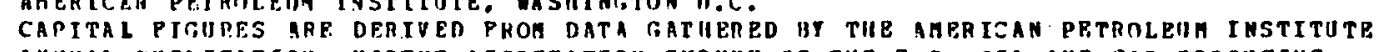
ANNIIAL PHBLICATION. "JOTNT ASSOCTATION SURVPY OF THE O.S. OIL AHN GAS PRONICING

INDIISTBY FOR 1975". THPSE OATA ARP ISED AS INPUT TO THE PIBS OIL AND GaS MODEL.

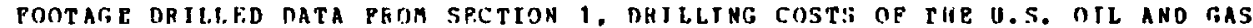

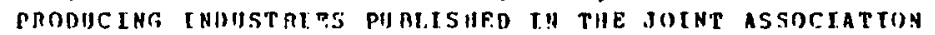

AMFRICGN PPTROLPUM COUNCIL. THE AMPRTCAN GAS ASSOCIATIOH. AND VARLOUS ORHER ENPRGY

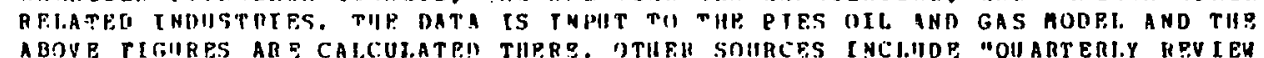

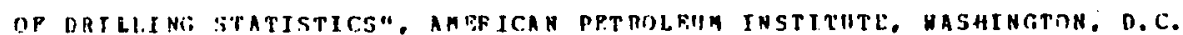

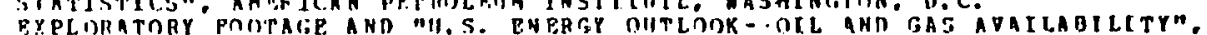
MASUINGTON, 11. C.. 1977 .

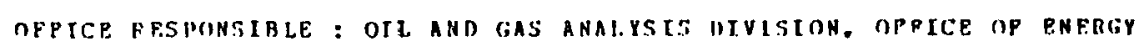

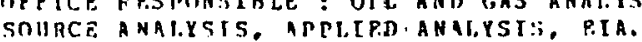


TABLE,

DISTRIBUTION OP/INTPRSTATE TAS IN 1985

(MMCP /D)

DOR REGION

NPC RFGINH

PACIFIC CHAST PACIPIC OCEAN WEST ROCKIPS F.AST ROCKI ES V. TFX. $/$ R. N. MEX. GULP COAST

GULP OP MEXICO

AIDCONTIHENT

HI. BASTN AND TNT.

APPALACHI.A

ATLANTIC COAST

A TLANTIC DCEAN

NOATH SLC.PE

SOUTH. ALASKA
IEW ENG. NY/NJ

MID-ATI.. S. -ATL.

MIDHEST

S. - HF.ST

CENTKAL.

H. - CNTRL nest

N. - HES r

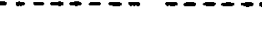

min-

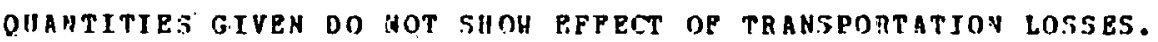

STATEA DTSTRIBITION OF EORTI SLOPE GAS TO THR BAST COAST REPRESENTS NEC EPPECT OP DISPLACERENT. DATA SOUPCE. : PRONICTION PIGURES ARE TAKEH PROA THE PIES CIL AND GAS MONEL WHICY ISZS DATA FROM "RPSERVES OP CPUDE OIL, NATURAL GAS LECUIDS, AND NATIRAL GAS IN THE TNTTED STATES AND CANADA AND CAPACITY AS OF DECEMBER 31. $1974 "$. AKERICAN PEZROLEUM INSTITUTE. MASUINGTON D.C.

DPPICE RZSPONSIALE : OIL AND GAS ANALYSIS DIVISION, OPPICE C:P ENERG SOURCE ANALSIS. APPLIED ANALYSIS, BIA. 
TABL? 10

INTERSTATE. GAS DPLIVERIRE IN 1985

DOE RPGIOH

NEH ENG. NY /NJ

MID-ATI. S. -ATL.

MEM

LOUFR 118 AND S. ALASKA

OUANTITY NEI.TVERED (MMCP/D)

AVERAGE PRICE ( $\$ / M C P)$

3 คก

1. 58

1.352

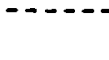

ALASKAN NORTH SLOPP

OUANTITY DELIVERED (HMCE/D)

DELIVEREN PIICF (E/MCP)

34
3.05

1.43

1.34

2486

MIDARST

S. - YEST

CENTRAL N. -CNTRL UEST

N. - 4 RS T

$10 B$

3.01

145
2.94

235
2.76

4445

1509
.85

บ1A

.68

518
.65

2039
1.38

45

1. 15

667
2.89

129
2.61

107
2.81

2

577

2.58

40 DATA SOI RCES : PRODUC?ION PIGURFS ARETAKPN

HIICH ISES DATA PHOY "RESERVES OP CRIUDE OLL, MATORAL GAS LIOUINS, AND NATHAAL

GAS IN PHE INITED STATES AND CAHADA ANI CAPACITY AS OP DECEBBER 31. 1974".

AHERICAN PZTROLEUM INSTITUTE, WASHINGTON D.C.

OFPICE RESPONSTELE : OIL AND GAS ANALYSIS DITISLON, OPPICE OP FNERGY SOIHCE AHALYSIS. APPLIED ANALYSIS. PITA. 
TAHLE 11

DISTREATIOH OP TNTERSTATE GAS IY 1990

(HACP /D)

DOE RPGION

\begin{tabular}{|c|c|c|c|c|c|c|c|c|c|c|}
\hline NTC RRGION & NEH ENG. & NY $/ N J$ & MLE-ATL. & S.-ATI. & MI DHEST & S. - DEST & CENTRAL & N. -CNTRI. & DEST & N. $-W$ ES T \\
\hline PACIFIC COAST & & & & & & & & & & \\
\hline PACTFIC OCEAN & & & & & & & & & 408 & \\
\hline WFST ROCKIES & & & & & & 19 & & 113 & 356 & 28 \\
\hline PAST ROCKIPS & & & & & 10 & & 30 & 178 & 4 & 5 \\
\hline H. TEX.YZ. N. MRX. & & & & 1.1 & 78 & 84 & 75 & 5 & 263 & \\
\hline GII,P COSST & 60 & 195 & 251 & 483 & 591 & 304 & 53 & & & \\
\hline GILF OP MEXICO & 236 & 752 & 1001 & 1.365 & 2411 & 56.7 & 45 & & & \\
\hline MIDCONTINENT & & 13 & 18 & 21 & 430 & 130. & 361 & 64 & 22 & \\
\hline MI. BASTN AND INT. & & & & & 10 & & & & & \\
\hline A P PATACAIA & & 55 & 74 & 14 & 62 & & & & & \\
\hline ATLANTIC COAST & & & & & & & & & & \\
\hline A TLANTIC OCEAN & 6,6 & 235 & 298 & 219 & & & & & & \\
\hline $\begin{array}{l}\text { NORTT SLOPE } \\
\text { SOUTH RLASKA }\end{array}$ & 111 & 131 & 174 & 274 & 793 & 197 & 126 & 24 & $\begin{array}{r}667 \\
1467\end{array}$ & 146 \\
\hline
\end{tabular}

OINATITIRS GIVEN DO NOT SHOH EPFECT OF TRAHSPORTATION LOSSES.

STATED OTSTAIBITION OP HORTH SLOPE GAS TI THE EAST COAST REPRESENTS NET. PAPPCF OP DISPLACERENT. DATA SOTRCES : PRODUCTION PIGURES ARP. TAKP.N PROA THE PIES OIL AND GAS MODEL WHICH USES DATA PROM "RESERUES OF CRUDE OIL. NATIHAL GAS LIQUIDS, AND NATURAL GAS IN THE UNITED STATES AND CANADA AND CAPACITY AS OP EBCEABER 31. 1974". AH FR ICAH PETROLFUM INSTITUTE, MA SHIMGTOH D.C.

OFPICF RESPONSIBLE: OIL AND GAS ANALYSTS DIVISION, OPPICB OP ENERGY SOORCF ANALYSIS, APPLIPD ARALISIS, RIA. 
$T \cap B L P, 12$

YNTERSTATE GAS DELIVERIP.S IN 1990

DOE REGION

NEW ENG. HY / NJ

MID-ATL.

- ATL.

MINHEST

S. - HPST

CENTRAL

N. -CNTRL UEST

N. - HEST

LONFR 4B AND S. ALASKA QUANTITY DELIVERED (KMCE; ADERAGE PRICP (\$/MCP)

$\begin{array}{rr}339 & 1183 \\ 1.80 & 1.65\end{array}$

1579

2060

1.35

3450

1. 38

1083
1.011

551

.73

359

.55

2325

1.60

31

ALASKAN NOHTH SLOPE

OIIANTSTY DEIIVERED (MMCP;O)

37
28

118
2.54

159

258

733
2.44

142

117
2.46

23

6.35

2.33

1.05

STATED DTSTRIBUT ION OF NORTII SLOPF GAS TO TIIE EAST COAST REPRESENTS NET EPPECT OP D.tSPLACEMENT.

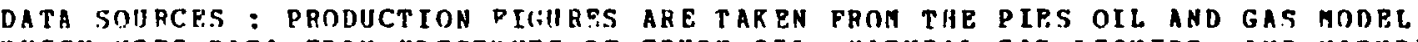

WHICH IISES DATA PROA "RESEIUVES OF CRUDE OIL. NATURAL GaS LIQUIDS. AND NaTURAL

GAS IN THE "INTED ST ATES AND CANADA AND CAPACITY AS OP DECEMBER 31, 1974",

AM ER ICAN RETROLEIM INSTITITE. HASHINGTON D.C.

OFPICP. RPSPONSIBLE : OTL AND GAS ANALYSIS DIVISTON. OPPICE OF PNERGY SOURC ANILSIS. APPI.IED ANALYSIS. EIA. 
TAB L.F. 13

OIL HPLI, DRELLINT; INVESTHENT AHO PRODUCTIOM SURAAIY POH 1985

CRUDE OTL INTERSTACE INTAASTATE CAPITAL ORILLING

\begin{tabular}{|c|c|c|c|c|}
\hline$(A B B L / D)$ & $\begin{array}{c}\text { GAS } \\
\text { (MACP/D) }\end{array}$ & $\begin{array}{l}\text { GAS } \\
\text { (ance }\end{array}$ & $\begin{array}{c}1977-1984 \\
\text { (nn\$) }\end{array}$ & $\begin{array}{c}\text { 1977-1984 } \\
\text { (NAPT) }\end{array}$ \\
\hline 316 & 131. & & 1404 & $h$ \\
\hline 831 & & & 4150 & $124^{\circ}$ \\
\hline 209 & $\begin{array}{l}295 \\
100\end{array}$ & & $\begin{array}{r}703 \\
907\end{array}$ & 6 \\
\hline 492 & 109 & & 1466 & 85 \\
\hline 1527 & 541 & . & 3316 & 103 \\
\hline 1949 & 939 & & 4582 & 169 \\
\hline 565 & 635 & & 1727 & 35 \\
\hline 544 & 320 & & $35 \mathrm{B2}$ & 228 \\
\hline 251 & 24 & & 1173 & B9 \\
\hline 112 & & & 3.33 & 22 \\
\hline $\begin{array}{r}270 \\
2072\end{array}$ & 205 & & $\begin{array}{r}759 \\
13705\end{array}$ & 6 \\
\hline
\end{tabular}

NPC REGIOH

SOUTH ALASKA

PACIPIC COAST

PACIPIC OCEAN

WEST ROCRIPS

RAST ROCKIES

h. Tex./e. n. nex.

Gutep const

GILP OP HEX ICO

ATDCONTI HPNT

HI. B., INT./ AP.P.

ATLANTTC OCEAH

AORTH SIOPE

3168

3795

DOP BEGTON

NP. ENGLAND

H.Y./H.J.

MID-ATLANTIC

SnIITH ATLATIC

MIOURST

SOIITHEP.ST

CENTRAI.

NOATII CENTRAL

HEST

NOHTHEPST

NATTONAL TOTAL

9769

646 A

1
47
57
25
2635
137
213
231

N

WICH. USES DATA PROA "RFSRRVES OF CRIDP OIL, NATURAI. GAS LIQIITS, AND NATIIRAL

"AS IN THE IINT TFD STATES ANO CANADA AND CAPACITT AS DP DECEMAER 31. 1974".

AM FR ICAN PETROLEUA INSTITUTE, HASHINGTUN D. C.

CAPITAL PIGIIES APP DFRIVPD FROM DATA GATHEPED OY THE AHERICAN PETROLEUA INSTITUTE

ANNIAL PIJLICATION. "JOINT ASSOCIATION SURVEY DE THE II.S. DTL AND GAS PRODUCING

INOUSTPY FOR 1975". THESE DATA ARE IISEN AS TNPUT TO TIE PIRS OII. ANA GAS MODEL.

FONTAGE DRTLLED DATA PROA SPCTIIN 1. DRLLLING COSTS OP THE IJ.S. OIL AHD GAS

PRODICING INDUST RIPS PURLISIIED IN THP, JOINT ASSOCIATION

AMERICAN PETROI.PUM COINCIL, THP AMERICAN GAS ASSOCIATLON, LND VARLOUS OTHPR ENERGT

RELATFD INDISTRIPS. THP DATA IS INPIT TO THP PIES OIL AHD LAS MODEL AND THE

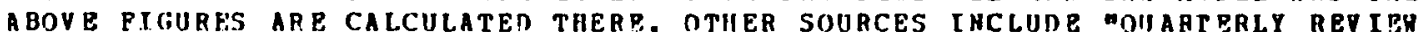

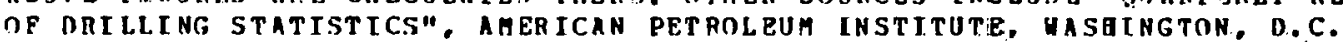

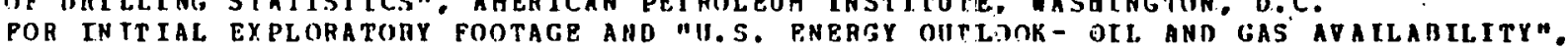
WASHITTON, D. C. . 1973 .

UPFICE RESPONSIALE : OIL AND GAS ABALSLS DIVISLON, OPPICE OF ENFRGY

SOURCE AMALYSIS, APPLTEN ANAIYSIS. FIA. 
TAUI.P. 14

OIL WHLL DEIIIITNG, INVESTMPAT AND PROUUCT.IOH SIJMAARY POR 1990

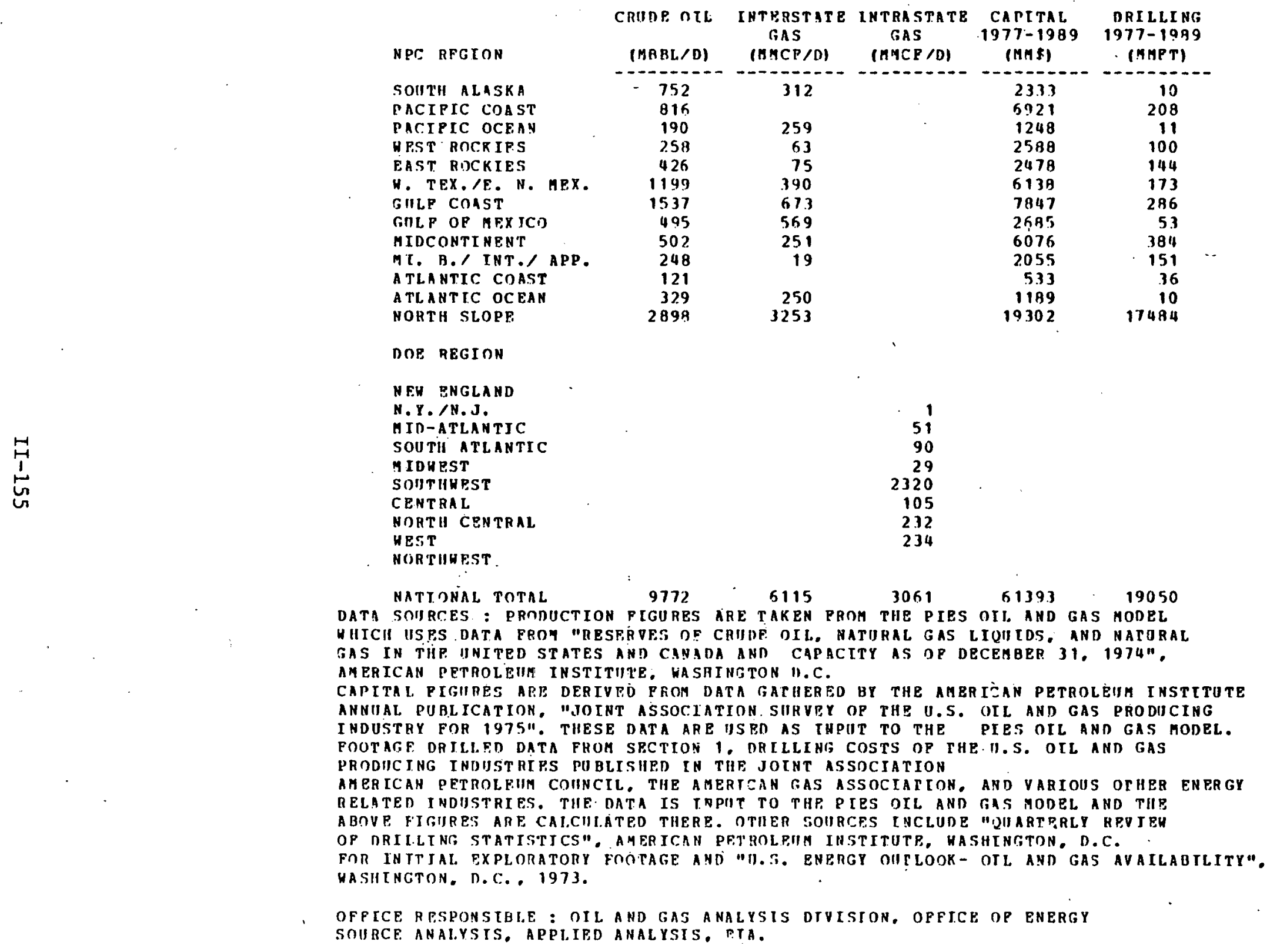


TABLE 15

NOH-ASSOCIATED INTRESTATP (IAS

PRODICT ION SIMAMARE

I N 19 a5

(AnCF/D)

PRICE STEP

\begin{tabular}{|c|c|c|c|c|c|c|c|c|c|}
\hline NPC RRGION & 1 & 2. & 3 & 4 & 5 & 6 & 7 & A & 9 \\
\hline $\begin{array}{l}\text { SOUT H ALASKA } \\
\text { PACIFIC OCEAN }\end{array}$ & $\begin{array}{r}385 \\
55\end{array}$ & $\begin{array}{r}424 \\
55\end{array}$ & $\begin{array}{r}492 \\
55\end{array}$ & $\begin{array}{r}633 \\
57\end{array}$ & $\begin{array}{r}697 \\
57\end{array}$ & 870 & $\begin{array}{r}890 \\
73\end{array}$ & $\begin{array}{r}992 \\
74\end{array}$ & $\begin{array}{r}992 \\
75\end{array}$ \\
\hline WEST ROCRIES & 66.3 & 663 & 663 & 663 & 663 & 653 & 663 & 663 & 661 \\
\hline EAST ROCKIES & 2411 & 244 & 244 & 244 & 244 & 244 & 2114 & 244 & 244 \\
\hline H. TEX./R. N. MPX. & 3.33 & 333 & 333 & 333 & 333 & 333 & 3.33 & 333 & 333 \\
\hline GUIT.P COAST & 2498 & 2498 & $249 n$ & 2498 & 2498 & 2499 & 2498 & 2498 & 2499 \\
\hline GIILP OP MEXICO & 6083 & 6086 & 6086 & 6016 & 5207 & 6207 & 6209 & 6209 & 6209 \\
\hline $\begin{array}{l}\text { MIDCONTINPAT } \\
\text { MI. DASIN AND INT. }\end{array}$ & 1513 & 1513 & 1513 & 1513 & 1513 & 1513 & 1513 & 1513 & 1513 \\
\hline APPALACHIA & 317 & 317 & 317 & 317 & 317 & 317 & 317 & 317 & 317 \\
\hline ATIANTIC OCPAB & 395 & 395 & 402 & 402 & 402 & 402 & 402 & 420 & 420 \\
\hline NATIONAL TOTAL & 12496 & 12528 & 12604 & 12746 & 12932 & 13140 & 13141 & 13162 & 13164 \\
\hline
\end{tabular}

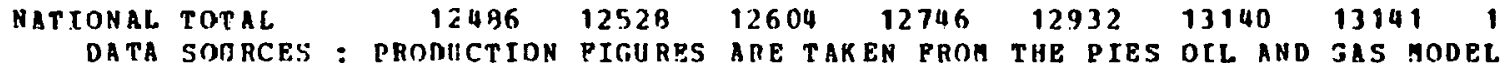

WIICH USRS DATA PROA "RESERVES OE CRUDE OIL. NATIRAL GAS LIOIFIDS. AND NATURAL

GAS IN TIB UNITFD STATBS AND CANADA AND CAPACITY AS OF OBCEMBER 31, 1974".

AMERICAN PBTROLEMA INSTITUTR. WASHINGTON D.C.

DPFICR RESPONGIBLE : OIE AND GAS ANALYSIS DIVISION, OPPICE OP ENERGY

SOURCP ANALYSIS, APQLID ANALYSIS, EIA. 
TABLE 16

NON-ASSIJCIATED INTERSTATE GAS

PRODUCTION SUIMART

IN 1990

(MACP/D)

PAICE STEP

NPC' REGION

SNITH ALASKA

PACIPIC OCEA

WEST ROCKIES

FAST ROCKIES

H. TEX./R. N. MEX.

GIILF COAST

GILF OP MEXICO

M.I DCONTI NENT

MI. BASIN AND INT.

APPAL ACIIA

ATI.ANTIC OCEAN

Mational TOTaI.

OSES DATA FROA "RESERY ES OP CRUDE OIL. NATORAL GAS LIOUIDS, AND NATURA

GAS IN THF IPNITED STATES AND CANARA AND CAPACTTT AS OF DPCERBR. 31, $1974 \%$

AMERICAN PETROLPIIM IHSTITUTB, HASHIGTON D.C.

OFPICE RESPONSTBLE : OIL AND GAS ANALYSIS DIVISION, OPPICR OP ENPRGY SOURCE ANALYSIS, APPLIED ANALYSIS, FIK. 
TABLE 1:

HON-ASSOCIATRD TNTEASTATE GAS

PBUDUCT ION SEMMABY

IN 19 Q⿱一

(AMCY $>$ ()

PRICE STEP

DOE REGION

N.Y.IN.J.

BID-ATLANTIC

SOUTH ATLAMTIC

MIDHEST

SOIITHEFST

CF.NTRAL

NORTH CENTRAL

WEST

$\begin{array}{ccccc}1 & 2 & 3 & 4 & \\ 2 & 2 & 2 & 2 & \\ 124 & 124 & 124 & 1214\end{array}$

5

6

$\begin{array}{lll}0 & 0\end{array}$

NATLONAI. TOTAL

$22.39523 .326 \quad 24710$

DATA SOURCES : PBODUCTION EIGURES ARE PAKEN PRCA THE DIES OIL AND GAS MODEL

MHLCH OSRS DATA JMON "RPSERVES OF CRTDE OIL, NATJRAL GAS LTOUIOS. AND NATURAL

GAS IN TII IINITED STATES AHD CANADA AND CAPACITY AS JP DECEABPB 31. 1974n.

AMEPICAN PETROIEOE INSTITUTE. HASHINGTON D. C.

OFPICE RESPONSTBLE : OII. AND GAS AHALYSIS DIVISION, OPPICE OP ENERGT

SOIBCE ANALYSIS. APPLIED AHAIYSIS, E.TA. 
TABLE 18

NON-ASSOCIATED INTRASTATE GAS

PONDICTION SUHABR

$$
\text { I* } 1990
$$

(AMCP/D)

PRICE STEP

DOE REGIC:N

N. P./N.J.

MII-ATLANT IC

MIintaThanT IC

IDHEST

SOITH HEST

CENTRAL

NORTH CENTRAI

UEST

NATIONAL TOTAL

MATA SOHCES : PRODICTION PIGURES ARE TAKEN PROM THE PIQS OLL AND GAS MODEL

A

GA.S IN THE JNITPD STATES AND CANADA AND CAPACITY AS OP DECRMBER $31.1974 \%$.

AMPRICAN PPTROLEUM INSTITUTE, HASHIHGTON D.C.

OPPICE PESPONSIBLE : OIL AND GAS ANALYSIS DIVISIOH. OPPICP OP ENERGY SOIIRCE ANALYSIS. APPLIED AHALYSIS. EIA.

$\begin{array}{rrrrrrrrr}1 & 2 & 3 & 4 & 5 & 6 & 7 & 9 & 9 \\ 105 & 138 & 159 & 176 & 182 & 188 & 196 & 208 & 221 \\ 22 & 46 & 46 & 46 & 46 & 46 & 46 & 46 & 46 \\ 14292 & 16456 & 18951 & 20920 & 21796 & 22448 & 23391 & 24934 & 26819 \\ 815 & 945 & 1039 & 1130 & 1189 & 1210 & 1262 & 1336 & 1443 \\ 205 & 205 & 205 & 541 & 642 & 761 & 898 & 1130 & 1445 \\ 129 & 136 & 338 & 460 & 503 & 546 & 607 & 697 & 828 \\ 15568 & 18014 & 20817 & 23350 & 241137 & 25278 & 26470 & 28428 & 30880\end{array}$


TABLE 19

CAPITAL IMVESTMPAT SUABARY FIOR GAS TBLLS

PQON 1977 THRO 1984.

(ดMe)

PAICE STRP

MPC REGION

SOUTH ALASKA

PACIPIC OCEAN

GiJl.F OF MEXICn

n tlantic ocean

$\begin{array}{rrrrrrrrr}1 & 2 & 3 & 4 & 5 & 6 & 7 & 8 & 9 \\ 143 & 193 & 290 & 489 & 588 & 882 & 892 & 884 & 884 \\ 30 & 30 & 30 & 31 & 31 & 31 & 31 & 31 & 31 \\ 1551 & 1552 & 1552 & 1552 & 1552 & 1552 & 1552 & 1552 & 1552 \\ 222 & 222 & 234 & 234 & 234 & 234 & 234 & 253 & 253\end{array}$

TOE REGION

כOIITH ATLANTIC

SOIITHURST

EENTRAL

MORTH CENTRAL

DEST

$\begin{array}{rrr}71 & 77 & 85 \\ 10906 & 11570 & 12629 \\ 610 & 644 & 696 \\ & 116 & 213\end{array}$

$\begin{array}{rrrrrr}96 & 98 & 160 & 102 & 106 & 108 \\ 13845 & 14991 & 14258 & 14490 & 14907 & 15441 \\ 749 & 778 & 778 & 735 & 810 & 851 \\ 331 & 422 & 530 & 650 & 845 & 1114 \\ 331 & 364 & 397 & 445 & 509 & 607\end{array}$

NATIONAL TOTAL

$\begin{array}{lllllllll}13533 & 14404 & 15729 & 17658 & 18157 & 18761 & 19181 & 19896 & 20839\end{array}$ DATA SOIIRCES: CAPITAL FIGURES ABE DERTVED PROA DATA GATIIERE BY THE AHERICAN PETROLEIIA

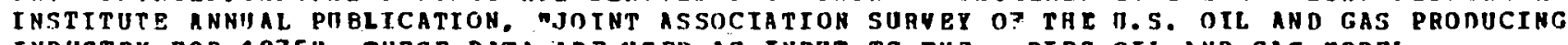
INDUSTIY POR 1975". GHESE DATA ARE USED AS INPIT TO THE PIES OIL AND GAS MODEL.

OPPICE RESPONSIBLE : OIL AND GAS RHALYSIS DIVISION, DPPICE OF EHERGY SOURCE ANALYSIS. APPLIPD ANALYSIS, EIA. 
TABLE 20

CAPITAL IN VESTMEHT SIIMAAT POR GAS UELLS

PROA 1977 TIIRU 1989

(Ans)

PRICE STR.P

NPC RFGION

SOUTH AI.ASKA

PACIFIC OCEAN

GIILP OF GPXICO

ATLAHTIC OCEAN

DOE RRGION

SOITH ATLANTTC

SOITH DEST

CENTR A L

NORTH CENTAA

WEST

NATIONAL TrTiL

$\stackrel{H}{H}$
$\stackrel{⿱ ⺊}{\sigma}$

$\begin{array}{rrrrrrrrr}1 & 2 & 3 & 4 & 5 & 6 & 7 & 9 & 9 \\ 59 & 69 & 255 & 556 & 725 & 1189 & 1189 & 1192 & 1192 \\ 2402 & 249 & 59 & 59 & 60 & 60 & 50 & 60 & 60 \\ 242 & 244 & 2405 & 2705 & 2405 & 2405 & 2405 & 2405 & 2405 \\ & 279 & 304 & 304 & 304 & 304 & 355 & 355\end{array}$

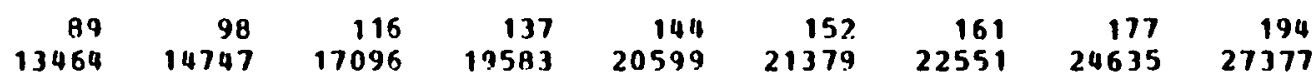

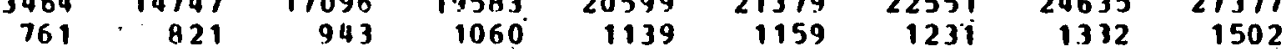

$\begin{array}{lllllllll}761 & 821 & 943 & 1060 & 1139 & 1159 & 1231 & 1332 & 1502\end{array}$

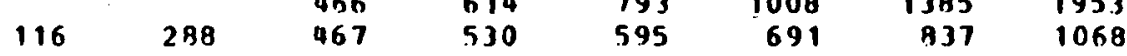

$\begin{array}{lllllllll}17016 & 18559 & 21440 & 25036 & 26520 & 28036 & 29600 & 32377 & 36105\end{array}$ DATA SOIIRCES:CAPITAL PIGIRES ARE DRRIVED PRON DATA GATHERED BY TIE ANBRICAN PETROLEIA

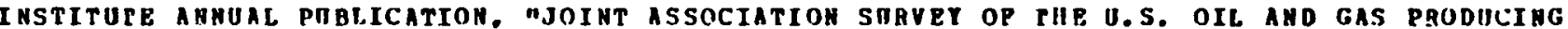
INDUSTEY PAR 1975". THESE DATA ARE USED AS INPUT TO THB PIBS OIL AND GAS MODBL.

OPPICE RPSPONSTBLE : OIL AND GAS ANALTSIS DIUISION. OPPICE DP ENFRG SOURCE ANALYSIS. APPLIED ANALYSIS. EIA. 
TABLP. 21

DRLLLIAS SUHAART POK GA.S VELLS

PROH 1977 THRU 1984 .

$$
\text { ( IMFT) }
$$

PRICP, STEP

HPC REGION

SO!̣TH A LASKa

GILP OF MEXICO

GLANTIC OCEAN

JOE REGION

SOUTH ATI.ANTIC

SOIITH WEST

CFNTRAT.

NOATH CENTRAL

JEST

WATIONAL TOTAL

554
590

$\begin{array}{rr}1 & 2 \\ 26 & 1 \\ 2 & 26 \\ 2 & 2\end{array}$

PRICP, STEP

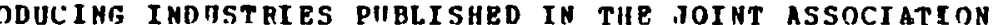

AMERICAN PETROLPUU CONNCIL. THR AMERICAN GAS ASSOCIATION, AND VARIODS OTHER BNERGY

RELATED INDDSTRIES. THP. DATA IS INPOT TO THE PIES OIL AND GAS MODEI. AND THE

ABOUE PIGURES ARE CALCIILATED THRRE. OTHER SOURCES INCLUDE "ONARTERLY REVIP.H

OP DRILLING STATISTICS". ANERICAN PETROLENA INSTITITE. DASHINGTOH, D. C.

POR INITTAL EXPLORATORT POOTAGP. AND "II.S. RNERGY OUTLOOK- OIL AND GAS AVATLABLITYN.

HASHINGTON, D.C.. 1973.

OPPICE RESPONSTBLE : OIL AND GAS AHALYSIS DIVISION, DPPICE OP ENRRY SOURCE ANALYSIS. APPLIED ANALYSIS, EIA. 
TABL.P 22

DAILI.ING SUMAARY POR GAS HDLLS

FROA 1977 THRI 1909

(AMPT)

PRICE STEP

NPC RETION

SOUTH ALASKA

PACIPIC OCEAN

GIILF OP KEXICO

ATI.ANTIC OCEAN

\begin{tabular}{|c|c|c|c|c|c|c|c|c|}
\hline 1 & ? & 3 & 4 & 5 & 6 & 7 & B & 9 \\
\hline & & 1 & 2 & 3 & 5 & 5 & 5 & 5 \\
\hline 1 & 1 & 1 & 1 & 1 & 1 & 1 & 1 & 1 \\
\hline $\begin{array}{r}39 \\
2\end{array}$ & $\begin{array}{r}39 \\
2\end{array}$ & $\begin{array}{r}39 \\
2\end{array}$ & $\begin{array}{r}39 \\
2\end{array}$ & $\begin{array}{r}39 \\
2\end{array}$ & $\begin{array}{r}39 \\
2\end{array}$ & $\begin{array}{r}39 \\
2\end{array}$ & 39 & $\begin{array}{r}39 \\
?\end{array}$ \\
\hline
\end{tabular}

DOP AEGTOH

SOUTH ATLANTIC

SOITHWFST

CENTAAL

NORTH CENTRAL

HEST

606

4
606

38

$\begin{array}{rr}4 & 5 \\ 663 & 765 \\ 41 & 47 \\ & \end{array}$

$\begin{array}{rr}5 & \\ 65 & 87 \\ 47 & 5 \\ & 2 \\ 12 & 19\end{array}$

$\begin{array}{rr}5 & 6 \\ 872 & 916 \\ 53 & 57 \\ 22 & 29 \\ 19 & 22\end{array}$

6
916
57
29
22

6
947
58
38
24

7
996
61
48
28

108

1200

$\begin{array}{lr}65 & 73 \\ 66 & 94\end{array}$

NATIONAL TOTAL

$690 \quad 756 \quad 073 \quad 1016 \quad 1075$

1120

$1186 \quad 1303$

SOURCE: POOTAGE DRILLED DATA PROA SECTION 1, DRILLING COSTS OP THE U.S. OIL AYD 3 AS PRODICING INDUSTRIPS PUBLISHED IN THE, JOINT ASSOCIATION

AMERICAN PETROLEUH COINCIL. THE ARPRICAN GAS ASSOCTATTOH, AND VARIOUS OTHER ENPRGP

RELATED INDUSTRIES. THE DATA IS INPIT TO TIIE PIES OIL AHD GAS HODEL AND THE

ABOVE PIGIHES ARE CALCMLATED THERE. OTHER SOURCES INCLUDE "OUARTEQLP REVIEH

OF DRILLING S'TATISTICS". AHERICAN PETROLEIA INSTITUTE, NASHIGTON, D.C.

POR IN IT TAI. RXPLORATORY POOTAGE AND "II.S. RNERGY OTILOOK- OLL AND GAS AVAILABILITY". MASHINGTON, D. C. . 1973.

OPFTCE RESPONSIBLF. : OIL AND GAS AHALYSIS DIVESION, OFPICE OP ENERGY SOURCE ARALYSIS. APPLIPD ANALYSIS. EIA. 
TABLE 23

OIL AND GAS SUPPLY AT TIE IITGHOST PRIEE STEPS IN 1925

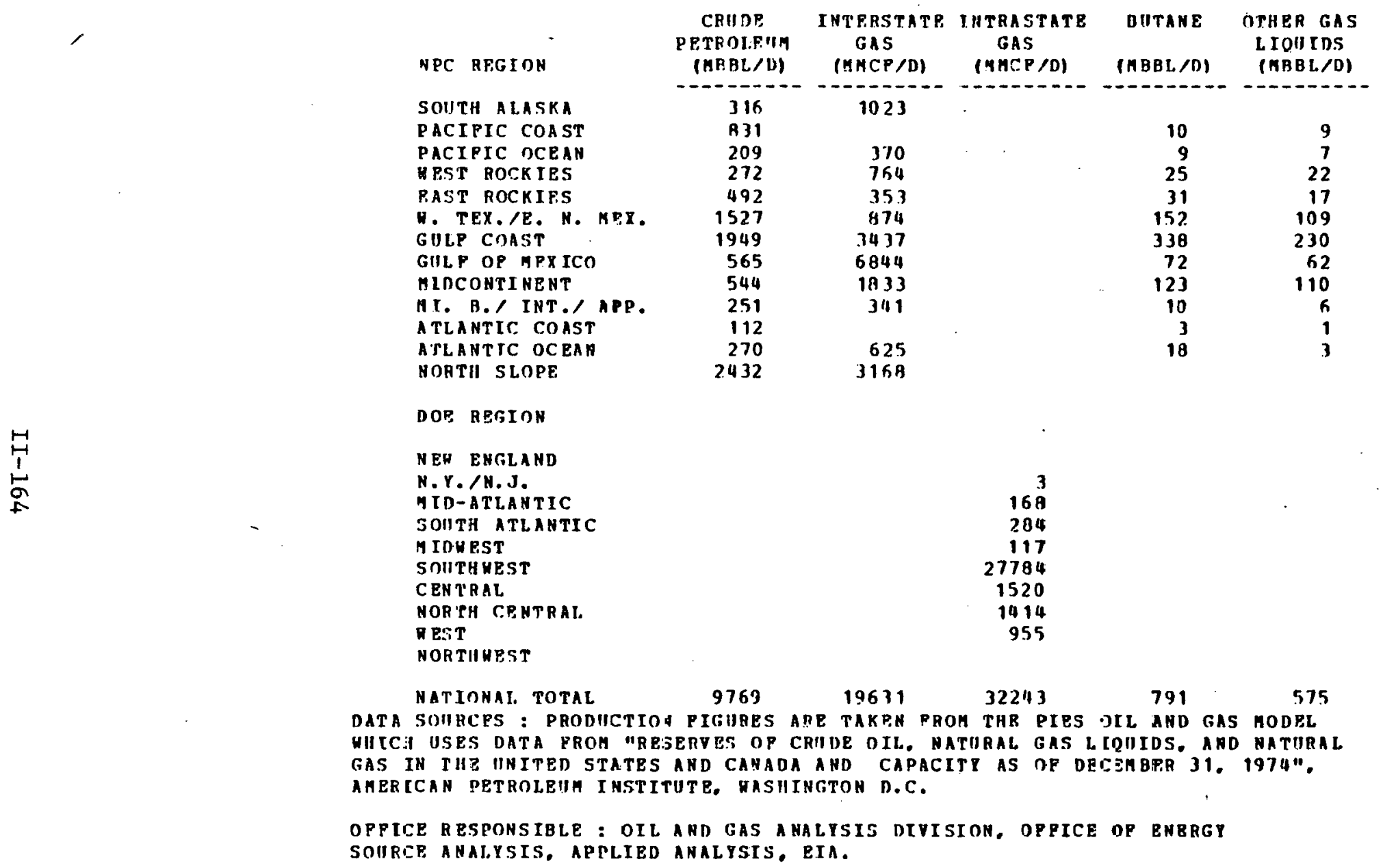


TABLE 24

OIL AND GA.T SUPPLY AT TIIR AIGHEST PEICP STPPS IN 1990

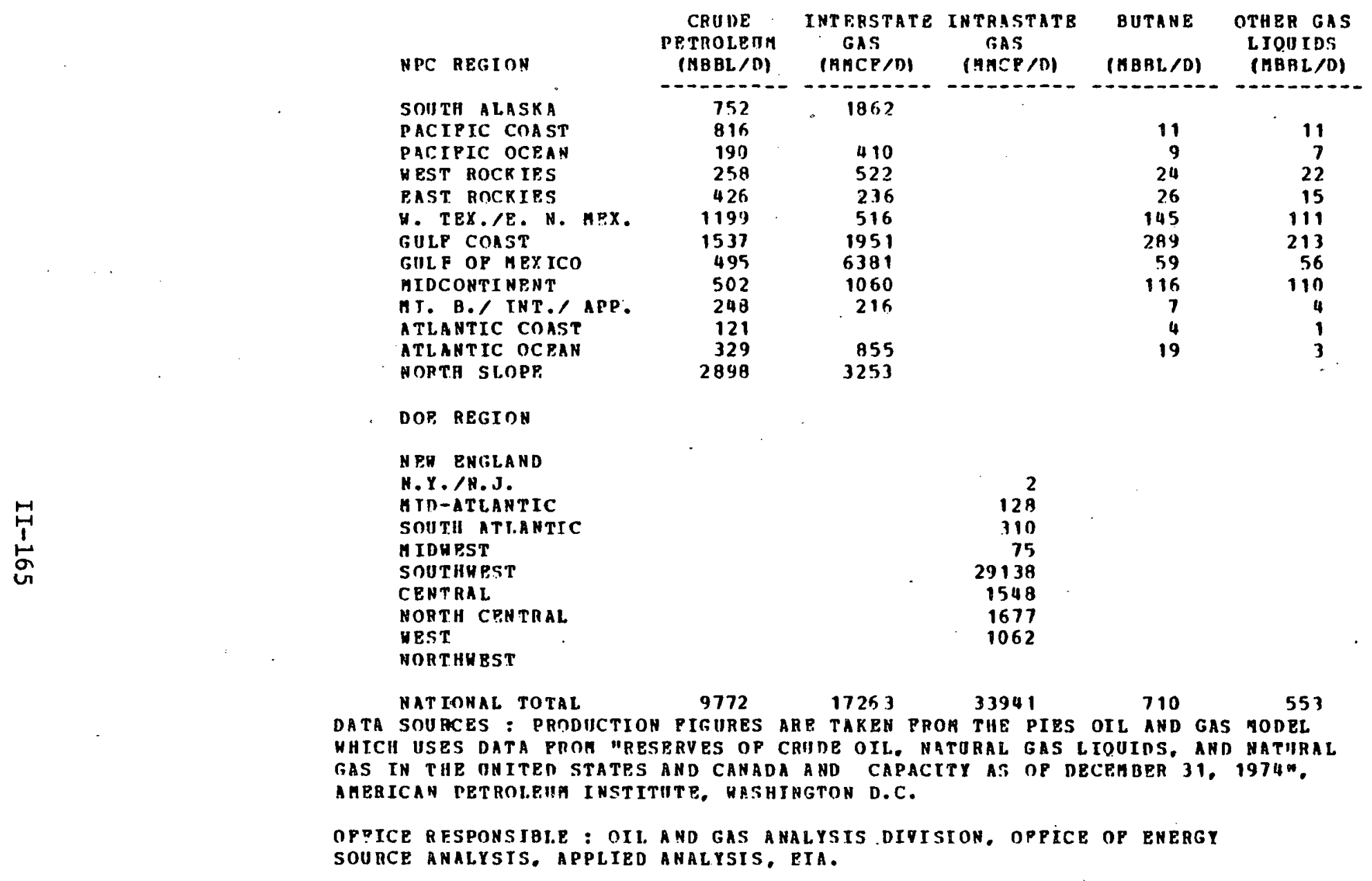


TABLE 25

BRPAKDOWH OP REGIOHAL PHODUCTION BY CRIJDE TYPE

(PRACT ION OP REGIONAL CRUDE PBODICTION)

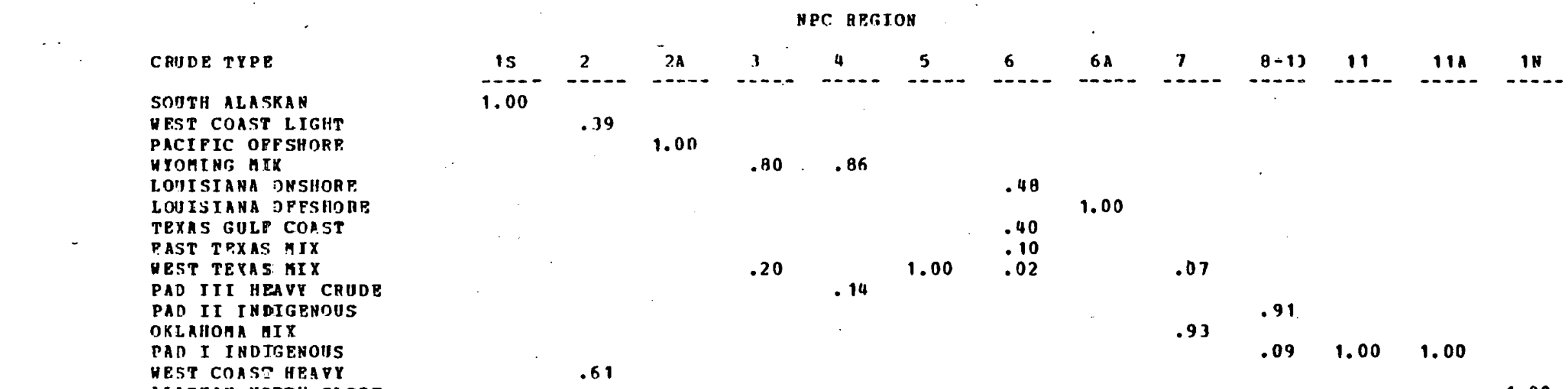

DATA SOIIRCES : OIL AND GAS JOTRNAL 'PETROLEUM RNCYCLOPRDIA POR 1975:

OPPICE RESPONSIBLE : OIL $E$ GAS ANALISIS DIVISTON. OPPICE OP EHPRG SOORCE ANALTSIS. APPLIED ANALYSIS. DOE

TARLB 26

SPLITTING PACTORS FOR INTRASTATE ASSOCIRTEN

AND DISSOLVED GAS

DOE REGION

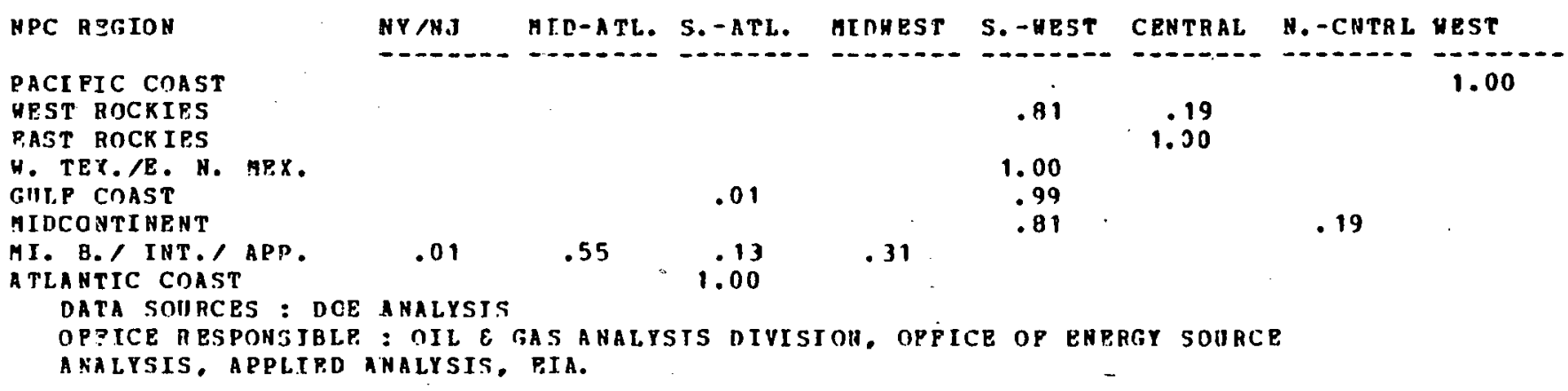


TABLE 27

SPLITTING FACTORS POR INTRASTATE CO-PRODUCTS

NPC HFGION

\begin{tabular}{|c|c|c|c|c|c|c|c|c|c|}
\hline DOE REGION & $\begin{array}{l}\text { PACIPIC } \\
\text { COAST }\end{array}$ & $\begin{array}{l}\text { VEST } \\
\text { RnCKI I ES }\end{array}$ & $\begin{array}{l}\text { EAST } \\
\text { ROCK IES }\end{array}$ & $\begin{array}{l}\text { H. } \\
\text { E. }\end{array}$ & $\begin{array}{l}\text { TEXAS/ } \\
\text { H. MEX. }\end{array}$ & $\begin{array}{l}\text { H. GIILF } \\
\text { BASIN }\end{array}$ & $\begin{array}{l}\text { MIDCON- } \\
\text { TIREHI }\end{array}$ & $\begin{array}{l}\text { MI. B. /I.'/ } \\
\text { APPLACRIA }\end{array}$ & $\begin{array}{l}\text { ATLANTIC } \\
\text { COAST }\end{array}$ \\
\hline $\begin{array}{l}\text { N.Y. IN.J. } \\
\text { MID-ATLANTIC }\end{array}$ & $\cdots--$ & $\cdots$ & $-\infty---\infty \cdots=--$ & $\cdots$ & & $-\cdots--$ & $-\cdots$ & 1.00 & \\
\hline $\begin{array}{l}\text { SOUTH ATLANTIC } \\
\text { MIDHEST }\end{array}$ & & & & & .49 & & & $\begin{array}{r}.33 \\
1.00\end{array}$ & .18 \\
\hline $\begin{array}{l}\text { SOUTHHEST } \\
\text { CENTRAL }\end{array}$ & & .04 & .26 & & .45 & $\therefore .00$ & & & \\
\hline $\begin{array}{l}\text { NORTH CPNTRAL } \\
\text { MPST }\end{array}$ & .99 & $\begin{array}{l}.26 \\
.01\end{array}$ & & & & & .74 & & \\
\hline
\end{tabular}

DATA SOIIRCES : DOR ANALYSIS

OPPICE: RPSPONSIBLE : OIL G GAS ANALYSIS DIVISION, OPFICE OP ENERGT SODRCE

ANALYSIS, APPLIED ANALYSIS, R.IA. 


\section{REFINERIES DATA}

The refinery data presented in this section were generated under the assumption that there would be no real increase in world oil prices between 1980 and the target years, 1985 and 1990. The data are given in two sets of four tables, one for each target year.

TABLE 1 gives the cost of converting one barrel of the crude type specified by the row heading into petroleum products in the refinery region specified by the column heading. Refinery regions are shown in Figure II-6. The petroleum products produced are liquefied petroleum gases, gasoline, jet fuel, distillate oil, residual oil, naphtha and other. Still gases are consumed in the refinery as fuel. Blanks in the table indicate the absence of links between the corresponding refinery region and crude producing regions. Not all crudes are available for processing in all regions.

TABLE 2 consists of seven sections - one for each refinery region - which give the ratio of barrels of refinery products produced per barrel of crude consumed. There is a volumetric gain indicated for each fuel type and each region since the ratio of total refined product to crude consumed exceeds unity in all tables. For example, refinery region 1 exhibits a gain of 4.2 percent in volume of refined product, since 1,042 barrels of product are produced for every barrel of crude.

TABLE 3 displays equations for price relations between pairs of petroleum products. TABLE 4 , shows existing capacity, build limits and build costs for each refinery region. All costs for this section are in 1975 constant dollars. 


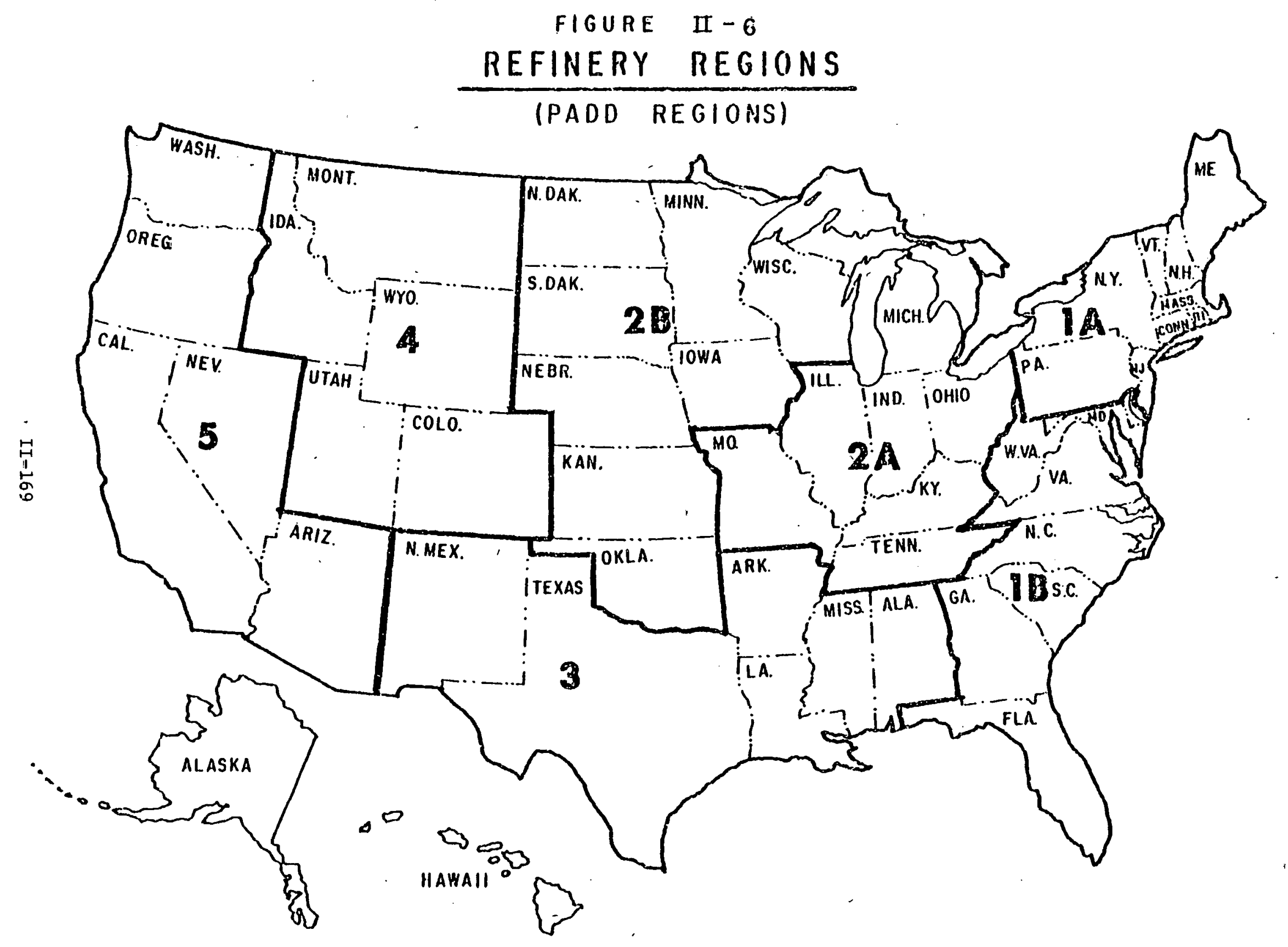


19H5 REFIMFPY DATA ASSUMING NO REAL INCAEASE IN WORLO OIL PRICF

GFNERATINT DATE I1878 


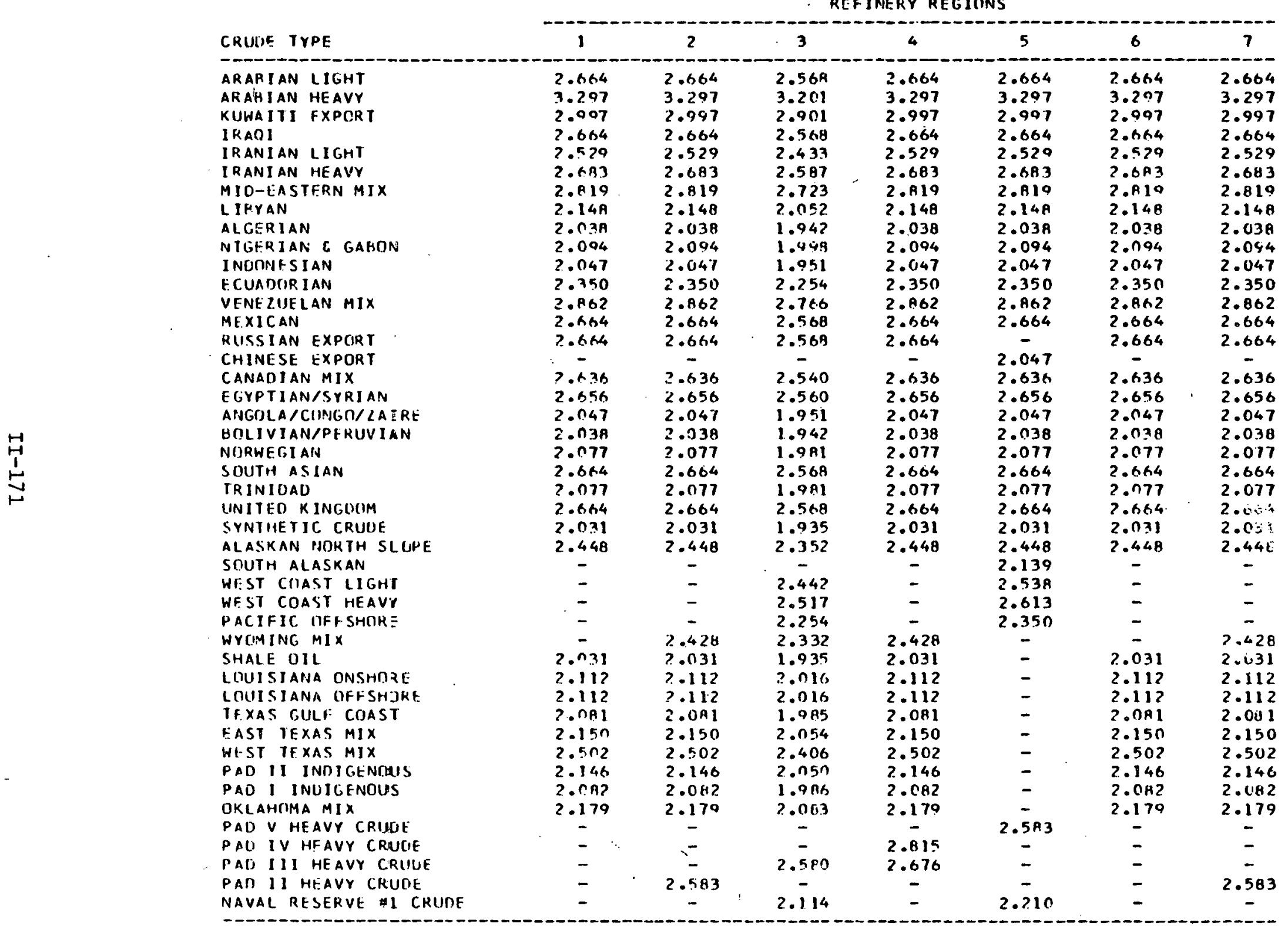

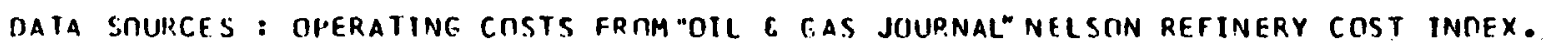

CAPACITY COSTS DERIVED FPIP "OIL AND GAS JUURNAL". COSTS FOR NEW CATALYTIC CRACKING AND

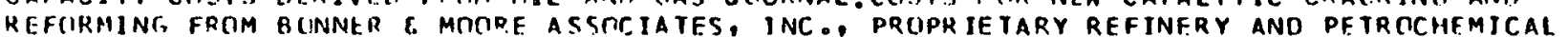

MIIOFLING SYSIEM (RPHS) DATA PAST.

RFFICE RFSPIJNSIHLE : MID-RANTE ANALYSIS IIIVISINN, OFFICE OF INTEGQATIVE ANALYSIS.

APPLIFD ANALYSBS, EIA. 
TAHLE 2: BARRELS OF REFINEPY PRADIITS PRIIDUCFD PIR HARREL OF CFUDF CONSUMEO

REFINEPY REGICN

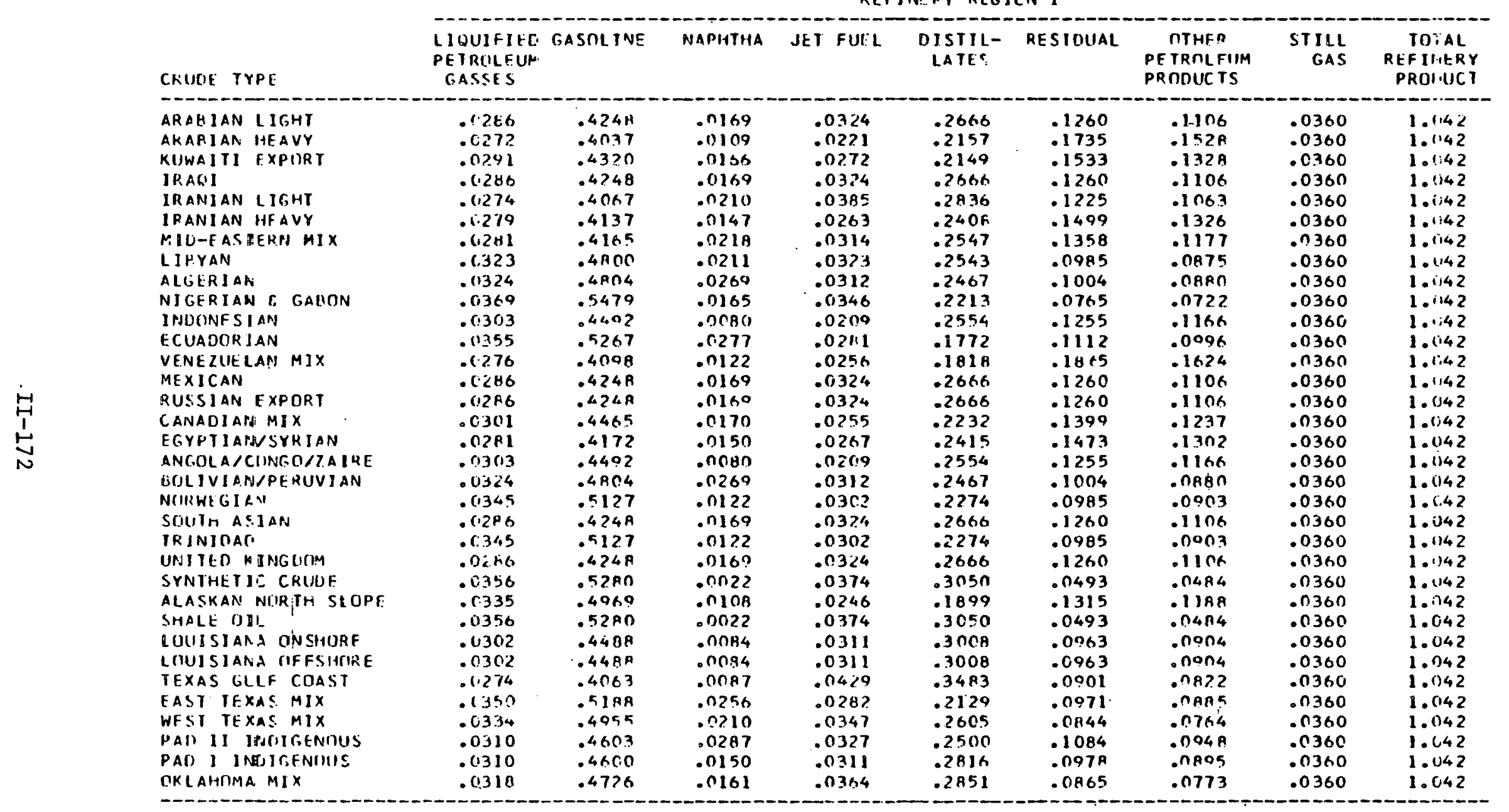


TAMLE 2: BAKRELS OF RFFINERY PRONIICTS PROUUCFD PFR BARREL OF CPIDE CONSUMED

REFINERY FEGION 2

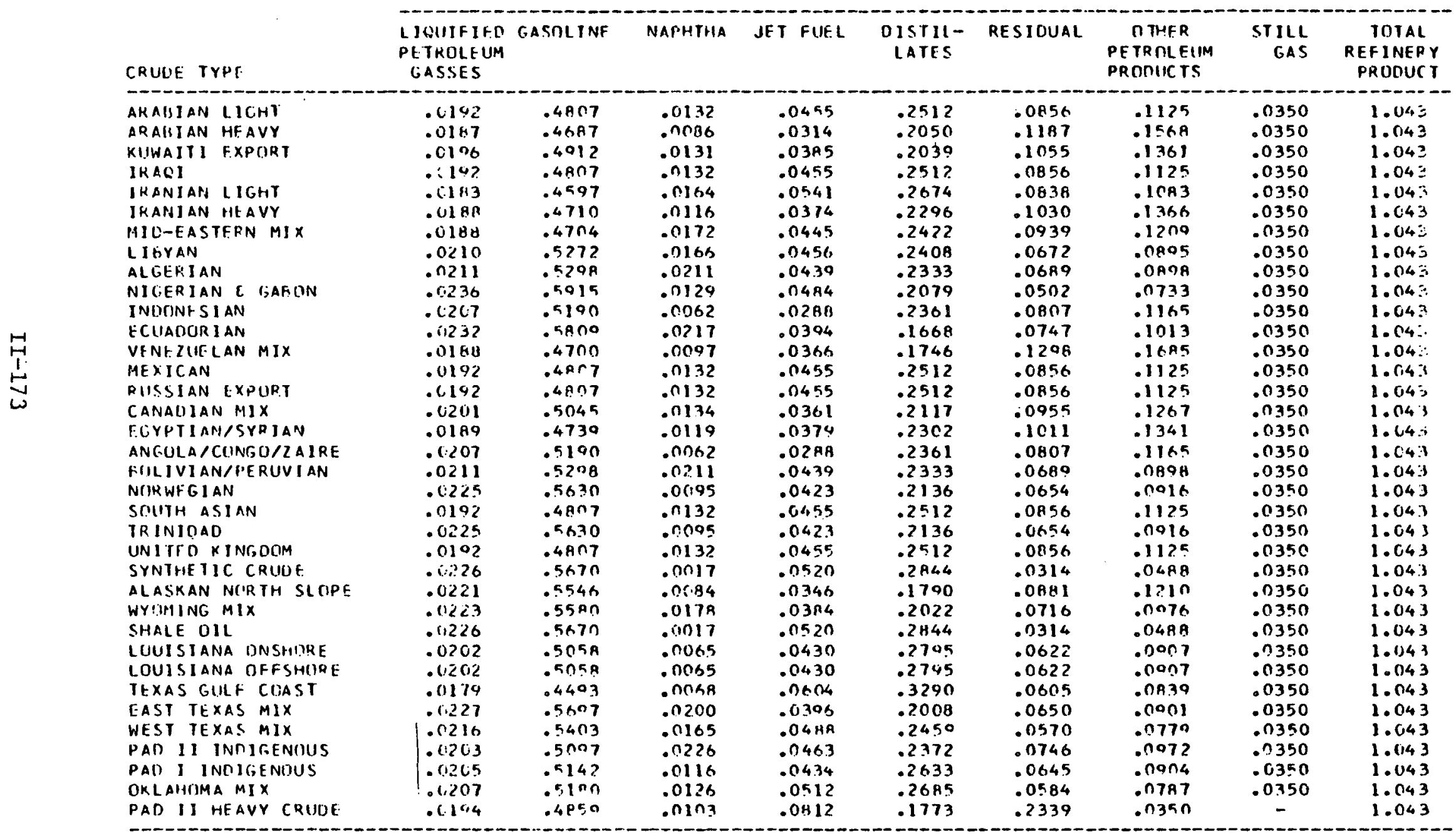


REFINFRY REGION 3

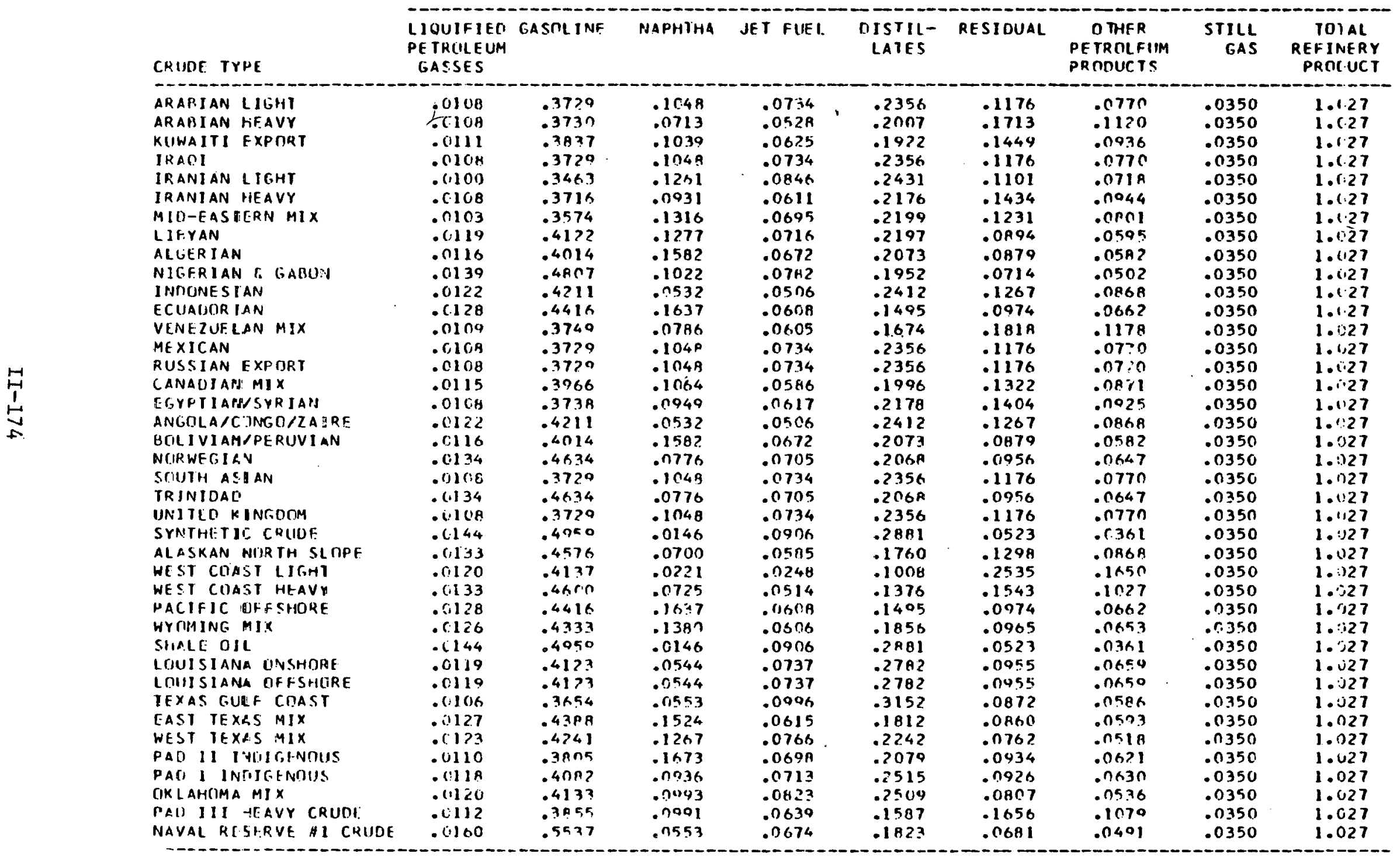


TAFILE 2: BARRELS IF REFINERY PROOIKTS PRIOUUCEO RER RAPREL OF CPUDE CONSUMED

REFINEPY REGION 4

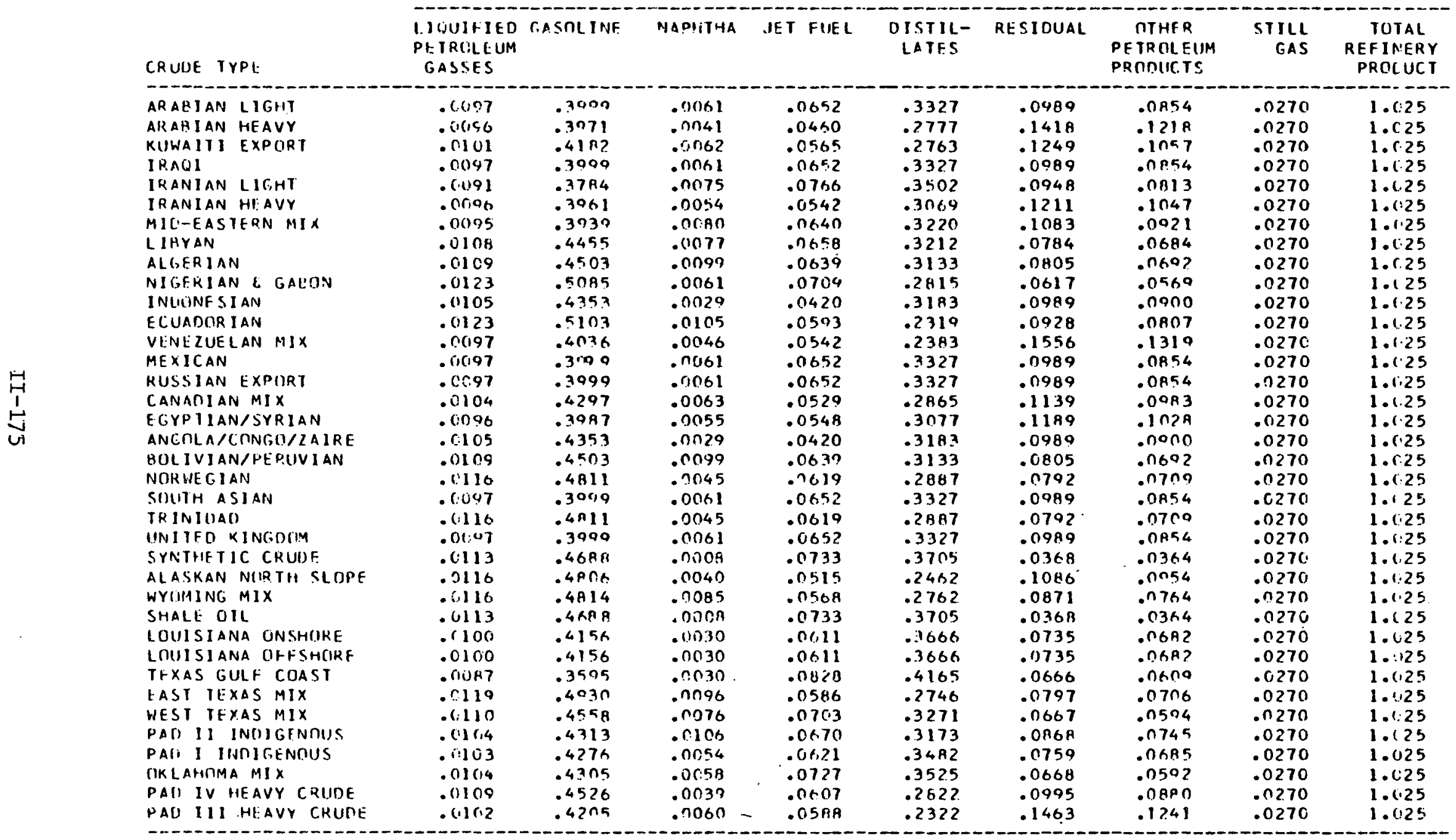


(CONTIHIIFD)

TABL = 2: HARRELS UF REFINERY PR ODUCTS PRODUCFO PER RARREL UF CRUDE CONSIJMFI

REFINERY REGION 5

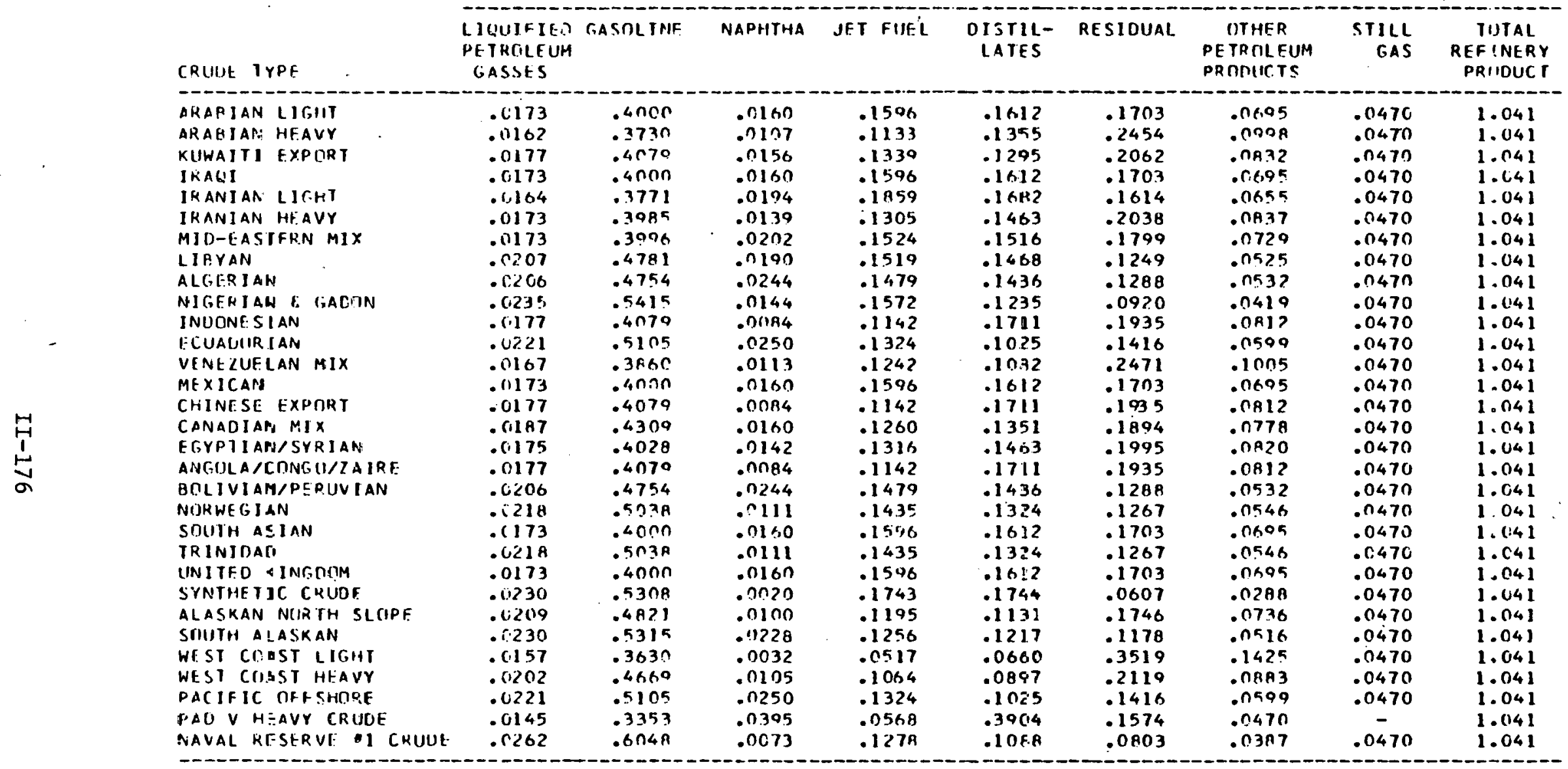


REFINERY REGION 6

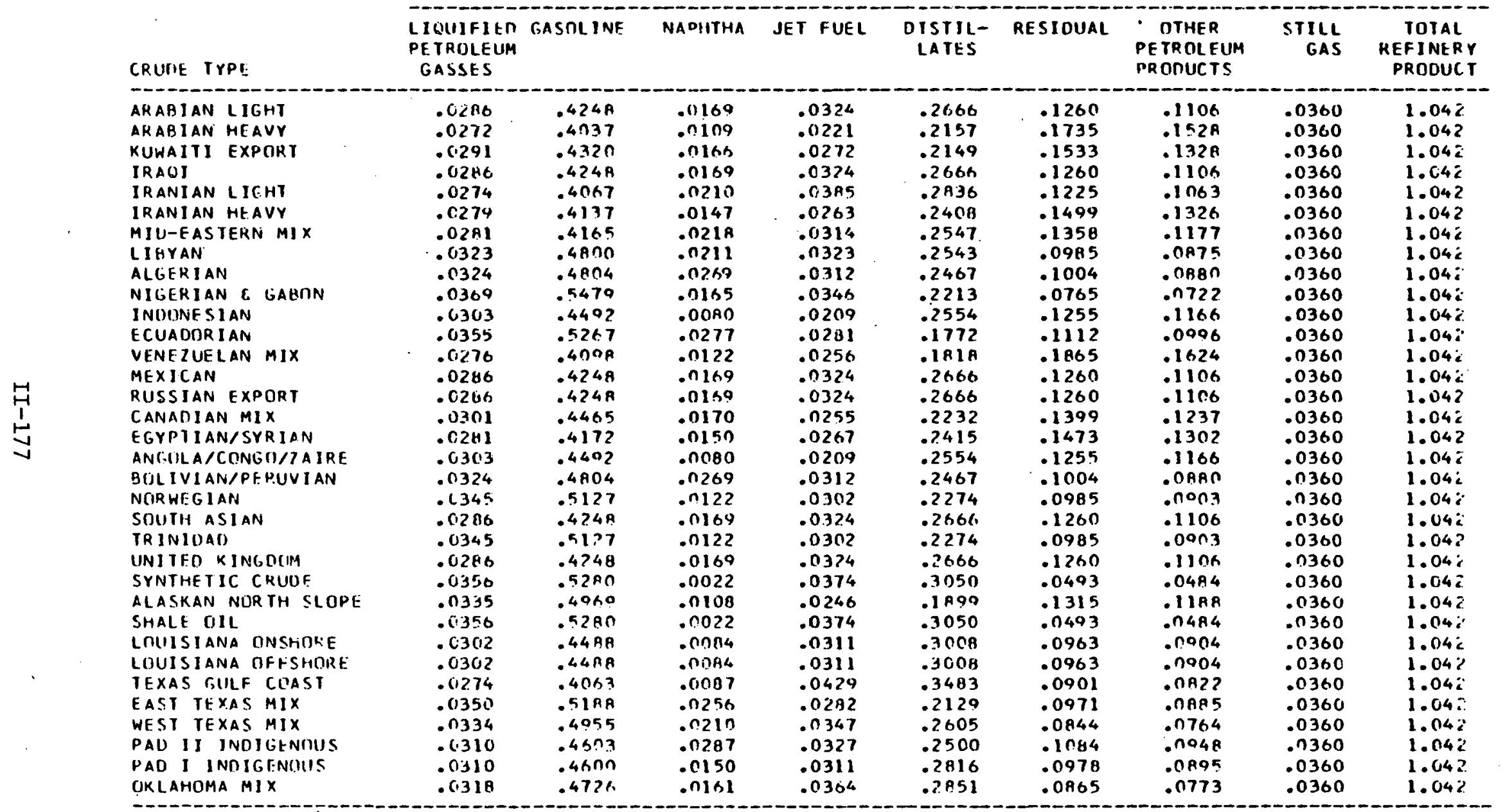


TAPLF 2: BARRELS OF REFINERY PRODUCTS PRIOUCED PER FARREL TIF CRUDE CONSUHA:D

REFINERY DEGION 7

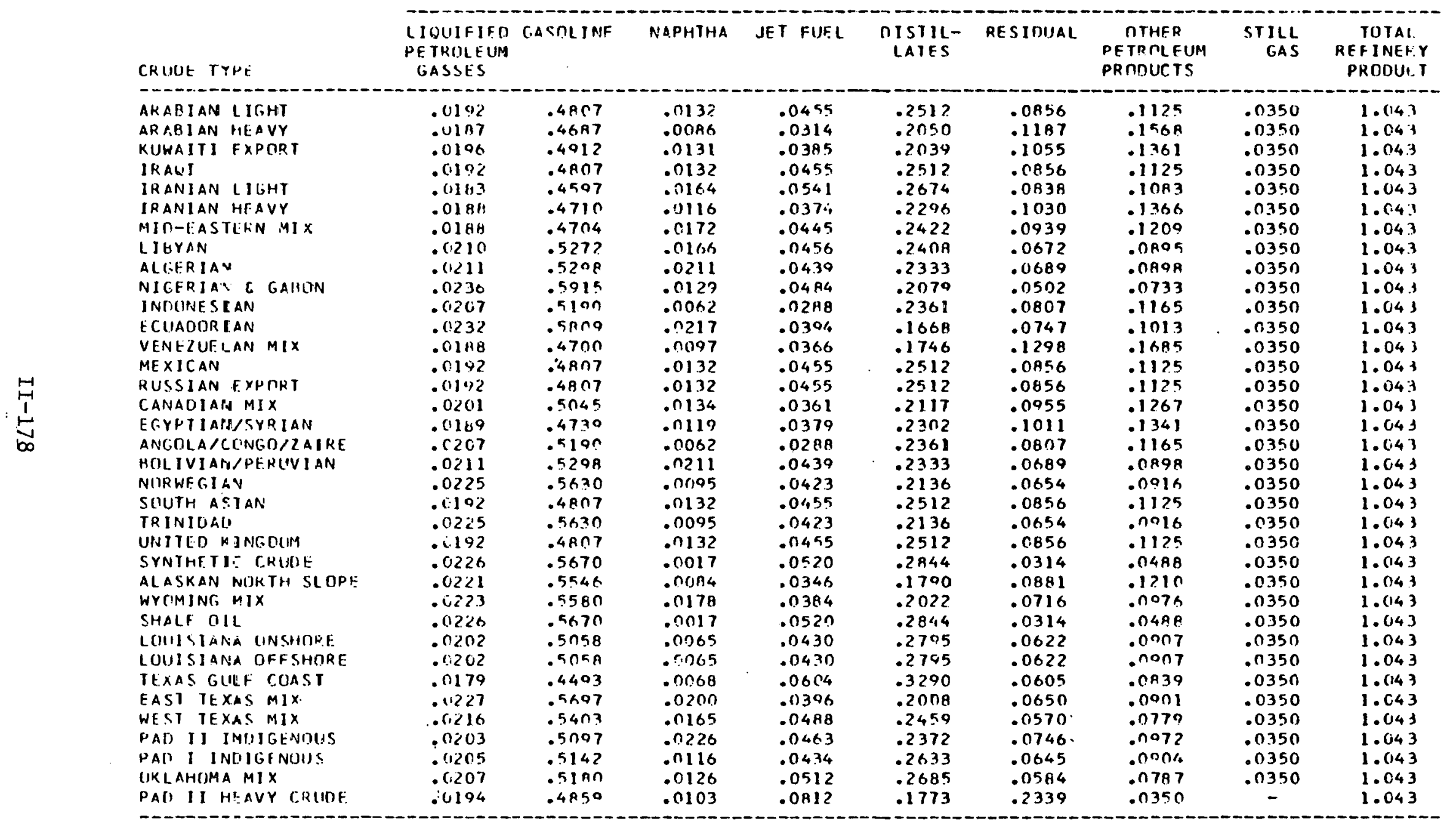

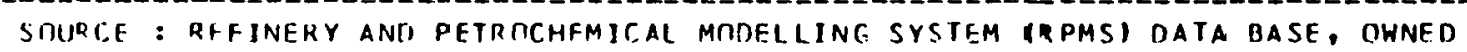

BY HOMNEP C. MOORE ASSOCIATFS, INC., WASHINGTIN, D.C.

OFF]CL RESPIINSIBLE: MIID-RANGE ANAL YSIS OIVISION, CIFFICE OF INTESRATIVE ANALYSIS,

APPLIED ANALYSIS, HIA. 


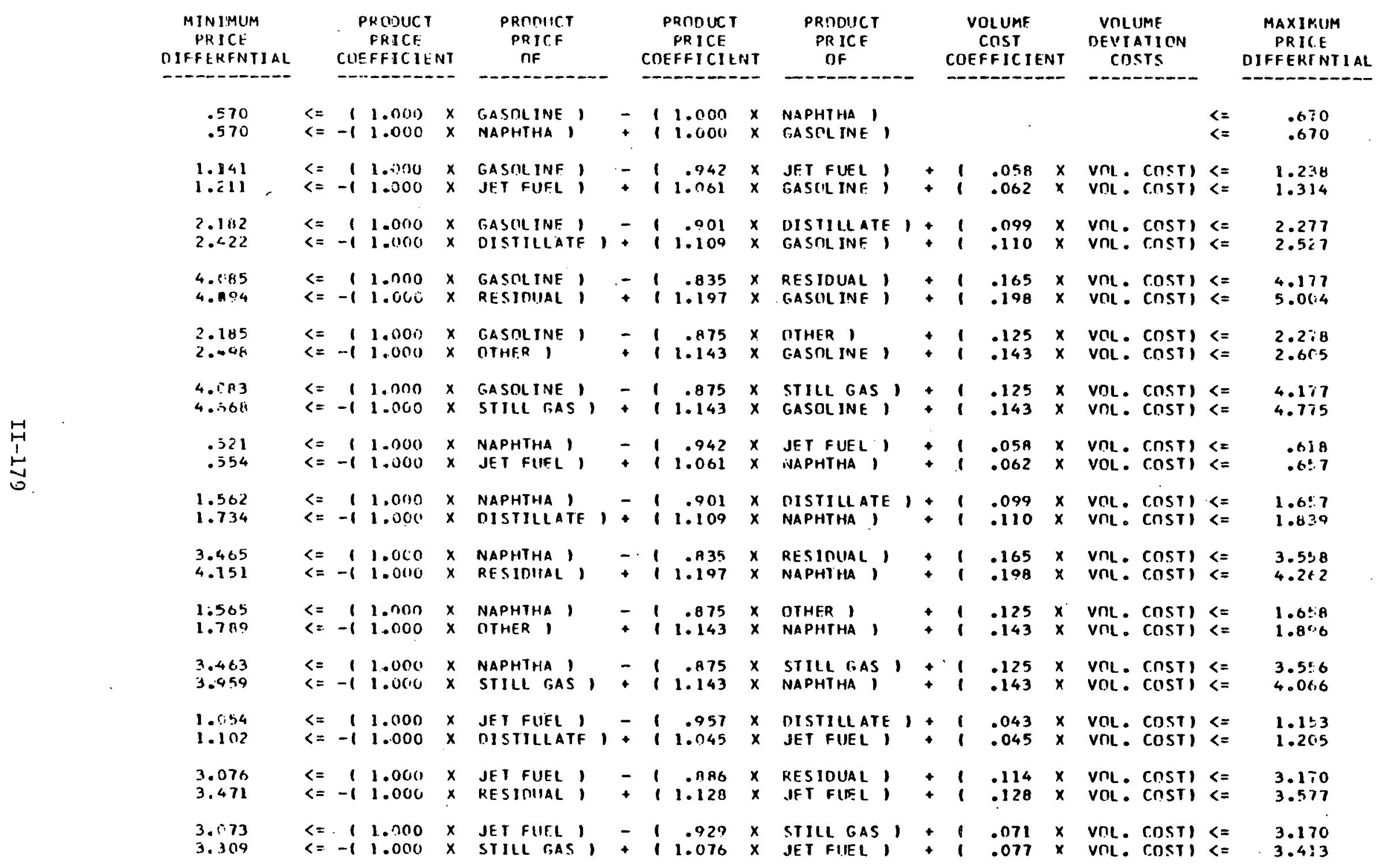


TABLF 3: RFLATIVE PROUUCT PPICING RILATIONSHIPS

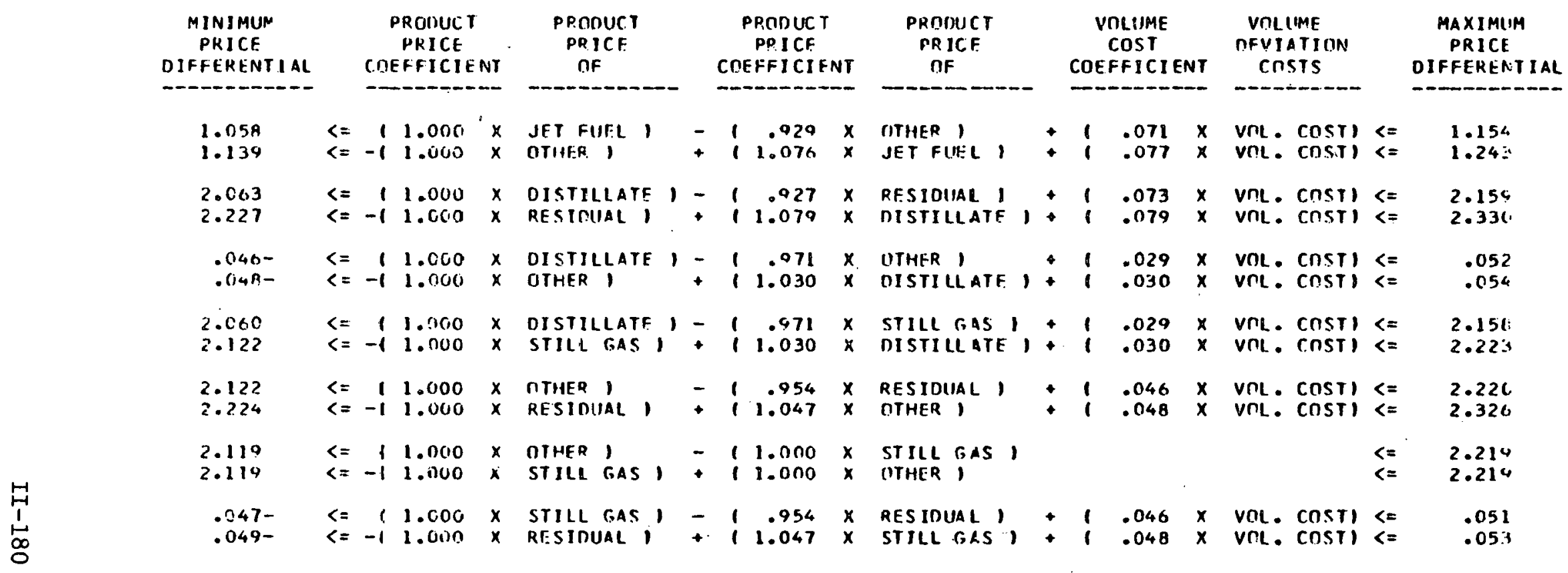

GATA SOUACE = CUSTS ANO PRICF RFLATIONSHIPS ARE OUTPUT FROM THE REFINERY CONVFRSION SUP-MODEL IMHEOULD IN P JES.

TFFTCE RESPINSIBIE : MILI-AANEF ANILYSIS DIVISION, OFFICE OF INTEGRATIVE ANALYSIS, APPLIED ANALYSIS, EIA. 
TARLE 4: REFINERY CAPAC ITY DATA

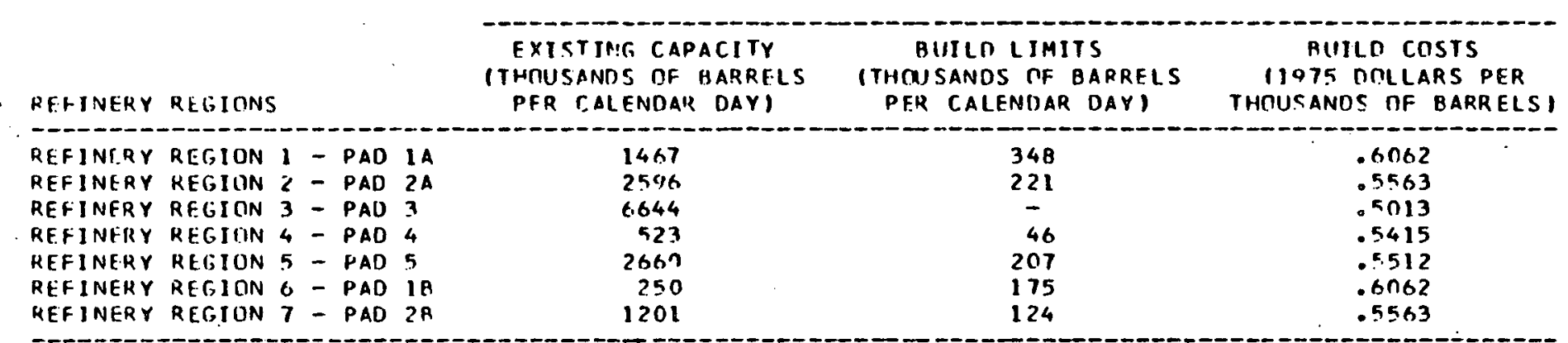

LATA SOURCE : CAPACITY ROINOS RASEO IN DATA. PUBLISHED IN FEA.S "TRENDS IN

REFINERY CARACITY ANO UTILIZATINN", BY E.L. PEER AND F.V. MARSIK,

UFFICE OF OIL E CAS, FEA, 1975. CAPACITY COSTS DERIVED FROM REFERENCES IN "OIL E. GAS JOURNAL".

RFFICE RESPONSIRLE : MIO-RANGF ANALYSIS OIVISION, OFFICE OF INTEGRATIVE ANALYSIS, EIA. 
1990 REFINFRY OATA ASSUMING NO PEAL INOFEASE IN WORLO OIL PRICF.

GENERATION DATE ILETB 
1ABLE 1: REFINFRY COSTS FOR BASE YIFLOS 11075 ONLLARS/BARREL)

REF INFRY REGIONS

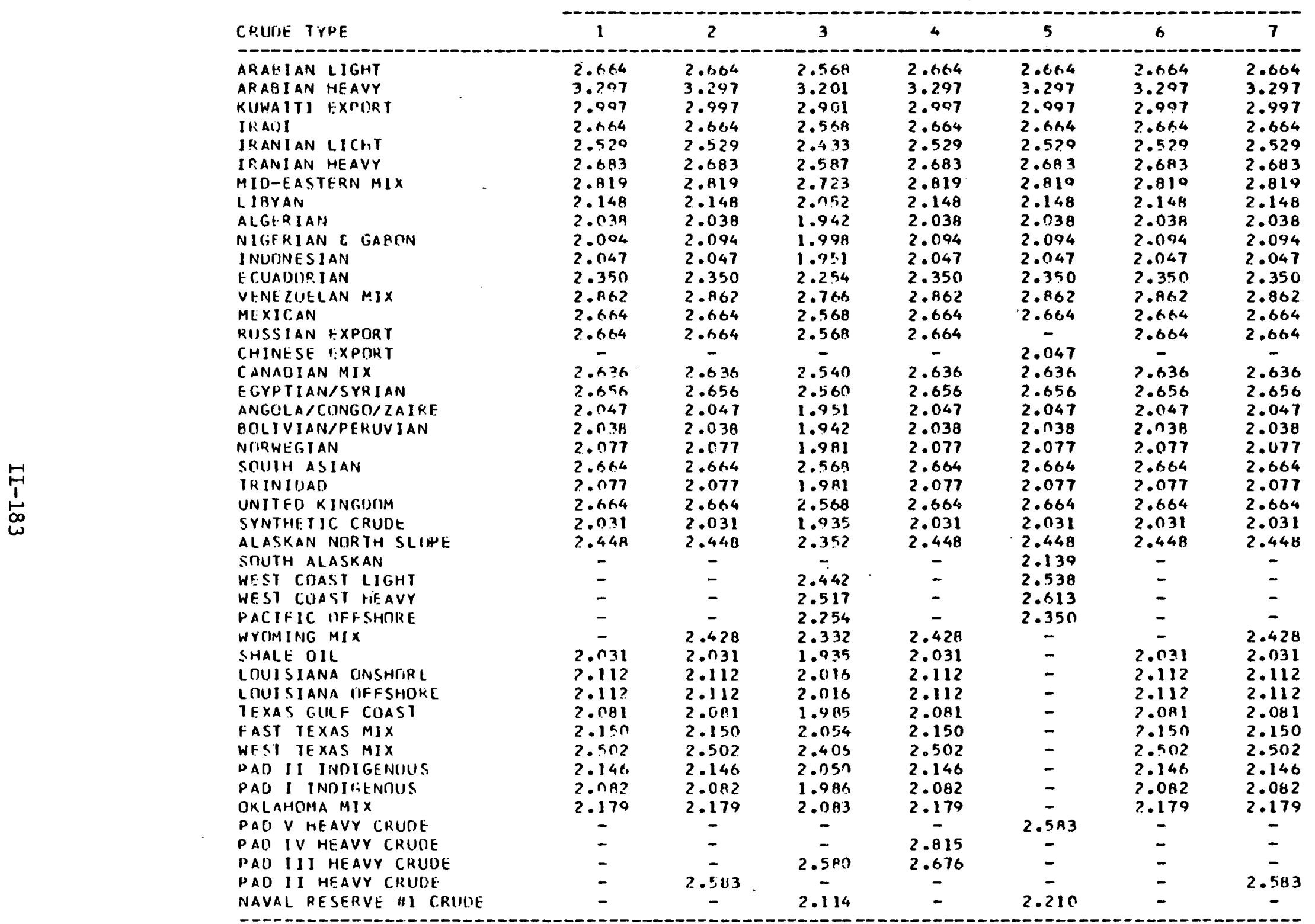

OATA SOURCES : OFLRAIING, COSTS FROM "OIL G GAS JOURNAL" NELSIIN REFINERY COST INDEX.

CAPACITY COSSTS OFRIVED FRGM "NIL AND GAS JOURNAL":COSTS FOR NEW CATALYTIC CRACKING AND

REFORMING FRIMM BINNER C. MOTRF. ASSIICIATES, INC., PROPRIETARY REFINERY ANU PETROCHFMICAL MOOUL ING SYSTEM (KPMSI MATA RASE. 
TABLE 2: BARRELS OF RFFINERY PROMUCTS PROOUCED PER BARREL OF CRUNE C IMMED

REFINERY RFGION

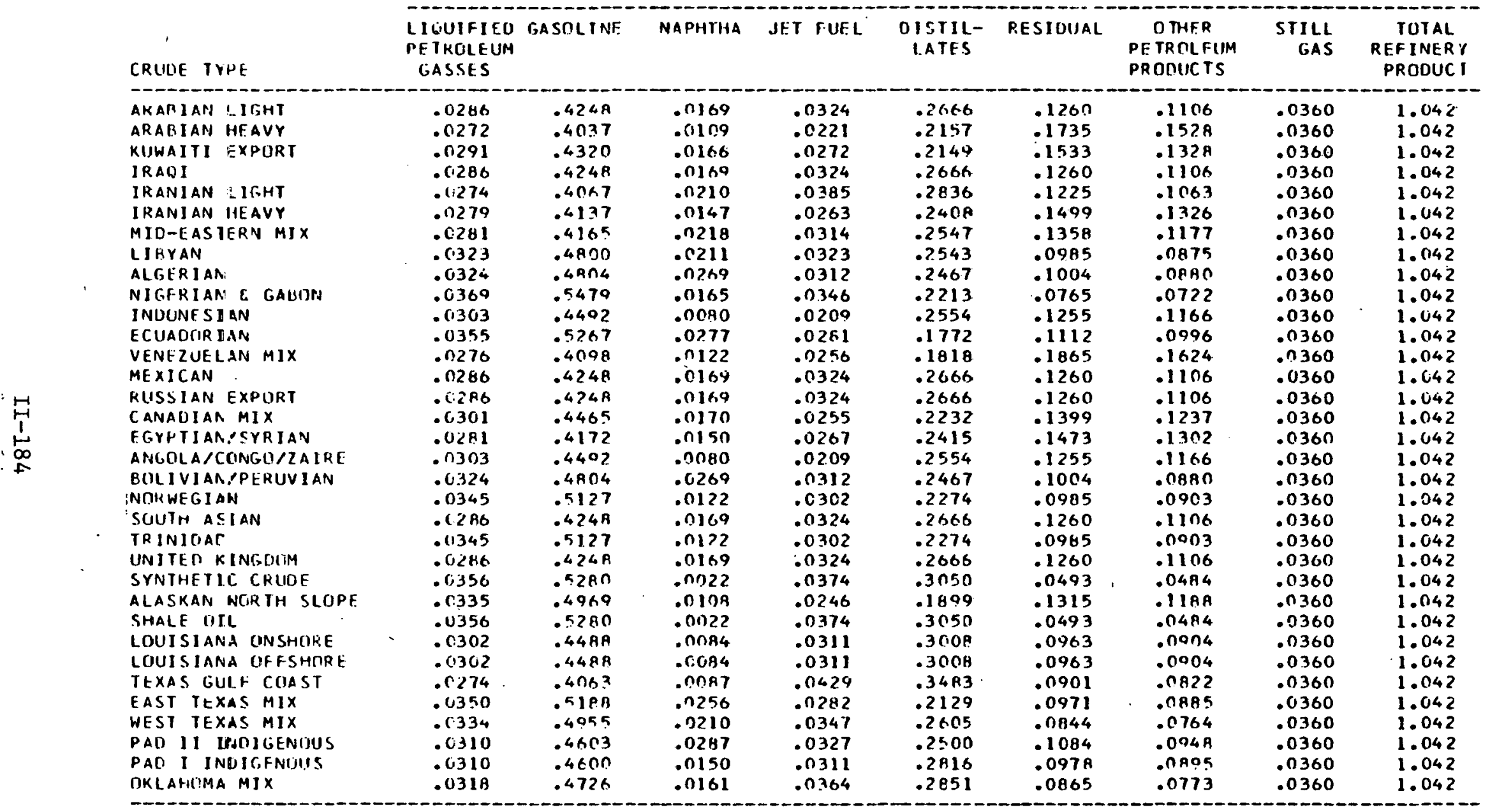


[ ronh'S JWIIED)

TABLE 2: GANRELS OF REFINFRY PROBICTS PRODUCEO PER BARREL CIF CRUN: CONSUMED

RFFINEPY REGION 2

\begin{tabular}{|c|c|c|c|c|c|c|c|c|c|c|}
\hline & CRUIDE TYPE & $\begin{array}{l}\text { LIOUIHIED } \\
\text { PEIRULEUM } \\
\text { GASSES }\end{array}$ & GASOLINF & NAPHTHA & JET FUEL & $\begin{array}{l}\text { UISTIL- } \\
\text { LATES }\end{array}$ & RESIDUAL & $\begin{array}{l}\text { OTHER } \\
\text { PETROLFIMM } \\
\text { PRDDUCTS }\end{array}$ & $\begin{array}{r}\text { STILL } \\
\text { GAS }\end{array}$ & $\begin{array}{l}\text { TOIAL } \\
\text { REFINERY } \\
\text { PRCIDUCT }\end{array}$ \\
\hline $\begin{array}{l}\stackrel{H}{-1} \\
\stackrel{1}{+} \\
\infty \\
\sim\end{array}$ & 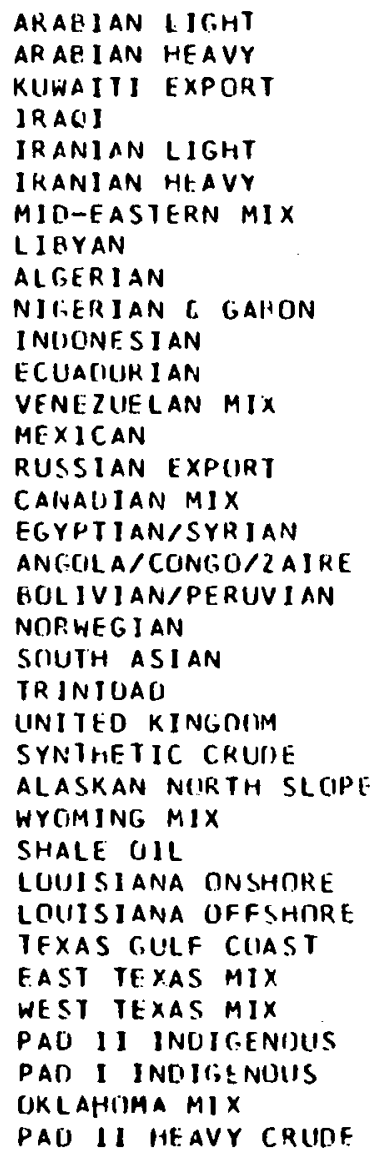 & $\begin{array}{l}.0142 \\
.01147 \\
.0196 \\
.0192 \\
.0113 \\
.0188 \\
.0186 \\
.01210 \\
.0211 \\
.0236 \\
.0207 \\
.0232 \\
.0188 \\
.0192 \\
.0192 \\
.0201 \\
.0189 \\
.0207 \\
.0211 \\
.0225 \\
.01192 \\
.0225 \\
.0192 \\
.01226 \\
.0221 \\
.11223 \\
.0226 \\
.01202 \\
.0202 \\
.01174 \\
.0227 \\
.0116 \\
.0203 \\
.0205 \\
.0207 \\
.0194\end{array}$ & $\begin{array}{l}.4807 \\
.4687 \\
.4912 \\
.4807 \\
.4597 \\
.4710 \\
.4704 \\
.5277 \\
.5208 \\
.5915 \\
.5190 \\
.5400 \\
.4700 \\
.4807 \\
.4807 \\
.5045 \\
.4739 \\
.5100 \\
.5298 \\
.5630 \\
.4807 \\
.5630 \\
.4807 \\
.567 n \\
.554 A \\
.5580 \\
.5670 \\
.505 A \\
.505 A \\
.4493 \\
.5697 \\
.6403 \\
.5097 \\
.5142 \\
.5190 \\
.4850\end{array}$ & $\begin{array}{l}.0132 \\
.0086 \\
.0131 \\
.0132 \\
.0164 \\
.0116 \\
.0172 \\
.0166 \\
.0211 \\
.0129 \\
.0062 \\
.0217 \\
.0097 \\
.0132 \\
.0132 \\
.0134 \\
.0119 \\
.0062 \\
.0211 \\
.0095 \\
.0132 \\
.0095 \\
.0132 \\
.0017 \\
.0094 \\
.0178 \\
.0117 \\
.01065 \\
.0065 \\
.0068 \\
.0200 \\
.0165 \\
.0226 \\
.0116 \\
.0126 \\
.0103\end{array}$ & $\begin{array}{l}.0455 \\
.0314 \\
.0385 \\
.0455 \\
.0541 \\
.0374 \\
.0445 \\
.0456 \\
.0439 \\
.0484 \\
.0288 \\
.0394 \\
.0366 \\
.0455 \\
.0455 \\
.0361 \\
.0379 \\
.0288 \\
.0439 \\
.0423 \\
.0455 \\
.0423 \\
.0455 \\
.0520 \\
.0346 \\
.0384 \\
.0520 \\
.0430 \\
.0430 \\
.0604 \\
.0306 \\
.0448 \\
.0463 \\
.0434 \\
.0512 \\
.0412\end{array}$ & $\begin{array}{l}.2512 \\
.2050 \\
.2039 \\
.2512 \\
.2674 \\
.2296 \\
.2422 \\
.2408 \\
.2333 \\
.2079 \\
.2361 \\
.1668 \\
.1746 \\
.2512 \\
.2512 \\
.2117 \\
.2302 \\
.2361 \\
.2333 \\
.2136 \\
.2512 \\
.2136 \\
.2512 \\
.2844 \\
.1790 \\
.2022 \\
.2844 \\
.2795 \\
.2795 \\
.3290 \\
.2008 \\
.2459 \\
.2372 \\
.2633 \\
.2685 \\
.1773\end{array}$ & $\begin{array}{l}.0856 \\
.1187 \\
.1055 \\
.0856 \\
.0838 \\
.1030 \\
.0939 \\
.0672 \\
.0689 \\
.0502 \\
.0807 \\
.0747 \\
.1298 \\
.0856 \\
.0856 \\
.0955 \\
.1011 \\
.0807 \\
.0689 \\
.0654 \\
.0856 \\
.0654 \\
.0856 \\
.0314 \\
.0881 \\
.0716 \\
.0314 \\
.0622 \\
.0622 \\
.0605 \\
.0650 \\
.0570 \\
.0746 \\
.0645 \\
.0584 \\
.2339\end{array}$ & 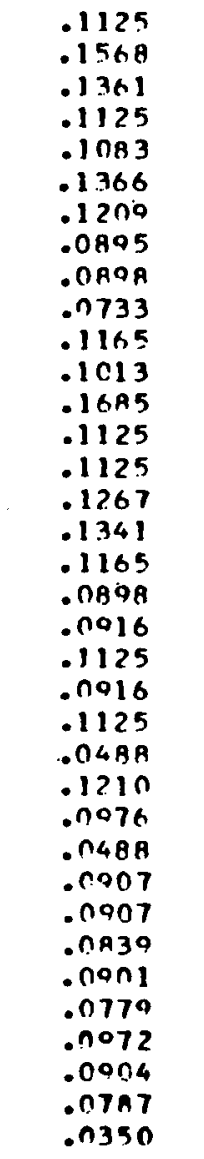 & $\begin{array}{r}.0350 \\
.0350 \\
.0350 \\
.0350 \\
.0350 \\
.0350 \\
.0350 \\
.0350 \\
.0350 \\
.0350 \\
.0350 \\
.0350 \\
.0350 \\
.0350 \\
.0350 \\
.0350 \\
.0350 \\
.0350 \\
.0350 \\
.0350 \\
.0350 \\
.0350 \\
.0350 \\
.0350 \\
.0350 \\
.0350 \\
.0350 \\
.0350 \\
.0350 \\
.0350 \\
.0350 \\
.0350 \\
.0350 \\
.0350 \\
.0350 \\
-030\end{array}$ & 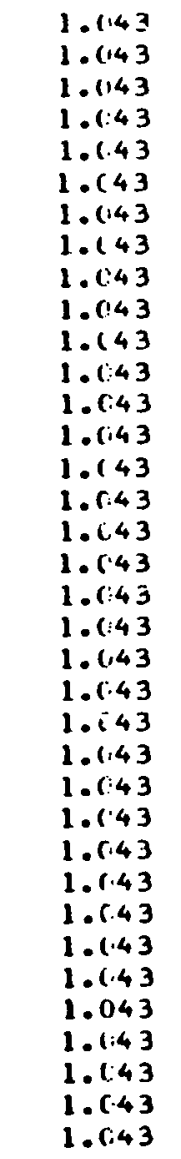 \\
\hline
\end{tabular}


TALLE ?: BARRELS IIF REFIVERY PRONIICTS PROUUCED PFR RARF.EL OF CRUDE CONSUMED

REFINERY REGION 3

\begin{tabular}{|c|c|c|c|c|c|c|c|c|c|}
\hline CRINOE TYPE & $\begin{array}{l}\text { LIOUIFIED } \\
\text { PITRULEUM } \\
\text { GASSES }\end{array}$ & GASOL INF & NAPHTHA & JET FIJEL & $\begin{array}{l}\text { OISTIL- } \\
\text { LATES }\end{array}$ & RESIDUAL & $\begin{array}{l}\text { OTHFR } \\
\text { PETRIILEUM } \\
\text { PRIJNIICTS }\end{array}$ & $\begin{array}{r}\text { STILL } \\
\text { GAS }\end{array}$ & $\begin{array}{l}\text { TOTAL } \\
\text { REF INERY } \\
\text { PROOUCT }\end{array}$ \\
\hline ARABIAN LIC;HT & .0108 & .3729 & .1040 & .07 .34 & .2356 & .1176 & .0770 & .0350 & $1.0,27$ \\
\hline ARABIAN HUY & $.010 p$ & .3730 & .0713 & $.052 A$ & .2007 & .1713 & .1120 & .0350 & 1.027 \\
\hline KUWAITI EXPחDT & .01111 & .3837 & .1039 & .0625 & .1922 & .1449 & .0936 & .0350 & 1.027 \\
\hline IRAOI & .0108 & .3720 & $.104 \mathrm{~B}$ & .0734 & .2356 & .1176 & .0770 & .0350 & 1.027 \\
\hline IRANIAN LIGHI & $.0110 n$ & .3463 & .1261 & .0846 & .2431 & .1101 & .0718 & .0350 & 1.027 \\
\hline IRANIAN MEAVY & .0108 & .3716 & .0931 & .0611 & .2176 & .1434 & .0944 & .0350 & 1.027 \\
\hline MID-EASTERN MIX & .0103 & .3574 & .1316 & .0695 & .2199 & .1231 & .0AC1 & .0350 & 1.027 \\
\hline LIHYAN & .0119 & .4122 & .1277 & .0716 & .2197 & .0894 & .0595 & .0350 & 1.027 \\
\hline ALGER IAN & $.0,116$ & .4014 & .1582 & .0672 & .2073 & .0879 & .0582 & .0350 & 1.027 \\
\hline NIGERIAN G GABON & .0139 & .4807 & .1022 & $.07 A 2$ & .1952 & .0714 & .0502 & .0350 & 1.027 \\
\hline INDONE S I AN & .0122 & .4211 & .0532 & .0506 & .2412 & .1267 &. . 968 & .0350 & 1.027 \\
\hline ECUADORRIAN & .0128 & .4416 & .1637 & .0600 & .1495 & .0974 & .0662 & $.035 n$ & 1.027 \\
\hline $\begin{array}{l}\text { VENEZUELAN MIX } \\
\text { MEXICAN }\end{array}$ & $\begin{array}{l}.0109 \\
.0108\end{array}$ & $\begin{array}{l}.3749 \\
.3729\end{array}$ & $\begin{array}{l}.0786 \\
.104 \mathrm{~A}\end{array}$ & $\begin{array}{l}.0605 \\
.0734\end{array}$ & $\begin{array}{l}.1674 \\
.2356\end{array}$ & $\begin{array}{l}.1818 \\
.1176\end{array}$ & $\begin{array}{l}.1178 \\
.0770\end{array}$ & $\begin{array}{l}.0350 \\
.0350\end{array}$ & $\begin{array}{l}1.027 \\
1.027\end{array}$ \\
\hline RUSSIAN EXPORT & .0108 & .3729 & .1048 & .0734 & .2356 & .1176 & .0770 & .0350 & 1.027 \\
\hline CANADIAN MIX & .01115 & .3966 & .1064 & $.05 A 6$ & .1996 & .1322 & .0871 & .0350 & 1.027 \\
\hline FGYPIIANASYRIANI & .0100 & .3738 & . & .0617 & .2178 & .1404 & .0925 & .0350 & 1.027 \\
\hline ANGIILA/CONGO/LAIRF & .0122 & .4211 & .0532 & .0506 & .2412 & .1267 & .0868 & .03511 & 1.027 \\
\hline BOLIVIANIPERUVIAPA & .0116 & .4014 & .1582 & .0672 & .2073 & .0879 & .0582 & .0350 & 1.027 \\
\hline NIIRWE G, I APH & .0134 & .46 .74 & .0776 & .0705 & .2068 & .0956 & .0547 & .0350 & 1.027 \\
\hline SUUTH AS:AN & $.0: 108$ & .3729 & .1049 & .0734 & .2356 & .1176 & .0770 & .0350 & 1.027 \\
\hline IRINIDAI] & .0134 & .4634 & .0776 & .0705 & $.206 A$ & .0956 & .0647 & .0350 & J.027 \\
\hline LNITED KINGOOM & $.0: 108$ & .3729 & .1048 & .0734 & .2356 & .1176 & $.077 n$ & $.0350^{\circ}$ & 1.027 \\
\hline SYNTHETIC CRUUE & $.0: 144$ & .4950 & .0146 & .0906 & .2881 & .0523 & .0361 & .0350 & 1.027 \\
\hline ALASKAN PIIKTH SLOPE & .0133 & .4576 & .0700 & $.05 A 5$ & .1760 & $.129 A$ & .0868 & .0350 & 1.027 \\
\hline WEST COAST LIGHT & .0120 & .4137 & .0221 & .0248 & $.10 n 8$ & .2535 & $.16,50$ & .0350 & 1.027 \\
\hline WEST CCAST HEAVY & .0133 & .4600 & .0725 & .0514 & .1376 & .1543 & .1027 & .0350 & 1.027 \\
\hline PACIFIC OFFSHNHE & .0128 & .4416 & .1637 & .0608 & .1495 & .0974 & .0662 & .0350 & 1.027 \\
\hline WYIIMING MIX & $.0,126$ & .4333 & .1380 & .0606 & .1856 & .0965 & .0653 &.$\cap 350$ & 1.027 \\
\hline SHALE OIE & .0144 & .4059 & .0146 & .0906 & .2801 & .0523 & .0361 & .0350 & 1.027 \\
\hline LDUISIANA ONSHOKE & .0119 & .4123 & .0544 & .0737 & .2782 & .0955 & .0650 & .0350 & 1.027 \\
\hline LOUISIANA NFFSHORE & .01129 & .4123 & .0544 & .0737 & .2782 & .0955 & $.0 \times 59$ & .0350 & 1.027 \\
\hline TEXAS GULF CIIAST & .0106 & .3654 & .0553 & .0046 & .3152 & .0872 & $.05 A 6$ & .0350 & 1.027 \\
\hline EAST IEXAS MIX & .0127 & $.438 A$ & .1524 & .0615 & .1812 & .0860 & .05093 & .0350 & 1.027 \\
\hline WFST TEXAS MIX & .0123 & .4241 & .1267 & .0766 & .2242 & .0762 & .0518 & .0350 & 1.027 \\
\hline PAL II IMUIGFNOUS & .0110 & $.3 A \cap 5$ & .1673 & .0698 & .2079 & .0934 & .0621 & .0350 & 1.027 \\
\hline PAO I INGIRENOUS & $.0(11 \mathrm{~A}$ & $.40 \times 2$ & .0936 & .0713 & .2515 & .0926 & $.0+30$ & .0350 & 1.027 \\
\hline OKLAHOHA MIX & .0120 & $.41 \times 3$ & .0443 & .0823 & .2509 & .0807 & .05 .36 & .0350 & 1.027 \\
\hline PAC, III HFAVY CRULF & .01112 & $.3 A 55$ &.$n 991$ & .0639 & .1587 & .1656 & .1079 & .0350 & 1.027 \\
\hline NAVAL KESERVE HI CRUHE & .0160 & .5537 & .7553 & .0674 & .1823 & .0681 & .0491 & .0350 & 1.027 \\
\hline
\end{tabular}


ICORIINUE:D)

IALLE ¿: BAFRELS OF KEFINFRY PROMUCTS PROQUCEO PER RARREL OF CRUOE CONSUMFO

REFINERY REGIINN 4

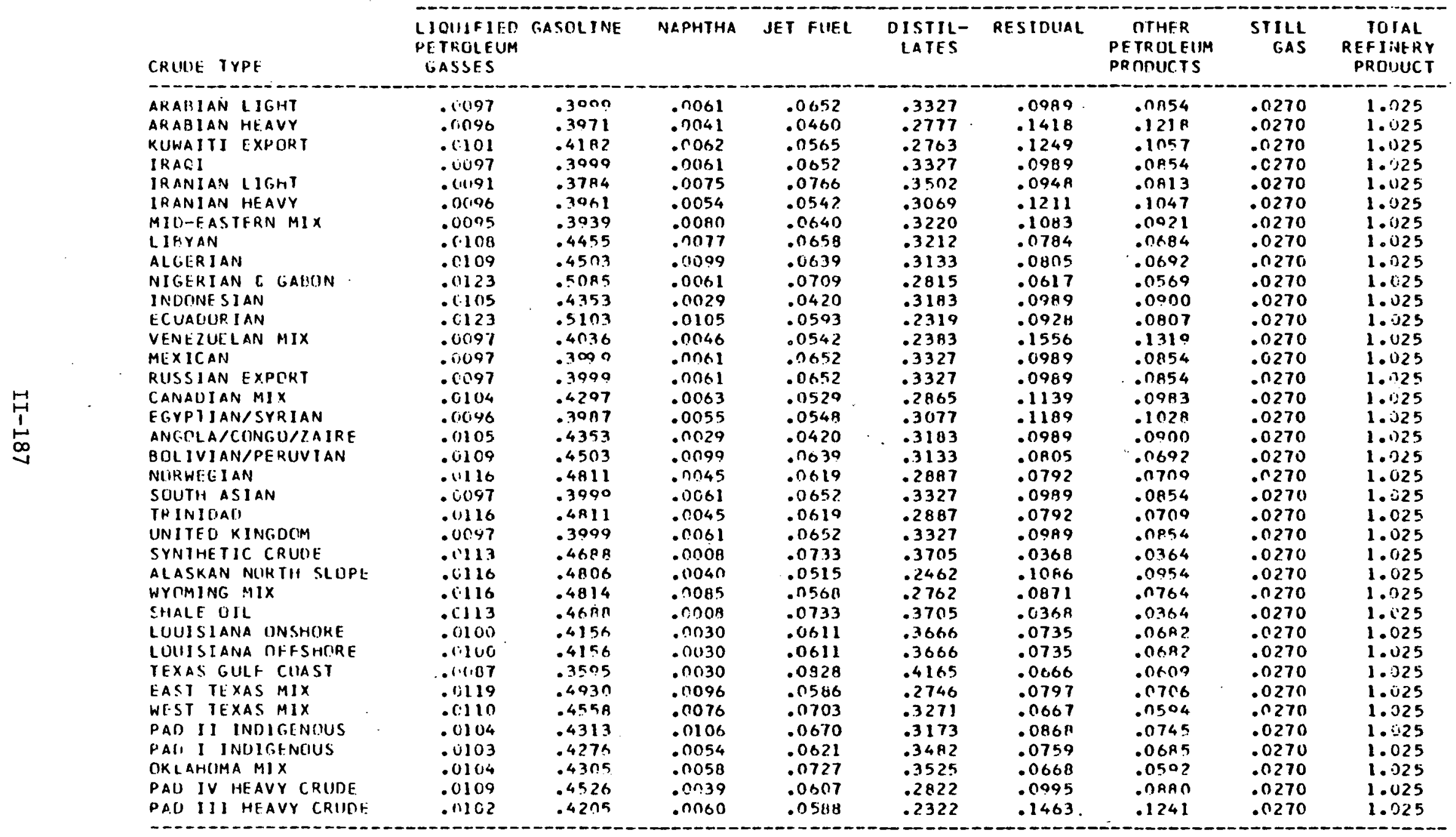


REFINERY REGIDN 5

\begin{tabular}{|c|c|c|c|c|c|c|c|c|c|}
\hline CRUDE TYPE & $\begin{array}{l}\text { LIUUIFIED } \\
\text { PETROLEUM } \\
\text { GASSES }\end{array}$ & GASOLINE & NAPHTHA & JET FUEL & $\begin{array}{l}\text { DISTIL- } \\
\text { LATES }\end{array}$ & RESIDUAL & $\begin{array}{c}\text { OTHFR } \\
\text { PFTRNLEUM } \\
\text { PRODUCTS }\end{array}$ & $\begin{array}{r}\text { STILL } \\
\text { GAS }\end{array}$ & $\begin{array}{l}\text { TOTAL } \\
\text { REFINERY } \\
\text { PRODUCT }\end{array}$ \\
\hline 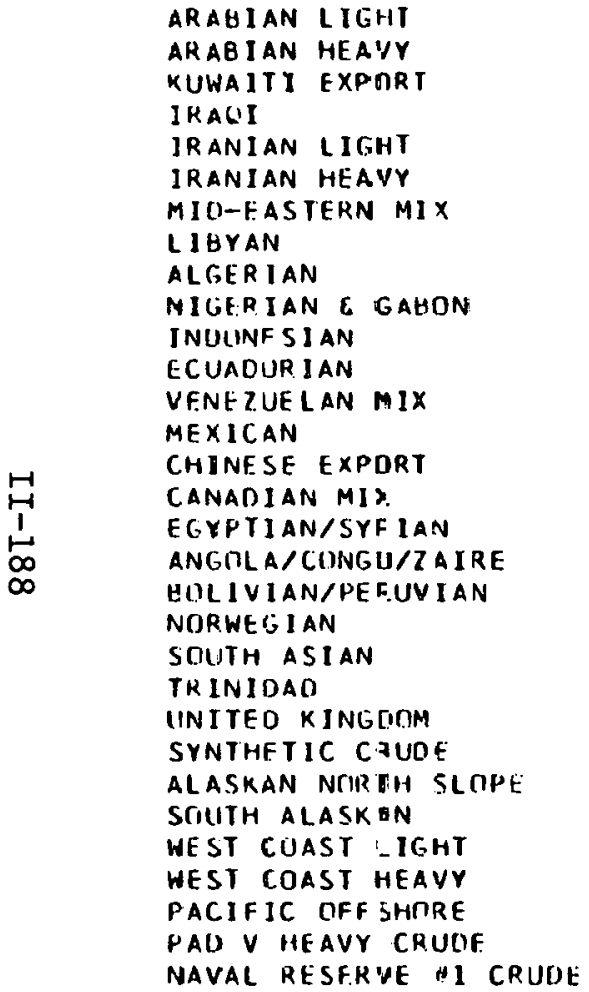 & $\begin{array}{l}.0173 \\
.0162 \\
.01177 \\
.0173 \\
.0104 \\
.0173 \\
.0173 \\
.0207 \\
.0206 \\
.0215 \\
.0177 \\
.0221 \\
.0167 \\
.01173 \\
.0177 \\
.0187 \\
.0175 \\
.0177 \\
.0206 \\
.0218 \\
.01173 \\
.0218 \\
.01173 \\
.0230 \\
.0209 \\
.0210 \\
.0157 \\
.01202 \\
.0221 \\
.0145 \\
.01262\end{array}$ & $\begin{array}{l}.4000 \\
.3730 \\
.4079 \\
.4000 \\
.3771 \\
.3985 \\
.3006 \\
.4781 \\
.4754 \\
.5415 \\
.4079 \\
.5105 \\
.3860 \\
.400 n \\
.4079 \\
.4309 \\
.402 A \\
.4079 \\
.4754 \\
.5038 \\
.4000 \\
.503 R \\
.4000 \\
.5308 \\
.4921 \\
.5315 \\
.3630 \\
.4669 \\
.5105 \\
.3353 \\
.6048\end{array}$ & $\begin{array}{l}.1160 \\
.0107 \\
.0156 \\
.0160 \\
.0194 \\
.0139 \\
.0202 \\
.0100 \\
.01244 \\
.0144 \\
.0084 \\
.0250 \\
.0113 \\
.0160 \\
.0004 \\
.0160 \\
.0142 \\
.0084 \\
.0244 \\
.0111 \\
.0160 \\
.0111 \\
.0160 \\
.0020 \\
.0100 \\
.0228 \\
.0032 \\
.0105 \\
.0250 \\
.0395 \\
.0073\end{array}$ & $\begin{array}{l}.1596 \\
.1133 \\
.1339 \\
.1546 \\
.1159 \\
.1305 \\
.1524 \\
.1519 \\
.1479 \\
.1572 \\
.1142 \\
.1324 \\
.1242 \\
.1596 \\
.1142 \\
.1260 \\
.1316 \\
.1142 \\
.1479 \\
.1435 \\
.1596 \\
.1435 \\
.1596 \\
.1743 \\
.1195 \\
.1256 \\
.0511 \\
.1064 \\
.1324 \\
.0568 \\
.1278\end{array}$ & 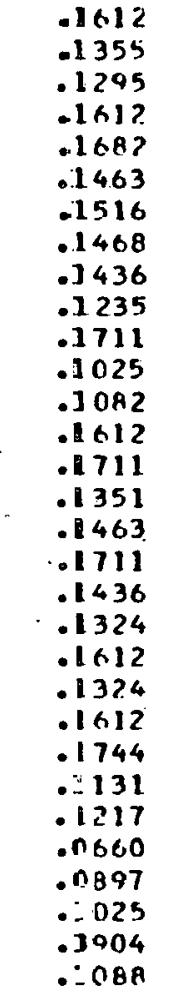 & $\begin{array}{l}.1703 \\
.1454 \\
.2062 \\
.1703 \\
.1614 \\
.2038 \\
11749 \\
.1249 \\
.1288 \\
.0920 \\
.1935 \\
.1416 \\
.2471 \\
.1703 \\
11935 \\
.1894 \\
.1995 \\
.1935 \\
.1288 \\
.1267 \\
.1703 \\
.1267 \\
.1703 \\
.0607 \\
.1746 \\
.1178 \\
.3519 \\
.2119 \\
.1416 \\
11574 \\
.0803\end{array}$ & $\begin{array}{l}.0605 \\
.0098 \\
.0837 \\
.0695 \\
.0655 \\
.0837 \\
.0720 \\
.0525 \\
.0532 \\
.0419 \\
.0112 \\
.0509 \\
.1005 \\
.0695 \\
.0812 \\
.0778 \\
.0820 \\
.0812 \\
.0532 \\
.0546 \\
.0695 \\
.0546 \\
.0695 \\
.0288 \\
.0736 \\
.0516 \\
.1425 \\
.0883 \\
.0599 \\
.0470 \\
.0387\end{array}$ & $\begin{array}{l}.0470 \\
.0470 \\
.0470 \\
.0470 \\
.0470 \\
.0470 \\
.0470 \\
.0470 \\
.0470 \\
.0470 \\
.0470 \\
.0470 \\
.0470 \\
.0470 \\
.0470 \\
.0470 \\
.0470 \\
.0470 \\
.0470 \\
.0470 \\
.0470 \\
.0470 \\
.0470 \\
.0470 \\
.0470 \\
.0470 \\
.0470 \\
.0470 \\
.0470 \\
. \\
.0470\end{array}$ & $\begin{array}{l}1.041 \\
1.041 \\
1.041 \\
1.041 \\
1.041 \\
1.041 \\
1.041 \\
1.041 \\
1.041 \\
1.041 \\
1.041 \\
1.041 \\
1.041 \\
1.041 \\
1.041 \\
1.041 \\
1.041 \\
1.041 \\
1.041 \\
1.041 \\
1.041 \\
1.041 \\
1.041 \\
1.041 \\
1.041 \\
1.041 \\
1.041 \\
1.041 \\
1.041 \\
1.041 \\
1.041\end{array}$ \\
\hline
\end{tabular}


REFINERY REGINN 6

\begin{tabular}{|c|c|c|c|c|c|c|c|c|c|}
\hline CRUDE TYPE & $\begin{array}{l}\text { LIOUIFIED } \\
\text { PETROLEUM } \\
\text { GASSES }\end{array}$ & GASNLINE & NAPHTHA & JET FUEL & $\begin{array}{l}\text { OISTIL- } \\
\text { LATES }\end{array}$ & RESIDUAL & $\begin{array}{l}\text { OTHFR } \\
\text { PETROLEUM } \\
\text { PROOICTS }\end{array}$ & $\begin{array}{l}\text { STILL } \\
\text { GAS }\end{array}$ & $\begin{array}{l}\text { TOTAL } \\
\text { REFINEKY } \\
\text { PRODIJCT }\end{array}$ \\
\hline 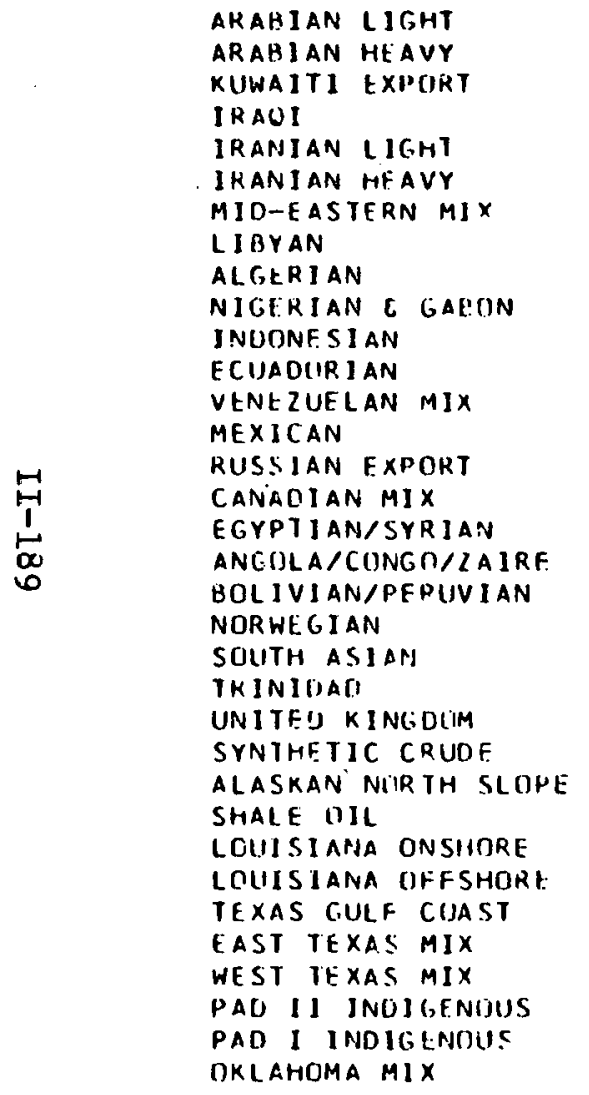 & $\begin{array}{l}.0286 \\
.0272 \\
.0241 \\
.0286 \\
.0274 \\
.0279 \\
.0281 \\
.0323 \\
.0324 \\
.0369 \\
.0303 \\
.0355 \\
.0276 \\
.0286 \\
.0286 \\
.0301 \\
.0281 \\
.0303 \\
.0324 \\
.0345 \\
.0286 \\
.0345 \\
.0286 \\
.0356 \\
.0335 \\
.0356 \\
.0302 \\
.0302 \\
.0274 \\
.0350 \\
.0334 \\
.0310 \\
.0310 \\
.0318\end{array}$ & $\begin{array}{l}.4248 \\
.4037 \\
.4320 \\
.4248 \\
.4067 \\
.4137 \\
.4165 \\
.4800 \\
.48144 \\
.5479 \\
.4492 \\
.5267 \\
.4098 \\
.4248 \\
.424 A \\
.4465 \\
.4172 \\
.4492 \\
.4804 \\
.5127 \\
.4248 \\
.5177 \\
.424 A \\
.5280 \\
.4969 \\
.5280 \\
.4488 \\
.44 A A \\
.4063 \\
.518 A \\
.4955 \\
.4603 \\
.4600 \\
.4726\end{array}$ & $\begin{array}{l}.0169 \\
.1109 \\
0166 \\
0169 \\
.0210 \\
.0147 \\
.0218 \\
01211 \\
.0269 \\
.0165 \\
.0010 \\
.0277 \\
.0122 \\
.0169 \\
.0169 \\
.0170 \\
.0150 \\
.0080 \\
.0269 \\
.0122 \\
.0169 \\
.0122 \\
.0169 \\
.0022 \\
.0108 \\
0022 \\
.0084 \\
.0014 \\
.0187 \\
.0256 \\
.0210 \\
.0287 \\
.0150 \\
.0161\end{array}$ & $\begin{array}{l}.0324 \\
.0221 \\
.0272 \\
.0324 \\
.0385 \\
.0263 \\
.0314 \\
.0323 \\
.0312 \\
.0346 \\
.0209 \\
.0281 \\
.0256 \\
.0324 \\
.0324 \\
.0255 \\
.0267 \\
.0209 \\
.0312 \\
.03112 \\
.0324 \\
.0302 \\
.0324 \\
.0374 \\
.0246 \\
.0374 \\
.0311 \\
.0311 \\
.0429 \\
.0282 \\
.0347 \\
.0327 \\
.0311 \\
.0364\end{array}$ & $\begin{array}{l}.2666 \\
.2157 \\
.2149 \\
.2666 \\
.2836 \\
.2408 \\
.2547 \\
.2543 \\
.2467 \\
.2213 \\
.2554 \\
.1772 \\
.1818 \\
.2666 \\
.2666 \\
.2232 \\
.2415 \\
.2554 \\
.2467 \\
.2274 \\
.2666 \\
.2274 \\
.2666 \\
.3050 \\
.1899 \\
.3050 \\
.3008 \\
.3609 \\
.3483 \\
.2129 \\
.2605 \\
.2500 \\
.2816 \\
.2851\end{array}$ & $\begin{array}{l}.1260 \\
.1735 \\
.1533 \\
.1260 \\
.1225 \\
.1499 \\
.1358 \\
.0985 \\
.1004 \\
.0765 \\
.1255 \\
.1112 \\
.1865 \\
.1260 \\
.1260 \\
.1399 \\
.1473 \\
.1255 \\
.1004 \\
.0985 \\
.1260 \\
.0985 \\
.1260 \\
.0493 \\
.1315 \\
.0493 \\
.0963 \\
.0963 \\
.0901 \\
.0971 \\
.0844 \\
.1084 \\
.0978 \\
.0865\end{array}$ & 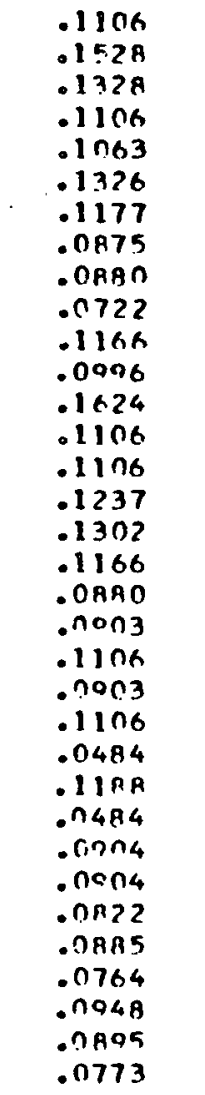 & $\begin{array}{l}.0360 \\
.0360 \\
.0360 \\
.0360 \\
.0360 \\
.0360 \\
.0360 \\
.0360 \\
.0360 \\
.0360 \\
.0360 \\
.0360 \\
.0360 \\
.0360 \\
.0360 \\
.0360 \\
.0360 \\
.0360 \\
.0360 \\
.0360 \\
.0360 \\
.0360 \\
.0360 \\
.0360 \\
.0360 \\
.0360 \\
.0360 \\
.0360 \\
.0360 \\
.0360 \\
.0360 \\
.0360 \\
.0360 \\
.0360\end{array}$ & $\begin{array}{l}1.042 \\
1.042 \\
1.042 \\
1.042 \\
1.042 \\
1.042 \\
1.042 \\
1.042 \\
1.042 \\
1.042 \\
1.042 \\
1.042 \\
1.042 \\
1.042 \\
1.042 \\
1.042 \\
1.042 \\
1.042 \\
1.042 \\
1.042 \\
1.042 \\
1.042 \\
1.042 \\
1.042 \\
1.042 \\
1.042 \\
1.042 \\
1.042 \\
1.042 \\
1.042 \\
1.042 \\
1.042 \\
1.042 \\
1.042\end{array}$ \\
\hline
\end{tabular}




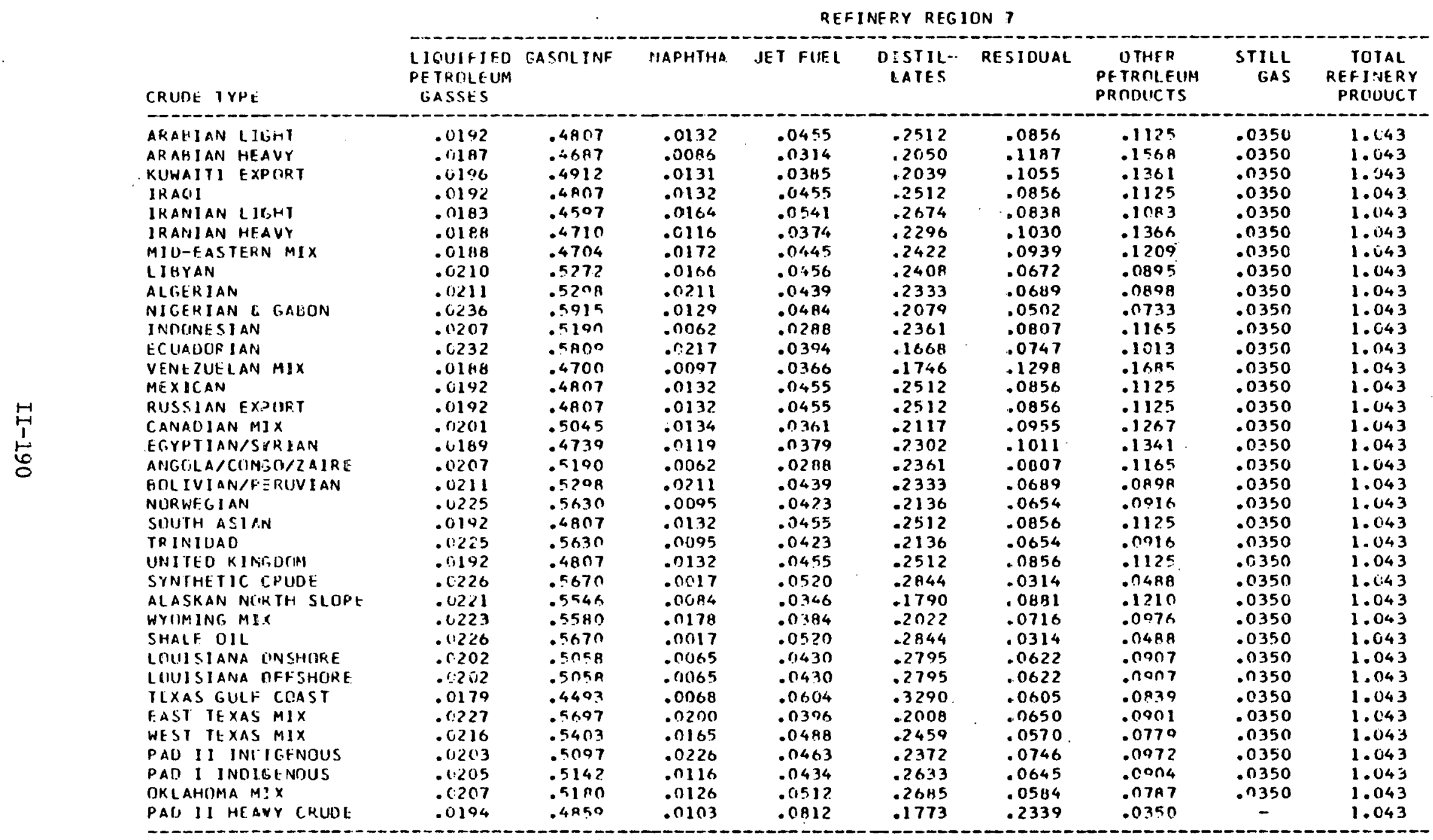

SOLIRCE : RFFINERY ANO PETROCHFMICAL MOOFLLING SYSTEM (RPMSI DATA EASE, OWNFO AY EONNER G MUCIRE ASSICIATES, INC., WASHINGTON, D.C.

DFFICE RESPONSIGLE: MIO-RANGF ANALYSIS OIVISICIN, OFFICE OF INTEGAATIVE ANAIYSIS, APPLIED ANALYSIS, FIA. 
TABLE 3: RFIATIVE PRCIOUCT PRICING, RFLATIONSHIPS

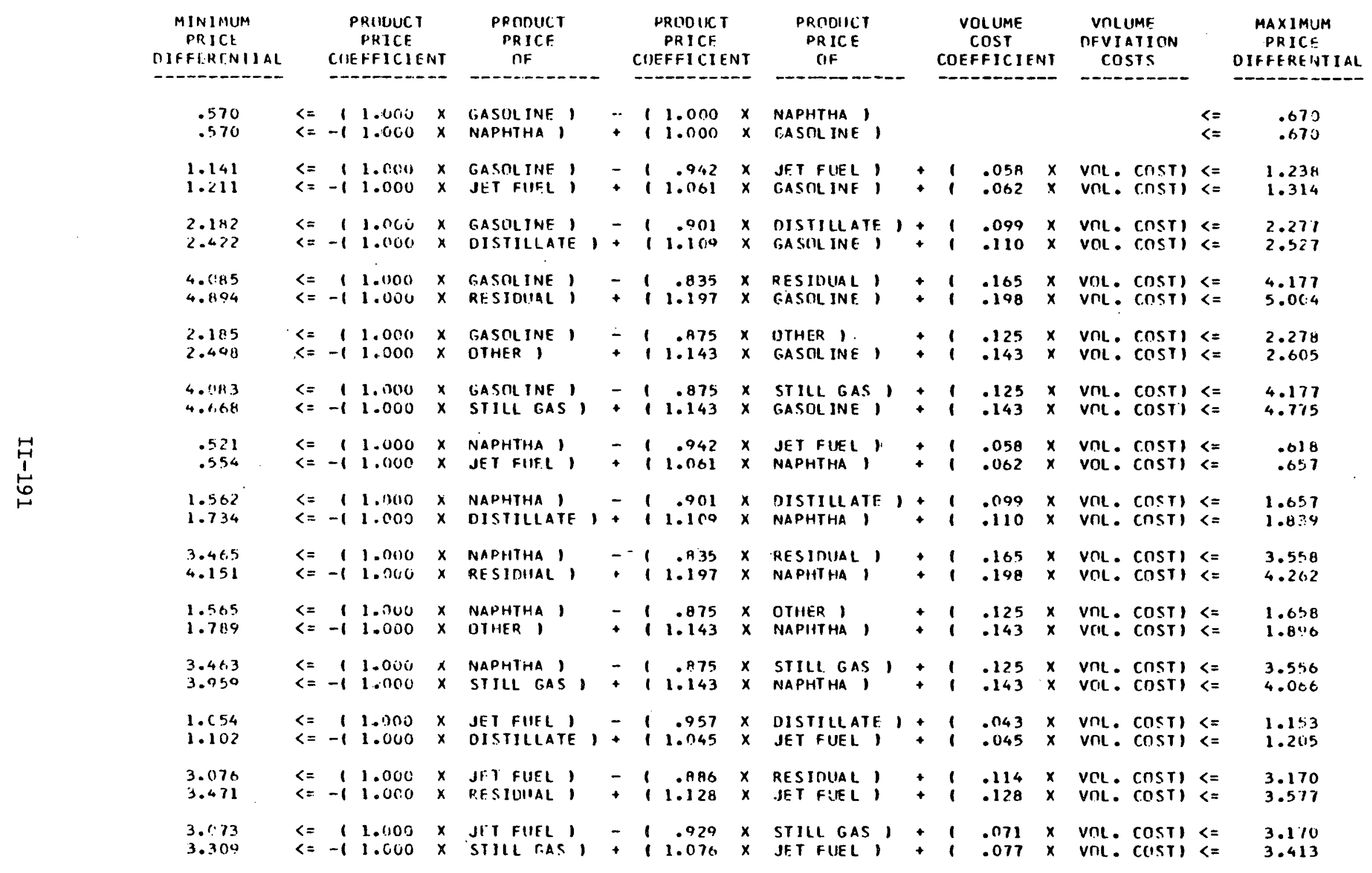


(CONTINUEO)

TARLF 3: RFLAIIZF PRUOIXT PRICING RFLATIONSHIPS

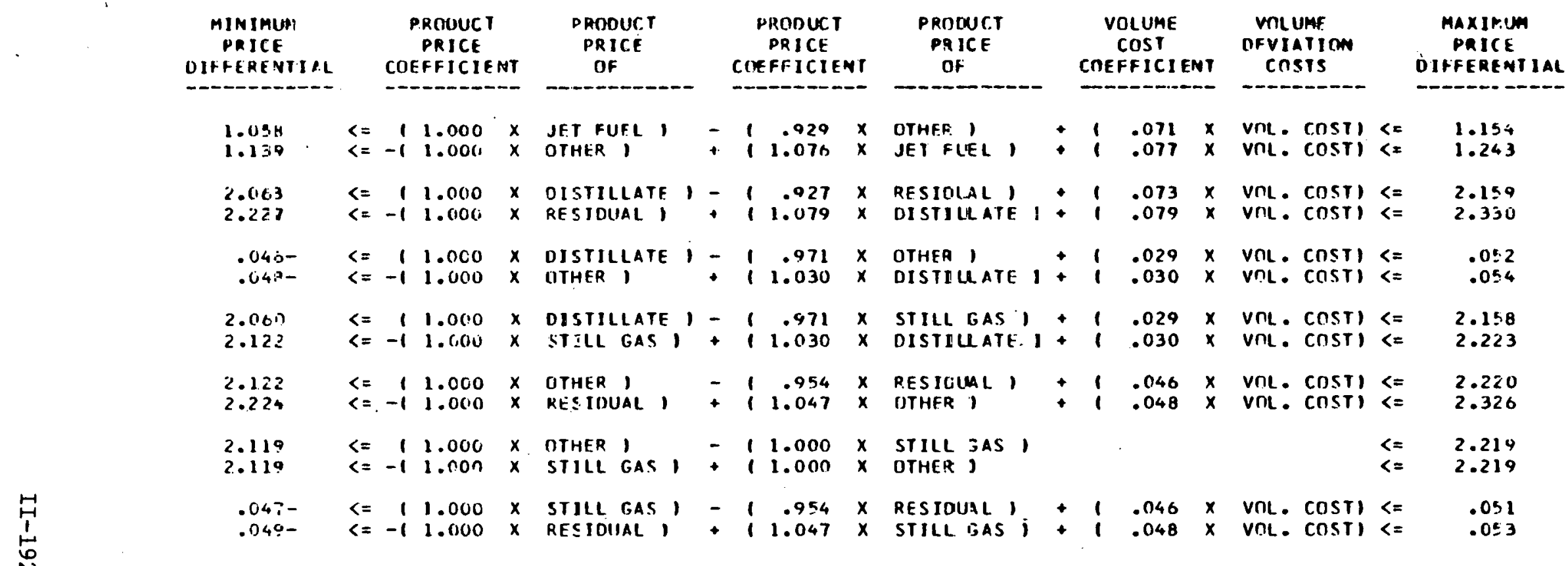

UATA SOURCE : COSIS AND PRICE RELATIONSHIPS ARE MUTPIT FROM THE REFINERY CONVERSION SUH-MUDEL IMESEODED IN PIFS.

DFFICE RESPONSIBLE : MIO-RANGE ANALYSIS DIVISION, DFFICE OF INTEGRATIVE ANALYSIS, APPL IED ANALYSIS, EIA. 
TABLF 4: REFINERY CAPACITY DATA

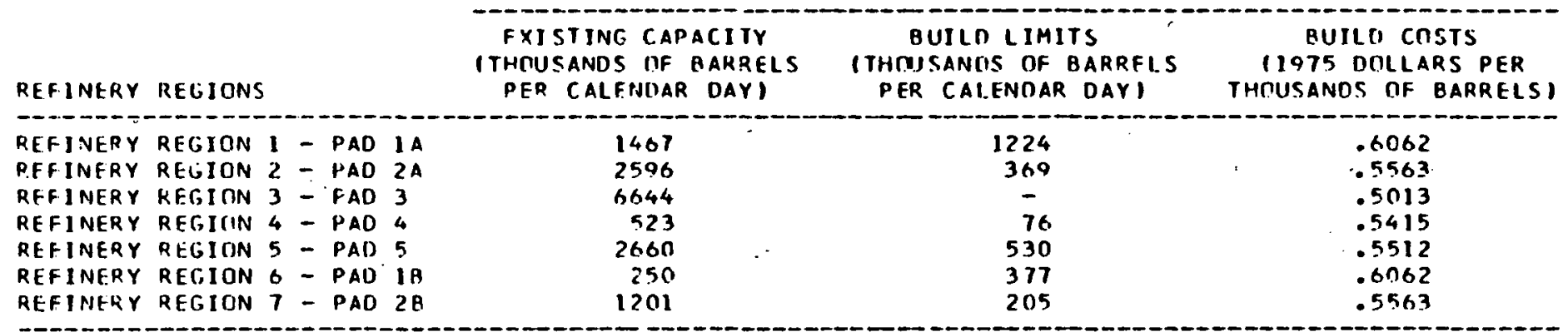

REFINERY CAPACITY ANI UTILIZATION", BY E.L. PEER AND F.V. MARSIK.

IFFICE OF OIL $T$ GAS, FEA, 1975. CAPACITY COSTS DERIVED FROM REFERENCES IN ¿OIL E GAS JOURNAL:

OFFICE RESPONSIBLE : MID-RANGE ANALYSIS DIVISION, OFFICE DF INTEGRATIVE ANALYSIS, EIA. 
This section contains data tables for three energy sources: synthetics, shale and solar/geothermal. These are three of the smaller supply sectors in PIES and are also referred to as "New and Emerging Technologies" in the 1978 Annual Administrator's Report.

In the synthetics data tables, $\$ / B O E$ means dollars per barrel of oil equivalents, $M B O E / D$ means thousands of barrels of oil equivalents per day, and $M \$ / B / D$ means thousands of dollars per barrel per day. The other units are the usual standard units. Synthetics Data

This section contains data from the synthetics analyses performed by DOE. These analyses yield figures relating to the production of synthetic fuels from high sulfur coal. The four modes of synthetic production permitted are: crude oil from coal, natural gas from coal, electricity from low Btu fuel gas (which in turn is produced from coal), and "syngas from other." The latter models synthetic natural gas production from naphtha.

There are four tables per target year containing the price, production and capital data for synthetic fuels, for both the mid-range supply and high growth supply scenarios.

The first table in each group gives production data for the conversion of coal to synthetic crude, syncrude. This conversion takes place in coal regions, which are the rows. Only three coal regions contain facilities for the production of syncrude.

The second table in each group gives data for the conversion of high Btu gas from coal by coal region. The third table gives the production of low and medium Btu gas by utility region. This synthetic gas is converted to electricity in the utility regions.

The last synthetics data table in each group contains data for the conversion of synthetic gas from sources other than coal. This activity takes place in demand regions. The other category refers to naphtha, a petroleum product produced in the refinery regions. 


\section{Shale Data}

Shale is a synthetic fuel, but is input to PIES by a separate process. One table is given for shale production. The table has production, cost and capital requirement data for the target years 1985 and 1990 .

Solar/Geothermal Data

There are four tables of solar and geothermal input data. They show price, quantity and capital data for geothermal, wind, solar thermal, and solar photovoltaic energy sources by DOE region for both 1985 and 1990. 
TABI.F 1: PLODUCTION OF SYITHETEC CRUOR PROY COAL IN 1935 BY COAL RBGION

COA: REGIOY

nonthent

NORTHERN APPLACHIAN

CENTPAL APPI.ACHIAI

SOIITHERN APPLACHIAN

MIDYEST

CENTRAL WEST

Gill, ?

N.E. GREAT PLATNS

N. H. GPPATT PLAENS

POCEIES

SNITHHEST

NOR?HHEST

A L. A.SK A

DATA SOUHCES : DOE ESTIMATES DEVELOPBD IN CONJINCZITN UHITH THE POLLONING STUDY: "SYNFI ELS MAFKET PUTFNTIAE". INSTITUTE OF GAS TECBNOLGGY, tTT CBNTER, CHICAgO,

ILLINOIS, PREPARE FOR THE II.S. EPA UNDER CONTRACT P-03-77-5810-0, JANIARY. 1979.

OFPICP RPSPONSIBLE : CONGERVATION AND RBNEMABLE RESTORCES DTVISION, OPFICP OF EHERGY USE ANALYSIS, EIA.

TABLE, 2: PRONICTION DF HJGII OTTU GAS PQOS COAL IN 1985 BY COAL IIEGION

- - - MIDRANGF, SUPPLY-----

\begin{tabular}{|c|c|c|}
\hline \multicolumn{3}{|c|}{ PROIS!CTION } \\
\hline P RICE & I. F.VELL & CAPITAL \\
\hline$\$ / B O P$ & MAOR D & $M \$ / B / D$ \\
\hline$-\cdots-$ & $\cdots-\cdots$ & $---\cdots$ \\
\hline 20.99 & & 16.30 \\
\hline $\begin{array}{l}21.75 \\
2.0 .79\end{array}$ & 23.50 & $\begin{array}{l}23.00 \\
16.30\end{array}$ \\
\hline 21.75 & 23.50 & 23.00 \\
\hline
\end{tabular}

\section{COAL REGION}

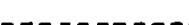

NORTHERN APPLACHTAN

CENTRAL APPLACHTAN

SOIITHERN APPLACHIAN

MIDARST'

C.N.TPAI. WP.ST

(i) $1 .=$

N.E. GRFAT PLALNS

N. 4 GREAT PLATNS

ROCEIRS

SกUフ्म ESTT

NORTHEST

21.75

23.50

23.00
-- -HIGH GROHTII SHPPLY--

\begin{tabular}{|c|c|c|}
\hline \multicolumn{3}{|c|}{ PRODUCE ION } \\
\hline ICE & LEVEI. & CAPITAL \\
\hline$\$ B \cap A$ & MBOE/D & $\triangle B / B / D$ \\
\hline---- & $\cdots-$ & \\
\hline 20.98 & & 16.30 \\
\hline 0.99 & & 16.30 \\
\hline 0.98 & & 16.30 \\
\hline 20.99 & & 16.30 \\
\hline
\end{tabular}

21.75

35.30

23.00

20.98

20.93

16.30

35.30

ALA SK A

DATA SOIIRCES : DOE ESTIMATES DEVELOPEO IA CONJUNCTI.ON AHTTH THE POLLOHINJ STINY:

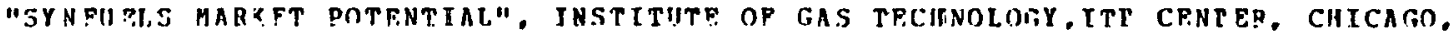

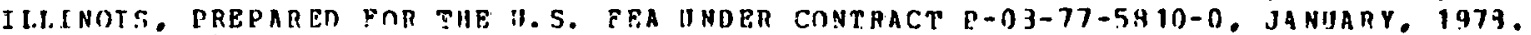

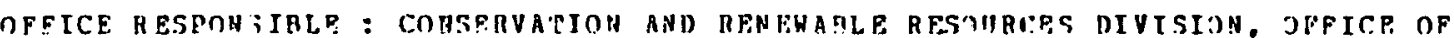
ENEHGY UIS ANALYSTS. EIA. 
TABLP 3: PRODUCTION OP LOW GND MROIIIA DTU SYNTHETIC GAS PROM COAL. IN 1985 DY ITII.TYY RE,G ION

- - - - - MI DRANGE SUPPLY -

UTTLITY REGION

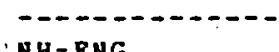

NH-BNG.

\section{NY $/$ N.}

MID-ATL.

S. - ATI.

MIDNEST

S. - HP.ST

CENTRAI.

N-CNTRL.

WFST

N.-WEST
PRODIICT ION

LEVEL
MBOE/D

MBOE/D

CAPITAL
$M \$ / B / D$
-

$\$ / B O E$

$--0-$

-

23. 30

2.3. 30

23. 30

23. 30

23. 30

23. 30

21.20
21.20
21.20
21.20
21.20
21.20

-D-HIGH GPOHT!I SIJ PPLY - -

\begin{tabular}{|c|c|c|}
\hline & איח & \\
\hline $\begin{array}{l}P \text { I CP, } \\
/ \mathrm{BOE}\end{array}$ & $\begin{array}{r}\text { LEVEL } \\
\text { MBOE } / 0\end{array}$ & $\begin{array}{l}\text { CAPITAL } \\
A \delta / B / D\end{array}$ \\
\hline
\end{tabular}

$\begin{array}{lll}16.80 & 7.83 & 13.00\end{array}$

$\begin{array}{lll}16.80 & 7.83 & 13.00\end{array}$

$\begin{array}{lll}16.80 & 7.83 & 13.00\end{array}$

23. $30 \quad 21.20$

$23.30 \quad 21.20$
$23.30 \quad 21.20$

23.30

DATA SOIRCES : nOR. ESTIMATPS DEVELOPRD IN CONJUNCTION HHITI THE POLLOHING STTOD: "SYNFII MLS MARKET POTENTIAL". INSTITUTB OF GAS TECHNOLOGY. ITT CRNTRR, CIICAGO, ILLINOIS. PREPARP POR THE IJ.S. PF. IINDER CONTRACT P-03-77-5810-0, JANUAR. 1979.

OPFTCP. RPSPONSIBLP : CONSERVATION AND RENEMABI, RESOURCES DIVISION, OFPICR OP PNERGY IISP, ANALYSIS, EIA.

TABLE 4: PRODUCTION DP SYNTHFTIC GaS PROA SOURCES OTHER THAN COAL IN 1985 BY DEMAN B REGION

DEMAN.P REGION

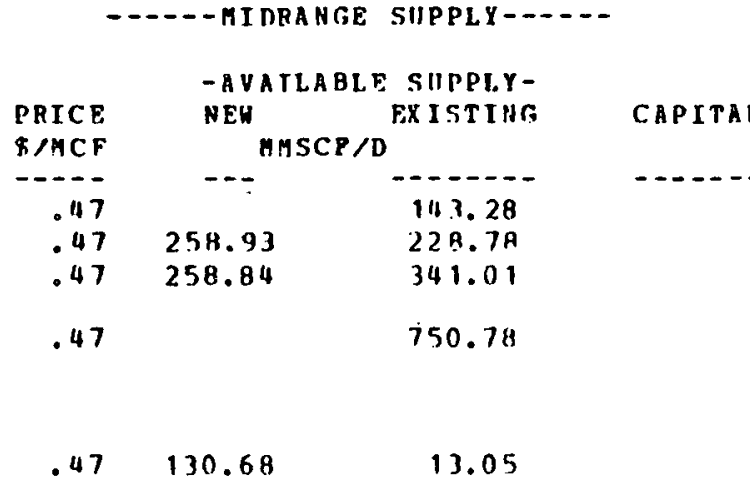

--- -IIIGH GROHTH SIJPPLY -...

NH - ENG.

NY $/$ NJ

A ID - A TL

S. -ATL

MIDHRST

S. - HEST

CENTPAL

N-CNTRI.

HEST

N. - H F.ST

\begin{tabular}{|c|c|c|}
\hline PBICE & $\begin{array}{l}\text { - AV A II } \\
\text { NP.H }\end{array}$ & $\begin{array}{r}\text { SUPPLY - } \\
\text { BXISTI NG }\end{array}$ \\
\hline$\triangle / M C F$ & \multicolumn{2}{|c|}{ MMSCF/D } \\
\hline$-\cdots$ & --- & $----\infty$ \\
\hline .47 & & 143.28 \\
\hline .47 & 258.93 & 228.78 \\
\hline .47 & 258.84 & 341.01 \\
\hline .47 & & 750.78 \\
\hline .47 & 130.68 & 13.05 \\
\hline
\end{tabular}

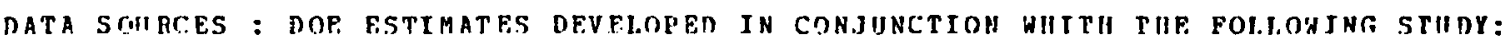
"SYNPIIFLS MARKET POTENT IAL". IHSTITUTE OP GAS TECUNOLOCY, I TT CENTER, CHICAGO

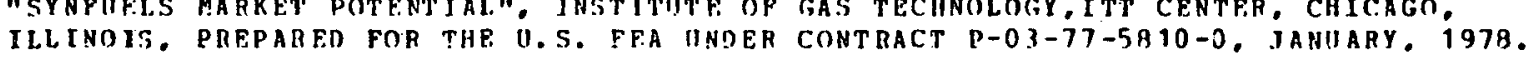

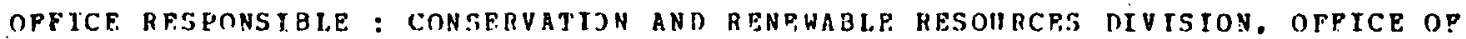
ENERGY IISF. ANALYSIS. EIA. 
TARLE 1: PRODICTJON OP SYNTHFTIC CRIDE PROA COAL TN 1990

BY COAL REGLON

- - MIDRANGE. SUPPLY-D.--

COAL REGION

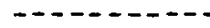

NORTHFRN APPLACHIAN

CFNTRAE APPLACHIAN

SOUTHERN APPLACHIAN

NIDHP.ST

CENTRAL HEST

GUI.F

N. E. GSFAT PLALNS

M. H. TREAT PLAINS

ROCKI.F.S

SOUTHHFST

MORTHUEST

ALASKA

DATA SOIICPS : DOE PSTIMATES DEVPLOPRD IN CONJUNCTION HHITH THF POLLOHING SIIDY: "SYNFUEI.S MARKET POTENT IAL", INST TTUTE DF GAS TECHNOLOGY, ITT CENTPR, CHICAGO.

ILLINOIS. PHEPARE POR THE II. S. FPA IINIER CONPRACT P-03-77-5A10-0, JANIJAY, 1978.

OPPICE HESPONSI BLF : CONGERVATIDN AND RENEHABLE RESOURCES DIVISION, OPFICE OF ENERGY IISE ANAIYSIS. EIA.

TABIE 2: PRODICTION OF HIGH BTU GAS FROH COAL IN 1990 BY COAJ. REGION

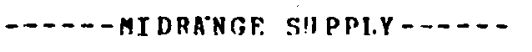

COAL REGION

- - - - - -

NOPTIIRRN APPLACUIAN

CENTRAL APPI,ACIIAN

SOIITIIRRN APBLACHIAN

MIDHR.ST

CENTAAL WRST

GIII.P

N.E. IGREAT PLAINS

H. H. GREAT PLAINS

BOCKI H.S

SOUTHUEST

NORT!IUEST

ALASKA
-- - -HISH SROHPH SIIPLY --- -

\begin{tabular}{|c|c|c|}
\hline \multicolumn{3}{|c|}{ PRODUCTION } \\
\hline PRICE & I. F.V PL & CAPITAL \\
\hline$\$ / B \cap E$ & MBOP, /D & $H \$ / B / D$ \\
\hline & & \\
\hline $\begin{array}{l}21.75 \\
21.75\end{array}$ & $\begin{array}{l}29.57 \\
14.79\end{array}$ & $\begin{array}{l}23.00 \\
23.00\end{array}$ \\
\hline 21.75 & 29.57 & 23.00 \\
\hline & - & \\
\hline $\begin{array}{l}21.75 \\
21.75\end{array}$ & $\begin{array}{l}36.10 \\
65.67\end{array}$ & $\begin{array}{l}23.00 \\
23.00\end{array}$ \\
\hline 1.75 & 36.10 & 23.00 \\
\hline
\end{tabular}

DATA SOIRCES : DDE ESTIMATFS DEVELOPFA IN CONJUNCTION DHITH THP POLLOHING SEUDY:

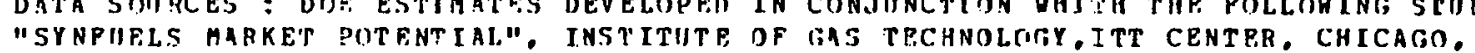

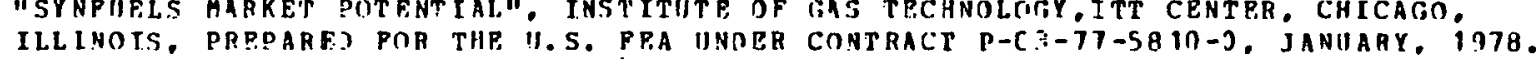

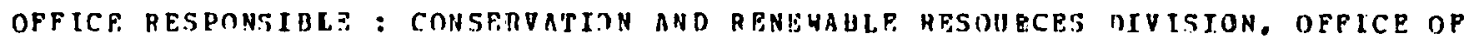
ENFHGY IISE AHALYSIS, EIA. 
TARI.P 3: PRODUCTION OF LOH AN MEOIJM BRO SYNIIETIC GNS FROM COAL IN 1990 WY ITTLITY D?

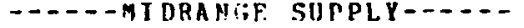

ITILITY REGION

NH-BNG.
NY/NJ
MID-ATL
S.-ATL
MINHFST
S.-RPST
CENTRAL
N-CNTRL
HEST
N. - TEST

PRODUCT TON

\begin{tabular}{|c|c|c|}
\hline $\begin{array}{l}\text { P RICE } \\
\$ / B \cap E\end{array}$ & $\begin{array}{r}\text { TEUEL } \\
\text { MDOP } / D\end{array}$ & $\begin{array}{l}\text { CAPITAL } \\
\text { HS/B/D }\end{array}$ \\
\hline
\end{tabular}

$\begin{array}{rrr}16.82 & 16.50 & 13.00 \\ 16.82 & 9.25 & 13.00 \\ 16.82 & B .25 & 13.00 \\ 23.30 & & 21.20 \\ 23.30 & & 21.20 \\ 16.82 & & 11.00\end{array}$

- - -HIGH GROHTH SUPPIYY - - -

\begin{tabular}{|c|c|c|}
\hline \multicolumn{3}{|c|}{ PROUUCT LON } \\
\hline Pก ICE & I. EVEJ. & CAPITAL \\
\hline $8 / B \cap P$. & MBOE/D & AE / B / D \\
\hline & $\cdots$ & $-\cdots$ \\
\hline $15.9 ?$ & 22.20 & 13.00 \\
\hline $16.8 ?$ & 111.80 & 13.00 \\
\hline 15.82 & 14.80 & 13.00 \\
\hline 16.82 & & 1.3 .00 \\
\hline 16.82 & & 13.00 \\
\hline 16.82 & & 13.00 \\
\hline
\end{tabular}

DATA SOURCES : DOE ESTIMATPS DEVELOPED IN COHJUNCTION HHITH THR POLLOHING STUNY: "SYMPUELS MARKET POTENTIAL". INST ITUTE DF GAS TECHNOLOGY ITT CPNTER. CHICAGO. ILLINOIS. ERFPARED POR THP. U.S. PEA IINDER CONTRACT P-03-77-5810-0, JANIARY. 1978.

OPPICE RESPONSIBLP : CONSFAVATION AND RFNEHALE RPSOURCES DIVISION, OPFICE OF ENERGY IRSE ANALYSIS. EIA.

TABLF 4: PRODIICTION OP SYNTIITTC FAS FROM SOHRCES OTHPA THAN COAL IN 1990 BY DEMAND REGION

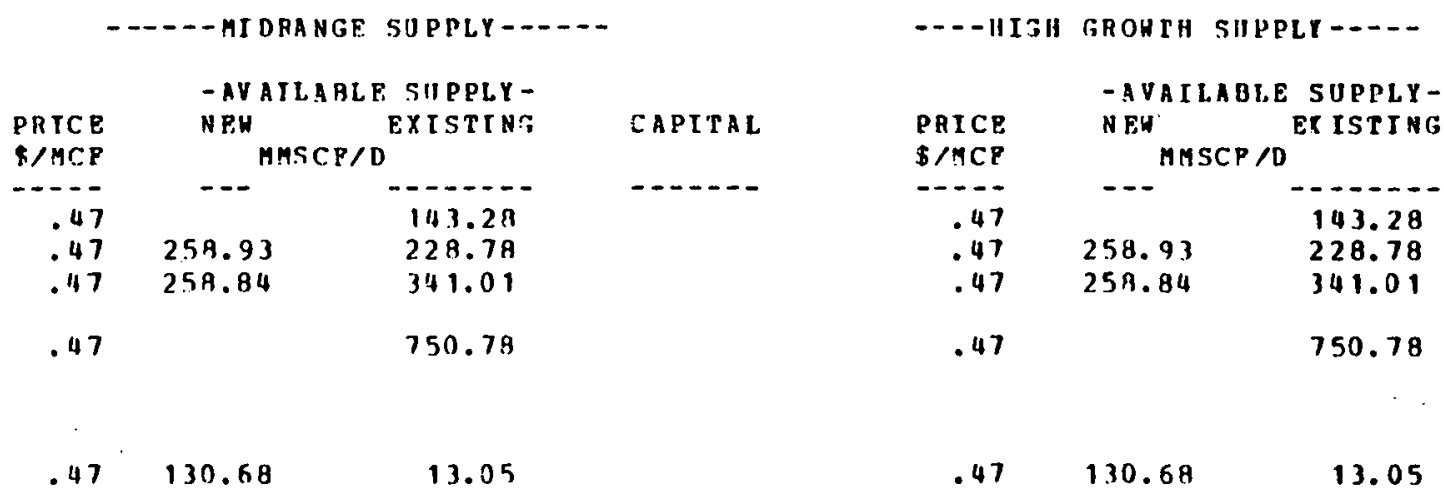

DATA SOURCES : DOF, RSTIMATES DEVELOPED IN CON.JUNCTION HHITH THP POLLOHING STUDY: "SYNPUELS MARRET POTENTIAL". INSTITITP OP GAS TECHNOLOGY ITT CENTBR, CHICAGO JILINOIS, PRFPAREO POR THP U.S. PEA IINDER CONTRACT P-03-77-5B10-0. JANJARY, i 978. OPFICE RESPONSIBLT : CONSERVATION AND RENEHABLF RESOURCES DIVISION, OPFICE OP ENERTY ESE ANALYSIS. FIA. 
SHALE IAUTT DATA

1978 A DAINISTRATOA'S ANNIJL REPORT

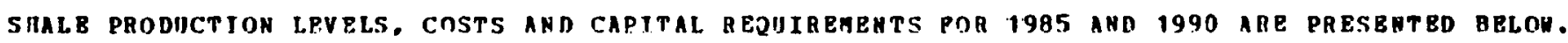
ALL SBALP PTGURES ARP. EXIGEHOIS INPUTS TO THP. MONgL.

TABLE 1: SHALE INDIT DATA

PRONICTION LEVRL

$\cos \mathrm{r}$

CAPITAL REQUIREAENT

(THOISAND BARRELS/DAY)

$(1975$ \$ /BARREL)

THOIJAND 1975 S/BAGRPL/DAY

1985

47.0

15.29

11.3

1990

164.8

15.00

12.0

DATA SOLRCES : DOE RSTIGATRS DEVELOPED IN CONJINCTION NIITH TIIR POLLONING STIIDY: "SYRPUPLS GARKET POTENTIAL". IHSTITUTE OP GAS TPCHNOI.OFY TTR TCENTER, CHICAGO, ILLINOIS. PREPAPED FOR THE U.S. PEA UNDER CONTRACT P-03-77-5810-3, JANUARY 1973.

DFPICE RESPOHSIULE : CONSERVATION AMD RENRHABLE RESTHRCPS DITLSION, OPPICE OP ENERGY ISE ANAI.YSIS, EIA. 
TABLF 1 : GEOTERAAT.

\section{SOUTHHAST
N. -CPNTRAL HEST}

NORTIUEST

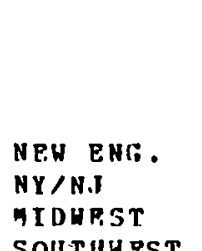

SOUTHH EST

\section{SOUTHMEST}

N. - CENTRAL

Y FST

Son

SOUTHHFST
N. - CENTRAL N. - CPN
HEST

\begin{tabular}{|c|c|c|}
\hline $\begin{array}{l}\text { OUANTITY } \\
\text { (KYRGH/DAY) }\end{array}$ & $\begin{array}{c}\text { PR.TCE } \\
\text { (MII.T.S/K HH) }\end{array}$ & $\begin{array}{l}\text { CAPITAL } \\
\text { (\$/KUII) }\end{array}$ \\
\hline 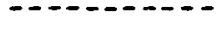 & 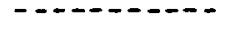 & $--\infty-\cdots$ \\
\hline
\end{tabular}

55. 20

19.30

TABLP. 2 : HIND

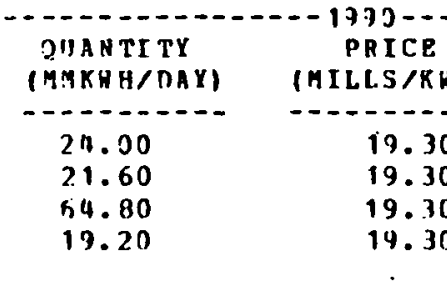

\begin{tabular}{cc} 
OIINTITY & PRICE \\
(HAKUILDAY) & (AILLS/KHH) \\
\hline 1.72 & 27.00 \\
1.72 & 27.00 \\
1.72 & 27.00 \\
1.72 & 27.00
\end{tabular}

CAPITAL.

(\$ $\$$ K 4 II)

29.17

29.17

29.17

29.17

TABLE $3=$

\begin{tabular}{|c|}
\hline $\begin{array}{l}\text { DIANTETY } \\
\text { ( IMRUH/DAY) }\end{array}$ \\
\hline $\begin{array}{l}13.72 \\
13.72 \\
13.72 \\
13.72\end{array}$ \\
\hline
\end{tabular}

$1990-=$

CAPITAL

PRICE
(HILLS/KWH)

(\$/KH H)

\begin{tabular}{|c|c|c|}
\hline $\begin{array}{l}\text { QHANTTTY } \\
\text { (HAKH }\end{array}$ & $\begin{array}{c}\text { PRICE } \\
\text { (MILLS/KYH) }\end{array}$ & $\begin{array}{l}\text { CAPITAC } \\
\text { (B/KWII) }\end{array}$ \\
\hline$\cdots \cdots$ & - - - - - - - & $----\cdots$ \\
\hline $\begin{array}{l}.46 \\
.46 \\
.46\end{array}$ & $\begin{array}{l}114.00 \\
114.00 \\
114.00\end{array}$ & $\begin{array}{l}125.00 \\
125.00 \\
125.00 \\
\text { TABLP I }\end{array}$ \\
\hline OUANTTY & PRICE & CAPITAL \\
\hline 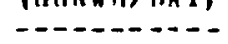 & 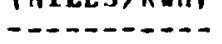 & \\
\hline
\end{tabular}

27.08

27.08

27.08

27.08

SOLAR THERHAL

25.00

25.00

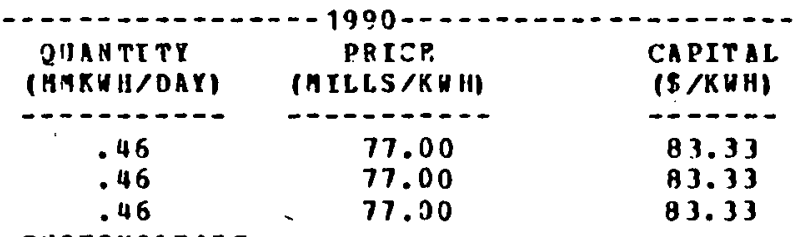

SULAR PHOTOVOI.TAIC

\begin{tabular}{|c|c|c|}
\hline $\begin{array}{l}\text { DOUANTITY } \\
\text { (TYKH /DAY) }\end{array}$ & $\begin{array}{c}\text { PRICE } \\
\text { (MTLLS/KHH) }\end{array}$ & $\begin{array}{l}\text { CAPLTAL } \\
\text { (E/KUII) }\end{array}$ \\
\hline $\begin{array}{l}6.86 \\
6.85 \\
6.86\end{array}$ & $\begin{array}{l}57.00 \\
57.00 \\
57.00\end{array}$ & $\begin{array}{l}62.50 \\
62.50 \\
62.50\end{array}$ \\
\hline
\end{tabular}

DATA SOIIRCPS : SOLAR THERMAL : ORIGINAL DATA BASFO ON 1985 PROJECTIONS OP ERDA-49. DRPINITION REPOHT DF THE NATIONAL SOLAR ENRHGY RBSEALCH, DEVELOPAPNT AND DPRONSTRAT ION PRIBRAH, JUNE 1975. PRESBNT VALIIES PREPARED BY DOE.

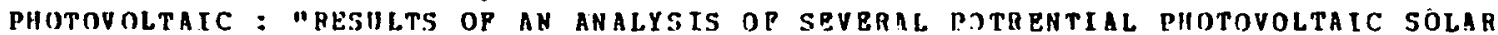

SYSTFM INTRODUCTION SCEHARIOS, MARTIN HOLP, DOR. JAHUARY, 1978

HIND : MAIND BNEAGY OSP IN THE UNITED STATES TO THP YPAR ?000". MARSHALI, F.

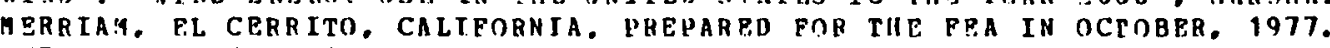

THE ENTRLES IN THE TABLE ARP. ESTIMATES DERTVER PROM TUIS SOURCP

SOLAR HEATING : "SOLAR HFATING ANR COOJ.ING DF BIILDINGS (SHACOB) MONEL, ARThIJR

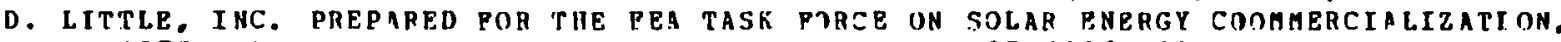

SP.PT. 1977. HASIIINGTON, D.C. FEA CONTRACT HIMBPR CR-05-70065-00.

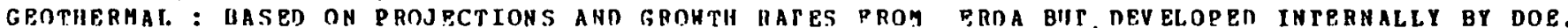

OFPTCE AESPONSIBLP: : CONSERVATION ANI) RBNPGABLE RPSOURCES DIVISION, OEPICP.

DF PR RHGY ISE ANAYSIS. PIA. 
The following tables give the transportation standard table inputs to the PIES Integrating Model as they existed for the Administrator's Annual Report of 1978. Transportation networks are described for coal, natural gas, crude oil and petroleum products. Links are also given for the transmission of electricity within DOE regions and the transportation of nuclear fuel.

Most data sources are given in the text preceding each group of tables. Additional sources and information are contained in footnotes. Note that data sources given in 1973 dollars have been converted to 1975 dollars by the conversion factor 1.212, where necessary. 
TRANSPORTAT ION DATA

INPUTS TO TIR PIPS I ATEGAATINA MODEL

1978 ANHIJAL ADMINISTRATDRS REPORT

THE TRANSPORTATIJN SUGMODEL IN PIES CONGISTS OP SPVERAL I NDEPENDENT NETUORKS TAAT IRE OISTINGIISHED BY THE HATERIAL. TRANSPORTEN AND THE MODE OP TRANSPORTATLON. THE MOST IMPORTANT OF THESE NETWORKS ARE LISTED BPLOK.

\begin{tabular}{ll} 
MATERIAL & MODE \\
\hline COAL & PATL AND BARGE \\
NATIURL GAS & PIPELINE \\
CRUDE OIL & PIPELINE \\
CRIIDE OIL & BARGE AHD TANKER \\
PETROLPUH PRODUCTS & PIPELINE \\
PPTROLEOH PRODUCTS & BARGE ARD TANKER
\end{tabular}

IN ADDITION. THE MODEL CONTAINS LENKS YOR THE TRANSMISSION OP ELECTRICITY ITHIN DOE RESIONS AND LINKS POR THE TRANSPORTATION OP NUCLEAR PUEL. MOST OP THE TRANSPORTATION NETHORKS SHIP DIRECTLY PROM ORIGIN TD PINAL DESTINATION. ONLY ALASKAN AND IMPORTPD CRIJDE OIL ARP TRANSHIPEED TILOUGH INTERMEDIATR LOLATIONS.

BESIDPS ORIGIN AND -DESTINATION, THE ONLY DATA ASSOCIATED HITH MOST LINKS IS UNIT COST. HOWEVPR. NATIRAL GAS LINKS ALSO INCLIIDE A LOSS PACTOR TO ACCOINT FOB PURL CONSUMPTION. THP. LINR RETRESENTING TIE TAPS PIPPILNE IS THE ONLY CAPACITATED LI NK IN THE MODEL.

ALL CNSTS ARE REPORTED IN 1975 DOLLARS. 
THE COAL TRANSPORTATION HETHORK IS DESCRIBPD IU TABLES 1 AND 2. DESAND POR COAL IS DETERAINED BY THE PIRS DEAAHD AND UT ILITIR.S HODEL.S POR RACII DOP RECITON. HOWEV?R, SINCF, THE DRLIV RRPD COST OP COAL IT IS IMPORTANT THAT ORIGINS AND DESTINATIONS BE SIPFICIPNTLY DISAGGREGATE TO ALLOH ACCORATE COSTTNG. POR THIS REASOH, GITH IUST THO BXCEPTIOHS. COAL DEAAND IN EACH DOE RPGION IS SPLIT AMONG REPRESENTATIVE CTTIES IN THE HEGION AND LIAKS AHE PROUIDED PROA TIIE COAL REGIONS TO THESP CITIES. THE DRAAND SPLITTING IS DONP IN PIXED PAOPORTIONS BASED UPON POPULATIONS OP STANTARD MPTROPOLT TAN AREAS. THE TRANSPORTATION COSTS ARE CALCILATPD POR THE CHEAPEST PATH ALONG AVAILABLE RAIL ANO BARGE ROUTES FROM CENTROIDS IN THE COAL REGIONS TO THE DESTINATION CITIPIS. RAIL COSTS ASSUHE A PIXED CHARTE DF \$1.60/SHORP TON AHO A MILEAGE CHARGE OP B.0094/TON-HILB IPROA LIKPAR RPGRESSION ANALYSIS OP 100 CAR UN TT TRAIN OPEHATIONS USIHS DATA FROA TIIE ICC REPORT - IHVESTIGATION OP PREIGHT RATE STRUCTORE -COAL**I. THESE CHARSES ARE FOR UNIT TRAIN OPERATIONS AND APPLY TO COAL MOVENENTS TO THE EL ECTRIC UTILITIES ONLY. OTHER CJAL CONSUMERS PAY AN ADDITIONAL 82.50 PER SHORT TON TS ACCONHT FOR THE IIGHER SINGLE CAR RATES. THE BARGP COSTS ARP TAKEN FRAM KEARNEY'S - DOAESTIC WATERBOHNE SHIPPING ANALYSIS' AND INPLATED BY 3.38 (IN REAL TPRAS) TO ACCOINT FOR INCREASED PIJPL COSTS. AAIL-TO-BARGE-TO-RAIL TRANSPER CHARGE OF \$. 45/5HORT TON IS INCLIDED WIIERR APPROPRIATE. * *

THP. THO EXCBPTIOHS ARP. ZOAL BORNED IN MINEHOETH PLANTS AND LIGNTE. IN TRESE THO INSTANCRS. LINKS RON DIBPCTIY TO THE DOE RRGIONS. COAI. DURNED IN MENEAOITTI PLANTS IS CHARGED A NOMINAL COST OF 5.0 I/SHORT TON. THE LINKS PROA THE FIILP TO THE CENTRAL STATES AND PROA THE NORTHEAST GRRAT PIAIHS TO TIIE MIDUEST AND NORTH CENTRAL STATES ARE POR SEIPPING IIGNITE OVPR SHORT DISTANCES. THE LINK TO THE MIDKEST IS ADDITIONALLY EXCEPTIOHAL IN THAT IT INCI.UDES A CHARGE POR TRANSPORTING LIGNITE FO TIIE DAKOTAS PLUS A CHARGE POR TKANSMITT ING ELECTRICITY PROA TIIERE TO MINHESOIA. THIS IS THE ONLY I NSTANCE OF INTER-REGIONAL TRANSAISSION OP RLBCTRICITY IN THE MODEL.

- icc appoat number 345

* THERE IS a \$.20/TON HANDLING CHARge to TRANSPER PROA BARGE TO RAIL AND A $\$ .25 /$ TON CHARGE TO TRANSPER PROM HARGE TO RAJL AND A $\$ .25 / T O N$ CHARGE TO TRANSPER PROA 
TABLE 1

ATION COSTS

(1975 DOLLARS PPI SHORT TON)

DESTINATION

\begin{tabular}{|c|c|c|c|c|c|c|c|c|c|c|c|}
\hline COAL REGION & $\begin{array}{l}\text { BOSTON } \\
\text { - }\end{array}$ & $\begin{array}{l}\text { NEW } \\
\text { YORK }\end{array}$ & $\begin{array}{l}\text { BALT. } \\
\text { PHIL. }\end{array}$ & $\begin{array}{l}\text { PI'TTS - } \\
\text { BIS RT;H }\end{array}$ & $\begin{array}{l}\text { MIAHI } \\
\text {. }\end{array}$ & $\begin{array}{l}\text { ATLANTA } \\
\text { - }\end{array}$ & $\begin{array}{l}\text { CINCI- } \\
\text { NATTI, S }\end{array}$ & $\begin{array}{l}\text { CINCI - } \\
\text { NATTI, N }\end{array}$ & $\begin{array}{l}\text { nERR I } \mathrm{T} \\
\text { - }\end{array}$ & $\begin{array}{l}\text { CHICAGO } \\
\text {. }\end{array}$ & $\begin{array}{l}\text { Sr. } \\
\text { Lriis IS, B }\end{array}$ \\
\hline $\begin{array}{l}\text { N. APPLACH. } \\
\text { CEN. APPLACH, } \\
\text { S. ADPLACH. } \\
\text { MIDHEST } \\
\text { CEN, HEST } \\
\text { GULP }\end{array}$ & $\begin{array}{r}9.30 \\
9.24 \\
13.67 \\
12.65 \\
15.90 \\
19.38\end{array}$ & $\begin{array}{r}6.89 \\
6.83 \\
11.26 \\
10.24 \\
13.49 \\
16.97\end{array}$ & $\begin{array}{r}5.14 \\
5.08 \\
9.51 \\
8.49 \\
11.74 \\
15.22\end{array}$ & $\begin{array}{r}1.67 \\
6.91 \\
10.73 \\
5.97 \\
9.18 \\
13.11\end{array}$ & $\begin{array}{r}14.77 \\
11.72 \\
9.89 \\
11.66 \\
11.27 \\
14.55\end{array}$ & $\begin{array}{l}8.04 \\
4.99 \\
3.16 \\
7.10 \\
6.95 \\
8.87\end{array}$ & $\begin{array}{r}3.44 \\
4.00 \\
7.76 \\
3.02 \\
6.27 \\
10.80\end{array}$ & $\begin{array}{r}3.44 \\
4.00 \\
7.76 \\
3.02 \\
5.27 \\
10.80\end{array}$ & $\begin{array}{r}4.47 \\
3.31 \\
9.63 \\
6.25 \\
7.34 \\
11.87\end{array}$ & $\begin{array}{l}6.08 \\
5.76 \\
7.08 \\
3.70 \\
4.79 \\
9.32\end{array}$ & $\begin{array}{l}6.33 \\
4.07 \\
5.39 \\
2.01 \\
3.10 \\
7.63\end{array}$ \\
\hline $\begin{array}{l}\text { NE GREAT PL. } \\
\text { NH GRBAT PI.. } \\
\text { ROCKIES } \\
\text { SOUTHUEST } \\
\text { NORTHUEST } \\
\text { ALASKA }\end{array}$ & $\begin{array}{l}21.14 \\
24.55 \\
24.61 \\
27.71 \\
33.88 \\
41.79\end{array}$ & $\begin{array}{l}18.73 \\
22.14 \\
22.20 \\
25.30 \\
31.47 \\
39.38\end{array}$ & $\begin{array}{l}17.11 \\
20.52 \\
20.45 \\
23.55 \\
29.85 \\
37.76\end{array}$ & $\begin{array}{l}13.75 \\
17.16 \\
17.89 \\
21.29 \\
26.49 \\
34.40\end{array}$ & $\begin{array}{l}19.64 \\
23.05 \\
22.98 \\
23.13 \\
32.39 \\
40.29\end{array}$ & $\begin{array}{l}14.2 .2 \\
17.63 \\
17.56 \\
17.20 \\
26.96 \\
34.87\end{array}$ & $\begin{array}{l}11.64 \\
15.05 \\
14.98 \\
18.37 \\
24.38 \\
32.29\end{array}$ & $\begin{array}{l}11.64 \\
15.35 \\
14.98 \\
18.37 \\
24.38 \\
32.29\end{array}$ & $\begin{array}{l}11.91 \\
15.32 \\
16.55 \\
19.44 \\
24.65 \\
32.56\end{array}$ & $\begin{array}{l}9.36 \\
12.77 \\
13.50 \\
16.89 \\
22.10 \\
30.01\end{array}$ & $\begin{array}{l}8.47 \\
11.88 \\
11.81 \\
15.20 \\
21.21 \\
29.12\end{array}$ \\
\hline
\end{tabular}

DESTINATION

COAL REGION

N. APPLACH.

CEN. APPLACH

S. APPLACH.

MIDHEST

CEN. NEST

NE GREAT PL

NG GREAT PL.

ROCKIRS

SOUTHWEST

NORTHWEST

ALASKA

\begin{tabular}{ll} 
ST. PAULI & NEH \\
HINN. & ORLEANS \\
\hline 9.80 & 8.00 \\
8.40 & 6.05 \\
9.72 & 3.35 \\
6.34 & 2.47 \\
6.20 & 5.08 \\
10.48 & 5.36 \\
5.64 & 10.45 \\
9.05 & 13.86 \\
12.50 & 13.79 \\
15.89 & 15.34 \\
19.38 & 23.19 \\
26.29 & 31.10
\end{tabular}

\begin{tabular}{l} 
HOHSTON \\
\hline \\
\hline 8.28 \\
8.22 \\
6.76 \\
5.88 \\
8.49 \\
1.95 \\
13.86 \\
14.99 \\
14.50 \\
11.93 \\
26.50 \\
74.51
\end{tabular}

\begin{tabular}{l} 
DALRAS \\
\hline \\
\hline 10.76 \\
10.70 \\
9.24 \\
8.35 \\
6.54 \\
1.12 \\
15.00 \\
12.51 \\
12.02 \\
9.45 \\
24.42 \\
32.33
\end{tabular}

Str.

Loll Is.

\begin{tabular}{l} 
KAHSAS \\
\hline \\
\hline 0.52 \\
6.26 \\
7.58 \\
4.20 \\
1.69 \\
5.97 \\
10.15 \\
10.64 \\
10.15 \\
13.54 \\
22.55 \\
311.46
\end{tabular}

DENVER

Los

ANGELE;

SAN

SEATTLE

14.-. - - -

6.33

14.49

23.70

2月. 11

16.

4.07

13.55

23.64
22.18

5.39
2.01

10.17

21.30

$7.66 \quad 20.26$

26.59

.4 .80

3.10

8.96

20.26

24.11

21.60

19.25

$$
\begin{array}{r}
7.63 \\
H .117
\end{array}
$$

H. 117
11.89

11.88
11.81

9.99

14.84
23.61

$4.67 \quad 11.40$

$4.18 \quad 12.68$

15.20

7.57

12.68
12.78

22.74

18.61

13.06

17. 19

24.17

24.49

22.76

18.35

?6. 12

22.74

$? 2.60$

$? 4.73$

14.28

14.26

15.55

$? 3.34$
1.98

9.89

DOE REGION

COAL RPGION

N. APPLACH.

CEN. ARPI.ACH. MIDURST

CEN. HEST

GiJle

NE GREAT PL.

NW GREAT PL.

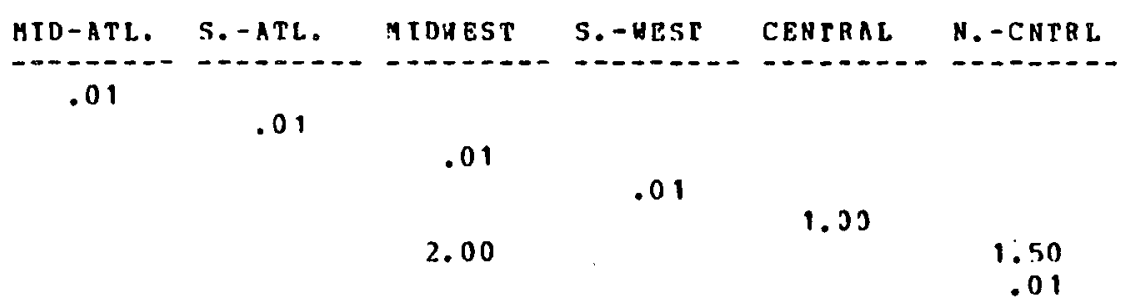




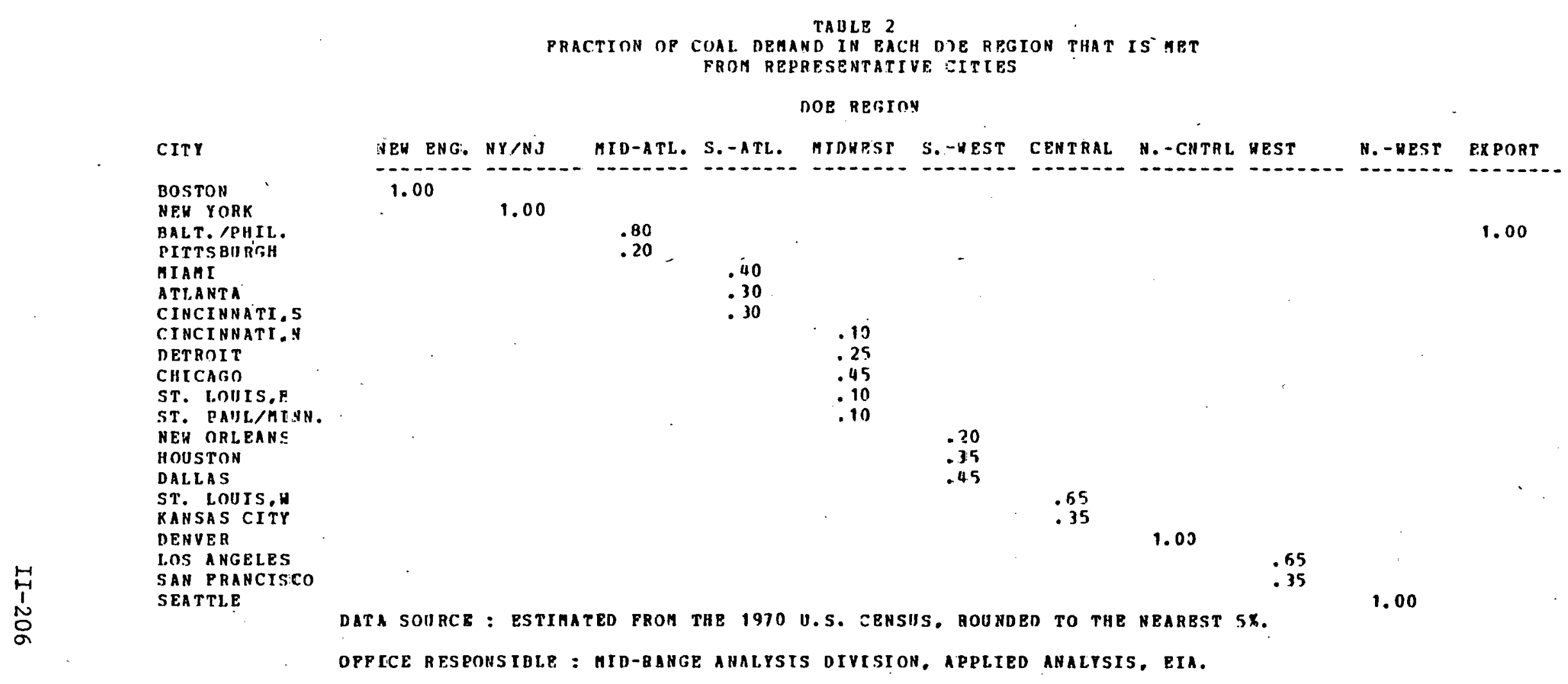


TARLES 3, 4 AND 5 DESCRIDE THE NATURAL (IAS TRANSTORTAT ION NETHORK. VOLUHES ARE ADJISTEO TO A STANDARD IIEAT VALUR OP 1032 BTH/CP. UNIT COSTS ARE THE BATIO OF TOTAL TRANSPORTAT TON COSTS, EXCLUDING FIIEL COSTS, TO VOLUH? OP INPIT. LINKS CORRESPONDING TO EXISTING PIPPI.IULS ARE COSTED AT $\$ .033 / \mathrm{Bz}$ PEA 100 MILES (A UEIGHTED AVERACE OP TRANSPORT COSTS POR RIGHT LARGE GAS PIPELINRS). USIN(; SHOTT LINE MILFAGE PROA CPNTROTDS OF NPC REGIONS TO CENTROIDS OP DOF RESIONS. OTHER LINKS ARP. CNSTED AT \$.04/HCF PER 100 MILES (NOTIONAL). LOSSES ARE CALCILATED AT 48 PRR 1000 AILES IPROA NORTHERN BORDER PIPELI NP PROPOSAL*I.

THP LINKS FROH THE NORTI SLOPB MODEL THE ALCAN PIPELINE.

ASSUAI NG A 30 CONSTRICTION COST OVERRIJ, HITH THOSE LINKS TD TIIE EAST COAST INTENDRD TO REPRESPRT DELIVERY BY DISPLACENENT THE COST OF SERVICE ESTIMATPS ARE TAKEN FROM THE FPC REPORT

- RRCOH TATION SYSTEHS (EXHIBIT IV-5). AlgMBNTED BY 30\% AND AD.JOSTE? TO GIVE REGTONAL COST RSTIAATES.

IMPORTED LNG AND CANADIAN GAS ARE ASSIGNED ZERO TRANSPORTATION CHARCES SINCE THFIR PUBCHASE PRICE, AS REPORTED IN THE IAPOAT TABLES. IS ALREADY THE DELIVERED CITT-GATE PRICE. THE

TRANSPOHTATION CHARGFS SHOKN FOR MEXICAN BAS BEGIN AT THE BORDER. BLANK ENTRY MEANS TIE CORRESPONDING LINK IS NOT PROVIOED IN THE MODEI.

I NTRASTATE Gas, NOT COVERED AY THE TABLES. IS TRANSPORTED A1 A COST OP $\$ .10 / M C P$ HITH 15 COSS (NOTIONAI.).

THE COSTS AND LOSS FACTORS IN THESE TAULES ALSO APPLY TO SYNTHETIC GAS MADE FROM COAL. SYNTIETIC GAS FROA COAL IS PRODIICEN IN COAL REGIONS. THP CORRESPONDENCE BETHEEN COAL REGIONS AND NPC REGIONS GIVEN BELOH IS OSED TO DETERMINE HHIOH COSTS AND LOSS PACTORS APPI.Y.

\begin{tabular}{l} 
COAL AEGION \\
\hline N. APPALACHIA \\
CEN. APPALACHIA \\
S. APPALACHIA \\
MIDHEST. \\
CENTRAL HEST \\
GILF \\
NE GREAT PLAINS \\
NH GRFAT PLAINS \\
ROCKIES \\
SOUTHEST \\
NORTHHEST \\
ALASKA
\end{tabular}

\begin{tabular}{l} 
NPC REGION \\
\hline APPALACHIA \\
APPALACHIA \\
HEST GULP BASIH \\
MI. BASIN /INTER. \\
MIDCONTINENT \\
HEST GIILF BASIH \\
EAST ROCKIES \\
EAST ROCKIES \\
HEST ROCKIES \\
WEST ROCKIPS \\
PACIFIC COAST \\
SOITH ALASKA
\end{tabular}

* "TIIE ALASKAN OIL DISPOSITION STIID". A REPORT TO FFA RPGION 9. SAN PRANCISCO. AUTROR, ASTL 3ERZPA OP THANSPGATATION AND ENERGY RESEARCH ASSOCIATES (TERA). IINUBL ISHPD. 1976 
TABLE 3

TRANSPORTATION COSTS OP DOMESTIC NATIRAL GAS

11975 DOI.LAIS PER THOUSAND CIIBIC FEETI

DOE REGION

NPC 3BRIOH

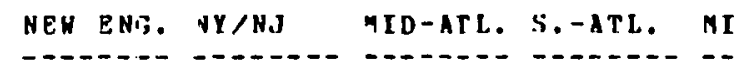

PAC. COAST

PAC. OCEAN

R. ROCKIES

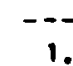

P. ROCKIES

1.

.41

$1.3 \%$

IOWFST

S. - HEST CBI

N. - CNTAL WEST

H. - XES T

E. ROCKIF,S N. MEX, 1.0

H. TIF BASIN MXX. 1.

H. GILF BASIN

GIII.P OF MEX.

IIDCONT.

MI. FASIN, INT.

APPLACHIA

ATL. COAST

ATL. OCEAN

NORTH SIOPE (1985)

NORTY SLOPE (1990)

1.48

1.33

1.19

1.01

. 8 ?

.99

.65

.16

.50

079

.73

.41

. .55

.25

.99

.85

.76

- 59

.52

.57

.48
.38

.59

.60

.44

.23

.50

.34

.40

.46

.35

.34
.27

.45
.50

.64
.79

.20

.33

$.05 \quad .35 \quad .23$

.51

.37

.23

.51

4.

1.77

.32

.30

.63

SOUTH ALASKA (1985)

1.73

.29

1.68

1.56

.04

.31

.56

.60

$1.77 \quad 1.73$

1. 58

1.56

1.56

1.44

1.44

.69

.72
1.59

1.59

1.59

.94

.9 A

1.49
1.48

.33
.55
.53

.14
.53

.53

$.6 \%$

.54

1.0. 1.26

$1.19 \quad 1.38$

1.76
1.07

$1.27 \quad 1.34$

$1.42 \quad 1.46$

$1.49 \quad 1.46$

1.45

SOITH ALASKA (1990)

DATA SOURCE : "INPTTS TO TILE PROJECT INORPENDENCE EVALUATION SYSTEM INT BGRATION MODEL FOR THE TRANSPORT OP ENERGY MATERIALS", VOLUHE II, PREPARED POR THE TASR

PORCE REPORT. FFDPRAI. ENERGT ADMINISTAATION PROJECT INDEPENDENCE BLUEPRINT

PINAL. INDER THE DIRECTION OF THE DEFARTAENT OP TAANSORTATION, NOVEMBR, 1974.

TABLE VIII-14, PG. VIJI-17.

OPYICP RESPONSIBLE : MID-RANGE ANALYSIS DIVISIOH, APPLIED ANALYSIJ, EIA.

TABLE 4

TRANSPORTATION ZOSTS POR IMPORTED NATUBAL GAS

(1975 DOLLARS PER THOISAND CHBIC PEETI

DOE REGION

POREIGN REGION

NEH ENG, WE/N.I

ATL. HIUWRT

- HeST

CFNTRAL N. -CNTRL HEST

N. - MRST

LANDED EAST COAST

LANDED HEST COAST

CANADA

.00

.00

.00

.00

$\begin{array}{ll}.00 & .00 \\ .89 & .78\end{array}$

.71

.50

.00
.51

.30

.47

.00
.64

.00

.00

.00
1.26

DATA SOURCES : APMCRANDUM POR THE RECORU FROA RICHARD THRASHER, DID-RANGE AHALYSIS DIVISIOH. OPFTCE OP INTEGEATIV ANAI.YSIS, EIA. SUBJECT : IMPLRMENTATION OP MEXICAN GAS:

DATED 10/26/77. NOTE : A \$.02/ACF CHARTE HAS BFF,N ADDED TO THE TBANSPORTATION COSTS

TO ACCOUNT PDR THE PACT THAT THE LARGEST NATIRAL GAS COMPANIES B!IY DIRECTLY PROM

GAS ?IPELINES AND DO NOT PAY THE CITY-GATE CHARGR.

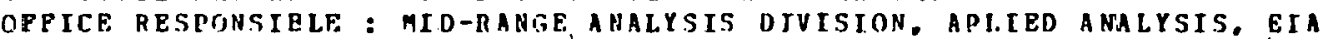


TABI. F. 5

LOSS FACTORS POR NATURAL GAS

(PERCENT LOST)

DOP. REGION

\begin{tabular}{|c|c|c|c|c|c|c|c|c|c|c|}
\hline MPC REGTON & N FH ENG. & $\begin{array}{l}\text { NY /NJ } \\
-\end{array}$ & FID-ATL. & $\begin{array}{l}\text { S. - A TL. } \\
-\end{array}$ & MIDHEST & 5. - RPST & CENTAAL & N!. - CNTRI. & UEST & N. - KeST \\
\hline PAC. COAST & 13 & 13 & 12 & $\begin{array}{l}11 \\
10\end{array}$ & $\begin{array}{r}9 \\
10\end{array}$ & $\begin{array}{l}8 \\
7\end{array}$ & $\begin{array}{l}\mathbf{8} \\
\mathbf{8}\end{array}$ & $\begin{array}{l}6 \\
6\end{array}$ & $\begin{array}{l}1 \\
2\end{array}$ & $\begin{array}{l}4 \\
6\end{array}$ \\
\hline PAC. OCEAN & 14 & 13 & 12 & 10 & 10 & 7 & $\begin{array}{l}8 \\
5\end{array}$ & 6 & 2 & 6 \\
\hline ה. ROCKIPS & 10 & 10 & 9 & 7 & 6 & 4 & 5 & 2 & 5 & 5 \\
\hline E. ROCKIPS & 9 & 9 & 9 & 8 & 5 & 5 & 4 & 2 & 5 & $\mathbf{5}$ \\
\hline M. TFXXS/R. N. MEX. & 9 & 8 & H & 5 & 5 & 2 & 4 & 5 & 7 & 10 \\
\hline W. GULP BASIN & 9 & 8 & 7 & 4 & 6 & 2 & 4 & 5 & 9 & 11 \\
\hline GULP OP MEX. & 7 & 6 & 5 & -2 & 4 & 2 & 4 & 6 & 10 & 12 \\
\hline nIDCONT. & 7 & 7 & 6 & $\|$ & 3 & 2 & 1 & 3 & A & 9 \\
\hline MI. BASIR. INT. & 5 & 4 & 4 & 3 & 2 & 4 & 2 & 5 & 10 & 10 \\
\hline APPLACHIA & 4 & 3 & 2 & $\mathbf{3}$ & 2 & 6 & 4 & 6 & 12 & 13 \\
\hline ATL. COAST & 6 & 5 & 5 & 2 & 5 & 5 & 6 & 9 & 13 & 14 \\
\hline ATL. OCBAN & 3 & 2 & 1 & 3 & 4 & 7 & 6 & $\boldsymbol{\theta}$ & 13 & 13 \\
\hline NORTH SLOPE & 10 & 10 & 9 & 6 & 8 & 4 & 7 & 6 & 5 & 5 \\
\hline SOUTH ALASKA & & & & & & & & & 1.1 & \\
\hline
\end{tabular}

$\stackrel{H}{1}$
N
0 
THE TAANSPORTATIOH NETWURK POR CRUDE OIL IS PDRTRAYED IN TABLES 6 THROUGH 9. FOR LINKS EORRESPONDING TO EXISTING

PIPELINES. ICC TARIPFS HAVE BEEN OSED TO ESTABLISH TRANSPORTATION COSTS. GATHERING CHARAFS. ALSO BASED ON ICC TARIPFS. ARE INCLODED. OTHR.R PIPELTNE LINKS ARE COSTED AT $\$ .06 / B$ L PEA 100 MILES INOTIONALI PLOS GATHFRTNG CHARGES. BARGE COSTS ARE FROM TIIF PFA REPORT 'INPUTS TO TIIF PROJECT INDEPENDENCE EVALIIATION SZSTEA INTEGRATION MODFL FOR THE TRANSPORT OF BNERGY MATERIALS, VOLHME II*I. TANKER COSTS ARE CALCULATED USING THP FORMILA

(TURN) * (PORT) + (MILEA(BE/SPRED) * (SEA)

I) NIT $\cos T=2 * \ldots$

(PAYLOAD) * (SCALE)

YHERP

TIIRN= TURNAROUND TIME IN PORT IUOURS\}.

PORT = COST/HOIR DP STAYING IN PORT

MILEAGE = MILEAGE ONE HAY (HAUTICAI. HILES) .

SPEED = SPEEN IN KNOTS.

$S E A=\operatorname{COST} / H O U R$ AT SEA.

PAYLOAD = CARGO CAPACITY (TONS). AND

SCALE= NOHBEP OP BARRELS OP CRIIIE PEB TOW.

TIIP FACTOR 2 IS OIR TO THF ASSUSPTION THAT THE TANKER MAKES THE RPTIRN TRIP EMPTY. DATA FOR IISF IN THIS PORIILA ÁRE TAKEM FRDA THE MARITIME ADATISTRATION RPPORT I BSTIMATED VRSSEL OPERATING PXPENSES- 1976 .

THR \$3.40/BEL LISTED POR THE TRANS-ALASKAN PIPELINP. SYSTEM IS THE TARIFP TIIE SHIPPERS ROULD HAVB TO CHARGE AT A VOLIIAE OP 2 MMBBL/D TO RECEIVF, THE SAMP RETURN AS TIEY YOILL UNDER THE EXISTING AVRRAGE TARIPF OP \$5.60/BAL AT A VOLUAE OP 1. K MBBBL/D. COSTS POR THP NORTHERA TIER AND EL PASO-SONIO ARE TAKRN FRON A STIDY BY TRANSPORTATION AND ENERGY RESEARCH ASSOCIATES ON THE DISPOSITION OP NORTH SLOPP, OIL**.

CRIDE OIL SHIPPED PROM POREIGN REGIONS IS ASSIGNEO ZERO TRANSPORTATION COSTS, SINCE ACTUAL TRANBPORTATION COSTS ARE ALREADY INCLUDED IN THE PISRCIIASP COST, AS PPPORTED EN THE IMPORT TABIES. TILE COSTS OF TRANSHIPPING FORFIGN CRIDP PROM THE GULF COAST (PAD 3) INLAND ARE ICC PIPELINE TARIBES. THE LINK FROA PAD 1A TO PAD IB IS POA CRUDE IMPORTS ACTUALLY LANDING IN PAD 1 B. A GLANK BNTRY MEAHS THE CORRESPOADING LINK IS NOT ATATLABLF, IN TIIP, MODEL.

THE COSTS IN THESE TABLES ALSO APPLY TO SYNTHETIC JIL

MADF FROM COAL. THE CORBESPONDENCE BETHEEN COAL REGIONS ANI) NPC REGIONS GIVEN AROVE FOR SYNTHETIC GAS IS USED TO DETERMINE WIICH COSTS APPI.Y.

* APPI.IES TC. PETROLFIHA PRODIICTS ONLY.

* "ThF alaskan OIL DISPOSITION STIDY", a REPORT to PFarejion 9. SAN PRANCISCO. AUTHOR, ASIL GERLEN OF TRANSPORTATION AND PRERGY RESEARCH ASSOCTATES ITERAI. UNPIJBLISHED. 1976 
T'ABLP, 6

COSTS OF TRANSPORTIHC CRIITE OIL BY PIPELINE 11975 DOLLARS PER BARRELI

REPINEITY REGION

\begin{tabular}{|c|c|c|c|c|c|c|c|}
\hline NPC REGION & PAD IA & PAD 1B & PAD 2A & PAD ?D & PAD 3 & PAD 4 & PA) 5 \\
\hline os & 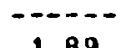 & 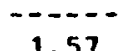 & $-\cdots$ & $\cdots$ & $-\cdots$ & $-\cdots---$ & $\cdots$ \\
\hline $\begin{array}{l}\text { PAC. COIST } \\
\text { PAC. OCEAN }\end{array}$ & $\begin{array}{l}1.89 \\
1.99\end{array}$ & $\begin{array}{l}1.57 \\
1.57\end{array}$ & $\begin{array}{l}1.26 \\
1.36\end{array}$ & $\begin{array}{l}.6 .1 \\
1.06\end{array}$ & $\begin{array}{r}.41 \\
1.13\end{array}$ & $\begin{array}{l}.75 \\
.85\end{array}$ & $\begin{array}{r}.10 \\
.32\end{array}$ \\
\hline R. RnCKIES & 1.50 & 1.24 & .86 & $.5 A$ & .72 & .50 & .55 \\
\hline E. ROCKTES & 1.45 & 1.39 & .43 & .26 & 1.03 & .10 & .79 \\
\hline H. TEX. / & & & & & & & \\
\hline E. N. MEX. & 1.2 .3 & .90 & .46 & .22 & .36 & .65 & .72 \\
\hline H. GULF ḄASIN & 1.02 & .56 & .33 & .51 & .10 & .91 & 1.08 \\
\hline GHLP OP $M P X$. & .97 & .61 & .60 & . An & .32 & 1. 16 & 1. 33 \\
\hline MIOCONT. & 1.05 & . 88 & .41 & .19 & .38 & .68 & 1.06 \\
\hline $\begin{array}{l}\text { MI. BASIN, INT/ } \\
\text { APPLACHIA }\end{array}$ & .70 & .62 & .24 & .52 & .79 & .92 & 1. 45 \\
\hline ATL. COAST & .87 & .20 & .77 & 1.09 & .76 & 1.45 & 1.72 \\
\hline ATL, OCEAN & .45 & .45 & .74 & 1.03 & 1.05 & 1.4 .3 & 1. 90 \\
\hline
\end{tabular}

DATA SOURCE : "I NPUTS TO THE PRDJECT INDBPENDENCE EVALUATION SYSTEM INTEGRAT ION AODRL POR THE TRANSPORT OP ENERFY MATERIALS". VJILIME II. PREPARED POR THE TASK FORCE REPORT. PEDERAL ENERGY ADKINISTRATION PRO.JCT INDEPENDENCP BLJEPAINT

PINAL, INDER THE DIRECTION. OF THE DEPARTHENT OF IRANSPORTATION, NOVEMBER. 1974, TABLE VIIT-20, PG. VIII-24; TABLE VIII-21. PG. VIIT-25; 1973 DOLLARS CHANFBD TO 1975 DOLLARS BY FACTOR 1.212

OPPICE RESPONSIBLE : hID-RANGE ANALYSIS DIVISION, APPLIED analysis, EIa.

TABLE 7

COSTS OP TRANSPORTING DOAPSTIC CRHDE DTL

BY BARGR AND TANKER

(1975 DOLLARS. PER BARREL)

\section{REFINPRY BEC;ION}

$\begin{array}{lcccc}\text { NPC REGION } & \text { CAD 1A } & \text { PAD 1B } & \text { PAD 3 } & \text { PAD } 5 \\ \text { PAC. COAST } & 2.04 & 1.92 & 1.90 & \\ \text { PAC. OCEAN } & 2.09 & 1.97 & 1.95 & \\ \text { H. GIILF BASTN } & .72 & .55 & & 1.90 \\ \text { GILF OP MFX. } & .72 & .55 & & 1.92 \\ \text { ATL. COAST } & .68 & .51 & .46 & \\ \text { ATL. OCFAN } & .35 & .11 & .76 & \end{array}$

DATA SOUBCE: "I NDITS TO THE PROJECT INDPPENDENCE EVALIIATION SYSTEM INTEGRATION MONEL FOQ THE TRANSPORT OP ENERGY MATFRIALS". VOLUME II. PREPARED POR THE TASK FORCE REPORT. FEDFRAL ENERGY ADMINISTRATIOH PROIECT INDEPENDENCF BLUEPRINT

FINAL. IIDER THE DI.RRCTION OP THE DEPARTAPNT OP TBANSPORTATTON, NOVPABER, 1974, TABLE VILI-10, PG. VIII -12

1973 DOLLARS CHANGED TO 1975 DOLLARS BY FACTOR 1.212.

OPPICE RESPONSIBLE : RID-RANGE ANALYSIS DIVISION. APPI.tPd ANALYST. EIA. 
TABLE 9

TRANSPOR TATIS! COSTS FOR ALASKAN CRIITR OIL

(1975 DOLLARS PER BARREL)

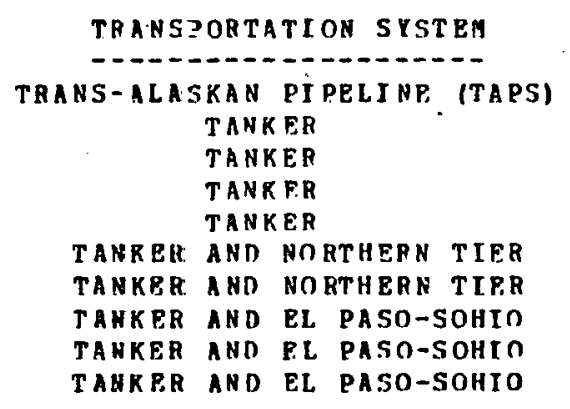

ORIGIN
NORYH SLORE
SOUTH ALASKA
SOUTH ALASKA
SOITH ALASKA
SOIITH ALASKA
SOIITH AIASKA
SOITI ALASKA
SOUTH ALASKA
SOUTH ALASKA
SOIITH ALASKA

\begin{tabular}{ll} 
& \\
DESTINETION & COST \\
\hline SOUTH ILASKA & 3.45 \\
PAN 1A & 2.53 \\
PAD 18 & 2.51 \\
PAD 3 & 2.49 \\
PAD 5 & .65 \\
PAD 2B & 1.13 \\
PAD 3 & .91 \\
PAD 2A & 1.12 \\
PAD 2B & 1.13 \\
PAD 4 & 1.07
\end{tabular}

TABLE $B$

TRANSPORTATION COSTS FOB POREI3N CRHDE OIL

11975 DOLLARS PER AARREL)

RPPINERY HEGION

POREIGN REGIION
LAMDED EAST COAST
LAMDED GQLP COAST
LANDEA HEST COAST
CAMADA
CARRIBEAN
FOREIGN OTHE,

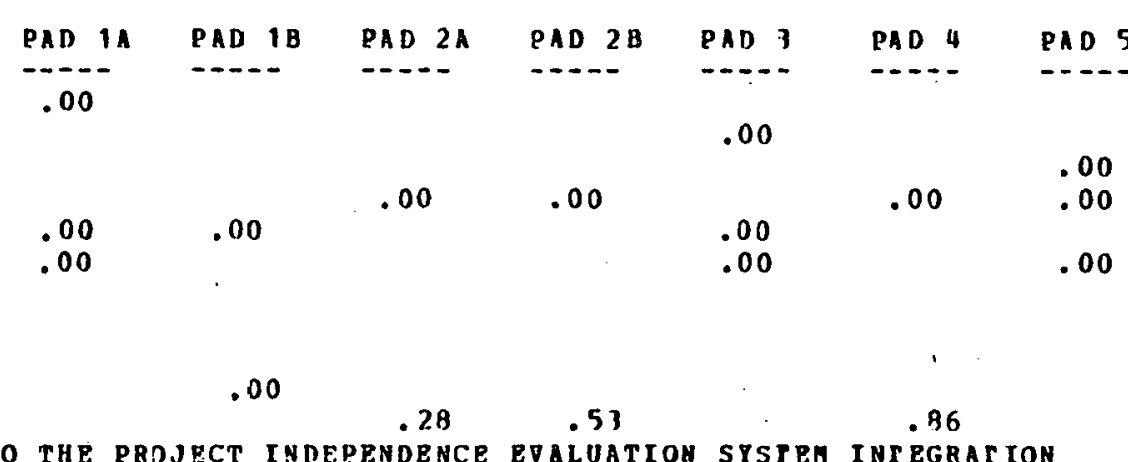

$\begin{array}{ll}\text { PAD } & 1 \\ \text { PAD } & 1\end{array}$

DATA SOURCE : "INPUTS TO THE PRDJPCT INDEPENDENCE EVALUATIOH STSTEM INTEGRATION HODEL POR TIIE TRANSPORT OP ENERGY MATBRIALS". VOLIIME II, PREPARED PJR THE TASK PORCE REPORT. FEDERAL. ENERGY ADIINISTPATION PROJECT INDEPENDENCE BLIE EPRINT

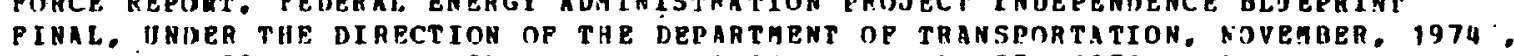
PABLE VIIL-20. PG. VIII-24: TABLE VIII-21. PG. VIII-25: 1973 DOL.LARS CHANG TD TO 1975 DOLLARS BY FACTOR 1.212 .

OPPICE RESPONSIBLP: MID-RANGE ANALYSIS DIVISION, APPLIED ANALYSIS, EIA. 
TABLES 10 THROUGU 14 DESCRIBE THE TRANSQORTATION RETWORR POB PETROLEUM PRODNCTS. RESIDUAL FUEL UTL REOUTRRS SEPARATE AARGE AND TANRER COSTS UFCAOSE OP ITS GREATPR DENSITY AND IS NOT SHIPPED IN PIPPI.INES BECAUSE OP ITS HIGH VISCOSITY. THE DATA SOURSES POR PRODICTS THANSPORTATION ARE THE SAME AS POR CRIINE OIL TRANSPORTATION, PRONUCT PIPELINE LINKS THAT DO ROT CORRESPOND TO EXISTING PIPELINES ARE COSTED AT $5.07 / \mathrm{BBL}$

INOT IONALI.

THE PURCHASE PRICES GIVPN IN THE IAPORT TABLES POA

PRONUCTS ARP. THE DELIVERED CITY-GATE PRICES FOR TLE DOR REgION OP ENTRY. ACCORDINGLY. THR. TRANGPORTATION COSTS SHOHN IN

TABLES 12 AND 14 POR THPSE LTHKS ARP ZERO. TIIE NON-ZRRO COSES ARE FOR ADDITINNAL TRANSPORTATION INLAND. 
TABLE 10

COSTS OF TRANSPJATING PFTROLEIM FBODULTS BY PIPELINE

(1975 DOLIARS PF.R BARKELI)

DOE RPGIOH

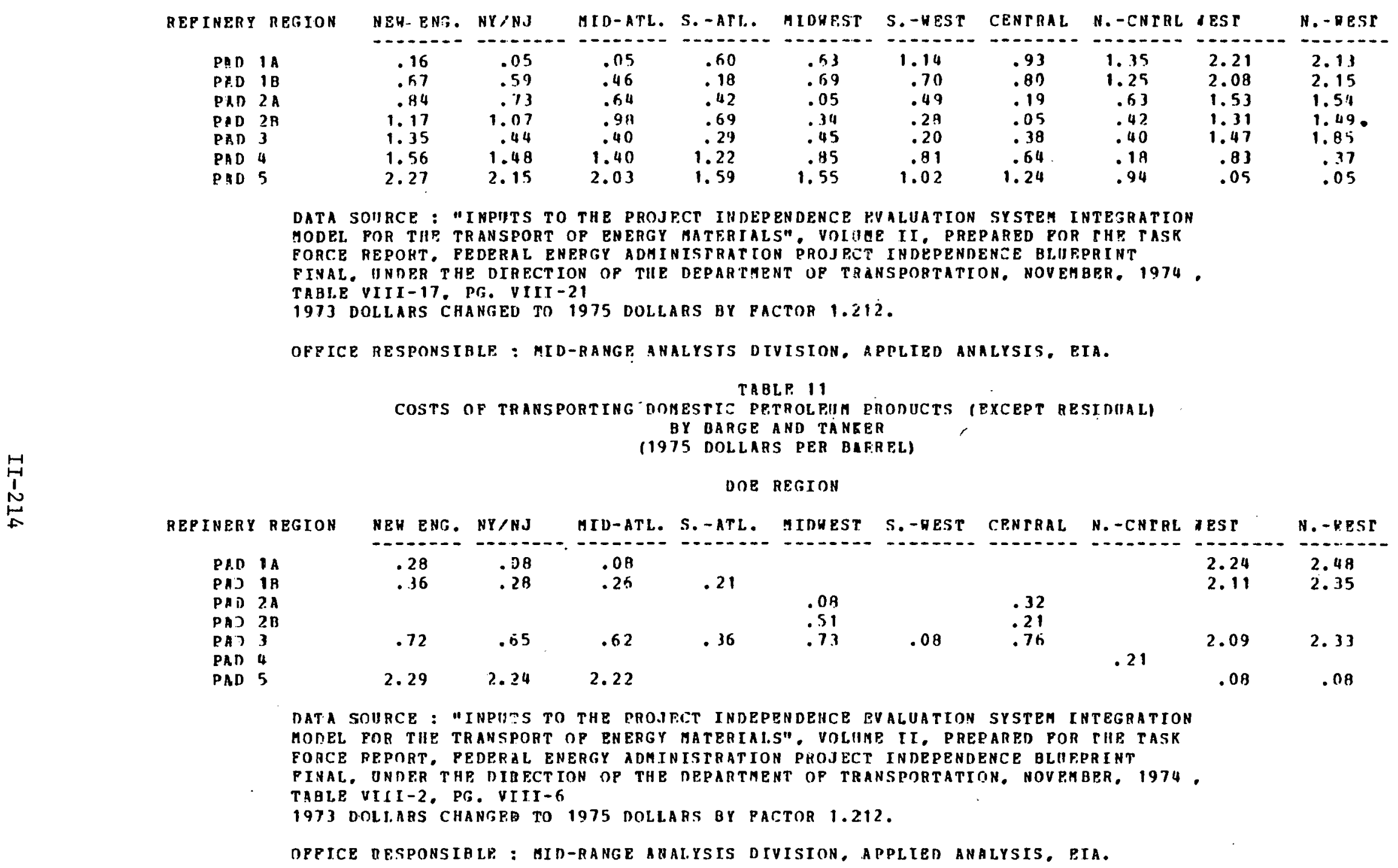


TABLE 12

COSTS OP TRANSPORTING IMPORTED PETPOL FIIM PHODUCTS (EXCEPT RESIDUAL)

BY BARGE AND TANKER

$(1975$ DOLLARS PER BARREL)

DOE REGION

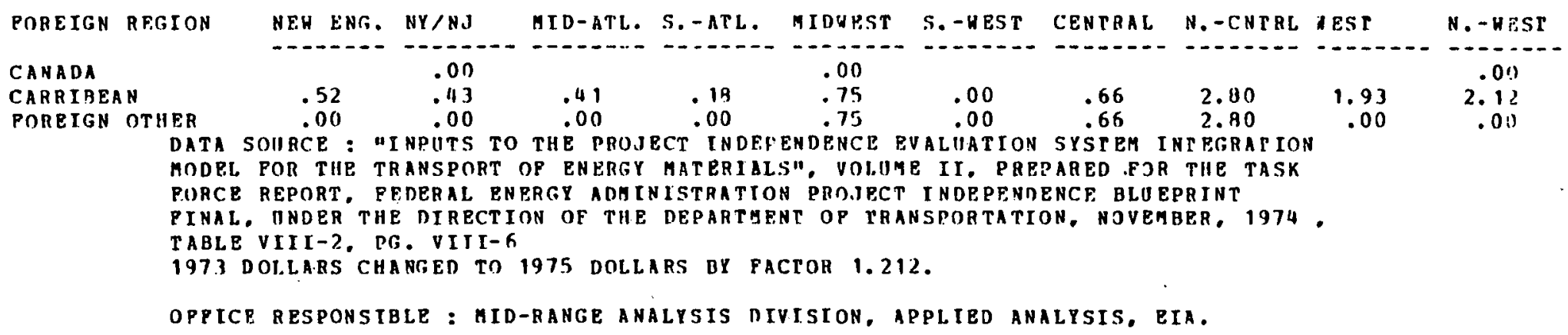


TABLE 13

COSTS OP TRANSPORTINF' DONESTIC RESIDUAL FIIEL

BY BARGP. AND TANKEF

(1975 DOLlars PER barFet.

DOE REGION

AEF INERY REGTHN

PAD IA

PAD IB

PAD 2A

PAD 2B

PAD 3

PAD 4
NP.W ENG. NY/N.J

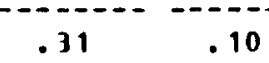

.31

.10
.31

HID-ATL . 5.-ATL.

MINHEST

.10

.25

$.80 \quad .71$

.69

.40

.10

.62

2.52

2.46

2.44 S.-WEst

CENTRAL

H. - CNTRL

NEST

N. - HEST

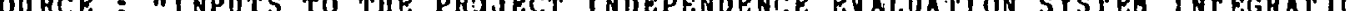

MODEL POR THP TRANSPOZT OP ENERGY MATERIALS", VOLUME II PREPADEN POB THE TASR

PORCE REPORT, PEDERAL ENERGY ADUINISTIATION PROJECT IHOEPENDENCE BLIOEPRINT

PINAL. UNDER THE DIRECTION OP THP DEPARTHENT OP TRANSPORTATION. NOVEMBER, 1974.

TABLE VIII-6. PG. VITI-9

1973 DOLLARS CHANGE TO 1975 DOLLARS BT PACTIR 1.212.

OPPICE RESPONSIBLE : MID-RANGE ANALYSIS DIVISIOH, AEPLIED ANALYSIS. EIA.

TABLE 14
COSTS OP TAANSPOATING IMPORTED RESIDIJAL PIIEL

BY BARGE AND TANKEA

(1975, DOLLARS PBR BARREL)

DDP. RPGION

\begin{tabular}{|c|c|c|c|c|c|c|c|c|c|c|}
\hline POREIGN REGION & N EH ENG. & $\mathrm{NY} / \mathrm{NJ}$ & HID-ATL. & S. - ATI.. & MIDGFST & S. - HEST & CENTRAL & N. - CNTAI. & Y EST & N. - HEST \\
\hline $\begin{array}{l}\text { CAN ADA } \\
\text { CARRIBEAM } \\
\text { POREIGN OTIIER }\end{array}$ & $\begin{array}{r}.55 \\
.00\end{array}$ & $\begin{array}{l}.00 \\
.46 \\
.00\end{array}$ & $\begin{array}{l}.44 \\
.00\end{array}$ & $\begin{array}{r}.20 \\
.00\end{array}$ & $\begin{array}{l}.00 \\
.80 \\
.80\end{array}$ & $\begin{array}{l}.00 \\
.00\end{array}$ & $\begin{array}{r}.70 \\
.70\end{array}$ & $\begin{array}{l}3.00 \\
3.00\end{array}$ & $\begin{array}{r}2.05 \\
.00\end{array}$ & $\begin{array}{r}.00 \\
2.26 \\
.00\end{array}$ \\
\hline
\end{tabular}

DATA SOURCE : "INPUTS TO THE PROJPCT INDEPENDFNCE EUALIITION SYSTEM INTEGRATION

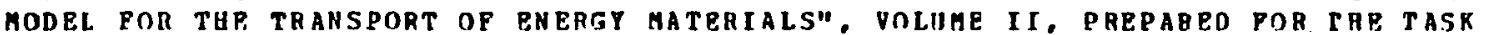

FORCE REPORT, FEDERAL. ENERGY ADAINISTRATION PROJECT INDEPENDENCP BLIIPPRINT

PINAL. DNDER THE DIRECTION OF THP DEPARTIENT OP TRANSPORTATION. NOVEHBER, 1974.

TABLE VIII-6, PG. VITI-9

1973 DOLlaRS CHANGED TO 1975 DOLlars BY PACTOR 1.212.

OFFICE RFSPONSIBI.P : MID-RANGE ANALYSIS DIVISION, AFPLIED ANALYSYS, RIA. 
This section has 21 tables of electric utilities input data. The tables fall inte four categories: load duration, capacity, cost and physical units.

TABLE 1 contains the load duration factors representing the various dand requirements by base, cycling, daily peak and seasonal peak loads. These four modis account for the fact that electricity cannot be stored and must be produced on doment and that demand changes daily and seasonally. The load duration factors are given by DOE region. Regional system load factors are given as well, and scheduled shut-downs for maintenance and other forced outages are also considered. DOE regions are shown in Figure $\Pi-1$.

Following TABLE 1 is a load duration curve approximation and ten unnumbered tables containing plant capacity factors -one for each DOE region. The capacity factors are given for both existing and new plants.

Capacity figures are given in TABLES 3,5,6,7,8, and 9. TABLE given the retirement rates for fossil-fuel-fired plants in percentage terms.

Cost data appear in TABLES 10 through 15. Heat rates and heat values appar in TABLES 16 through 21. 
1995 ELECTHT: ITIEITIPS INPUT MATA

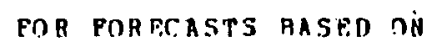

CURAENO LAY 
THERE ARE POUR CATECORIES OP INPUT CATA BLEAENTS

LOAD-DURATION- FACTORS MHICI REPRESPNT NONONIZORM DPHAND REOUIRPAENTS

II.E. BASP. CYCI.ING. DATLY PEAR, ANO SEASONAL PBAK LOADSI:

CAPACITY - EXISTING ANI NEH CAPACITY POA PACII PLAHT TRPB. AHD

¿nst -

BANDATED CONVEHSIDA:

CAPITAL. CHARGES, OPERATION AND haINTEAANCE CJITS.

SCRIBBING COSTS. AND TRANSHISSIOR AND DISTAIDITION COSTS:

PIITICAL-: HEAT RATZS AND VALUES POP. RACII PI.ANT AND POEL:

TH MOST CASES THE DATA YARIPS OY OOP REGION. THE DATA ELBMENTS.THEREPORE.

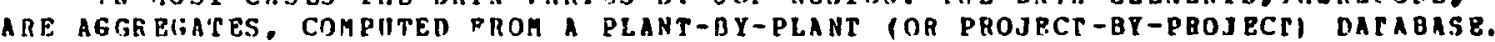

TAALE 1 SHOWS REGIONA: COAPOSITION AND CAPACITY PACTOAS POR BASE. EYZLIRT. DAILY PEAR, AND SEASONAL PQAK LOADS AS WELL AS THE SPSTEM LOAD PACTOBS II. E. THE RATIO OP AVERAGE TO PEAK LOAD). THE RPSERVE HARGIN IS 20 POR PACH REgION. COAPOSITION FACTORS ARE TIIE FRACTIONS OP ELECTRIC. POHER IN EACH LOAD. POR BXAMPLE. IN REGION 1 OF ALL ELECTRICITY DEANNDED DIIRING FHE YEAR. 5A.78 IS BASE. 24.78.5 CICLIJG, 4.4X IS DATLY PEAR, AND 2. 2X IS SEASONAL PBAK. PITURE 1 SHOHS A

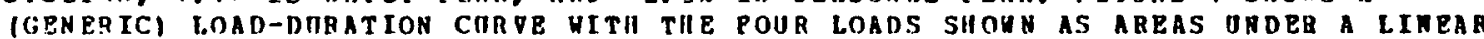
IGPRETIMATION OF THF ACTIHL CURVE.

GENPRATING FACILIPIES ARE CLASSIPIPD AS AASE, CRCLIHS. DALLT PQAK OR SEASOHAL PFAR JPPEHDING UPON THE ORDER IN HIICH TIIET ARF, BROUGHT INTO ORERAT COA. AS DBAAMD INCREISES. CAPACITY PACTORS, ARE TIIR. PAACIIONS DF TIMR A PLATT IS IZTUALLY OPERA?ED ANR NOT SHOT DOKN PJR RPPAIQS OB SZREDIILPD HAIHTENANCE

OA UNISED POR LACK OP SUFPICIBNT DEMAND. POS EXAMPLE, IP AN EXISTIN3 RESIDIALPIRED PLANT IN RPGLON 1 IS OPERATED IN BRSE LOAO POR A TEAR. TAB ACTIAL NIMBER OP KILONATT-YEARS OP PLECTRICITT GENEAATED. ON THE AVERAGR. UTLL BB ITS CAPACITY (IN KILONATTS) BILTIPLIRD UX ITS CAPACITY PACTOR, I THLS CASE .70. SI RC BASE PLAHIS ARE ORERATED ALMOST CONTIMTOJSLY MHEN THEY

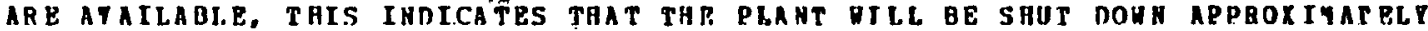
30X O? THE TIMP. POA TIIIS SAHE. PLANT OPPAATING IN CYCLING LOAD. TII CAPACITY PACTOB IS . 55 , REPI.FCTING THE PERIODS TIIE PLANT WILL MOT OPERATE BFCAIISR TIIE DEMAND DORS NOT HARAAT BRINGING TH CYCLING PLANTS AS HELL AS PPRIOOS DHPN IT IS PORCEJ OU'T OP SERVICE OR SHIT DONN POR HAINTENANCE. 
TABLE 1: LOAD-DIIATION CURYE DATA PDF 1985

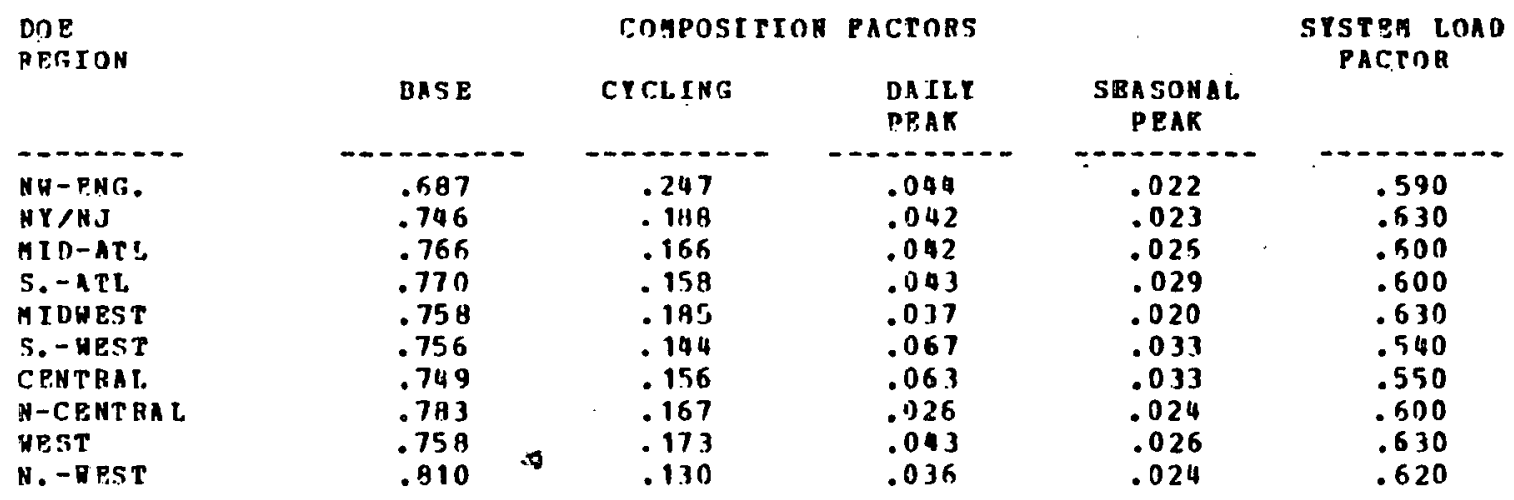

DAT SOUACES : COMPOSTTION FACTOAS; 1971 ANNIJLIZBD LOAN DIRATION CORVES

PROA "RESPECIPICATION OP THE ELECTRIC ITILITY HODEL IN PIES". SENBAL BLECTRIC

COMPANT. ALSO, THE BREAK POINT CIVING BOUNDS POR THE POII GODES ARE ASJIGNED REJGENOUSLY

AND IRE. BASED ON GISTORICAL AND BCONONTC COHSIDEBATIOHS.

OPPICE BPSPONSIBLE : OPPICE OP INTEFRAPIVE ANALISIS, APPLI ZD ANALYSIS, EIA APPLIED ANALSIS. FIIA 
FIGURP 1: LIURAR APPROXIMATIOH OP A LOAD-DIBATIOH CURVB

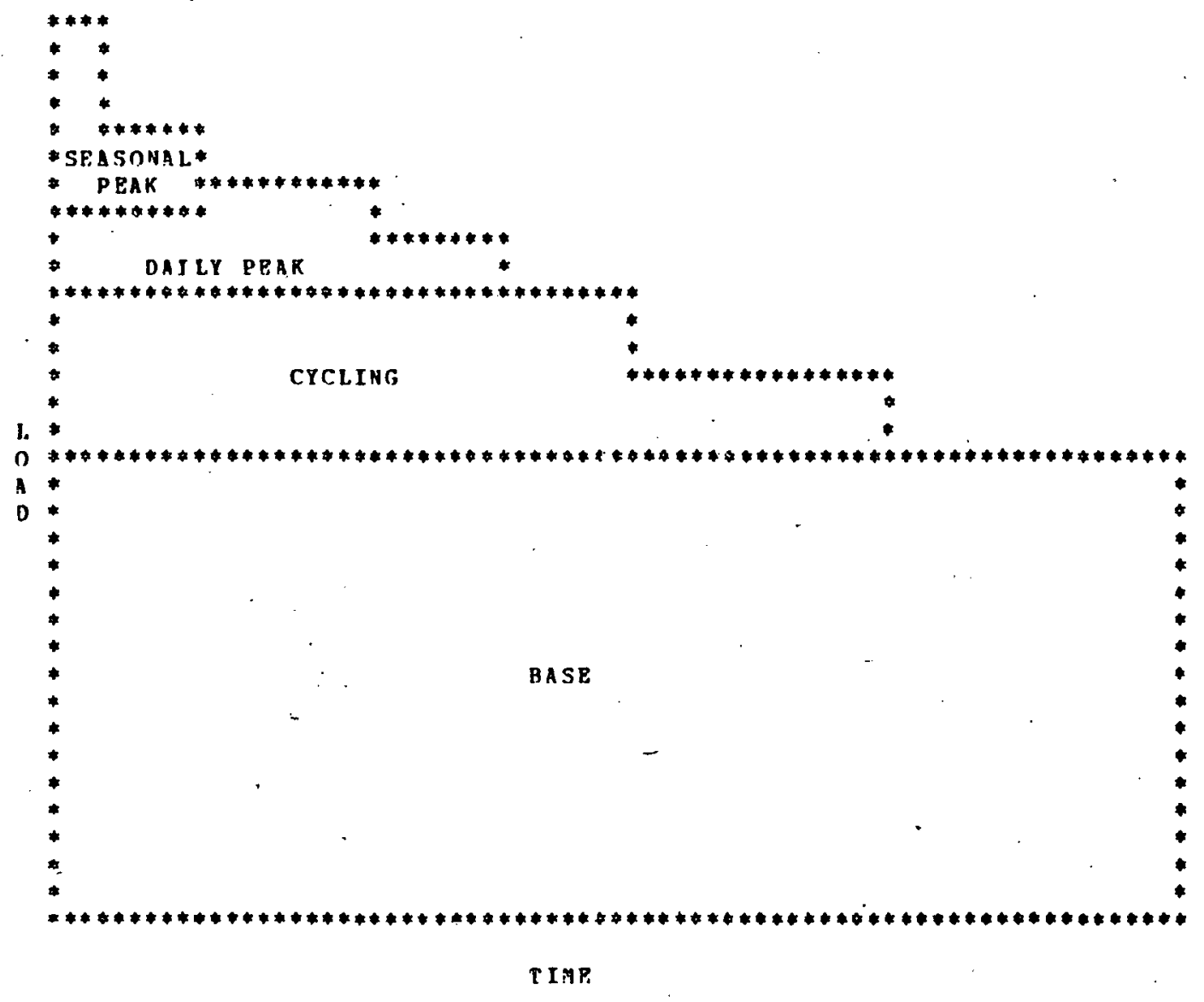




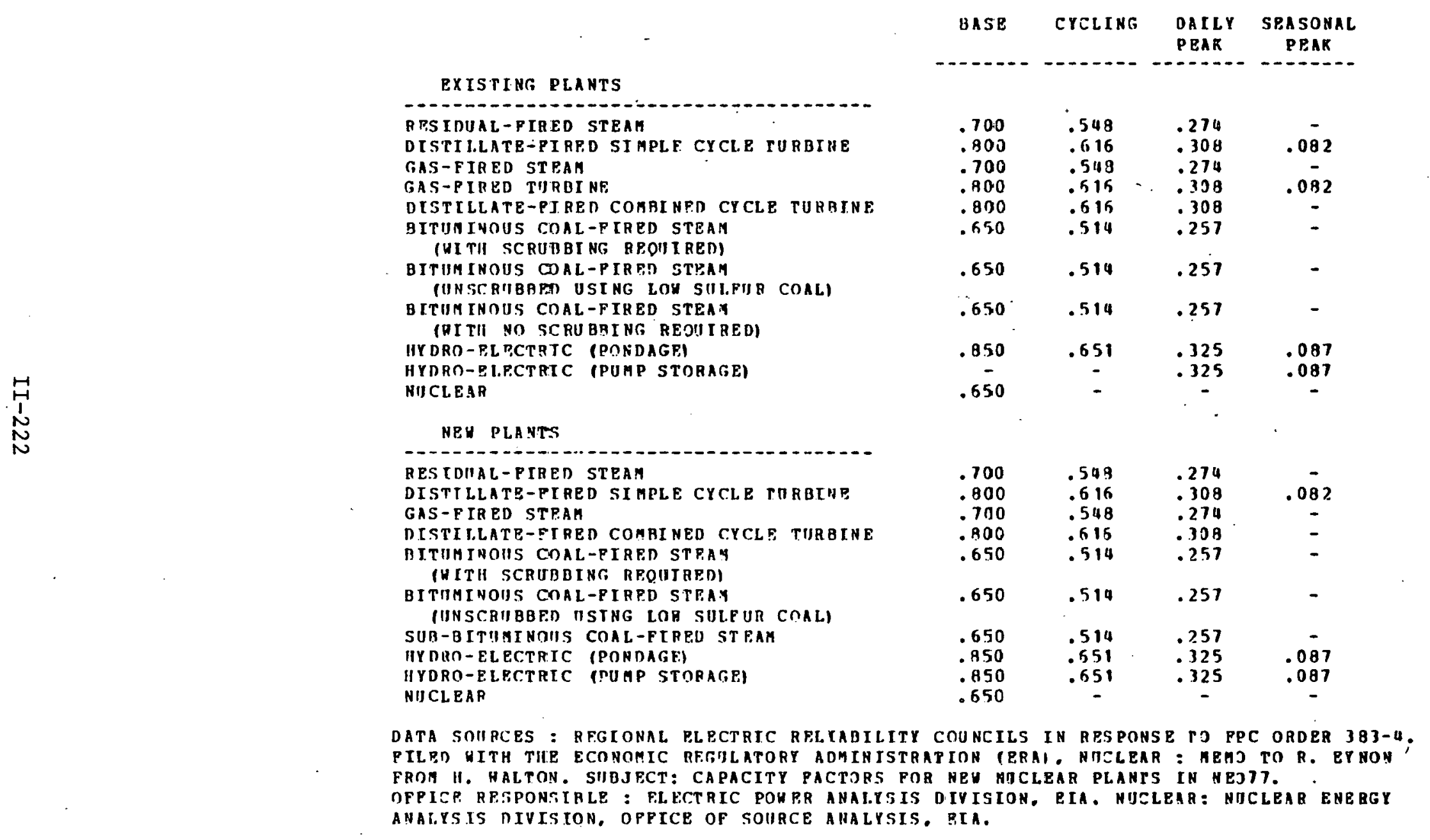


CAPACJTY FACTOHS PUB DOE REGION 2 (NEM YORK AND NEM JERSEY)

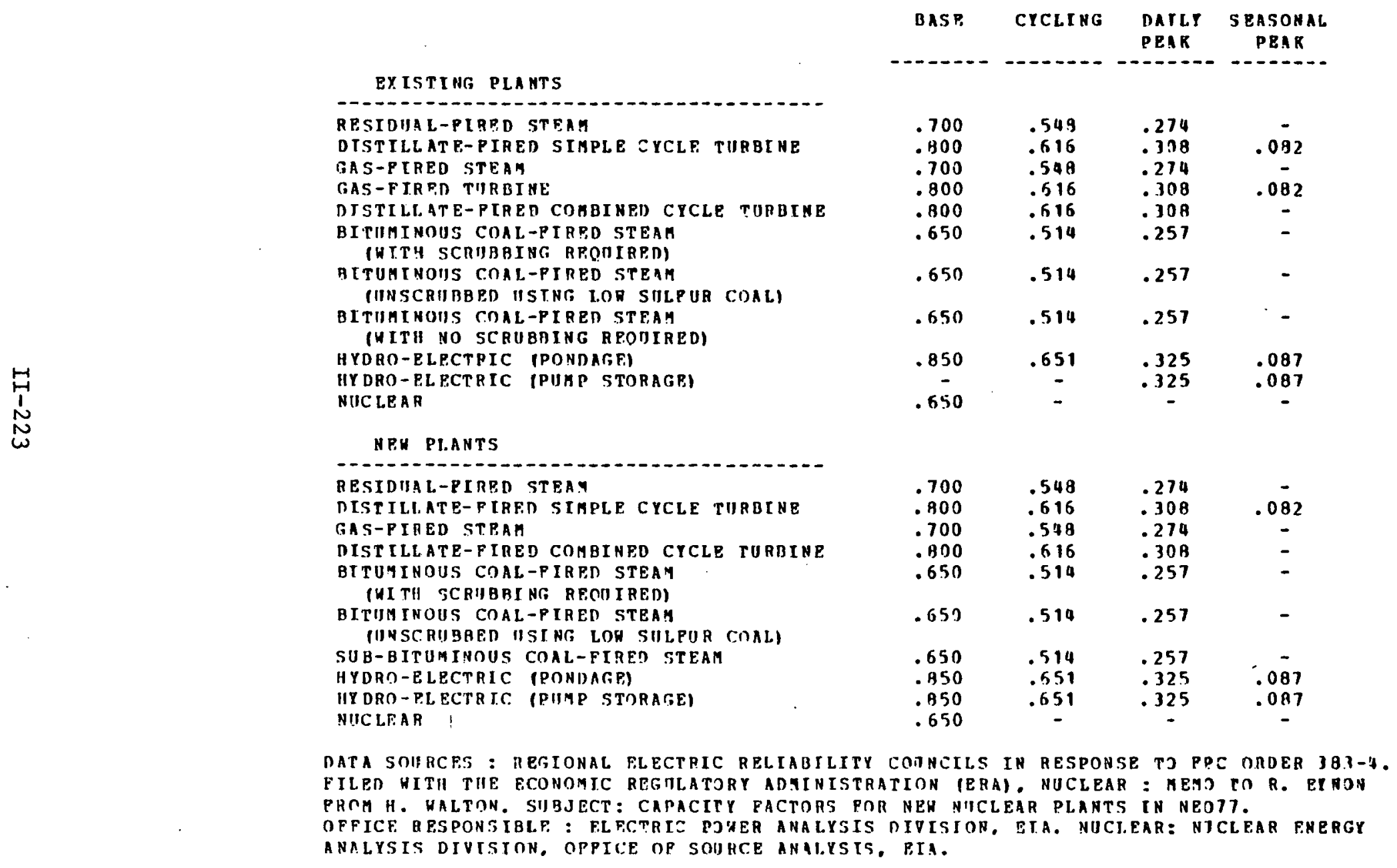




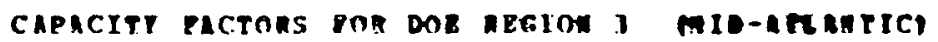

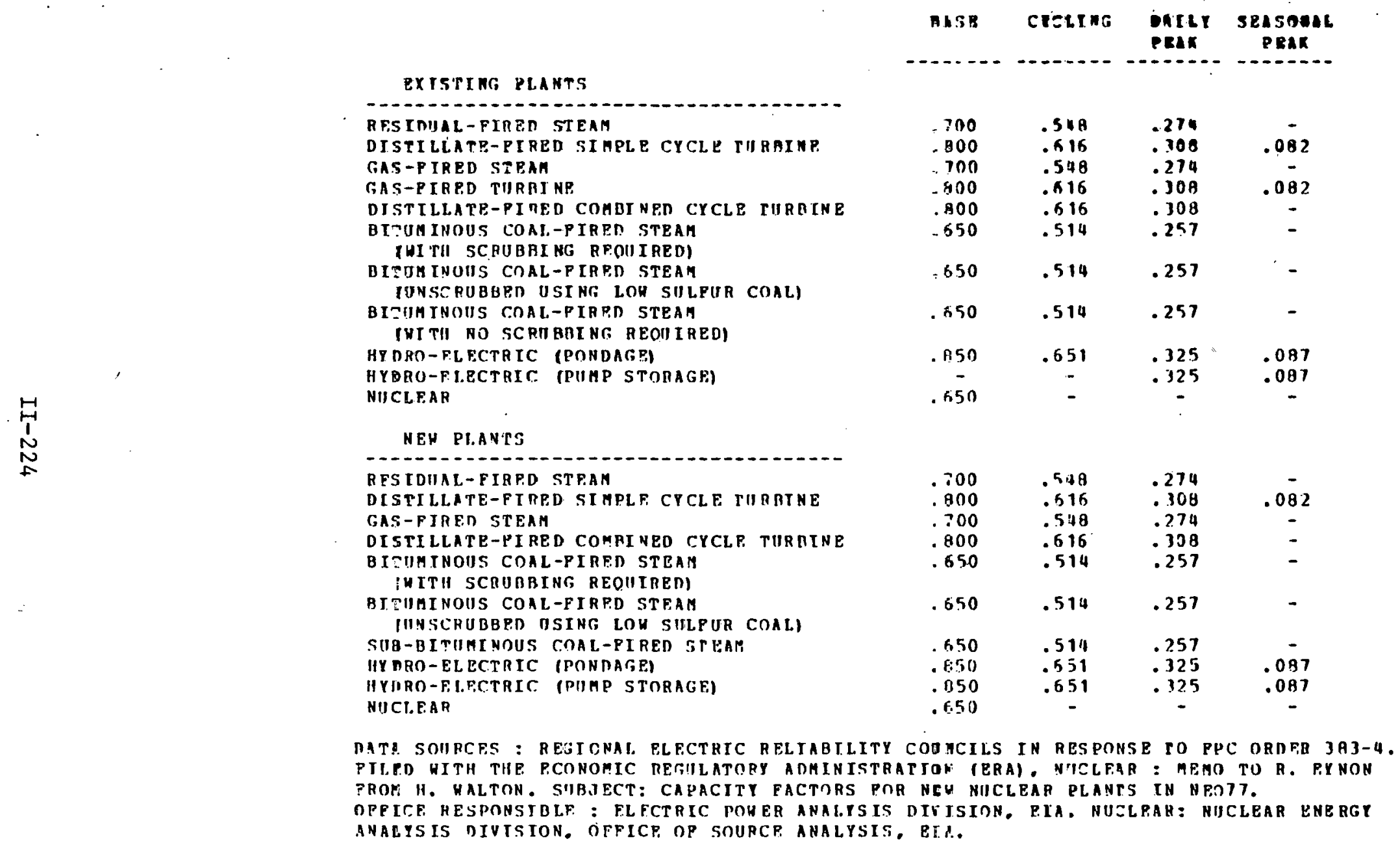


CAPACITY FACTORS POB DOE REgion 4 (SOUTH atLantic)

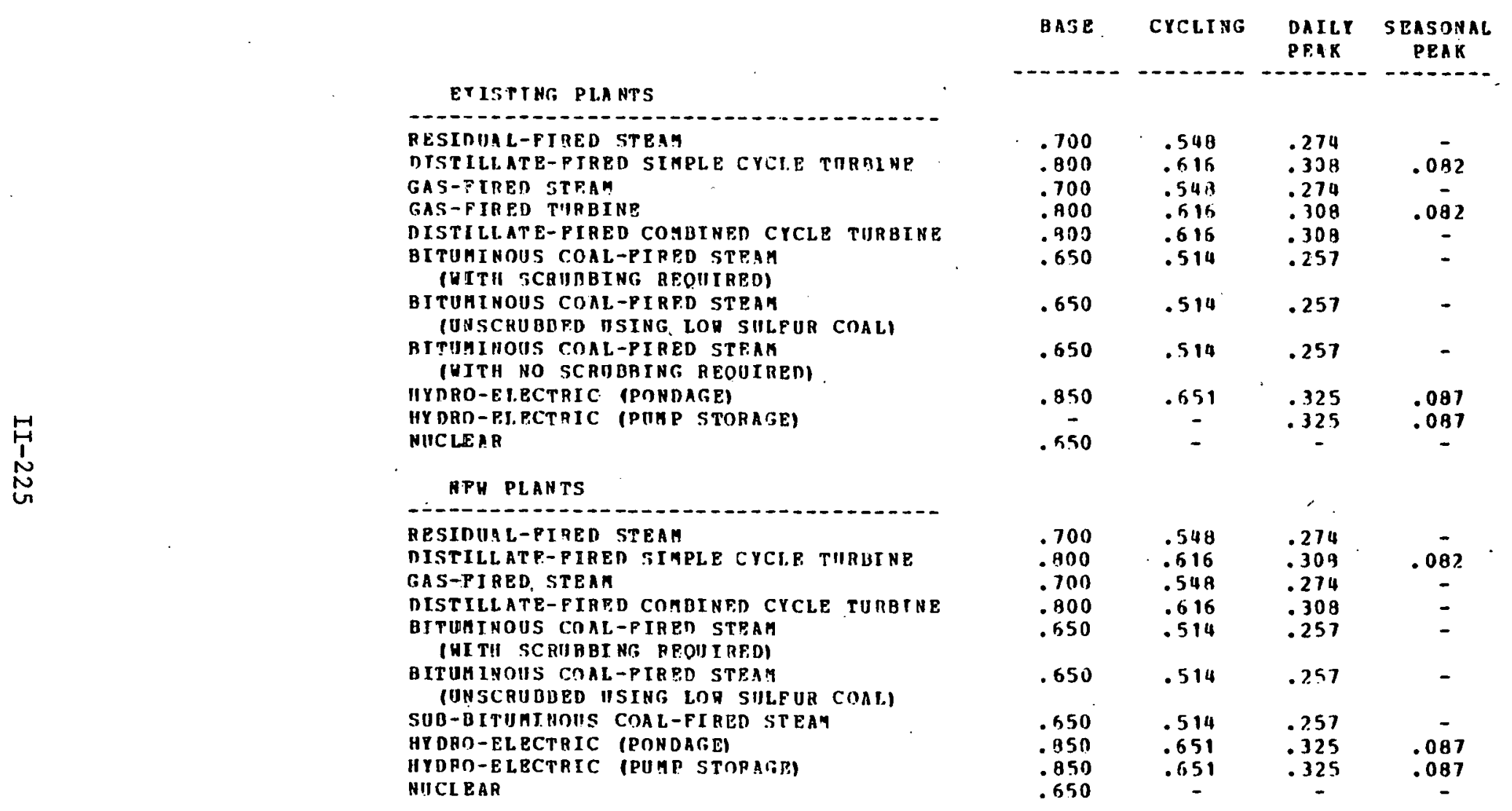

DATA SOIRCES : RECIONAL ELECTRIC ARLTABILITY CONNCLLS IN RESPONSP TO PPC ORDPR 383-4.

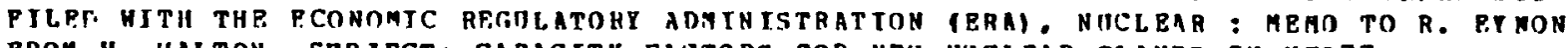
PROM H. HALTON, STB.JECT: CAPACITY FACTORS POR NPW NIICLBAR PLANTS IN NBJ77.

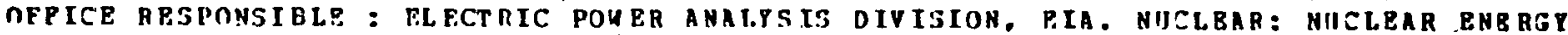
ANALYS IS DIVISION, OFPICE OP SOURCP ANALYSIS, PIA. 
CAPACITY PACTORS gOR DOE REgION 5 (HIDUEST)

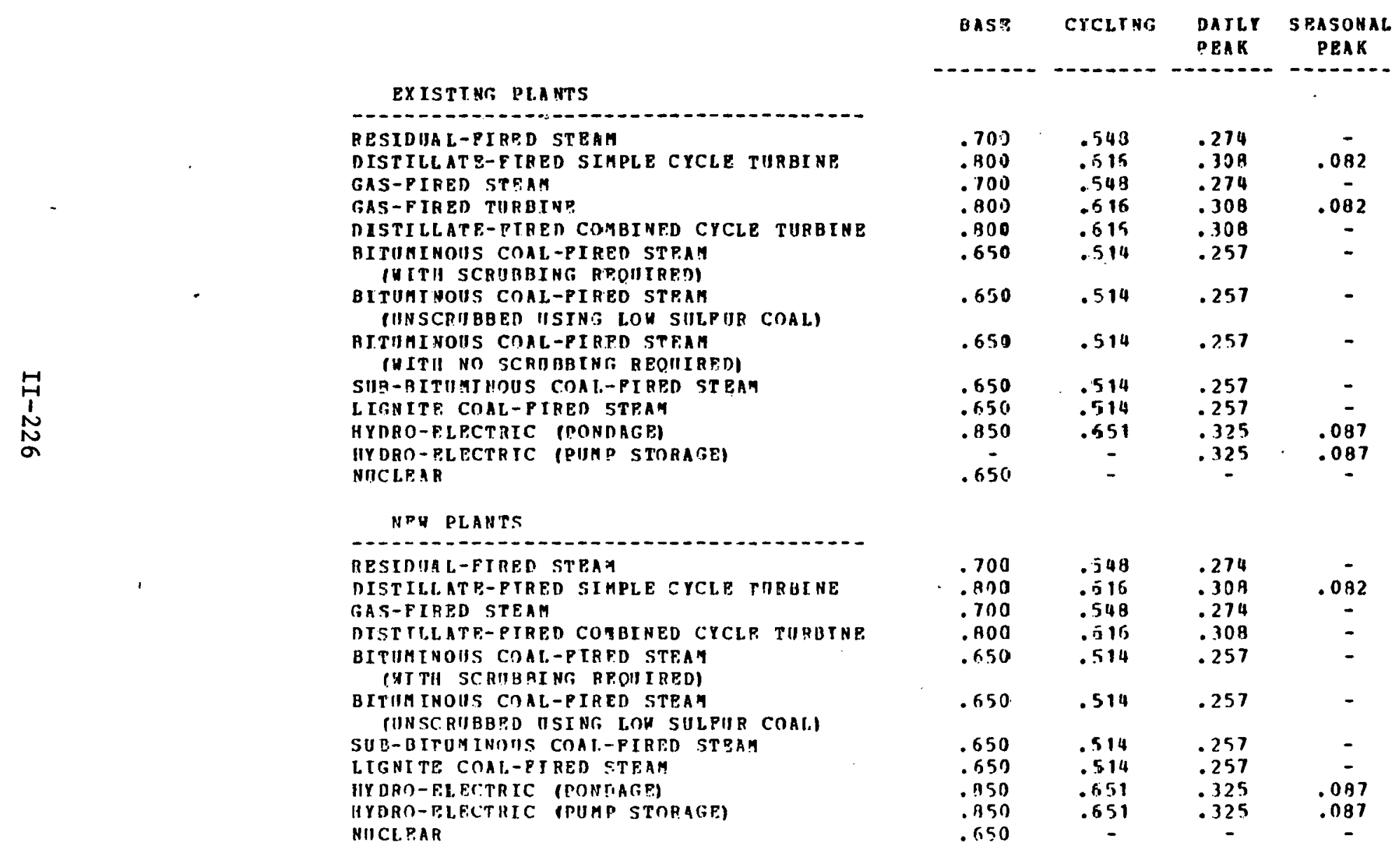

DATA SOIBCFA : REGIOMAL FLECTRIC REI.TABIITTY COINCIL.S TN BPSPONSF TO PQC ORDER 3AT-4.

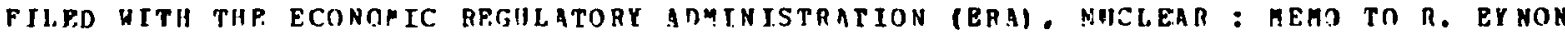
F?OM II. NALTON. SIIBIECT: CAPACITY FACTORS FOR NBW NILLEAR PLANTS IN NBO77.

OPPICP RPSPONSIPLE : EL FCTRIS POH PR ANALYSIS DIVISIOR. PIA. NIIZLEAR: NICLEAR ENERGY ANALYSIS DIVISION, ORFICE OF SNIJCE AHALYSIS. FIA. 
CAPACITY PACTORS POP DOE REGION 6 ISOUTHUESTI

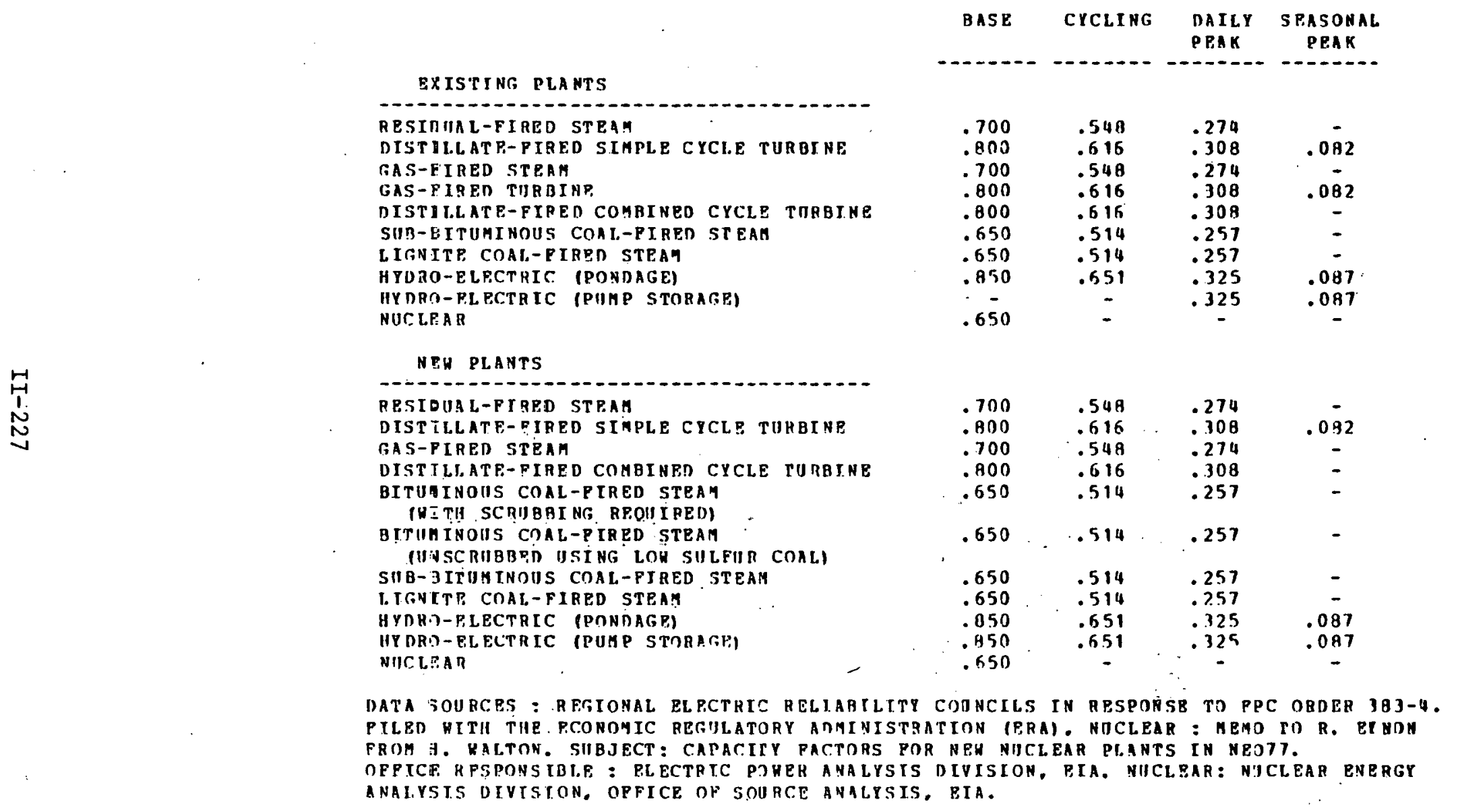


CAPACITY FACTORS FOR DOE REGION $?$ (CRMTRAL:

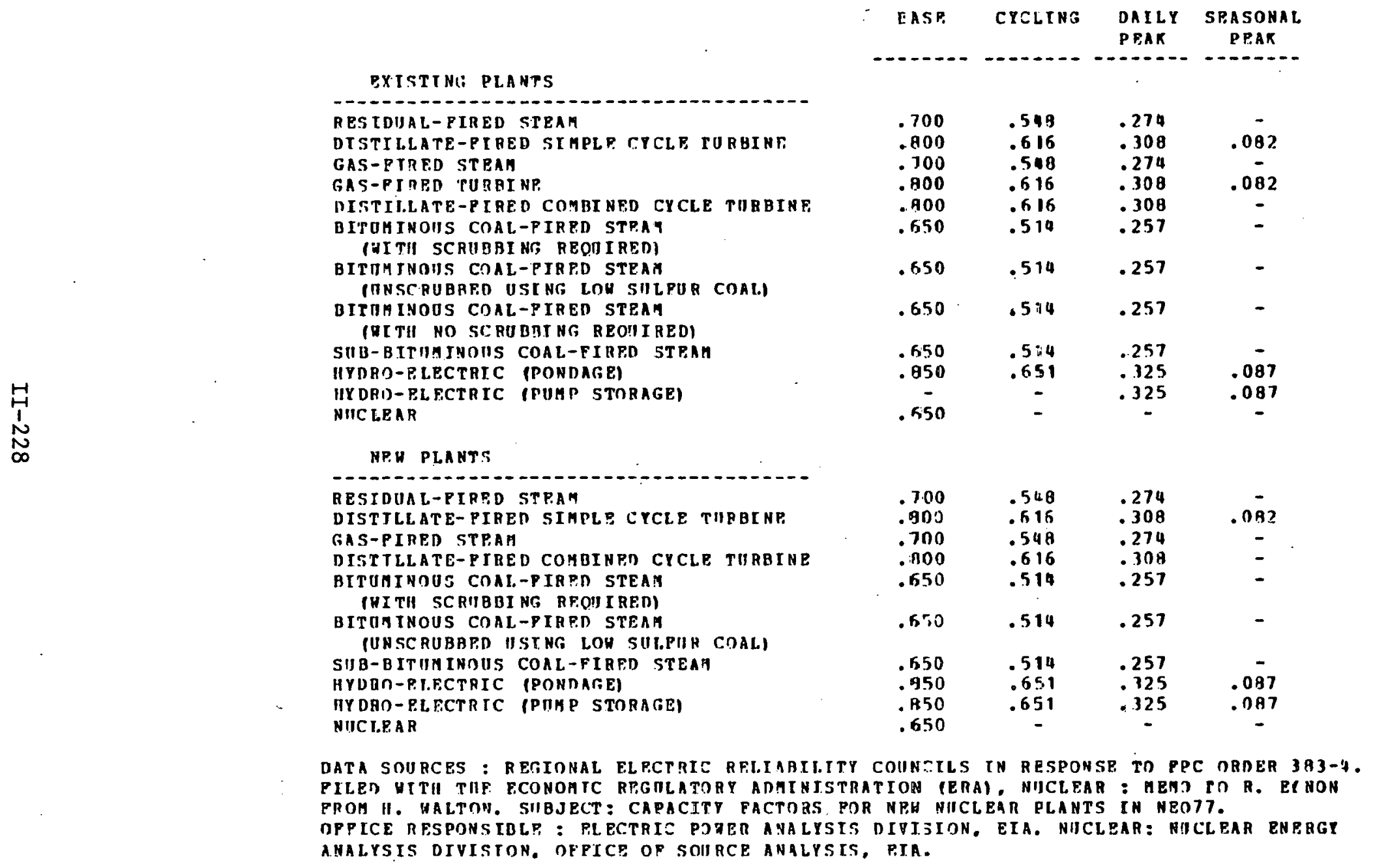


CAPACITY PACTOAS FOR DOE REgTON $\theta$ (HORTH CEH ROAL)

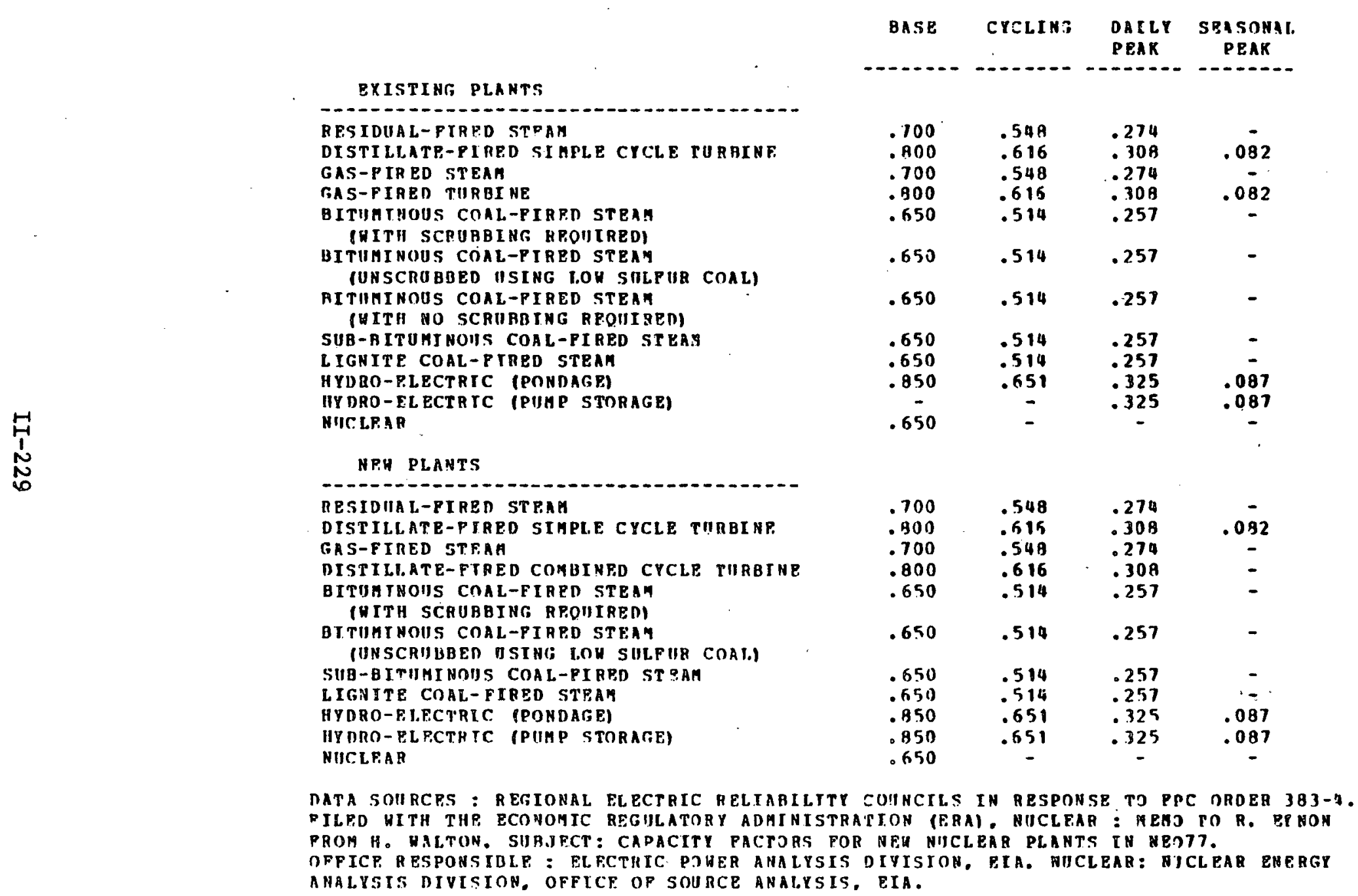


CAPACITY FACTORS FOR DOE RHAION 9 (UEST)

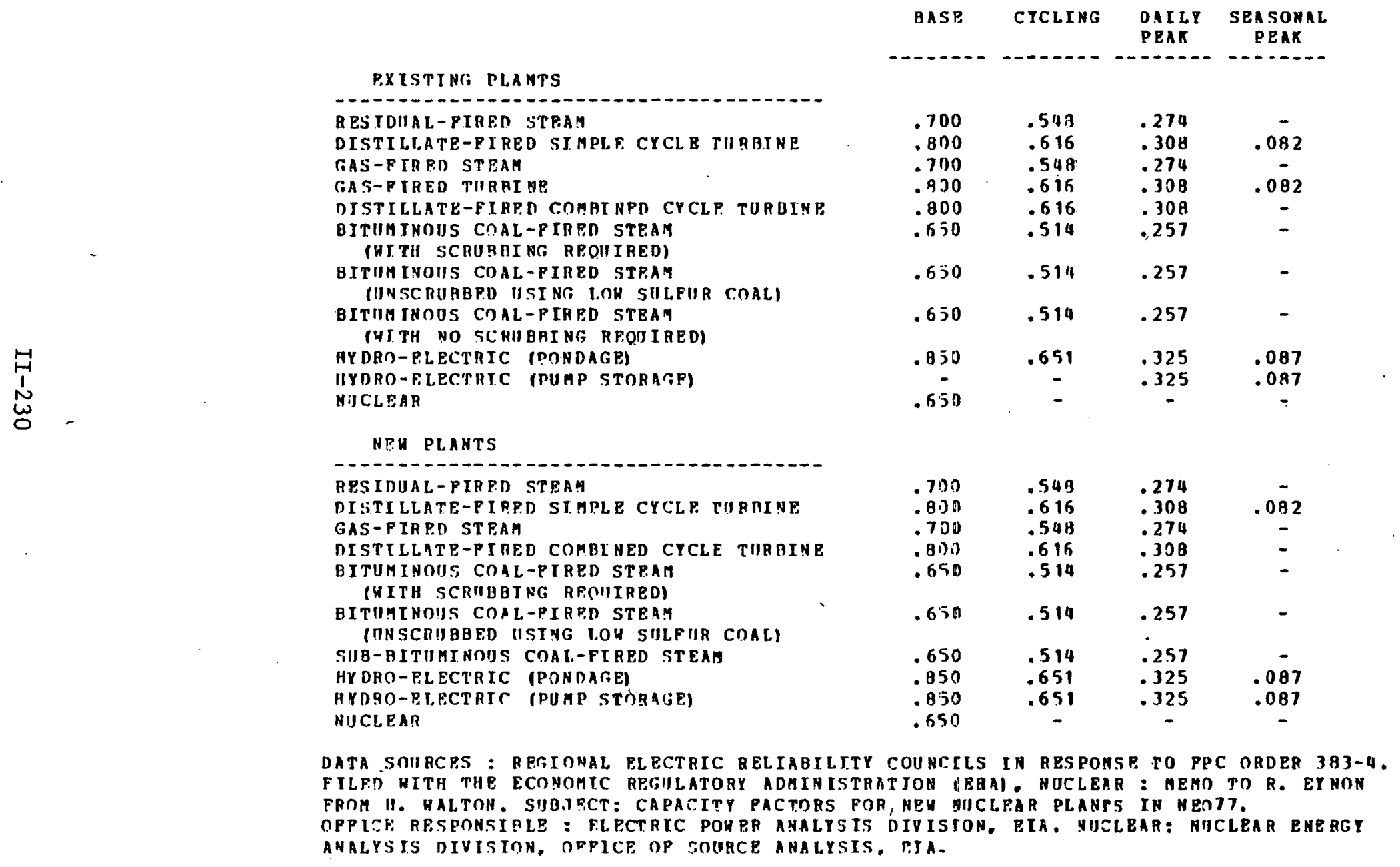


CAPACITY FACTORS FOR DOE HEGIUN IO (NOBTHESTT)

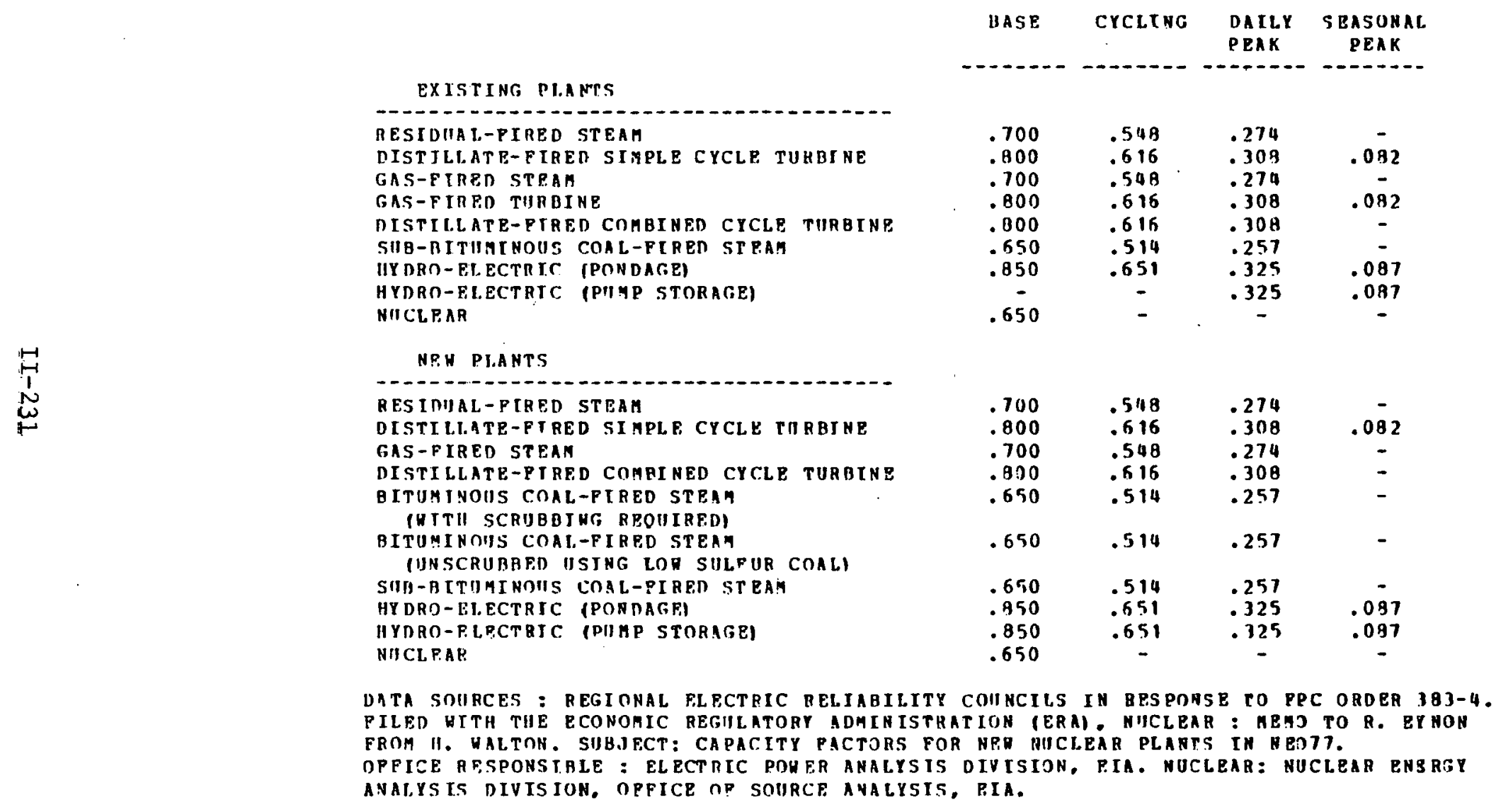


TABL 2. BELOA SHOHS RXISTLNA CAPACITI POB BACH PLANT TYPE IN EACH DOB ARGIDN. TABLP. 3 SHOUS THP RXISTING; CAPACITIES WHICH MAY BE CONVERTEN EO ALTRRATE PUEL TYPES AS GELL AS TIR MANDATED CONYPRSIONS ACCORDING TO THE BH BRTY SUPPLY AND EUVIRONAPNTAL COORDIHATION ACT (ESECAI AS OP JTHE 30.1977.

TABLE 2: RYISTIHG CAPACITY (MEGAHATTSI *

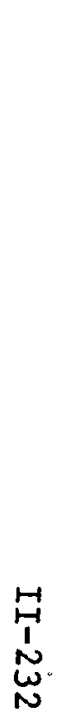

PLANT TYPE

AESI DIIAL, - FIRBD STBA.

TIMLL CYCLE TUBBINE 1276

DISTTLLATE-PIAF.D

GAS-FIRED CDRBINE

GAS- FIRED CORBINE

PIRED STEAH

IUHSCRUBGED ISTIG LOH SULPAB COAL)

BITUIINOIS COAL-PIREO STEAT

IUITH SCAUBAIHG BPONIAEDI

BITUNTNOIIS COAL-PIRED STEAH

(HITH NO SCRUBBING REOUIRETI

SUB-BITURIDOUS COAL-PIRED STEAM

LIGNITE COHL-FIBED STEA

HYDRO-PL F.CTRIC POONDAGEI

MYORO-ELRCFRIC (PUMP STORAGP.)

NIICLEAR

REGIONAL AND MATIONAL, TOTALS

- AS of $1 ; 1 / 78$

DATA SOIACES : REGIONAL ELECTRIC RELIABII. ITY COUNCILS IN BESPONSE TO PPC ORDER 383-4.

PILPD WITH THF PCONOYIC REgIILATORY ANMINLSTRATION (RRA).

NICLEAR : HEMO TO D. HULETT AND H. HALTON FRON R.F. CLARK AND A. REYNOLOS DATED $12 / 5 / 77$

SUBJECT: NICLPAR POHER PLANT BIJLD LIMITS AND CAPLTAL COSTS POR PIES. ATTACHMENT IA.

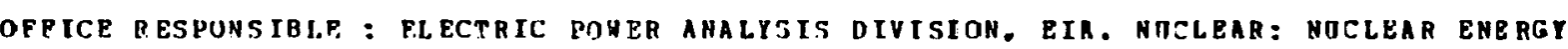

ANALYSIS DIVISION, OPFICE, OR SOURCE ANALYSIS, RTA.

\begin{tabular}{rr}
10 & [OTAL \\
\hdashline 204 & 102202 \\
960 & 39145 \\
612 & 5153 \\
35 & 65411 \\
50 & 5731 \\
& 22687 \\
& \\
- & 8558 \\
- & 132697 \\
& \\
1300 & 25825 \\
- & 7037 \\
23861 & 61491 \\
100 & 10355 \\
1130 & 165073 \\
\hline 565041064710
\end{tabular}


TABI. P. 3: P.TISTING CAPACITIES HHIC! haY BE COHYERTED

TO BURN ALTERNATE FUP.L TTPES

\begin{tabular}{|c|c|c|c|c|c|c|c|c|c|c|c|}
\hline PLANT TYPR. & & & & D.) & & PCION & & & & & \\
\hline & 1 & 2 & 3 & 4 & 5 & h & 7 & B & 9 & 10 & TOTALS \\
\hline PESIJUAL-PIRFD STEAM PLANTS UIICH & $\cdots--$ & $\cdots$ & $\cdots-$ & $\ldots-$. & $\cdots$ & $\ldots-$ & & ---- & & & \\
\hline $\begin{array}{l}\text { MUST BR CONVERTBN TO COAT. } \\
\text { GAS-PIRED STPAN PI.ANTS WHICH }\end{array}$ & 2490 & 6822 & 1061 & 1794 & 656 & - & 46 & $6 H$ & - & - & $\cdot 159.17$ \\
\hline $\begin{array}{l}\text { CAN BE CONVFATED TO RESIDMAL } \\
\text { RESIDUAL-PIRED STEAM WII ICII CAN }\end{array}$ & - & - & - & 1764 & 68 & 45958 & 2700 & 168 & 744 & 35 & 51147 \\
\hline $\begin{array}{l}\text { BE CONNUERTER TO GAS } \\
\text { GAS-FIRED TURBINFS WHCF }\end{array}$ & 18.5 & 1539 & 443 & 6513 & $2.28 A$ & 1578 & 231 & 12.3 & 22936 & 53 & З НАด? \\
\hline $\begin{array}{l}\text { CAN BE CONVERTED TO NISTILLATE. } \\
\text { DISTTLLATE-PIRED THRBTES WICH }\end{array}$ & - & - & - & 2396 & 629 & 327 & 5.31 & 31 & 59 & 50 & 4022 \\
\hline $\begin{array}{l}\text { CAN AE CONVERTF TO GIS } \\
\text { GAS-PINED STEAn PI.ANTS WHICII }\end{array}$ & 291 & 3208 & 386 & 2851 & 2610 & 106 & 616 & 314 & 2199 & 256 & 13037 \\
\hline 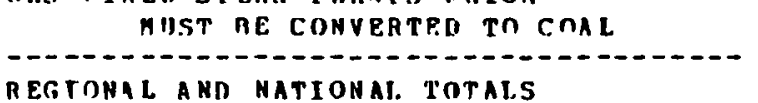 & 2964 & 14569 & 4992 & 15318 & 6250 & $\begin{array}{r}- \\
-27979\end{array}$ & $\begin{array}{r}685 \\
\hdashline 009\end{array}$ & $\frac{-}{104}$ & $\overline{26138}$ & 394 & $\begin{array}{r}695 \\
---0- \\
124017\end{array}$ \\
\hline
\end{tabular}

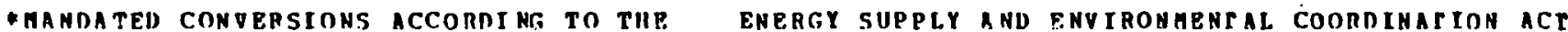
(ESECA) AS OP JIHP. 10. 1977.

DATA SONRCP : OPPICE OP COAL UTILILATION.

OFPICE R ZSPOHSIBLE : ELECTRIC POWER ANALYSIS DIVISIOH, EIA

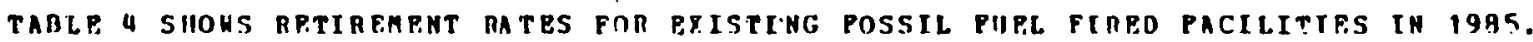
POSSIL PIIP. PLANTSCOAL, OTL AND GAS PIRED FACILTTIES:

TABLE 4: RFTIREKENT RATPS POP FOSSTI, FIIEL FIRPA PLANTS (PBHCENTI

YEAR

$\overrightarrow{1985}$

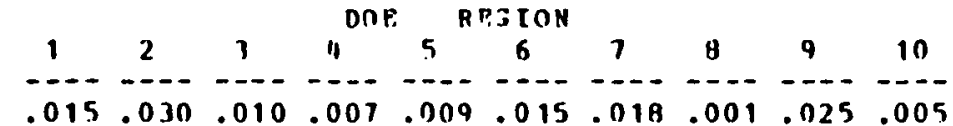

OATA SOIIRCE : OPDER 393, RPPORT BT THD REGTONAL PLPCTRIC RELIABILITY COUNCILS

IN RESPANSE TO PPC ORDER 383-4. PILED WITII THE ECONORIC REGIILATORY ADMIMISFATION IEAAI PRO.IECTRD ADDITIONS AND RETIRFMFNTS.

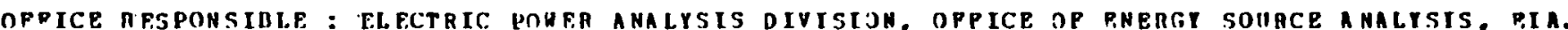




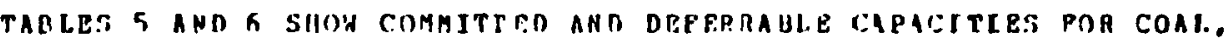
RESTDUAL DIST ILLATE. NATHBAT GAS AHI NIICIEAII PLANPS. TA3LET

SHOHS HIIIID LIAITS POR NIICI,PAR. COAL. HPSIDIAL ANO IYDHO-ELECTAIZ PLANTS.

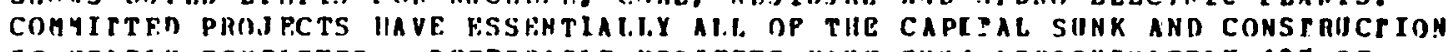

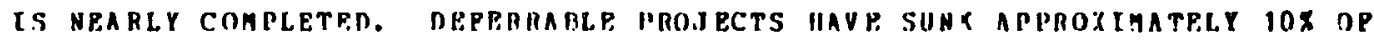

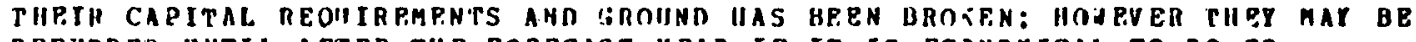
DEFERRED IINTL A PTFR TIIE FORECAST YEAR IP IT IS EC.JHONCCAL TO DO SO.

NFH PROJECTS IIAVE NO CAPITAI. SIINK, DIIT THEY MAY HATE ENTER PD TIIP PLANMING STAGR. SINCE AI.L NICLEAR PLANTS TIIAT WOULD BE CORPLETE F.Y 1945 IIAVE

ALRP.ATY BPEN AFIIISTERED UITI THP U.S. NICLEAR REGNI.RTORY COMASSSION, THERE ARE ASSOCIATED I.TSTS IN THP. MODEL. THE LIAITS POR NUClgar CAPACITy IN 1990. FOR COAL-FIRPD STPAM IN IOAS. FIR RESINIIAL-PIRED STEAT AND UYDKO-ELPCPRIC IN 1985 AND 1990 ARF. PSTIMATEN BOH HDS ON THP GROUTI OP NUCLFAR, COAL-PIRED STEAH. RESIDJALFIRED STPAM AND HYDHO-EI, PCTRTC CAPACTTY, RESPFCTIVELY. ALL OT IFR PLANT TYPES HAYZ HO I.IMITS FOR 1985 AND 1990 BFCA IISP THOSF. YFARS ARE DEYONA TIIETR PLANMING CYCLES. PXCBPT.

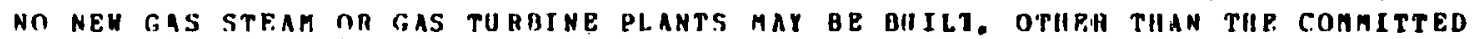
PLANTS SHOWN IN TABLP 5 .

TABIF 5: COMMTTTED* CAPACITY FOR 1985

(MPGANATSS)

\begin{tabular}{|c|c|c|c|c|c|c|c|c|c|c|c|}
\hline PI,ANT TYPE & & & & & $\mathbf{E}$ & ; I r.N & & & & & \\
\hline & 1 & 2. & 3 & 4 & 5 & 6 & 7 & \$ & 9 & 10 & rotaLs \\
\hline $\begin{array}{l}n_{1} \\
n_{1}\end{array}$ & & & $-\cdots-$ & & $-\cdots$ & & & & & & \\
\hline NIICLEAR & - & 2001 & 5925 & $1 \mathrm{J226}$ & 7579 & 3227 & - & 330 & 4470 & 1103 & 37861 \\
\hline RESIDIAL-FI'HED STEAY & 600 & BSO & - & 688 & 2.340 & 480 & - & - & 292 & 198 & 5448 \\
\hline $\begin{array}{l}\text { ATTUMINOUS COAL-EIRED STEAH } \\
\text { (HITHOUT SCRUGREUS) }\end{array}$ & - & - & 400 & 1532 & 1892 & - & 1250 & 600 & 1200 & - & 6874 \\
\hline BITIMINOIS COAL-FIRED STEAM & - & - & 2077 & 2905 & 4953 & - & - & - & - & - & 98.35 \\
\hline (HETH SCHUBBERS) & & & & & & & & & & & \\
\hline SUB-HITIMINUISS COAL-PIRED STEAH & - & - & - & - & 897 & 4541 & 2066 & 2090 & - & - & 9594 \\
\hline I. TGNITE COAL-PINED STEAM & - & - & - & - & 641 & 25115 & - & 339 & - & - & 3665 \\
\hline DISTIILATP-FIRBD SIMPLE CYCLF TURBI NE & 6 & - & 200 & 300 & 405 & 239 & 770 & 246 & 199 & - & 2.372 \\
\hline DISTILLATE-PIRBD COMBTHED CYCLP TURBTHE & 230 & - & - & - & - & - & - & - & 809 & - & 1039 \\
\hline GAS-PIRED STEAM & - & - & - & - & - - & 550 & - & - & - & - & 550 \\
\hline REGIONAL AND NATIONAL TOTALS & 836 & $---\overline{1}$ & 8602 & 18651 & 13607 & 11722 & 4094 & 3605 & 6969 & 1301 & 77238 \\
\hline
\end{tabular}

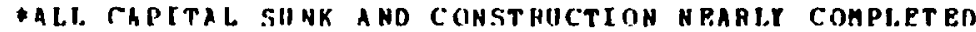

DATA SOHACES POA TABIES 5.6 .7 .8 .9 :

FORH 4 IHONTIIY PUBLICATIONI GENERATION OP ENERGY. CONSUHPTION OP ENPRGY AND FIIEL. STOCKS. PILEO YITH PRA. DATA FOR SCRIBBEIIS DPRIVED PROA THE P.NVTRONA RATAL PBOTECTION AGFACY STANARRDS POR ALL COAL PLANTS.

PRODIETION PICIINES AMP. TAKF.N PAOM THP. PIES OIL. AND GAS HODGL WHICII USES OATA

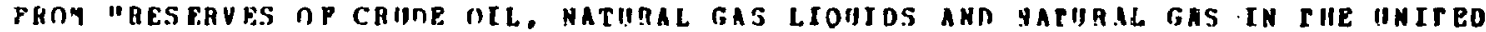
STATES AND CANADA AND CAPACITY AS OP DECEMBER 31, 19J4", ATER IZAH PETROLEIJA INSTTTUTF. WASHIHGTON D.C.

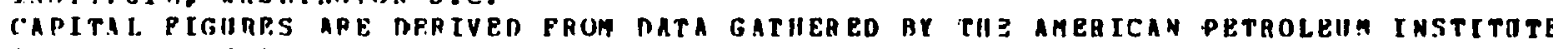
ANNIAL PIIBLCATION. "JOINT ASSOCIATION SIIRVEY OP THE A.S DIL ANO GAS PRODICING INIISTPY FOH 1975". TIIESE DATA APQ IISED AS INPUT POR IHE PIES OTL ANO SAS HODEL.

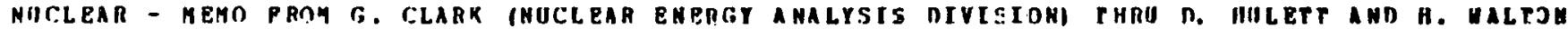

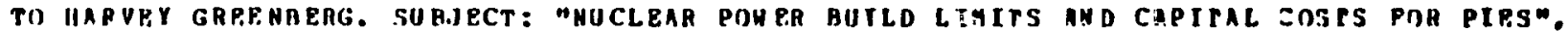
IINDATED BIIT C. 12/5/77, RPVISFD 1/13/7H. THE MUCLEAR EMRBSY ANALYSIS DIVISION OP

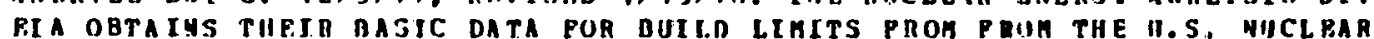
HERULATORY COMNISSIOA. 
TABLP, 6: DEFERRABLE * CAPACITY POR 1985 (HETA MATTS)

\begin{tabular}{|c|c|c|c|c|c|c|c|c|c|c|c|}
\hline PLANT TYPB & & & & & RE & ON & & & & & \\
\hline & 1 & 2 & $\mathbf{3}$ & 4 & 5 & 6 & 7 & $\mathbf{\theta}$ & 9 & 10 & PCITALS \\
\hline A IICLPAB & & 1067 & 3065 & 1145 & 4154 & 3.367 & & & 340 & 1267 & \\
\hline BITUM INOUS COAL-PTREN STEAM & - & - & 1300 & 2710 & & & & 40 & 700 & - & \\
\hline $\begin{array}{l}\text { SMP-RITUNINOUS COAL-PIGED STEAM } \\
\text { LIGNTTE COAL-PIRED STEAM }\end{array}$ & - & - & - & - & $\begin{array}{l}590 \\
556\end{array}$ & $\begin{array}{l}6346 \\
1978\end{array}$ & 1910 & $\begin{array}{r}2780 \\
294\end{array}$ & - & 500 & $\begin{array}{r}12116 \\
2828\end{array}$ \\
\hline EGIONAL AND NATIONAL TOTALS & - & 1067 & 4365 & 3855 & 7433 & $\overline{12791}$ & 4335 & 3474 & 1040 & 1767 & 40127 \\
\hline
\end{tabular}

- APPROXI GATELY 10\% OF CAPITAL SINK.GROIND IIAS

BEEN BROKEY AND PLANT SEHPDOLED TO OPERATE

AY 19.85

TABLE 7: REM PLANT CAPACITY LIATS (1985)

(HEGanatTS)

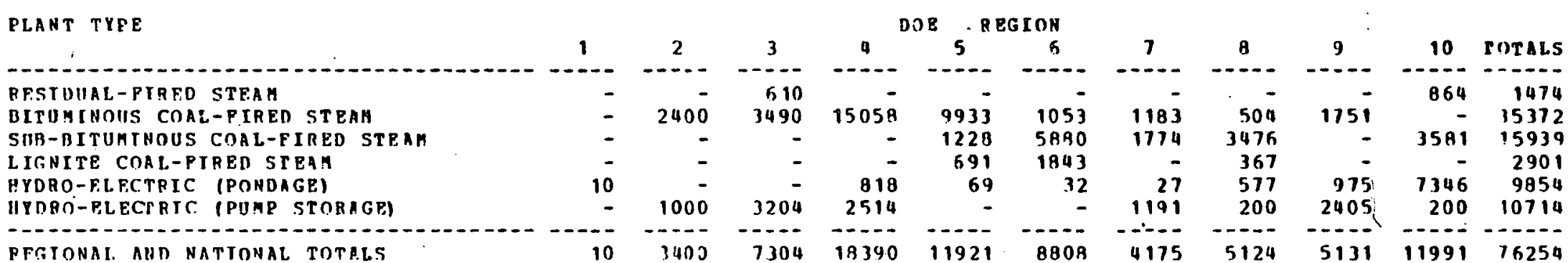




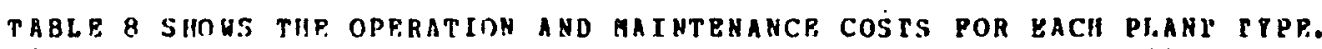
TABLE 9 SHOHS AEVPNUE REOII IREHENTS PUR EXISTING FOUTPHEAT. TADLE 10

SHOKS THE CAPITAL COSTS POR NEW PI.ANIS IN 19A5. TABLES 11 AHD 12 SUON ASSOCIATBD CHARGE PACTORS. THP REVENIIE REPIIIREMENT CHALGE PACTOR IS USED IN THE RATE BASE

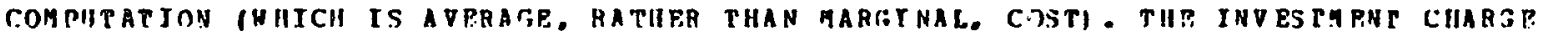
FACTOR IS USPD IN DRTERMINING TIL DYCTSION OP HHICH PLANT IS TO BP BUILP IP MORE

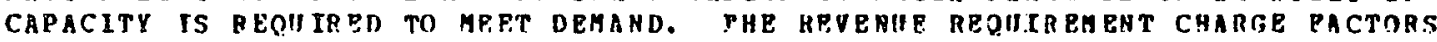
ARE REPORTAD BY REGION. BPCAIISE THEST. CIIARGR FACTORS ARP BASED ON ATERAGE COSTS OF PRODHCING BI.ECTRICITY, RATHF, TIIAN THE MABGINAJ. COSTS POR ANY PART ICILAR PLANT TYPE. ON THE OTHER IAND. THE INV BSTHENT CHARGE IS PF.POATED BY PLANT TY PE BECAUS P OP THP DIPPERPNT [NCOMP TAX TAEATARAT FACII PLANT TYPE RECRIYPS UNDFR TIIE IRS CODF. TAULE 13 SHOHS TRANSAISSION AND DISTRIBITION COSTS AS WRLI. AS EPPICIENCY BATPS RE.G. IN RRTYON I POR RVPRY KILOYATT GENERATED. OHLY. 914 KILORATTS IS DISTRIBUTP.D.

TABLE R: OPERATION AND MAINTENANCE COSTS 1975 ATLLS PER KILOUATT ROII

\begin{tabular}{|c|c|}
\hline PLANT TTPP & DE, \\
\hline RPSIDIIAL-PIRPD STEAH & .90 \\
\hline 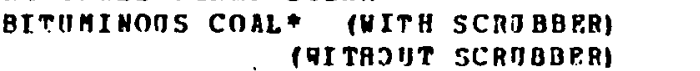 & $\begin{array}{l}2.80 \\
1.10\end{array}$ \\
\hline $\begin{array}{l}\text { SUB-BITUMTBOUS COAL* (DITH SCRIJBBER) } \\
\text { (UITHOUT SCHIBBER) }\end{array}$ & $\begin{array}{l}2.00 \\
1.10\end{array}$ \\
\hline $\begin{array}{r}\text { LIGNITP. COAL*** (HITH SCRIIBBER) } \\
\text { (WITTIOUT SCRIBBPR) }\end{array}$ & $\begin{array}{l}2.00 \\
1.10\end{array}$ \\
\hline $\begin{array}{l}\text { DISTILLATR-PIREN SIMPI.E CYCLE } \\
\text { DISTILLATE-PIAED COABINED CYCLE }\end{array}$ & $\begin{array}{l}2.75 \\
1.25\end{array}$ \\
\hline GAS-FIFFD TURAINE & 2.75 \\
\hline GAS-P IRED STEAM & .50 \\
\hline IVORO-RI.FCTRIC (PONDAGR) & .70 \\
\hline $\begin{array}{l}\text { UYYRO-ELFCTRIC (PUSP STORAFE) } \\
\text { HUCI.EAR }\end{array}$ & $\begin{array}{r}.70 \\
1.65\end{array}$ \\
\hline
\end{tabular}

* hitiminous plants hat ae scribaed or unscribaed R.YCEPT POR NEN PLANTS J.N DOE REGTONS H. 9 M.ND 10 HIIICH MIIST BE SCAUBBF.D AND IJSP. LOW SULOEIIR COAL

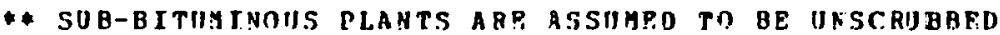
F.XCEPT FOR NEA PLANTS IN DNE REGIONS B, 9 AND 10 HHICH ARE ASSUHED TO DP SCRIBBE?

* * NPG lignitf: plants arf. assumed to be scrilbBed:

WHLE FXISTING PLANTS ARP ASSHAED TO BR UNSCRIBDRD 
TABLE 9: REVENUE REQTIREIAENTS IN 1985 FOR EXISTINI ISSETS

DOE REGION

1
2
3
4
5
6
7
6
9
10

TABLE 10: CADITAL COSTS OP NRU PLANTS IN 1985

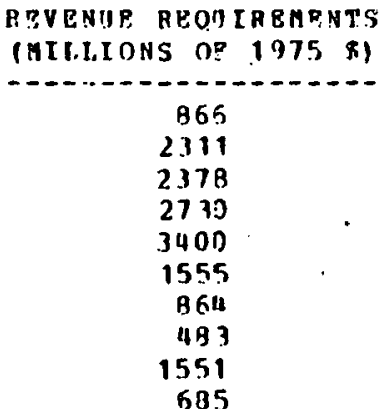

PLANT TYPE

RESTDIIAL-FIRED STEAM

BETTIAL-PIRED STBAM

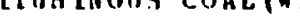

SIIB-BITIIINOUS COAL

I. IrinITP COAL

DISTILLATE-PIRED SIMPLF, CYCLE

DTSTTLLATE-FTRED COMBINED CYCLE

GAS-PIREA STPAM

HYDR()-FI. F.CTRIC (PONARGE)

HYDRO-ELPCTRIC. IPUMP STORAFEI

N!)CLEAP (DEPERAABLE)

1975 DOLLARS PRA KILOMATT)

* Capitat. cost of defferrabie coal plants ls . go of neh plant costs

NOTR: RETROPITTING, AN EXISTING BITHAINOUS COAL PLANT ISING HIGH SIIFIUR COAI. IN BASELOAD COSTS 8131 PER KLLOHATT.

DATA SOURCPS : ANNUAL REPORT FOR DETATLEO PINANCTAL AND OPERATING DAPA

SIBBITTED BY ALL CLASS A AND B PRIVAT, ELECTBIE ITILITIBS. PILBD NITH ETA. PPE PUBLICATION,

PRODICTION PIGURFS ARE TAKEN PROM THE PIES OIL AND JAS HODEL UHICH USES DATA

PROM "RESERVES OP CRIDE OIL. MATI RAL GAS ILIOIIDS AND HATIIRAL GAS IN THE UNITPD STATRS

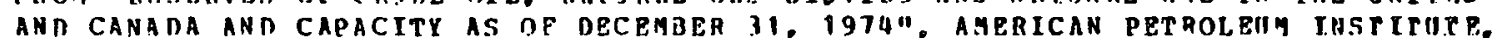

WA SHI INGTON, D. C.

CAPITAL PICIIRES ARE DERIURD FIOA DATA TATHERED BY THE AMPRICAN PETROLFI A INSTIPOTE

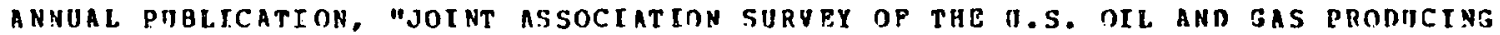

INDIISCRY FOR 1975". TIESF. DATA ARE USED AS INPIJ FOR THE PIBS OIL AND GAS HODBL. 
TART.E 11: CAPITAL CHARGP RATES (PEACENTI POB GEVEHUE REOUTREMPATS

CAPITAL CHARGE UATES PJP REV RNIIS REOII IREAENTS

\begin{tabular}{cr} 
DDF REGION & 1995 \\
\hline 1 & 15.6 \\
2 & 18.8 \\
3 & 14.2 \\
4 & 11.2 \\
5 & 14.7 \\
6 & 13.5 \\
7 & 14.3 \\
8 & 11.1 \\
9 & 13.1 \\
10 & 7.2
\end{tabular}

DATA SOURCZS : STIDY CONDICTED AY THE OAKRIDGE NATIONAL LABORATORIES USING THB COYPUTER HONEL CONCEPT

NUCLEAR: HFMO PROA G. CLARK TO H. GREENBERG DATED 5/24/7?

PRODICTION PIGIBRES ARE TAKPN PROM TIE PIES OIL AND GAS MODEL HHICH ISES DATA

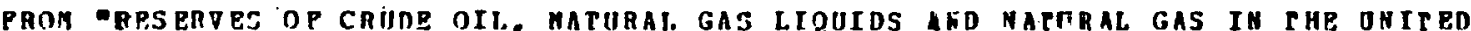

STATES AND CANADA AND CAPACITY AS DF DRCEYBER 31. 1974n LGERCAN PETROLEUM INSTETUTE

WASHENGTON. D.C.

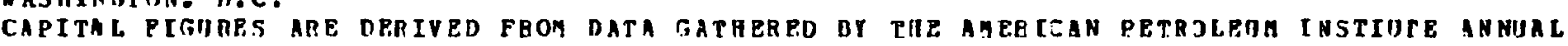

PUBL FCATION, "JOINT ASSOCIATION SURVFY OP TKB I. S. OTL AED GAS PRODUCING INDUSTRT POR I975". THESE JATA ARE IISEA AS THPUT EOR THE PIPS OTI. AND GIS HOLEL. 
TABLE 12: CAPITAL CIIARGE RATES POR INVPGTMETT

DECISTONS IANAIAL. PERCENTI

\section{PLANT TYPF}

RP.S ITUAL-PIREA STEAM

BITUMINOISS COAL-FIRED STEA

(HITH SCR HBBER)

BITIITH NOIS COAL-FIRED STEAM

(H J THOIIT SCRUBBER)

DISTILLATE-PIRED SIMPI.B CYCLE TIIABINE

DISTILIATR-FTREA CORBINED CYCLE TJRBINB

GAS-EIRED STP.AM

II YDRO

SUB-BITIMINOIS COAL-PIRED STEAM

LITNITE COAL-PIREN STPAM

HIICLEAR

DATA SOIIRCES : STIDT CONDIICTED BY TILP OAKRING? NATIONAL LABORATORIES IISTNG THE

COMPUTER HODEL. CONCEPT 4

NICLEAR: MEMO FROM G. CLARK TO II. GREENAERG DATEL 5/24/77

PRODICTIOR FIGIIRES ARE TAKP,N PRDM TIE, DIRS OIL AND GAS MODRL YHICH ISES DATA

PROM "RPSERVES OF CAIIDE OIL. NATIRAL GAS LIOUIDS AND NATURAL GAS IN THE UNITED

STATES AN T CANADA AND CAPACITY AS OF DPCEMBPR 31, $1974 "$ ANBRICAN PPIROLEUM INSTITUTE

HASH THETON. D. C.

CAPITAL FIGURES ARE DERTIED PROA DATA. SATHERED BY THF AMPRICAN PETROLEUG INSTLUPE ANNIAL

PIBLTCATION, "JOINT ASS ACIATION SURV BY OP THR U.S. OTL AND GAS PRODUCING INDISTRT POR 1975". THESE DATA AHE USED AS INPIIT POR THE PLES OIL AND GAS MONEL.
CAPITAL CIIARGE RATE rOR

11.4

11.4 
TABLE 13: TRAISMISSION AND DISTRIBITCON DAEA

DOE RPGION

1
2
3
4
5
6
7
8
9
10

AVFRAG: ZPPICIENCY RATE (PERCENTAGE)

91.40
91.80
92.40
91.40
92.30
92.50
91.20
88.30
90.80
91.00

MPERATION AN MATHERANCE COST (MILLS/KILOHATT-HOTR) (M. 5.7 4.7 4.0
4.3
4.6 4.6 4.6
5.5
5.6 5. 3 5.6
CAPITAL cost

(\$/KILONATT)

493
626
304
262
361
228
154
309
514
279

DATA SOIIRCPS : AJUIAL BPPOST POR DETAILED FINANCIAL AND OPERATING DATA

SHBHTTED BY ALL CIASS A AND B PRIVARP PLECTRIC UTILTIES. PTLED RITH EIA PPC PIIALICATION. PRODICTION FIGIIRES ARE TAKEN PROA THE PIRS OIL AND GAS MODEL HHICH ISES DATA

PROM "RESFRVES OF CRIIDE OIL. HATURAL GAS LIOIIDS AND NATURAL GAS IN THE INITBD STATPS AND CANADA AND CAPACITY AS OP DECERBER 31. 1974". AMERTCAN PPTROLPISA TNSTITUTE. WA SHI NGTON, D. C.

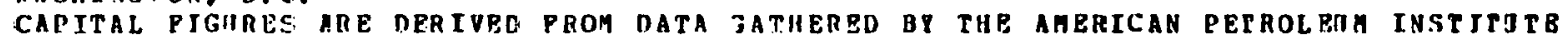

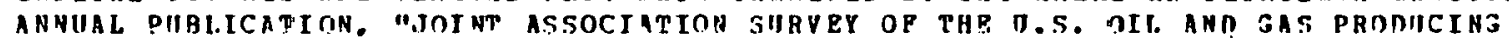
INNUSFGY FOR 1975". THESE TATA ARE USPD AS INPUT POR THE PIRS OII. AND GAS MODEL.

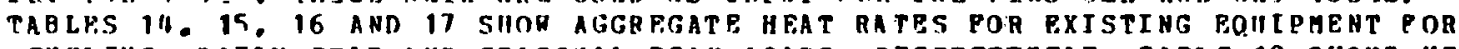

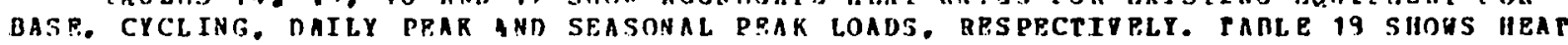
RATES* FOR NPH FACILITIES ISAME POR EACII REgIONI. AND TABLB 19 SHJHS HEAT VALUES FOR FOSSIL PIIEI.S. THE RATIO OP IIFAT PATF TO HEAT VALIE DETENHINPS THR AHOUNT OP PIIEL UPEDPD TO GENERATE OHP K ILOUATT SPFCIPTCALLP THP. PUEL HEOIIIRPD TO OPEAATE

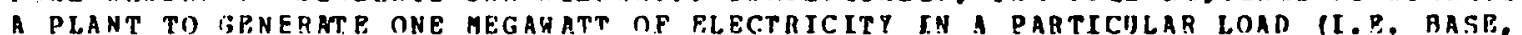
CYCLING, DATLY PEAR OR SPASONAL PEAKI IS:

PIEL REOIIREAPNT = (HFAT RATE)/(HEAT VALUE)

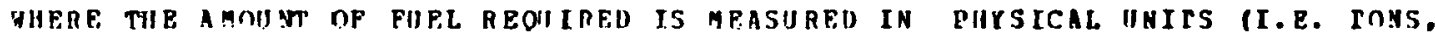
BARRELS. THOIISANDS OP STANDABD CUUIC FFRT. ETC.I

TABLE 20 SHOHS NUELEA FIIEL SUPPLY nATA.

* the heat rate is the enehgy reouigen pri hOIR of operation. WHERE FNERGY IS MEASURED BY RRITTSII THERHAL INITS (BTII 
PLANT TYPP.

\section{RESIDIIAL-PIRED ST PAF}

DISTILLATE-FIRPD SIMUTE CYCL NIST TLLATE-PIRED COMBINF.D CYCLE GAS-PIRED STEAM

GAS - FTRPD TIRBIN

BITUAINIIS COAL (HITH SCPUBUER)

BITUMTNOUS COAL (HITAOUT SCAIBQER

SUB-BITUAINOUS COAL

I. IGNITR

\begin{tabular}{|c|c|c|c|c|c|c|c|c|c|}
\hline & & & $D O E$ & REC & ION & & & & \\
\hline 1 & 2 & 3 & 4 & 5 & 6 & 7 & 8 & 9 & 10 \\
\hline & $\cdots$ & $-\cdots$ & $\cdots$ & $\cdots$ & $-\infty+$ & - & $\cdots-$ & & \\
\hline $\begin{array}{l}10000 \\
1451) 0\end{array}$ & $\begin{array}{l}10300 \\
14600\end{array}$ & $\begin{array}{l}10700 \\
12530\end{array}$ & $\begin{array}{l}10000 \\
13000\end{array}$ & & $\begin{array}{l}10300 \\
12800\end{array}$ & & & & \\
\hline 8500 & R500 & 8500 & 8500 & 8500 & 8500 & & 19000 & & \\
\hline 12500 & 10900 & 10300 & 11100 & 13400 & 10100 & 11300 & 11900 & 10400 & \\
\hline 14000 & 15400 & 14300 & 16500 & 14700 & 13000 & 14700 & 12200 & 13700 & \\
\hline 10510 & 11000 & 10300 & 10.300 & 10500 & - & 10900 & 11500 & 10700 & - \\
\hline 10110 & 10500 & 9930 & 9900 & 10200 & - & 10 & 112 & 10.300 & - \\
\hline & - & - & - & 10300 & 10100 & 10 & & - & 10700 \\
\hline- & - & - & - & 12400 & 10500 & & 11900 & - & \\
\hline
\end{tabular}

DATA SOIRCES : COMPUTER MODPL. DEVELOPPD EY ROBERT BORLTCK ANO DAUID GATCHER IELCCTRIC PONER ANALYSIS DIVISION OP F.IA)

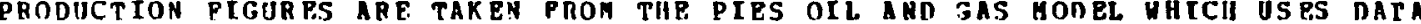
PRDM "RESERUES OP CRIIDE OLL. NATIBAL GAS LLOUIDS AND NATIRAL GAS IN THP ONITPO STATES

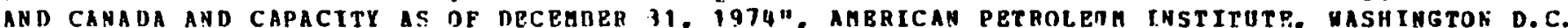
CAPITAL. FIGIIRES ARE DERIVFD FROA DATA GATUPRED OT THE AMPRICAN PPRROLEOM INSTIPJTE

ANNIIL PUBLICATIOH, "JOINT ASSOCIATION SURVEY OP THE O.S. OIL AND GAS PBNDIJCING INDUSTRY POR 1975". THESE DATA ARP. USEN AS INPUT TO THE PIES OLL AND GAS NODEL.

-ABLE 15: HEAT RATES POR EX ISTTHG EOUTPGENT OPPRATEN IN CYCLIHJ LOAD

(RTI) PER KTLOHATT-HOUR)

PLANT TYPE

RESTDIIAL- FIRED STBAH

DTSTILLATE-EIRED SIMDLP. CYCLP

DISTILLATF-FJRED COH 3 INED CYCLE

GAS-PIRED STRAM

GAS-PIRFN TURBIN

BTTUMINOHS COAL. (HITH SCBUBBER)

HITUMINOUS COAL IHITHOUT SCR!JBBPI

SHB-BITUMINOUS COAL

LIGNITE

\begin{tabular}{cccccccccc}
1 & 2 & 1 & 4 & 5 & 6 & 7 & 8 & 9 & 10 \\
\hline 10500 & 10800 & 11200 & 10500 & 12600 & 10800 & 11700 & 15000 & 10300 & 11500 \\
14500 & 15100 & 13000 & 13500 & 15000 & 13300 & 13000 & 12500 & 16200 & 12500 \\
9000 & 9000 & 9000 & 9000 & 9000 & 9000 & 9000 & - & 9000 & 9300 \\
13000 & 11400 & 11400 & 11600 & 13900 & 10600 & 11800 & 12430 & 10900 & 12300 \\
14500 & 15900 & 14830 & 17000 & 15200 & 13500 & 15200 & 12700 & 14200 & 13100 \\
11000 & 11500 & 10800 & 10900 & 11000 & - & 11400 & 12130 & 11200 & - \\
$101, i) 0$ & 11900 & 10400 & 10400 & 10700 & - & 11000 & 11700 & 10800 & - \\
- & - & - & - & 10800 & 10500 & 11100 & 11200 & - & 11200 \\
- & - & - & - & 12900 & 11000 & - & 12400 & - & -
\end{tabular}

DATA SOURCES : FINANCIAL STATEAENT, ANNIIL REPORT. PPC PIORM 1

PRODTCTION PIGURES ARP TAKEN FROM THE PIES OLL AND GAS MODEL HHTEH USPS DATA

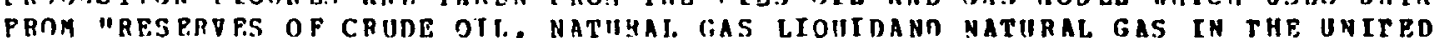

STATES AND CANADA AND CAPACIYY AS OP DFCEMRPR 31. 1974". AMERICAN PRTROLBUE INSTITURB HASHTNGTON, D.C.

CAPITAL PIGURPS ARE DFRIVED FROM DATA GATIIFRED BY THE AMERICAN PETROLPIIM INSTITUTE

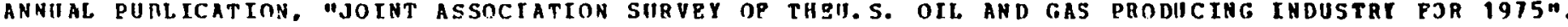
THESE DATA ARE ISED A.S INEUT FOR. THF. PIES OIL AND GAS MODEL. 


\begin{tabular}{rccccccccc}
1 & \multicolumn{1}{c}{ DOE } & \multicolumn{1}{c}{$R P G I O N$} & & & \\
1 & 2 & 3 & 14 & 5 & 6 & 7 & 9 & 9 & 10 \\
\hdashline 10500 & 10800 & 11200 & 10500 & 12600 & 10800 & 11700 & 15000 & 10300 & 11500 \\
14500 & 15100 & 13020 & 13500 & 15000 & 13300 & 13000 & 12500 & 16200 & 12500 \\
9000 & 9000 & 9000 & 4000 & 9000 & 9000 & 9000 & - & 9000 & 9300 \\
13000 & 11400 & 11400 & 11600 & 13900 & 10600 & 11800 & 12400 & 10900 & 12300 \\
11500 & 15900 & 14900 & 17000 & 15200 & 13500 & 15200 & 12700 & 14200 & 13100 \\
11000 & 11500 & 10900 & 10900 & 11000 & - & 11400 & 12100 & 11200 & - \\
10600 & 11000 & 10400 & 101100 & 10700 & - & 11000 & 11700 & 10900 & - \\
- & - & - & - & 10800 & 10600 & 11100 & 11200 & - & 11200 \\
- & - & - & - & 12900 & 11000 & - & 12400 & - & -
\end{tabular}

RES IDIIAL -FI RED STRAM

SIMPLP CYCLA

DISTILLATE-FIRED COMBINED CYCLP

GAS-PIRED STR.AY

GAS-FIREA TURBINP.

BITIMINOUS COAL IHITH SCRIBBERI

BITUMINOUS COAL IWITHOUT SCRIOBERI

SIIB-DITI:MINOUS COAL

LIGNITP.

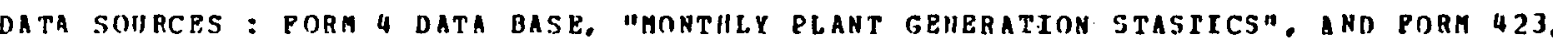
"COST AND QHAT. ITY OP PIIEL DELIVEREO". BOTH ARE PEDERAL POWRR COMHISSION PUBLICATIOHS. MOST MOST RECFNT ISSIJES USFD POR EIA ANNUAL ADHINISTRATOR'S REPORT.

PRODUCTION PIGIIRES ARE TAKEN FAOH THE PIES OLL AND GAS MODEL OHICH USES DATA PROA

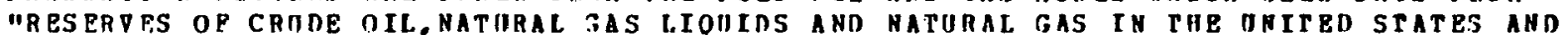

CANADA ANO CAPACITY AS OP DECPMABR 31, 1974". AMPRICAN PETROLEUI INSTITUTE. HASTINGTON D.C. CAPITAL PIGIIRES ARE DFRIVED PROM DATA GATHERPO BY TIL PROM DATA GAFHERED BY THE AMERICAN PRTROLEIM INSTITUTE ANNIIAI. PUBLICACLON "JOINT ASSOCIATION SURVEP OP THE OLL AND GAS PRODICING INDUSTRY POR 19\%". TIIFSE DATA ARE OSBD AS INPOF POR THE PIBS OIL AND GAS MODEL.

TABLE 17: HEAT RATES POR EXISTING ROUTPAENT OPP.RATED IN SEASTRAL PBAR LOAD (RTII PFR KILOHATT-HOIRI

PLANT TYPE

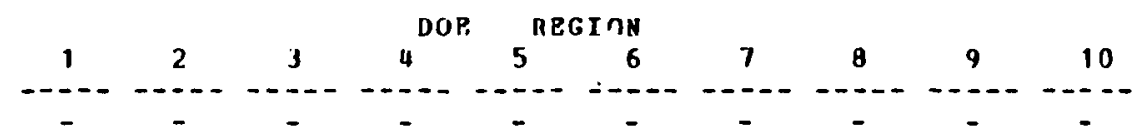

RESTDIIAL-PTRED STEAM

DISTILIATF-PIREN SIBPLE, CYCLE

DISTILLATE-FIRFD COMBINED CYCLE

GAS-FIRFD STEAM

GAS - FITED TURBINE

BITUMINOOS COAL (WITH SCRIBBRBI

BITUMINMIS COAT. (UTTHOIT SCAIBBERI

SUB-BITUMINDIIS COAI.

I. IAN IT D

DATA SOURCFS : PORM 4 DATA RISE MMONTHLY PLANT GFHPRATION STASTICSN IMD PORM 423

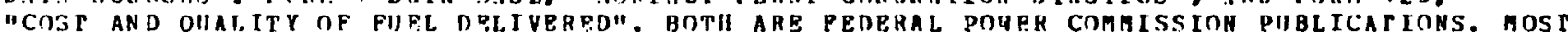
MOST RECENT ISSUES IISED FOR EIA ANNIAL ADATMISTRATOR'S GEPORT.

PRODIETTON FIGISRES ARE TAKPN PROM TUE FIF.S OIL AND GAS MODET. WHTCH OSES DATA PROM

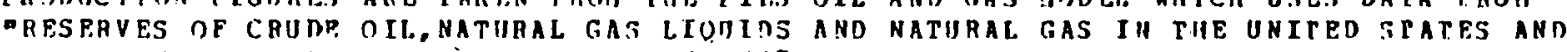

CANADA AND CAPACITY AS OF DECEHBER 31. 1974". ARPRICAN PPTROLEIIT INSTITHTE. HASTINGTOR D.C.

CAPITAL PIGHRFS ARE DERIVED FROM DATA 3ATHERED BY THF. ERDM DATA GATHERPD HE THE

AMERICAN PETROLEIIK TNSTITITE ANNUAL PIINITCATION OJOTNT ASSOCIATION SURVEY OP THE

OIL AND GAS PRODICTNG INDISTRY FOR 1975,". TIESE DATA ARE ISPD AS INPUT FOR THE PIBS

OIL AND GAS MODRL. 
TARLE 1A: HEAT RATES POR NPH EOH IPMPNT

(HILLIONS OP BTII. PEN KLLOWATT)

\section{PLANT TYQF}

RESIDUAL-FIRED STPAA

DISTILLATE-PIRED SIMPLF. CYCL

DISTILLATE- PT RED COMBINPD CYCLP

GAS - FTRED STEAT

GAS-PTRED TIRBI NE

GITUA INOUS CDAL. (HITII SCRIBBER)

ATTUAINOHS COAL (HITHCHT SCRUDBER)

S'JB-BTTUATNO!IS COAL

LIFNITE.

\begin{tabular}{cccc} 
AASE & CYCLING & $\begin{array}{c}\text { DAILY } \\
\text { PEAK }\end{array}$ & $\begin{array}{c}\text { SPASONAL } \\
\text { PEAK }\end{array}$ \\
\hline 9650 & 10300 & 19300 & - \\
10000 & 10500 & 10500 & 10750 \\
7500 & 3000 & 9000 & - \\
10010 & 10750 & 10760 & - \\
10090 & 10500 & 10500 & 10750 \\
9840 & 10300 & 10300 & - \\
9870 & 10350 & 10350 & - \\
10230 & 10710 & 10710 & - \\
10500 & 11000 & 11000 & -
\end{tabular}

10500

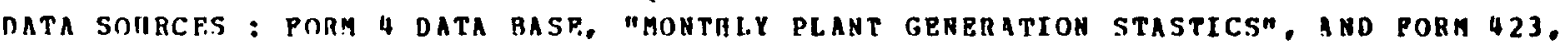

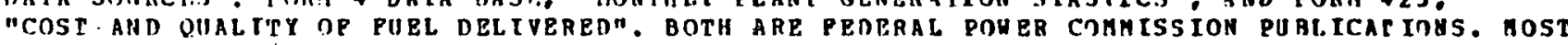
MOST RECPNT ISSUES USED FOR EIA ANNITL ADHINISTIATOR'S RBPORT.

PRODICTION PIGURFS ARE TAKEN PROM THE PIES OIL AND FAS MODEL HIICH OSES DATA PRJA

"RESERVFS OP CRIIDE OIL. NATURAL GAS LIQUIIDS AND NATHGAL GAS IN THE UNITED STRTBS AND

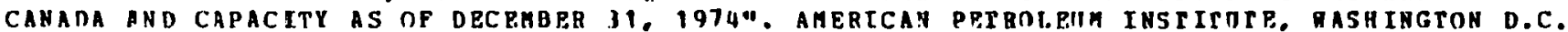
CAPITAL FIGIIRPS ARF. DERIVEN PHOM DATA GATHERPR BT TIIP PROM OATA JATHEBED BT THE

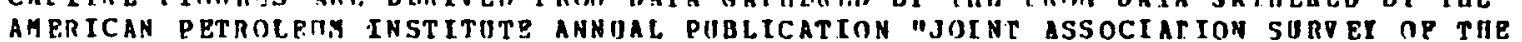

OIL AND GAS PRODICING INDUSTRY FOR 1975". THESE DATA ARE USEN as INPUT FOR THE PIES

OTL AND GAS MODEI.

TABI.E 19: HFAT VALIJES OF FOSSIL POBLS

(AILLIONS OP BTU PER PHYSICAL. INITSI

FII R L

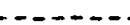

RESINII.L OIL

DISTILIATE OIL

NATIRAL GAS.

COAL

\begin{tabular}{c} 
HEAT VALUE \\
\hline 5.287 \\
5.825 \\
1.032 \\
22.500
\end{tabular}

\section{PIIYSICAL INITS}

BARRELS

BARBELS

THOUSANOS OP STANOART COBIC PRET

TnNS

DATA SOIIRCE : PORM 4 DATA BATF, MMONTHLY PLANT GFNBAATION STASTICS" AND FORM 423

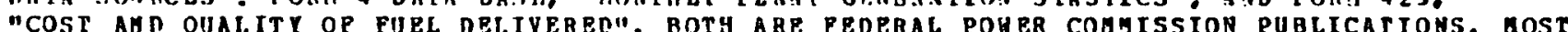
MOST RRCENT ISSURS IJSED POR EIA ANHIAL ADHINISTRATOR'S REPORT.

PRODICTION PIGURFS ARE TAKEN FROM THP PIES OIL AKD GAS MODRL HIICH USES DATA PAOM "RESERV RS OP CAIIDE OIL. NATURAI. GAS LIOUIOS AND NATURAL GAS IN THE ONITED STAPES AND

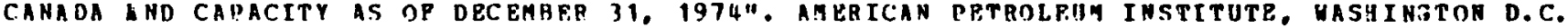
CAPITAL. PIGURES ARE DERIVED PROY DATA 3ATHERPD BY TIL PROA DATA GATHFAED BT THE AMPBICAB PETROLETA INSTITITE AYPUA PIIBL OII. AND GAS PRODUCING INDUSTRY PAB 1975". PHESE DATA ARE USED AS IMPUT FOR THE PIES OIL AN!) GAS MUDEL. 
TABLE 20: NICLEAR PIEL. SIIPPLI CURVR

INCRPMFINTAL

CAPACITY SUPPORTPR (MEGAHATTS)

costs

84.0
17.0

11975 HILLS/KUHI

37.0

DATA SOIRCES : FORM 4 DATA BASE, "KONTHLY PLAMT GEYRRATION STASTICS". ANO PORH 423.

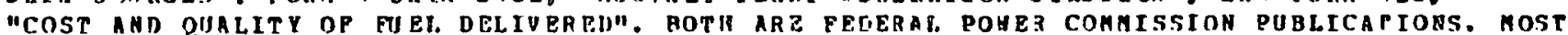
MOST RECENT ISSIIFS USED FOR FIA ANNUAL ADMINISTRITOI'S REPOBT.

PRODOCTTRN PIGURES ARE TAKEN FROM THE PIES OIL ABE EAS RODEL NIICH OSES DATA PROA

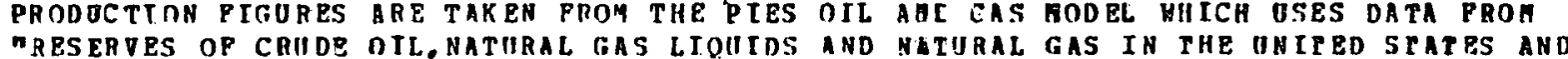

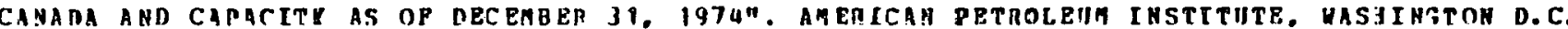

CAPITAL PIGTRES ARE DERIVEN PROM DATA GATHERED BT THR PAOM DATA GAPHERPD BP THP.

AMERICAN PETPOLFUA INSTITITE ANNUAL PUBLICATION MJOINT ASSOCIATIOH SURVEY OP THE

OTL UAD GAS PRODUCING INDIISTRY POR 1975". THESE RATA RRE USEO AS IAPUT POR THR PIES

OT IND GAS MODEL. 
1990 ELECTRIC ITILITIES INPUT DATA

BOA PORECASTS BASED ON

CURAENT LAH

点 
THRRE ALF, FOUR CATEGORIES JP INUUT DATA BLPHENTS:

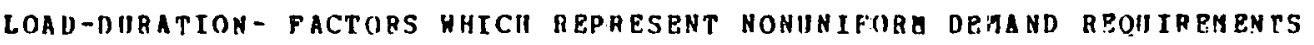

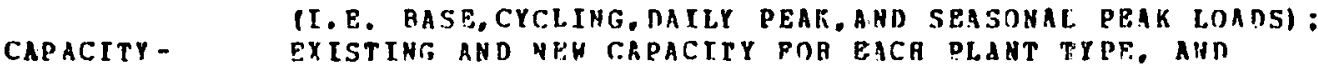

MANDATED CONUERS[IIN;

COST- CAPITAL CHARGES. OPPRATION AND MAINTRIANCR COSTS.

SCRUBBING COSTS. AND PARASAISSIOH AND OLSTR IBITIOH COSTS:

IEAT RATES AND VALUES POR BACH PLANT AND PUBL:

I H MOST CASES THE DATA VARIES BY DOE RPGION. THE DATA RI.EMPNTS,THEREPORE.

AR.E AGGREGATES. COMPIJTPD PROA A PLANT-RY-PLANT (OR PBDJBCT-BY-PROJECT) DATABASE.

TABLP. 1 SIOHS REGIOHAL COMPOSITTON AND CAPACITY PACTORS POR BASB. CYCLIKT,

DAILY PEAK. AND SFASONAL PEAK LOADS AS NEIL AS THF SISTEA LOAD PACTORS II. R. THE RATIO OP AV RBAGP TO PEAK LOAMI. THE PBSERPQ AARGIN IS 20X POR EACH REGION.

CONPOSITION PACTORS ARE THE PRACTIONS OP ELPCT ZIC POHER IN EACH LOAD. POR EXAGPLE. IN REGION 1 OF ALL ELPCTRICITY DEHANDED DURING THE YEAR. 68.7\% IS BASE. 24.7X I.S CYCLING, 4.48 IS DAILY PFAK, AND 2.2\% IS SBASTNAL PBAK. PTrIIRP 1 SHOHS A (GENERIC) LOAN-DURATION CIIRVE WITII TIIE FOUR LOADS SHCINA AS AREAS INDER A LINEAR APEROX IMATION OP TIIE ACTUAL CURVP.

GENERATING FACILITIES ARE CLASSTFIED AS BASE, CYCLIŃ̈, DAILY PRAK OR SEASONAL PEAK DEPENDING DPON THE ORDER IN GIILCH THET ARZ BROOGHT INTO OPEAATION AS DBHAND INCBEASES. CAPACITY PACTORS ARE TUP. PRACTIONS DF TIME A PLANT IS ACTUALLY OPEATEO AND NOT SHUT DOHN POR REDAIRS OR SCHEDIIED PAT HTENANCP

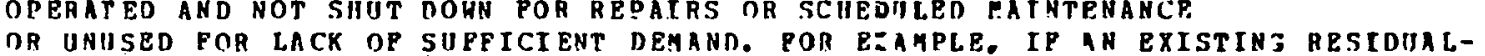
PI TPA PLANT IN REGION 1 IS OPERATED IN BASE LOAD POR A YEAR. TIIE ACTOAL NUMBER OF KILONATT-YEARS OP ELECTRICITY GENBRATED, ON THE AYERAGE, HILL BE ITS CADACITT (IN RILOHATTS) MOLTIPLIBD BY TTS CAPACITY PACTOR. IN

THIS CASE .70 . STRCE BASP PIANTS ARE OPERAT ED ALMOST CONTINOOIJSLY WHEM THEY

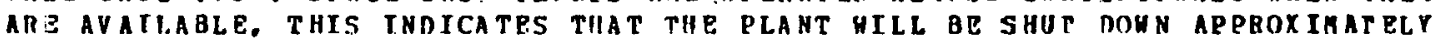
10: OP THF TIME. POR THIS SAME PLANT OPBRATTUG IK CYCIING LOAD. THE CAPACITY FACTOR TS . 55 , REPLECTING THE PERIODS TUB PLANT UILL NOT OPBRATE BECAUSE THB

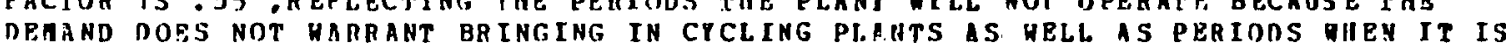
POACEN OIIT OP SERVICE OA SHUT DORN POR MALNTENANCE. 
TABLE 1: LOAD-DURATLON CUROE DATA FOR 1990

DOE

REGION

- - -

HH- ENG.

NY/N.J

MID-ATL

S. $-A T L$

MI DWEST

S. - HRST

CENTRAI.

N-CENTRA L

YEST

N. - HEST
COMPOSTTION FACTOKS

\begin{tabular}{l} 
RASE \\
\hline .787 \\
.746 \\
.766 \\
.770 \\
.758 \\
.756 \\
.749 \\
.783 \\
.758 \\
.810
\end{tabular}

\begin{tabular}{c} 
CYCLING \\
\hline $.1-$ \\
.247 \\
.188 \\
.156 \\
.158 \\
.185 \\
.144 \\
.156 \\
.167 \\
.173 \\
.130
\end{tabular}

\begin{tabular}{l} 
DAILY \\
PEAK \\
\hline .044 \\
.042 \\
.042 \\
.047 \\
.037 \\
.067 \\
.063 \\
.026 \\
.043 \\
.036
\end{tabular}

\begin{tabular}{cc} 
& $\begin{array}{c}\text { SYSTEG LOAD } \\
\text { PACTOR }\end{array}$ \\
SEASONAL & \\
PEAK & \\
\hline .022 & .570 \\
.023 & .610 \\
.026 & .580 \\
.029 & .580 \\
.020 & .610 \\
.031 & .520 \\
.033 & .530 \\
.024 & .580 \\
.026 & .610 \\
.024 & .600
\end{tabular}

DATA SOIIRCPS - COMPOSITION PACTORS: 1971 ANNUALLZED LOAD DURATION CORVES

FROA "RESPECIFICATION OP TIIR ELCCTRT. IITILITY MODEL IN PIES". GENERAL ELECTRIC

CORPANY. ALSO. THE BREAK POINT GIVJHG GOUNDS FOR THE FOIIR MODES ARE ASSIGNED EXJGENOUSLT

AND ARE BASEO ON IIISTORICAL AND ECONOHIC CONSIDERATIONS.

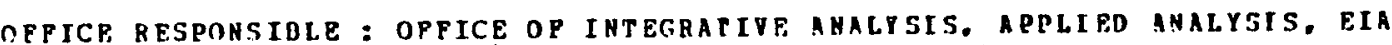
APPLIED ANALYSIS. EIA 
PIG:IRE 1: LINFAR APOPROXTMATION OP A I.OAD-DUGATION CUNVE

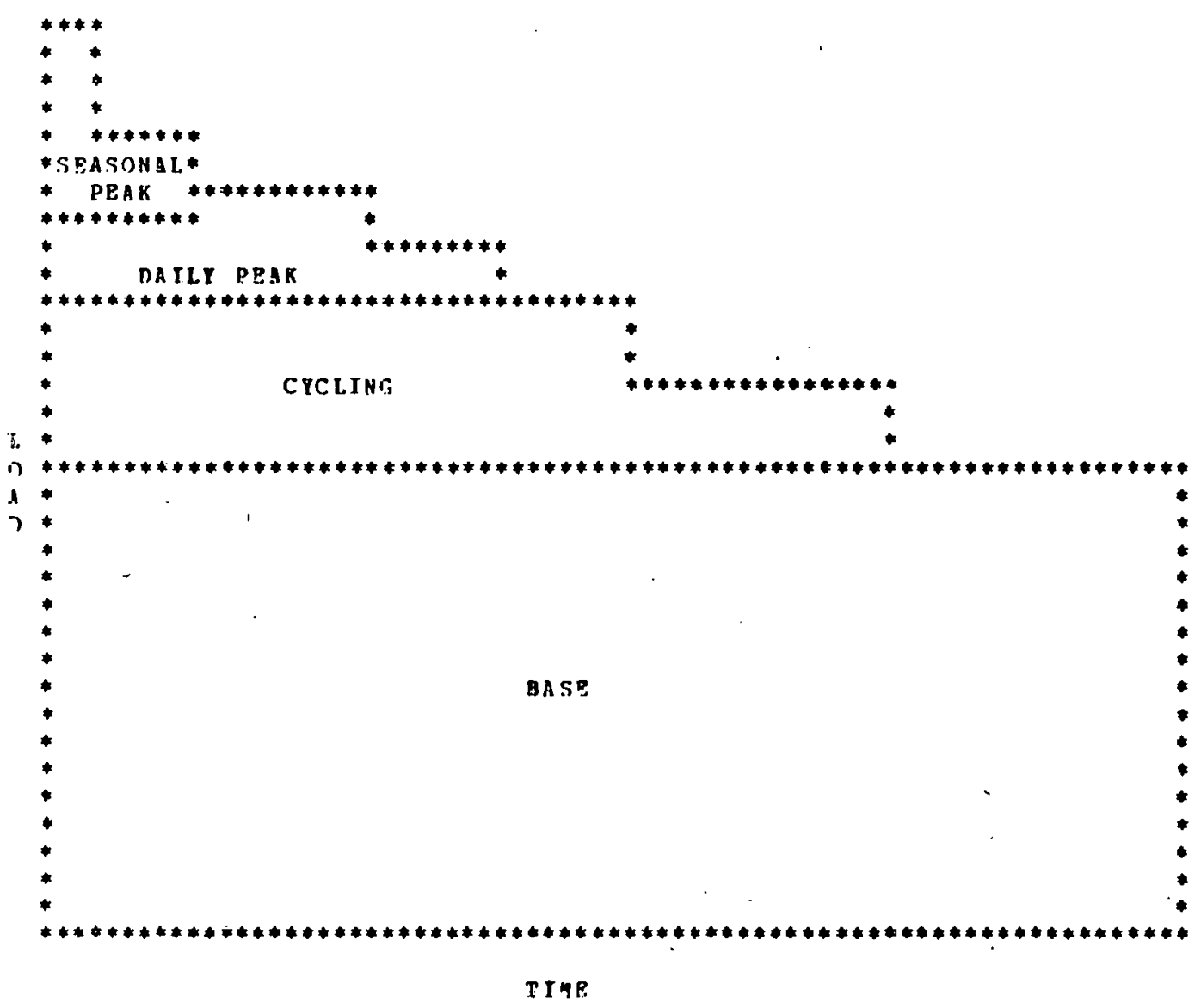


CAPACITY YACTORS POR DOE REgION 1 (NEM RNGLAND)

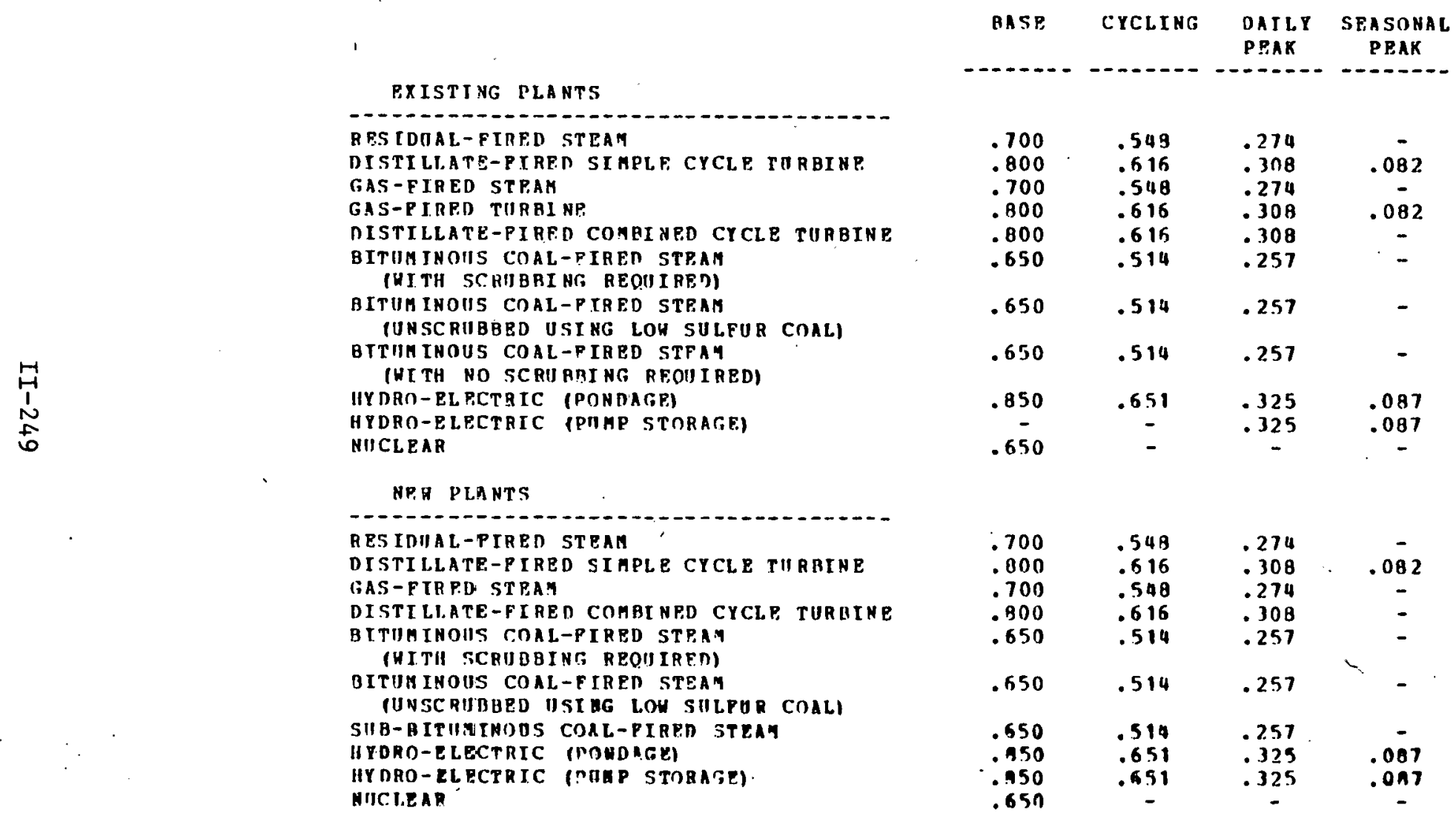

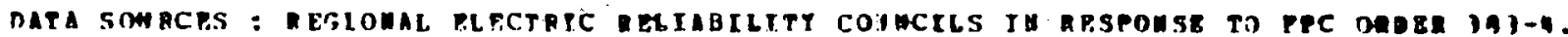

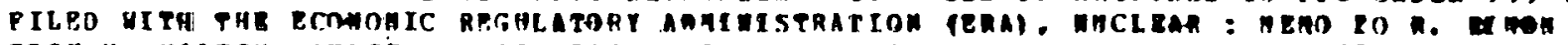

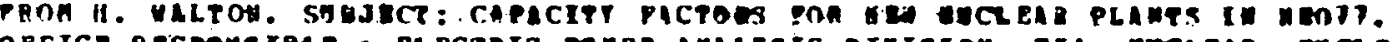

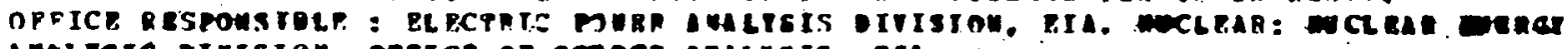

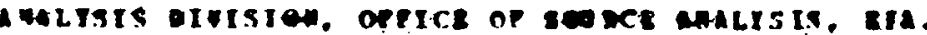


CAPACITY FACTORS FOR DOE REGIDU 2 (TEA YORK AND NEW JBRSEY)

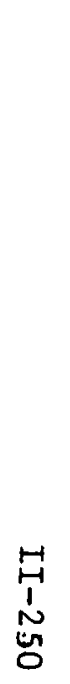

\begin{tabular}{|c|c|c|c|}
\hline AASE & CYCLING & $\begin{array}{l}\text { DAILY } \\
\text { PEAK }\end{array}$ & $\begin{array}{l}\text { SEASONAL } \\
\text { PEAK }\end{array}$ \\
\hline .700 & .549 & .274 & -1 \\
\hline .900 & .696 & .308 & .082 \\
\hline $\begin{array}{l}.700 \\
.800\end{array}$ & $\begin{array}{l}.548 \\
.616\end{array}$ & $\begin{array}{r}.274 \\
.308\end{array}$ & $.0 \overline{2}$ \\
\hline .800 & .616 & .308 & - \\
\hline .650 & .5111 & .257 & - \\
\hline .650 & .514 & .257 & - \\
\hline .650 & .514 & .257 & - \\
\hline .850 & .651 & $\begin{array}{r}.325 \\
.225\end{array}$ & .087 \\
\hline .650 & - & - & - \\
\hline $\begin{array}{l}.700 \\
.800\end{array}$ & $\begin{array}{l}.5+8 \\
.616\end{array}$ & $\begin{array}{l}.274 \\
.308\end{array}$ & $.0 \overline{82}$ \\
\hline 800 & .615 & $\begin{array}{r}.274 \\
309\end{array}$ & - \\
\hline .650 & .514 & .257 & - \\
\hline .650 & .504 & .257 & - \\
\hline $\begin{array}{r}.650 \\
.850 \\
.850\end{array}$ & $\begin{array}{l}.514 \\
.651 \\
.651\end{array}$ & $\begin{array}{r}.257 \\
.325 \\
.325\end{array}$ & $\begin{array}{c}\overline{0} \\
.087 \\
.087\end{array}$ \\
\hline .650 & - & - & - \\
\hline
\end{tabular}

BXISTING PLANTS

-

RESIDUAL-FIRPD STEAK

DISTI JLATE-PIRED SIMPLE CYCLE TURALH

GAS-PIRED STPAM

GAS-FIRED TISBBINR.

DISTILLATE-PI RED COMAI NED CYCLE TUIBINE

BITUA J.HOUS COAL-PIRPD STRAM

(HI TII SCR!IBUING RP.OUIREDI

B ITIIM INOUS COAL-PIRED STEAM

IUNSCBUBROO USI HF LOH SIILPUR COALI

BITUA INOUS COAL-FIRED STEAM

(HITH NO SCRI) BBING RROTIRED)

IIY DRO-PLECTRIC (PONDAGE)

HYDRO-ELECTRTC (PI)MP STORAGEI

niJCLEAR

\section{HP. PLANTS}

RESIOUAL-FIRED STEAH

DISTILI,ATT-PIRED SIMPLE, CYCIE TURBINR

GAS-FIRED STFAH

DISTI LIATE-PIRED COMRINRD CYCLF TURATNE

BITININOTS COAL-PIRPD STEAM

IHITH SCRIIDBINS, REQUITEDI

HITIMTNOUS COAL-FIRED STEAM

IINSCRIIBBED IISTAG LOR SULFUR COAH.

SHB-BITIMINOIS COAL-PIRFA STEAM

HYDRO-ELECTRTC (PONDAGPI

HYDRO-F,LECTRIC POIHP STORARE

NITLEAR

DATA SNIIRCES : REFIONAL ELPCTRIC REI.IABILITY COIINCILS IN HESPONSP TO PPC ORDER 383-4.

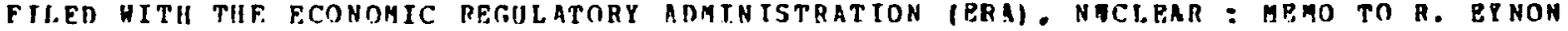
PROM H. YALTON, SIJBJECT: CAPACITY FACTOES POR NPW NIIELEAR DLANTS IN NEOT7.

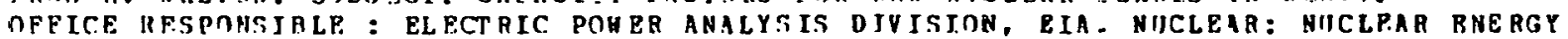
ANALISIS DIVISION. DFPTCF OF SOURCE ANALYSTS, EIA. 
CAPACITY PACTORS POR DOE REGIOH J (MIO-ATLANTICI

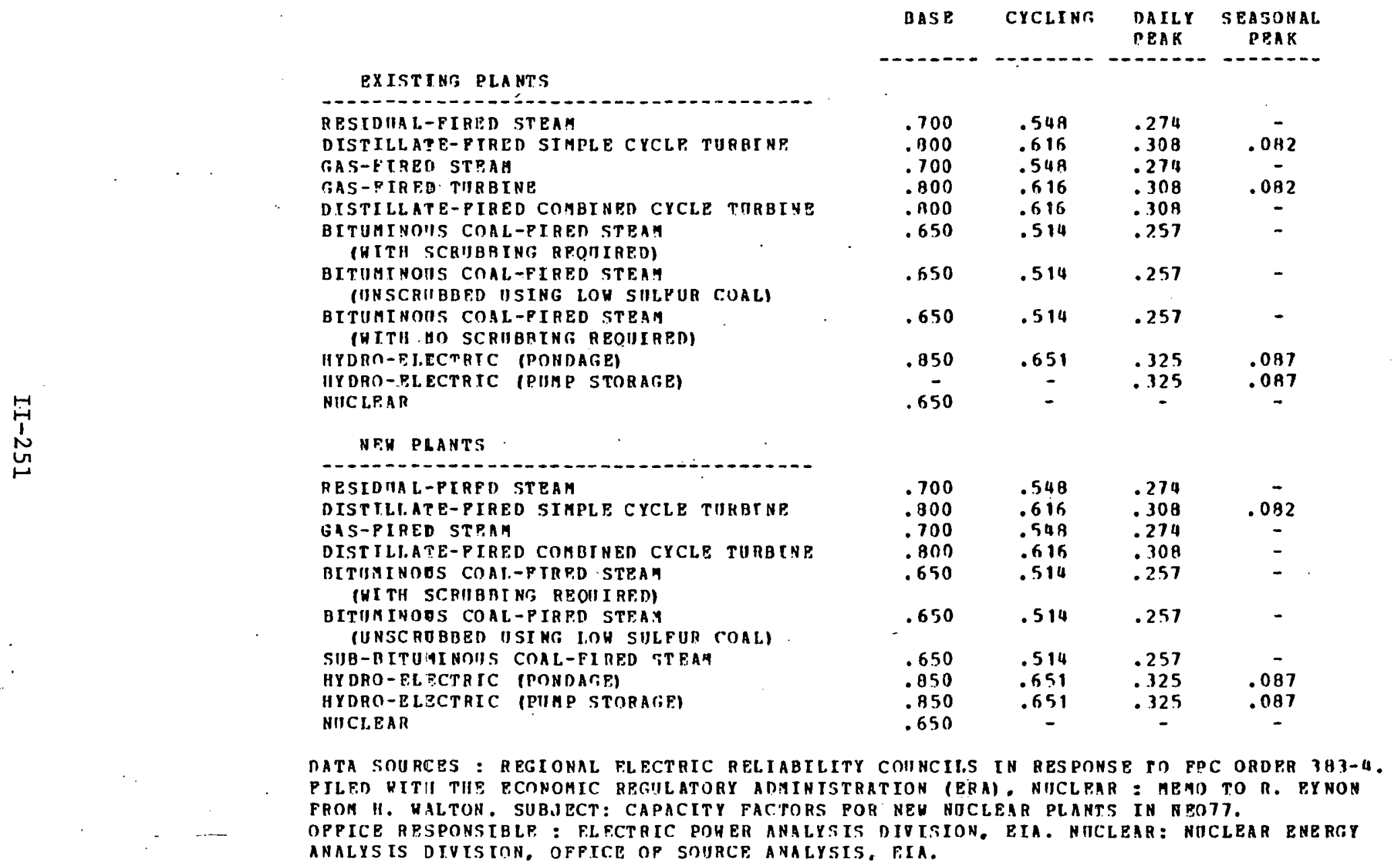


CAPACITY PACTORS YOR DOF REgLON (SOITH ATLATIC)

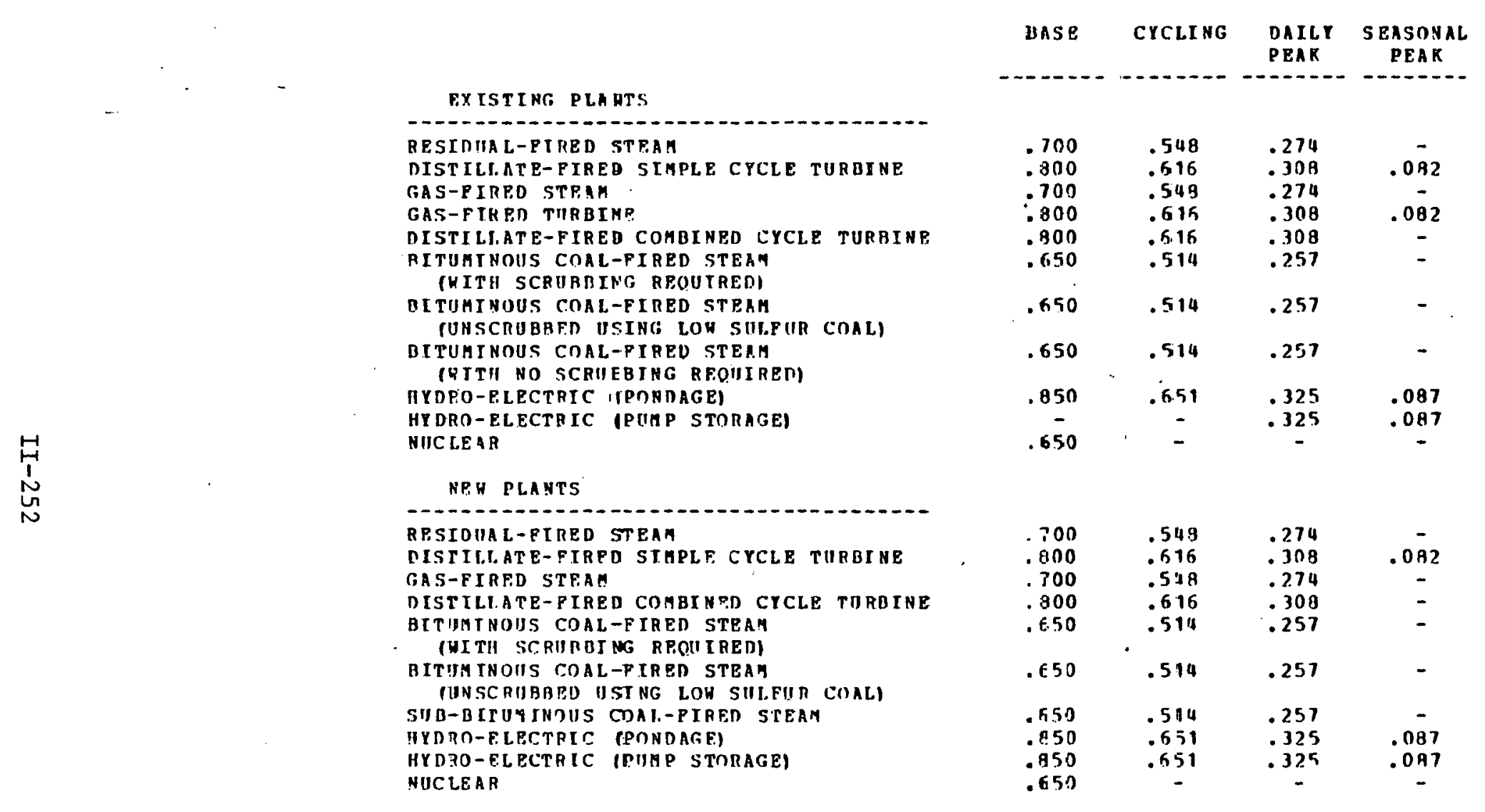

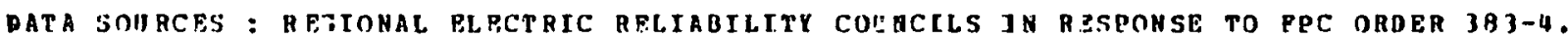

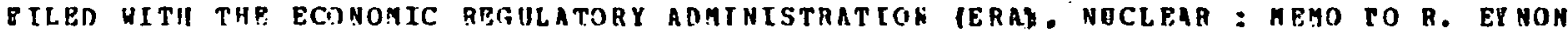
PROM H. YALTON. SIBIPCT: CAPACITY FACTORS POR NEW NIICLFiR PLANTS IN NEOT7.

OPPICE RESPONSIBLE : RIECTRIE POHFR ANALYSIS DIVISION. EIA, NIICLEAR: NICL GAR BNERGY PALYSIS DIVISION, DFFICF, OF SOURCE ANATSIS, BIA. 
CAPACITY PACTONS YOH DUE REGIOA 5 (MIDUEST)

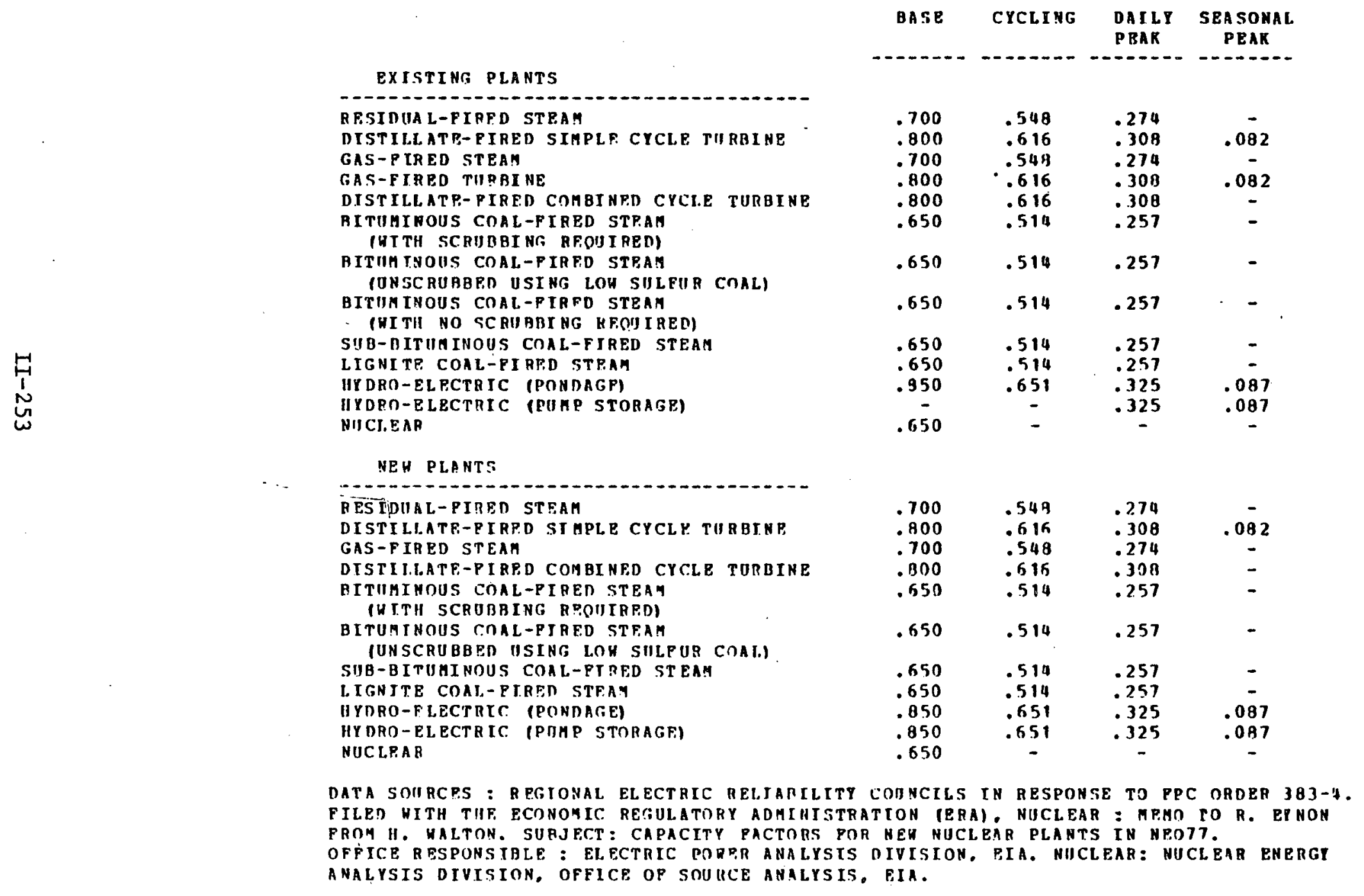


CAPACITY FACTORS POR DOE REGTON 5 (SOUTAUESTI

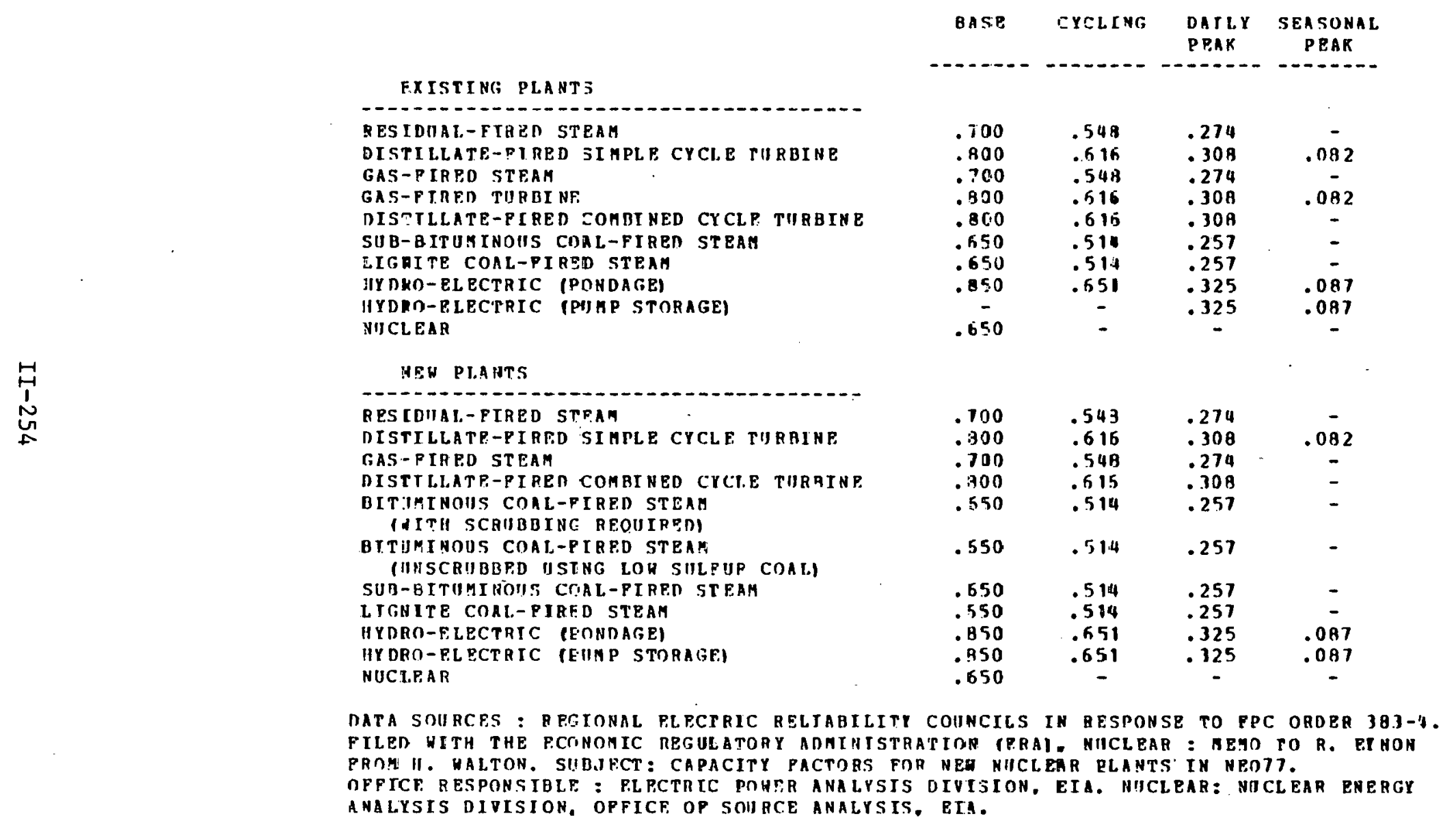


CAPACITY PACTOFS POA DOF RPGIOH 7 (CENTRAL)

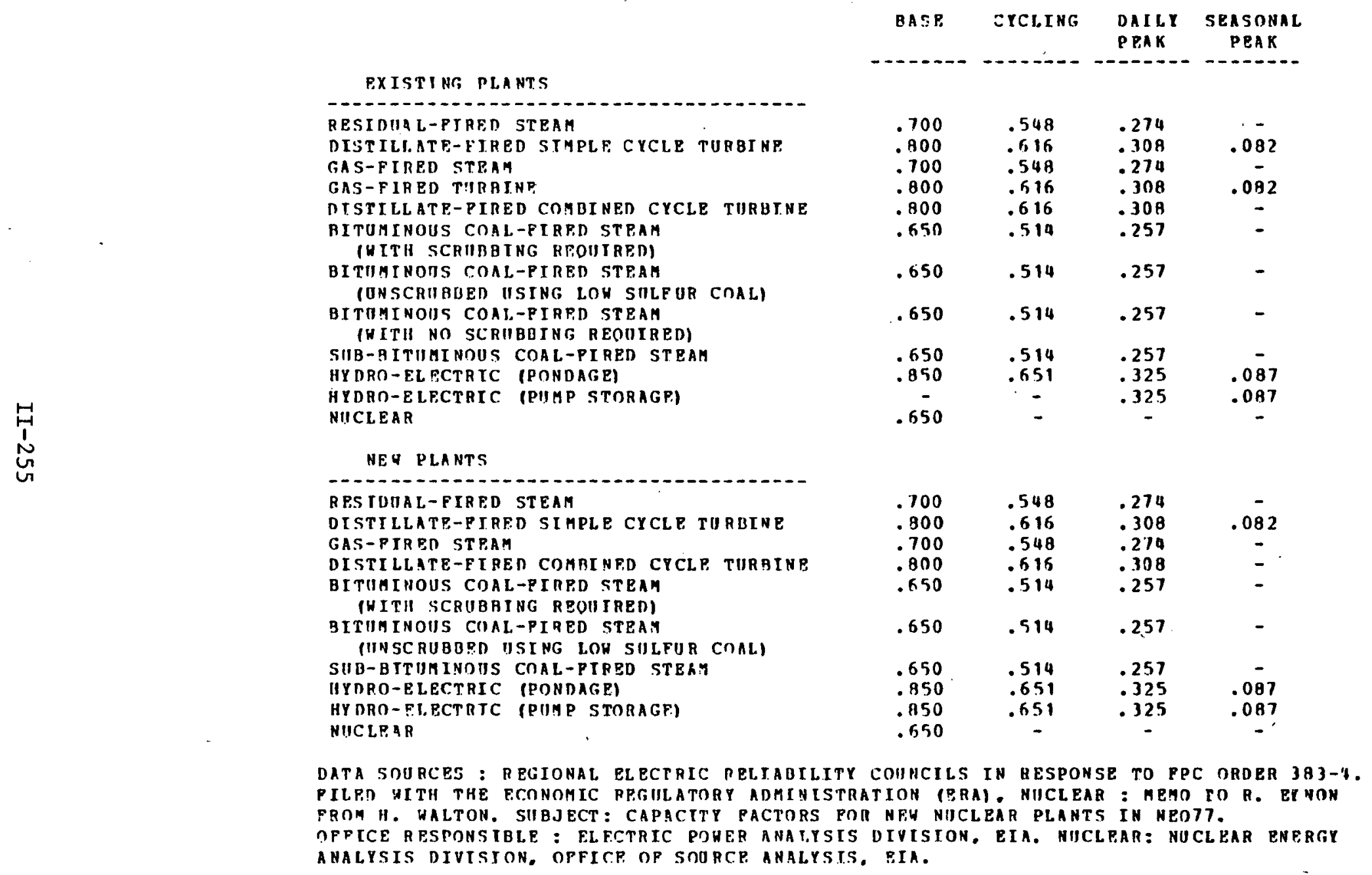




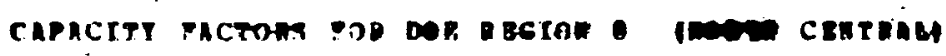

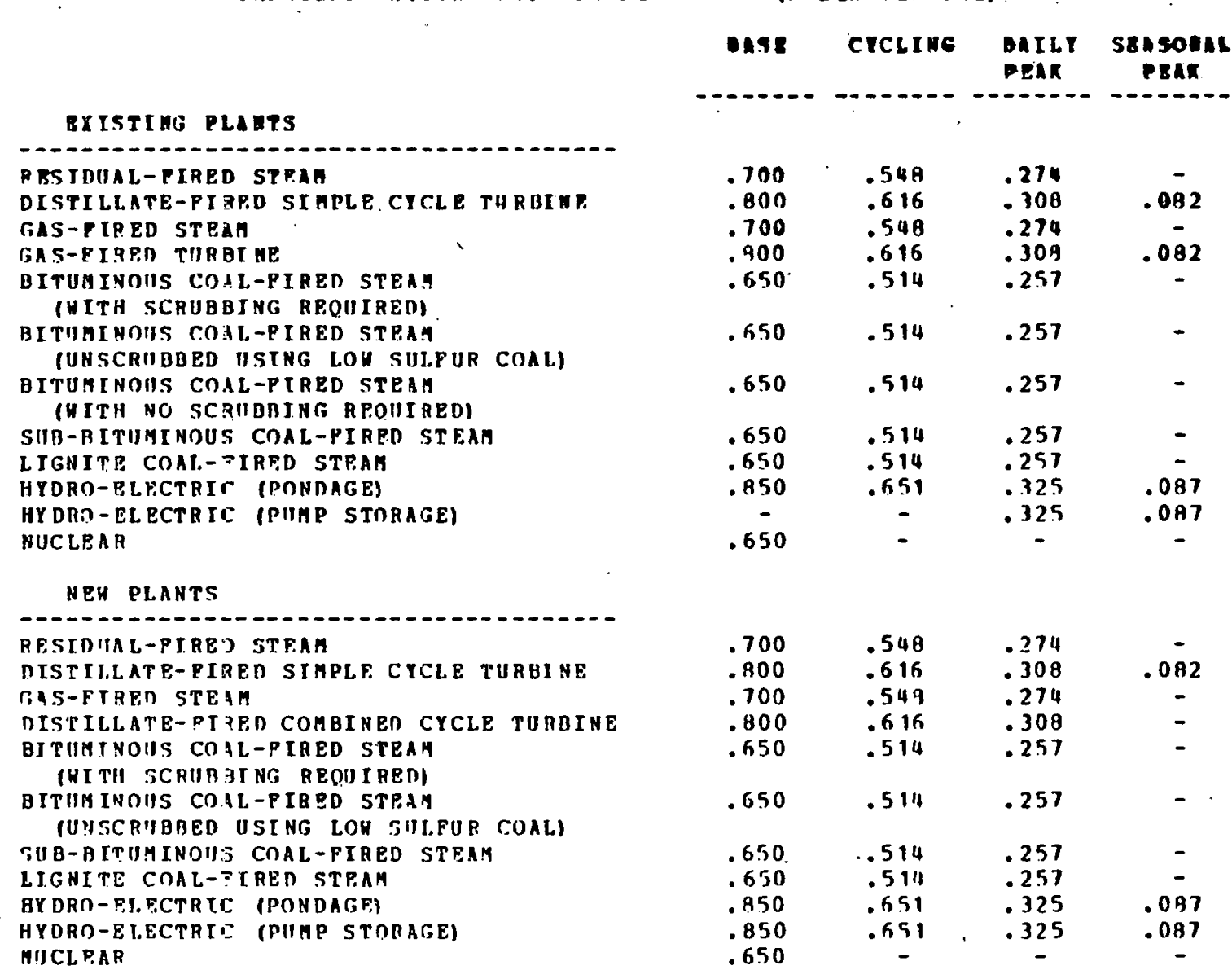

FATA SNIIRCRS : REGIONAL PLECTRIC RFITABILITY COONCILS IN RESPONSE TO PPC ORDER 383-4.

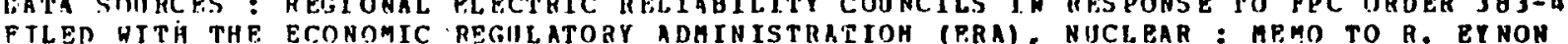
ERON II. HALTON. SIBB.JECT: CAPACITT FACTORS FOQ NEW NICLEAR PLANTS IN NEOIT.

CIPPIER RESPONSIBLE : FLECTRIC POHPR ANALYSIS DIVISION, EIA. NIICLEAR: NICLEAR RHERTY ARALPSIS DIVISION, OPFICE. OF SOIRCE ANALYSIS, EIA. 
CAPACITY FACTORS.POR DOE ABGJON ") (GRST)

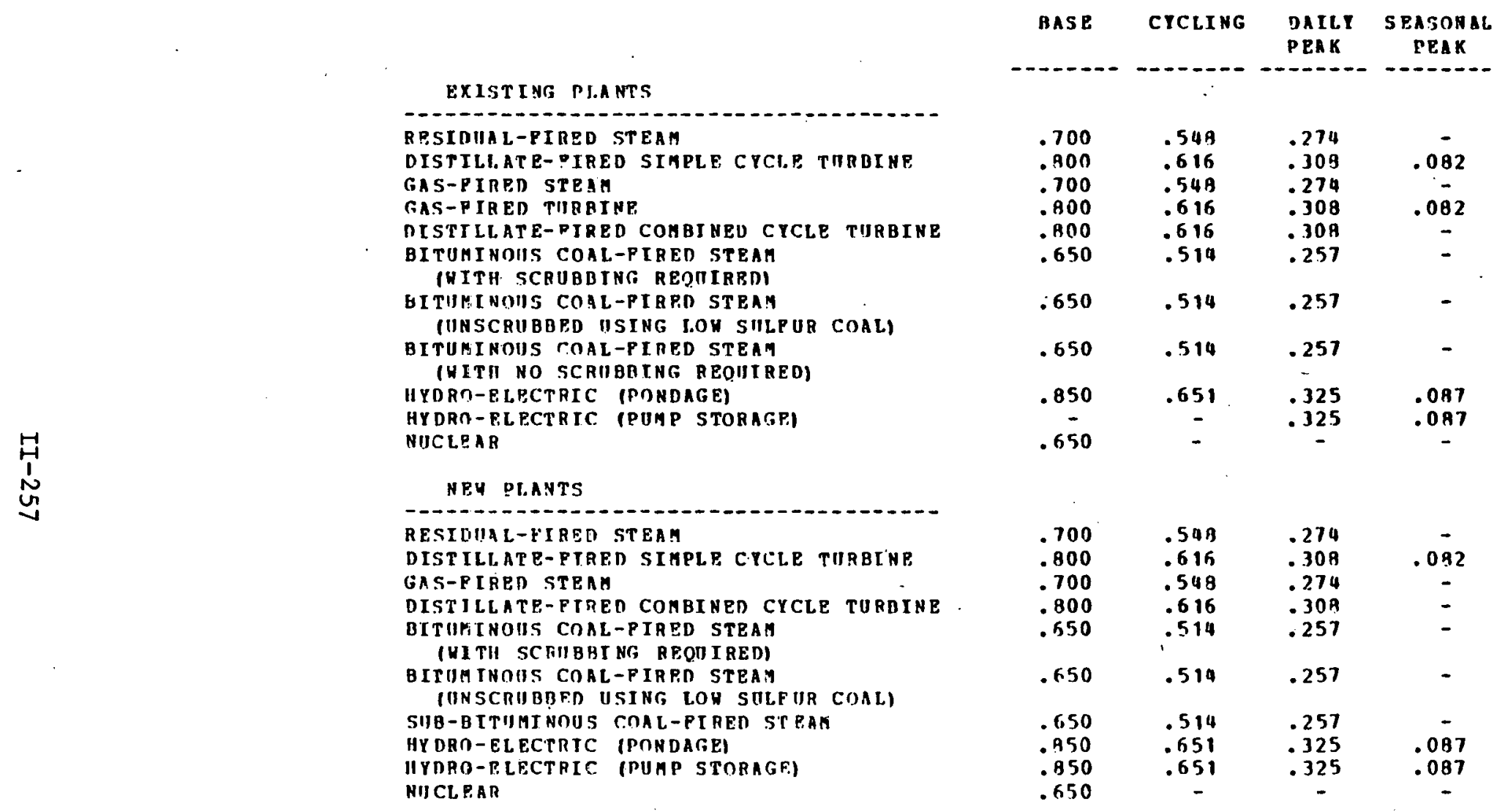

DATA SOIIRES : REGTONAL ELPCTRIC RELIABII.TTY COUNCLLS IN RESPONSE TD PPC ORDER 3B3-4 PIL.ED NITII TIIP ECONOAIC PRGULATORY ADAINISTRATTON (ERAI. NIICLEAR : HENO TO R. EY NOH FROM A. HALTON. SIIBJECT: CAPACITY PACTORS POR NEW YMCLEAR PLAHTS IN NEOT7.

OPPICE RPSPONSIBLE: FLFCTRIC POW ER AIRIYS IS DIVISIOR, EIA. NIICLBAR: NIJLEAR ENERG ANALYSIS DIVISTON, OPFICF, OP SOIRCP ANAIYYIS, P.IA. 
CAPACTTY PACTORS POR DOE RFGLON 10 (NORTHHEST)

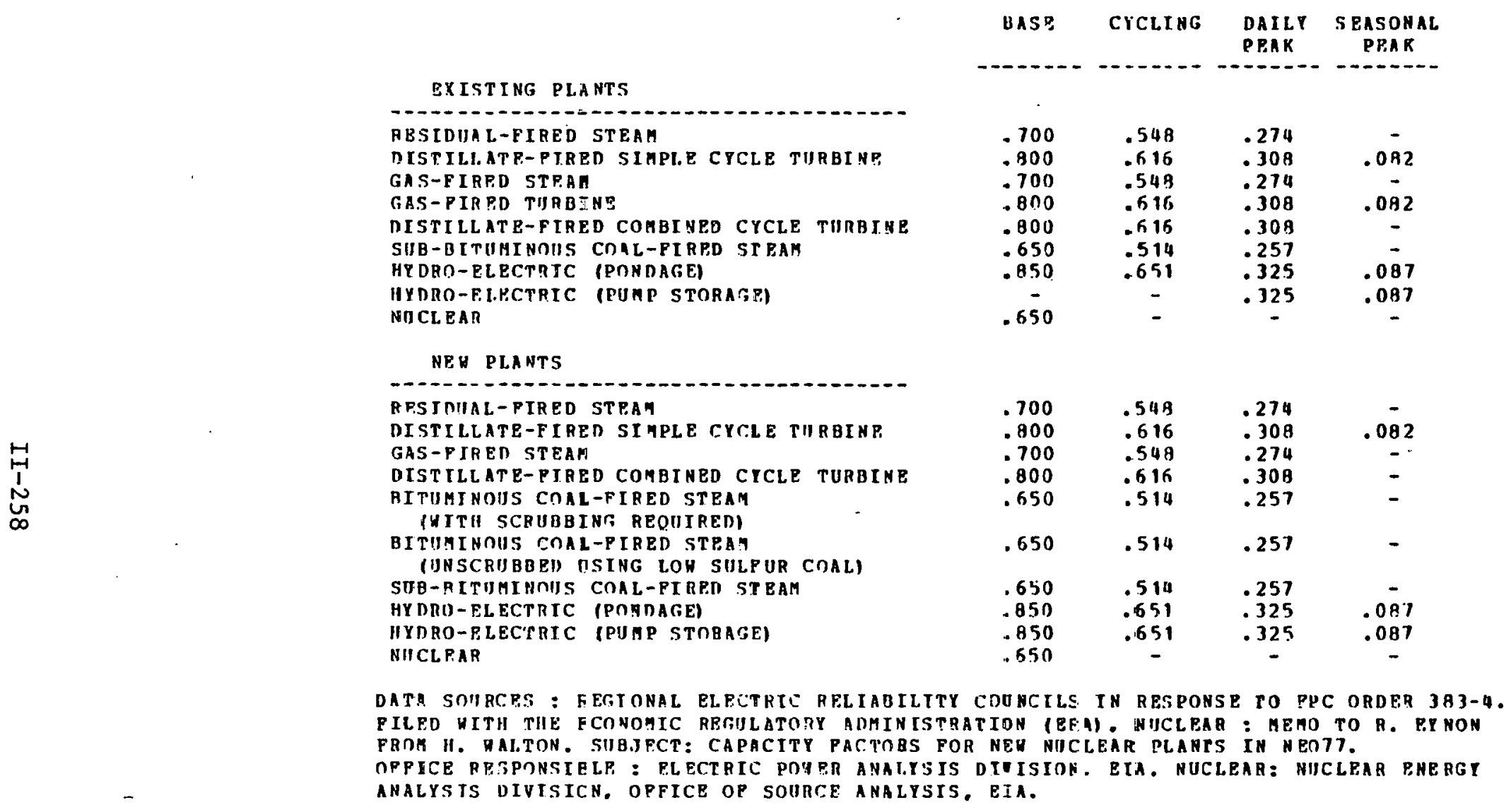


TABLE ? BPLOH SHOUS EXTSTING CAPACITY POR EACH PLANT TYPE IH BACH DOE REGION. TABLE 3 SHOYS THE EXISTING CAPACITIBS MHICH YAY BE CONYERTED PO

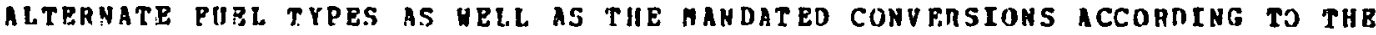
PNERGP SIIPPLY AND ENVTRONKENTAL COOHDINATIOH ACT (BSECA) AS OF IONP 30. 1977.

TABLF: 2: EXISTING CAPACITY (MPGANATTS) *

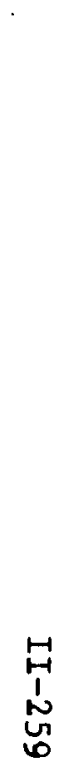

PLANT TYPE

RFEIDUAL-FIBED STEAM

DISTIILATE-FIRPA SIMPLD CICLF. TIRBIHE 1276 8583 4473

DISTILLATE-FIRED COHBINED CYCLE TIRAINB

GAS - PTRED STFAM

(GAS-PIAB) TIIRAINB

BITIMINOIIS COAL-PIRED STPAM

(UNSCROHBED ISING LOW SULPUR COAL)

BITIYINOUS CDAL-PIRED STPAA

(HITH SCRUBBING BEOHIFEA)

BITOMINOSS COAL-PIRED STEAM

(HTTH DO SCRUBBING RECIIIIRED)

SUB-AITUGINOUS COAL-PIRED STEAM

LIGNITE COAL-FIRED STEA

HYIRO-ELECTRIC (PONMAGP)

HYDRT-EI.FCTBIC (PUAP STRRAGE

NICLEAR

RPG [INAL. AND NATIONAI, TÓTAI.S

$\begin{array}{ccc}1 & 2 & 3 \\ -10286 & 21489 & 13690\end{array}$

\begin{tabular}{|c|c|c|}
\hline \multicolumn{2}{|c|}{ DOP } & REGIO! \\
\hline 4 & 5 & 6 \\
\hline & & \\
\hline 6117 & 10122 & \\
\hline 7900 & 6534 & \\
\hline 619 & 217 & \\
\hline 2981 & 10.35 & \\
\hline 2418 & 1110 & \\
\hline 3589 & $12 A 02$ & \\
\hline 2370 & 1470 & \\
\hline 8731 & 40296 & \\
\hline - & 13385 & \\
\hline & 141 & \\
\hline 10445 & 1436 & \\
\hline 921 & 1979 & \\
\hline 12838 & 11391 & \\
\hline & & \\
\hline
\end{tabular}

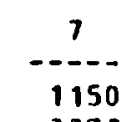

1150

931

50

1.30

1024

1624

$-$

485

2950

26909

-
$130 \overline{3}$
1607

4199

$\begin{array}{r}- \\ 5187 \\ 1629 \\ 6132 \\ - \\ \hline\end{array}$

1101
1432
6112

40700

*A3 OP $1 / 1 / 78$

DATA SOUREBS : REGIONAL FLPCTRIC RPLIAOTLITY COJNCILS IN RBSPONSE TO PPC ORDER IA3-4. PILER HITA THE FCONOHIC REGIJLATORY ADHINISTHATLON (BRA).

NISCLEAR : MEMO TO D. HULETT AND H. WALTON PROK R.G. CLARK AND A.R. REYHOLDS DATED $12 / 5 / 77$

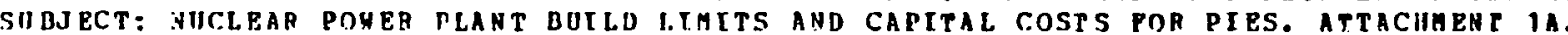

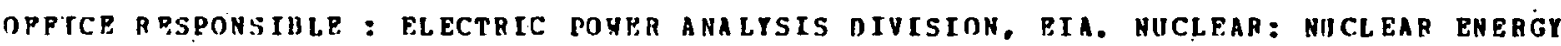
ANALYSIS DIVISION. OEPICE OF SOURCE ANALYSIS, BIA. 
TABI.E 3: FXISTING CAPACITIES HHICH haY Be COHVERTE TO BIJRN ALTEgNATE PUP. TYPES

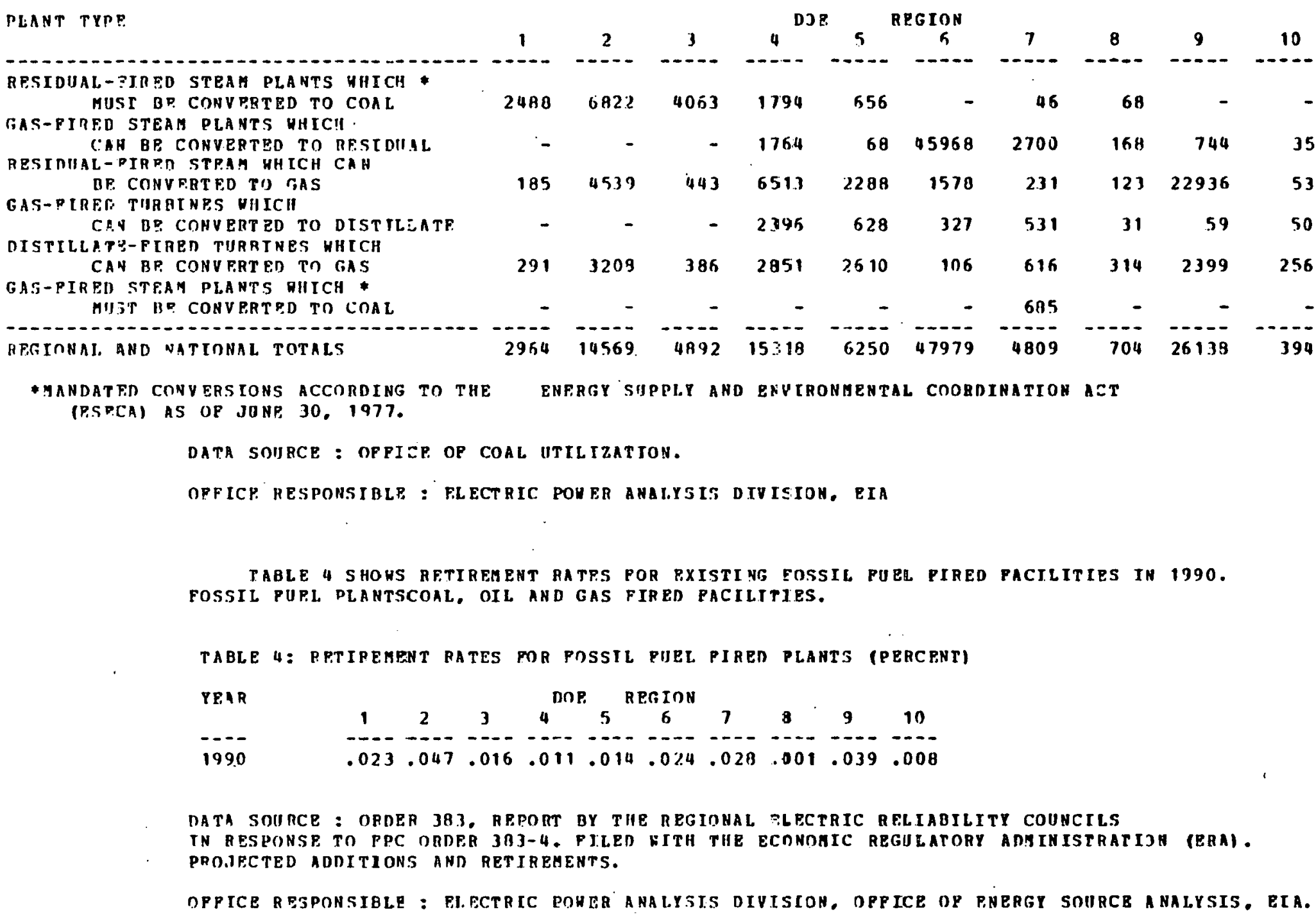

- yandatFa CONVERs ions according to the (P.SPCA) AS OP JONP 30, 1977.

DATA SOJRCE : OFPIEA OP COAL UTILTZATTON.

OPFICP. RESPONSIBLP : FLECTRIC POH ER ANAIYYIS DIVISIOH, RIA

TABLE 4 SHOWS RFTIREMENT RATPS POR RXISTI NG EOSSIL PUEL PIRED FACILITIES IN 1390. FOSSIL PUPL. PLANTSCOAL, OTL AND GAS FIRED PACILITIES.

TABLE 4: PPTIPEMRNT PATES POR POSSIL PJEL PIREN PLANTS (PERCBNT)

YEAR

1990

DOR. RERION

DATA SOURCE : ORDER 383. REPORT BY THE REGIOHAL GLECTRIC RELIABILTTY COUNCILS

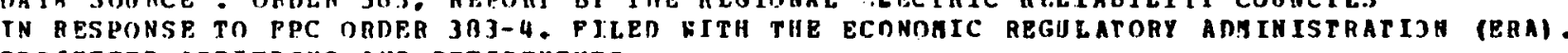
PRODECTED ADDITIONS AND RETIREHENTS.

DPPICE RESPONSIBLE : EI. ECTRIC POHER ANALYSIS DIVISION, OPPICE OP PNERGY SOURCB ANALYSIS. EIA. 
TAFLES 5 AND 5 SHOH CCIMHITTED ANH DPPERTABLR CAPACITIES POR COAL.

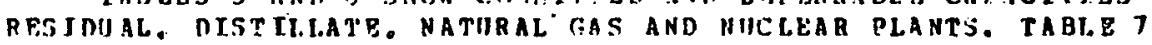

SHOYS BIILD LIAITS POR NIJCLPAR, COAL, RESID'IAL AND IYYDO-BLECTRIC PLANTS. CONAITTED PROJ ECTS HAVE ESSPNTIALLY AL!. OP THE CAPITAL. SIINK AND CONSTRICCION IS NEARI.T COMPI.PTRD. DPFYRRABLE PROI ECTS HAVR STNK APPROXIMATELY 10X OP

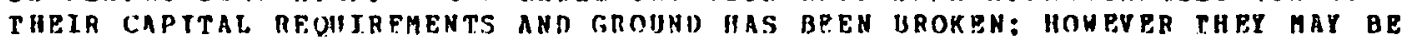
DEPERAPO UNTIL AFTPA THE FORBCAST YEAR IP IT IS ECONOAICAL TO DO SO.

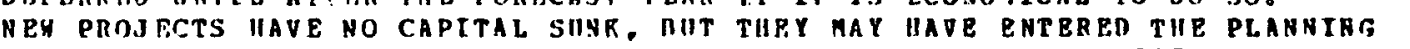

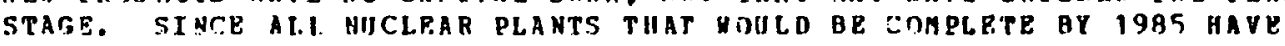

ALREADY NEEN REG ISTERED HITH THE U.S. NILLEAR REGILATOHY COHAISSION, TIERE APE ASSOC.IATRD LIMITS IN THE AODRL. THE LIHITS POB NDCLEAR CAPACITY IH 1990.

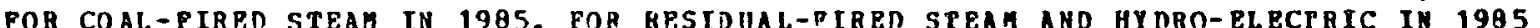
AND 1990 ARP, PSTIHATED BOTNDS ON THP GROHTH OF NICLEAR, COAL-PIREDSTEAH. RESTDJALPIPPO STEAM AND HYDRO-ELECTRTC CAPACITY. RESPECTIVELY. ALL OTHPR PLANT TYPES HAOE NO

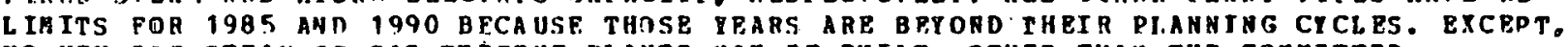

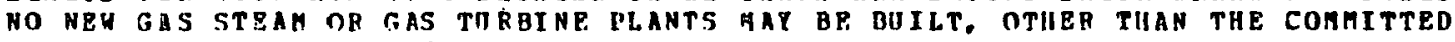

PLANTS SHOHN IN TABLE 5.

TABLE 5: COAYITTED* CAPACITY POP 1990 InPGA HATTS

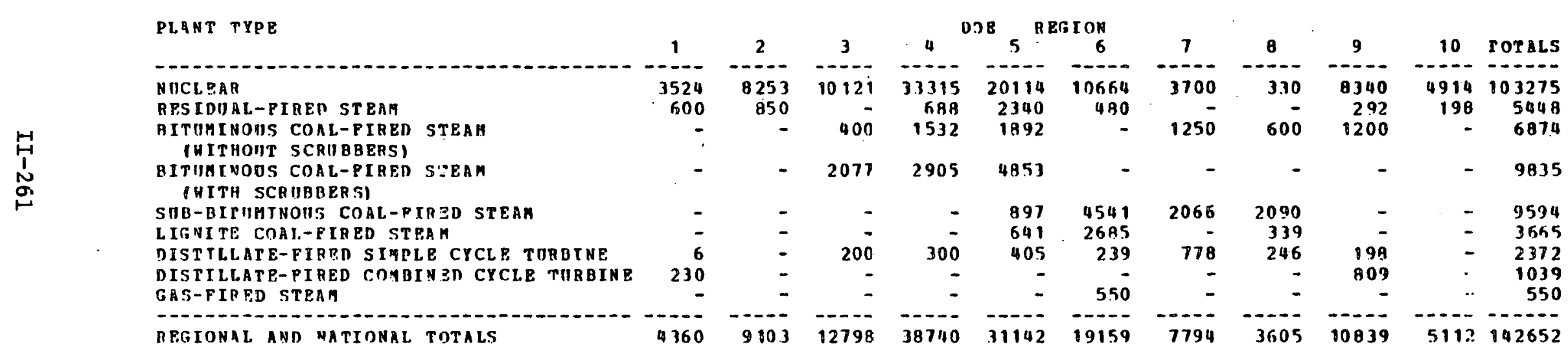

*all capital sunk and construction heahly completed

DATA SOIRCES POR TABLES 5,6,7,8,9:

PORM 4 IMONTHLY PJBLICATION) GEHERATIOH OP ENERSY, CONSHAPTION OP ENERGY AND EUEL STOCKS. PILED WITH RRA. DATR PDR SCRIBBERS DPRIVED PROA THE FNVIRONM 3 TALA PROTECTYON AGFACY STANOARDS FOR ALL COAL PLANYS.

PRODUCTION PIGURES ARE TAKEN PANA THP. PIES OIL AND GAS BODEL UHICH USES DAT PROM "HESERVPS OP CRIIDE OTI. NATUAAL GES LTOUIDS AND NATURAL GAS TH THE UHITED STATFS AND CANADA AND CAPACITY AS OF NECEMBRR 31, 1974n. AMERICAR PETROLEUA INSTITIIT 3 , WASUINGTON D.C.

CAPITAL PIGURPS ARF DFRTVED PROM DATA GATHRRPD BY THE AMERICAN PETROLEIIA INSTITOTE ANNIIA PIRLICATION. "JOINT ASSOCIATION SURVPY OP THP U.S OIL AND GAS PRODUCING INDUSTRY POP 1975". TIESE DATA ARP IISED AS INPIJT POR TIIE PIES OIL AND GAS MONEL.

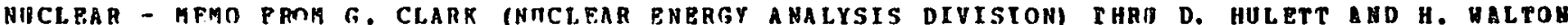
TO HARVEY FERENBERG. SIIBJECT: "NIICLEAR POHER BUILD LIMITS AMD CAPITAL COSTS POR PIES O UNDATED BITT C. 12/5/77, GEVISED 1/13/78. THE NICLEAR ENERGY ANALYSIS DIVISION OP BIA OBTALNS THEIR BASIC DATA POR BUTLD IITITS PROA PROA THE I. S. NICLEAR. REGULATORY COMHISSION. 
TABI.E 6: DEPERRABI, P. CAPACITY POR 1990 (MEGA RATTS)

\begin{tabular}{|c|c|c|c|c|c|c|c|c|c|c|c|}
\hline PLANT TYPE & & & & & $\mathbf{R z}$ & ION & & & & & 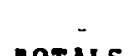 \\
\hline & 1 & 2 & $\mathbf{1}$ & 4 & 5 & 6 & 7 & $\mathbf{8}$ & 9 & 10 & rotals \\
\hline----------1 & $=$ & 2134 & & 900 & - & & & - & & 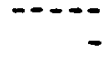 & .30 .34 \\
\hline BIIUNTNOUS COAL-FIRED STEAN & - & - & 1300 & 2710 & 2143 & 1100 & 1275 & 400 & 700 & - & \\
\hline SUE-DITUAINOUS COAL-PIRED STEAT & - & - & - & - & 58) & 6346 & 1910 & 2780 & - & 500 & 12116 \\
\hline LLSNITE COAL-PIRED STEAM & - & - & - & - & 555 & 1978 & - & 294 & - & - & 2 \\
\hline E.JIOUAL AND NATIONAL TOTA & - & 2134 & 1300 & 3610 & 3279 & 9424 & 3185 & 3470 & 700 & 503 & \\
\hline
\end{tabular}

* appaoximately 10\% op capttal sunk, riound tas BPEN BROKEN AND PLANT SCHEDILLPD TO DPEBATE BY 1990

TABLE 7: NEH PLAHT CAPACITY LIMETS (1990) (HEGAMATTS)

\begin{tabular}{|c|c|c|c|c|c|c|c|c|c|c|c|}
\hline PI.ANT TYPE & & & & & $P_{\text {P }}$ & ION & & & & & \\
\hline & 1 & 2 & 3 & 4 & 5 & 6 & 7 & 8 & 9 & 10 & TOTALS \\
\hline NICLEAR & 2344 & 1150 & 191 & - & $37 E$ & 2113 & 250 & - & 974 & 3761 & 14557 \\
\hline RESIDUAL-PTRED STEAM & - & - & 510 & - & - & - & - & - & - & 864 & 1474 \\
\hline LIGNTTE COAL - PIRED ST EAM & - & - & - & - & 170. & - & - & - & - & - & 1703 \\
\hline HYDRO-ELECTRLC (PONDAGF) & 384 & 32 & 287 & 1181 & 150 & 174 & 42 & 1349 & 1416 & 9476 & 14490 \\
\hline UIPDAO-PLECTRJC (PUMP STORAGF) & 36 & 2350 & 6054 & 4415 & - & 446 & 1199 & 200 & 2961 & 650 & 18311 \\
\hline REGTONAL ARD HATIONAL TOTALS & 2764 & $353 ?$ & 7132 & 5595 & $56 \vdots 7$ & 2733 & 1491 & 1549 & 5351 & 14751 & 50535 \\
\hline
\end{tabular}


TABLE G SHOUS THE OPERATION AND MAINTENANCQ COSTS FOL EACH PLAHT TYPE. TABLE 9 SHOHS REVENII RFOUIREMENTS FOR EXISTING EOIJIPHENT. TABLE 10

SHOHS TIIB CAPTTAL COSTS FOI NEK PLANTS IN 1990. TABIBS 11 AND 12 SHOH ASSOCIATED CHARGE PACTORS. THP, REV FNIS. R.POJ TREMENT CHARGE PACTOR IS USPD IN THE RATE BASE COAPUTATION IWHICH IS AVERAGE, RATHER. TIIAN AARGINAL. COSTI. THE INVESTABHT CHARGE PACTOR IS ISED I D DETERHINING TIE DECISION OP HHICH PLANT IS TS BE. BIILT IP MORE

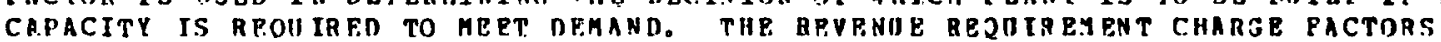
ARP. REPORTEO BY RECIINN. BECAUSE THESE CHARCBE FACTORS ARE BASPD ON AVERAGE CrISTS OF PRODICING ELECTRICITY, RATHER THAN THE MARGINAL COSTS POR ANY PABT ICILAR PLANT TYPR. ON THE OTHER HAND, TIIP INUESTAENT CIIARG IS RBPORTED BY PLANT TYPF BECAUSE OP TIIZ DIPPERENT INCOMR TAX TRBATHENT FECH PLANT TYPE RECEIVES HHOER TRE IRS CONE. TABLP. 13 SHORS TRANSATSSIOH AND DISTREBTTION COSTS AS HEI.L AS EPPICIENCY RATRS IZ.G. IN REGTON 1 POR ERRRY RILONAT GBNERATED, ONLY. 914 KILONATTS IS DISTHIBITED.)

TABLE 8: OQERATION AND MATHTBNANCE COSTS 1975 AILLS PER KILOHATT HOUR)

\begin{tabular}{|c|c|}
\hline PLANT TYPP. & OEH Cos $\mathrm{r}$ \\
\hline RESIDUAL-PIREN STEAB & $\cdots-$ \\
\hline BITUMTHOUS COAL* (HITH SCRIBBERI & 2.80 \\
\hline 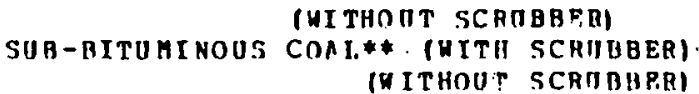 & $\begin{array}{l}1.10 \\
2.00 \\
1.10\end{array}$ \\
\hline 1. IGNITE COAL * ** (YITH SCRUBNER) & 2.00 \\
\hline (H ITHOUT SCRI BRPR) & 1.10 \\
\hline DISTITI.ATF-FIRED SIMPI.P CYCI.P & 2.75 \\
\hline DISTILLATE-FIRED COHBINED CYCLE & 1.25 \\
\hline GAS-PIRED TURATHR & 2.75 \\
\hline GAS-FTRRD STEAM & .50 \\
\hline HXDPO-FLECTRIC (PONDAFE) & .70 \\
\hline $\begin{array}{l}\text { HYDRO-ELECTRIC IUUMP STORAGEI } \\
\text { NISCLEAR }\end{array}$ & .70 \\
\hline
\end{tabular}

* ditumingus plants may de scribagd or unscriaged FXCPPT POR NEW PLANTS IN DOF. KEGIONS A, 9 and 10 HIICII MUST BE GCRIIBBED AND USE LOW SILLPIJR COAL

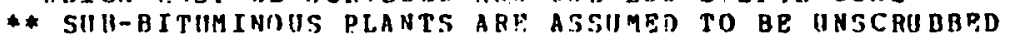
excrpt for nen plants Iy dof regions 9.9 and 10 HHTCH ARE ASSIMED TO BE SCRUBAPR

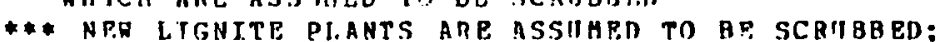

WHLE EXISTIUI. PIANTS ARE ASSIJED TO GE INSCRIIBPD 
TABIP, 9: REVPNIE REOIITEMPNTS IN 1990 FOR PXISTING ASSFTS

DOP HBGLOH

REVPNIIR REOTIREARNTS INILI.TONS OP 1975 \$1

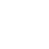

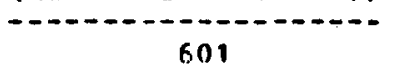

TABLE 10: CAPITAL COSTS OE HEY PLANTS IN 1990

(1975 DOLLARS PER KLLOUNTT)

\begin{tabular}{|c|c|c|c|c|c|c|c|c|c|}
\hline & & & $D O B$ & REG & & & & & \\
\hline 1 & 2 & 3 & 4 & 5 & 6 & 7 & $\theta$ & 9 & 10 \\
\hline & $\cdots$ & -- & $\cdots$ & -- & $\cdots$ & --- & --- & $-\cdot-$ & \\
\hline 415 & 438 & 393 & 373 & 409 & 375 & 406 & 398 & 322 & 403 \\
\hline 556 & $58 \mathrm{~B}$ & 529 & 501 & 549 & 503 & 545 & 524 & & 541 \\
\hline 447 & 473 & 424 & 402 & 442 & 405 & 438 & 524 & 567 & 541 \\
\hline 485 & 513 & 461 & 436 & 479 & 439 & 476 & 560 & 505 & 578 \\
\hline 591 & 591 & 591 & 591 & 591 & 591 & 591 & 591 & 591 & 591 \\
\hline 163 & 163 & 163 & 163 & 163 & 16.3 & 163 & 163 & 163 & 163 \\
\hline 312 & 312 & 312 & 312 & 312 & 312 & 312 & 312 & 312 & 312 \\
\hline 50 & 350 & 350 & 285 & 315 & 285 & 315 & 330 & & 325 \\
\hline 400 & 3110 & 470 & 420 & 450 & 520 & 490 & 130 & 520 & 220 \\
\hline 400 & 340 & 470 & 420 & 450 & 520 & 490 & 330 & 620 & 220 \\
\hline 695 & ค 10 & $695 i$ & 570 & 695 & 535 & 670 & - & 705 & 770 \\
\hline- & 585 & - & 599 & - & - & - & - & - & \\
\hline
\end{tabular}

\section{RESIDIAL-FIRED STEAH}

$\left(-\frac{10}{20}\right.$

1596

1685

2326

1061

590

369
1072

493 STIS BBERI

DISTILLATE-PIRED 5 IHPLZ CYCLE DISTILLATE-PIRED CONBINBD CYCLE GAS-PIRED STPAA

HYDRO-P.LECTRIC (PONDACE)

HYDRO-BJ.PCTRIC IPUMP STORAGEI

NICLEAP (NEW)

HIICLPAR (CEPPRPPABLE)

- capital cost of depperahale coal plagts is .90 op peu plant costs

NOTE: HFTROPITTING AN EXISTING RITUMINOUS COAL PEANT IISING HITH SIIL PUR COAL. IN BASPLOAD COSTS $\$ 131$ PER KILDHATT

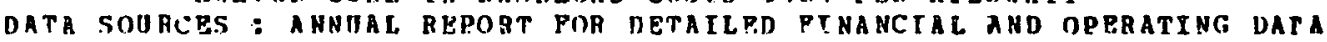

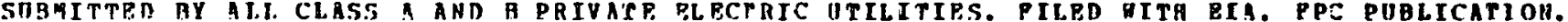
PRODHCT TON FIGIIR RS ARE TAKEN PROA TIFE PIES OIL AND GAS MODEL MHICH USES DATA

PROA "RESERYES OP CRIIDE OIL. NATIIRAI. GAS LIOUIDG AMD NATURAL GAS IN THE INITED STATES

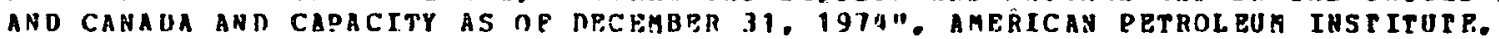

HASHINGTON, D. C.

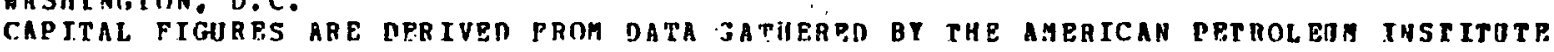

ANHUA PIBLICATION. "JOINT ASSOCIATIOH SURUEY DF THE U.S. OIL AND GAS PRODICI RG

INDUSTRY FOR 1975". TIIESE DATA ARE IISED AS INPUT POR THE PIES OIL AND GAS MONEL. 
TABLE 11: CAPITAL CIMAGE RATES (PRRCBAT) POR REVENUE REOTIREABNTS

\begin{tabular}{|c|c|}
\hline & $\begin{array}{l}\text { CAPITAI. CUARGE RATPS POR } \\
\text { REVENIJ PROT IRBHENTS }\end{array}$ \\
\hline DOE RPGION & 1990 \\
\hline$\cdots$ & $-\infty$ \\
\hline 1 & 14.0 \\
\hline 2 & 17.4 \\
\hline 3 & 12.7 \\
\hline 4 & 10.0 \\
\hline 5 & 13.3 \\
\hline $\boldsymbol{6}$ & 18.9 \\
\hline 7 & 12.9 \\
\hline 8 & 9.9 \\
\hline 9 & 11.7 \\
\hline 10 & 6.3 \\
\hline
\end{tabular}

DATA SODRCES : STULY CONDICTED BY THE OAKRIDGE NATIOHAL LABORATORIES USTHG FHE COMPUT BR MODEI CONCEPT 4

NIICLEAR: GEMO PROA G. CLARK TO II. GREENBERG DATEN 5/24/77

PRODICTION FIGURES ARE TAK FW PROA THE PIFS OIL AND GAS MODEL NHICH IISES DATA

PRAM "RES RRVES OP CAIIDE OIL. NATIRAL GAS LIOUIDJ AND NATIRAL GAS IA IHP UNTRED

STATES IND CANADA AND CAFACITY AS OP DRCEMBER 31, 1974" AMEAICAN PETQDLEUA IRSTCTUTE

WASHIRGTON, D.C.

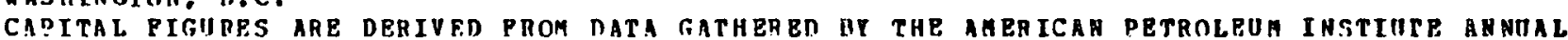

PIIBLICATINH. "JOINT ASSOCIATIOR SURVRT OP THE II.S. OLL AND GAS PRODICING IHDOSTET POR 1975". THPSP DATA ATE USFD AS INPTT TOR THE PIES OIL AND GAS MODEL. 
TABLE 12: CAPITAL CHARTE RATES FOR IYJESTHEHT DECISIONS IANNUAL PERCENTI

PLANT TYPP

RPSIDUAL-FIRPO STEAR
BITUMINOUS COAT. - FIRED STEAM

(IITH SCRUABER)

B ITIMINOUS COAL-EJRRD STEAM

(HITHOUT SCRUBRER)

DISTILLATE-PIPP.D SIRIPL CYCI. F TIABIAP

GAS-PIFED STPAM

HYDRO

SUB-DITUAINOUS COAL-FIRED STEAM

LIGNITE COAL-FIREI STPAM

NIICLEAR
DATA SOIRCPS: STIDY CONDUCTED BY THE OAKRIDGE NaTIONAL LABORATORIES USTHG THP COMPUTER MODEL COMCEPT 4

NIICLEAH: MEAO FRON G. CLARK TO H. GREENBERE DATPR $5.24 / 77$

PRONICTION PIIIIRES ARF. TAKEN EHOM THE PIBS OIL AND GAS HODRL HHICH DSES DATA

PROM "RPSERVES OP CRIIDE OII. NATIRAL GAS LIOUTDS AHA HATURAL GAS IN THE UNITED

STATRS AND CANADA AND CAPACITY AS OF nECEMBEH 31, 1974n AMERICAN PETBOLEUE INSTETUTB

HASUINGTON, D.C.

CAPTTAL PIGIIRES APQ DERIVEA FROY DATA GaTHER BY THE AMPRICAN PETROLEUA IHSTIUTE AHNIAL

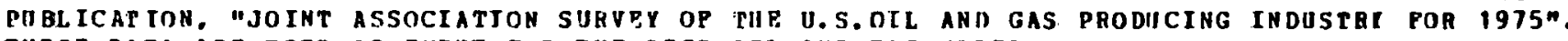

THESE DATA ARE OSPD AS INPUT PUR THE PIRS OIL AND GAS MODEL.

CATITAL CHARGE RATE POR

IN RETHENT DECISTONS

11. 1

11.4

10.5

11.4

10.2

11.4

11.4

BORATORIPS USING THP
DISTILLATE-FIRED COMBINED CYCI,E TIJRBINF 
TABLP. 13: TRANSMISSION ANO DTSTRIBUTIOH DATA

DOE REST ION

3
1
3
4
5
5
7
8
9
10

Avenig.

RPFICIENCY RATE
(PERCFATAGEI
[PERCFNTAGB]

91. 40

91.30

92.40

91.40

92.30

92.50

91.20

88. 30

90.30

91.00

\begin{tabular}{l} 
OPERATION AND \\
MAINTRNANCR. COST \\
MILIS/RILOART-GOURI \\
\hline 5.7 \\
5.2 \\
4.7 \\
5.0 \\
4.3 \\
4.6 \\
5.5 \\
5.6 \\
5.3 \\
5.6
\end{tabular}

Capital $\cos r$

(s/KILOUATE)

\section{3}

\section{6}

304

262

361
228

354

309

514

DATA SOUICES : ANHIJAL REPORT TOR DETAII,PD PINANCIAL AND OPERATING DATA

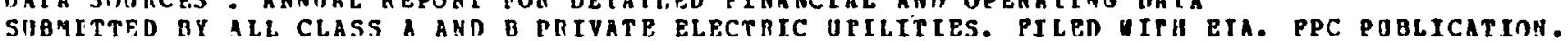
PRODTCTYON PIGIIRES IRE TAKEN PROM THE PIPS OIL AND GAS MODEL WHICH OSES DATA

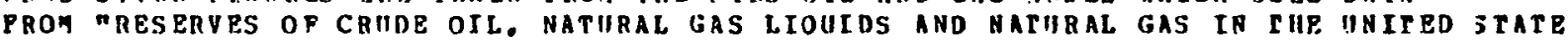

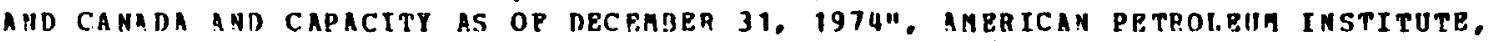

WASHINGTON, D, C.

CAPITAI. PIGIIARS ARE DERIVPD PROM DATA GATHEAED BY TIE AMPRICAN PETROLPOA INSTITUTE ANNIIL PUBLICATION. "JOINT ASSOCTATION SURVEY OP TIID U.S. OII. AND GAS PRODIJCING

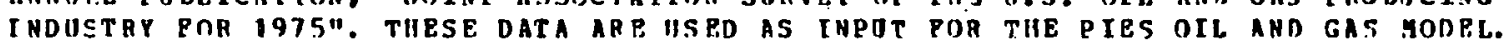

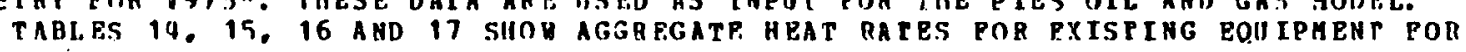

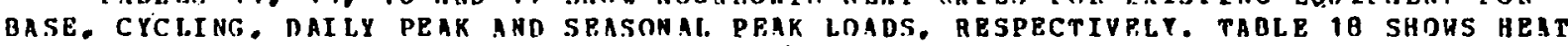
RATES* FOR NEQ PACILTTIES ISAMF FOK FACH REGIOH). AHD TABLP 19 SHOHS HEAT VALIIPS

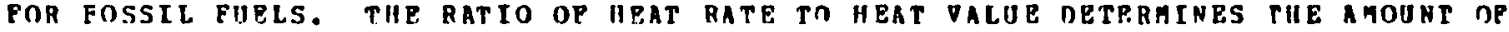
PUEL NEPDED TO GENFRATE ONE KILOHATT. SPECIPICALLT, THP PORL REOULAED TO OPRRATE A PIANT TO GEHPRATE ONE GEGAHATT DP ELRCTHICITY IN PARTICIILAB LOAD II. B. BASE. CYCLDHG. DAILT PPAR OR SEASTHAL PEAKI IS:

PHEL REOUIPRMENT = (HEAT PATF)/(HEAT VALUE)

DHFRE THE AMOIINT OP PIIEL REOIIHEO IS MEASURED IN PHYSTCAL IJNITS (I.B. TONS BARRELS. TIONSANDS OP STANDARD CIIBIC PEET, ETC.I

TABLE 20 SHOHS HUCLEAR PIJEL SUPPI. DATA.

- the heat rate Is thf. penergt reonifed pen holl op operation.

WHERE ENFRGY TS MEASTRED BY BRITISH THERMAL NMITS (BTII) 


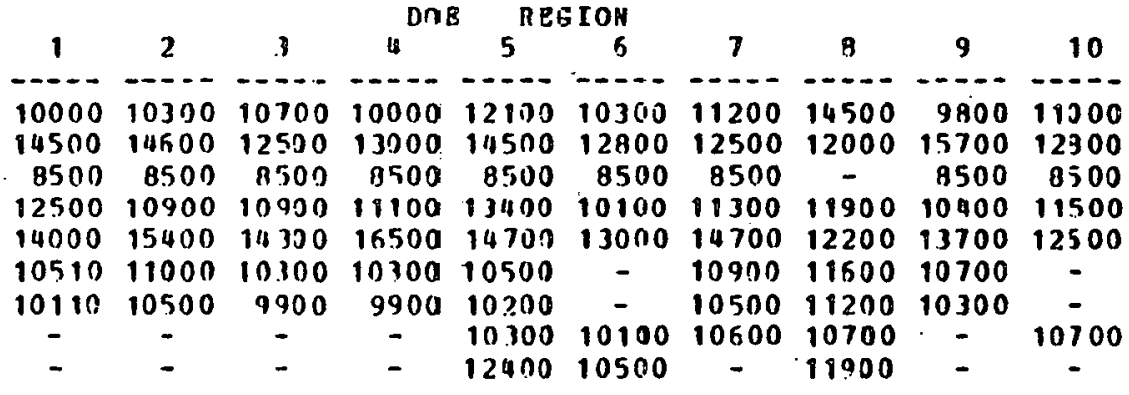

RESTDUIL -PIRED STBAM

DISTILLATE-PIHED SIMPLE CICLE

DISTILIATE-PIPED CORBINAD CYCL.

GAS-PIAED STEA

GAS-PTZPR TIIRBIND

BITIMINOOS COAL (WITH SCRIIBBP.BI

BITUMIHOIS COAL (HITHOIIT SCRIIBBERI

SUB-BITIIIAOUS COAL

LIGNIT:

DATA SOIRCES : COAPUTER MODEL DEV ELOPPD BY ROOEAT BORLICF AND DATID HATCHER (ELFCTRIC POYEB ANALYSIS DIVISION OP PITA)

PRODUCTION FIGORES ARE TAKPA FROM THE PIES OLL AND GRS MODRE HATCh OSES DATA

PROM "RFSERVES DP CRIDE OIL, NATURAL GAS LIQUIOS AMD HATURAL GAS IN THE IJITED 3 TATES

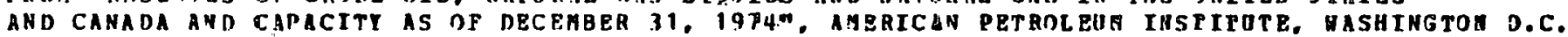

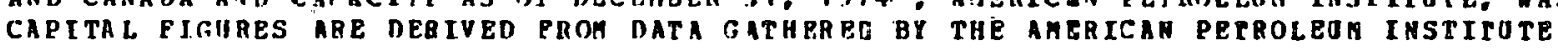

ANNIAL PUBLTCATION. "JOINT ASSOCIATION SURVBY OP THE IJ.S. OIL AND GAS PRODUCING

TABLE 15: REAT RATES POB EXISTING ROUIPMENT OPEAATEO IN CYCLING LOAD

$$
\text { (BTO PER KILONATT-HOJB) }
$$

PIANT TYPE

\section{RESIDHAL-FIRPO STEAH}

DISTILEATE-FIRED SIAPLE CYCLE

DISTII.LATE-PIP.EA CONBINRD CTCLE

GAS - PI PED STEAH

GAS-PIRPD TIIRATNE

BITIMINOUS COAL (WITH SCRUBBERI

BITUAI VOUS COAL (WTTHOOT SCRUBB BRI

SUA-BITUMINOIS COAL

LIGNIT ?

\begin{tabular}{cccccccccc}
1 & 7 & 7 & 4 & 5 & 6 & 7 & 8 & 9 & 10 \\
\hdashline 10500 & 10800 & 11200 & 10500 & 12600 & 10800 & 11700 & 15000 & 10300 & 11500 \\
14500 & 15100 & 13000 & 13500 & 15000 & 13300 & 13000 & 12500 & 16200 & 12500 \\
9000 & 9000 & 9000 & 9000 & 9000 & 9000 & 9000 & - & 9000 & 9000 \\
13000 & 111100 & 11400 & 11600 & 13900 & 10500 & 11400 & 12400 & 10900 & 12300 \\
14500 & 15900 & 11400 & 17000 & 15200 & 13500 & 15200 & 12700 & 14200 & 13100 \\
11000 & 11500 & 10800 & 10900 & 11000 & - & 11400 & 12100 & 11200 & - \\
10600 & 11000 & 10400 & 10400 & 10700 & - & 11000 & 11700 & 10800 & - \\
- & - & - & - & 10900 & 10500 & 11100 & 11200 & - & 11200 \\
- & - & - & - & 12900 & 11000 & - & 12400 & - & -
\end{tabular}

DATA SOIIRCES : FINANCIAL STATERENT ANNIIAL REPORT. PPC FORM 1

PRODIICTION FIGIIRPS ARE TAKEN PROM THE PIES OIL AHD GAS MONEL HHICH USES DATA PROM "RESERVPS DP CATDE OIL, NATIRAL GAS LIOHIOANI NATURAL GAS IH THP OATTED

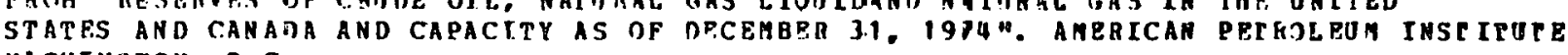
HASHIHGTON. D.C.

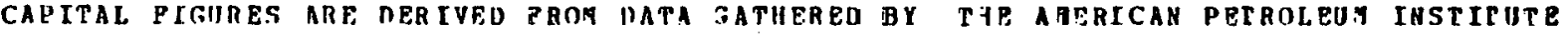

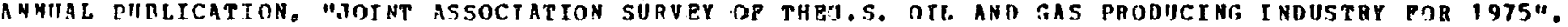
THESE, DATA ARE DSER AS INPITT FOR THP PIRS OIL AND GAS MODRL. 
DOP. HRGION

RESIDIIAL - FIRED STEA

DISTILLATE-PIRTD SIAPLE CTCLE DISTILIATE-PIRED CNMBINRD CYCLE GAS-PTHEN ST EAM

GAS-PIREN TURBINB

BITUMINOUS COAL (HITH SCRIBRER)

BITUMINOIS COAI. (UITHOIT SCRIIBBERI

SUR-BITUMINOUS COA. I. ITNITS

\begin{tabular}{|c|c|c|c|c|c|c|c|c|c|}
\hline 1 & 2 & 3 & 4 & 5 & 6 & 7 & 8 & 9 & 10 \\
\hline & & & & & & & & & \\
\hline $\begin{array}{r}10500 \\
14500 \\
90 \mathrm{nO}\end{array}$ & $\begin{array}{r}10800 \\
15100 \\
9000\end{array}$ & $\begin{array}{r}11200 \\
13000 \\
9000\end{array}$ & $\begin{array}{r}10500 \\
13500 \\
9000\end{array}$ & $\begin{array}{r}12600 \\
15000 \\
9000\end{array}$ & $\begin{array}{r}10800 \\
13300 \\
9000\end{array}$ & $\begin{array}{r}11700 \\
13000 \\
9000\end{array}$ & $\begin{array}{c}15000 \\
12500 \\
-\end{array}$ & $\begin{array}{r}10300 \\
16200 \\
9000\end{array}$ & \\
\hline $\begin{array}{l}13000 \\
14500\end{array}$ & $\begin{array}{l}11400 \\
15900\end{array}$ & $\begin{array}{l}11400 \\
14800\end{array}$ & $\begin{array}{l}11600 \\
17000\end{array}$ & $\begin{array}{l}13400 \\
15200\end{array}$ & $\begin{array}{l}10600 \\
13500\end{array}$ & $\begin{array}{l}11800 \\
15200\end{array}$ & $\begin{array}{l}12400 \\
12700\end{array}$ & $\begin{array}{l}10900 \\
14200\end{array}$ & $\begin{array}{l}12300 \\
13100\end{array}$ \\
\hline 11000 & 11500 & 10800 & 10800 & 11000 & - & 11400 & 12100 & 11200 & - \\
\hline 10600 & 11000 & 10400 & 10400 & 10700 & - & 11000 & 11700 & 10800 & - \\
\hline - & - & - & - & 10800 & 10600 & 11100 & 11200 & - & 200 \\
\hline - & - & - & - & 12900 & 11000 & & 12400 & - & \\
\hline
\end{tabular}

DATA SOllRCES : FORM 4 DATA BASE. "YONTRT.Y PLANT GPHERATION STASTICS", AHD FORB 423.

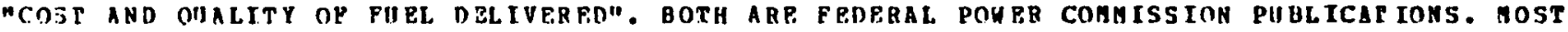
IIOST RECENT ISSOES USF.D POR E.IA ANHOAL ADAINISTRATOR'S RF.PORT.

PRONUCTION FIGURES ARE TAKEN PPOA THE PIES OTL AND GAS HODEI. UHICII USPS DATA PRJA "RESERVES OP CRUDE OIL. NATUPAL GAS L IOII IDS AND NATURAL GRS IN THE UNITED STATES AND CANADA AND CAPACITY AS OP DPCEMBPR 31. 1974". AMERTCAN PPTROLETH IBSTITUTE. MASHINGTOH D.C.

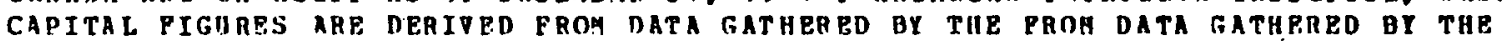
AHER ICAN PETROLPIIK INSTITUTE AHNHL PUBLICATION MJOINT ASSOCIAT ION SUROEY DF THE DIL AND GAS PRODUCTNG INDUSTRY POR 1975". THESE DATA ARE OSPD AS IHPIIT POB THE PIP.S OIL AYD GAS MODP.L.

TAALE 17: HEAT RATES FOR EXISTING FOIIPAENT OPERATED IN SEASOHAL PEAR LOAD (BTII PEB KILONATT-HOIIR)

\section{PLANT IYPE}

RESIDIAL - FIMPD STEIM

DIST ILLATR-PIRED STMBLE CYCLB

DISTTILLATE-FIRED COJMBINED CYCLE

GAS-FIRED STPAM

GAS- PIPB.D TII RBIN

BITUMINOTS COAL (WITH SCRIJBAFI

BITUMINOIS COAL. (HETHOIIT SCRIIBBERI

SUB-BITUNINOUS COA:

L. IGN I TPB

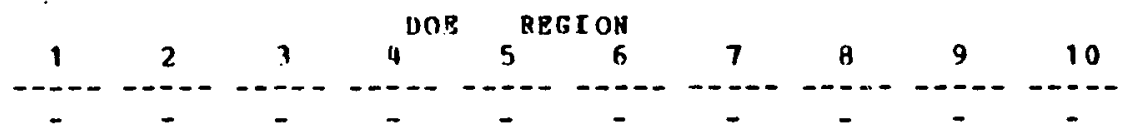

$145001510013000 \quad 13500 \quad 15000 \quad 13300 \quad 13000 \quad 12500 \quad 1620012500$

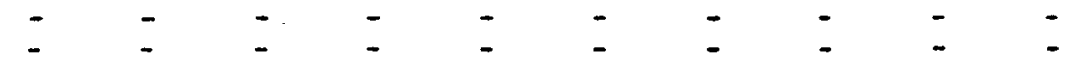

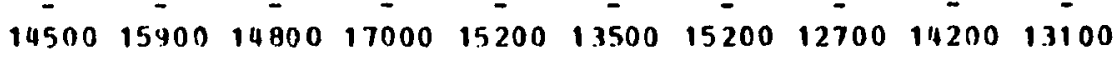

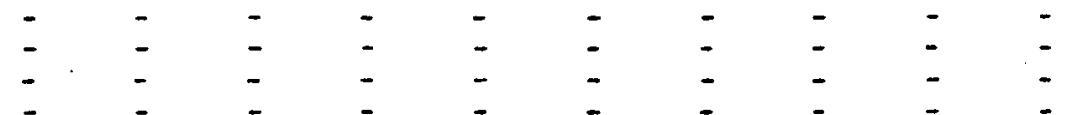

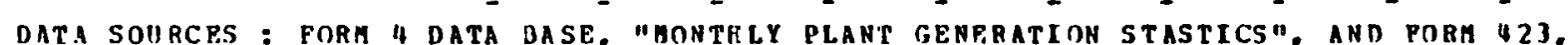
"COST AND OIIALITY OF FIEL DELIVERED". BOTH ARE PRERRAL POHER CONHISSION PIIBLICATIONS. MOSI MOS: FECENT ISSUES OSED POR EIA ANNULL ADAINISTRATOR'S REPORT.

PROJUCTION FIGURES ARF TAKEN PROM TIIP PIES OIL AND GAS NODEI. HHTCH USES DATA PROM

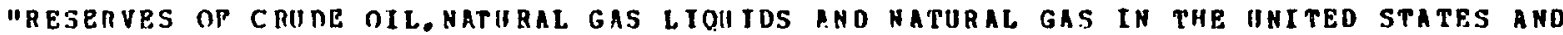

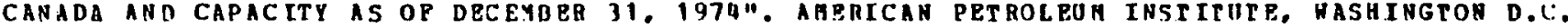
CAPITAL PIGIJRES ARF DERIVPA FBOH DATA GATHRRED HY THR FROA DATA SATIIEPRD BI THE AMERICAN DETROLEUM TNSTITUTP ABNIIA PIIBLICATION NJOINT ASSOCIATION STRVEY OE PHE OII. AND GAS PRODUCING INDUSTRY POR 1975". THPSP DATA ARE ISEO AS INPIIT POR TIIE PSPS OII. A!I D GAS MODEL. 
TABLE 16: HEAT RATES POR NEU EOUIPMENT

(HI I.LIONS OP BTU P.ER KILOUATTI

\section{PLANT TYPP}

STE-PIRED STMPLE CXCI.

DISTILLATE-PIRED CCIMUINTD CYCLP

GAS-PIRED STFAM

GAS-FIRED TIIRBINE

BITISTNOUS COAL (W.ITII SCRIBBF?

BITUMINOIS COAL (HITHOUT SCRIIBUER

SUB-DJTUMINOUS COAL

L IGN I T P.

DASE
9650
9000
7000
10010
9000
9840
9870
10230
10500

\section{CYCLING}

\section{0}

9500

7500

10760

9500

10300

10350

10710

11000

$\begin{array}{cc}\text { DAILY } & \text { SPASOHAL } \\ \text { PRAR } & \begin{array}{c}\text { PEAR } \\ \hline 10305\end{array} \\ 9500 & - \\ 7500 & 9750 \\ 10760 & - \\ 9500 & 9750 \\ 10300 & - \\ 10350 & - \\ 10710 & - \\ 11000 & -\end{array}$

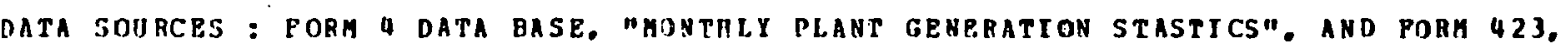
"COST AND O!IAITTY OF FUIL DRLIVERFD". BOTH ARE FERERAL PORER CONAISSION PIJBLICATIONS. MOST MOST RECENT ISSIIES ISF, FOR P.IA ANNUAL ADHINISTRATOR'S REPORT.

PRODICTION EIGIIRES GRE TAKFN PROM TIIE PIES OII. AND GAS MODRL NHTCH USES DATA PROA "P. PSERVES OF CRUDE OIL. HAT!IRAL GAS LIOUIDS AND NATURAL GaS IN THE UHITED STATES AHD CANADA AND CAPACITY AS OP DECEHBER 11, 1974". AMERTCAN PETROLEUA INSTITUTE. HASIINGTON D.C. CAPITAL EIGIIRES ARE DERIVED PROM DATA GATHERPD BY THP PROB DATA GATHERED BY THE AMPRICAN PETROI.FIM INSTITUTE ANNIIL POBLICATLON "JOINT ASSOCIATION SURVET OP TIIE OTL AND GAS PRONICIUG IHDUSTRT POR 1975". TIIPSE DATA ARE USPE AS IHPOT POR PHE PIES OTL AND GAS MODF.L.

TABLE 1.9: HEAT VALURS OE POSSIL FU BLS

(MILLIONS OP BTII PER PHYSICAL INITS)

\begin{tabular}{lcl} 
FURL. & HFAT VALUE & PUYSICAL IINITS \\
\hline RESIDIIL OIL & 6.297 & BARAELS \\
DISTILLATE OIL & 5.825 & BARRLS \\
NATURAL GAS & 1.032 & THOUSANDS OP STANDARD CIBIC FEET \\
COAL & 22.500 & TONS
\end{tabular}

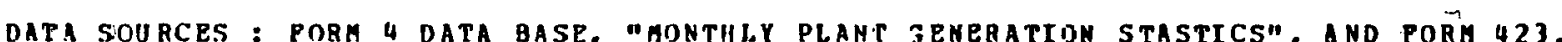

"COST AND OIALITY OF FUEL DPLIVEREO". BUTH ARE PRDERAL POHER COMHISSION PUBLICATIONS. MOST "CTST AND OIALITY OP PURL OTLTVEREO

PRADUCTION FIGIRES ARE TAREN PROA THE PIES OII. AND GAS MODPL UHICH USBS DATA PRJA

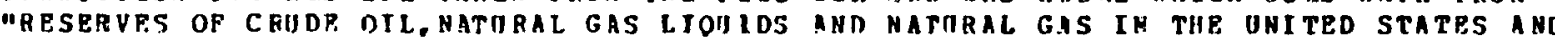
CANADA AND CAPACITY AS OP DPCEMBER 31, 1974". AMERTCAN PRTROLEUA IRSTICUTE, OASHINGTON D.C.

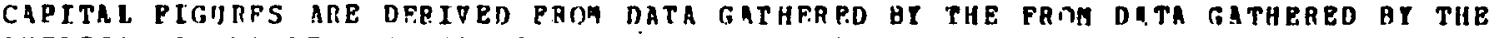
AMPRICAN PETHOLEOM TNSTITITE ANNUAL PIBLICATION $J O L N T$ ASSOCTAT TON SORTET OP THE OIL AND GAS PRODUCT IS INDIISTRE FOR 1975". TURSE DATA ARE USED AS INPUT FOR THP PIES OIL AND GAS MODFL. 
TABLB 20: HICLEAR POEL SUPPLY CUROE

IACRTHENTAL

CAFACITY SUPPORTED

(MBRAHATTS)

costs

-...-...-

79.0

(KW)

6.29

$40.0 \quad 6.41$

$12.0 \quad 6.53$

$13.0 \quad 6.90$

$15.0 \quad 7.15$

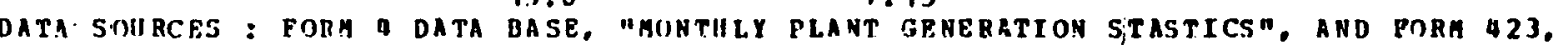

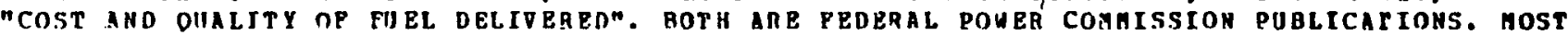
MOST RECPNT ISSUES OSED POR EIA ANBIAL ADMLAISTRATOR'S REPORT.

PRODICTION PIGURES ARE TAKEN FROA THE PIES OIL AND GAS HODEL. WHICH USES DATE PROA

"RES ERVES OP CPIIDE OII. NATURA. GAS LIOUIDS AHO HATIRAL GAS IN THE UNTTPD STATPS ANO

CANADA. AND CAPACITY AS OP DEC EMBRP 39. 197U". ARERICAH PETROLEUA IRSTITOTE. RASTIRSTON D.C.

CAPITAE PICURES ARE DERIVRD FROH DATA TATHERED BY TIIR PQOM DATA GATHERED BT THE

AHERICAN PBTROLEIIM IHSTITOTP ANAIIL PUBLICATION "JOINT ASSOCTATIOH SURVEY OP THE

OJ AND GAS PRONUCING INDUSTRT POR 1975". THESE DATA ARE OSED AS INPOT POR TRE PIES

OIL AND GAS MODEL. 
THIS PAGE

\section{WAS INTENTIONALLY LEFT BLANK}

